S1 - Data for Table 1 (note: numbering scheme differs from that of the
text)

\title{
A Visual Method to Predict the Nucleophilic Asymmetric Induction of Prochiral Electrophiles
}

\author{
Nathan Wilmot, Michael J. Marsella* \\ Deparment of Chemisty, University of California- Riverside, Riverside, CA 92521 \\ michael.marsella@ucr.edu
}

Complete Table of Test Compounds......

S1-2

Gaussian Output File of $\mathbf{1}$

Gaussian Output File of 2

Gaussian Output File of 3.

Gaussian Output File of 4 S1-25

Gaussian Output File of 5 S1-35

Gaussian Output File of 6 $\mathrm{S} 1-44$

Gaussian Output File of 7 S1-54

Gaussian Output File of 8 S1-64

Gaussian Output File of $\mathbf{9}$ S1-73

Gaussian Output File of $\mathbf{1 0}$ S1-79

Gaussian Output File of $\mathbf{1 1}$ S1-93

Gaussian Output File of $\mathbf{1 2}$ S1-99

Gaussian Output File of 13 S1-111

Gaussian Output File of $\mathbf{1 4}$ S1-125

Gaussian Output File of 15 S1-135

Gaussian Output File of $\mathbf{1 6}$ S1-145 S1-158 
Table 1a: Complete Table of Test Compounds (Note: Compound numbering differs from article text.)

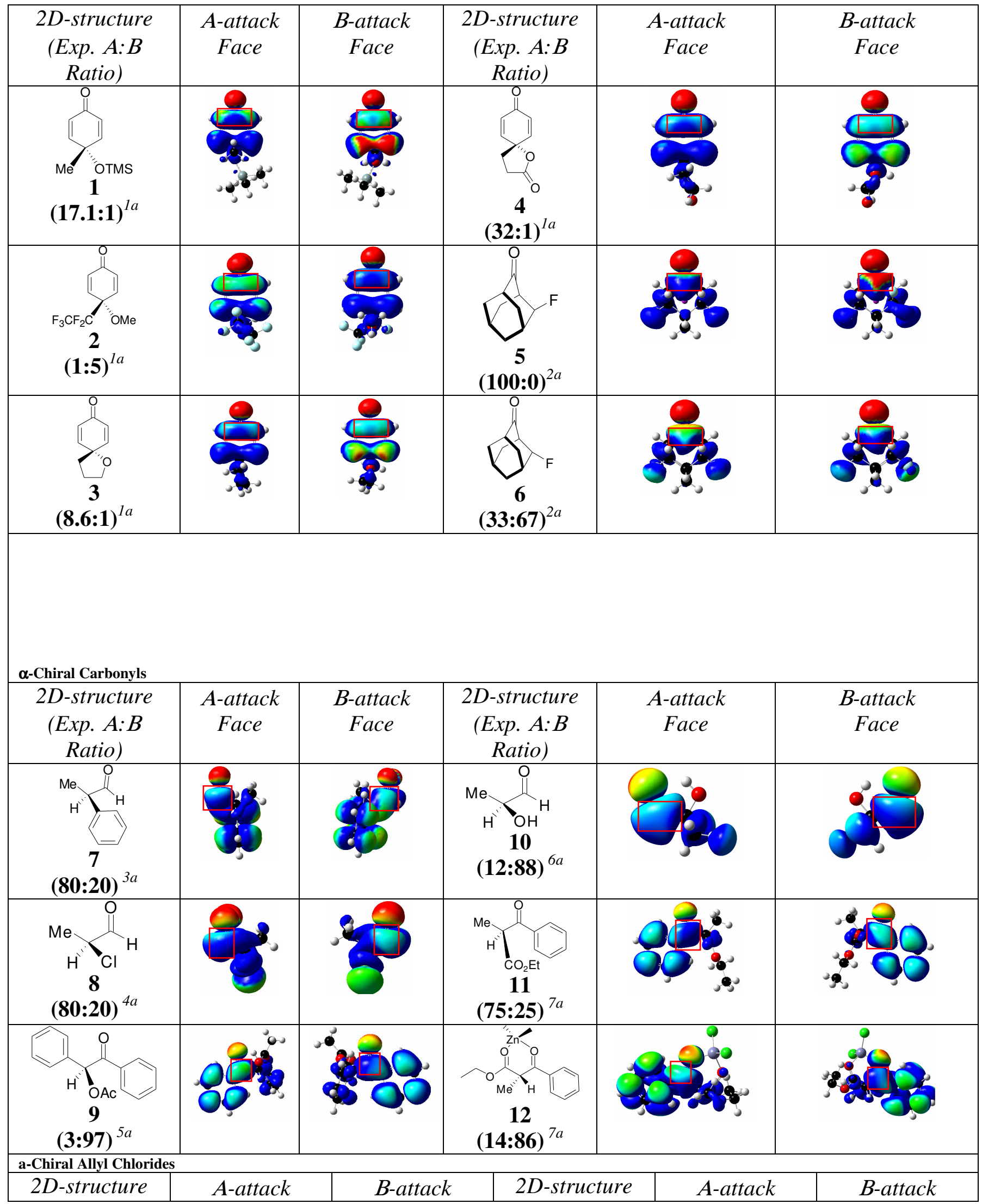




\begin{tabular}{|c|c|c|c|c|c|}
\hline $\begin{array}{c}\text { (Exp. A: } B \\
\text { Ratio })\end{array}$ & Face & Face & $\begin{array}{c}\text { (Exp. A:B } \\
\text { Ratio) }\end{array}$ & Face & Face \\
\hline $\begin{array}{c}\text { Me } \\
13 \\
(\mathbf{9 5 : 5})^{8 a}\end{array}$ & & & $\begin{array}{c}\text { ŌMOM } \\
\mathbf{1 5} \\
(\mathbf{1 0 0 : 0})^{8 a}\end{array}$ & & \\
\hline$=\operatorname{(78:22)}^{8 a}$ & & & ${ }_{(\mathbf{6 5 : 3 5})^{8 a}}$ & & \\
\hline
\end{tabular}

Table la Reference information:

1a. Wipf, P. and Kim, Y.T. J. Am. Chem. Soc. 1994, 116, 11678-11688.

2a. Kaselj, M.; leNoble, W. J. J. Org. Chem. 1996, 61, 4157-4160.

3a. Nakada, M.; Urano, Y.; Kobayashi, S.; Ohno, M. J. Am. Chem. Soc. 1988, 110, 4826-4827.

4a. $\quad$ Frenking, G.; Kohler, K. F.; Reetz, M. T. Tetrahedron 1991, 47, 9005-9018.

5a. Stocker, J. H.; Sidisunthorn, P.; Benjamin, B. M.; Collins, C. J. J. Am. Chem. Soc. 1960, 82, 3913-3918.

6a. Paquette, L. A.; Mitzel, T. M. J.Org. Chem. 1996, 61, 8799-8804.

7a. Taniguchi, M.; Fujii, H.; Oshima, K.; Utimoto, K. Tetrahedron. 1993, 49, 11169-11182.

8a. Arai, M.; Kawasuji, T.; Nakamura, E. J. Org. Chem. 1993, 58, 5121-5129. 


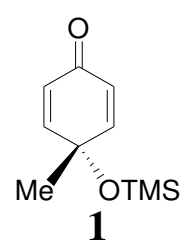

Entering Link 1 = C: $\backslash$ G03W $\backslash 11$ exe PID $=3008$.

Copyright (c) 1988,1990,1992,1993,1995,1998,2003, Gaussian, Inc. All Rights Reserved.

This is the Gaussian(R) 03 program. It is based on the the Gaussian(R) 98 system (copyright 1998, Gaussian, Inc.), the Gaussian(R) 94 system (copyright 1995, Gaussian, Inc.), the Gaussian 92(TM) system (copyright 1992, Gaussian, Inc.), the Gaussian 90(TM) system (copyright 1990, Gaussian, Inc.), the Gaussian 88(TM) system (copyright 1988, Gaussian, Inc.), the Gaussian 86(TM) system (copyright 1986, Carnegie Mellon University), and the Gaussian 82(TM) system (copyright 1983, Carnegie Mellon University). Gaussian is a federally registered trademark of Gaussian, Inc.

This software contains proprietary and confidential information, including trade secrets, belonging to Gaussian, Inc.

This software is provided under written license and may be used, copied, transmitted, or stored only in accord with that written license.

The following legend is applicable only to US Government contracts under DFARS:

\section{RESTRICTED RIGHTS LEGEND}

Use, duplication or disclosure by the US Government is subject to restrictions as set forth in subparagraph (c)(1)(ii) of the

Rights in Technical Data and Computer Software clause at DFARS 252.227-7013.

Gaussian, Inc.

Carnegie Office Park, Building 6, Pittsburgh, PA 15106 USA

The following legend is applicable only to US Government contracts under FAR:

\section{RESTRICTED RIGHTS LEGEND}

Use, reproduction and disclosure by the US Government is subject to restrictions as set forth in subparagraph (c) of the

Commercial Computer Software - Restricted Rights clause at FAR 52.227-19.

Gaussian, Inc.

Carnegie Office Park, Building 6, Pittsburgh, PA 15106 USA

Warning -- This program may not be used in any manner that competes with the business of Gaussian, Inc. or will provide 
assistance to any competitor of Gaussian, Inc. The licensee of this program is prohibited from giving any competitor of Gaussian, Inc. access to this program. By using this program, the user acknowledges that Gaussian, Inc. is engaged in the business of creating and licensing software in the field of computational chemistry and represents and warrants to the licensee that it is not a competitor of Gaussian, Inc. and that it will not use this program in any manner prohibited above.

Cite this work as:

Gaussian 03, Revision B.05,

M. J. Frisch, G. W. Trucks, H. B. Schlegel, G. E. Scuseria,

M. A. Robb, J. R. Cheeseman, J. A. Montgomery, Jr., T. Vreven,

K. N. Kudin, J. C. Burant, J. M. Millam, S. S. Iyengar, J. Tomasi,

V. Barone, B. Mennucci, M. Cossi, G. Scalmani, N. Rega,

G. A. Petersson, H. Nakatsuji, M. Hada, M. Ehara, K. Toyota,

R. Fukuda, J. Hasegawa, M. Ishida, T. Nakajima, Y. Honda, O. Kitao,

H. Nakai, M. Klene, X. Li, J. E. Knox, H. P. Hratchian, J. B. Cross,

C. Adamo, J. Jaramillo, R. Gomperts, R. E. Stratmann, O. Yazyev,

A. J. Austin, R. Cammi, C. Pomelli, J. W. Ochterski, P. Y. Ayala,

K. Morokuma, G. A. Voth, P. Salvador, J. J. Dannenberg,

V. G. Zakrzewski, S. Dapprich, A. D. Daniels, M. C. Strain,

O. Farkas, D. K. Malick, A. D. Rabuck, K. Raghavachari,

J. B. Foresman, J. V. Ortiz, Q. Cui, A. G. Baboul, S. Clifford,

J. Cioslowski, B. B. Stefanov, G. Liu, A. Liashenko, P. Piskorz,

I. Komaromi, R. L. Martin, D. J. Fox, T. Keith, M. A. Al-Laham,

C. Y. Peng, A. Nanayakkara, M. Challacombe, P. M. W. Gill,

B. Johnson, W. Chen, M. W. Wong, C. Gonzalez, and J. A. Pople, Gaussian, Inc., Pittsburgh PA, 2003.

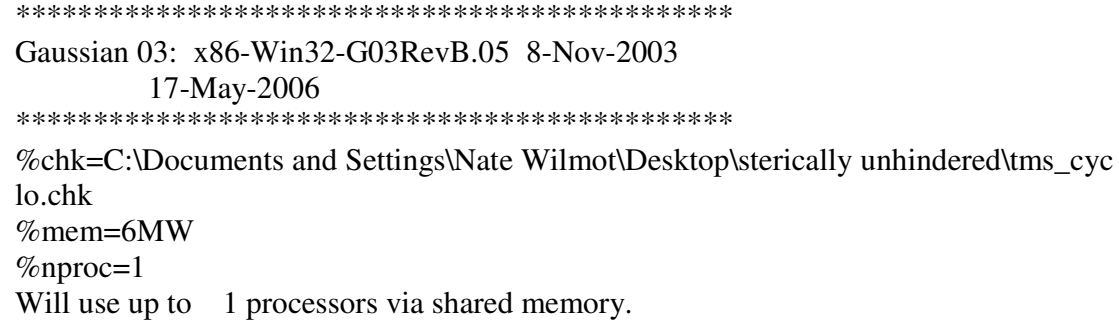

\# rhf/6-31g(d) geom=connectivity

$1 / 38=1,57=2 / 1$;

$2 / 17=6,18=5,40=1 / 2$;

$3 / 5=1,6=6,7=1,11=1,16=1,25=1,30=1 / 1,2,3$;

$4 / 7=1 / 1$;

$5 / 5=2,32=1,38=5 / 2$;

$6 / 7=2,8=2,9=2,10=2,28=1 / 1$;

$99 / 5=1,9=1 / 99$;

Title Card Required

Symbolic Z-matrix:

Charge $=0$ Multiplicity $=1$

$\begin{array}{llll}\mathrm{C} & 3.32604 & -0.22668 & -0.2041 \\ \mathrm{C} & 1.05862 & -1.03501 & 0.29747 \\ \mathrm{C} & 1.44488 & 1.33957 & -0.44386 \\ \mathrm{C} & 0.47159 & 0.35702 & 0.17684 \\ \mathrm{C} & 2.74375 & 1.07725 & -0.5973 \\ \mathrm{C} & 2.35637 & -1.29514 & 0.14071 \\ \mathrm{H} & 3.42777 & 1.81932 & -1.0259 \\ \mathrm{H} & 2.75398 & -2.31086 & 0.25663\end{array}$




\begin{tabular}{lrrl}
$\mathrm{H}$ & 0.34173 & -1.83499 & 0.5309 \\
$\mathrm{H}$ & 1.01784 & 2.29971 & -0.76059 \\
$\mathrm{O}$ & 4.52983 & -0.41522 & -0.17399 \\
$\mathrm{C}$ & 0.14146 & 0.85769 & 1.59553 \\
$\mathrm{H}$ & -0.54705 & 0.16552 & 2.1119 \\
$\mathrm{H}$ & -0.33828 & 1.84606 & 1.56714 \\
$\mathrm{H}$ & 1.04949 & 0.94308 & 2.20422 \\
$\mathrm{O}$ & -0.63983 & 0.33264 & -0.68556 \\
$\mathrm{Si}$ & -2.21008 & -0.10978 & -0.17611 \\
$\mathrm{C}$ & -3.02279 & -0.72925 & -1.77065 \\
$\mathrm{H}$ & -4.09917 & -0.86324 & -1.6301 \\
$\mathrm{H}$ & -2.60032 & -1.68855 & -2.08155 \\
$\mathrm{H}$ & -2.87527 & -0.01744 & -2.58786 \\
$\mathrm{C}$ & -2.29039 & -1.46503 & 1.15113 \\
$\mathrm{H}$ & -3.31759 & -1.59807 & 1.49873 \\
$\mathrm{H}$ & -1.67024 & -1.19682 & 2.01756 \\
$\mathrm{H}$ & -1.93871 & -2.42659 & 0.7656 \\
$\mathrm{C}$ & -3.13128 & 1.42513 & 0.44685 \\
$\mathrm{H}$ & -4.20049 & 1.21867 & 0.54689 \\
$\mathrm{H}$ & -3.01486 & 2.25974 & -0.24946 \\
$\mathrm{H}$ & -2.75721 & 1.74871 & 1.42207 \\
& & & \\
& Input orientation: & \\
\hline & & &
\end{tabular}

\begin{tabular}{|c|c|c|c|c|}
\hline \multirow{2}{*}{$\begin{array}{l}\text { Center } \\
\text { Number }\end{array}$} & \multirow{2}{*}{$\begin{array}{l}\text { Atomic } \\
\text { Number }\end{array}$} & \multirow{2}{*}{$\begin{array}{l}\text { Atomic } \\
\text { Type }\end{array}$} & \multicolumn{2}{|c|}{ Coordinates (Angstroms) } \\
\hline & & & $\begin{array}{ll}\mathrm{X} & \mathrm{Y}\end{array}$ & $\mathrm{Z}$ \\
\hline 1 & 6 & 3.326039 & -0.226681 & -0.204104 \\
\hline 2 & 6 & 1.058617 & -1.035007 & 0.297468 \\
\hline 3 & 6 & 1.444883 & 1.339569 & -0.443860 \\
\hline 4 & 6 & 0.471588 & 0.357019 & 0.176842 \\
\hline 5 & 6 & 2.743748 & 1.077249 & -0.597299 \\
\hline 6 & 6 & 2.356374 & -1.295140 & 0.140713 \\
\hline 7 & 1 & 3.427770 & 1.819320 & -1.025898 \\
\hline 8 & 1 & 2.753984 & -2.310864 & 0.256628 \\
\hline 9 & 1 & 0.341733 & -1.834991 & 0.530895 \\
\hline 10 & 1 & 1.017839 & 2.299711 & -0.760587 \\
\hline 11 & 8 & 4.529831 & -0.415216 & -0.173992 \\
\hline 12 & 6 & 0.141457 & 0.857691 & 1.595529 \\
\hline 13 & 1 & -0.547052 & 0.165521 & 2.111899 \\
\hline 14 & 1 & -0.338284 & 1.846060 & 1.567142 \\
\hline 15 & 1 & 1.049488 & 0.943077 & 2.204215 \\
\hline 16 & 8 & -0.639826 & 0.332638 & -0.685557 \\
\hline 17 & 14 & -2.210078 & -0.109781 & -0.176111 \\
\hline 18 & 6 & -3.022794 & -0.729246 & -1.770649 \\
\hline 19 & 1 & -4.099168 & -0.863235 & -1.630104 \\
\hline 20 & 1 & -2.600318 & -1.688549 & -2.081546 \\
\hline 21 & 1 & -2.875271 & -0.017439 & -2.587859 \\
\hline 22 & 6 & -2.290391 & -1.465027 & 1.151134 \\
\hline 23 & 1 & -3.317592 & -1.598074 & 1.498735 \\
\hline 24 & 1 & -1.670241 & -1.196825 & 2.017555 \\
\hline 25 & 1 & -1.938706 & -2.426588 & 0.765601 \\
\hline 26 & 6 & -3.131282 & 1.425125 & 0.446852 \\
\hline 27 & 1 & -4.200490 & 1.218669 & 0.546886 \\
\hline 28 & 1 & -3.014860 & 2.259740 & -0.249460 \\
\hline \multirow[t]{3}{*}{29} & 1 & -2.757214 & 1.748708 & 1.422072 \\
\hline & Distance & ce matrix (angstro & oms): & \\
\hline & 1 & $\begin{array}{llll}2 & 3 & 4\end{array}$ & 5 & \\
\hline $1 \mathrm{C}$ & 0.000000 & & & \\
\hline $2 \mathrm{C}$ & 2.458895 & 0.000000 & & \\
\hline $3 \mathrm{C}$ & 2.459546 & $2.517415 \quad 0.00$ & 0000 & \\
\hline $4 \mathrm{C}$ & 2.938319 & $1.515550 \quad 1.51$ & $15908 \quad 0.000$ & 0000 \\
\hline $5 \mathrm{C}$ & 1.481182 & $2.846384 \quad 1.33$ & $33944 \quad 2.506$ & $6140 \quad 0.000000$ \\
\hline
\end{tabular}


$\begin{array}{lllllll}6 & \mathrm{C} & 1.483494 & 1.332822 & 2.848549 & 2.506662 & 2.514548\end{array}$

$\begin{array}{lllllll}7 & \mathrm{H} & 2.207218 & 3.938447 & 2.121502 & 3.510544 & 1.096472\end{array}$

$\begin{array}{lllllll}8 & \mathrm{H} & 2.209828 & 2.122204 & 3.940823 & 3.511880 & 3.494082\end{array}$

$\begin{array}{lllllll}9 \mathrm{H} & 3.468856 & 1.099266 & 3.499273 & 2.224213 & 3.940005\end{array}$

$\begin{array}{lllllll}10 \mathrm{H} & 3.467004 & 3.498783 & 1.097523 & 2.225133 & 2.121282\end{array}$

$\begin{array}{lllllll}11 & \mathrm{O} & 1.218839 & 3.557492 & 3.559354 & 4.145935 & 2.365742\end{array}$

$\begin{array}{lllllll}12 & \mathrm{C} & 3.815246 & 2.471527 & 2.467839 & 1.540237 & 3.410076\end{array}$

$\begin{array}{lllllll}13 & \mathrm{H} & 4.529737 & 2.703996 & 3.446461 & 2.195163 & 4.358941\end{array}$

$\begin{array}{lllllll}14 & \mathrm{H} & 4.567365 & 3.444408 & 2.735022 & 2.192275 & 3.843800\end{array}$

$\begin{array}{lllllll}15 & \mathrm{H} & 3.514402 & 2.747468 & 2.706630 & 2.188075 & 3.276737\end{array}$

$\begin{array}{lllllll}16 & \mathrm{O} & 4.033945 & 2.391965 & 2.327732 & 1.406970 & 3.465661\end{array}$

$\begin{array}{llllllll}17 & \mathrm{Si} & 5.537421 & 3.429968 & 3.940945 & 2.744778 & 5.111441\end{array}$

$\begin{array}{llllllll}18 & \mathrm{C} & 6.558530 & 4.585686 & 5.099069 & 4.145286 & 6.155744\end{array}$

$\begin{array}{llllllll}19 & \mathrm{H} & 7.587646 & 5.508882 & 6.082436 & 5.064176 & 7.187327\end{array}$

$\begin{array}{llllllll}20 & \mathrm{H} & 6.386200 & 4.413007 & 5.311795 & 4.326808 & 6.197715\end{array}$

$\begin{array}{llllllll}21 & \mathrm{H} & 6.646978 & 4.983576 & 5.010183 & 4.357207 & 6.060862\end{array}$

$\begin{array}{llllllll}22 & \mathrm{C} & 5.908846 & 3.482746 & 4.935792 & 3.449294 & 5.904469\end{array}$

$\begin{array}{llllllll}23 & \mathrm{H} & 6.994155 & 4.572887 & 5.923225 & 4.464042 & 6.949141\end{array}$

$\begin{array}{lllllll}24 & \mathrm{H} & 5.553355 & 3.229791 & 4.711248 & 3.223366 & 5.611789\end{array}$

$\begin{array}{llllllll}25 & \mathrm{H} & 5.787699 & 3.337603 & 5.205325 & 3.728890 & 6.004977\end{array}$

$\begin{array}{lllllll}26 & \mathrm{C} & 6.696954 & 4.861051 & 4.662829 & 3.767549 & 5.977227\end{array}$

$\begin{array}{lllllll}27 & \mathrm{H} & 7.700757 & 5.727082 & 5.732926 & 4.765258 & 7.039290\end{array}$

$\begin{array}{llllllll}28 & \mathrm{H} & 6.811119 & 5.267608 & 4.557830 & 3.994670 & 5.889044\end{array}$

$\begin{array}{llllllll}29 & \mathrm{H} & 6.599438 & 4.855344 & 4.615920 & 3.729954 & 5.898246\end{array}$

$6 \mathrm{C} \quad 0.000000$

$\begin{array}{llll}7 \mathrm{H} & 3.494100 & 0.000000\end{array}$

$\begin{array}{lllll}8 & \mathrm{H} & 1.096916 & 4.376903 & 0.000000\end{array}$

$\begin{array}{llllll}9 \mathrm{H} & 2.121900 & 5.030031 & 2.473991 & 0.000000\end{array}$

$\begin{array}{lllllll}10 & \mathrm{H} & 3.940428 & 2.471626 & 5.030540 & 4.384154 & 0.000000\end{array}$

$\begin{array}{llllllll}11 & \mathrm{O} & 2.365846 & 2.633141 & 2.632974 & 4.478035 & 4.477612\end{array}$

$\begin{array}{lllllll}12 & \mathrm{C} & 3.414239 & 4.312361 & 4.319455 & 2.902428 & 2.898059\end{array}$

$\begin{array}{llllllll}13 & \mathrm{H} & 3.801182 & 5.327291 & 4.524528 & 2.700289 & 3.905742\end{array}$

$\begin{array}{lllllll}14 & \mathrm{H} & 4.377558 & 4.572498 & 5.344117 & 3.884117 & 2.731884\end{array}$

$\begin{array}{lllllll}15 & \mathrm{H} & 3.312945 & 4.105808 & 4.157708 & 3.319424 & 3.260599\end{array}$

$\begin{array}{lllllll}16 & \mathrm{O} & 3.508503 & 4.344122 & 4.403835 & 2.672420 & 2.573491\end{array}$

$\begin{array}{llllllll}17 & \mathrm{Si} & 4.728417 & 6.019045 & 5.447379 & 3.160371 & 4.070223\end{array}$

$\begin{array}{llllllll}18 & \mathrm{C} & 5.736635 & 6.975645 & 6.323174 & 4.223721 & 5.149905\end{array}$

$\begin{array}{lllllll}19 & \mathrm{H} & 6.707932 & 8.013486 & 7.254039 & 5.033470 & 6.078162\end{array}$

$\begin{array}{lllllll}20 \mathrm{H} & 5.446283 & 7.053891 & 5.875617 & 3.937252 & 5.544566\end{array}$

$\begin{array}{lllllll}21 & \mathrm{H} & 6.037196 & 6.748462 & 6.711141 & 4.835208 & 4.885122\end{array}$

$\begin{array}{lllllll}22 & \mathrm{C} & 4.758386 & 6.944334 & 5.192427 & 2.729404 & 5.363984\end{array}$

$\begin{array}{llllllll}23 & \mathrm{H} & 5.842078 & 7.971967 & 6.238184 & 3.792559 & 6.252458\end{array}$

$\begin{array}{lllllll}24 & \mathrm{H} & 4.443627 & 6.659538 & 4.890369 & 2.581754 & 5.212448\end{array}$

$\begin{array}{lllllll}25 & \mathrm{H} & 4.485350 & 7.073632 & 4.721629 & 2.367588 & 5.779993\end{array}$

$\begin{array}{llllllll}26 & \mathrm{C} & 6.132530 & 6.733910 & 6.973532 & 4.764163 & 4.408856\end{array}$

$\begin{array}{llllllll}27 & \mathrm{H} & 7.033966 & 7.811837 & 7.804266 & 5.473288 & 5.487176\end{array}$

$\begin{array}{lllllll}28 & \mathrm{H} & 6.452872 & 6.504176 & 7.377405 & 5.351867 & 4.065158\end{array}$

$\begin{array}{llllllll}29 & \mathrm{H} & 6.087337 & 6.652186 & 6.943464 & 4.820847 & 4.395297\end{array}$

$11 \mathrm{O} \quad 0.000000$

$\begin{array}{llll}12 & \mathrm{C} & 4.899931 & 0.000000\end{array}$

$\begin{array}{lllll}13 & \mathrm{H} & 5.597972 & 1.104437 & 0.000000\end{array}$

$\begin{array}{llllll}14 \mathrm{H} & 5.643001 & 1.099013 & 1.778919 & 0.000000\end{array}$

$\begin{array}{lllllll}15 & \mathrm{H} & 4.428727 & 1.096499 & 1.778216 & 1.774021 & 0.000000\end{array}$

$\begin{array}{lllllll}16 & \mathrm{O} & 5.248461 & 2.467678 & 2.803978 & 2.730572 & 3.402528\end{array}$

$\begin{array}{llllllll}17 & \mathrm{Si} & 6.746827 & 3.099101 & 2.841907 & 3.219916 & 4.171238\end{array}$

$\begin{array}{llllllll}18 \mathrm{C} & 7.725936 & 4.884875 & 4.690851 & 4.997965 & 5.931247\end{array}$

$\begin{array}{llllllll}19 & \mathrm{H} & 8.762455 & 5.599035 & 5.261032 & 5.630890 & 6.668839\end{array}$

$\begin{array}{lllllll}20 & \mathrm{H} & 7.489938 & 5.246098 & 5.023789 & 5.560862 & 6.214039\end{array}$

$\begin{array}{llllllll}21 & \mathrm{H} & 7.798751 & 5.231370 & 5.248028 & 5.212770 & 6.268197\end{array}$

$\begin{array}{lllllll}22 & \mathrm{C} & 7.026628 & 3.392108 & 2.573129 & 3.866146 & 4.250028\end{array}$

$\begin{array}{llllllll}23 & \mathrm{H} & 8.110439 & 4.243250 & 3.340978 & 4.554450 & 5.101621\end{array}$

$\begin{array}{lllllll}24 & \mathrm{H} & 6.622287 & 2.771532 & 1.768175 & 3.352034 & 3.465681\end{array}$ 


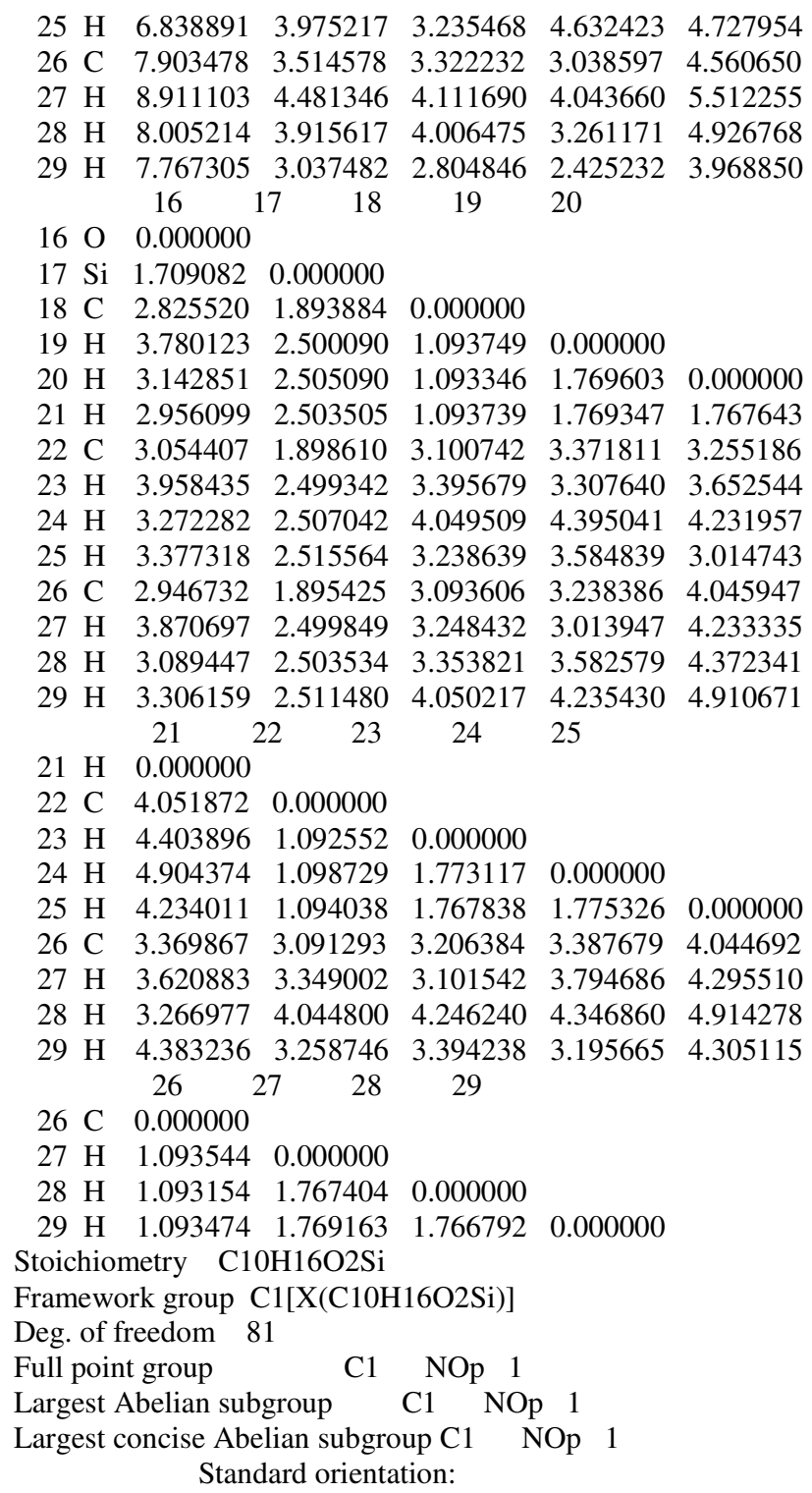

$\begin{array}{cccccc}1 & 6 & 0 & 3.326039 & -0.226681 & -0.204104 \\ 2 & 6 & 0 & 1.058617 & -1.035007 & 0.297468 \\ 3 & 6 & 0 & 1.444883 & 1.339569 & -0.443860 \\ 4 & 6 & 0 & 0.471588 & 0.357019 & 0.176842 \\ 5 & 6 & 0 & 2.743748 & 1.077249 & -0.597299 \\ 6 & 6 & 0 & 2.356374 & -1.295140 & 0.140713 \\ 7 & 1 & 0 & 3.427770 & 1.819320 & -1.025898 \\ 8 & 1 & 0 & 2.753984 & -2.310864 & 0.256628 \\ 9 & 1 & 0 & 0.341733 & -1.834991 & 0.530895 \\ 10 & 1 & 0 & 1.017839 & 2.299711 & -0.760587 \\ 11 & 8 & 0 & 4.529831 & -0.415216 & -0.173992 \\ 12 & 6 & 0 & 0.141457 & 0.857691 & 1.595529 \\ 13 & 1 & 0 & -0.547052 & 0.165521 & 2.111899 \\ 14 & 1 & 0 & -0.338284 & 1.846060 & 1.567142 \\ 15 & 1 & 0 & 1.049488 & 0.943077 & 2.204215 \\ 16 & 8 & 0 & -0.639826 & 0.332638 & -0.685557 \\ 17 & 14 & 0 & -2.210078 & -0.109781 & -0.176111 \\ 18 & 6 & 0 & -3.022794 & -0.729246 & -1.770649\end{array}$




$\begin{array}{rrrrrr}19 & 1 & 0 & -4.099168 & -0.863235 & -1.630104 \\ 20 & 1 & 0 & -2.600318 & -1.688549 & -2.081546 \\ 21 & 1 & 0 & -2.875271 & -0.017439 & -2.587859 \\ 22 & 6 & 0 & -2.290391 & -1.465027 & 1.151134 \\ 23 & 1 & 0 & -3.317592 & -1.598074 & 1.498735 \\ 24 & 1 & 0 & -1.670241 & -1.196825 & 2.017555 \\ 25 & 1 & 0 & -1.938706 & -2.426588 & 0.765601 \\ 26 & 6 & 0 & -3.131282 & 1.425125 & 0.446852 \\ 27 & 1 & 0 & -4.200490 & 1.218669 & 0.546886 \\ 28 & 1 & 0 & -3.014860 & 2.259740 & -0.249460 \\ 29 & 1 & 0 & -2.757214 & 1.748708 & 1.422072\end{array}$

Rotational constants (GHZ): $\quad 1.5458247 \quad 0.3775331 \quad 0.3652947$

Standard basis: 6-31G(d) $(6 \mathrm{D}, 7 \mathrm{~F})$

There are 231 symmetry adapted basis functions of A symmetry.

Integral buffers will be 262144 words long.

Raffenetti 1 integral format.

Two-electron integral symmetry is turned on.

231 basis functions, 452 primitive gaussians, 231 cartesian basis functions

53 alpha electrons 53 beta electrons

nuclear repulsion energy $\quad 919.0408278157$ Hartrees.

NAtoms $=29$ NActive $=29 \mathrm{NUniq}=29 \mathrm{SFac}=1.00 \mathrm{D}+00 \mathrm{NAtFMM}=60 \mathrm{Big}=\mathrm{F}$

One-electron integrals computed using PRISM.

NBasis $=231$ RedAO $=\mathrm{T}$ NBF $=231$

NBsUse $=231$ 1.00D-06 NBFU $=231$

Harris functional with IExCor $=205$ diagonalized for initial guess.

ExpMin= 7.78D-02 ExpMax $=1.61 \mathrm{D}+04 \mathrm{ExpMxC}=2.43 \mathrm{D}+03 \mathrm{IAcc}=2 \mathrm{IRadAn}=\quad 4$ AccDes $=0.00 \mathrm{D}+00$
HarFok: IExCor $=205$ AccDes $=0.00 \mathrm{D}+00 \mathrm{IRadAn}=\quad 4 \mathrm{IDOV}=1$

ScaDFX $=1.0000001 .0000001 .0000001 .000000$

Initial guess orbital symmetries:

Occupied (A) (A) (A) (A) (A) (A) (A) (A) (A) (A) $(A)(A)$

(A) $(\mathrm{A})(\mathrm{A})(\mathrm{A})(\mathrm{A})(\mathrm{A})(\mathrm{A})(\mathrm{A})(\mathrm{A})(\mathrm{A})(\mathrm{A})(\mathrm{A})$

(A) $(\mathrm{A})(\mathrm{A})(\mathrm{A})(\mathrm{A})(\mathrm{A})(\mathrm{A})(\mathrm{A})(\mathrm{A})(\mathrm{A})(\mathrm{A})(\mathrm{A})$

(A) $(\mathrm{A})(\mathrm{A})(\mathrm{A})(\mathrm{A})(\mathrm{A})(\mathrm{A})(\mathrm{A})(\mathrm{A})(\mathrm{A})(\mathrm{A})(\mathrm{A})$

(A) $(\mathrm{A})(\mathrm{A})(\mathrm{A})(\mathrm{A})$

Virtual (A) (A) (A) (A) $(\mathrm{A})(\mathrm{A})(\mathrm{A})(\mathrm{A})(\mathrm{A})(\mathrm{A})(\mathrm{A})(\mathrm{A})$

(A) $(\mathrm{A})(\mathrm{A})(\mathrm{A})(\mathrm{A})(\mathrm{A})(\mathrm{A})(\mathrm{A})(\mathrm{A})(\mathrm{A})(\mathrm{A})(\mathrm{A})$

(A) $(\mathrm{A})(\mathrm{A})(\mathrm{A})(\mathrm{A})(\mathrm{A})(\mathrm{A})(\mathrm{A})(\mathrm{A})(\mathrm{A})(\mathrm{A})(\mathrm{A})$

(A) (A) (A) (A) (A) (A) (A) (A) (A) (A) (A) (A)

(A) $(\mathrm{A})(\mathrm{A})(\mathrm{A})(\mathrm{A})(\mathrm{A})(\mathrm{A})(\mathrm{A})(\mathrm{A})(\mathrm{A})(\mathrm{A})(\mathrm{A})$

(A) $(\mathrm{A})(\mathrm{A})(\mathrm{A})(\mathrm{A})(\mathrm{A})(\mathrm{A})(\mathrm{A})(\mathrm{A})(\mathrm{A})(\mathrm{A})(\mathrm{A})$

(A) (A) (A) (A) (A) (A) (A) (A) (A) (A) (A) (A)

(A) $(\mathrm{A})(\mathrm{A})(\mathrm{A})(\mathrm{A})(\mathrm{A})(\mathrm{A})(\mathrm{A})(\mathrm{A})(\mathrm{A})(\mathrm{A})(\mathrm{A})$

(A) (A) (A) (A) (A) (A) (A) (A) (A) (A) (A) (A)

(A) $(A)(A)(A)(A)(A)(A)(A)(A)(A)(A)(A)$

(A) $(\mathrm{A})(\mathrm{A})(\mathrm{A})(\mathrm{A})(\mathrm{A})(\mathrm{A})(\mathrm{A})(\mathrm{A})(\mathrm{A})(\mathrm{A})(\mathrm{A})$

(A) (A) (A) (A) (A) (A) (A) (A) (A) (A) (A) (A)

(A) $(\mathrm{A})(\mathrm{A})(\mathrm{A})(\mathrm{A})(\mathrm{A})(\mathrm{A})(\mathrm{A})(\mathrm{A})(\mathrm{A})(\mathrm{A})(\mathrm{A})$

(A) $(\mathrm{A})(\mathrm{A})(\mathrm{A})(\mathrm{A})(\mathrm{A})(\mathrm{A})(\mathrm{A})(\mathrm{A})(\mathrm{A})(\mathrm{A})(\mathrm{A})$

(A) (A) (A) (A) (A) (A) (A) (A) (A) (A)

The electronic state of the initial guess is $1-\mathrm{A}$.

Warning! Cutoffs for single-point calculations used.

Requested convergence on RMS density matrix=1.00D-04 within 128 cycles.

Requested convergence on MAX density matrix $=1.00 \mathrm{D}-02$.

Requested convergence on energy=5.00D-05.

No special actions if energy rises.

SCF Done: $\mathrm{E}(\mathrm{RHF})=-826.670090094 \quad$ A.U. after 7 cycles

Convg $=0.5859 \mathrm{D}-05 \quad-\mathrm{V} / \mathrm{T}=2.0015$

$\mathrm{S} * 2=0.0000$

***************************************************************************

Population analysis using the SCF density. 
Orbital symmetries:

Occupied (A) (A) (A) (A) (A) (A) (A) (A) (A) (A) (A) (A)

(A) $(\mathrm{A})(\mathrm{A})(\mathrm{A})(\mathrm{A})(\mathrm{A})(\mathrm{A})(\mathrm{A})(\mathrm{A})(\mathrm{A})(\mathrm{A})(\mathrm{A})$

(A) (A) (A) (A) (A) (A) (A) (A) (A) (A) (A) (A)

$(\mathrm{A})(\mathrm{A})(\mathrm{A})(\mathrm{A})(\mathrm{A})(\mathrm{A})(\mathrm{A})(\mathrm{A})(\mathrm{A})(\mathrm{A})(\mathrm{A})(\mathrm{A})$

(A) $(\mathrm{A})(\mathrm{A})(\mathrm{A})(\mathrm{A})$

Virtual (A) (A) (A) (A) (A) (A) (A) (A) (A) (A) (A) (A)

(A) $(\mathrm{A})(\mathrm{A})(\mathrm{A})(\mathrm{A})(\mathrm{A})(\mathrm{A})(\mathrm{A})(\mathrm{A})(\mathrm{A})(\mathrm{A})(\mathrm{A})$

(A) $(\mathrm{A})(\mathrm{A})(\mathrm{A})(\mathrm{A})(\mathrm{A})(\mathrm{A})(\mathrm{A})(\mathrm{A})(\mathrm{A})(\mathrm{A})(\mathrm{A})$

(A) $(\mathrm{A})(\mathrm{A})(\mathrm{A})(\mathrm{A})(\mathrm{A})(\mathrm{A})(\mathrm{A})(\mathrm{A})(\mathrm{A})(\mathrm{A})(\mathrm{A})$

(A) $(\mathrm{A})(\mathrm{A})(\mathrm{A})(\mathrm{A})(\mathrm{A})(\mathrm{A})(\mathrm{A})(\mathrm{A})(\mathrm{A})(\mathrm{A})(\mathrm{A})$

(A) $(\mathrm{A})(\mathrm{A})(\mathrm{A})(\mathrm{A})(\mathrm{A})(\mathrm{A})(\mathrm{A})(\mathrm{A})(\mathrm{A})(\mathrm{A})(\mathrm{A})$

(A) (A) (A) (A) (A) (A) (A) (A) (A) (A) (A) (A)

(A) $(\mathrm{A})(\mathrm{A})(\mathrm{A})(\mathrm{A})(\mathrm{A})(\mathrm{A})(\mathrm{A})(\mathrm{A})(\mathrm{A})(\mathrm{A})(\mathrm{A})$

(A) $(\mathrm{A})(\mathrm{A})(\mathrm{A})(\mathrm{A})(\mathrm{A})(\mathrm{A})(\mathrm{A})(\mathrm{A})(\mathrm{A})(\mathrm{A})(\mathrm{A})$

(A) (A) (A) (A) (A) (A) (A) (A) (A) (A) (A) (A)

(A) $(\mathrm{A})(\mathrm{A})(\mathrm{A})(\mathrm{A})(\mathrm{A})(\mathrm{A})(\mathrm{A})(\mathrm{A})(\mathrm{A})(\mathrm{A})(\mathrm{A})$

(A) $(\mathrm{A})(\mathrm{A})(\mathrm{A})(\mathrm{A})(\mathrm{A})(\mathrm{A})(\mathrm{A})(\mathrm{A})(\mathrm{A})(\mathrm{A})(\mathrm{A})$

(A) (A) (A) (A) (A) (A) (A) (A) (A) (A) (A) (A)

(A) $(\mathrm{A})(\mathrm{A})(\mathrm{A})(\mathrm{A})(\mathrm{A})(\mathrm{A})(\mathrm{A})(\mathrm{A})(\mathrm{A})(\mathrm{A})(\mathrm{A})$

(A) (A) (A) (A) (A) (A) (A) (A) (A) (A)

The electronic state is 1-A.

Alpha occ. eigenvalues -- -68.79325 -20.56673 -20.54500 -11.32978 -11.32231

Alpha occ. eigenvalues -- -11.26344 -11.26091 -11.24382 -11.24285 -11.23977

Alpha occ. eigenvalues -- $-11.22060-11.20992-11.20399-6.14235-4.24967$

Alpha occ. eigenvalues -- $-4.24868 \quad-4.24835 \quad-1.37456 \quad-1.36569-1.13646$

$\begin{array}{llllll}\text { Alpha occ. eigenvalues -- } & -1.04238 & -1.02450 & -0.97742 & -0.93838 & -0.92841\end{array}$

$\begin{array}{lllllll}\text { Alpha occ. eigenvalues -- } & -0.91019 & -0.83588 & -0.81423 & -0.71116 & -0.68563\end{array}$

$\begin{array}{lllllll}\text { Alpha occ. eigenvalues -- } & -0.66699 & -0.65853 & -0.62229 & -0.60736 & -0.59789\end{array}$

Alpha occ. eigenvalues -- $\quad-0.59236 \quad-0.58166 \quad-0.57520 \quad-0.56535 \quad-0.56349$

$\begin{array}{lllllll}\text { Alpha occ. eigenvalues -- } & -0.55003 & -0.54793 & -0.54012 & -0.53803 & -0.53508\end{array}$

$\begin{array}{llllll}\text { Alpha occ. eigenvalues -- } & -0.53024 & -0.47317 & -0.46870 & -0.43590 & -0.42181\end{array}$

Alpha occ. eigenvalues -- $-0.40834-0.38114-0.37449$

$\begin{array}{lllllll}\text { Alpha virt. eigenvalues -- } & 0.08167 & 0.16567 & 0.19220 & 0.21255 & 0.22290\end{array}$

$\begin{array}{lllllll}\text { Alpha virt. eigenvalues -- } & 0.22956 & 0.24464 & 0.25021 & 0.26612 & 0.27111\end{array}$

$\begin{array}{lllllll}\text { Alpha virt. eigenvalues -- } & 0.28744 & 0.29327 & 0.30207 & 0.30460 & 0.30984\end{array}$

$\begin{array}{lllllll}\text { Alpha virt. eigenvalues -- } & 0.31962 & 0.32185 & 0.33511 & 0.34313 & 0.35513\end{array}$

$\begin{array}{lllllll}\text { Alpha virt. eigenvalues -- } & 0.37223 & 0.37463 & 0.38238 & 0.40863 & 0.43207\end{array}$

$\begin{array}{lllllll}\text { Alpha virt. eigenvalues -- } & 0.43657 & 0.46526 & 0.47729 & 0.50424 & 0.52525\end{array}$

$\begin{array}{lllllll}\text { Alpha virt. eigenvalues -- } & 0.55394 & 0.55524 & 0.56410 & 0.60027 & 0.64652\end{array}$

$\begin{array}{lllllll}\text { Alpha virt. eigenvalues -- } & 0.66898 & 0.68884 & 0.69935 & 0.72036 & 0.74186\end{array}$

$\begin{array}{lllllll}\text { Alpha virt. eigenvalues -- } & 0.74565 & 0.75988 & 0.78135 & 0.80016 & 0.80756\end{array}$

$\begin{array}{lllllll}\text { Alpha virt. eigenvalues -- } & 0.83505 & 0.84035 & 0.85367 & 0.86236 & 0.86834\end{array}$

$\begin{array}{lllllll}\text { Alpha virt. eigenvalues -- } & 0.89744 & 0.92316 & 0.93693 & 0.94621 & 0.95099\end{array}$

$\begin{array}{lllllll}\text { Alpha virt. eigenvalues -- } & 0.95926 & 0.97635 & 0.98077 & 0.98442 & 1.00528\end{array}$

$\begin{array}{lllllll}\text { Alpha virt. eigenvalues -- } & 1.01310 & 1.01656 & 1.04057 & 1.08167 & 1.10546\end{array}$

$\begin{array}{llllll}\text { Alpha virt. eigenvalues -- } & 1.11411 & 1.11723 & 1.12512 & 1.14067 & 1.14608\end{array}$

$\begin{array}{lllllll}\text { Alpha virt. eigenvalues -- } & 1.15045 & 1.16004 & 1.17081 & 1.17907 & 1.18518\end{array}$

$\begin{array}{lllllll}\text { Alpha virt. eigenvalues -- } & 1.19273 & 1.19682 & 1.20019 & 1.20206 & 1.22016\end{array}$

$\begin{array}{lllllll}\text { Alpha virt. eigenvalues -- } & 1.22824 & 1.23567 & 1.24845 & 1.27068 & 1.27413\end{array}$

$\begin{array}{lllllll}\text { Alpha virt. eigenvalues -- } & 1.30258 & 1.31223 & 1.31440 & 1.33854 & 1.36097\end{array}$

$\begin{array}{lllllll}\text { Alpha virt. eigenvalues -- } & 1.37276 & 1.38964 & 1.44098 & 1.46264 & 1.48749\end{array}$

$\begin{array}{llllll}\text { Alpha virt. eigenvalues -- } & 1.51029 & 1.53995 & 1.56433 & 1.56974 & 1.61111\end{array}$

$\begin{array}{lllllll}\text { Alpha virt. eigenvalues -- } & 1.64206 & 1.67290 & 1.71342 & 1.76142 & 1.77805\end{array}$

$\begin{array}{lllllll}\text { Alpha virt. eigenvalues -- } & 1.78521 & 1.85055 & 1.90544 & 1.93082 & 1.94483\end{array}$

$\begin{array}{lllllll}\text { Alpha virt. eigenvalues -- } & 1.98143 & 1.99764 & 2.00931 & 2.03848 & 2.05878\end{array}$

$\begin{array}{lllllll}\text { Alpha virt. eigenvalues -- } & 2.08183 & 2.09558 & 2.10602 & 2.11892 & 2.13617\end{array}$

$\begin{array}{llllll}\text { Alpha virt. eigenvalues -- } & 2.14820 & 2.16551 & 2.21077 & 2.23054 & 2.23625\end{array}$

$\begin{array}{lllllll}\text { Alpha virt. eigenvalues -- } & 2.25375 & 2.26545 & 2.29322 & 2.32556 & 2.35226\end{array}$

$\begin{array}{lllllll}\text { Alpha virt. eigenvalues -- } & 2.37438 & 2.39431 & 2.41091 & 2.44380 & 2.50069\end{array}$

$\begin{array}{llllll}\text { Alpha virt. eigenvalues -- } & 2.50268 & 2.52412 & 2.54211 & 2.55332 & 2.57028\end{array}$

Alpha virt. eigenvalues -- $2.60260 \quad 2.61414 \quad 2.61565 \quad 2.62507 \quad 2.63087$ 
$\begin{array}{lllllll}\text { Alpha virt. eigenvalues -- } & 2.63730 & 2.65299 & 2.66699 & 2.70692 & 2.75319\end{array}$

$\begin{array}{lllllll}\text { Alpha virt. eigenvalues -- } & 2.75636 & 2.85856 & 2.91476 & 2.94907 & 2.95589\end{array}$

$\begin{array}{lllllll}\text { Alpha virt. eigenvalues -- } & 2.97846 & 3.03629 & 3.11575 & 3.13085 & 3.15957\end{array}$

$\begin{array}{lllllll}\text { Alpha virt. eigenvalues -- } & 3.23178 & 3.28158 & 3.38000 & 3.43909 & 3.51443\end{array}$

$\begin{array}{lllllll}\text { Alpha virt. eigenvalues -- } & 3.64330 & 4.36930 & 4.47865 & 4.57093 & 4.57871\end{array}$

$\begin{array}{lllllll}\text { Alpha virt. eigenvalues -- } & 4.60584 & 4.64371 & 4.67658 & 4.70894 & 4.80242\end{array}$

Alpha virt. eigenvalues -- $4.84173 \quad 4.98467 \quad 5.21366$

Condensed to atoms (all electrons):
$\begin{array}{llllll}1 & 2 & 3 & 4 & 5 & 6\end{array}$

$\begin{array}{llllllll}1 & C & 4.258620 & -0.038653 & -0.037491 & -0.011895 & 0.412678 & 0.411510\end{array}$

$\begin{array}{llllllll}2 & \mathrm{C} & -0.038653 & 5.102261 & -0.077125 & 0.386490 & 0.013297 & 0.559506\end{array}$

$\begin{array}{llllllll}3 & \mathrm{C} & -0.037491 & -0.077125 & 5.038882 & 0.420694 & 0.558563 & 0.012406\end{array}$

$\begin{array}{llllllll}4 & \mathrm{C} & -0.011895 & 0.386490 & 0.420694 & 4.655434 & -0.044322 & -0.039240\end{array}$

$\begin{array}{llllllll}5 & \mathrm{C} & 0.412678 & 0.013297 & 0.558563 & -0.044322 & 5.144511 & -0.114918\end{array}$

$\begin{array}{llllllll}6 \mathrm{C} & 0.411510 & 0.559506 & 0.012406 & -0.039240 & -0.114918 & 5.131188\end{array}$

$\begin{array}{llllllll}7 & \mathrm{H} & -0.024440 & -0.000367 & -0.029165 & 0.002494 & 0.377185 & 0.003603\end{array}$

$\begin{array}{llllllll}8 \mathrm{H} & -0.023053 & -0.030574 & -0.000301 & 0.002152 & 0.003518 & 0.377526\end{array}$

$\begin{array}{llllllll}9 \mathrm{H} & 0.001146 & 0.376188 & 0.002607 & -0.029904 & -0.000263 & -0.017422\end{array}$

$\begin{array}{llllllll}10 \mathrm{H} & 0.001221 & 0.002980 & 0.374998 & -0.028313 & -0.020498 & -0.000302\end{array}$

$\begin{array}{lllllllll}11 & \mathrm{O} & 0.530693 & 0.004315 & 0.004101 & -0.000020 & -0.060516 & -0.060507\end{array}$

$\begin{array}{llllllll}12 & \mathrm{C} & 0.000333 & -0.086349 & -0.079210 & 0.358464 & -0.002635 & -0.002337\end{array}$

$\begin{array}{llllllll}13 \mathrm{H} & -0.000021 & -0.008549 & 0.006021 & -0.033187 & -0.000005 & 0.000198\end{array}$

$\begin{array}{llllllll}14 \mathrm{H} & -0.000019 & 0.006093 & -0.005986 & -0.032577 & 0.000332 & -0.000015\end{array}$

$\begin{array}{llllllll}15 \mathrm{H} & 0.000061 & 0.001158 & -0.000349 & -0.026734 & 0.000921 & 0.000739\end{array}$

$\begin{array}{llllllll}16 & \mathrm{O} & 0.000207 & -0.047296 & -0.053956 & 0.159193 & 0.001263 & 0.001103\end{array}$

$\begin{array}{llllllll}17 \mathrm{Si} & -0.000024 & -0.002670 & 0.000181 & -0.026806 & 0.000180 & 0.000571\end{array}$

$\begin{array}{llllllll}18 & \mathrm{C} & 0.000000 & -0.000174 & -0.000003 & 0.001234 & 0.000000 & -0.000009\end{array}$

$\begin{array}{llllllll}19 \mathrm{H} & 0.000000 & 0.000001 & 0.000000 & -0.000011 & 0.000000 & 0.000000\end{array}$

$\begin{array}{llllllll}20 \mathrm{H} & 0.000000 & 0.000028 & -0.000001 & -0.000071 & 0.000000 & 0.000000\end{array}$

$\begin{array}{llllllll}21 & \mathrm{H} & 0.000000 & 0.000003 & 0.000004 & -0.000038 & 0.000000 & 0.000000\end{array}$

$\begin{array}{llllllll}22 & \mathrm{C} & -0.000002 & -0.001291 & -0.000075 & -0.000511 & 0.000005 & 0.000012\end{array}$

$\begin{array}{llllllll}23 \mathrm{H} & 0.000000 & 0.000071 & 0.000001 & 0.000000 & 0.000000 & 0.000000\end{array}$

$\begin{array}{llllllll}24 \mathrm{H} & 0.000000 & 0.000039 & -0.000034 & 0.000698 & 0.000000 & -0.000060\end{array}$

$\begin{array}{llllllll}25 & \mathrm{H} & 0.000000 & -0.000928 & -0.000007 & -0.000208 & 0.000000 & -0.000013\end{array}$

$\begin{array}{llllllll}26 \mathrm{C} & 0.000000 & -0.000122 & -0.000052 & -0.000188 & 0.000000 & 0.000000\end{array}$

$\begin{array}{llllllll}27 & \mathrm{H} & 0.000000 & 0.000001 & 0.000001 & 0.000003 & 0.000000 & 0.000000\end{array}$

$\begin{array}{llllllll}28 \mathrm{H} & 0.000000 & -0.000001 & 0.000019 & 0.000072 & 0.000000 & 0.000000\end{array}$

$\begin{array}{llllllll}29 & \mathrm{H} & 0.000000 & -0.000014 & 0.000008 & -0.000005 & 0.000000 & 0.000000\end{array}$ $\begin{array}{llllll}7 & 8 & 9 & 10 & 11 & 12\end{array}$

$\begin{array}{llllllll}1 & \mathrm{C} & -0.024440 & -0.023053 & 0.001146 & 0.001221 & 0.530693 & 0.000333\end{array}$

$\begin{array}{llllllll}2 & \mathrm{C} & -0.000367 & -0.030574 & 0.376188 & 0.002980 & 0.004315 & -0.086349\end{array}$

$\begin{array}{llllllll}3 & \mathrm{C} & -0.029165 & -0.000301 & 0.002607 & 0.374998 & 0.004101 & -0.079210\end{array}$

$\begin{array}{llllllll}4 & \mathrm{C} & 0.002494 & 0.002152 & -0.029904 & -0.028313 & -0.000020 & 0.358464\end{array}$

$\begin{array}{llllllll}5 & \mathrm{C} & 0.377185 & 0.003518 & -0.000263 & -0.020498 & -0.060516 & -0.002635\end{array}$

$\begin{array}{llllllll}6 & \mathrm{C} & 0.003603 & 0.377526 & -0.017422 & -0.000302 & -0.060507 & -0.002337\end{array}$

$\begin{array}{llllllll}7 & \mathrm{H} & 0.446545 & -0.000054 & 0.000007 & -0.002231 & 0.001357 & -0.000158\end{array}$

$\begin{array}{llllllll}8 \mathrm{H} & -0.000054 & 0.444751 & -0.001768 & 0.000007 & 0.001406 & -0.000162\end{array}$

$\begin{array}{llllllll}9 \mathrm{H} & 0.000007 & -0.001768 & 0.453499 & -0.000068 & -0.000050 & -0.001286\end{array}$

$\begin{array}{llllllll}10 \mathrm{H} & -0.002231 & 0.000007 & -0.000068 & 0.447063 & -0.000046 & -0.001212\end{array}$

$\begin{array}{lllllllll}11 & \mathrm{O} & 0.001357 & 0.001406 & -0.000050 & -0.000046 & 8.153202 & 0.000025\end{array}$

$\begin{array}{llllllll}12 & \mathrm{C} & -0.000158 & -0.000162 & -0.001286 & -0.001212 & 0.000025 & 5.282897\end{array}$

$\begin{array}{llllllll}13 & \mathrm{H} & 0.000003 & -0.000001 & 0.000405 & -0.000095 & 0.000000 & 0.383038\end{array}$

$\begin{array}{llllllll}14 \mathrm{H} & -0.000003 & 0.000002 & -0.000064 & 0.001230 & 0.000000 & 0.383331\end{array}$

$\begin{array}{llllllll}15 \mathrm{H} & 0.000000 & 0.000003 & 0.000208 & 0.000247 & -0.000018 & 0.377267\end{array}$

$\begin{array}{llllllll}16 & \mathrm{O} & -0.000058 & -0.000052 & -0.001139 & 0.000295 & 0.000000 & -0.044691\end{array}$

$\begin{array}{lllllll}17 \mathrm{Si} & -0.000005 & -0.000050 & 0.000661 & 0.000491 & 0.000000 & -0.006816\end{array}$

$\begin{array}{llllllll}18 & \mathrm{C} & 0.000000 & 0.000000 & -0.000033 & -0.000001 & 0.000000 & -0.000075\end{array}$

$\begin{array}{llllllll}19 \mathrm{H} & 0.000000 & 0.000000 & 0.000001 & 0.000000 & 0.000000 & 0.000001\end{array}$

$\begin{array}{llllllll}20 \mathrm{H} & 0.000000 & 0.000000 & -0.000001 & 0.000000 & 0.000000 & -0.000003\end{array}$

$\begin{array}{llllllll}21 \mathrm{H} & 0.000000 & 0.000000 & 0.000000 & 0.000000 & 0.000000 & -0.000004\end{array}$

$\begin{array}{llllllll}22 & \mathrm{C} & 0.000000 & 0.000002 & -0.002416 & 0.000001 & 0.000000 & -0.008118\end{array}$

$\begin{array}{llllllll}23 & \mathrm{H} & 0.000000 & 0.000000 & 0.000174 & 0.000000 & 0.000000 & 0.000204\end{array}$

$\begin{array}{llllllll}24 \mathrm{H} & 0.000000 & 0.000001 & -0.000731 & 0.000001 & 0.000000 & -0.007511\end{array}$

$\begin{array}{llllllll}25 & \mathrm{H} & 0.000000 & -0.000002 & 0.000818 & 0.000000 & 0.000000 & 0.000422\end{array}$ 


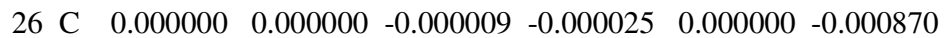

$\begin{array}{llllllll}27 & \mathrm{H} & 0.000000 & 0.000000 & 0.000000 & 0.000000 & 0.000000 & 0.000056\end{array}$

$\begin{array}{llllllll}28 \mathrm{H} & 0.000000 & 0.000000 & 0.000000 & 0.000014 & 0.000000 & 0.000069\end{array}$

$\begin{array}{llllllll}29 & \mathrm{H} & 0.000000 & 0.000000 & -0.000001 & -0.000009 & 0.000000 & -0.000482\end{array}$ $\begin{array}{llllll}13 & 14 & 15 & 16 & 17 & 18\end{array}$

$\begin{array}{llllllll}1 & \mathrm{C} & -0.000021 & -0.000019 & 0.000061 & 0.000207 & -0.000024 & 0.000000\end{array}$

$\begin{array}{llllllll}2 & \mathrm{C} & -0.008549 & 0.006093 & 0.001158 & -0.047296 & -0.002670 & -0.000174\end{array}$

$\begin{array}{llllllll}3 & C & 0.006021 & -0.005986 & -0.000349 & -0.053956 & 0.000181 & -0.000003\end{array}$

$\begin{array}{llllllll}4 & \mathrm{C} & -0.033187 & -0.032577 & -0.026734 & 0.159193 & -0.026806 & 0.001234\end{array}$

$\begin{array}{llllllll}5 & \mathrm{C} & -0.000005 & 0.000332 & 0.000921 & 0.001263 & 0.000180 & 0.000000\end{array}$

$\begin{array}{llllllll}6 \mathrm{C} & 0.000198 & -0.000015 & 0.000739 & 0.001103 & 0.000571 & -0.000009\end{array}$

$\begin{array}{llllllll}7 & \mathrm{H} & 0.000003 & -0.000003 & 0.000000 & -0.000058 & -0.000005 & 0.000000\end{array}$

$\begin{array}{llllllll}8 & \mathrm{H} & -0.000001 & 0.000002 & 0.000003 & -0.000052 & -0.000050 & 0.000000\end{array}$

$\begin{array}{llllllll}9 \mathrm{H} & 0.000405 & -0.000064 & 0.000208 & -0.001139 & 0.000661 & -0.000033\end{array}$

$\begin{array}{llllllll}10 \mathrm{H} & -0.000095 & 0.001230 & 0.000247 & 0.000295 & 0.000491 & -0.000001\end{array}$

$\begin{array}{llllllll}11 & \mathrm{O} & 0.000000 & 0.000000 & -0.000018 & 0.000000 & 0.000000 & 0.000000\end{array}$

$\begin{array}{llllllll}12 & \mathrm{C} & 0.383038 & 0.383331 & 0.377267 & -0.044691 & -0.006816 & -0.000075\end{array}$

$\begin{array}{llllllll}13 & \mathrm{H} & 0.543629 & -0.029014 & -0.023293 & -0.000744 & 0.002798 & -0.000007\end{array}$

$\begin{array}{llllllll}14 & \mathrm{H} & -0.029014 & 0.511410 & -0.020088 & 0.000624 & -0.000795 & -0.000001\end{array}$

$\begin{array}{llllllll}15 \mathrm{H} & -0.023293 & -0.020088 & 0.483621 & 0.002562 & 0.001874 & 0.000001\end{array}$

$\begin{array}{llllllll}16 & \mathrm{O} & -0.000744 & 0.000624 & 0.002562 & 8.537214 & 0.307178 & -0.025890\end{array}$

$\begin{array}{lllllllll}17 & \text { Si } & 0.002798 & -0.000795 & 0.001874 & 0.307178 & 11.412693 & 0.396132\end{array}$

$\begin{array}{llllllll}18 & \mathrm{C} & -0.000007 & -0.000001 & 0.000001 & -0.025890 & 0.396132 & 5.355840\end{array}$

$\begin{array}{llllllll}19 & \mathrm{H} & 0.000001 & 0.000000 & 0.000000 & 0.000526 & -0.012007 & 0.366921\end{array}$

$\begin{array}{llllllll}20 \mathrm{H} & -0.000001 & 0.000000 & 0.000000 & -0.000088 & -0.016821 & 0.375375\end{array}$

$\begin{array}{llllllll}21 & \mathrm{H} & 0.000000 & 0.000000 & 0.000000 & -0.000233 & -0.015662 & 0.377010\end{array}$

$\begin{array}{llllllll}22 & \mathrm{C} & -0.003897 & 0.000277 & 0.000090 & -0.010920 & 0.369407 & -0.028563\end{array}$

$\begin{array}{llllllll}23 & \mathrm{H} & 0.000198 & -0.000007 & -0.000003 & 0.000254 & -0.009698 & -0.000057\end{array}$

$\begin{array}{llllllll}24 & \mathrm{H} & -0.000573 & 0.000243 & 0.000257 & -0.000244 & -0.027222 & 0.001048\end{array}$

$\begin{array}{llllllll}25 \mathrm{H} & -0.000070 & 0.000000 & -0.000010 & -0.000146 & -0.016779 & -0.000692\end{array}$

$\begin{array}{llllllll}26 & \mathrm{C} & -0.000905 & -0.001378 & 0.000032 & -0.017647 & 0.382040 & -0.030978\end{array}$

$\begin{array}{llllllll}27 & \mathrm{H} & 0.000011 & 0.000058 & -0.000001 & 0.000439 & -0.009981 & -0.000379\end{array}$

$\begin{array}{llllllll}28 \mathrm{H} & 0.000037 & 0.000096 & -0.000005 & -0.000416 & -0.016659 & -0.000511\end{array}$

$\begin{array}{llllllll}29 & \mathrm{H} & 0.000152 & 0.000100 & 0.000029 & -0.000182 & -0.020221 & 0.000924\end{array}$
$\begin{array}{llllll}19 & 20 & 21 & 22 & 23 & 24\end{array}$

$\begin{array}{llllllll}1 & \mathrm{C} & 0.000000 & 0.000000 & 0.000000 & -0.000002 & 0.000000 & 0.000000\end{array}$

$\begin{array}{llllllll}2 & \mathrm{C} & 0.000001 & 0.000028 & 0.000003 & -0.001291 & 0.000071 & 0.000039\end{array}$

$\begin{array}{llllllll}3 \mathrm{C} & 0.000000 & -0.000001 & 0.000004 & -0.000075 & 0.000001 & -0.000034\end{array}$

$\begin{array}{llllllll}4 & \mathrm{C} & -0.000011 & -0.000071 & -0.000038 & -0.000511 & 0.000000 & 0.000698\end{array}$

$\begin{array}{llllllll}5 \mathrm{C} & 0.000000 & 0.000000 & 0.000000 & 0.000005 & 0.000000 & 0.000000\end{array}$

$\begin{array}{llllllll}6 \mathrm{C} & 0.000000 & 0.000000 & 0.000000 & 0.000012 & 0.000000 & -0.000060\end{array}$

$\begin{array}{llllllll}7 \mathrm{H} & 0.000000 & 0.000000 & 0.000000 & 0.000000 & 0.000000 & 0.000000\end{array}$

$\begin{array}{llllllll}8 \mathrm{H} & 0.000000 & 0.000000 & 0.000000 & 0.000002 & 0.000000 & 0.000001\end{array}$

$\begin{array}{llllllll}9 \mathrm{H} & 0.000001 & -0.000001 & 0.000000 & -0.002416 & 0.000174 & -0.000731\end{array}$

$\begin{array}{llllllll}10 \mathrm{H} & 0.000000 & 0.000000 & 0.000000 & 0.000001 & 0.000000 & 0.000001\end{array}$

$\begin{array}{llllllll}11 & \mathrm{O} & 0.000000 & 0.000000 & 0.000000 & 0.000000 & 0.000000 & 0.000000\end{array}$

$\begin{array}{llllllll}12 & \mathrm{C} & 0.000001 & -0.000003 & -0.000004 & -0.008118 & 0.000204 & -0.007511\end{array}$

$\begin{array}{llllllll}13 \mathrm{H} & 0.000001 & -0.000001 & 0.000000 & -0.003897 & 0.000198 & -0.000573\end{array}$

$\begin{array}{llllllll}14 \mathrm{H} & 0.000000 & 0.000000 & 0.000000 & 0.000277 & -0.000007 & 0.000243\end{array}$

$\begin{array}{llllllll}15 \mathrm{H} & 0.000000 & 0.000000 & 0.000000 & 0.000090 & -0.000003 & 0.000257\end{array}$

$\begin{array}{llllllll}16 & \mathrm{O} & 0.000526 & -0.000088 & -0.000233 & -0.010920 & 0.000254 & -0.000244\end{array}$

$\begin{array}{llllllll}17 & \mathrm{Si} & -0.012007 & -0.016821 & -0.015662 & 0.369407 & -0.009698 & -0.027222\end{array}$

$\begin{array}{llllllll}18 & \mathrm{C} & 0.366921 & 0.375375 & 0.377010 & -0.028563 & -0.000057 & 0.001048\end{array}$

$\begin{array}{llllllll}19 \mathrm{H} & 0.524494 & -0.024950 & -0.024281 & 0.000122 & 0.000044 & -0.000025\end{array}$

$\begin{array}{llllllll}20 \mathrm{H} & -0.024950 & 0.510098 & -0.021872 & -0.000786 & 0.000021 & -0.000011\end{array}$

$\begin{array}{llllllll}21 & \mathrm{H} & -0.024281 & -0.021872 & 0.497097 & 0.000869 & -0.000009 & -0.000014\end{array}$

$\begin{array}{llllllll}22 & \mathrm{C} & 0.000122 & -0.000786 & 0.000869 & 5.435439 & 0.374675 & 0.373668\end{array}$

$\begin{array}{lllllllll}23 \mathrm{H} & 0.000044 & 0.000021 & -0.000009 & 0.374675 & 0.485746 & -0.020864\end{array}$

$\begin{array}{llllllll}24 \mathrm{H} & -0.000025 & -0.000011 & -0.000014 & 0.373668 & -0.020864 & 0.520870\end{array}$

$\begin{array}{llllllll}25 \mathrm{H} & 0.000017 & 0.000679 & 0.000000 & 0.373499 & -0.022638 & -0.022588\end{array}$

$\begin{array}{llllllll}26 \mathrm{C} & 0.000066 & 0.000886 & -0.000463 & -0.033957 & -0.000494 & -0.000249\end{array}$

$\begin{array}{llllllll}27 & \mathrm{H} & 0.000220 & -0.000003 & 0.000030 & 0.000152 & 0.000154 & 0.000039\end{array}$

$\begin{array}{llllllll}28 \mathrm{H} & 0.000029 & -0.000010 & 0.000223 & 0.000886 & 0.000006 & -0.000019\end{array}$

$\begin{array}{llllllll}29 & \mathrm{H} & -0.000012 & -0.000013 & -0.000014 & -0.000577 & 0.000039 & 0.000235\end{array}$ 


\begin{abstract}
$\begin{array}{lllll}25 & 26 & 27 & 28 & 29\end{array}$
$\begin{array}{lllllll}1 & \mathrm{C} & 0.000000 & 0.000000 & 0.000000 & 0.000000 & 0.000000\end{array}$

$\begin{array}{lllllll}2 & \mathrm{C} & -0.000928 & -0.000122 & 0.000001 & -0.000001 & -0.000014\end{array}$

$\begin{array}{lllllll}3 & \mathrm{C} & -0.000007 & -0.000052 & 0.000001 & 0.000019 & 0.000008\end{array}$

$\begin{array}{lllllll}4 \mathrm{C} & -0.000208 & -0.000188 & 0.000003 & 0.000072 & -0.000005\end{array}$

$\begin{array}{lllllll}5 \mathrm{C} & 0.000000 & 0.000000 & 0.000000 & 0.000000 & 0.000000\end{array}$

$\begin{array}{lllllll}6 & \mathrm{C} & -0.000013 & 0.000000 & 0.000000 & 0.000000 & 0.000000\end{array}$

$\begin{array}{lllllll}7 & \mathrm{H} & 0.000000 & 0.000000 & 0.000000 & 0.000000 & 0.000000\end{array}$

$\begin{array}{lllllll}8 \mathrm{H} & -0.000002 & 0.000000 & 0.000000 & 0.000000 & 0.000000\end{array}$

$\begin{array}{lllllll}9 \mathrm{H} & 0.000818 & -0.000009 & 0.000000 & 0.000000 & -0.000001\end{array}$

$\begin{array}{lllllll}10 \mathrm{H} & 0.000000 & -0.000025 & 0.000000 & 0.000014 & -0.000009\end{array}$

$\begin{array}{llllllll}11 & \mathrm{O} & 0.000000 & 0.000000 & 0.000000 & 0.000000 & 0.000000\end{array}$

$\begin{array}{lllllll}12 & \mathrm{C} & 0.000422 & -0.000870 & 0.000056 & 0.000069 & -0.000482\end{array}$

$\begin{array}{lllllll}13 \mathrm{H} & -0.000070 & -0.000905 & 0.000011 & 0.000037 & 0.000152\end{array}$

$\begin{array}{lllllll}14 \mathrm{H} & 0.000000 & -0.001378 & 0.000058 & 0.000096 & 0.000100\end{array}$

$\begin{array}{lllllll}15 \mathrm{H} & -0.000010 & 0.000032 & -0.000001 & -0.000005 & 0.000029\end{array}$

$\begin{array}{lllllll}16 & \mathrm{O} & -0.000146 & -0.017647 & 0.000439 & -0.000416 & -0.000182\end{array}$

$\begin{array}{lllllll}17 & \mathrm{Si} & -0.016779 & 0.382040 & -0.009981 & -0.016659 & -0.020221\end{array}$

$\begin{array}{lllllll}18 & \mathrm{C} & -0.000692 & -0.030978 & -0.000379 & -0.000511 & 0.000924\end{array}$

$\begin{array}{lllllll}19 & \mathrm{H} & 0.000017 & 0.000066 & 0.000220 & 0.000029 & -0.000012\end{array}$

$\begin{array}{lllllll}20 \mathrm{H} & 0.000679 & 0.000886 & -0.000003 & -0.000010 & -0.000013\end{array}$

$\begin{array}{lllllll}21 \mathrm{H} & 0.000000 & -0.000463 & 0.000030 & 0.000223 & -0.000014\end{array}$

$\begin{array}{lllllll}22 & \mathrm{C} & 0.373499 & -0.033957 & 0.000152 & 0.000886 & -0.000577\end{array}$

$\begin{array}{lllllll}23 & \mathrm{H} & -0.022638 & -0.000494 & 0.000154 & 0.000006 & 0.000039\end{array}$

$\begin{array}{lllllll}24 \mathrm{H} & -0.022588 & -0.000249 & 0.000039 & -0.000019 & 0.000235\end{array}$

$\begin{array}{llllllll}25 & \mathrm{H} & 0.506453 & 0.000899 & -0.000019 & -0.000012 & -0.000004\end{array}$

$\begin{array}{llllllll}26 & \mathrm{C} & 0.000899 & 5.386487 & 0.369789 & 0.377868 & 0.376219\end{array}$

$\begin{array}{llllllll}27 & \mathrm{H} & -0.000019 & 0.369789 & 0.510542 & -0.023618 & -0.024187\end{array}$

$\begin{array}{lllllll}28 \mathrm{H} & -0.000012 & 0.377868 & -0.023618 & 0.501390 & -0.023199\end{array}$

$\begin{array}{lllllll}29 & \mathrm{H} & -0.000004 & 0.376219 & -0.024187 & -0.023199 & 0.520032\end{array}$

Mulliken atomic charges:
\end{abstract}

$$
1
$$

$\begin{array}{lll}1 & \mathrm{C} & 0.519127\end{array}$

2 C -0.158320

3 C -0.134732

4 C 0.287102

5 C -0.269295

6 C -0.263541

$\begin{array}{lll}7 & \mathrm{H} & 0.225289\end{array}$

$8 \mathrm{H} \quad 0.226649$

9 H 0.219438

$10 \mathrm{H} \quad 0.224252$

1100.573941

12 C -0.544189

$\begin{array}{lll}13 & \mathrm{H} & 0.163871\end{array}$

$14 \mathrm{H} \quad 0.186152$

$15 \mathrm{H} \quad 0.201430$

$16 \mathrm{O} \quad-0.807154$

$17 \mathrm{Si} 1.308009$

18 C -0.787113

$19 \mathrm{H} \quad 0.168842$

$20 \mathrm{H} \quad 0.177541$

$\begin{array}{lll}21 & \mathrm{H} & 0.187351\end{array}$

$\begin{array}{lll}22 & \mathrm{C} & -0.837991\end{array}$

$23 \mathrm{H} \quad 0.192181$

$24 \mathrm{H} \quad 0.183046$

$\begin{array}{lll}25 \mathrm{H} & 0.181329\end{array}$

$\begin{array}{lll}26 \mathrm{C} & -0.806948\end{array}$

$\begin{array}{lll}27 & \mathrm{H} & 0.176693\end{array}$

$28 \mathrm{H} \quad 0.183741$

$29 \mathrm{H} \quad 0.171180$

Sum of Mulliken charges $=0.00000$

Atomic charges with hydrogens summed into heavy atoms: 

$\begin{array}{lll}1 & \mathrm{C} & 0.519127\end{array}$
2 C 0.061118
3 C 0.089520
4 C 0.287102
5 C -0.044007
$\begin{array}{lll}6 & \mathrm{C} & -0.036892\end{array}$
$\begin{array}{lll}7 \mathrm{H} & 0.000000\end{array}$
$8 \mathrm{H} \quad 0.000000$
$9 \mathrm{H} \quad 0.000000$
$\begin{array}{lll}10 \mathrm{H} & 0.000000\end{array}$
$\begin{array}{lll}11 & \mathrm{O} & -0.573941\end{array}$
$12 \mathrm{C} \quad 0.007264$
$13 \mathrm{H} \quad 0.000000$
$14 \mathrm{H} \quad 0.000000$
$\begin{array}{lll}15 \mathrm{H} & 0.000000\end{array}$
$\begin{array}{lll}16 \mathrm{O} & -0.807154\end{array}$
$17 \mathrm{Si} 1.308009$
$\begin{array}{lll}18 \mathrm{C} & -0.253378\end{array}$
$19 \mathrm{H} \quad 0.000000$
$20 \mathrm{H} \quad 0.000000$
$\begin{array}{lll}21 \mathrm{H} & 0.000000\end{array}$
22 C -0.281435
$23 \mathrm{H} \quad 0.000000$
$24 \mathrm{H} \quad 0.000000$
$25 \mathrm{H} \quad 0.000000$
$\begin{array}{lll}26 \mathrm{C} & -0.275334\end{array}$
$\begin{array}{lll}27 \mathrm{H} & 0.000000\end{array}$
$28 \mathrm{H} \quad 0.000000$
$29 \mathrm{H} \quad 0.000000$

Sum of Mulliken charges $=0.00000$

Electronic spatial extent $(\mathrm{au}):<\mathrm{R} * * 2>=3264.7097$

Charge $=0.0000$ electrons

Dipole moment (field-independent basis, Debye):

$\mathrm{X}=-4.8835 \mathrm{Y}=0.4464 \mathrm{Z}=1.5940$ Tot $=5.1565$

Quadrupole moment (field-independent basis, Debye-Ang):

$\mathrm{XX}=-108.8038 \quad \mathrm{YY}=-81.8360 \quad \mathrm{ZZ}=-88.0825$

$\mathrm{XY}=4.2017 \quad \mathrm{XZ}=-0.9017 \quad \mathrm{YZ}=-1.1842$

Traceless Quadrupole moment (field-independent basis, Debye-Ang):

$\mathrm{XX}=-15.8964 \mathrm{YY}=11.0714 \mathrm{ZZ}=4.8249$

$\mathrm{XY}=4.2017 \quad \mathrm{XZ}=-0.9017 \quad \mathrm{YZ}=-1.1842$

Octapole moment (field-independent basis, Debye-Ang**2):

$\mathrm{XXX}=-140.2097 \mathrm{YYY}=-0.7018 \mathrm{ZZZ}=0.3928 \mathrm{XYY}=12.4356$

$\mathrm{XXY}=13.3330 \mathrm{XXZ}=0.8519 \mathrm{XZZ}=6.1479 \mathrm{YZZ}=4.2058$

$\mathrm{YYZ}=-5.4876 \mathrm{XYZ}=-1.6800$

Hexadecapole moment (field-independent basis, Debye-Ang**3):

$\mathrm{XXXX}=-3890.5325 \mathrm{YYYY}=-621.0123 \mathrm{ZZZZ}=-535.0371 \mathrm{XXXY}=89.4176$

$\mathrm{XXXZ}=-4.8163 \mathrm{YYYX}=-9.2328 \mathrm{YYYZ}=-11.5235 \mathrm{ZZZX}=-3.7697$

$Z Z Z Y=-0.4167 X X Y Y=-639.1221 X X Z Z=-630.6249 \mathrm{YYZZ}=-199.3009$

$\mathrm{XXYZ}=-22.3973 \mathrm{YYXZ}=-1.4616 \mathrm{ZZXY}=-4.2698$

$\mathrm{N}-\mathrm{N}=9.190408278157 \mathrm{D}+02 \mathrm{E}-\mathrm{N}=-3.784201245320 \mathrm{D}+03 \quad \mathrm{KE}=8.254001655822 \mathrm{D}+02$

1/1IUNPC-UNKISP|RHF|6-31G(d)|C10H16O2Si1|PCUSERI17-May-2006|0|\#\# RHF/6

-31G(D) GEOM=CONNECTIVITY॥Title Card Required\|0,1|C,0,3.32603865,-0.

22668107,-0.2041039|C,0,1.05861676,-1.03500659,0.29746841IC,0,1.444883

23,1.33956871,-0.4438597|C,0,0.4715875,0.35701937,0.17684199|C,0,2.743

$7482,1.07724941,-0.5972991|\mathrm{C}, 0,2.3563735,-1.29514026,0.14071315| \mathrm{H}, 0,3$.

$42777044,1.81931953,-1.02589763 \mathrm{lH}, 0,2.75398382,-2.31086408,0.25662836 \mathrm{l}$

$\mathrm{H}, 0,0.34173275,-1.83499066,0.530895 \mathrm{IH}, 0,1.01783913,2.29971117,-0.76058$

$668|\mathrm{O}, 0,4.52983147,-0.4152158,-0.17399187| \mathrm{C}, 0,0.14145711,0.85769108,1$.

$59552875|\mathrm{H}, 0,-0.54705179,0.16552143,2.11189865| \mathrm{H}, 0,-0.33828365,1.84606$

$004,1.56714243|\mathrm{H}, 0,1.04948757,0.94307673,2.20421517| \mathrm{O}, 0,-0.63982577,0$.

$33263831,-0.68555665|\mathrm{Si}, 0,-2.21007781,-0.10978105,-0.1761108| \mathrm{C}, 0,-3.02$

$279414,-0.7292463,-1.77064883 \mid \mathrm{H}, 0,-4.0991678,-0.86323544,-1.63010408 \mathrm{lH}$

$, 0,-2.60031786,-1.68854929,-2.08154553 \mid \mathrm{H}, 0,-2.87527101,-0.01743887,-2$.

$5878589|\mathrm{C}, 0,-2.29039145,-1.4650273,1.15113403| \mathrm{H}, 0,-3.31759231,-1.59807$ 
$373,1.4987347|\mathrm{H}, 0,-1.67024081,-1.1968248,2.01755528| \mathrm{H}, 0,-1.93870583,-2$ $.42658837,0.76560098|\mathrm{C}, 0,-3.1312818,1.42512533,0.44685241| \mathrm{H}, 0,-4.20049$ $041,1.21866872,0.54688631 \mathrm{lH}, 0,-3.01485965,2.25973958,-0.24946011 \mathrm{IH}, 0,-$ $2.75721424,1.74870831,1.42207223 \|$ Version $=x 86-$ Win32-G03RevB.05|State $=1$ $-\mathrm{A}|\mathrm{HF}=-826.6700901| \mathrm{RMSD}=5.859 \mathrm{e}-006 \mid \mathrm{Dipole}=-1.9213269,0.1756436,0.62714$ 61|PG=C01 [X(C10H16O2Si1)]\|@

IF IT'S NOT TRUE, IT'S WELL INVENTED -- DANTE

Job cpu time: 0 days 0 hours 2 minutes 49.0 seconds.

File lengths (MBytes): RWF $=38 \mathrm{Int}=0 \mathrm{D} 2 \mathrm{E}=0 \mathrm{Chk}=9 \mathrm{Scr}=1$

Normal termination of Gaussian 03 at Wed May 17 13:54:30 2006. 


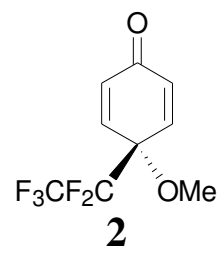

Entering Link $1=\mathrm{C}: \mathrm{\backslash G} 03 \mathrm{~W} \backslash 11$.exe PID $=3208$.

Copyright (c) 1988,1990,1992,1993,1995,1998,2003, Gaussian, Inc. All Rights Reserved.

This is the Gaussian(R) 03 program. It is based on the the Gaussian(R) 98 system (copyright 1998, Gaussian, Inc.), the Gaussian(R) 94 system (copyright 1995, Gaussian, Inc.), the Gaussian 92(TM) system (copyright 1992, Gaussian, Inc.), the Gaussian 90(TM) system (copyright 1990, Gaussian, Inc.), the Gaussian 88(TM) system (copyright 1988, Gaussian, Inc.), the Gaussian 86(TM) system (copyright 1986, Carnegie Mellon University), and the Gaussian 82(TM) system (copyright 1983, Carnegie Mellon University). Gaussian is a federally registered trademark of Gaussian, Inc.

This software contains proprietary and confidential information, including trade secrets, belonging to Gaussian, Inc.

This software is provided under written license and may be used, copied, transmitted, or stored only in accord with that written license.

The following legend is applicable only to US Government contracts under DFARS:

\section{RESTRICTED RIGHTS LEGEND}

Use, duplication or disclosure by the US Government is subject to restrictions as set forth in subparagraph (c)(1)(ii) of the Rights in Technical Data and Computer Software clause at DFARS 252.227-7013.

Gaussian, Inc.

Carnegie Office Park, Building 6, Pittsburgh, PA 15106 USA

The following legend is applicable only to US Government contracts under FAR:

\section{RESTRICTED RIGHTS LEGEND}

Use, reproduction and disclosure by the US Government is subject to restrictions as set forth in subparagraph (c) of the Commercial Computer Software - Restricted Rights clause at FAR 52.227-19.

Gaussian, Inc.

Carnegie Office Park, Building 6, Pittsburgh, PA 15106 USA

Warning -- This program may not be used in any manner that competes with the business of Gaussian, Inc. or will provide assistance to any competitor of Gaussian, Inc. The licensee of this program is prohibited from giving any competitor of Gaussian, Inc. access to this program. By using this program, 
the user acknowledges that Gaussian, Inc. is engaged in the business of creating and licensing software in the field of computational chemistry and represents and warrants to the licensee that it is not a competitor of Gaussian, Inc. and that it will not use this program in any manner prohibited above.

Cite this work as:

Gaussian 03, Revision B.05,

M. J. Frisch, G. W. Trucks, H. B. Schlegel, G. E. Scuseria,

M. A. Robb, J. R. Cheeseman, J. A. Montgomery, Jr., T. Vreven,

K. N. Kudin, J. C. Burant, J. M. Millam, S. S. Iyengar, J. Tomasi,

V. Barone, B. Mennucci, M. Cossi, G. Scalmani, N. Rega,

G. A. Petersson, H. Nakatsuji, M. Hada, M. Ehara, K. Toyota,

R. Fukuda, J. Hasegawa, M. Ishida, T. Nakajima, Y. Honda, O. Kitao,

H. Nakai, M. Klene, X. Li, J. E. Knox, H. P. Hratchian, J. B. Cross,

C. Adamo, J. Jaramillo, R. Gomperts, R. E. Stratmann, O. Yazyev,

A. J. Austin, R. Cammi, C. Pomelli, J. W. Ochterski, P. Y. Ayala,

K. Morokuma, G. A. Voth, P. Salvador, J. J. Dannenberg,

V. G. Zakrzewski, S. Dapprich, A. D. Daniels, M. C. Strain,

O. Farkas, D. K. Malick, A. D. Rabuck, K. Raghavachari,

J. B. Foresman, J. V. Ortiz, Q. Cui, A. G. Baboul, S. Clifford,

J. Cioslowski, B. B. Stefanov, G. Liu, A. Liashenko, P. Piskorz,

I. Komaromi, R. L. Martin, D. J. Fox, T. Keith, M. A. Al-Laham,

C. Y. Peng, A. Nanayakkara, M. Challacombe, P. M. W. Gill,

B. Johnson, W. Chen, M. W. Wong, C. Gonzalez, and J. A. Pople,

Gaussian, Inc., Pittsburgh PA, 2003.

$* * * * * * * * * * * * * * * * * * * * * * * * * * * * * * * * * * * * * * * * * * * * * *$

Gaussian 03: x86-Win32-G03RevB.05 8-Nov-2003

17-May-2006

*******************************************************

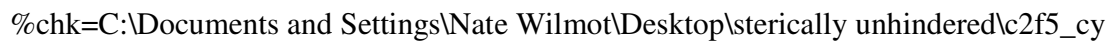

clo.chk

$\%$ mem $=64 \mathrm{MB}$

$\%$ nproc $=1$

Will use up to 1 processors via shared memory.

\# rhf/6-31g(d) geom=connectivity

$1 / 38=1,57=2 / 1$

$2 / 17=6,18=5,40=1 / 2$;

$3 / 5=1,6=6,7=1,11=1,16=1,25=1,30=1 / 1,2,3$;

$4 / 7=1 / 1$;

$5 / 5=2,32=1,38=5 / 2$;

$6 / 7=2,8=2,9=2,10=2,28=1 / 1$;

$99 / 5=1,9=1 / 99$;

Title Card Required

Symbolic Z-matrix:

Charge $=0$ Multiplicity $=1$

$\begin{array}{llll}\mathrm{C} & 2.96414 & -0.69075 & -0.22077 \\ \mathrm{C} & 0.85084 & 0.1012 & -1.20923 \\ \mathrm{C} & 1.40035 & 0.57425 & 1.19836 \\ \mathrm{C} & 0.33657 & 0.57522 & 0.12645 \\ \mathrm{C} & 2.5899 & -0.00923 & 1.0442 \\ \mathrm{C} & 2.04024 & -0.48237 & -1.36479 \\ \mathrm{H} & 3.33252 & -0.00737 & 1.85317 \\ \mathrm{H} & 2.37698 & -0.82787 & -2.35036 \\ \mathrm{H} & 0.19552 & 0.27926 & -2.07226 \\ \mathrm{H} & 1.13265 & 1.08291 & 2.13499 \\ \mathrm{O} & 3.9717 & -1.36494 & -0.31845\end{array}$




$\begin{array}{llll}\text { C } & -0.84674 & -0.3729 & 0.62649 \\ \text { O } & -0.29351 & 1.85784 & 0.02388 \\ \text { C } & 0.55165 & 2.9081 & -0.37229 \\ \text { H } & 1.36297 & 3.07181 & 0.34654 \\ \text { H } & -0.12955 & 3.76328 & -0.3814 \\ \text { H } & 0.9698 & 2.74114 & -1.37131 \\ \text { C } & -2.15257 & -0.41677 & -0.32695 \\ \text { F } & -1.91043 & -0.92904 & -1.54563 \\ \text { F } & -3.1037 & -1.20358 & 0.21299 \\ \text { F } & -2.72957 & 0.78044 & -0.51394 \\ \text { F } & -1.27691 & -0.01492 & 1.85925 \\ \text { F } & -0.43192 & -1.65146 & 0.78604\end{array}$

Input orientation:

\begin{tabular}{|c|c|c|c|c|}
\hline \multirow{2}{*}{$\begin{array}{l}\text { Center } \\
\text { Number }\end{array}$} & \multirow{2}{*}{$\begin{array}{l}\text { Atomic } \\
\text { Number }\end{array}$} & \multirow{2}{*}{$\begin{array}{r}\text { Atomic } \\
\text { r Type }\end{array}$} & \multicolumn{2}{|c|}{ Coordinates (Angstro } \\
\hline & & & $\begin{array}{ll}X & Y\end{array}$ & $\mathrm{Z}$ \\
\hline 1 & 6 & 2.964138 & -0.690749 & -0.220774 \\
\hline 2 & 6 & 0.850842 & 0.101197 & -1.209227 \\
\hline 3 & 6 & 1.400350 & 0.574254 & 1.198360 \\
\hline 4 & 6 & 73 & 0.575224 & 0.126449 \\
\hline 5 & 6 & 2.589901 & -0.009225 & 1.044203 \\
\hline 6 & 6 & 2.040243 & -0.482369 & -1.364788 \\
\hline 7 & 1 & 3.332516 & -0.007373 & 1.853167 \\
\hline 8 & 1 & 2.376 & -0.827872 & -2.350355 \\
\hline 9 & 1 & 0.195525 & 0.279260 & -2.072263 \\
\hline 10 & 1 & 2646 & 1.082910 & 2.134995 \\
\hline 11 & 8 & 0 & -1.364940 & -0.318447 \\
\hline 12 & 6 & -0.846738 & -0.372897 & 0.626492 \\
\hline 13 & 8 & -0.293508 & 1.857842 & 0.023875 \\
\hline 14 & 6 & 0.551649 & 2.908097 & -0.372287 \\
\hline 15 & 1 & 1.3629 & 3.071805 & 0.346536 \\
\hline 16 & 1 & -0.129548 & 3.763285 & -0.381396 \\
\hline 17 & 1 & 0.969803 & 2.741144 & -1.371311 \\
\hline 18 & 6 & -2.152571 & -0.416771 & -0.326952 \\
\hline 19 & 9 & -1.910429 & -0.929035 & -1.545632 \\
\hline 20 & 9 & -3.103696 & -1.203577 & 0.212989 \\
\hline 21 & 9 & -2.729573 & 0.780445 & -0.513939 \\
\hline 22 & 9 & -1.276906 & -0.014923 & 1.859252 \\
\hline 23 & 9 & -0.431924 & -1.651458 & 0.786035 \\
\hline
\end{tabular}


$\begin{array}{lllllll}21 & \mathrm{~F} & 5.888013 & 3.710009 & 4.475572 & 3.139023 & 5.598945\end{array}$

$\begin{array}{lllllll}22 & \mathrm{~F} & 4.771761 & 3.735821 & 2.819859 & 2.440122 & 3.951776\end{array}$

$\begin{array}{lllllll}23 & F & 3.670132 & 2.949298 & 2.912221 & 2.446171 & 3.448914\end{array}$ $\begin{array}{lllll}6 & 7 & 8 & 9 & 10\end{array}$

6 C 0.000000

$\begin{array}{llll}7 & \mathrm{H} & 3.500118 & 0.000000\end{array}$

$\begin{array}{lllll}8 & \mathrm{H} & 1.097318 & 4.388152 & 0.000000\end{array}$

$\begin{array}{llllll}9 \mathrm{H} & 2.117448 & 5.033079 & 2.462075 & 0.000000\end{array}$

$\begin{array}{lllllll}10 & \mathrm{H} & 3.939837 & 2.471351 & 5.031682 & 4.384641 & 0.000000\end{array}$

$\begin{array}{lllllll}11 & \mathrm{O} & 2.367338 & 2.639594 & 2.638226 & 4.476469 & 4.480133\end{array}$

$\begin{array}{llllllll}12 & \mathrm{C} & 3.508824 & 4.370870 & 4.411459 & 2.965620 & 2.883213\end{array}$

$\begin{array}{llllllll}13 & \mathrm{O} & 3.584881 & 4.469160 & 4.470071 & 2.669246 & 2.662942\end{array}$

$\begin{array}{lllllll}14 & \mathrm{C} & 3.833567 & 4.602807 & 4.604568 & 3.150798 & 3.155206\end{array}$

$\begin{array}{lllllll}15 & \mathrm{H} & 4.002436 & 3.953530 & 4.848600 & 3.874509 & 2.684648\end{array}$

$\begin{array}{lllllll}16 & \mathrm{H} & 4.868330 & 5.585430 & 5.589114 & 3.886275 & 3.887128\end{array}$

$\begin{array}{llllllll}17 & \mathrm{H} & 3.396605 & 4.851188 & 3.959362 & 2.674269 & 3.882067\end{array}$

$\begin{array}{lllllll}18 & \mathrm{C} & 4.319850 & 5.916647 & 4.977950 & 3.007346 & 4.370684\end{array}$

$\begin{array}{lllllll}19 & F & 3.979953 & 6.315835 & 4.363448 & 2.484424 & 5.182205\end{array}$

$\begin{array}{llllllll}20 \mathrm{~F} & 5.428593 & 6.748771 & 6.062151 & 4.278556 & 5.183505\end{array}$

$\begin{array}{llllllll}21 & \mathrm{~F} & 5.006974 & 6.555362 & 5.660033 & 3.351978 & 4.693088\end{array}$

$\begin{array}{llllllll}22 & \mathrm{~F} & 4.649346 & 4.609433 & 5.633165 & 4.208492 & 2.662182\end{array}$

$\begin{array}{lllllll}23 & \mathrm{~F} & 3.479141 & 4.244149 & 4.290125 & 3.505886 & 3.427002\end{array}$ $\begin{array}{lllll}11 & 12 & 13 & 14 & 15\end{array}$

$\begin{array}{lll}11 & \mathrm{O} & 0.000000\end{array}$

$\begin{array}{llll}12 & \mathrm{C} & 5.009434 & 0.000000\end{array}$

$\begin{array}{lllll}13 & \mathrm{O} & 5.356821 & 2.376007 & 0.000000\end{array}$

$\begin{array}{llllll}14 & \mathrm{C} & 5.473436 & 3.703777 & 1.405087 & 0.000000\end{array}$

$\begin{array}{lllllll}15 \mathrm{H} & 5.189645 & 4.102090 & 2.078876 & 1.096240 & 0.000000\end{array}$

$\begin{array}{lllllll}16 & \mathrm{H} & 6.566803 & 4.317198 & 1.954952 & 1.093371 & 1.798786\end{array}$

$\begin{array}{lllllll}17 & \mathrm{H} & 5.194213 & 4.121685 & 2.079116 & 1.095799 & 1.793018\end{array}$

$\begin{array}{lllllll}18 & \mathrm{C} & 6.197243 & 1.617461 & 2.958557 & 4.285978 & 4.998275\end{array}$

$\begin{array}{llllllll}19 & \mathrm{~F} & 6.024571 & 2.481703 & 3.583919 & 4.707670 & 5.504738\end{array}$

$\begin{array}{lllllll}20 \mathrm{~F} & 7.097162 & 2.440260 & 4.159952 & 5.532626 & 6.184480\end{array}$

$\begin{array}{lllllll}21 & \mathrm{~F} & 7.039032 & 2.485125 & 2.717432 & 3.913232 & 4.768608\end{array}$

$\begin{array}{llllllll}22 & \mathrm{~F} & 5.840617 & 1.353841 & 2.800523 & 4.106997 & 4.334181\end{array}$

$\begin{array}{llllllll}23 & F & 4.549054 & 1.353604 & 3.593777 & 4.806107 & 5.071884\end{array}$ $\begin{array}{lllll}16 & 17 & 18 & 19 & 20\end{array}$

$\begin{array}{ll}16 \mathrm{H} & 0.000000\end{array}$

$\begin{array}{llll}17 & \mathrm{H} & 1.798131 & 0.000000\end{array}$

$\begin{array}{lllll}18 & \mathrm{C} & 4.644184 & 4.562053 & 0.000000\end{array}$

$\begin{array}{llllll}19 & \text { F } & 5.152169 & 4.668655 & 1.343960 & 0.000000\end{array}$

$\begin{array}{lllllll}20 \mathrm{~F} & 5.819670 & 5.887632 & 1.347307 & 2.142896 & 0.000000\end{array}$

$\begin{array}{lllllll}21 & \mathrm{~F} & 3.959170 & 4.273734 & 1.342095 & 2.158172 & 2.145865\end{array}$

$\begin{array}{llllllll}22 & \mathrm{~F} & 4.540021 & 4.804180 & 2.389091 & 3.581924 & 2.731344\end{array}$

$\begin{array}{lllllll}23 & F & 5.547411 & 5.090573 & 2.392451 & 2.853865 & 2.768997\end{array}$ $\begin{array}{lll}21 & 22 & 23\end{array}$

$21 \mathrm{~F} \quad 0.000000$

$\begin{array}{llll}22 & \mathrm{~F} & 2.893940 & 0.000000\end{array}$

$\begin{array}{lllll}23 & \mathrm{~F} & 3.589328 & 2.131674 & 0.000000\end{array}$

Stoichiometry C9H7F5O2

Framework group $\mathrm{C} 1[\mathrm{X}(\mathrm{C} 9 \mathrm{H} 7 \mathrm{~F} 5 \mathrm{O} 2)]$

Deg. of freedom 63

Full point group $\quad \mathrm{C} 1 \quad \mathrm{NOp} 1$

Largest Abelian subgroup $\quad \mathrm{C} 1 \quad$ NOp 1

Largest concise Abelian subgroup C1 NOp 1 Standard orientation:

\begin{tabular}{|c|c|c|c|c|}
\hline \multirow{2}{*}{$\begin{array}{l}\text { Center } \\
\text { Number }\end{array}$} & \multirow{2}{*}{$\begin{array}{l}\text { Atomic } \\
\text { Number }\end{array}$} & \multirow{2}{*}{$\begin{array}{r}\text { Atomic } \\
\text { Type }\end{array}$} & \multicolumn{2}{|c|}{ Coordinates (Angstroms) } \\
\hline & & & $\begin{array}{ll}X & Y\end{array}$ & Y Z \\
\hline 1 & 6 & 2.964138 & -0.690749 & -0.220774 \\
\hline 2 & 6 & 0.850842 & 0.101197 & -1.209227 \\
\hline 3 & 6 & 1.400350 & 0.574254 & 1.198360 \\
\hline 4 & 6 & 0.336573 & 0.575224 & 0.126449 \\
\hline
\end{tabular}




$\begin{array}{cccccc}5 & 6 & 0 & 2.589901 & -0.009225 & 1.044203 \\ 6 & 6 & 0 & 2.040243 & -0.482369 & -1.364788 \\ 7 & 1 & 0 & 3.332516 & -0.007373 & 1.853167 \\ 8 & 1 & 0 & 2.376978 & -0.827872 & -2.350355 \\ 9 & 1 & 0 & 0.195525 & 0.279260 & -2.072263 \\ 10 & 1 & 0 & 1.132646 & 1.082910 & 2.134995 \\ 11 & 8 & 0 & 3.971702 & -1.364940 & -0.318447 \\ 12 & 6 & 0 & -0.846738 & -0.372897 & 0.626492 \\ 13 & 8 & 0 & -0.293508 & 1.857842 & 0.023875 \\ 14 & 6 & 0 & 0.551649 & 2.908097 & -0.372286 \\ 15 & 1 & 0 & 1.362966 & 3.071805 & 0.346536 \\ 16 & 1 & 0 & -0.129548 & 3.763285 & -0.381396 \\ 17 & 1 & 0 & 0.969803 & 2.741144 & -1.371311 \\ 18 & 6 & 0 & -2.152571 & -0.416771 & -0.326952 \\ 19 & 9 & 0 & -1.910429 & -0.929035 & -1.545632 \\ 20 & 9 & 0 & -3.103696 & -1.203577 & 0.212989 \\ 21 & 9 & 0 & -2.729573 & 0.780445 & -0.513939 \\ 22 & 9 & 0 & -1.276906 & -0.014923 & 1.859252 \\ 23 & 9 & 0 & -0.431924 & -1.651458 & 0.786035\end{array}$

Rotational constants (GHZ): $0.8777578 \quad 0.3985345 \quad 0.3646919$

Standard basis: $6-31 \mathrm{G}(\mathrm{d})(6 \mathrm{D}, 7 \mathrm{~F})$

There are 254 symmetry adapted basis functions of A symmetry.

Integral buffers will be 262144 words long.

Raffenetti 1 integral format.

Two-electron integral symmetry is turned on.

254 basis functions, 476 primitive gaussians, 254 cartesian basis functions

61 alpha electrons 61 beta electrons

nuclear repulsion energy 1256.5335304494 Hartrees.

NAtoms $=23$ NActive $=23$ NUniq $=23 \mathrm{SFac}=1.00 \mathrm{D}+00 \mathrm{NAtFMM}=60 \mathrm{Big}=\mathrm{F}$

One-electron integrals computed using PRISM.

NBasis $=254 \operatorname{Red} \mathrm{AO}=\mathrm{T} \mathrm{NBF}=254$

NBsUse $=254$ 1.00D-06 NBFU $=254$

Harris functional with IExCor= 205 diagonalized for initial guess.

ExpMin= 1.61D-01 ExpMax= 7.00D+03 ExpMxC=1.05D+03 IAcc=1 IRadAn= 1 AccDes=1.00D-06

HarFok: IExCor $=205$ AccDes $=1.00 \mathrm{D}-06 \mathrm{IRadAn}=\quad 1 \mathrm{IDoV}=1$

ScaDFX $=1.0000001 .0000001 .0000001 .000000$

Initial guess orbital symmetries:

Occupied (A) (A) (A) (A) (A) (A) (A) (A) (A) (A) (A) (A)

(A) $(A)(A)(A)(A)(A)(A)(A)(A)(A)(A)(A)$

(A) $(A)(A)(A)(A)(A)(A)(A)(A)(A)(A)(A)$

(A) $(\mathrm{A})(\mathrm{A})(\mathrm{A})(\mathrm{A})(\mathrm{A})(\mathrm{A})(\mathrm{A})(\mathrm{A})(\mathrm{A})(\mathrm{A})(\mathrm{A})$

(A) $(\mathrm{A})(\mathrm{A})(\mathrm{A})(\mathrm{A})(\mathrm{A})(\mathrm{A})(\mathrm{A})(\mathrm{A})(\mathrm{A})(\mathrm{A})(\mathrm{A})$

(A)

Virtual (A) (A) (A) (A) (A) (A) (A) (A) (A) (A) (A) (A)

(A) $(\mathrm{A})(\mathrm{A})(\mathrm{A})(\mathrm{A})(\mathrm{A})(\mathrm{A})(\mathrm{A})(\mathrm{A})(\mathrm{A})(\mathrm{A})(\mathrm{A})$

(A) (A) (A) (A) (A) (A) (A) (A) (A) (A) (A) (A)

(A) (A) (A) (A) (A) (A) (A) (A) (A) (A) (A) (A)

(A) (A) (A) (A) (A) (A) (A) (A) (A) (A) (A) (A)

(A) (A) (A) (A) (A) (A) (A) (A) (A) (A) (A) (A)

(A) (A) (A) (A) (A) (A) (A) (A) (A) (A) (A) (A)

(A) $(A)(A)(A)(A)(A)(A)(A)(A)(A)(A)(A)$

(A) (A) (A) (A) (A) (A) (A) (A) (A) (A) (A) (A)

(A) $(\mathrm{A})(\mathrm{A})(\mathrm{A})(\mathrm{A})(\mathrm{A})(\mathrm{A})(\mathrm{A})(\mathrm{A})(\mathrm{A})(\mathrm{A})(\mathrm{A})$

(A) $(A)(A)(A)(A)(A)(A)(A)(A)(A)(A)(A)$

(A) (A) (A) (A) (A) (A) (A) (A) (A) (A) (A) (A)

(A) $(\mathrm{A})(\mathrm{A})(\mathrm{A})(\mathrm{A})(\mathrm{A})(\mathrm{A})(\mathrm{A})(\mathrm{A})(\mathrm{A})(\mathrm{A})(\mathrm{A})$

(A) (A) (A) (A) (A) (A) (A) (A) (A) (A) (A) (A)

(A) $(\mathrm{A})(\mathrm{A})(\mathrm{A})(\mathrm{A})(\mathrm{A})(\mathrm{A})(\mathrm{A})(\mathrm{A})(\mathrm{A})(\mathrm{A})(\mathrm{A})$

(A) $(\mathrm{A})(\mathrm{A})(\mathrm{A})(\mathrm{A})(\mathrm{A})(\mathrm{A})(\mathrm{A})(\mathrm{A})(\mathrm{A})(\mathrm{A})(\mathrm{A})$

(A)

The electronic state of the initial guess is 1-A.

Warning! Cutoffs for single-point calculations used.

Requested convergence on RMS density matrix=1.00D-04 within 128 cycles. 
Requested convergence on MAX density matrix=1.00D-02.

Requested convergence on energy $=5.00 \mathrm{D}-05$.

No special actions if energy rises.

SCF Done: $\mathrm{E}(\mathrm{RHF})=-991.751571312$ A.U. after 7 cycles Convg $=0.9044 \mathrm{D}-05 \quad-\mathrm{V} / \mathrm{T}=2.0026$ $\mathrm{S} * * 2=0.0000$

****************************************************************************

Population analysis using the SCF density.

*******************************************************************************

Orbital symmetries:

Occupied (A) (A) (A) (A) (A) (A) (A) (A) (A) (A) (A) (A)

(A) $(\mathrm{A})(\mathrm{A})(\mathrm{A})(\mathrm{A})(\mathrm{A})(\mathrm{A})(\mathrm{A})(\mathrm{A})(\mathrm{A})(\mathrm{A})(\mathrm{A})$

(A) $(\mathrm{A})(\mathrm{A})(\mathrm{A})(\mathrm{A})(\mathrm{A})(\mathrm{A})(\mathrm{A})(\mathrm{A})(\mathrm{A})(\mathrm{A})(\mathrm{A})$

(A) $(\mathrm{A})(\mathrm{A})(\mathrm{A})(\mathrm{A})(\mathrm{A})(\mathrm{A})(\mathrm{A})(\mathrm{A})(\mathrm{A})(\mathrm{A})(\mathrm{A})$

$(\mathrm{A})(\mathrm{A})(\mathrm{A})(\mathrm{A})(\mathrm{A})(\mathrm{A})(\mathrm{A})(\mathrm{A})(\mathrm{A})(\mathrm{A})(\mathrm{A})(\mathrm{A})$

(A)

Virtual (A) (A) (A) (A) (A) (A) (A) (A) (A) (A) (A) (A)

(A) (A) (A) (A) (A) (A) (A) (A) (A) (A) (A) (A)

(A) (A) (A) (A) (A) (A) (A) (A) (A) (A) (A) (A)

(A) $(\mathrm{A})(\mathrm{A})(\mathrm{A})(\mathrm{A})(\mathrm{A})(\mathrm{A})(\mathrm{A})(\mathrm{A})(\mathrm{A})(\mathrm{A})(\mathrm{A})$

(A) $(\mathrm{A})(\mathrm{A})(\mathrm{A})(\mathrm{A})(\mathrm{A})(\mathrm{A})(\mathrm{A})(\mathrm{A})(\mathrm{A})(\mathrm{A})(\mathrm{A})$

(A) (A) (A) (A) (A) (A) (A) (A) (A) (A) (A) (A)

(A) $(\mathrm{A})(\mathrm{A})(\mathrm{A})(\mathrm{A})(\mathrm{A})(\mathrm{A})(\mathrm{A})(\mathrm{A})(\mathrm{A})(\mathrm{A})(\mathrm{A})$

(A) (A) (A) (A) (A) (A) (A) (A) (A) (A) (A) (A)

(A) (A) (A) (A) (A) (A) (A) (A) (A) (A) (A) (A)

(A) (A) (A) (A) (A) (A) (A) (A) (A) (A) (A) (A)

(A) $(\mathrm{A})(\mathrm{A})(\mathrm{A})(\mathrm{A})(\mathrm{A})(\mathrm{A})(\mathrm{A})(\mathrm{A})(\mathrm{A})(\mathrm{A})(\mathrm{A})$

(A) (A) (A) (A) (A) (A) (A) (A) (A) (A) (A) (A)

(A) (A) (A) (A) (A) (A) (A) (A) (A) (A) (A) (A)

(A) $(\mathrm{A})(\mathrm{A})(\mathrm{A})(\mathrm{A})(\mathrm{A})(\mathrm{A})(\mathrm{A})(\mathrm{A})(\mathrm{A})(\mathrm{A})(\mathrm{A})$

(A) $(\mathrm{A})(\mathrm{A})(\mathrm{A})(\mathrm{A})(\mathrm{A})(\mathrm{A})(\mathrm{A})(\mathrm{A})(\mathrm{A})(\mathrm{A})(\mathrm{A})$

(A) $(\mathrm{A})(\mathrm{A})(\mathrm{A})(\mathrm{A})(\mathrm{A})(\mathrm{A})(\mathrm{A})(\mathrm{A})(\mathrm{A})(\mathrm{A})(\mathrm{A})$

(A)

The electronic state is 1-A.

Alpha occ. eigenvalues -- -26.36228 -26.35296 -26.35062 -26.35005 -26.34895

Alpha occ. eigenvalues -- -20.60754 -20.57380 -11.53767 -11.47447 -11.36406

Alpha occ. eigenvalues -- -11.35585 -11.29972 -11.28759 -11.28714 -11.27444

Alpha occ. eigenvalues -- $-11.27341 \quad-1.74122 \quad-1.69925 \quad-1.64495 \quad-1.63574$

Alpha occ. eigenvalues -- $-1.62857 \quad-1.42287 \quad-1.40222-1.17088-1.07502$

Alpha occ. eigenvalues -- $\quad-1.06950-1.00984-0.94378 \quad-0.92920-0.87252$

Alpha occ. eigenvalues -- $\quad-0.85325 \quad-0.83484 \quad-0.83261 \quad-0.82602 \quad-0.78484$

$\begin{array}{lllllll}\text { Alpha occ. eigenvalues -- } & -0.78173 & -0.73078 & -0.72619 & -0.71506 & -0.71085\end{array}$

Alpha occ. eigenvalues -- $-0.70898 \quad-0.69271 \quad-0.68394 \quad-0.66894 \quad-0.66361$

Alpha occ. eigenvalues -- $-0.66210 \quad-0.65207 \quad-0.65005 \quad-0.63734 \quad-0.63539$

Alpha occ. eigenvalues -- $-0.63011-0.60590 \quad-0.59745 \quad-0.56743 \quad-0.55150$

Alpha occ. eigenvalues -- $-0.53715 \quad-0.50534 \quad-0.46817 \quad-0.43493 \quad-0.41041$

Alpha occ. eigenvalues -- -0.40573

$\begin{array}{lllllll}\text { Alpha virt. eigenvalues -- } & 0.04951 & 0.13197 & 0.21418 & 0.24058 & 0.24665\end{array}$

$\begin{array}{lllllll}\text { Alpha virt. eigenvalues -- } & 0.25690 & 0.27006 & 0.28354 & 0.29173 & 0.30206\end{array}$

$\begin{array}{lllllll}\text { Alpha virt. eigenvalues -- } & 0.31260 & 0.32181 & 0.33062 & 0.36137 & 0.37636\end{array}$

$\begin{array}{lllllll}\text { Alpha virt. eigenvalues -- } & 0.39547 & 0.41482 & 0.43031 & 0.44076 & 0.45875\end{array}$

$\begin{array}{lllllll}\text { Alpha virt. eigenvalues -- } & 0.47878 & 0.49727 & 0.52900 & 0.54671 & 0.62506\end{array}$

$\begin{array}{lllllll}\text { Alpha virt. eigenvalues -- } & 0.63872 & 0.66195 & 0.67822 & 0.68857 & 0.69194\end{array}$

$\begin{array}{lllllll}\text { Alpha virt. eigenvalues -- } & 0.72262 & 0.73587 & 0.74754 & 0.75550 & 0.76212\end{array}$

$\begin{array}{lllllll}\text { Alpha virt. eigenvalues -- } & 0.76980 & 0.79585 & 0.80215 & 0.82916 & 0.84771\end{array}$

$\begin{array}{lllllll}\text { Alpha virt. eigenvalues -- } & 0.87346 & 0.89012 & 0.90940 & 0.92459 & 0.93848\end{array}$

$\begin{array}{lllllll}\text { Alpha virt. eigenvalues -- } & 0.96997 & 0.97813 & 1.00568 & 1.01443 & 1.04801\end{array}$

$\begin{array}{llllll}\text { Alpha virt. eigenvalues -- } & 1.07749 & 1.08936 & 1.09043 & 1.10896 & 1.11198\end{array}$

$\begin{array}{lllllll}\text { Alpha virt. eigenvalues -- } & 1.12902 & 1.14002 & 1.15732 & 1.17549 & 1.18191\end{array}$

$\begin{array}{lllllll}\text { Alpha virt. eigenvalues -- } & 1.19773 & 1.20213 & 1.22915 & 1.25722 & 1.29177\end{array}$ 

$\begin{array}{llllll}\text { Alpha virt. eigenvalues -- } & 1.30696 & 1.32484 & 1.33376 & 1.34771 & 1.35535 \\ \text { Alpha virt. eigenvalues -- } & 1.38828 & 1.40557 & 1.42002 & 1.43711 & 1.45411 \\ \text { Alpha virt. eigenvalues -- } & 1.46593 & 1.48709 & 1.52558 & 1.55572 & 1.57225 \\ \text { Alpha virt. eigenvalues -- } & 1.60220 & 1.60994 & 1.62982 & 1.64027 & 1.64586 \\ \text { Alpha virt. eigenvalues -- } & 1.65517 & 1.66856 & 1.67399 & 1.67662 & 1.68346 \\ \text { Alpha virt. eigenvalues -- } & 1.69558 & 1.70675 & 1.72676 & 1.73572 & 1.74818 \\ \text { Alpha virt. eigenvalues -- } & 1.76528 & 1.77819 & 1.78805 & 1.83128 & 1.86162 \\ \text { Alpha virt. eigenvalues -- } & 1.89595 & 1.90991 & 1.93278 & 1.94947 & 1.97336 \\ \text { Alpha virt. eigenvalues -- } & 1.99227 & 2.04543 & 2.06186 & 2.07034 & 2.07793 \\ \text { Alpha virt. eigenvalues -- } & 2.08062 & 2.10436 & 2.11408 & 2.12488 & 2.13840 \\ \text { Alpha virt. eigenvalues -- } & 2.15896 & 2.16858 & 2.17841 & 2.20563 & 2.21045 \\ \text { Alpha virt. eigenvalues -- } & 2.23547 & 2.24208 & 2.25845 & 2.27409 & 2.30618 \\ \text { Alpha virt. eigenvalues -- } & 2.31821 & 2.34611 & 2.34962 & 2.36133 & 2.36858 \\ \text { Alpha virt. eigenvalues -- } & 2.38073 & 2.39634 & 2.41019 & 2.42275 & 2.42925 \\ \text { Alpha virt. eigenvalues -- } & 2.44030 & 2.46312 & 2.48210 & 2.49549 & 2.53885 \\ \text { Alpha virt. eigenvalues -- } & 2.56000 & 2.57956 & 2.59227 & 2.62745 & 2.63189 \\ \text { Alpha virt. eigenvalues -- } & 2.64367 & 2.64781 & 2.67968 & 2.69754 & 2.70936 \\ \text { Alpha virt. eigenvalues -- } & 2.74593 & 2.79074 & 2.80236 & 2.86080 & 2.92185 \\ \text { Alpha virt. eigenvalues -- } & 2.92948 & 2.93378 & 2.94958 & 2.96053 & 3.03232 \\ \text { Alpha virt. eigenvalues -- } & 3.08967 & 3.10258 & 3.12347 & 3.13876 & 3.17144 \\ \text { Alpha virt. eigenvalues -- } & 3.18952 & 3.24081 & 3.27448 & 3.34256 & 3.36598 \\ \text { Alpha virt. eigenvalues -- } & 3.39486 & 3.42803 & 3.45654 & 3.47626 & 3.49298 \\ \text { Alpha virt. eigenvalues -- } & 3.53736 & 3.66042 & 4.33853 & 4.37352 & 4.51171 \\ \text { Alpha virt. eigenvalues -- } & 4.56168 & 4.61063 & 4.64641 & 4.77141 & 4.78609 \\ \text { Alpha virt. eigenvalues -- } & 4.88902 & 5.02047 & 5.02688 & 5.13543 & 5.24823 \\ \text { Alpha virt. eigenvalues -- } & 5.51302 & 5.73039 & 6.11005 & & \\ & \end{array}$

Condensed to atoms (all electrons):

$$
\begin{array}{llllll}
1 & 2 & 3 & 4 & 5 & 6
\end{array}
$$

$\begin{array}{llllllll}1 & C & 4.265796 & -0.040440 & -0.040325 & -0.009451 & 0.406502 & 0.405972\end{array}$

$\begin{array}{llllllll}2 & \mathrm{C} & -0.040440 & 5.136049 & -0.072909 & 0.353233 & 0.014365 & 0.543569\end{array}$

$\begin{array}{llllllll}3 & C & -0.040325 & -0.072909 & 5.094399 & 0.373637 & 0.543262 & 0.014039\end{array}$

$\begin{array}{llllllll}4 & C & -0.009451 & 0.353233 & 0.373637 & 4.928425 & -0.043886 & -0.039152\end{array}$

$\begin{array}{llllllll}5 & \mathrm{C} & 0.406502 & 0.014365 & 0.543262 & -0.043886 & 5.133351 & -0.115716\end{array}$

$\begin{array}{llllllll}6 & \mathrm{C} & 0.405972 & 0.543569 & 0.014039 & -0.039152 & -0.115716 & 5.126508\end{array}$

$\begin{array}{llllllll}7 & \mathrm{H} & -0.022288 & -0.000308 & -0.027170 & 0.002063 & 0.375239 & 0.003280\end{array}$

$\begin{array}{llllllll}8 \mathrm{H} & -0.021922 & -0.027219 & -0.000294 & 0.001868 & 0.003299 & 0.375533\end{array}$

$\begin{array}{llllllll}9 \mathrm{H} & 0.001166 & 0.372785 & 0.002831 & -0.030020 & -0.000375 & -0.015810\end{array}$

$\begin{array}{llllllll}10 \mathrm{H} & 0.001179 & 0.003115 & 0.376244 & -0.030169 & -0.018147 & -0.000388\end{array}$

$\begin{array}{llllllll}11 & \mathrm{O} & 0.533888 & 0.004256 & 0.004163 & -0.000104 & -0.058895 & -0.058890\end{array}$

$\begin{array}{llllllll}12 & \mathrm{C} & -0.000262 & -0.066609 & -0.056212 & 0.360514 & -0.002807 & -0.001589\end{array}$

$\begin{array}{llllllll}13 & \mathrm{O} & 0.000149 & -0.035585 & -0.034645 & 0.150965 & 0.001768 & 0.001484\end{array}$

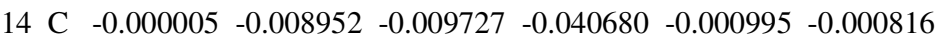

$\begin{array}{llllllll}15 \mathrm{H} & -0.000046 & -0.000523 & 0.005586 & -0.005033 & -0.000751 & 0.000292\end{array}$

$\begin{array}{llllllll}16 \mathrm{H} & 0.000001 & -0.000010 & 0.000032 & 0.005040 & 0.000011 & 0.000009\end{array}$

$\begin{array}{llllllll}17 & \mathrm{H} & -0.000049 & 0.005562 & -0.000574 & -0.005676 & 0.000329 & -0.000617\end{array}$

$\begin{array}{llllllll}18 & \mathrm{C} & -0.000006 & -0.004479 & 0.003886 & -0.055103 & -0.000029 & 0.000180\end{array}$

$\begin{array}{llllllll}19 & \mathrm{~F} & 0.000003 & 0.001348 & -0.000045 & -0.000067 & -0.000002 & -0.000098\end{array}$

$\begin{array}{llllllll}20 \mathrm{~F} & 0.000000 & 0.000015 & -0.000025 & 0.001263 & 0.000000 & 0.000000\end{array}$

$\begin{array}{llllllll}21 & \mathrm{~F} & 0.000000 & -0.000303 & -0.000024 & 0.002522 & 0.000000 & -0.000003\end{array}$

$\begin{array}{llllllll}22 & \mathrm{~F} & 0.000006 & 0.001903 & 0.004440 & -0.037753 & 0.000201 & -0.000016\end{array}$

$\begin{array}{lllllllll}23 & \mathrm{~F} & 0.000303 & 0.006872 & 0.003786 & -0.040099 & 0.000041 & -0.000262\end{array}$

$$
\begin{array}{llllll}
7 & 8 & 9 & 10 & 11 & 12
\end{array}
$$

$\begin{array}{llllllll}1 & \mathrm{C} & -0.022288 & -0.021922 & 0.001166 & 0.001179 & 0.533888 & -0.000262\end{array}$

$\begin{array}{llllllll}2 & \mathrm{C} & -0.000308 & -0.027219 & 0.372785 & 0.003115 & 0.004256 & -0.066609\end{array}$

$\begin{array}{llllllll}3 & C & -0.027170 & -0.000294 & 0.002831 & 0.376244 & 0.004163 & -0.056212\end{array}$

$\begin{array}{llllllll}4 & \mathrm{C} & 0.002063 & 0.001868 & -0.030020 & -0.030169 & -0.000104 & 0.360514\end{array}$

$\begin{array}{llllllll}5 & \mathrm{C} & 0.375239 & 0.003299 & -0.000375 & -0.018147 & -0.058895 & -0.002807\end{array}$

$\begin{array}{llllllll}6 & \mathrm{C} & 0.003280 & 0.375533 & -0.015810 & -0.000388 & -0.058890 & -0.001589\end{array}$

$\begin{array}{llllllll}7 & \mathrm{H} & 0.425090 & -0.000049 & 0.000006 & -0.001817 & 0.001228 & -0.000057\end{array}$

$\begin{array}{llllllll}8 \mathrm{H} & -0.000049 & 0.423569 & -0.001809 & 0.000006 & 0.001235 & -0.000032\end{array}$

$\begin{array}{llllllll}9 \mathrm{H} & 0.000006 & -0.001809 & 0.415863 & -0.000059 & -0.000042 & -0.002710\end{array}$

$\begin{array}{llllllll}10 \mathrm{H} & -0.001817 & 0.000006 & -0.000059 & 0.418114 & -0.000041 & -0.002625\end{array}$

$\begin{array}{llllllll}11 & \mathrm{O} & 0.001228 & 0.001235 & -0.000042 & -0.000041 & 8.121531 & 0.000020\end{array}$

$\begin{array}{llllllll}12 & \mathrm{C} & -0.000057 & -0.000032 & -0.002710 & -0.002625 & 0.000020 & 4.329590\end{array}$ 
$\begin{array}{llllllll}13 & \mathrm{O} & -0.000039 & -0.000039 & 0.000862 & 0.000916 & 0.000000 & -0.055979\end{array}$

$\begin{array}{llllllll}14 \mathrm{C} & -0.000007 & -0.000005 & -0.000084 & -0.000130 & 0.000000 & 0.003123\end{array}$

$\begin{array}{llllllll}15 & \mathrm{H} & 0.000054 & 0.000000 & 0.000098 & -0.000288 & 0.000000 & -0.000223\end{array}$

$\begin{array}{llllllll}16 & \mathrm{H} & 0.000000 & 0.000000 & -0.000037 & -0.000039 & 0.000000 & 0.000018\end{array}$

$\begin{array}{llllllll}17 & \mathrm{H} & -0.000001 & 0.000049 & -0.000340 & 0.000099 & 0.000000 & -0.000110\end{array}$

$\begin{array}{llllllll}18 & \mathrm{C} & 0.000001 & -0.000003 & 0.001549 & -0.000025 & 0.000000 & 0.380946\end{array}$

$\begin{array}{llllllll}19 & \mathrm{~F} & 0.000000 & -0.000003 & 0.003381 & 0.000000 & 0.000000 & -0.030262\end{array}$

$\begin{array}{llllllll}20 \mathrm{~F} & 0.000000 & 0.000000 & 0.000015 & 0.000000 & 0.000000 & -0.025204\end{array}$

$\begin{array}{llllllll}21 \mathrm{~F} & 0.000000 & 0.000000 & 0.000179 & -0.000001 & 0.000000 & -0.031242\end{array}$

$\begin{array}{llllllll}22 & \mathrm{~F} & -0.000001 & 0.000000 & -0.000005 & 0.002181 & 0.000000 & 0.225776\end{array}$

$\begin{array}{lllllllll}23 & F & 0.000001 & -0.000003 & 0.000111 & 0.000109 & -0.000002 & 0.227265\end{array}$

$\begin{array}{llllll}13 & 14 & 15 & 16 & 17 & 18\end{array}$

$\begin{array}{llllllll}1 & C & 0.000149 & -0.000005 & -0.000046 & 0.000001 & -0.000049 & -0.000006\end{array}$

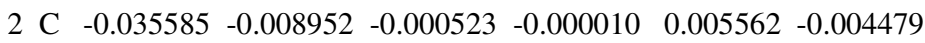

$\begin{array}{llllllll}3 \mathrm{C} & -0.034645 & -0.009727 & 0.005586 & 0.000032 & -0.000574 & 0.003886\end{array}$

$\begin{array}{llllllll}4 \mathrm{C} & 0.150965 & -0.040680 & -0.005033 & 0.005040 & -0.005676 & -0.055103\end{array}$

$\begin{array}{llllllll}5 & \mathrm{C} & 0.001768 & -0.000995 & -0.000751 & 0.000011 & 0.000329 & -0.000029\end{array}$

$\begin{array}{llllllll}6 \mathrm{C} & 0.001484 & -0.000816 & 0.000292 & 0.000009 & -0.000617 & 0.000180\end{array}$

$\begin{array}{lllllllll}7 & \mathrm{H} & -0.000039 & -0.000007 & 0.000054 & 0.000000 & -0.000001 & 0.000001\end{array}$

$\begin{array}{llllllll}8 & \mathrm{H} & -0.000039 & -0.000005 & 0.000000 & 0.000000 & 0.000049 & -0.000003\end{array}$

$\begin{array}{llllllll}9 \mathrm{H} & 0.000862 & -0.000084 & 0.000098 & -0.000037 & -0.000340 & 0.001549\end{array}$

$\begin{array}{llllllll}10 \mathrm{H} & 0.000916 & -0.000130 & -0.000288 & -0.000039 & 0.000099 & -0.000025\end{array}$

$\begin{array}{lllllllll}11 & \mathrm{O} & 0.000000 & 0.000000 & 0.000000 & 0.000000 & 0.000000 & 0.000000\end{array}$

$\begin{array}{llllllll}12 & \mathrm{C} & -0.055979 & 0.003123 & -0.000223 & 0.000018 & -0.000110 & 0.380946\end{array}$

$\begin{array}{llllllll}13 & \mathrm{O} & 8.527314 & 0.187798 & -0.037787 & -0.038205 & -0.037546 & 0.006821\end{array}$

$\begin{array}{llllllll}14 & \mathrm{C} & 0.187798 & 4.868262 & 0.390241 & 0.403259 & 0.390433 & -0.000290\end{array}$

$\begin{array}{llllllll}15 & \mathrm{H} & -0.037787 & 0.390241 & 0.549351 & -0.026024 & -0.040335 & 0.000023\end{array}$

$\begin{array}{llllllll}16 \mathrm{H} & -0.038205 & 0.403259 & -0.026024 & 0.483725 & -0.026025 & -0.000006\end{array}$

$\begin{array}{llllllll}17 & \mathrm{H} & -0.037546 & 0.390433 & -0.040335 & -0.026025 & 0.550114 & -0.000042\end{array}$

$\begin{array}{llllllll}18 & \mathrm{C} & 0.006821 & -0.000290 & 0.000023 & -0.000006 & -0.000042 & 3.776394\end{array}$

$\begin{array}{lllllll}19 \mathrm{~F} & -0.000217 & 0.000000 & 0.000000 & 0.000000 & 0.000000 & 0.266310\end{array}$

$\begin{array}{lllllllll}20 \mathrm{~F} & -0.000007 & 0.000000 & 0.000000 & 0.000000 & 0.000000 & 0.282671\end{array}$

$\begin{array}{llllllll}21 & \mathrm{~F} & -0.001610 & 0.000144 & -0.000003 & 0.000015 & 0.000003 & 0.281073\end{array}$

$\begin{array}{llllllll}22 & \mathrm{~F} & 0.002734 & -0.000056 & 0.000004 & 0.000000 & -0.000001 & -0.015868\end{array}$

$\begin{array}{lllllllll}23 & \mathrm{~F} & 0.000578 & -0.000013 & 0.000000 & 0.000000 & 0.000000 & -0.016148\end{array}$

$\begin{array}{lllll}19 & 20 & 21 & 22 & 23\end{array}$

$\begin{array}{lllllll}1 & \mathrm{C} & 0.000003 & 0.000000 & 0.000000 & 0.000006 & 0.000303\end{array}$

$\begin{array}{lllllll}2 & \mathrm{C} & 0.001348 & 0.000015 & -0.000303 & 0.001903 & 0.006872\end{array}$

$\begin{array}{lllllll}3 \mathrm{C} & -0.000045 & -0.000025 & -0.000024 & 0.004440 & 0.003786\end{array}$

$\begin{array}{lllllll}4 & \mathrm{C} & -0.000067 & 0.001263 & 0.002522 & -0.037753 & -0.040099\end{array}$

$\begin{array}{lllllll}5 & \mathrm{C} & -0.000002 & 0.000000 & 0.000000 & 0.000201 & 0.000041\end{array}$

$\begin{array}{lllllll}6 & \mathrm{C} & -0.000098 & 0.000000 & -0.000003 & -0.000016 & -0.000262\end{array}$

$\begin{array}{lllllll}7 & \mathrm{H} & 0.000000 & 0.000000 & 0.000000 & -0.000001 & 0.000001\end{array}$

$\begin{array}{llllllll}8 \mathrm{H} & -0.000003 & 0.000000 & 0.000000 & 0.000000 & -0.000003\end{array}$

$\begin{array}{lllllll}9 \mathrm{H} & 0.003381 & 0.000015 & 0.000179 & -0.000005 & 0.000111\end{array}$

$\begin{array}{lllllll}10 \mathrm{H} & 0.000000 & 0.000000 & -0.000001 & 0.002181 & 0.000109\end{array}$

$\begin{array}{llllllll}11 & \mathrm{O} & 0.000000 & 0.000000 & 0.000000 & 0.000000 & -0.000002\end{array}$

$\begin{array}{lllllll}12 & \mathrm{C} & -0.030262 & -0.025204 & -0.031242 & 0.225776 & 0.227265\end{array}$

$\begin{array}{lllllll}13 & \mathrm{O} & -0.000217 & -0.000007 & -0.001610 & 0.002734 & 0.000578\end{array}$

$\begin{array}{lllllll}14 \mathrm{C} & 0.000000 & 0.000000 & 0.000144 & -0.000056 & -0.000013\end{array}$

$\begin{array}{lllllll}15 \mathrm{H} & 0.000000 & 0.000000 & -0.000003 & 0.000004 & 0.000000\end{array}$

$\begin{array}{llllll}16 \mathrm{H} & 0.000000 & 0.000000 & 0.000015 & 0.000000 & 0.000000\end{array}$

$\begin{array}{llllllll}17 & \mathrm{H} & 0.000000 & 0.000000 & 0.000003 & -0.000001 & 0.000000\end{array}$

$\begin{array}{lllllll}18 & \mathrm{C} & 0.266310 & 0.282671 & 0.281073 & -0.015868 & -0.016148\end{array}$

$\begin{array}{llllllll}19 & \text { F } & 9.188474 & -0.035357 & -0.032954 & 0.000225 & 0.000639\end{array}$

$\begin{array}{lllllll}20 \mathrm{~F} & -0.035357 & 9.174592 & -0.035518 & 0.000577 & 0.000382\end{array}$

$\begin{array}{lllllll}21 & \mathrm{~F} & -0.032954 & -0.035518 & 9.164928 & 0.000514 & 0.000221\end{array}$

$\begin{array}{llllllll}22 & \mathrm{~F} & 0.000225 & 0.000577 & 0.000514 & 9.229634 & -0.036009\end{array}$

$\begin{array}{lllllll}23 & F & 0.000639 & 0.000382 & 0.000221 & -0.036009 & 9.228512\end{array}$

Mulliken atomic charges:

1

$\begin{array}{lll}1 & \mathrm{C} & 0.519829\end{array}$

2 C -0.185736

3 C -0.184355 

4 C 0.157665
5 C -0.236767
6 C -0.237510
$\begin{array}{lll}7 \mathrm{H} & 0.244775\end{array}$
$8 \mathrm{H} \quad 0.245818$
$9 \mathrm{H} \quad 0.252442$
$10 \mathrm{H} \quad 0.251764$
$\begin{array}{lll}11 & \mathrm{O} & -0.548347\end{array}$
$\begin{array}{lll}12 & \mathrm{C} & 0.748671\end{array}$
13 O -0.639729
14 C -0.181502
$15 \mathrm{H} \quad 0.165365$
$16 \mathrm{H} \quad 0.198236$
$17 \mathrm{H} \quad 0.164726$
$18 \mathrm{C} \quad 1.092145$
$19 \mathrm{~F}-0.361376$
$20 \mathrm{~F}-0.363403$
$21 \mathrm{~F}-0.347941$
$22 \mathrm{~F}-0.378486$
$23 \mathrm{~F}-0.376284$

Sum of Mulliken charges $=0.00000$

Atomic charges with hydrogens summed into heavy atoms:
1 C 0.519829
2 C 0.066706
3 C 0.067409
4 C 0.157665
5 C 0.008008
6 C 0.008308
$7 \mathrm{H} \quad 0.000000$
$8 \mathrm{H} \quad 0.000000$
$9 \mathrm{H} \quad 0.000000$
$10 \mathrm{H} \quad 0.000000$
$\begin{array}{lll}11 & \mathrm{O} & -0.548347\end{array}$
$\begin{array}{lll}12 & \mathrm{C} & 0.748671\end{array}$
13 O -0.639729
$14 \mathrm{C} \quad 0.346825$
$15 \mathrm{H} \quad 0.000000$
$16 \mathrm{H} \quad 0.000000$
$17 \mathrm{H} \quad 0.000000$
$18 \mathrm{C} \quad 1.092145$
$19 \mathrm{~F}-0.361376$
$20 \mathrm{~F}-0.363403$
$\begin{array}{llll}21 & \mathrm{~F} & -0.347941\end{array}$
$22 \mathrm{~F}-0.378486$
$23 \mathrm{~F}-0.376284$

Sum of Mulliken charges $=0.00000$

Electronic spatial extent $(\mathrm{au}):\langle\mathrm{R} * * 2>=3163.1471$

Charge $=0.0000$ electrons

Dipole moment (field-independent basis, Debye):

$\mathrm{X}=0.6854 \mathrm{Y}=3.6320 \mathrm{Z}=-0.6167$ Tot $=3.7472$

Quadrupole moment (field-independent basis, Debye-Ang):

$\mathrm{XX}=-106.5336 \quad \mathrm{YY}=-88.5736 \quad \mathrm{ZZ}=-83.6114$

$\mathrm{XY}=9.2172 \mathrm{XZ}=2.8646 \quad \mathrm{YZ}=0.1001$

Traceless Quadrupole moment (field-independent basis, Debye-Ang):

$\mathrm{XX}=-13.6274 \mathrm{YY}=4.3326 \quad \mathrm{ZZ}=9.2948$

$\mathrm{XY}=9.2172 \mathrm{XZ}=2.8646 \mathrm{YZ}=0.1001$

Octapole moment (field-independent basis, Debye-Ang $* * 2$ ):

$\mathrm{XXX}=-119.4459 \mathrm{YYY}=10.0618 \mathrm{ZZZ}=-0.4504 \mathrm{XYY}=-25.1600$

$\mathrm{XXY}=29.6453 \mathrm{XXZ}=13.3930 \mathrm{XZZ}=-4.2418 \mathrm{YZZ}=-2.6005$

$\mathrm{YYZ}=-0.8338 \mathrm{XYZ}=0.1321$

Hexadecapole moment (field-independent basis, Debye-Ang**3):

$X X X X=-2601.9900 \mathrm{YYYY}=-788.1785 \mathrm{ZZZZ}=-521.7721 \mathrm{XXXY}=154.0125$

$\mathrm{XXXZ}=55.6111 \mathrm{YYYX}=36.0961 \mathrm{YYYZ}=-13.6578 \mathrm{ZZZX}=6.0212$ 
$Z Z Z Y=7.8898 X X Y Y=-577.4437 X X Z Z=-435.5433 \mathrm{YYZZ}=-234.5860$

$\mathrm{XXYZ}=-6.1299 \mathrm{YYXZ}=4.1164 \mathrm{ZZXY}=0.8341$

$\mathrm{N}-\mathrm{N}=1.256533530449 \mathrm{D}+03 \mathrm{E}-\mathrm{N}=-4.858724791727 \mathrm{D}+03 \mathrm{KE}=9.891710935653 \mathrm{D}+02$

1|1|UNPC-UNKISP|RHF|6-31G(d)|C9H7F5O2|PCUSER|17-May-2006|0|\#\# RHF/6-31

G(D) GEOM=CONNECTIVITYIITitle Card Required $\| 0,1 \mid \mathrm{C}, 0,2.96413824,-0.690$

$74875,-0.22077444|\mathrm{C}, 0,0.85084187,0.10119748,-1.20922652| \mathrm{C}, 0,1.40034951$

$, 0.57425383,1.19836024|\mathrm{C}, 0,0.33657335,0.57522398,0.12644901| \mathrm{C}, 0,2.5899$

$01,-0.00922535,1.0442025|\mathrm{C}, 0,2.04024324,-0.48236944,-1.36478774| \mathrm{H}, 0,3$.

$33251624,-0.0073731,1.85316749|\mathrm{H}, 0,2.37697837,-0.82787186,-2.35035545|$

$\mathrm{H}, 0,0.19552489,0.27925979,-2.07226341 \mid \mathrm{H}, 0,1.13264591,1.08291022,2.1349$

$9498|\mathrm{O}, 0,3.97170151,-1.36494047,-0.31844737| \mathrm{C}, 0,-0.84673838,-0.3728965$

$7,0.62649217|\mathrm{O}, 0,-0.29350835,1.8578424,0.02387531| \mathrm{C}, 0,0.55164901,2.908$

$09672,-0.3722865|\mathrm{H}, 0,1.36296626,3.07180511,0.34653621| \mathrm{H}, 0,-0.12954822$,

$3.76328472,-0.38139646|\mathrm{H}, 0,0.9698026,2.74114387,-1.37131128| \mathrm{C}, 0,-2.152$

$57129,-0.41677051,-0.32695154|\mathrm{~F}, 0,-1.91042941,-0.92903546,-1.54563165|$

F,0,-3.1036956,-1.20357695,0.21298869|F,0,-2.72957253,0.78044474,-0.51

$393949|\mathrm{~F}, 0,-1.27690631,-0.01492349,1.85925172| \mathrm{F}, 0,-0.43192403,-1.65145$

$799,0.78603531 \|$ Version $=x 86-$ Win32-G03RevB.05|State $=1-\mathrm{AlHF}=-991.7515713$

$|\mathrm{RMSD}=9.044 \mathrm{e}-006| \mathrm{Dipole}=0.2696625,1.42895,-0.2426093 \mid \mathrm{PG}=\mathrm{C} 01$ [X(C9H7F5O

2)]॥@

A COMPUTER IS LIKE A HORSE, IT WILL SENSE WEAKNESS.

-- GREG WETTSTEIN

Job cpu time: 0 days 0 hours 3 minutes 2.0 seconds.

File lengths (MBytes): $\mathrm{RWF}=43 \mathrm{Int}=0 \mathrm{D} 2 \mathrm{E}=0 \mathrm{Chk}=9 \mathrm{Scr}=1$

Normal termination of Gaussian 03 at Wed May 17 14:04:47 2006. 


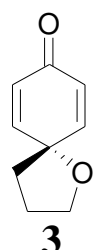

Entering Link 1 = C:IG03WV11.exe PID= 2004

Copyright (c) 1988,1990,1992,1993,1995,1998,2003, Gaussian, Inc. All Rights Reserved.

This is the Gaussian(R) 03 program. It is based on the the Gaussian(R) 98 system (copyright 1998, Gaussian, Inc.), the Gaussian(R) 94 system (copyright 1995, Gaussian, Inc.), the Gaussian 92(TM) system (copyright 1992, Gaussian, Inc.), the Gaussian 90(TM) system (copyright 1990, Gaussian, Inc.), the Gaussian 88(TM) system (copyright 1988, Gaussian, Inc.), the Gaussian 86(TM) system (copyright 1986, Carnegie Mellon University), and the Gaussian 82(TM) system (copyright 1983, Carnegie Mellon University). Gaussian is a federally registered trademark of Gaussian, Inc.

This software contains proprietary and confidential information, including trade secrets, belonging to Gaussian, Inc.

This software is provided under written license and may be used, copied, transmitted, or stored only in accord with that written license.

The following legend is applicable only to US Government contracts under DFARS:

\section{RESTRICTED RIGHTS LEGEND}

Use, duplication or disclosure by the US Government is subject to restrictions as set forth in subparagraph (c)(1)(ii) of the

Rights in Technical Data and Computer Software clause at DFARS 252.227-7013.

Gaussian, Inc.

Carnegie Office Park, Building 6, Pittsburgh, PA 15106 USA

The following legend is applicable only to US Government contracts under FAR:

\section{RESTRICTED RIGHTS LEGEND}

Use, reproduction and disclosure by the US Government is subject to restrictions as set forth in subparagraph (c) of the

Commercial Computer Software - Restricted Rights clause at FAR 52.227-19.

Gaussian, Inc.

Carnegie Office Park, Building 6, Pittsburgh, PA 15106 USA

Warning -- This program may not be used in any manner that competes with the business of Gaussian, Inc. or will provide assistance to any competitor of Gaussian, Inc. The licensee of this program is prohibited from giving any competitor of Gaussian, Inc. access to this program. By using this program, the user acknowledges that Gaussian, Inc. is engaged in the 
business of creating and licensing software in the field of computational chemistry and represents and warrants to the licensee that it is not a competitor of Gaussian, Inc. and that it will not use this program in any manner prohibited above.

Cite this work as:

Gaussian 03, Revision B.05,

M. J. Frisch, G. W. Trucks, H. B. Schlegel, G. E. Scuseria,

M. A. Robb, J. R. Cheeseman, J. A. Montgomery, Jr., T. Vreven,

K. N. Kudin, J. C. Burant, J. M. Millam, S. S. Iyengar, J. Tomasi,

V. Barone, B. Mennucci, M. Cossi, G. Scalmani, N. Rega,

G. A. Petersson, H. Nakatsuji, M. Hada, M. Ehara, K. Toyota,

R. Fukuda, J. Hasegawa, M. Ishida, T. Nakajima, Y. Honda, O. Kitao,

H. Nakai, M. Klene, X. Li, J. E. Knox, H. P. Hratchian, J. B. Cross,

C. Adamo, J. Jaramillo, R. Gomperts, R. E. Stratmann, O. Yazyev,

A. J. Austin, R. Cammi, C. Pomelli, J. W. Ochterski, P. Y. Ayala,

K. Morokuma, G. A. Voth, P. Salvador, J. J. Dannenberg,

V. G. Zakrzewski, S. Dapprich, A. D. Daniels, M. C. Strain,

O. Farkas, D. K. Malick, A. D. Rabuck, K. Raghavachari,

J. B. Foresman, J. V. Ortiz, Q. Cui, A. G. Baboul, S. Clifford,

J. Cioslowski, B. B. Stefanov, G. Liu, A. Liashenko, P. Piskorz,

I. Komaromi, R. L. Martin, D. J. Fox, T. Keith, M. A. Al-Laham,

C. Y. Peng, A. Nanayakkara, M. Challacombe, P. M. W. Gill,

B. Johnson, W. Chen, M. W. Wong, C. Gonzalez, and J. A. Pople, Gaussian, Inc., Pittsburgh PA, 2003.

$* * * * * * * * * * * * * * * * * * * * * * * * * * * * * * * * * * * * * * * * * * * * * *$

Gaussian 03: x86-Win32-G03RevB.05 8-Nov-2003

18-May-2006

***************************************************

$\%$ chk=spiro2.chk

$\%$ mem $=64 \mathrm{MB}$

$\%$ nproc $=1$

Will use up to 1 processors via shared memory.

\# hf/6-31g(d) geom=connectivity

$1 / 38=1,57=2 / 1$;

$2 / 17=6,18=5,40=1 / 2$;

$3 / 5=1,6=6,7=1,11=9,16=1,25=1,30=1 / 1,2,3$;

$4 / / 1$

$5 / 5=2,32=1,38=5 / 2$;

$6 / 7=2,8=2,9=2,10=2,28=1 / 1$;

$99 / 5=1,9=1 / 99$;

Title Card Required

Symbolic Z-matrix:

Charge $=0$ Multiplicity $=1$

C $\quad 2.02385-1.09655 \quad 0.74525$

$\begin{array}{llll}\text { C } & -0.02215 & -1.05455 & -0.60775\end{array}$

$\begin{array}{llll}\mathrm{C} & 0.61785 & 0.91145 & 0.80225\end{array}$

$\begin{array}{llll}\mathrm{C} & -0.42115 & 0.30945 & -0.10375\end{array}$

$\begin{array}{llll}\mathrm{C} & 1.72985 & 0.27945 & 1.20325\end{array}$

$\begin{array}{llll}\mathrm{C} & 1.08385 & -1.70755 & -0.22075\end{array}$

$\begin{array}{llll}\mathrm{H} & 2.45985 & 0.73745 & 1.85825\end{array}$

$\mathrm{H} \quad 1.34585-2.68855-0.59675$

$\mathrm{H} \quad \begin{array}{llll}\mathrm{H} & -0.67715 & -1.52455 & -1.33575\end{array}$

$\begin{array}{llll}\mathrm{H} & 0.44585 & 1.93145 & 1.13625\end{array}$

$\begin{array}{llll}\mathrm{O} & 3.00885 & -1.70455 & 1.14525\end{array}$

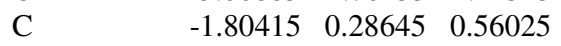

O $\quad-0.54015 \quad 1.16045-1.25675$ 


$\begin{array}{llrr}\mathrm{H} & -2.04415 & -0.66255 & 1.05125 \\ \mathrm{H} & -1.89715 & 1.07745 & 1.31625 \\ \mathrm{C} & -1.90615 & 1.57245 & -1.39575 \\ \mathrm{H} & -1.99215 & 2.59045 & -1.00075 \\ \mathrm{H} & -2.18115 & 1.58345 & -2.45475 \\ \mathrm{C} & -2.73315 & 0.59945 & -0.58875 \\ \mathrm{H} & -2.92715 & -0.30755 & -1.17275 \\ \mathrm{H} & -3.69415 & 1.01545 & -0.27475\end{array}$

Input orientation:

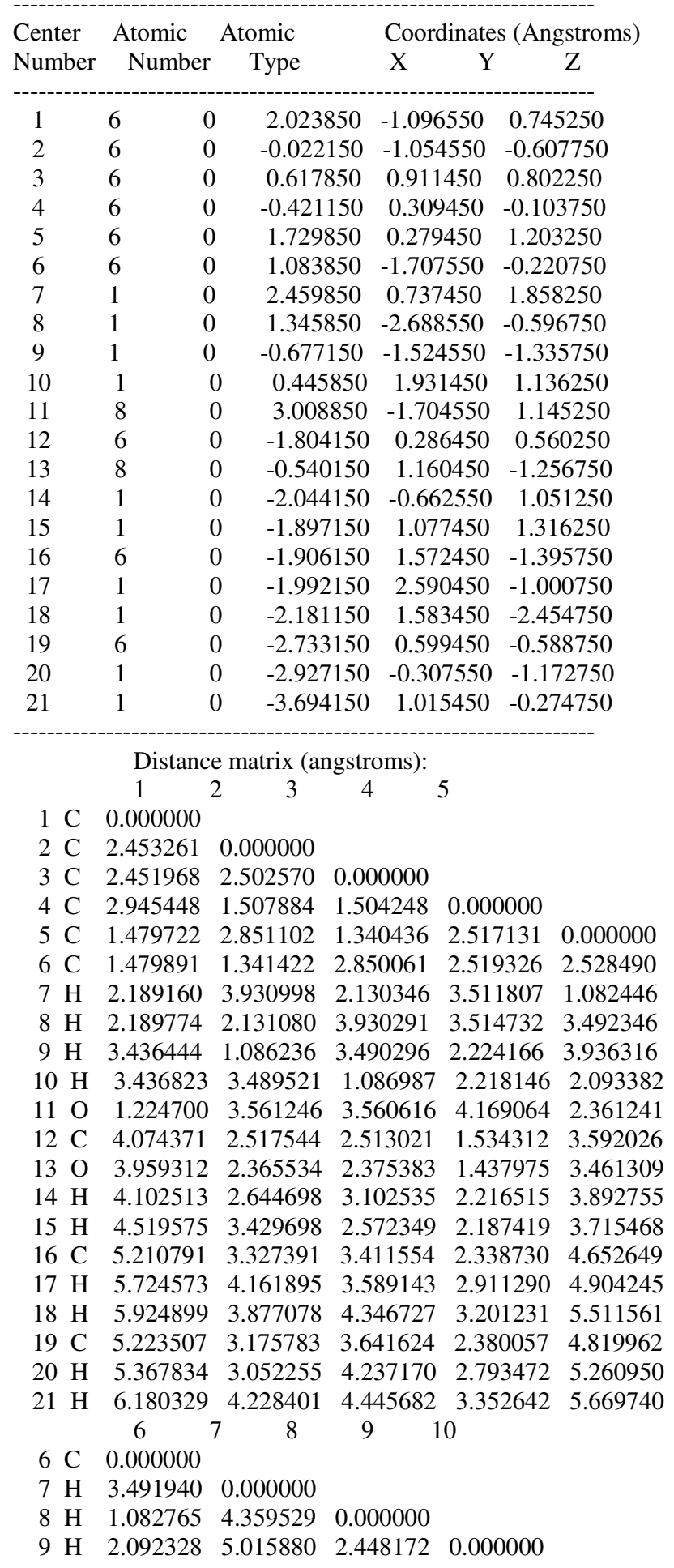


$\begin{array}{lllllll}10 & \mathrm{H} & 3.935837 & 2.450126 & 5.015744 & 4.394980 & 0.000000\end{array}$

$\begin{array}{lllllll}11 & \mathrm{O} & 2.360422 & 2.602525 & 2.601613 & 4.446837 & 4.448544\end{array}$

$\begin{array}{lllllll}12 & \mathrm{C} & 3.595350 & 4.479944 & 4.484615 & 2.853886 & 2.846103\end{array}$

$\begin{array}{llllllll}13 & \mathrm{O} & 3.454866 & 4.345360 & 4.336750 & 2.689653 & 2.700571\end{array}$

$\begin{array}{llllllll}14 & \mathrm{H} & 3.534741 & 4.785109 & 4.279332 & 2.882621 & 3.596688\end{array}$

$\begin{array}{llllllll}15 & \mathrm{H} & 4.359467 & 4.403727 & 5.325352 & 3.910487 & 2.500273\end{array}$

$\begin{array}{lllllll}16 \mathrm{C} & 4.591201 & 5.508874 & 5.419412 & 3.332484 & 3.474451\end{array}$

$\begin{array}{llllllll}17 & \mathrm{H} & 5.342563 & 5.606050 & 6.258858 & 4.332975 & 3.308307\end{array}$

$\begin{array}{lllllll}18 & \mathrm{H} & 5.146034 & 6.391914 & 5.843105 & 3.629579 & 4.462904\end{array}$

$\begin{array}{llllllll}19 & \mathrm{C} & 4.475172 & 5.742308 & 5.239203 & 3.049020 & 3.854334\end{array}$

$\begin{array}{llllllll}20 \mathrm{H} & 4.353668 & 6.268872 & 4.925390 & 2.563232 & 4.660658\end{array}$

$\begin{array}{llllllll}21 & \mathrm{H} & 5.499721 & 6.519102 & 6.262979 & 4.084068 & 4.468733\end{array}$

$\begin{array}{llll}12 & \mathrm{C} & 5.241305 & 0.000000\end{array}$

$\begin{array}{lllll}13 & \mathrm{O} & 5.154923 & 2.379719 & 0.000000\end{array}$

$\begin{array}{llllll}14 & \mathrm{H} & 5.160175 & 1.095117 & 3.303363 & 0.000000\end{array}$

$\begin{array}{lllllll}15 & \mathrm{H} & 5.642482 & 1.098119 & 2.910097 & 1.766192 & 0.000000\end{array}$

$\begin{array}{lllllll}16 & \mathrm{C} & 6.430601 & 2.343104 & 1.433534 & 3.316938 & 2.756819\end{array}$

$\begin{array}{llllllll}17 & \mathrm{H} & 6.932701 & 2.789351 & 2.053957 & 3.846481 & 2.768878\end{array}$

$\begin{array}{lllllll}18 & \mathrm{H} & 7.120888 & 3.303720 & 2.075335 & 4.165972 & 3.815381\end{array}$

$\begin{array}{lllllll}19 & \mathrm{C} & 6.425398 & 1.510368 & 2.360126 & 2.181047 & 2.134574\end{array}$

$\begin{array}{lllllll}20 \mathrm{H} & 6.523866 & 2.148780 & 2.803542 & 2.419068 & 3.028902\end{array}$

$\begin{array}{lllllll}21 & \mathrm{H} & 7.371907 & 2.191065 & 3.306519 & 2.701196 & 2.400903\end{array}$

$16 \mathrm{C} \quad 0.000000$

$\begin{array}{llll}17 \mathrm{H} & 1.095329 & 0.000000\end{array}$

$\begin{array}{lllll}18 \mathrm{H} & 1.094179 & 1.778732 & 0.000000\end{array}$

$\begin{array}{llllll}19 & \mathrm{C} & 1.510598 & 2.164002 & 2.180577 & 0.000000\end{array}$

$\begin{array}{lllllll}20 & \mathrm{H} & 2.150946 & 3.049953 & 2.403315 & 1.096057 & 0.000000\end{array}$

$\begin{array}{lllllll}21 & \mathrm{H} & 2.182621 & 2.429919 & 2.713705 & 1.093240 & 1.773421\end{array}$ 21

$\begin{array}{lll}21 \mathrm{H} & 0.000000\end{array}$

Stoichiometry $\mathrm{C} 9 \mathrm{H} 10 \mathrm{O} 2$

Framework group $\mathrm{C} 1[\mathrm{X}(\mathrm{C} 9 \mathrm{H} 10 \mathrm{O} 2)]$

Deg. of freedom 57

Full point group $\quad \mathrm{C} 1 \quad \mathrm{NOp} 1$

Largest Abelian subgroup $\quad \mathrm{C} 1 \quad \mathrm{NOp} 1$

Largest concise Abelian subgroup C1 NOp 1

Standard orientation:

\begin{tabular}{|c|c|c|c|c|}
\hline \multirow{2}{*}{$\begin{array}{l}\text { Center } \\
\text { Number }\end{array}$} & Atomic & Atomic & \multicolumn{2}{|c|}{ Coordinates (Angstroms) } \\
\hline & Numbe & Type & $\begin{array}{ll}X & Y\end{array}$ & $\mathrm{Z}$ \\
\hline 1 & 6 & 2.418562 & -0.065785 & -0.004415 \\
\hline 2 & 6 & 0.239856 & -1.182570 & -0.161005 \\
\hline 3 & 6 & 0.386592 & 1.303728 & 0.083218 \\
\hline 4 & 6 & -0.521759 & 0.106487 & 0.017838 \\
\hline 5 & 6 & 1.725447 & 1.238639 & 0.083014 \\
\hline 6 & 6 & 1.578190 & -1.273509 & -0.163341 \\
\hline 7 & 1 & 2.354501 & 2.118105 & 0.133210 \\
\hline 8 & 1 & 2.100551 & -2.213273 & -0.291256 \\
\hline 9 & 1 & -0.344345 & -2.087235 & -0.303132 \\
\hline 10 & 1 & -0.090258 & 2.279778 & 0.121707 \\
\hline 11 & 8 & 3.639710 & -0.143309 & 0.047309 \\
\hline 12 & 6 & -1.463468 & 0.045604 & 1.227628 \\
\hline 13 & 8 & -1.360444 & 0.258157 & -1.140339 \\
\hline 14 & 1 & -1.098531 & -0.589625 & 2.041622 \\
\hline 15 & 1 & -1.639020 & 1.044988 & 1.647484 \\
\hline 16 & 6 & -2.734124 & 0.194779 & -0.735359 \\
\hline 17 & 1 & -3.117963 & 1.219501 & -0.686815 \\
\hline 18 & 1 & -3.312015 & -0.360607 & -1.480218 \\
\hline 19 & 6 & -2.751734 & -0.462382 & 0.624691 \\
\hline 20 & 1 & -2.712649 & -1.552606 & 0.518737 \\
\hline
\end{tabular}


Rotational constants (GHZ): $\quad 3.0317571 \quad 0.7152621 \quad 0.6751577$

Standard basis: 6-31G(d) $(6 \mathrm{D}, 7 \mathrm{~F})$

There are 185 symmetry adapted basis functions of A symmetry.

Integral buffers will be 262144 words long.

Raffenetti 1 integral format.

Two-electron integral symmetry is turned on.

185 basis functions, 348 primitive gaussians, 185 cartesian basis functions

40 alpha electrons 40 beta electrons nuclear repulsion energy 602.6479923302 Hartrees.

NAtoms $=21$ NActive $=21 \mathrm{NUniq}=21 \mathrm{SFac}=1.00 \mathrm{D}+00 \mathrm{NAtFMM}=60 \mathrm{Big}=\mathrm{F}$

One-electron integrals computed using PRISM.

NBasis $=185$ RedAO $=\mathrm{T}$ NBF $=185$

$\mathrm{NBsUse}=185$ 1.00D-06 $\mathrm{NBFU}=185$

Harris functional with IExCor $=205$ diagonalized for initial guess.

ExpMin= 1.61D-01 ExpMax= 5.48D+03 ExpMxC= 8.25D+02 IAcc=1 IRadAn=

HarFok: IExCor $=205$ AccDes $=1.00 \mathrm{D}-06 \mathrm{IRadAn}=\quad 1 \mathrm{IDoV}=1$

ScaDFX $=1.0000001 .0000001 .0000001 .000000$

Initial guess orbital symmetries:

Occupied (A) (A) (A) (A) (A) (A) (A) (A) (A) (A) (A) (A)

(A) (A) (A) (A) (A) (A) (A) (A) (A) (A) (A) (A)

(A) $(\mathrm{A})(\mathrm{A})(\mathrm{A})(\mathrm{A})(\mathrm{A})(\mathrm{A})(\mathrm{A})(\mathrm{A})(\mathrm{A})(\mathrm{A})(\mathrm{A})$

(A) $(A)(A)(A)$

Virtual (A) (A) (A) (A) (A) (A) (A) (A) (A) (A) (A) (A)

(A) $(\mathrm{A})(\mathrm{A})(\mathrm{A})(\mathrm{A})(\mathrm{A})(\mathrm{A})(\mathrm{A})(\mathrm{A})(\mathrm{A})(\mathrm{A})(\mathrm{A})$

(A) (A) (A) (A) (A) (A) (A) (A) (A) (A) (A) (A)

(A) (A) (A) (A) (A) (A) (A) (A) (A) (A) (A) (A)

(A) (A) (A) (A) (A) (A) (A) (A) (A) (A) (A) (A)

(A) (A) (A) (A) (A) (A) (A) (A) (A) (A) (A) (A)

(A) (A) (A) (A) (A) (A) (A) (A) (A) (A) (A) (A)

(A) (A) (A) (A) (A) (A) (A) (A) (A) (A) (A) (A)

(A) (A) (A) (A) (A) (A) (A) (A) (A) (A) (A) (A)

(A) $(\mathrm{A})(\mathrm{A})(\mathrm{A})(\mathrm{A})(\mathrm{A})(\mathrm{A})(\mathrm{A})(\mathrm{A})(\mathrm{A})(\mathrm{A})(\mathrm{A})$

(A) (A) (A) (A) (A) (A) (A) (A) (A) (A) (A) (A)

(A) $(A)(A)(A)(A)(A)(A)(A)(A)(A)(A)(A)$

(A)

The electronic state of the initial guess is 1-A.

Warning! Cutoffs for single-point calculations used.

Requested convergence on RMS density matrix=1.00D-04 within 128 cycles.

Requested convergence on MAX density matrix=1.00D-02.

Requested convergence on energy $=5.00 \mathrm{D}-05$.

No special actions if energy rises.

SCF Done: $\mathrm{E}(\mathrm{RHF})=-496.320576223 \quad$ A.U. after 6 cycles

Convg $=0.2026 \mathrm{D}-04 \quad-\mathrm{V} / \mathrm{T}=2.0018$

$\mathrm{S} * * 2=0.0000$

$* * * * * * * * * * * * * * * * * * * * * * * * * * * * * * * * * * * * * * * * * * * * * * * * * * * * * * * * * * * * * * * * * * * * * *$

Population analysis using the SCF density.

***************************************************************************

Orbital symmetries:

Occupied (A) (A) (A) (A) (A) (A) (A) (A) (A) (A) (A) (A)

(A) $(\mathrm{A})(\mathrm{A})(\mathrm{A})(\mathrm{A})(\mathrm{A})(\mathrm{A})(\mathrm{A})(\mathrm{A})(\mathrm{A})(\mathrm{A})(\mathrm{A})$

(A) $(\mathrm{A})(\mathrm{A})(\mathrm{A})(\mathrm{A})(\mathrm{A})(\mathrm{A})(\mathrm{A})(\mathrm{A})(\mathrm{A})(\mathrm{A})(\mathrm{A})$

(A) $(\mathrm{A})(\mathrm{A})(\mathrm{A})$

Virtual (A) (A) (A) (A) (A) (A) (A) (A) (A) (A) (A) (A)

(A) (A) (A) (A) (A) (A) (A) (A) (A) (A) (A) (A)

(A) $(\mathrm{A})(\mathrm{A})(\mathrm{A})(\mathrm{A})(\mathrm{A})(\mathrm{A})(\mathrm{A})(\mathrm{A})(\mathrm{A})(\mathrm{A})(\mathrm{A})$

(A) (A) (A) (A) (A) (A) (A) (A) (A) (A) (A) (A)

(A) (A) (A) (A) (A) (A) (A) (A) (A) (A) (A) (A)

(A) $(\mathrm{A})(\mathrm{A})(\mathrm{A})(\mathrm{A})(\mathrm{A})(\mathrm{A})(\mathrm{A})(\mathrm{A})(\mathrm{A})(\mathrm{A})(\mathrm{A})$ 
(A) $(\mathrm{A})(\mathrm{A})(\mathrm{A})(\mathrm{A})(\mathrm{A})(\mathrm{A})(\mathrm{A})(\mathrm{A})(\mathrm{A})(\mathrm{A})(\mathrm{A})$

(A) $(\mathrm{A})(\mathrm{A})(\mathrm{A})(\mathrm{A})(\mathrm{A})(\mathrm{A})(\mathrm{A})(\mathrm{A})(\mathrm{A})(\mathrm{A})(\mathrm{A})$

(A) $(\mathrm{A})(\mathrm{A})(\mathrm{A})(\mathrm{A})(\mathrm{A})(\mathrm{A})(\mathrm{A})(\mathrm{A})(\mathrm{A})(\mathrm{A})(\mathrm{A})$

(A) $(\mathrm{A})(\mathrm{A})(\mathrm{A})(\mathrm{A})(\mathrm{A})(\mathrm{A})(\mathrm{A})(\mathrm{A})(\mathrm{A})(\mathrm{A})(\mathrm{A})$

(A) $(\mathrm{A})(\mathrm{A})(\mathrm{A})(\mathrm{A})(\mathrm{A})(\mathrm{A})(\mathrm{A})(\mathrm{A})(\mathrm{A})(\mathrm{A})(\mathrm{A})$

(A) $(\mathrm{A})(\mathrm{A})(\mathrm{A})(\mathrm{A})(\mathrm{A})(\mathrm{A})(\mathrm{A})(\mathrm{A})(\mathrm{A})(\mathrm{A})(\mathrm{A})$

(A)

The electronic state is 1-A.

Alpha occ. eigenvalues -- -20.57515 -20.54274 -11.32682 -11.31594 -11.28804

Alpha occ. eigenvalues -- -11.26200 -11.26195 -11.24925 -11.24355 -11.24178

Alpha occ. eigenvalues -- $-11.24140 \quad-1.39369-1.36874-1.13924-1.08686$

Alpha occ. eigenvalues -- $-1.04171 \quad-1.01301 \quad-0.88938 \quad-0.84438 \quad-0.82556$

Alpha occ. eigenvalues -- $-0.80451 \quad-0.71225 \quad-0.70179 \quad-0.66741 \quad-0.65099$

$\begin{array}{lllllll}\text { Alpha occ. eigenvalues -- } & -0.64546 & -0.61170 & -0.60610 & -0.58553 & -0.57445\end{array}$

Alpha occ. eigenvalues -- $-0.54387 \quad-0.53989-0.52406 \quad-0.50776 \quad-0.49421$

Alpha occ. eigenvalues -- $-0.49088 \quad-0.43958 \quad-0.40736 \quad-0.37796-0.37141$

$\begin{array}{lllllll}\text { Alpha virt. eigenvalues -- } & 0.08067 & 0.16551 & 0.21062 & 0.23613 & 0.25048\end{array}$

$\begin{array}{lllllll}\text { Alpha virt. eigenvalues -- } & 0.27067 & 0.28349 & 0.28680 & 0.30041 & 0.30490\end{array}$

$\begin{array}{lllllll}\text { Alpha virt. eigenvalues -- } & 0.32487 & 0.32877 & 0.34159 & 0.36550 & 0.37527\end{array}$

$\begin{array}{lllllll}\text { Alpha virt. eigenvalues -- } & 0.38298 & 0.39823 & 0.42712 & 0.44172 & 0.46509\end{array}$

$\begin{array}{lllllll}\text { Alpha virt. eigenvalues -- } & 0.50169 & 0.54642 & 0.55343 & 0.65420 & 0.68342\end{array}$

$\begin{array}{lllllll}\text { Alpha virt. eigenvalues -- } & 0.70774 & 0.71112 & 0.71773 & 0.72755 & 0.75517\end{array}$

$\begin{array}{lllllll}\text { Alpha virt. eigenvalues -- } & 0.77607 & 0.78996 & 0.79886 & 0.82232 & 0.84414\end{array}$

$\begin{array}{lllllll}\text { Alpha virt. eigenvalues -- } & 0.85589 & 0.88905 & 0.89654 & 0.90634 & 0.92733\end{array}$

$\begin{array}{lllllll}\text { Alpha virt. eigenvalues -- } & 0.93581 & 0.94407 & 0.95058 & 0.98441 & 0.99692\end{array}$

$\begin{array}{llllll}\text { Alpha virt. eigenvalues -- } & 1.01844 & 1.03875 & 1.07105 & 1.08113 & 1.09585\end{array}$

$\begin{array}{llllll}\text { Alpha virt. eigenvalues -- } & 1.12145 & 1.12871 & 1.13169 & 1.14044 & 1.15105\end{array}$

$\begin{array}{lllllll}\text { Alpha virt. eigenvalues -- } & 1.16570 & 1.18208 & 1.18956 & 1.20019 & 1.20741\end{array}$

$\begin{array}{lllllll}\text { Alpha virt. eigenvalues -- } & 1.21789 & 1.26277 & 1.27706 & 1.31350 & 1.33796\end{array}$

$\begin{array}{lllllll}\text { Alpha virt. eigenvalues -- } & 1.36758 & 1.37920 & 1.39509 & 1.43290 & 1.47227\end{array}$

$\begin{array}{lllllll}\text { Alpha virt. eigenvalues -- } & 1.48215 & 1.50659 & 1.55513 & 1.61353 & 1.67033\end{array}$

$\begin{array}{lllllll}\text { Alpha virt. eigenvalues -- } & 1.70212 & 1.70794 & 1.73756 & 1.76871 & 1.80955\end{array}$

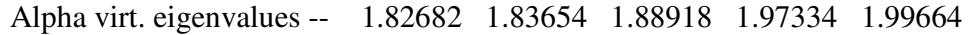

$\begin{array}{lllllll}\text { Alpha virt. eigenvalues -- } & 2.00518 & 2.05648 & 2.10425 & 2.11008 & 2.11641\end{array}$

$\begin{array}{llllll}\text { Alpha virt. eigenvalues -- } & 2.14439 & 2.16885 & 2.19768 & 2.20447 & 2.23488\end{array}$

$\begin{array}{llllll}\text { Alpha virt. eigenvalues -- } & 2.23905 & 2.24900 & 2.26494 & 2.27909 & 2.33187\end{array}$

$\begin{array}{lllllll}\text { Alpha virt. eigenvalues -- } & 2.35756 & 2.37073 & 2.38039 & 2.40075 & 2.41789\end{array}$

$\begin{array}{lllllll}\text { Alpha virt. eigenvalues -- } & 2.46093 & 2.48355 & 2.49932 & 2.52843 & 2.59710\end{array}$

$\begin{array}{lllllll}\text { Alpha virt. eigenvalues -- } & 2.64437 & 2.66667 & 2.67355 & 2.68360 & 2.72117\end{array}$

$\begin{array}{llllll}\text { Alpha virt. eigenvalues -- } & 2.73131 & 2.78649 & 2.82525 & 2.88624 & 2.93385\end{array}$

$\begin{array}{lllllll}\text { Alpha virt. eigenvalues -- } & 2.95228 & 2.95562 & 2.98045 & 3.01298 & 3.07886\end{array}$

$\begin{array}{llllll}\text { Alpha virt. eigenvalues -- } & 3.09137 & 3.10645 & 3.14396 & 3.17651 & 3.29906\end{array}$

$\begin{array}{lllllll}\text { Alpha virt. eigenvalues -- } & 3.34915 & 3.39906 & 3.50895 & 3.66303 & 4.36062\end{array}$

$\begin{array}{lllllll}\text { Alpha virt. eigenvalues -- } & 4.42795 & 4.57126 & 4.58541 & 4.62253 & 4.71468\end{array}$

$\begin{array}{lllllll}\text { Alpha virt. eigenvalues -- } & 4.78561 & 4.87000 & 4.94647 & 5.03458 & 5.22073\end{array}$ Condensed to atoms (all electrons):
$\begin{array}{llllll}1 & 2 & 3 & 4 & 5 & 6\end{array}$

$\begin{array}{llllllll}1 & C & 4.262286 & -0.038675 & -0.038979 & -0.012356 & 0.416684 & 0.416320\end{array}$

$\begin{array}{llllllll}2 & \mathrm{C} & -0.038675 & 5.033274 & -0.072006 & 0.390699 & 0.010206 & 0.557192\end{array}$

$\begin{array}{llllllll}3 & \mathrm{C} & -0.038979 & -0.072006 & 5.001501 & 0.420177 & 0.560595 & 0.009952\end{array}$

$\begin{array}{llllllll}4 & \mathrm{C} & -0.012356 & 0.390699 & 0.420177 & 4.754651 & -0.040507 & -0.037187\end{array}$

$\begin{array}{llllllll}5 & \mathrm{C} & 0.416684 & 0.010206 & 0.560595 & -0.040507 & 5.123107 & -0.107242\end{array}$

$\begin{array}{llllllll}6 \mathrm{C} & 0.416320 & 0.557192 & 0.009952 & -0.037187 & -0.107242 & 5.125251\end{array}$

$\begin{array}{llllllll}7 & \mathrm{H} & -0.024633 & -0.000407 & -0.028193 & 0.002290 & 0.377971 & 0.003604\end{array}$

$\begin{array}{llllllll}8 \mathrm{H} & -0.024320 & -0.028873 & -0.000352 & 0.002160 & 0.003546 & 0.378503\end{array}$

$\begin{array}{llllllll}9 \mathrm{H} & 0.001443 & 0.375125 & 0.002628 & -0.029413 & -0.000254 & -0.019781\end{array}$

$\begin{array}{llllllll}10 \mathrm{H} & 0.001504 & 0.002928 & 0.374678 & -0.030264 & -0.020450 & -0.000306\end{array}$

$\begin{array}{lllllllll}11 & \mathrm{O} & 0.521813 & 0.004343 & 0.004295 & 0.000051 & -0.059766 & -0.059923\end{array}$

$\begin{array}{llllllll}12 & \mathrm{C} & 0.000354 & -0.062163 & -0.059293 & 0.350523 & 0.000593 & -0.002712\end{array}$

$\begin{array}{llllllll}13 & \mathrm{O} & 0.000031 & -0.052454 & -0.049754 & 0.177742 & 0.000556 & 0.001376\end{array}$

$\begin{array}{llllllll}14 & \mathrm{H} & -0.000001 & 0.000146 & 0.000934 & -0.030055 & 0.000108 & 0.000584\end{array}$

$\begin{array}{llllllll}15 & \mathrm{H} & -0.000058 & 0.004240 & -0.006068 & -0.044105 & 0.000850 & 0.000087\end{array}$

$\begin{array}{llllllll}16 & \mathrm{C} & -0.000001 & 0.003625 & 0.002550 & -0.051733 & -0.000057 & -0.000215\end{array}$

$\begin{array}{llllllll}17 & \mathrm{H} & -0.000001 & -0.000223 & 0.000543 & 0.000341 & 0.000007 & 0.000017\end{array}$ 
$\begin{array}{llllllll}18 \mathrm{H} & 0.000001 & 0.000048 & -0.000190 & 0.003488 & 0.000001 & -0.000009\end{array}$

$\begin{array}{llllllll}19 & \mathrm{C} & -0.000009 & -0.002799 & 0.006516 & -0.075820 & -0.000139 & 0.000190\end{array}$

$\begin{array}{llllllll}20 & \mathrm{H} & -0.000005 & 0.001818 & -0.000080 & -0.003870 & 0.000009 & 0.000199\end{array}$

$\begin{array}{llllllll}21 \mathrm{H} & 0.000000 & -0.000141 & -0.000045 & 0.005483 & -0.000001 & 0.000001\end{array}$ $\begin{array}{llllll}7 & 8 & 9 & 10 & 11 & 12\end{array}$

$\begin{array}{llllllll}1 & \mathrm{C} & -0.024633 & -0.024320 & 0.001443 & 0.001504 & 0.521813 & 0.000354\end{array}$

$\begin{array}{llllllll}2 & C & -0.000407 & -0.028873 & 0.375125 & 0.002928 & 0.004343 & -0.062163\end{array}$

$\begin{array}{llllllll}3 & C & -0.028193 & -0.000352 & 0.002628 & 0.374678 & 0.004295 & -0.059293\end{array}$

$\begin{array}{llllllll}4 & \mathrm{C} & 0.002290 & 0.002160 & -0.029413 & -0.030264 & 0.000051 & 0.350523\end{array}$

$\begin{array}{llllllll}5 & \mathrm{C} & 0.377971 & 0.003546 & -0.000254 & -0.020450 & -0.059766 & 0.000593\end{array}$

$\begin{array}{llllllll}6 \mathrm{C} & 0.003604 & 0.378503 & -0.019781 & -0.000306 & -0.059923 & -0.002712\end{array}$

$\begin{array}{llllllll}7 \mathrm{H} & 0.440224 & -0.000057 & 0.000008 & -0.002181 & 0.001726 & -0.000092\end{array}$

$\begin{array}{llllllll}8 & \mathrm{H} & -0.000057 & 0.440559 & -0.002058 & 0.000008 & 0.001737 & -0.000110\end{array}$

$\begin{array}{llllllll}9 \mathrm{H} & 0.000008 & -0.002058 & 0.457637 & -0.000073 & -0.000058 & -0.001463\end{array}$

$\begin{array}{llllllll}10 \mathrm{H} & -0.002181 & 0.000008 & -0.000073 & 0.458440 & -0.000057 & -0.002602\end{array}$

$\begin{array}{llllllll}11 & \mathrm{O} & 0.001726 & 0.001737 & -0.000058 & -0.000057 & 8.167707 & 0.000005\end{array}$

$\begin{array}{llllllll}12 & \mathrm{C} & -0.000092 & -0.000110 & -0.001463 & -0.002602 & 0.000005 & 5.259810\end{array}$

$\begin{array}{llllllll}13 & \mathrm{O} & -0.000045 & -0.000048 & 0.000359 & 0.001023 & 0.000000 & -0.052863\end{array}$

$\begin{array}{llllllll}14 \mathrm{H} & 0.000007 & -0.000018 & 0.000624 & -0.000013 & -0.000001 & 0.391321\end{array}$

$\begin{array}{llllllll}15 \mathrm{H} & -0.000011 & 0.000003 & -0.000096 & 0.001584 & 0.000000 & 0.406016\end{array}$

$\begin{array}{llllllll}16 & \mathrm{C} & 0.000002 & 0.000002 & 0.000390 & 0.000310 & 0.000000 & -0.083504\end{array}$

$\begin{array}{llllllll}17 \mathrm{H} & 0.000000 & 0.000000 & 0.000001 & -0.000182 & 0.000000 & -0.003303\end{array}$

$\begin{array}{llllllll}18 \mathrm{H} & 0.000000 & 0.000000 & -0.000055 & 0.000010 & 0.000000 & 0.005780\end{array}$

$\begin{array}{llllllll}19 \mathrm{C} & 0.000002 & 0.000001 & 0.000426 & -0.000071 & 0.000000 & 0.300171\end{array}$

$\begin{array}{llllllll}20 \mathrm{H} & 0.000000 & 0.000000 & 0.000070 & -0.000008 & 0.000000 & -0.042027\end{array}$

$\begin{array}{lllllllll}21 \mathrm{H} & 0.000000 & 0.000000 & 0.000019 & -0.000002 & 0.000000 & -0.031377\end{array}$

$\begin{array}{llllll}13 & 14 & 15 & 16 & 17 & 18\end{array}$

$\begin{array}{llllllll}1 & \mathrm{C} & 0.000031 & -0.000001 & -0.000058 & -0.000001 & -0.000001 & 0.000001\end{array}$

$\begin{array}{llllllll}2 & \mathrm{C} & -0.052454 & 0.000146 & 0.004240 & 0.003625 & -0.000223 & 0.000048\end{array}$

$\begin{array}{llllllll}3 & \mathrm{C} & -0.049754 & 0.000934 & -0.006068 & 0.002550 & 0.000543 & -0.000190\end{array}$

$\begin{array}{llllllll}4 & C & 0.177742 & -0.030055 & -0.044105 & -0.051733 & 0.000341 & 0.003488\end{array}$

$\begin{array}{llllllll}5 \mathrm{C} & 0.000556 & 0.000108 & 0.000850 & -0.000057 & 0.000007 & 0.000001\end{array}$

$\begin{array}{llllllll}6 & \mathrm{C} & 0.001376 & 0.000584 & 0.000087 & -0.000215 & 0.000017 & -0.000009\end{array}$

$\begin{array}{llllllll}7 & \mathrm{H} & -0.000045 & 0.000007 & -0.000011 & 0.000002 & 0.000000 & 0.000000\end{array}$

$\begin{array}{llllllll}8 \mathrm{H} & -0.000048 & -0.000018 & 0.000003 & 0.000002 & 0.000000 & 0.000000\end{array}$

$\begin{array}{llllllll}9 \mathrm{H} & 0.000359 & 0.000624 & -0.000096 & 0.000390 & 0.000001 & -0.000055\end{array}$

$\begin{array}{llllllll}10 \mathrm{H} & 0.001023 & -0.000013 & 0.001584 & 0.000310 & -0.000182 & 0.000010\end{array}$

$\begin{array}{lllllllll}11 & \mathrm{O} & 0.000000 & -0.000001 & 0.000000 & 0.000000 & 0.000000 & 0.000000\end{array}$

$\begin{array}{llllllll}12 & \mathrm{C} & -0.052863 & 0.391321 & 0.406016 & -0.083504 & -0.003303 & 0.005780\end{array}$

$\begin{array}{llllllll}13 & \mathrm{O} & 8.535309 & 0.002237 & 0.001819 & 0.206660 & -0.043424 & -0.032434\end{array}$

$\begin{array}{llllllll}14 & \mathrm{H} & 0.002237 & 0.482295 & -0.020970 & 0.005160 & -0.000120 & -0.000069\end{array}$

$\begin{array}{llllllll}15 & \mathrm{H} & 0.001819 & -0.020970 & 0.514166 & 0.000328 & 0.001222 & -0.000077\end{array}$

$\begin{array}{llllllll}16 \mathrm{C} & 0.206660 & 0.005160 & 0.000328 & 4.816831 & 0.400632 & 0.405051\end{array}$

$\begin{array}{llllllll}17 & \mathrm{H} & -0.043424 & -0.000120 & 0.001222 & 0.400632 & 0.566721 & -0.037186\end{array}$

$\begin{array}{llllllll}18 & \mathrm{H} & -0.032434 & -0.000069 & -0.000077 & 0.405051 & -0.037186 & 0.519233\end{array}$

$\begin{array}{llllllll}19 & \mathrm{C} & -0.044703 & -0.029824 & -0.044298 & 0.342418 & -0.045420 & -0.037723\end{array}$

$\begin{array}{llllllll}20 \mathrm{H} & 0.001817 & -0.004138 & 0.003998 & -0.039757 & 0.004290 & -0.005748\end{array}$

$\begin{array}{llllllll}21 & \mathrm{H} & 0.002380 & 0.000125 & -0.004298 & -0.030204 & -0.001495 & 0.000860\end{array}$

$\begin{array}{lll}19 & 20 & 21\end{array}$

$\begin{array}{lllll}1 & \mathrm{C} & -0.000009 & -0.000005 & 0.000000\end{array}$

$\begin{array}{lllll}2 & \mathrm{C} & -0.002799 & 0.001818 & -0.000141\end{array}$

$\begin{array}{lllll}3 \text { C } & 0.006516 & -0.000080 & -0.000045\end{array}$

$\begin{array}{lllll}4 & \mathrm{C} & -0.075820 & -0.003870 & 0.005483\end{array}$

$\begin{array}{lllll}5 & \mathrm{C} & -0.000139 & 0.000009 & -0.000001\end{array}$

$\begin{array}{lllll}6 \mathrm{C} & 0.000190 & 0.000199 & 0.000001\end{array}$

$\begin{array}{lllll}7 & \mathrm{H} & 0.000002 & 0.000000 & 0.000000\end{array}$

$\begin{array}{lllll}8 \mathrm{H} & 0.000001 & 0.000000 & 0.000000\end{array}$

$\begin{array}{llllll}9 & \mathrm{H} & 0.000426 & 0.000070 & 0.000019\end{array}$

$10 \mathrm{H}-0.000071 \quad-0.000008-0.000002$

$\begin{array}{lllll}11 & \mathrm{O} & 0.000000 & 0.000000 & 0.000000\end{array}$

$\begin{array}{lllll}12 & \mathrm{C} & 0.300171 & -0.042027 & -0.031377\end{array}$

$\begin{array}{lllll}13 & \mathrm{O} & -0.044703 & 0.001817 & 0.002380\end{array}$

$\begin{array}{lllll}14 \mathrm{H} & -0.029824 & -0.004138 & 0.000125\end{array}$

$\begin{array}{lllll}15 & \mathrm{H} & -0.044298 & 0.003998 & -0.004298\end{array}$

S1- 32 
$\begin{array}{lllll}16 & \mathrm{C} & 0.342418 & -0.039757 & -0.030204\end{array}$

$\begin{array}{lllll}17 & \mathrm{H} & -0.045420 & 0.004290 & -0.001495\end{array}$

$\begin{array}{lllll}18 \mathrm{H} & -0.037723 & -0.005748 & 0.000860\end{array}$

$\begin{array}{lllll}19 & \mathrm{C} & 5.204389 & 0.401129 & 0.393654\end{array}$

$\begin{array}{llllll}20 \mathrm{H} & 0.401129 & 0.527616 & -0.021833\end{array}$

$\begin{array}{lllll}21 & \mathrm{H} & 0.393654 & -0.021833 & 0.498855\end{array}$

Mulliken atomic charges: 1

$1 \mathrm{C} \quad 0.518601$

$\begin{array}{lll}2 & C & -0.125904\end{array}$

3 C -0.129409

$\begin{array}{lll}4 & \mathrm{C} & 0.247702\end{array}$

5 C -0.265818

6 C -0.265900

$\begin{array}{lll}7 \mathrm{H} & 0.229787\end{array}$

$8 \mathrm{H} \quad 0.229317$

$9 \mathrm{H} \quad 0.214525$

$\begin{array}{lll}10 \mathrm{H} & 0.215723\end{array}$

$\begin{array}{lll}11 & \mathrm{O} & -0.581872\end{array}$

$\begin{array}{lll}12 & \mathrm{C} & -0.373063\end{array}$

13 O -0.655585

$\begin{array}{ll}14 \mathrm{H} & 0.201668\end{array}$

$\begin{array}{lll}15 \mathrm{H} & 0.185667\end{array}$

$16 \mathrm{C} \quad 0.021510$

$\begin{array}{ll}17 \mathrm{H} & 0.157580\end{array}$

$\begin{array}{lll}18 \mathrm{H} & 0.179020\end{array}$

19 C -0.368090

$20 \mathrm{H} \quad 0.176520$

$21 \mathrm{H} \quad 0.188020$

Sum of Mulliken charges $=0.00000$

Atomic charges with hydrogens summed into heavy atoms:

$$
1
$$

1 C 0.518601

2 C 0.088620

3 C 0.086314

4 C 0.247702

5 C -0.036031

6 C -0.036583

$7 \mathrm{H} \quad 0.000000$

$8 \mathrm{H} \quad 0.000000$

$9 \mathrm{H} \quad 0.000000$

$10 \mathrm{H} \quad 0.000000$

$\begin{array}{lll}11 & \mathrm{O} & -0.581872\end{array}$

12 C 0.014272

$13 \mathrm{O}-0.655585$

$14 \mathrm{H} \quad 0.000000$

$\begin{array}{lll}15 \mathrm{H} & 0.000000\end{array}$

$16 \mathrm{C} \quad 0.358111$

$\begin{array}{lll}17 \mathrm{H} & 0.000000\end{array}$

$18 \mathrm{H} \quad 0.000000$

19 C -0.003550

$20 \mathrm{H} \quad 0.000000$

$21 \mathrm{H} \quad 0.000000$

Sum of Mulliken charges $=0.00000$

Electronic spatial extent $(\mathrm{au}):<\mathrm{R} * * 2>=1761.9629$

Charge $=0.0000$ electrons

Dipole moment (field-independent basis, Debye):

$\mathrm{X}=-5.6156 \mathrm{Y}=-0.0456 \mathrm{Z}=1.6978$ Tot $=5.8669$

Quadrupole moment (field-independent basis, Debye-Ang):

$\mathrm{XX}=-76.3809 \quad \mathrm{YY}=-58.5700 \quad \mathrm{ZZ}=-67.7484$

$\mathrm{XY}=1.3429 \mathrm{XZ}=-2.2902 \quad \mathrm{YZ}=1.3603$

Traceless Quadrupole moment (field-independent basis, Debye-Ang):

$\mathrm{XX}=-8.8144 \mathrm{YY}=8.9964 \mathrm{ZZ}=-0.1820$

$\mathrm{XY}=1.3429 \mathrm{XZ}=-2.2902 \quad \mathrm{YZ}=1.3603$ 
The best way to pay for a lovely moment is to enjoy it. -- Richard Bach Job cpu time: 0 days 0 hours 1 minutes 30.0 seconds. File lengths (MBytes): RWF $=26 \mathrm{Int}=0 \mathrm{D} 2 \mathrm{E}=0 \mathrm{Chk}=8 \mathrm{Scr}=1$ Normal termination of Gaussian 03 at Thu May 18 15:25:09 2006. 


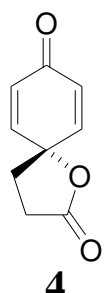

Entering Link 1 = C: $\backslash$ G03W 11 .exe PID $=520$.

Copyright (c) 1988,1990,1992,1993,1995,1998,2003, Gaussian, Inc. All Rights Reserved.

This is the Gaussian(R) 03 program. It is based on the the Gaussian(R) 98 system (copyright 1998, Gaussian, Inc.), the Gaussian(R) 94 system (copyright 1995, Gaussian, Inc.), the Gaussian 92(TM) system (copyright 1992, Gaussian, Inc.), the Gaussian 90(TM) system (copyright 1990, Gaussian, Inc.), the Gaussian 88(TM) system (copyright 1988, Gaussian, Inc.), the Gaussian 86(TM) system (copyright 1986, Carnegie Mellon University), and the Gaussian 82(TM) system (copyright 1983, Carnegie Mellon University). Gaussian is a federally registered trademark of Gaussian, Inc.

This software contains proprietary and confidential information, including trade secrets, belonging to Gaussian, Inc.

This software is provided under written license and may be used, copied, transmitted, or stored only in accord with that written license.

The following legend is applicable only to US Government contracts under DFARS:

\section{RESTRICTED RIGHTS LEGEND}

Use, duplication or disclosure by the US Government is subject to restrictions as set forth in subparagraph (c)(1)(ii) of the Rights in Technical Data and Computer Software clause at DFARS 252.227-7013.

Gaussian, Inc.

Carnegie Office Park, Building 6, Pittsburgh, PA 15106 USA

The following legend is applicable only to US Government contracts under FAR

\section{RESTRICTED RIGHTS LEGEND}

Use, reproduction and disclosure by the US Government is subject to restrictions as set forth in subparagraph (c) of the Commercial Computer Software - Restricted Rights clause at FAR 52.227-19.

Gaussian, Inc.

Carnegie Office Park, Building 6, Pittsburgh, PA 15106 USA

Warning -- This program may not be used in any manner that competes with the business of Gaussian, Inc. or will provide assistance to any competitor of Gaussian, Inc. The licensee of this program is prohibited from giving any competitor of 
Gaussian, Inc. access to this program. By using this program, the user acknowledges that Gaussian, Inc. is engaged in the business of creating and licensing software in the field of computational chemistry and represents and warrants to the licensee that it is not a competitor of Gaussian, Inc. and that it will not use this program in any manner prohibited above.

Cite this work as:

Gaussian 03, Revision B.05,

M. J. Frisch, G. W. Trucks, H. B. Schlegel, G. E. Scuseria,

M. A. Robb, J. R. Cheeseman, J. A. Montgomery, Jr., T. Vreven,

K. N. Kudin, J. C. Burant, J. M. Millam, S. S. Iyengar, J. Tomasi,

V. Barone, B. Mennucci, M. Cossi, G. Scalmani, N. Rega,

G. A. Petersson, H. Nakatsuji, M. Hada, M. Ehara, K. Toyota,

R. Fukuda, J. Hasegawa, M. Ishida, T. Nakajima, Y. Honda, O. Kitao,

H. Nakai, M. Klene, X. Li, J. E. Knox, H. P. Hratchian, J. B. Cross,

C. Adamo, J. Jaramillo, R. Gomperts, R. E. Stratmann, O. Yazyev,

A. J. Austin, R. Cammi, C. Pomelli, J. W. Ochterski, P. Y. Ayala,

K. Morokuma, G. A. Voth, P. Salvador, J. J. Dannenberg,

V. G. Zakrzewski, S. Dapprich, A. D. Daniels, M. C. Strain,

O. Farkas, D. K. Malick, A. D. Rabuck, K. Raghavachari,

J. B. Foresman, J. V. Ortiz, Q. Cui, A. G. Baboul, S. Clifford,

J. Cioslowski, B. B. Stefanov, G. Liu, A. Liashenko, P. Piskorz,

I. Komaromi, R. L. Martin, D. J. Fox, T. Keith, M. A. Al-Laham,

C. Y. Peng, A. Nanayakkara, M. Challacombe, P. M. W. Gill,

B. Johnson, W. Chen, M. W. Wong, C. Gonzalez, and J. A. Pople, Gaussian, Inc., Pittsburgh PA, 2003.

$* * * * * * * * * * * * * * * * * * * * * * * * * * * * * * * * * * * * * * * * * * * * * *$

Gaussian 03: x86-Win32-G03RevB.05 8-Nov-2003 18-May-2006

****************************************************

$\%$ chk=spiro1.chk

$\%$ mem $=64 \mathrm{MB}$

$\%$ nproc $=1$

Will use up to 1 processors via shared memory.

\# hf/6-31g(d) geom=connectivity

$1 / 38=1,57=2 / 1$;

$2 / 17=6,18=5,40=1 / 2$;

$3 / 5=1,6=6,7=1,11=9,16=1,25=1,30=1 / 1,2,3$;

$4 / / 1$

$5 / 5=2,32=1,38=5 / 2$;

$6 / 7=2,8=2,9=2,10=2,28=1 / 1$;

$99 / 5=1,9=1 / 99$;

Title Card Required

Symbolic Z-matrix:

Charge $=0$ Multiplicity $=1$

$\begin{array}{lrrr}\mathrm{C} & 2.18828 & -1.20335 & 0.96097 \\ \mathrm{C} & 0.14728 & -1.16135 & -0.40403 \\ \mathrm{C} & 0.78028 & 0.80565 & 1.01197 \\ \mathrm{C} & -0.25672 & 0.20565 & 0.09897 \\ \mathrm{C} & 1.89028 & 0.17165 & 1.41797 \\ \mathrm{C} & 1.25228 & -1.81235 & -0.01003 \\ \mathrm{H} & 2.61728 & 0.62965 & 2.07797 \\ \mathrm{H} & 1.51728 & -2.79435 & -0.38303 \\ \mathrm{H} & -0.49872 & -1.64235 & -1.13403 \\ \mathrm{H} & 0.61028 & 1.82465 & 1.35197 \\ \mathrm{O} & 3.17028 & -1.81135 & 1.36597\end{array}$




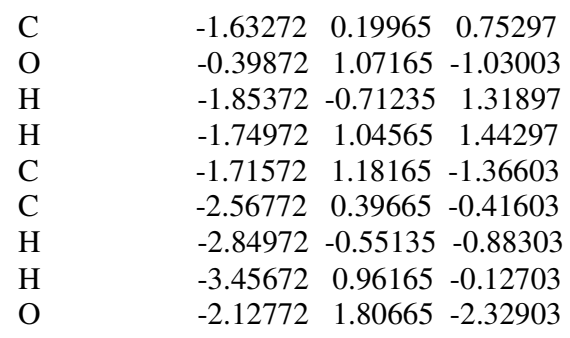

Input orientation:

\begin{tabular}{|c|c|c|c|c|c|}
\hline \multirow{2}{*}{$\begin{array}{l}\text { Center } \\
\text { Number }\end{array}$} & \multirow{2}{*}{$\begin{array}{l}\text { Atomic } \\
\text { Number }\end{array}$} & \multirow{2}{*}{\multicolumn{2}{|c|}{$\begin{array}{r}\text { Atomic } \\
\text { Type }\end{array}$}} & \multicolumn{2}{|c|}{ Coordinates (Angstrom } \\
\hline & & & & X $\quad Y$ & $\mathrm{Z}$ \\
\hline 1 & 6 & 0 & 2.188279 & -1.203349 & 0.960965 \\
\hline 2 & 6 & 0 & 0.147279 & -1.161349 & -0.404035 \\
\hline 3 & 6 & 0 & 0.780279 & 0.805651 & 1.011965 \\
\hline 4 & 6 & 0 & -0.256721 & 0.205651 & 0.098965 \\
\hline 5 & 6 & 0 & 1.890279 & 0.171651 & 1.417965 \\
\hline 6 & 6 & 0 & 1.252279 & -1.812349 & -0.010035 \\
\hline 7 & 1 & 0 & 2.617279 & 0.629651 & 2.077965 \\
\hline 8 & 1 & 0 & 1.517279 & -2.794349 & -0.383035 \\
\hline 9 & 1 & 0 & -0.498721 & -1.642349 & -1.134035 \\
\hline 10 & 1 & 0 & 0.610279 & 1.824651 & 1.351965 \\
\hline 11 & 8 & 0 & 3.170279 & -1.811349 & 1.365965 \\
\hline 12 & 6 & 0 & -1.632721 & 0.199651 & 0.752965 \\
\hline 13 & 8 & 0 & -0.398721 & 1.071651 & -1.030035 \\
\hline 14 & 1 & 0 & -1.853721 & -0.712349 & 1.318965 \\
\hline 15 & 1 & 0 & -1.749721 & 1.045651 & 1.442965 \\
\hline 16 & 6 & 0 & -1.715721 & 1.181651 & -1.366035 \\
\hline 17 & 6 & 0 & -2.567721 & 0.396651 & -0.416035 \\
\hline 18 & 1 & 0 & -2.849721 & -0.551349 & -0.883035 \\
\hline 19 & 1 & 0 & -3.456721 & 0.961651 & -0.127035 \\
\hline 20 & 8 & 0 & -2.127721 & 1.806651 & -2.329035 \\
\hline
\end{tabular}

\footnotetext{
Distance matrix (angstroms):

$1 \mathrm{C} \quad 0.000000$

$\begin{array}{llll}2 & \mathrm{C} & 2.455742 & 0.000000\end{array}$

$\begin{array}{lllll}3 \mathrm{C} & 2.453802 & 2.504962 & 0.000000\end{array}$

$\begin{array}{llllll}4 & \mathrm{C} & 2.950652 & 1.511593 & 1.506299 & 0.000000\end{array}$

$\begin{array}{lllllll}5 & \mathrm{C} & 1.479283 & 2.852126 & 1.341228 & 2.520025 & 0.000000\end{array}$

$\begin{array}{lllllll}6 \mathrm{C} & 1.479803 & 1.341664 & 2.849771 & 2.522159 & 2.526358\end{array}$

$\begin{array}{lllllll}7 & \mathrm{H} & 2.188977 & 3.933053 & 2.131174 & 3.515123 & 1.083463\end{array}$

$\begin{array}{lllllll}8 & \mathrm{H} & 2.188117 & 2.131673 & 3.930546 & 3.518437 & 3.489969\end{array}$

$\begin{array}{lllllll}9 & \mathrm{H} & 3.435362 & 1.087004 & 3.497694 & 2.234716 & 3.938353\end{array}$

$\begin{array}{lllllll}10 \mathrm{H} & 3.436823 & 3.494868 & 1.087594 & 2.223254 & 2.091690\end{array}$

$\begin{array}{llllllll}11 & \mathrm{O} & 1.223933 & 3.562854 & 3.561756 & 4.173477 & 2.360803\end{array}$

$\begin{array}{lllllll}12 & \mathrm{C} & 4.075747 & 2.521779 & 2.501377 & 1.523525 & 3.585323\end{array}$

$\begin{array}{lllllll}13 & \mathrm{O} & 3.978979 & 2.382495 & 2.372880 & 1.429951 & 3.470191\end{array}$

$\begin{array}{lllllll}14 & \mathrm{H} & 4.087421 & 2.678494 & 3.055573 & 2.209419 & 3.848219\end{array}$

$\begin{array}{lllllll}15 & \mathrm{H} & 4.560501 & 3.446863 & 2.577646 & 2.177380 & 3.743541\end{array}$

$\begin{array}{lllllll}16 & \mathrm{C} & 5.132677 & 3.144179 & 3.467892 & 2.286369 & 4.666261\end{array}$

$\begin{array}{llllllll}17 & \mathrm{C} & 5.203428 & 3.130293 & 3.662727 & 2.375379 & 4.825758\end{array}$

$\begin{array}{lllllll}18 \mathrm{H} & 5.404339 & 3.095731 & 4.313858 & 2.874199 & 5.318358\end{array}$

$\begin{array}{lllllll}19 & \mathrm{H} & 6.143044 & 4.191977 & 4.390197 & 3.295848 & 5.621524\end{array}$

$\begin{array}{llllllll}20 & \mathrm{O} & 6.205808 & 4.205981 & 4.541007 & 3.458182 & 5.732151\end{array}$

6 C 0.000000

$\begin{array}{lllll}6 & 7 & 8 & 9 & 10\end{array}$

$\begin{array}{llll}7 \mathrm{H} & 3.490893 & 0.000000\end{array}$

$\begin{array}{lllll}8 \mathrm{H} & 1.083364 & 4.357786 & 0.000000\end{array}$

$\begin{array}{lllllll}9 \mathrm{H} & 2.087649 & 5.018803 & 2.440361 & 0.000000\end{array}$

$\begin{array}{lllllll}10 \mathrm{H} & 3.936366 & 2.446048 & 5.016775 & 4.407966 & 0.000000\end{array}$
} 
$\begin{array}{lllllll}11 & \mathrm{O} & 2.360530 & 2.602159 & 2.599557 & 4.442986 & 4.446829\end{array}$

$\begin{array}{llllllll}12 & \mathrm{C} & 3.599102 & 4.472474 & 4.491885 & 2.870486 & 2.833809\end{array}$

$\begin{array}{llllllll}13 & \mathrm{O} & 3.476155 & 4.353307 & 4.362983 & 2.717832 & 2.694256\end{array}$

$\begin{array}{lllllll}14 & \mathrm{H} & 3.552953 & 4.729364 & 4.312212 & 2.952649 & 3.536772\end{array}$

$\begin{array}{lllllll}15 & \mathrm{H} & 4.392195 & 4.432490 & 5.362198 & 3.928266 & 2.486910\end{array}$

$\begin{array}{llllllll}16 & \mathrm{C} & 4.428521 & 5.562439 & 5.217965 & 3.083811 & 3.634728\end{array}$

$\begin{array}{lllllll}17 \mathrm{C} & 4.431356 & 5.758346 & 5.183705 & 2.992291 & 3.907006\end{array}$

$\begin{array}{llllllll}18 & \mathrm{H} & 4.379344 & 6.328536 & 4.934748 & 2.603936 & 4.755229\end{array}$

$\begin{array}{llllllll}19 & \mathrm{H} & 5.466575 & 6.470373 & 6.238088 & 4.067509 & 4.412788\end{array}$

$\begin{array}{llllllll}20 & \mathrm{O} & 5.468027 & 6.581945 & 6.184023 & 3.997157 & 4.587671\end{array}$ $\begin{array}{lllll}11 & 12 & 13 & 14 & 15\end{array}$

$\begin{array}{lll}11 & \mathrm{O} & 0.000000\end{array}$

$\begin{array}{llll}12 & \text { C } & 5.242967 & 0.000000\end{array}$

$\begin{array}{lllll}13 & \mathrm{O} & 5.175931 & 2.337141 & 0.000000\end{array}$

$\begin{array}{llllll}14 & \mathrm{H} & 5.143013 & 1.095875 & 3.288994 & 0.000000\end{array}$

$\begin{array}{lllllll}15 \mathrm{H} & 5.689884 & 1.097955 & 2.818086 & 1.765434 & 0.000000\end{array}$

$\begin{array}{lllllll}16 & \mathrm{C} & 6.347824 & 2.336958 & 1.363629 & 3.288694 & 2.812496\end{array}$

$\begin{array}{llllllll}17 & \mathrm{C} & 6.401206 & 1.509833 & 2.353122 & 2.179427 & 2.132183\end{array}$

$\begin{array}{lllllll}18 & \mathrm{H} & 6.548740 & 2.172921 & 2.943321 & 2.422136 & 3.028314\end{array}$

$\begin{array}{lllllll}19 & \mathrm{H} & 7.337282 & 2.163798 & 3.190435 & 2.731813 & 2.320734\end{array}$

$\begin{array}{llllllll}20 & \mathrm{O} & 7.403496 & 3.510869 & 2.284090 & 4.441660 & 3.866522\end{array}$

$16 \mathrm{C} \quad 0.000000$

$\begin{array}{llll}17 \mathrm{C} & 1.498209 & 0.000000\end{array}$

$\begin{array}{lllll}18 & \mathrm{H} & 2.126625 & 1.093763 & 0.000000\end{array}$

$\begin{array}{llllll}19 & \mathrm{H} & 2.148162 & 1.092276 & 1.796985 & 0.000000\end{array}$

$\begin{array}{lllllll}20 & \mathrm{O} & 1.219729 & 2.416872 & 2.858735 & 2.707226 & 0.000000\end{array}$

Stoichiometry C9H8O3

Framework group $\mathrm{C} 1[\mathrm{X}(\mathrm{C} 9 \mathrm{H} 8 \mathrm{O} 3)]$

Deg. of freedom 54

Full point group $\quad \mathrm{C} 1 \quad \mathrm{NOp} 1$

Largest Abelian subgroup $\quad \mathrm{C} 1 \quad \mathrm{NOp} 1$

Largest concise Abelian subgroup C1 NOp 1 Standard orientation:

\begin{tabular}{|c|c|c|c|c|}
\hline \multirow{2}{*}{$\begin{array}{l}\text { Center } \\
\text { Number }\end{array}$} & Atomic & Atomic & \multicolumn{2}{|c|}{ Coordinates (Angstroms) } \\
\hline & Numbe & Type & $\begin{array}{ll}X & Y\end{array}$ & Z $\quad \mathrm{Z}$ \\
\hline 1 & 6 & 2.670183 & -0.118776 & -0.126827 \\
\hline 2 & 6 & 0.449025 & -1.153605 & 0.035212 \\
\hline 3 & 6 & 0.700248 & 1.338517 & 0.002792 \\
\hline 4 & 6 & -0.252430 & 0.180701 & 0.147032 \\
\hline 5 & 6 & 2.030773 & 1.215105 & -0.112815 \\
\hline 6 & 6 & 1.777854 & -1.298350 & -0.080212 \\
\hline 7 & 1 & 2.690901 & 2.068417 & -0.212719 \\
\hline 8 & 1 & 2.256709 & -2.267294 & -0.154536 \\
\hline 9 & 1 & -0.165735 & -2.050012 & 0.045258 \\
\hline 10 & 1 & 0.267590 & 2.336186 & -0.015208 \\
\hline 11 & 8 & 3.886753 & -0.242429 & -0.178597 \\
\hline 12 & 6 & -1.076201 & 0.297702 & 1.423292 \\
\hline 13 & 8 & -1.211628 & 0.269615 & -0.909753 \\
\hline 14 & 1 & -0.623038 & -0.191032 & 2.293190 \\
\hline 15 & 1 & -1.245455 & 1.348349 & 1.693474 \\
\hline 16 & 6 & -2.452573 & -0.037726 & $5-0.435327$ \\
\hline 17 & 6 & -2.397881 & -0.320331 & 1.034970 \\
\hline 18 & 1 & -2.410336 & -1.402207 & 1.195300 \\
\hline 19 & 1 & -3.231099 & 0.154809 & 1.557518 \\
\hline 20 & 8 & -3.454314 & -0.104266 & -1.128022 \\
\hline
\end{tabular}

Rotational constants (GHZ): $2.6656622 \quad 0.5553229 \quad 0.5447105$

Standard basis: 6-31G(d) $(6 \mathrm{D}, 7 \mathrm{~F})$

There are 196 symmetry adapted basis functions of A symmetry.

Integral buffers will be 262144 words long.

Raffenetti 1 integral format. 
Two-electron integral symmetry is turned on.

196 basis functions, 368 primitive gaussians, 196 cartesian basis functions

43 alpha electrons 43 beta electrons nuclear repulsion energy $\quad 675.6964334328$ Hartrees.

NAtoms $=20$ NActive $=20 \mathrm{NUniq}=20 \mathrm{SFac}=1.00 \mathrm{D}+00 \mathrm{NAtFMM}=60 \mathrm{Big}=\mathrm{F}$

One-electron integrals computed using PRISM.

NBasis $=196$ RedAO $=\mathrm{T}$ NBF $=196$

$\mathrm{NBsUse}=196$ 1.00D-06 $\mathrm{NBFU}=196$

Harris functional with IExCor= 205 diagonalized for initial guess.

ExpMin= 1.61D-01 ExpMax= 5.48D+03 ExpMxC= 8.25D+02 IAcc $=1$ IRadAn $=\quad 1$ AccDes $=1.00 \mathrm{D}-06$
HarFok: IExCor= 205 AccDes= $1.00 \mathrm{D}-06 \mathrm{IRadAn}=\quad 1 \mathrm{IDoV}=1$

ScaDFX $=1.0000001 .0000001 .0000001 .000000$

Initial guess orbital symmetries:

Occupied (A) (A) (A) (A) (A) (A) (A) (A) (A) (A) (A) (A)

(A) (A) (A) (A) (A) (A) (A) (A) (A) (A) (A) (A)

(A) $(\mathrm{A})(\mathrm{A})(\mathrm{A})(\mathrm{A})(\mathrm{A})(\mathrm{A})(\mathrm{A})(\mathrm{A})(\mathrm{A})(\mathrm{A})(\mathrm{A})$

(A) $(\mathrm{A})(\mathrm{A})(\mathrm{A})(\mathrm{A})(\mathrm{A})(\mathrm{A})$

Virtual (A) (A) (A) (A) (A) (A) (A) (A) (A) (A) (A) (A)

(A) (A) (A) (A) (A) (A) (A) (A) (A) (A) (A) (A)

(A) (A) (A) (A) (A) (A) (A) (A) (A) (A) (A) (A)

(A) (A) (A) (A) (A) (A) (A) (A) (A) (A) (A) (A)

(A) $(\mathrm{A})(\mathrm{A})(\mathrm{A})(\mathrm{A})(\mathrm{A})(\mathrm{A})(\mathrm{A})(\mathrm{A})(\mathrm{A})(\mathrm{A})(\mathrm{A})$

(A) $(A)(A)(A)(A)(A)(A)(A)(A)(A)(A)(A)$

(A) (A) (A) (A) (A) (A) (A) (A) (A) (A) (A) (A)

(A) $(\mathrm{A})(\mathrm{A})(\mathrm{A})(\mathrm{A})(\mathrm{A})(\mathrm{A})(\mathrm{A})(\mathrm{A})(\mathrm{A})(\mathrm{A})(\mathrm{A})$

(A) $(\mathrm{A})(\mathrm{A})(\mathrm{A})(\mathrm{A})(\mathrm{A})(\mathrm{A})(\mathrm{A})(\mathrm{A})(\mathrm{A})(\mathrm{A})(\mathrm{A})$

(A) (A) (A) (A) (A) (A) (A) (A) (A) (A) (A) (A)

(A) $(\mathrm{A})(\mathrm{A})(\mathrm{A})(\mathrm{A})(\mathrm{A})(\mathrm{A})(\mathrm{A})(\mathrm{A})(\mathrm{A})(\mathrm{A})(\mathrm{A})$

(A) (A) (A) (A) (A) (A) (A) (A) (A) (A) (A) (A)

(A) (A) (A) (A) (A) (A) (A) (A) (A)

The electronic state of the initial guess is 1-A.

Warning! Cutoffs for single-point calculations used.

Requested convergence on RMS density matrix=1.00D-04 within 128 cycles.

Requested convergence on MAX density matrix=1.00D-02.

Requested convergence on energy=5.00D-05.

No special actions if energy rises.

SCF Done: $\mathrm{E}(\mathrm{RHF})=-570.040882297$ A.U. after 7 cycles

Convg $=0.1253 \mathrm{D}-04 \quad-\mathrm{V} / \mathrm{T}=2.0022$

$\mathrm{S} * * 2=0.0000$

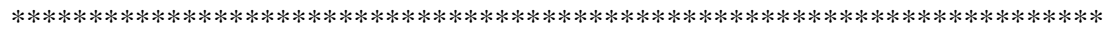

Population analysis using the SCF density.

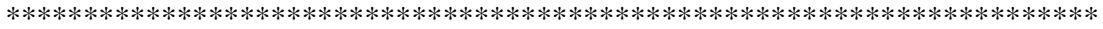

Orbital symmetries:

Occupied (A) (A) (A) (A) (A) (A) (A) (A) (A) (A) (A) (A)

(A) $(A)(A)(A)(A)(A)(A)(A)(A)(A)(A)(A)$

(A) $(A)(A)(A)(A)(A)(A)(A)(A)(A)(A)(A)$

(A) $(\mathrm{A})(\mathrm{A})(\mathrm{A})(\mathrm{A})(\mathrm{A})(\mathrm{A})$

Virtual (A) (A) (A) (A) (A) (A) (A) (A) (A) (A) (A) (A)

(A) (A) (A) (A) (A) (A) (A) (A) (A) (A) (A) (A)

(A) $(\mathrm{A})(\mathrm{A})(\mathrm{A})(\mathrm{A})(\mathrm{A})(\mathrm{A})(\mathrm{A})(\mathrm{A})(\mathrm{A})(\mathrm{A})(\mathrm{A})$

(A) $(\mathrm{A})(\mathrm{A})(\mathrm{A})(\mathrm{A})(\mathrm{A})(\mathrm{A})(\mathrm{A})(\mathrm{A})(\mathrm{A})(\mathrm{A})(\mathrm{A})$

(A) (A) (A) (A) (A) (A) (A) (A) (A) (A) (A) (A)

(A) $(\mathrm{A})(\mathrm{A})(\mathrm{A})(\mathrm{A})(\mathrm{A})(\mathrm{A})(\mathrm{A})(\mathrm{A})(\mathrm{A})(\mathrm{A})(\mathrm{A})$

(A) (A) (A) (A) (A) (A) (A) (A) (A) (A) (A) (A)

(A) (A) (A) (A) (A) (A) (A) (A) (A) (A) (A) (A)

(A) $(\mathrm{A})(\mathrm{A})(\mathrm{A})(\mathrm{A})(\mathrm{A})(\mathrm{A})(\mathrm{A})(\mathrm{A})(\mathrm{A})(\mathrm{A})(\mathrm{A})$

(A) (A) (A) (A) (A) (A) (A) (A) (A) (A) (A) (A)

(A) (A) (A) (A) (A) (A) (A) (A) (A) (A) (A) (A)

(A) $(\mathrm{A})(\mathrm{A})(\mathrm{A})(\mathrm{A})(\mathrm{A})(\mathrm{A})(\mathrm{A})(\mathrm{A})(\mathrm{A})(\mathrm{A})(\mathrm{A})$

(A) $(A)(A)(A)(A)(A)(A)(A)(A)$ 
The electronic state is 1-A.

Alpha occ. eigenvalues -- -20.63432 -20.56293 -20.56249 -11.40017 -11.34764

Alpha occ. eigenvalues -- -11.34489 -11.28351 -11.28163 -11.27142 -11.26959

Alpha occ. eigenvalues -- -11.26395 -11.26222 -1.48161 -1.38785 -1.37419

Alpha occ. eigenvalues -- $-1.16495-1.11337-1.06205-1.01817-0.90100$

Alpha occ. eigenvalues -- $\quad-0.86005 \quad-0.84828 \quad-0.80188 \quad-0.74622 \quad-0.72118$

$\begin{array}{lllllll}\text { Alpha occ. eigenvalues -- } & -0.68582 & -0.67162 & -0.66195 & -0.63627 & -0.62659\end{array}$

Alpha occ. eigenvalues -- $-0.61387 \quad-0.59919-0.58821 \quad-0.56856 \quad-0.56095$

$\begin{array}{lllllll}\text { Alpha occ. eigenvalues -- } & -0.55868 & -0.53280 & -0.50961 & -0.46958 & -0.44722\end{array}$

Alpha occ. eigenvalues -- $-0.42686-0.39879-0.39412$

$\begin{array}{lllllll}\text { Alpha virt. eigenvalues -- } & 0.06010 & 0.14002 & 0.15872 & 0.20280 & 0.22978\end{array}$

$\begin{array}{llllllll}\text { Alpha virt. eigenvalues -- } & 0.23378 & 0.26700 & 0.27339 & 0.28798 & 0.29672\end{array}$

$\begin{array}{lllllll}\text { Alpha virt. eigenvalues -- } & 0.32926 & 0.33251 & 0.34611 & 0.35523 & 0.37753\end{array}$

$\begin{array}{lllllll}\text { Alpha virt. eigenvalues -- } & 0.39360 & 0.42113 & 0.43376 & 0.45241 & 0.48917\end{array}$

$\begin{array}{lllllll}\text { Alpha virt. eigenvalues -- } & 0.51781 & 0.53086 & 0.55772 & 0.63005 & 0.66225\end{array}$

$\begin{array}{llllllll}\text { Alpha virt. eigenvalues -- } & 0.68414 & 0.69460 & 0.70984 & 0.72934 & 0.75300\end{array}$

$\begin{array}{lllllll}\text { Alpha virt. eigenvalues -- } & 0.75644 & 0.77092 & 0.80029 & 0.81500 & 0.82429\end{array}$

$\begin{array}{lllllll}\text { Alpha virt. eigenvalues -- } & 0.84459 & 0.85633 & 0.86469 & 0.86982 & 0.89818\end{array}$

$\begin{array}{lllllll}\text { Alpha virt. eigenvalues -- } & 0.92250 & 0.92467 & 0.93840 & 0.95601 & 0.96319\end{array}$

$\begin{array}{lllllll}\text { Alpha virt. eigenvalues -- } & 0.98305 & 1.01450 & 1.03778 & 1.05406 & 1.07225\end{array}$

$\begin{array}{lllllll}\text { Alpha virt. eigenvalues -- } & 1.09873 & 1.10367 & 1.11001 & 1.11072 & 1.13364\end{array}$

$\begin{array}{lllllll}\text { Alpha virt. eigenvalues -- } & 1.15684 & 1.15851 & 1.17090 & 1.18999 & 1.21804\end{array}$

$\begin{array}{lllllll}\text { Alpha virt. eigenvalues -- } & 1.23797 & 1.25712 & 1.28094 & 1.29465 & 1.31429\end{array}$

$\begin{array}{lllllll}\text { Alpha virt. eigenvalues -- } & 1.32197 & 1.34777 & 1.35156 & 1.37139 & 1.40210\end{array}$

$\begin{array}{lllllll}\text { Alpha virt. eigenvalues -- } & 1.41977 & 1.46081 & 1.49436 & 1.54749 & 1.58368\end{array}$

$\begin{array}{lllllll}\text { Alpha virt. eigenvalues -- } & 1.62200 & 1.66092 & 1.67596 & 1.69445 & 1.71687\end{array}$

$\begin{array}{lllllll}\text { Alpha virt. eigenvalues -- } & 1.74611 & 1.81529 & 1.82644 & 1.83607 & 1.91595\end{array}$

$\begin{array}{lllllll}\text { Alpha virt. eigenvalues -- } & 1.91942 & 1.94750 & 1.98634 & 2.00073 & 2.06108\end{array}$

$\begin{array}{lllllll}\text { Alpha virt. eigenvalues -- } & 2.07330 & 2.08555 & 2.08946 & 2.10498 & 2.13192\end{array}$

$\begin{array}{lllllll}\text { Alpha virt. eigenvalues -- } & 2.14372 & 2.15535 & 2.16154 & 2.17980 & 2.21154\end{array}$

$\begin{array}{llllll}\text { Alpha virt. eigenvalues -- } & 2.22559 & 2.24332 & 2.25369 & 2.26189 & 2.32815\end{array}$

$\begin{array}{lllllll}\text { Alpha virt. eigenvalues -- } & 2.33528 & 2.35166 & 2.36625 & 2.38770 & 2.42302\end{array}$

$\begin{array}{lllllll}\text { Alpha virt. eigenvalues -- } & 2.44787 & 2.47009 & 2.49109 & 2.56339 & 2.58642\end{array}$

$\begin{array}{lllllll}\text { Alpha virt. eigenvalues -- } & 2.61107 & 2.64781 & 2.66162 & 2.68142 & 2.70769\end{array}$

$\begin{array}{lllllll}\text { Alpha virt. eigenvalues -- } & 2.73870 & 2.76786 & 2.86092 & 2.89484 & 2.92115\end{array}$

$\begin{array}{lllllll}\text { Alpha virt. eigenvalues -- } & 2.93492 & 2.96141 & 2.98417 & 3.00775 & 3.07088\end{array}$

$\begin{array}{lllllll}\text { Alpha virt. eigenvalues -- } & 3.07847 & 3.09962 & 3.12987 & 3.15901 & 3.25837\end{array}$

$\begin{array}{lllllll}\text { Alpha virt. eigenvalues -- } & 3.27127 & 3.36455 & 3.39874 & 3.49065 & 3.52467\end{array}$

$\begin{array}{lllllll}\text { Alpha virt. eigenvalues -- } & 3.64091 & 4.34173 & 4.37860 & 4.52055 & 4.54949\end{array}$

$\begin{array}{lllllll}\text { Alpha virt. eigenvalues -- } & 4.60919 & 4.69436 & 4.75977 & 4.78907 & 4.88851\end{array}$

Alpha virt. eigenvalues -- $4.98551 \quad 5.05153 \quad 5.20156$

Condensed to atoms (all electrons):

$$
\begin{array}{llllll}
1 & 2 & 3 & 4 & 5 & 6
\end{array}
$$

$\begin{array}{llllllll}1 & C & 4.270561 & -0.039161 & -0.038919 & -0.012266 & 0.413287 & 0.413647\end{array}$

$\begin{array}{llllllll}2 & \mathrm{C} & -0.039161 & 5.067187 & -0.070970 & 0.372283 & 0.010227 & 0.561646\end{array}$

$\begin{array}{llllllll}3 & \mathrm{C} & -0.038919 & -0.070970 & 5.012578 & 0.421346 & 0.561907 & 0.009892\end{array}$

$\begin{array}{llllllll}4 & C & -0.012266 & 0.372283 & 0.421346 & 4.814887 & -0.038840 & -0.035602\end{array}$

$\begin{array}{llllllll}5 & \mathrm{C} & 0.413287 & 0.010227 & 0.561907 & -0.038840 & 5.110904 & -0.108054\end{array}$

$\begin{array}{llllllll}6 & \mathrm{C} & 0.413647 & 0.561646 & 0.009892 & -0.035602 & -0.108054 & 5.103715\end{array}$

$\begin{array}{llllllll}7 \mathrm{H} & -0.023962 & -0.000394 & -0.027113 & 0.002251 & 0.377393 & 0.003505\end{array}$

$\begin{array}{llllllll}8 \mathrm{H} & -0.023490 & -0.027984 & -0.000314 & 0.002044 & 0.003444 & 0.377758\end{array}$

$\begin{array}{llllllll}9 \mathrm{H} & 0.001390 & 0.373527 & 0.002443 & -0.030525 & -0.000263 & -0.015869\end{array}$

$\begin{array}{llllllll}10 \mathrm{H} & 0.001477 & 0.002871 & 0.374382 & -0.031222 & -0.018855 & -0.000324\end{array}$

$\begin{array}{lllllllll}11 & \mathrm{O} & 0.522797 & 0.004396 & 0.004273 & 0.000039 & -0.059006 & -0.059021\end{array}$

$\begin{array}{lllllllll}12 & \mathrm{C} & 0.000379 & -0.064181 & -0.059556 & 0.332787 & 0.000304 & -0.002715\end{array}$

$\begin{array}{llllllll}13 & \mathrm{O} & 0.000077 & -0.051604 & -0.048192 & 0.149746 & -0.000028 & 0.000809\end{array}$

$\begin{array}{llllllll}14 \mathrm{H} & -0.000006 & 0.001908 & -0.000247 & -0.030275 & 0.000232 & 0.000479\end{array}$

$\begin{array}{llllllll}15 \mathrm{H} & -0.000048 & 0.004431 & -0.006153 & -0.043446 & 0.000756 & 0.000053\end{array}$

$\begin{array}{llllllll}16 & \mathrm{C} & 0.000005 & -0.001068 & 0.000881 & -0.039014 & -0.000032 & -0.000238\end{array}$

$\begin{array}{llllllll}17 & \mathrm{C} & 0.000004 & -0.004742 & 0.006024 & -0.049782 & -0.000154 & -0.000053\end{array}$

$\begin{array}{llllllll}18 \mathrm{H} & -0.000002 & 0.002247 & -0.000148 & -0.001864 & 0.000007 & 0.000116\end{array}$

$\begin{array}{llllllll}19 \mathrm{H} & 0.000000 & -0.000060 & -0.000055 & 0.004639 & 0.000000 & 0.000004\end{array}$

$\begin{array}{llllllll}20 & \mathrm{O} & 0.000000 & -0.000123 & -0.000074 & 0.001394 & 0.000001 & -0.000001\end{array}$

$\begin{array}{llllll}7 & 8 & 9 & 10 & 11 & 12\end{array}$ 
$\begin{array}{llllllll}1 & \mathrm{C} & -0.023962 & -0.023490 & 0.001390 & 0.001477 & 0.522797 & 0.000379\end{array}$

$\begin{array}{llllllll}2 & \mathrm{C} & -0.000394 & -0.027984 & 0.373527 & 0.002871 & 0.004396 & -0.064181\end{array}$

$\begin{array}{llllllll}3 & C & -0.027113 & -0.000314 & 0.002443 & 0.374382 & 0.004273 & -0.059556\end{array}$

$\begin{array}{llllllll}4 & \mathrm{C} & 0.002251 & 0.002044 & -0.030525 & -0.031222 & 0.000039 & 0.332787\end{array}$

$\begin{array}{llllllll}5 & \mathrm{C} & 0.377393 & 0.003444 & -0.000263 & -0.018855 & -0.059006 & 0.000304\end{array}$

$\begin{array}{llllllll}6 & \mathrm{C} & 0.003505 & 0.377758 & -0.015869 & -0.000324 & -0.059021 & -0.002715\end{array}$

$\begin{array}{llllllll}7 & \mathrm{H} & 0.427619 & -0.000054 & 0.000007 & -0.002051 & 0.001607 & -0.000087\end{array}$

$\begin{array}{llllllll}8 \mathrm{H} & -0.000054 & 0.427654 & -0.001885 & 0.000007 & 0.001691 & -0.000107\end{array}$

$\begin{array}{llllllll}9 \mathrm{H} & 0.000007 & -0.001885 & 0.448032 & -0.000064 & -0.000058 & -0.001472\end{array}$

$\begin{array}{llllllll}10 \mathrm{H} & -0.002051 & 0.000007 & -0.000064 & 0.443774 & -0.000055 & -0.002503\end{array}$

$\begin{array}{llllllll}11 & \mathrm{O} & 0.001607 & 0.001691 & -0.000058 & -0.000055 & 8.149781 & 0.000005\end{array}$

$\begin{array}{llllllll}12 & \mathrm{C} & -0.000087 & -0.000107 & -0.001472 & -0.002503 & 0.000005 & 5.307995\end{array}$

$\begin{array}{llllllll}13 & \mathrm{O} & -0.000037 & -0.000039 & 0.000283 & 0.001414 & 0.000000 & -0.039459\end{array}$

$\begin{array}{llllllll}14 & \mathrm{H} & 0.000006 & -0.000013 & 0.000459 & 0.000031 & -0.000001 & 0.389701\end{array}$

$\begin{array}{llllllll}15 \mathrm{H} & -0.000010 & 0.000002 & -0.000091 & 0.001650 & 0.000000 & 0.407268\end{array}$

$\begin{array}{llllllll}16 & \mathrm{C} & 0.000000 & -0.000002 & 0.000546 & 0.000205 & 0.000000 & -0.076549\end{array}$

$\begin{array}{llllllll}17 & \mathrm{C} & 0.000001 & 0.000001 & 0.000812 & -0.000043 & 0.000000 & 0.247156\end{array}$

$\begin{array}{llllllll}18 \mathrm{H} & 0.000000 & 0.000001 & -0.000057 & -0.000003 & 0.000000 & -0.035068\end{array}$

$\begin{array}{llllllll}19 & \mathrm{H} & 0.000000 & 0.000000 & 0.000019 & -0.000006 & 0.000000 & -0.034411\end{array}$

$\begin{array}{lllllllll}20 & \mathrm{O} & 0.000000 & 0.000000 & -0.000050 & -0.000009 & 0.000000 & 0.003224\end{array}$

$\begin{array}{llllll}13 & 14 & 15 & 16 & 17 & 18\end{array}$

$\begin{array}{llllllll}1 & \mathrm{C} & 0.000077 & -0.000006 & -0.000048 & 0.000005 & 0.000004 & -0.000002\end{array}$

$\begin{array}{llllllll}2 & \mathrm{C} & -0.051604 & 0.001908 & 0.004431 & -0.001068 & -0.004742 & 0.002247\end{array}$

$\begin{array}{llllllll}3 \mathrm{C} & -0.048192 & -0.000247 & -0.006153 & 0.000881 & 0.006024 & -0.000148\end{array}$

$\begin{array}{llllllll}4 & \mathrm{C} & 0.149746 & -0.030275 & -0.043446 & -0.039014 & -0.049782 & -0.001864\end{array}$

$\begin{array}{llllllll}5 & \mathrm{C} & -0.000028 & 0.000232 & 0.000756 & -0.000032 & -0.000154 & 0.000007\end{array}$

$\begin{array}{llllllll}6 \mathrm{C} & 0.000809 & 0.000479 & 0.000053 & -0.000238 & -0.000053 & 0.000116\end{array}$

$\begin{array}{llllllll}7 & \mathrm{H} & -0.000037 & 0.000006 & -0.000010 & 0.000000 & 0.000001 & 0.000000\end{array}$

$\begin{array}{llllllll}8 \mathrm{H} & -0.000039 & -0.000013 & 0.000002 & -0.000002 & 0.000001 & 0.000001\end{array}$

$\begin{array}{llllllll}9 \mathrm{H} & 0.000283 & 0.000459 & -0.000091 & 0.000546 & 0.000812 & -0.000057\end{array}$

$\begin{array}{llllllll}10 \mathrm{H} & 0.001414 & 0.000031 & 0.001650 & 0.000205 & -0.000043 & -0.000003\end{array}$

$\begin{array}{lllllllll}11 & \mathrm{O} & 0.000000 & -0.000001 & 0.000000 & 0.000000 & 0.000000 & 0.000000\end{array}$

$\begin{array}{lllllllll}12 & \mathrm{C} & -0.039459 & 0.389701 & 0.407268 & -0.076549 & 0.247156 & -0.035068\end{array}$

$\begin{array}{lllllllll}13 & \mathrm{O} & 8.515633 & 0.002049 & 0.002157 & 0.250264 & -0.108426 & 0.001932\end{array}$

$\begin{array}{llllllll}14 \mathrm{H} & 0.002049 & 0.468000 & -0.017594 & 0.004783 & -0.029091 & -0.003640\end{array}$

$\begin{array}{llllllll}15 \mathrm{H} & 0.002157 & -0.017594 & 0.490529 & 0.000124 & -0.042208 & 0.003158\end{array}$

$\begin{array}{llllllll}16 & \mathrm{C} & 0.250264 & 0.004783 & 0.000124 & 4.128678 & 0.349295 & -0.029028\end{array}$

$\begin{array}{llllllll}17 & \mathrm{C} & -0.108426 & -0.029091 & -0.042208 & 0.349295 & 5.376408 & 0.389660\end{array}$

$\begin{array}{llllllll}18 \mathrm{H} & 0.001932 & -0.003640 & 0.003158 & -0.029028 & 0.389660 & 0.459362\end{array}$

$\begin{array}{llllllll}19 \mathrm{H} & 0.002116 & 0.000175 & -0.004110 & -0.025145 & 0.391532 & -0.010252\end{array}$

$\begin{array}{llllllll}20 & \mathrm{O} & -0.058732 & -0.000036 & -0.000054 & 0.610722 & -0.074278 & -0.000639\end{array}$

$19 \quad 20$

$\begin{array}{llll}1 \mathrm{C} & 0.000000 & 0.000000\end{array}$

2 C $-0.000060-0.000123$

3 C $-0.000055-0.000074$

$\begin{array}{llll}4 & \mathrm{C} & 0.004639 & 0.001394\end{array}$

$\begin{array}{llll}5 \mathrm{C} & 0.000000 & 0.000001\end{array}$

$\begin{array}{llll}6 \mathrm{C} & 0.000004 & -0.000001\end{array}$

$\begin{array}{llll}7 \mathrm{H} & 0.000000 & 0.000000\end{array}$

$\begin{array}{llll}8 \mathrm{H} & 0.000000 & 0.000000\end{array}$

$\begin{array}{llll}9 \mathrm{H} & 0.000019 & -0.000050\end{array}$

$10 \mathrm{H}-0.000006-0.000009$

$\begin{array}{llll}11 & \mathrm{O} & 0.000000 & 0.000000\end{array}$

$\begin{array}{llll}12 & \mathrm{C} & -0.034411 & 0.003224\end{array}$

$13 \mathrm{O} \quad 0.002116-0.058732$

$14 \mathrm{H} \quad 0.000175-0.000036$

$15 \mathrm{H} \quad-0.004110-0.000054$

$\begin{array}{llll}16 \mathrm{C} & -0.025145 & 0.610722\end{array}$

$\begin{array}{llll}17 & \mathrm{C} & 0.391532 & -0.074278\end{array}$

$18 \mathrm{H} \quad-0.010252-0.000639$

$\begin{array}{llll}19 \mathrm{H} & 0.440058 & 0.000792\end{array}$

$\begin{array}{llll}20 & \mathrm{O} & 0.000792 & 8.082775\end{array}$

Mulliken atomic charges:

1 

1 C 0.514229
2 C -0.140437
3 C -0.141987
4 C 0.211419
5 C -0.253229
6 C -0.249750
$\begin{array}{lll}7 & \mathrm{H} & 0.241319\end{array}$
$8 \mathrm{H} \quad 0.241285$
$9 \mathrm{H} \quad 0.222815$
$10 \mathrm{H} \quad 0.229325$
$\begin{array}{lll}11 & \mathrm{O} & -0.566450\end{array}$
12 C -0.372713
13 O -0.619961
$14 \mathrm{H} \quad 0.213083$
$\begin{array}{lll}15 \mathrm{H} & 0.203585\end{array}$
16 C 0.825574
$\begin{array}{lll}17 & \mathrm{C} & -0.452117\end{array}$
$\begin{array}{lll}18 \mathrm{H} & 0.224220\end{array}$
$19 \mathrm{H} \quad 0.234704$
$200 \quad-0.564914$

Sum of Mulliken charges $=0.00000$

Atomic charges with hydrogens summed into heavy atoms:

\begin{tabular}{ccc}
\multicolumn{3}{c}{1} \\
1 & $\mathrm{C}$ & 0.514229 \\
2 & $\mathrm{C}$ & 0.082378 \\
3 & $\mathrm{C}$ & 0.087339 \\
4 & $\mathrm{C}$ & 0.211419 \\
5 & $\mathrm{C}$ & -0.011910 \\
6 & $\mathrm{C}$ & -0.008465 \\
7 & $\mathrm{H}$ & 0.000000 \\
8 & $\mathrm{H}$ & 0.000000 \\
9 & $\mathrm{H}$ & 0.000000 \\
10 & $\mathrm{H}$ & 0.000000 \\
11 & $\mathrm{O}$ & -0.566450 \\
12 & $\mathrm{C}$ & 0.043955 \\
13 & $\mathrm{O}$ & -0.619961 \\
14 & $\mathrm{H}$ & 0.000000 \\
15 & $\mathrm{H}$ & 0.000000 \\
16 & $\mathrm{C}$ & 0.825574 \\
17 & $\mathrm{C}$ & 0.006807 \\
18 & $\mathrm{H}$ & 0.000000 \\
19 & $\mathrm{H}$ & 0.000000 \\
20 & $\mathrm{O}$ & -0.564914
\end{tabular}

Sum of Mulliken charges $=0.00000$

Electronic spatial extent $(\mathrm{au}):<\mathrm{R} * * 2\rangle=2098.5591$

Charge $=0.0000$ electrons

Dipole moment (field-independent basis, Debye):

$\mathrm{X}=-1.1861 \mathrm{Y}=0.2049 \mathrm{Z}=4.3272 \mathrm{Tot}=4.4915$

Quadrupole moment (field-independent basis, Debye-Ang):

$\mathrm{XX}=-98.4576 \quad \mathrm{YY}=-60.3810 \quad \mathrm{ZZ}=-71.8756$

$\mathrm{XY}=1.7413 \quad \mathrm{XZ}=-10.5316 \quad \mathrm{YZ}=0.1753$

Traceless Quadrupole moment (field-independent basis, Debye-Ang):

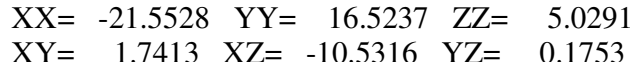

Octapole moment (field-independent basis, Debye-Ang**2):

$\mathrm{XXX}=-41.6874 \mathrm{YYY}=-0.2690 \mathrm{ZZZ}=2.6880 \mathrm{XYY}=2.0733$

$\mathrm{XXY}=10.4084 \mathrm{XXZ}=34.0776 \mathrm{XZZ}=3.6597 \mathrm{YZZ}=-0.6150$

$\mathrm{YYZ}=-2.0110 \mathrm{XYZ}=-0.8055$

Hexadecapole moment (field-independent basis, Debye-Ang**3):

$\mathrm{XXXX}=-2617.1076 \mathrm{YYYY}=-317.0977 \mathrm{ZZZZ}=-290.1878 \mathrm{XXXY}=19.0902$

$\mathrm{XXXZ}=-87.0725 \mathrm{YYYX}=-0.2302 \mathrm{YYYZ}=-0.7992 \mathrm{ZZZX}=-7.1457$

$Z Z Z Y=-0.3511 X X Y Y=-361.7066 X X Z Z=-404.2864 Y Y Z Z=-113.1618$

$\mathrm{XXYZ}=-2.9889 \mathrm{YYXZ}=-2.2879 \mathrm{ZZXY}=-2.7923$

$\mathrm{N}-\mathrm{N}=6.756964334328 \mathrm{D}+02 \mathrm{E}-\mathrm{N}=-2.686440353902 \mathrm{D}+03 \mathrm{KE}=5.687918070628 \mathrm{D}+02$ 
1|1|UNPC-UNKISP|RHF|6-31G(d)|C9H8O3|PCUSER|18-May-2006|0||\# HF/6-31G(D ) GEOM=CONNECTIVITY॥Title Card Required $\| 0,1 \mid \mathrm{C}, 0,2.18827907,-1.203348$ $84,0.96096512|\mathrm{C}, 0,0.14727907,-1.16134884,-0.40403488| \mathrm{C}, 0,0.78027907,0$. $80565116,1.01196512 \mathrm{lC}, 0,-0.25672093,0.20565116,0.09896512 \mathrm{lC}, 0,1.890279$ $07,0.17165116,1.41796512|\mathrm{C}, 0,1.25227907,-1.81234884,-0.01003488| \mathrm{H}, 0,2$. $61727907,0.62965116,2.07796512 \mathrm{lH}, 0,1.51727907,-2.79434884,-0.38303488 \mathrm{|}$ $\mathrm{H}, 0,-0.49872093,-1.64234884,-1.13403488 \mid \mathrm{H}, 0,0.61027907,1.82465116,1.35$ $196512|\mathrm{O}, 0,3.17027907,-1.81134884,1.36596512| \mathrm{C}, 0,-1.63272093,0.1996511$ 6,0.75296512|O,0,-0.39872093,1.07165116,-1.03003488|H,0,-1.85372093,-0 $.71234884,1.31896512 \mathrm{lH}, 0,-1.74972093,1.04565116,1.44296512 \mathrm{lC}, 0,-1.7157$ 2093,1.18165116,-1.36603488|C,0,-2.56772093,0.39665116,-0.41603488|H,0 ,-2.84972093,-0.55134884,-0.88303488|H,0,-3.45672093,0.96165116,-0.127 03488IO,0,-2.12772093,1.80665116,-2.32903488॥Version=x86-Win32-G03Rev B.05|State $=1-\mathrm{A}|\mathrm{HF}=-570.0408823| \mathrm{RMSD}=1.253 \mathrm{e}-005 \mid \mathrm{Dipole}=-1.3425542,-0.34$ 28792,1.0966092|PG=C01 [X(C9H8O3)]\|@

I take a simple view of life: keep your eyes open and get on with it. -- Laurence Olivier Job cpu time: 0 days 0 hours 1 minutes 47.0 seconds.

File lengths (MBytes): RWF $=28 \mathrm{Int}=0 \mathrm{D} 2 \mathrm{E}=0 \mathrm{Chk}=8 \mathrm{Scr}=1$ Normal termination of Gaussian 03 at Thu May 18 15:22:05 2006. 


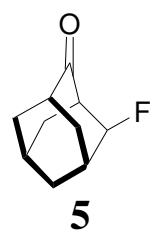

Entering Link 1 = C: $\backslash$ G03WV11.exe PID= 3700

Copyright (c) 1988,1990,1992,1993,1995,1998,2003, Gaussian, Inc. All Rights Reserved.

This is the Gaussian(R) 03 program. It is based on the the Gaussian(R) 98 system (copyright 1998, Gaussian, Inc.), the Gaussian(R) 94 system (copyright 1995, Gaussian, Inc.), the Gaussian 92(TM) system (copyright 1992, Gaussian, Inc.), the Gaussian 90(TM) system (copyright 1990, Gaussian, Inc.), the Gaussian 88(TM) system (copyright 1988, Gaussian, Inc.), the Gaussian 86(TM) system (copyright 1986, Carnegie Mellon University), and the Gaussian 82(TM) system (copyright 1983, Carnegie Mellon University). Gaussian is a federally registered trademark of Gaussian, Inc.

This software contains proprietary and confidential information, including trade secrets, belonging to Gaussian, Inc.

This software is provided under written license and may be used, copied, transmitted, or stored only in accord with that written license.

The following legend is applicable only to US Government contracts under DFARS:

\section{RESTRICTED RIGHTS LEGEND}

Use, duplication or disclosure by the US Government is subject to restrictions as set forth in subparagraph (c)(1)(ii) of the Rights in Technical Data and Computer Software clause at DFARS 252.227-7013.

Gaussian, Inc.

Carnegie Office Park, Building 6, Pittsburgh, PA 15106 USA

The following legend is applicable only to US Government contracts under FAR:

\section{RESTRICTED RIGHTS LEGEND}

Use, reproduction and disclosure by the US Government is subject to restrictions as set forth in subparagraph (c) of the

Commercial Computer Software - Restricted Rights clause at FAR 52.227-19.

Gaussian, Inc.

Carnegie Office Park, Building 6, Pittsburgh, PA 15106 USA

Warning -- This program may not be used in any manner that competes with the business of Gaussian, Inc. or will provide assistance to any competitor of Gaussian, Inc. The licensee of this program is prohibited from giving any competitor of Gaussian, Inc. access to this program. By using this program, the user acknowledges that Gaussian, Inc. is engaged in the 
business of creating and licensing software in the field of computational chemistry and represents and warrants to the licensee that it is not a competitor of Gaussian, Inc. and that it will not use this program in any manner prohibited above.

Cite this work as:

Gaussian 03, Revision B.05,

M. J. Frisch, G. W. Trucks, H. B. Schlegel, G. E. Scuseria,

M. A. Robb, J. R. Cheeseman, J. A. Montgomery, Jr., T. Vreven,

K. N. Kudin, J. C. Burant, J. M. Millam, S. S. Iyengar, J. Tomasi,

V. Barone, B. Mennucci, M. Cossi, G. Scalmani, N. Rega,

G. A. Petersson, H. Nakatsuji, M. Hada, M. Ehara, K. Toyota,

R. Fukuda, J. Hasegawa, M. Ishida, T. Nakajima, Y. Honda, O. Kitao,

H. Nakai, M. Klene, X. Li, J. E. Knox, H. P. Hratchian, J. B. Cross,

C. Adamo, J. Jaramillo, R. Gomperts, R. E. Stratmann, O. Yazyev,

A. J. Austin, R. Cammi, C. Pomelli, J. W. Ochterski, P. Y. Ayala,

K. Morokuma, G. A. Voth, P. Salvador, J. J. Dannenberg,

V. G. Zakrzewski, S. Dapprich, A. D. Daniels, M. C. Strain,

O. Farkas, D. K. Malick, A. D. Rabuck, K. Raghavachari,

J. B. Foresman, J. V. Ortiz, Q. Cui, A. G. Baboul, S. Clifford,

J. Cioslowski, B. B. Stefanov, G. Liu, A. Liashenko, P. Piskorz,

I. Komaromi, R. L. Martin, D. J. Fox, T. Keith, M. A. Al-Laham,

C. Y. Peng, A. Nanayakkara, M. Challacombe, P. M. W. Gill,

B. Johnson, W. Chen, M. W. Wong, C. Gonzalez, and J. A. Pople, Gaussian, Inc., Pittsburgh PA, 2003.

$* * * * * * * * * * * * * * * * * * * * * * * * * * * * * * * * * * * * * * * * * * * * * *$

Gaussian 03: x86-Win32-G03RevB.05 8-Nov-2003

17-May-2006

*****************************************************

$\%$ chk=C:|Documents and Settings\Nate Wilmot|Desktoplsterically unhinderedlfluoada m.chk

$\%$ mem=6MW

$\%$ nproc $=1$

Will use up to 1 processors via shared memory.

\# rhf/6-31g(d) geom=connectivity

$1 / 38=1,57=2 / 1$;

$2 / 17=6,18=5,40=1 / 2$;

$3 / 5=1,6=6,7=1,11=1,16=1,25=1,30=1 / 1,2,3$;

$4 / 7=1 / 1$

$5 / 5=2,32=1,38=5 / 2$;

$6 / 7=2,8=2,9=2,10=2,28=1 / 1$;

$99 / 5=1,9=1 / 99$;

Title Card Required

Symbolic Z-matrix:

Charge $=0$ Multiplicity $=1$

$\begin{array}{llll}\mathrm{H} & -2.14701 & 0 . & 1.31694\end{array}$

$\begin{array}{llll}\mathrm{C} & -1.27682 & 0 . & 0.65389\end{array}$

$\begin{array}{llll}\mathrm{C} & 0 . & -1.22442 & -1.16514\end{array}$

$\begin{array}{llll}\mathrm{C} & 0 . & 1.22442 & -1.16514\end{array}$

$\begin{array}{lllr}\mathrm{C} & 0 . & 0 . & -1.99433\end{array}$

$\begin{array}{llll}\text { C } & -1.25683 & 1.2541 & -0.16825\end{array}$

C $\quad-1.25683-1.2541-0.16825$

$\begin{array}{llll}\mathrm{H} & -0.88386 & 0 . & -2.64406\end{array}$

$\begin{array}{llll}\mathrm{H} & -2.15872 & 1.31719 & -0.78857\end{array}$

$\mathrm{H} \quad-2.15872-1.31719-0.78857$

$\mathrm{H} \quad 0 . \quad-2.10097-1.82305$

$\mathrm{H} \quad 0.2 .10097-1.82305$ 


\begin{tabular}{|c|c|}
\hline $\mathrm{H}$ & 0.883860 \\
\hline $\mathrm{H}$ & $\begin{array}{lll}-1.26263 & 2.12422 & 0.50042\end{array}$ \\
\hline $\mathrm{H}$ & $\begin{array}{lll}-1.26263 & -2.12422 & 0.50042\end{array}$ \\
\hline $\mathrm{C}$ & $\begin{array}{lll}1.25683 & 1.2541 & -0.16825\end{array}$ \\
\hline $\mathrm{H}$ & $\begin{array}{lll}1.26263 & 2.12422 & 0.50042\end{array}$ \\
\hline $\mathrm{H}$ & $\begin{array}{llll}2.15872 & 1.31719 & -0.78857\end{array}$ \\
\hline $\mathrm{C}$ & $\begin{array}{lll}0 . & 0 . & 1.48018\end{array}$ \\
\hline $\mathrm{C}$ & $\begin{array}{lll}1.25683 & -1.2541 & -0.16825\end{array}$ \\
\hline $\mathrm{H}$ & $\begin{array}{llll}2.15872 & -1.31719 & -0.78857\end{array}$ \\
\hline $\mathrm{C}$ & $\begin{array}{lll}1.27682 & 0 . & 0.65389\end{array}$ \\
\hline $\mathrm{H}$ & $\begin{array}{lll}2.14701 & 0 . & 1.31694\end{array}$ \\
\hline $\mathrm{O}$ & $\begin{array}{lll}0 . & 0 . & 2.70727\end{array}$ \\
\hline $\mathrm{F}$ & $\begin{array}{lll}1.26397 & -2.32451 & 0.65435\end{array}$ \\
\hline
\end{tabular}

\begin{tabular}{|c|c|c|c|c|}
\hline \multirow{2}{*}{$\begin{array}{l}\text { Center } \\
\text { Number }\end{array}$} & \multirow{2}{*}{$\begin{array}{l}\text { Atomic } \\
\text { Number }\end{array}$} & \multirow{2}{*}{$\begin{array}{r}\text { Atomic } \\
\text { Type }\end{array}$} & \multicolumn{2}{|c|}{ Coordinates (Angstroms) } \\
\hline & & & X $\quad Y$ & $\mathrm{Z}$ \\
\hline 1 & 1 & -2.147013 & 0.000000 & 1.316938 \\
\hline 2 & 6 & -1.276823 & 0.000000 & 0.653894 \\
\hline 3 & 6 & 0.000000 & -1.224422 & -1.165139 \\
\hline 4 & 6 & 0.000000 & 1.224422 & -1.165139 \\
\hline 5 & 6 & 0.000000 & 0.000000 & -1.994326 \\
\hline 6 & 6 & -1.256833 & 1.254101 & -0.168252 \\
\hline 7 & 6 & -1.256833 & -1.254101 & -0.168252 \\
\hline 8 & 1 & -0.883857 & 0.000000 & -2.644064 \\
\hline 9 & 1 & -2.158719 & 1.317186 & -0.788567 \\
\hline 10 & 1 & -2.158719 & -1.317186 & -0.788567 \\
\hline 11 & 1 & 0.000000 & -2.100971 & -1.823053 \\
\hline 12 & 1 & 0.000000 & 2.100971 & -1.823053 \\
\hline 13 & 1 & 0.883857 & 0.000000 & -2.644064 \\
\hline 14 & 1 & -1.262632 & 2.124220 & 0.500423 \\
\hline 15 & 1 & -1.262632 & -2.124220 & 0.500423 \\
\hline 16 & 6 & 1.256833 & 1.254101 & -0.168252 \\
\hline 17 & 1 & 1.262632 & 2.124220 & 0.500423 \\
\hline 18 & 1 & 2.158719 & 1.317186 & -0.788567 \\
\hline 19 & 6 & 0.000000 & 0.000000 & 1.480183 \\
\hline 20 & 6 & 1.256833 & -1.254101 & -0.168252 \\
\hline 21 & 1 & 2.158719 & -1.317186 & -0.788567 \\
\hline 22 & 6 & 1.276823 & 0.000000 & 0.653894 \\
\hline 23 & 1 & 2.147013 & 0.000000 & 1.316938 \\
\hline 24 & 8 & 0.000000 & 0.000000 & 2.707265 \\
\hline 25 & 9 & 1.263967 & -2.324513 & 0.654346 \\
\hline
\end{tabular}

\begin{tabular}{|c|c|c|c|c|c|}
\hline \multicolumn{6}{|c|}{ Distance matrix (angstroms): } \\
\hline & 12 & 23 & 45 & 5 & \\
\hline $1 \mathrm{H}$ & 0.000000 & & & & \\
\hline $2 \mathrm{C}$ & 1.094010 & 0.000000 & & & \\
\hline $3 \mathrm{C}$ & 3.502797 & 2.537394 & 0.000000 & & \\
\hline $4 \mathrm{C}$ & 3.502797 & 2.537394 & 2.448845 & 0.000000 & \\
\hline $5 \mathrm{C}$ & 3.946408 & 2.939957 & 1.478770 & 1.478770 & 0.000000 \\
\hline $6 \mathrm{C}$ & 2.137984 & 1.499697 & 2.952371 & 1.604461 & 2.546949 \\
\hline $7 \mathrm{C}$ & 2.137984 & 1.499697 & 1.604461 & 2.952371 & 2.546949 \\
\hline $8 \mathrm{H}$ & 4.157535 & 3.321287 & 2.113677 & 2.113677 & 1.096979 \\
\hline $9 \mathrm{H}$ & 2.483600 & 2.143225 & 3.355838 & 2.193281 & 2.801589 \\
\hline $10 \mathrm{H}$ & 2.483600 & 2.143225 & 2.193281 & 3.355838 & 2.801589 \\
\hline $11 \mathrm{H}$ & 4.345491 & 3.489932 & 1.095987 & 3.389851 & 2.107941 \\
\hline $12 \mathrm{H}$ & 4.345491 & 3.489932 & 3.389851 & 1.095987 & 2.107941 \\
\hline $13 \mathrm{H}$ & 4.987556 & 3.942723 & 2.113677 & 2.113677 & 1.096979 \\
\hline $14 \mathrm{H}$ & 2.441544 & 2.129804 & 3.947372 & 2.275516 & 3.511456 \\
\hline $15 \mathrm{H}$ & 2.441544 & 2.129804 & 2.275516 & 3.947372 & 3.511456 \\
\hline $16 \mathrm{C}$ & 3.919787 & 2.944165 & 2.952371 & 1.604461 & 2.546949 \\
\hline $17 \mathrm{H}$ & 4.099352 & 3.314317 & 3.947372 & 2.275516 & 3.511456 \\
\hline
\end{tabular}


$\begin{array}{lllllll}18 & \mathrm{H} & 4.970660 & 3.952040 & 3.355838 & 2.193281 & 2.801589\end{array}$

$\begin{array}{lllllll}19 & \mathrm{C} & 2.153210 & 1.520865 & 2.914951 & 2.914951 & 3.474509\end{array}$

$\begin{array}{lllllll}20 & \mathrm{C} & 3.919787 & 2.944165 & 1.604461 & 2.952371 & 2.546949\end{array}$

$\begin{array}{lllllll}21 & \mathrm{H} & 4.970660 & 3.952040 & 2.193281 & 3.355838 & 2.801589\end{array}$

$\begin{array}{llllllll}22 & \mathrm{C} & 3.487446 & 2.553645 & 2.537394 & 2.537394 & 2.939957\end{array}$

$\begin{array}{llllllll}23 & \mathrm{H} & 4.294026 & 3.487446 & 3.502797 & 3.502797 & 3.946408\end{array}$

$\begin{array}{llllllll}24 & \mathrm{O} & 2.557865 & 2.417976 & 4.061370 & 4.061370 & 4.701591\end{array}$

$\begin{array}{lllllll}25 & \mathrm{~F} & 4.180572 & 3.443686 & 2.473528 & 4.183669 & 3.743853\end{array}$

6 C 0.000000

$$
\begin{array}{lllll}
6 & 7 & 8 & 9 & 10
\end{array}
$$

$\begin{array}{llll}7 & \mathrm{C} & 2.508201 & 0.000000\end{array}$

$\begin{array}{lllll}8 \mathrm{H} & 2.800272 & 2.800272 & 0.000000\end{array}$

$\begin{array}{llllll}9 \mathrm{H} & 1.096435 & 2.794585 & 2.608279 & 0.000000\end{array}$

$\begin{array}{lllllll}10 \mathrm{H} & 2.794585 & 1.096435 & 2.608279 & 2.634373 & 0.000000\end{array}$

$\begin{array}{lllllll}11 & \mathrm{H} & 3.946455 & 2.243923 & 2.422672 & 4.173012 & 2.518839\end{array}$

$\begin{array}{lllllll}12 & \mathrm{H} & 2.243923 & 3.946455 & 2.422672 & 2.518839 & 4.173012\end{array}$

$\begin{array}{lllllll}13 & \mathrm{H} & 3.504992 & 3.504992 & 1.767715 & 3.799358 & 3.799358\end{array}$

$\begin{array}{lllllll}14 & \mathrm{H} & 1.097391 & 3.443865 & 3.813604 & 1.765154 & 3.782558\end{array}$

$\begin{array}{lllllll}15 & \mathrm{H} & 3.443865 & 1.097391 & 3.813604 & 3.782558 & 1.765154\end{array}$

$\begin{array}{lllllll}16 & \mathrm{C} & 2.513667 & 3.550999 & 3.504992 & 3.471998 & 4.319989\end{array}$

$\begin{array}{llllllll}17 & \mathrm{H} & 2.748079 & 4.267069 & 4.359762 & 3.744121 & 5.020998\end{array}$

$\begin{array}{lllllll}18 & \mathrm{H} & 3.471998 & 4.319989 & 3.799358 & 4.317438 & 5.057687\end{array}$

$\begin{array}{llllllll}19 & \mathrm{C} & 2.422754 & 2.422754 & 4.217892 & 3.397392 & 3.397392\end{array}$

$\begin{array}{lllllll}20 & \mathrm{C} & 3.550999 & 2.513667 & 3.504992 & 4.319989 & 3.471998\end{array}$

$\begin{array}{lllllll}21 & \mathrm{H} & 4.319989 & 3.471998 & 3.799358 & 5.057687 & 4.317438\end{array}$

$\begin{array}{lllllll}22 & \mathrm{C} & 2.944165 & 2.944165 & 3.942723 & 3.952040 & 3.952040\end{array}$

$\begin{array}{lllllll}23 & \mathrm{H} & 3.919787 & 3.919787 & 4.987556 & 4.970660 & 4.970660\end{array}$

$\begin{array}{lllllll}24 & \mathrm{O} & 3.379496 & 3.379496 & 5.423829 & 4.314614 & 4.314614\end{array}$

$\begin{array}{llllllll}25 & \mathrm{~F} & 4.453940 & 2.859525 & 4.571216 & 5.201803 & 3.848569\end{array}$

$11 \mathrm{H} \quad 0.000000$

$\begin{array}{lllll}11 & 12 & 13 & 14 & 15\end{array}$

$\begin{array}{llll}12 & \mathrm{H} & 4.201942 & 0.000000\end{array}$

$\begin{array}{lllll}13 & \mathrm{H} & 2.422672 & 2.422672 & 0.000000\end{array}$

$\begin{array}{llllll}14 & \mathrm{H} & 4.984478 & 2.644489 & 4.359762 & 0.000000\end{array}$

$\begin{array}{lllllll}15 & \mathrm{H} & 2.644489 & 4.984478 & 4.359762 & 4.248440 & 0.000000\end{array}$

$\begin{array}{lllllll}16 \mathrm{C} & 3.946455 & 2.243923 & 2.800272 & 2.748079 & 4.267069\end{array}$

$\begin{array}{llllllll}17 & \mathrm{H} & 4.984478 & 2.644489 & 3.813604 & 2.525265 & 4.942287\end{array}$

$\begin{array}{lllllll}18 \mathrm{H} & 4.173012 & 2.518839 & 2.608279 & 3.744121 & 5.020998\end{array}$

$\begin{array}{lllllll}19 & \mathrm{C} & 3.914773 & 3.914773 & 4.217892 & 2.658285 & 2.658285\end{array}$

$\begin{array}{lllllll}20 \mathrm{C} & 2.243923 & 3.946455 & 2.800272 & 4.267069 & 2.748079\end{array}$

$\begin{array}{lllllll}21 & \mathrm{H} & 2.518839 & 4.173012 & 2.608279 & 5.020998 & 3.744121\end{array}$

$\begin{array}{lllllll}22 & \mathrm{C} & 3.489932 & 3.489932 & 3.321287 & 3.314317 & 3.314317\end{array}$

$\begin{array}{llllllll}23 & \mathrm{H} & 4.345491 & 4.345491 & 4.157535 & 4.099352 & 4.099352\end{array}$

$\begin{array}{llllllll}24 & \mathrm{O} & 4.993783 & 4.993783 & 5.423829 & 3.313111 & 3.313111\end{array}$

$\begin{array}{lllllll}25 & \mathrm{~F} & 2.790177 & 5.226857 & 4.053067 & 5.118459 & 2.539196\end{array}$ $\begin{array}{lllll}16 & 17 & 18 & 19 & 20\end{array}$

$\begin{array}{ll}16 \mathrm{C} & 0.000000\end{array}$

$\begin{array}{llll}17 & \mathrm{H} & 1.097391 & 0.000000\end{array}$

$\begin{array}{lllll}18 & \mathrm{H} & 1.096435 & 1.765154 & 0.000000\end{array}$

$\begin{array}{lllllll}19 & \mathrm{C} & 2.422754 & 2.658285 & 3.397392 & 0.000000\end{array}$

$\begin{array}{lllllll}20 & \mathrm{C} & 2.508201 & 3.443865 & 2.794585 & 2.422754 & 0.000000\end{array}$

$\begin{array}{llllllll}21 & \mathrm{H} & 2.794585 & 3.782558 & 2.634373 & 3.397392 & 1.096435\end{array}$

$\begin{array}{lllllll}22 & \mathrm{C} & 1.499697 & 2.129804 & 2.143225 & 1.520865 & 1.499697\end{array}$

$\begin{array}{lllllll}23 & \mathrm{H} & 2.137984 & 2.441544 & 2.483600 & 2.153210 & 2.137984\end{array}$

$\begin{array}{lllllll}24 & \mathrm{O} & 3.379496 & 3.313111 & 4.314614 & 1.227082 & 3.379496\end{array}$

$\begin{array}{lllllll}25 & \text { F } & 3.671947 & 4.451395 & 4.018029 & 2.771819 & 1.350000\end{array}$ $\begin{array}{lllll}21 & 22 & 23 & 24 & 25\end{array}$

$\begin{array}{lll}21 \mathrm{H} & 0.000000\end{array}$

$\begin{array}{llll}22 & \mathrm{C} & 2.143225 & 0.000000\end{array}$

$\begin{array}{lllll}23 & \mathrm{H} & 2.483600 & 1.094010 & 0.000000\end{array}$

$\begin{array}{llllll}24 & \mathrm{O} & 4.314614 & 2.417976 & 2.557865 & 0.000000\end{array}$

$\begin{array}{lllllll}25 & F & 1.974154 & 2.324549 & 2.573356 & 3.348948 & 0.000000\end{array}$

Stoichiometry $\mathrm{C} 10 \mathrm{H} 13 \mathrm{FO}$

Framework group $\mathrm{C} 1[\mathrm{X}(\mathrm{C} 10 \mathrm{H} 13 \mathrm{FO})]$ 


\begin{tabular}{|c|c|c|c|c|c|}
\hline \multicolumn{6}{|c|}{$\begin{array}{lll}\text { Deg. of freedom } 69 & \\
\text { Full point group } & \mathrm{C} 1 & \mathrm{NO} \\
\text { Largest Abelian subgroup } & \mathrm{C} 1\end{array}$} \\
\hline Center & Atomic & & Atomic & Coordinate & s (Angstroms) \\
\hline Number & Numbe & & Type & $\begin{array}{ll}X & Y\end{array}$ & $\mathrm{Z}$ \\
\hline 1 & 1 & 0 & -0.175633 & 1.814109 & -1.838596 \\
\hline 2 & 6 & 0 & 0.078995 & 1.056705 & -1.091360 \\
\hline 3 & 6 & 0 & 0.459323 & -1.406759 & -0.616949 \\
\hline 4 & 6 & 0 & 1.775613 & 0.250789 & 0.614616 \\
\hline 5 & 6 & 0 & 1.768994 & -1.090847 & -0.007260 \\
\hline 6 & 6 & 0 & 1.392968 & 1.389083 & -0.449417 \\
\hline 7 & 6 & 0 & 0.044773 & -0.308642 & -1.710834 \\
\hline 8 & 1 & 0 & 2.550056 & -1.139958 & -0.775956 \\
\hline 9 & 1 & 0 & 2.190340 & 1.408065 & -1.201752 \\
\hline 10 & 1 & 0 & 0.774325 & -0.375061 & -2.526623 \\
\hline 11 & 1 & 0 & 0.505115 & -2.406996 & -1.062615 \\
\hline 12 & 1 & 0 & 2.763722 & 0.437171 & 1.050612 \\
\hline 13 & 1 & 0 & 2.008984 & -1.845476 & 0.751886 \\
\hline 14 & 1 & 0 & 1.337040 & 2.393937 & -0.011917 \\
\hline 15 & 1 & 0 & -0.946559 & -0.481702 & -2.148529 \\
\hline 16 & 6 & 0 & 0.623572 & 0.385846 & 1.723154 \\
\hline 17 & 1 & 0 & 0.564094 & 1.386071 & 2.170678 \\
\hline 18 & 1 & 0 & 0.868834 & -0.315080 & 2.529825 \\
\hline 19 & 6 & 0 & -0.961071 & 1.058178 & 0.018275 \\
\hline 20 & 6 & 0 & -0.724624 & -1.311879 & 0.461737 \\
\hline 21 & 1 & 0 & -0.547180 & -2.098206 & 1.204955 \\
\hline 22 & 6 & 0 & -0.702638 & 0.037512 & 1.115765 \\
\hline 23 & 1 & 0 & -1.489972 & 0.100308 & 1.872746 \\
\hline 24 & 8 & 0 & -1.925240 & 1.817142 & 0.027293 \\
\hline 25 & 9 & 0 & -1.948519 & -1.530470 & -0.064380 \\
\hline
\end{tabular}

Rotational constants (GHZ): $1.3022853 \quad 1.1486512 \quad 0.9560881$

Standard basis: $6-31 \mathrm{G}(\mathrm{d})(6 \mathrm{D}, 7 \mathrm{~F})$

There are 206 symmetry adapted basis functions of A symmetry.

Integral buffers will be 262144 words long.

Raffenetti 1 integral format.

Two-electron integral symmetry is turned on.

206 basis functions, 388 primitive gaussians, 206 cartesian basis functions

45 alpha electrons 45 beta electrons nuclear repulsion energy $\quad 789.5126009488$ Hartrees.

NAtoms $=25$ NActive $=25$ NUniq $=25 \mathrm{SFac}=1.00 \mathrm{D}+00 \mathrm{NAtFMM}=60 \mathrm{Big}=\mathrm{F}$

One-electron integrals computed using PRISM.

NBasis $=206$ RedAO $=\mathrm{T}$ NBF $=206$

NBsUse $=206$ 1.00D-06 NBFU $=206$

Harris functional with IExCor= 205 diagonalized for initial guess.

ExpMin= 1.61D-01 ExpMax= 7.00D+03 ExpMxC=1.05D+03 IAcc=1 IRadAn $=1$ AccDes=1.00D-06

HarFok: IExCor $=205$ AccDes= 1.00D-06 IRadAn $=\quad 1 \mathrm{IDoV}=1$

ScaDFX= 1.0000001 .0000001 .0000001 .000000

Initial guess orbital symmetries:

Occupied (A) (A) (A) (A) (A) (A) (A) (A) (A) (A) (A) (A)

$(\mathrm{A})(\mathrm{A})(\mathrm{A})(\mathrm{A})(\mathrm{A})(\mathrm{A})(\mathrm{A})(\mathrm{A})(\mathrm{A})(\mathrm{A})(\mathrm{A})(\mathrm{A})$

$(\mathrm{A})(\mathrm{A})(\mathrm{A})(\mathrm{A})(\mathrm{A})(\mathrm{A})(\mathrm{A})(\mathrm{A})(\mathrm{A})(\mathrm{A})(\mathrm{A})(\mathrm{A})$

(A) $(\mathrm{A})(\mathrm{A})(\mathrm{A})(\mathrm{A})(\mathrm{A})(\mathrm{A})(\mathrm{A})(\mathrm{A})$

Virtual (A) (A) (A) (A) (A) (A) (A) (A) (A) (A) (A) (A)

(A) (A) (A) (A) (A) (A) (A) (A) (A) (A) (A) (A)

(A) (A) (A) (A) (A) (A) (A) (A) (A) (A) (A) (A)

(A) (A) (A) (A) (A) (A) (A) (A) (A) (A) (A) (A)

(A) $(\mathrm{A})(\mathrm{A})(\mathrm{A})(\mathrm{A})(\mathrm{A})(\mathrm{A})(\mathrm{A})(\mathrm{A})(\mathrm{A})(\mathrm{A})(\mathrm{A})$

(A) $(\mathrm{A})(\mathrm{A})(\mathrm{A})(\mathrm{A})(\mathrm{A})(\mathrm{A})(\mathrm{A})(\mathrm{A})(\mathrm{A})(\mathrm{A})(\mathrm{A})$

(A) $(\mathrm{A})(\mathrm{A})(\mathrm{A})(\mathrm{A})(\mathrm{A})(\mathrm{A})(\mathrm{A})(\mathrm{A})(\mathrm{A})(\mathrm{A})(\mathrm{A})$ 
(A) $(\mathrm{A})(\mathrm{A})(\mathrm{A})(\mathrm{A})(\mathrm{A})(\mathrm{A})(\mathrm{A})(\mathrm{A})(\mathrm{A})(\mathrm{A})(\mathrm{A})$

(A) $(\mathrm{A})(\mathrm{A})(\mathrm{A})(\mathrm{A})(\mathrm{A})(\mathrm{A})(\mathrm{A})(\mathrm{A})(\mathrm{A})(\mathrm{A})(\mathrm{A})$

(A) $(A)(A)(A)(A)(A)(A)(A)(A)(A)(A)(A)$

(A) $(\mathrm{A})(\mathrm{A})(\mathrm{A})(\mathrm{A})(\mathrm{A})(\mathrm{A})(\mathrm{A})(\mathrm{A})(\mathrm{A})(\mathrm{A})(\mathrm{A})$

(A) $(\mathrm{A})(\mathrm{A})(\mathrm{A})(\mathrm{A})(\mathrm{A})(\mathrm{A})(\mathrm{A})(\mathrm{A})(\mathrm{A})(\mathrm{A})(\mathrm{A})$

(A) $(\mathrm{A})(\mathrm{A})(\mathrm{A})(\mathrm{A})(\mathrm{A})(\mathrm{A})(\mathrm{A})(\mathrm{A})(\mathrm{A})(\mathrm{A})(\mathrm{A})$

(A) (A) (A) (A) (A)

The electronic state of the initial guess is 1-A.

Warning! Cutoffs for single-point calculations used.

Requested convergence on RMS density matrix=1.00D-04 within 128 cycles.

Requested convergence on MAX density matrix=1.00D-02.

Requested convergence on energy=5.00D-05.

No special actions if energy rises.

SCF Done: $\mathrm{E}(\mathrm{RHF})=-560.550576015 \quad$ A.U. after 7 cycles

Convg $=0.6988 \mathrm{D}-05 \quad-\mathrm{V} / \mathrm{T}=2.0012$

$\mathrm{S} * * 2=0.0000$

******************************************************************************

Population analysis using the SCF density.

$* * * * * * * * * * * * * * * * * * * * * * * * * * * * * * * * * * * * * * * * * * * * * * * * * * * * * * * * * * * * * * * * * * * * * *$

Orbital symmetries:

Occupied (A) (A) (A) (A) (A) (A) (A) (A) (A) (A) (A) (A)

(A) $(\mathrm{A})(\mathrm{A})(\mathrm{A})(\mathrm{A})(\mathrm{A})(\mathrm{A})(\mathrm{A})(\mathrm{A})(\mathrm{A})(\mathrm{A})(\mathrm{A})$

$(\mathrm{A})(\mathrm{A})(\mathrm{A})(\mathrm{A})(\mathrm{A})(\mathrm{A})(\mathrm{A})(\mathrm{A})(\mathrm{A})(\mathrm{A})(\mathrm{A})(\mathrm{A})$

(A) $(A)(A)(A)(A)(A)(A)(A)(A)$

Virtual (A) (A) (A) (A) (A) (A) (A) (A) (A) (A) (A) (A)

$(\mathrm{A})(\mathrm{A})(\mathrm{A})(\mathrm{A})(\mathrm{A})(\mathrm{A})(\mathrm{A})(\mathrm{A})(\mathrm{A})(\mathrm{A})(\mathrm{A})(\mathrm{A})$

(A) $(A)(A)(A)(A)(A)(A)(A)(A)(A)(A)(A)$

(A) $(\mathrm{A})(\mathrm{A})(\mathrm{A})(\mathrm{A})(\mathrm{A})(\mathrm{A})(\mathrm{A})(\mathrm{A})(\mathrm{A})(\mathrm{A})(\mathrm{A})$

(A) $(\mathrm{A})(\mathrm{A})(\mathrm{A})(\mathrm{A})(\mathrm{A})(\mathrm{A})(\mathrm{A})(\mathrm{A})(\mathrm{A})(\mathrm{A})(\mathrm{A})$

(A) $(A)(A)(A)(A)(A)(A)(A)(A)(A)(A)(A)$

(A) $(\mathrm{A})(\mathrm{A})(\mathrm{A})(\mathrm{A})(\mathrm{A})(\mathrm{A})(\mathrm{A})(\mathrm{A})(\mathrm{A})(\mathrm{A})(\mathrm{A})$

(A) $(\mathrm{A})(\mathrm{A})(\mathrm{A})(\mathrm{A})(\mathrm{A})(\mathrm{A})(\mathrm{A})(\mathrm{A})(\mathrm{A})(\mathrm{A})(\mathrm{A})$

(A) $(A)(A)(A)(A)(A)(A)(A)(A)(A)(A)(A)$

(A) $(\mathrm{A})(\mathrm{A})(\mathrm{A})(\mathrm{A})(\mathrm{A})(\mathrm{A})(\mathrm{A})(\mathrm{A})(\mathrm{A})(\mathrm{A})(\mathrm{A})$

(A) $(\mathrm{A})(\mathrm{A})(\mathrm{A})(\mathrm{A})(\mathrm{A})(\mathrm{A})(\mathrm{A})(\mathrm{A})(\mathrm{A})(\mathrm{A})(\mathrm{A})$

(A) $(A)(A)(A)(A)(A)(A)(A)(A)(A)(A)(A)$

(A) $(\mathrm{A})(\mathrm{A})(\mathrm{A})(\mathrm{A})(\mathrm{A})(\mathrm{A})(\mathrm{A})(\mathrm{A})(\mathrm{A})(\mathrm{A})(\mathrm{A})$

(A) $(\mathrm{A})(\mathrm{A})(\mathrm{A})(\mathrm{A})$

The electronic state is $1-\mathrm{A}$.

Alpha occ. eigenvalues -- -26.28986 -20.54703 -11.32203 -11.31915 -11.24856

Alpha occ. eigenvalues -- -11.24856 -11.24349 -11.23810 -11.23756 -11.23596

Alpha occ. eigenvalues -- $-11.23459-11.23456-1.60829-1.36782-1.18493$

Alpha occ. eigenvalues -- $-1.07593-1.05964-1.04104-0.92681 \quad-0.88850$

Alpha occ. eigenvalues -- $-0.83829 \quad-0.82735 \quad-0.79563 \quad-0.72158 \quad-0.70609$

Alpha occ. eigenvalues -- $-0.68290 \quad-0.67771 \quad-0.65311 \quad-0.63320 \quad-0.61917$

Alpha occ. eigenvalues -- $\quad-0.60610 \quad-0.58751 \quad-0.57822 \quad-0.56995 \quad-0.55934$

Alpha occ. eigenvalues -- $-0.54743 \quad-0.53779-0.51785-0.50330 \quad-0.48451$

$\begin{array}{lllllll}\text { Alpha occ. eigenvalues -- } & -0.45506 & -0.44854 & -0.43844 & -0.42021 & -0.37972\end{array}$

$\begin{array}{lllllll}\text { Alpha virt. eigenvalues -- } & 0.15961 & 0.19779 & 0.25134 & 0.26149 & 0.26701\end{array}$

$\begin{array}{lllllll}\text { Alpha virt. eigenvalues -- } & 0.27146 & 0.27727 & 0.28119 & 0.29117 & 0.31756\end{array}$

$\begin{array}{lllllll}\text { Alpha virt. eigenvalues -- } & 0.32166 & 0.33086 & 0.34157 & 0.34795 & 0.35243\end{array}$

$\begin{array}{lllllll}\text { Alpha virt. eigenvalues -- } & 0.36441 & 0.37230 & 0.38792 & 0.39574 & 0.41459\end{array}$

$\begin{array}{lllllll}\text { Alpha virt. eigenvalues -- } & 0.42577 & 0.43748 & 0.44664 & 0.45609 & 0.49205\end{array}$

$\begin{array}{lllllll}\text { Alpha virt. eigenvalues -- } & 0.51029 & 0.53346 & 0.55254 & 0.70431 & 0.71967\end{array}$

$\begin{array}{lllllll}\text { Alpha virt. eigenvalues -- } & 0.73894 & 0.75300 & 0.77306 & 0.79330 & 0.80717\end{array}$

$\begin{array}{lllllll}\text { Alpha virt. eigenvalues -- } & 0.82963 & 0.83781 & 0.85136 & 0.88304 & 0.89981\end{array}$

$\begin{array}{llllllll}\text { Alpha virt. eigenvalues -- } & 0.91757 & 0.92152 & 0.93972 & 0.95689 & 0.96756\end{array}$

$\begin{array}{llllll}\text { Alpha virt. eigenvalues -- } & 0.97164 & 1.01284 & 1.01744 & 1.02407 & 1.03995\end{array}$

$\begin{array}{lllllll}\text { Alpha virt. eigenvalues -- } & 1.04263 & 1.05382 & 1.07696 & 1.10944 & 1.11572\end{array}$

$\begin{array}{llllll}\text { Alpha virt. eigenvalues -- } & 1.12252 & 1.12569 & 1.13055 & 1.13358 & 1.14214\end{array}$ 

$\begin{array}{llllll}\text { Alpha virt. eigenvalues -- } & 1.17799 & 1.18324 & 1.19521 & 1.19973 & 1.21117 \\ \text { Alpha virt. eigenvalues -- } & 1.24038 & 1.24537 & 1.26028 & 1.31958 & 1.34797 \\ \text { Alpha virt. eigenvalues -- } & 1.38333 & 1.41951 & 1.42175 & 1.43810 & 1.44298 \\ \text { Alpha virt. eigenvalues -- } & 1.45267 & 1.47056 & 1.56183 & 1.57562 & 1.62938 \\ \text { Alpha virt. eigenvalues -- } & 1.65683 & 1.67507 & 1.69954 & 1.73643 & 1.75986 \\ \text { Alpha virt. eigenvalues -- } & 1.82100 & 1.86544 & 1.87649 & 1.90678 & 1.92868 \\ \text { Alpha virt. eigenvalues -- } & 1.93902 & 1.94942 & 1.98631 & 2.00418 & 2.04062 \\ \text { Alpha virt. eigenvalues -- } & 2.06158 & 2.06476 & 2.09717 & 2.11647 & 2.13304 \\ \text { Alpha virt. eigenvalues -- } & 2.15867 & 2.19065 & 2.19987 & 2.22627 & 2.25228 \\ \text { Alpha virt. eigenvalues -- } & 2.27306 & 2.27627 & 2.29561 & 2.33901 & 2.35004 \\ \text { Alpha virt. eigenvalues -- } & 2.36014 & 2.38322 & 2.40620 & 2.41549 & 2.42563 \\ \text { Alpha virt. eigenvalues -- } & 2.44293 & 2.45945 & 2.50879 & 2.53780 & 2.56142 \\ \text { Alpha virt. eigenvalues -- } & 2.59881 & 2.61739 & 2.63690 & 2.65266 & 2.66638 \\ \text { Alpha virt. eigenvalues -- } & 2.67805 & 2.71440 & 2.73521 & 2.75380 & 2.77552 \\ \text { Alpha virt. eigenvalues -- } & 2.82755 & 2.84070 & 2.85494 & 2.87211 & 2.88012 \\ \text { Alpha virt. eigenvalues -- } & 3.00338 & 3.01347 & 3.04035 & 3.04884 & 3.07238 \\ \text { Alpha virt. eigenvalues -- } & 3.07798 & 3.09464 & 3.13321 & 3.14379 & 3.16359 \\ \text { Alpha virt. eigenvalues -- } & 3.24331 & 3.38397 & 3.39264 & 3.54829 & 4.50691 \\ \text { Alpha virt. eigenvalues -- } & 4.56149 & 4.68652 & 4.73021 & 4.75235 & 4.94109 \\ \text { Alpha virt. eigenvalues -- } & 4.98185 & 5.11146 & 5.13798 & 5.18642 & 5.28690 \\ \text { Alpha virt. eigenvalues -- } & 5.46485 & & & & \\ \text { Condensed to atoms (all electrons): } & & & & \end{array}$
1

$\begin{array}{llllllll}1 \mathrm{H} & 0.481716 & 0.403946 & 0.003914 & 0.004000 & -0.000400 & -0.032837\end{array}$

$\begin{array}{llllllll}2 & \mathrm{C} & 0.403946 & 5.308437 & -0.045275 & -0.049010 & -0.000741 & 0.303826\end{array}$

$\begin{array}{llllllll}3 & C & 0.003914 & -0.045275 & 5.271957 & -0.051205 & 0.289955 & -0.003703\end{array}$

$\begin{array}{llllllll}4 & \mathrm{C} & 0.004000 & -0.049010 & -0.051205 & 5.160814 & 0.324103 & 0.340668\end{array}$

$\begin{array}{llllllll}5 & \mathrm{C} & -0.000400 & -0.000741 & 0.289955 & 0.324103 & 5.185946 & -0.061193\end{array}$

$\begin{array}{llllllll}6 \mathrm{C} & -0.032837 & 0.303826 & -0.003703 & 0.340668 & -0.061193 & 5.212882\end{array}$

$\begin{array}{llllllll}7 & \mathrm{C} & -0.031961 & 0.301618 & 0.315610 & -0.004678 & -0.054889 & -0.058441\end{array}$

$\begin{array}{llllllll}8 & \mathrm{H} & -0.000081 & 0.000201 & -0.038650 & -0.043920 & 0.402883 & -0.003920\end{array}$

$\begin{array}{llllllll}9 \mathrm{H} & -0.004269 & -0.037921 & 0.000242 & -0.040501 & -0.001313 & 0.392136\end{array}$

$\begin{array}{llllllll}10 \mathrm{H} & -0.004175 & -0.037935 & -0.039657 & 0.000284 & -0.001708 & -0.004324\end{array}$

$\begin{array}{lllllllll}11 & \mathrm{H} & -0.000056 & 0.003882 & 0.404909 & 0.005128 & -0.041376 & -0.000347\end{array}$

$\begin{array}{llllllll}12 \mathrm{H} & -0.000054 & 0.003672 & 0.005196 & 0.409876 & -0.044315 & -0.028905\end{array}$

$\begin{array}{llllllll}13 \mathrm{H} & 0.000016 & -0.000864 & -0.047305 & -0.045199 & 0.406736 & 0.005112\end{array}$

$\begin{array}{llllllll}14 \mathrm{H} & -0.001943 & -0.047349 & -0.000496 & -0.029794 & 0.003470 & 0.403353\end{array}$

$\begin{array}{llllllll}15 \mathrm{H} & -0.001782 & -0.045544 & -0.028456 & -0.000407 & 0.003480 & 0.004876\end{array}$

$\begin{array}{llllllll}16 & \mathrm{C} & -0.000487 & 0.001460 & 0.003086 & 0.339170 & -0.067750 & -0.076373\end{array}$

$\begin{array}{llllllll}17 & \mathrm{H} & -0.000077 & 0.001667 & -0.000531 & -0.029010 & 0.003572 & -0.004275\end{array}$

$\begin{array}{llllllll}18 & \mathrm{H} & 0.000017 & -0.000865 & 0.000095 & -0.040772 & -0.001137 & 0.005122\end{array}$

$\begin{array}{llllllll}19 & \mathrm{C} & -0.036358 & 0.327447 & -0.015237 & -0.007089 & 0.000577 & -0.060597\end{array}$

$\begin{array}{llllllll}20 & \mathrm{C} & -0.000427 & 0.003781 & 0.357567 & -0.006303 & -0.053384 & -0.000378\end{array}$

$\begin{array}{llllllll}21 \mathrm{H} & 0.000016 & -0.001082 & -0.055924 & 0.000569 & 0.001598 & 0.000055\end{array}$

$\begin{array}{llllllll}22 & \mathrm{C} & 0.003744 & -0.097570 & -0.059825 & -0.050926 & 0.007304 & 0.004344\end{array}$

$\begin{array}{llllllll}23 & \mathrm{H} & -0.000087 & 0.003988 & 0.004530 & 0.003771 & -0.000490 & -0.000521\end{array}$

$\begin{array}{lllllllll}24 & \mathrm{O} & 0.003865 & -0.062794 & 0.000312 & 0.000178 & -0.000039 & 0.000998\end{array}$

$\begin{array}{llllllll}25 & \mathrm{~F} & -0.000010 & -0.002483 & -0.028943 & 0.000008 & 0.001420 & -0.000007\end{array}$ $\begin{array}{llllll}7 & 8 & 9 & 10 & 11 & 12\end{array}$

$\begin{array}{lllllll}1 \mathrm{H} & -0.031961 & -0.000081 & -0.004269 & -0.004175 & -0.000056 & -0.000054\end{array}$

$\begin{array}{llllllll}2 & \mathrm{C} & 0.301618 & 0.000201 & -0.037921 & -0.037935 & 0.003882 & 0.003672\end{array}$

$\begin{array}{llllllll}3 \mathrm{C} & 0.315610 & -0.038650 & 0.000242 & -0.039657 & 0.404909 & 0.005196\end{array}$

$\begin{array}{llllllll}4 \text { C } & -0.004678 & -0.043920 & -0.040501 & 0.000284 & 0.005128 & 0.409876\end{array}$

$\begin{array}{llllllll}5 & \mathrm{C} & -0.054889 & 0.402883 & -0.001313 & -0.001708 & -0.041376 & -0.044315\end{array}$

$\begin{array}{llllllll}6 \mathrm{C} & -0.058441 & -0.003920 & 0.392136 & -0.004324 & -0.000347 & -0.028905\end{array}$

$\begin{array}{llllllll}7 & \mathrm{C} & 5.228371 & -0.004352 & -0.004166 & 0.393382 & -0.029364 & -0.000312\end{array}$

$\begin{array}{llllllll}8 \mathrm{H} & -0.004352 & 0.526985 & 0.002994 & 0.002845 & -0.003632 & -0.003361\end{array}$

$\begin{array}{llllllll}9 \mathrm{H} & -0.004166 & 0.002994 & 0.537345 & 0.003454 & -0.000047 & -0.002143\end{array}$

$\begin{array}{llllllll}10 & \mathrm{H} & 0.393382 & 0.002845 & 0.003454 & 0.533641 & -0.002154 & -0.000047\end{array}$

$\begin{array}{llllllll}11 \mathrm{H} & -0.029364 & -0.003632 & -0.000047 & -0.002154 & 0.505205 & -0.000112\end{array}$

$\begin{array}{llllllll}12 & \mathrm{H} & -0.000312 & -0.003361 & -0.002143 & -0.000047 & -0.000112 & 0.515165\end{array}$

$\begin{array}{llllllll}13 \mathrm{H} & 0.005191 & -0.024126 & -0.000168 & -0.000154 & -0.003583 & -0.003501\end{array}$

$\begin{array}{llllllll}14 \mathrm{H} & 0.005144 & -0.000064 & -0.024112 & -0.000108 & 0.000010 & -0.001712\end{array}$

$\begin{array}{llllllll}15 & \mathrm{H} & 0.403088 & -0.000076 & -0.000106 & -0.023372 & -0.001422 & 0.000009\end{array}$ 


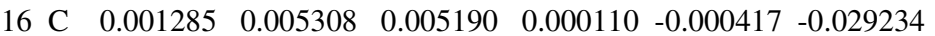

$\begin{array}{llllllll}17 & \mathrm{H} & -0.000043 & -0.000070 & 0.000049 & 0.000002 & 0.000011 & -0.001633\end{array}$

$\begin{array}{llllllll}18 \mathrm{H} & 0.000109 & -0.000183 & -0.000131 & -0.000003 & -0.000049 & -0.002095\end{array}$

$\begin{array}{llllllll}19 & \mathrm{C} & -0.054886 & 0.000160 & 0.005468 & 0.005482 & -0.000561 & -0.000393\end{array}$

$\begin{array}{llllllll}20 & \mathrm{C} & -0.065637 & 0.004118 & 0.000119 & 0.004645 & -0.026919 & -0.000199\end{array}$

$\begin{array}{llllllll}21 \mathrm{H} & 0.005982 & -0.000249 & -0.000002 & -0.000100 & -0.003463 & -0.000026\end{array}$

$\begin{array}{llllllll}22 & \mathrm{C} & -0.005079 & -0.000876 & -0.000903 & -0.000883 & 0.004256 & 0.003694\end{array}$

$\begin{array}{llllllll}23 & \mathrm{H} & -0.000717 & 0.000017 & 0.000016 & 0.000016 & -0.000056 & -0.000053\end{array}$

$\begin{array}{llllllll}24 & \mathrm{O} & 0.000669 & 0.000000 & -0.000081 & -0.000080 & 0.000000 & -0.000002\end{array}$

$\begin{array}{llllllll}25 & \mathrm{~F} & 0.004145 & -0.000019 & 0.000000 & 0.000065 & 0.000422 & 0.000001\end{array}$
$\begin{array}{llllll}13 & 14 & 15 & 16 & 17 & 18\end{array}$

$\begin{array}{llllllll}1 \mathrm{H} & 0.000016 & -0.001943 & -0.001782 & -0.000487 & -0.000077 & 0.000017\end{array}$

$\begin{array}{llllllll}2 & \mathrm{C} & -0.000864 & -0.047349 & -0.045544 & 0.001460 & 0.001667 & -0.000865\end{array}$

$\begin{array}{llllllll}3 \mathrm{C} & -0.047305 & -0.000496 & -0.028456 & 0.003086 & -0.000531 & 0.000095\end{array}$

$\begin{array}{llllllll}4 & \mathrm{C} & -0.045199 & -0.029794 & -0.000407 & 0.339170 & -0.029010 & -0.040772\end{array}$

$\begin{array}{llllllll}5 & \mathrm{C} & 0.406736 & 0.003470 & 0.003480 & -0.067750 & 0.003572 & -0.001137\end{array}$

$\begin{array}{llllllll}6 & \mathrm{C} & 0.005112 & 0.403353 & 0.004876 & -0.076373 & -0.004275 & 0.005122\end{array}$

$\begin{array}{lllllllll}7 & \mathrm{C} & 0.005191 & 0.005144 & 0.403088 & 0.001285 & -0.000043 & 0.000109\end{array}$

$\begin{array}{llllllll}8 \mathrm{H} & -0.024126 & -0.000064 & -0.000076 & 0.005308 & -0.000070 & -0.000183\end{array}$

$\begin{array}{lllllllll}9 \mathrm{H} & -0.000168 & -0.024112 & -0.000106 & 0.005190 & 0.000049 & -0.000131\end{array}$

$\begin{array}{lllllll}10 \mathrm{H} & -0.000154 & -0.000108 & -0.023372 & 0.000110 & 0.000002 & -0.000003\end{array}$

$\begin{array}{llllllll}11 \mathrm{H} & -0.003583 & 0.000010 & -0.001422 & -0.000417 & 0.000011 & -0.000049\end{array}$

$\begin{array}{llllllll}12 & \mathrm{H} & -0.003501 & -0.001712 & 0.000009 & -0.029234 & -0.001633 & -0.002095\end{array}$

$\begin{array}{llllllll}13 & \mathrm{H} & 0.537203 & -0.000064 & -0.000059 & -0.003912 & -0.000074 & 0.003049\end{array}$

$\begin{array}{llllllll}14 \mathrm{H} & -0.000064 & 0.511263 & -0.000104 & -0.004309 & 0.001460 & 0.000047\end{array}$

$\begin{array}{llllllll}15 \mathrm{H} & -0.000059 & -0.000104 & 0.483325 & -0.000059 & -0.000001 & 0.000002\end{array}$

$\begin{array}{llllllll}16 & \mathrm{C} & -0.003912 & -0.004309 & -0.000059 & 5.238658 & 0.400244 & 0.393483\end{array}$

$\begin{array}{lllllllll}17 & \mathrm{H} & -0.000074 & 0.001460 & -0.000001 & 0.400244 & 0.500776 & -0.023697\end{array}$

$\begin{array}{llllllll}18 & \mathrm{H} & 0.003049 & 0.000047 & 0.000002 & 0.393483 & -0.023697 & 0.535233\end{array}$

$\begin{array}{llllllll}19 & \text { C } & 0.000251 & -0.002690 & -0.002301 & -0.051857 & -0.003109 & 0.005495\end{array}$

$\begin{array}{llllllll}20 & \mathrm{C} & -0.003382 & 0.000030 & -0.005759 & -0.048064 & 0.004120 & -0.003803\end{array}$

$\begin{array}{lllllllll}21 & \mathrm{H} & 0.003314 & 0.000003 & 0.000174 & -0.001335 & -0.000194 & 0.003674\end{array}$

$\begin{array}{llllllll}22 & \mathrm{C} & -0.000209 & 0.001487 & 0.001772 & 0.273127 & -0.041718 & -0.040830\end{array}$

$\begin{array}{llllllll}23 & \mathrm{H} & -0.000083 & -0.000073 & -0.000078 & -0.028969 & -0.002085 & -0.004424\end{array}$

$\begin{array}{lllllllll}24 & \mathrm{O} & 0.000000 & 0.000763 & 0.000696 & 0.000197 & 0.000839 & -0.000078\end{array}$

$\begin{array}{lllllllll}25 & \mathrm{~F} & -0.000003 & 0.000000 & 0.004262 & 0.002258 & -0.000031 & -0.000009\end{array}$ $\begin{array}{llllll}19 & 20 & 21 & 22 & 23 & 24\end{array}$

$\begin{array}{llllllll}1 \mathrm{H} & -0.036358 & -0.000427 & 0.000016 & 0.003744 & -0.000087 & 0.003865\end{array}$

$\begin{array}{llllllll}2 & \mathrm{C} & 0.327447 & 0.003781 & -0.001082 & -0.097570 & 0.003988 & -0.062794\end{array}$

$\begin{array}{llllllll}3 & C & -0.015237 & 0.357567 & -0.055924 & -0.059825 & 0.004530 & 0.000312\end{array}$

$\begin{array}{llllllll}4 \mathrm{C} & -0.007089 & -0.006303 & 0.000569 & -0.050926 & 0.003771 & 0.000178\end{array}$

$\begin{array}{llllllll}5 & \mathrm{C} & 0.000577 & -0.053384 & 0.001598 & 0.007304 & -0.000490 & -0.000039\end{array}$

$\begin{array}{llllllll}6 & \mathrm{C} & -0.060597 & -0.000378 & 0.000055 & 0.004344 & -0.000521 & 0.000998\end{array}$

$\begin{array}{llllllll}7 & \mathrm{C} & -0.054886 & -0.065637 & 0.005982 & -0.005079 & -0.000717 & 0.000669\end{array}$

$\begin{array}{llllllll}8 \mathrm{H} & 0.000160 & 0.004118 & -0.000249 & -0.000876 & 0.000017 & 0.000000\end{array}$

$\begin{array}{llllllll}9 \mathrm{H} & 0.005468 & 0.000119 & -0.000002 & -0.000903 & 0.000016 & -0.000081\end{array}$

$\begin{array}{llllllll}10 \mathrm{H} & 0.005482 & 0.004645 & -0.000100 & -0.000883 & 0.000016 & -0.000080\end{array}$

$\begin{array}{llllllll}11 \mathrm{H} & -0.000561 & -0.026919 & -0.003463 & 0.004256 & -0.000056 & 0.000000\end{array}$

$\begin{array}{llllllll}12 \mathrm{H} & -0.000393 & -0.000199 & -0.000026 & 0.003694 & -0.000053 & -0.000002\end{array}$

$\begin{array}{llllllll}13 \mathrm{H} & 0.000251 & -0.003382 & 0.003314 & -0.000209 & -0.000083 & 0.000000\end{array}$

$\begin{array}{llllllll}14 \mathrm{H} & -0.002690 & 0.000030 & 0.000003 & 0.001487 & -0.000073 & 0.000763\end{array}$

$\begin{array}{llllllll}15 \mathrm{H} & -0.002301 & -0.005759 & 0.000174 & 0.001772 & -0.000078 & 0.000696\end{array}$

$\begin{array}{llllllll}16 & \mathrm{C} & -0.051857 & -0.048064 & -0.001335 & 0.273127 & -0.028969 & 0.000197\end{array}$

$\begin{array}{llllllll}17 & \mathrm{H} & -0.003109 & 0.004120 & -0.000194 & -0.041718 & -0.002085 & 0.000839\end{array}$

$\begin{array}{llllllll}18 \mathrm{H} & 0.005495 & -0.003803 & 0.003674 & -0.040830 & -0.004424 & -0.000078\end{array}$

$\begin{array}{llllllll}19 & \mathrm{C} & 4.567759 & -0.052046 & 0.006412 & 0.299244 & -0.037037 & 0.552674\end{array}$

$\begin{array}{llllllll}20 \mathrm{C} & -0.052046 & 4.689536 & 0.406179 & 0.331348 & -0.027688 & 0.001114\end{array}$

$\begin{array}{lllllllll}21 & \mathrm{H} & 0.006412 & 0.406179 & 0.560331 & -0.053189 & -0.005685 & -0.000120\end{array}$

$\begin{array}{llllllll}22 & \mathrm{C} & 0.299244 & 0.331348 & -0.053189 & 5.413907 & 0.395899 & -0.061143\end{array}$

$\begin{array}{llllllll}23 & \mathrm{H} & -0.037037 & -0.027688 & -0.005685 & 0.395899 & 0.469082 & 0.004309\end{array}$

$\begin{array}{llllllll}24 & \mathrm{O} & 0.552674 & 0.001114 & -0.000120 & -0.061143 & 0.004309 & 8.113878\end{array}$

$\begin{array}{llllllll}25 & \mathrm{~F} & 0.011874 & 0.200855 & -0.028749 & -0.042821 & 0.000879 & -0.000555\end{array}$

25

$1 \mathrm{H}-0.000010$ 
2 C -0.002483

3 C -0.028943

4 C 0.000008

5 C 0.001420

$\begin{array}{lll}6 & \mathrm{C} & -0.000007\end{array}$

7 C 0.004145

$8 \mathrm{H} \quad-0.000019$

$9 \mathrm{H} \quad 0.000000$

$10 \mathrm{H} \quad 0.000065$

$\begin{array}{lll}11 \mathrm{H} & 0.000422\end{array}$

$12 \mathrm{H} \quad 0.000001$

$13 \mathrm{H}-0.000003$

$14 \mathrm{H} \quad 0.000000$

$\begin{array}{lll}15 \mathrm{H} & 0.004262\end{array}$

$16 \mathrm{C} \quad 0.002258$

$17 \mathrm{H}-0.000031$

$18 \mathrm{H}-0.000009$

19 C 0.011874

$20 \mathrm{C} \quad 0.200855$

$21 \mathrm{H}-0.028749$

$22 \mathrm{C}-0.042821$

$23 \mathrm{H} \quad 0.000879$

$24 \mathrm{O} \quad-0.000555$

$25 \mathrm{~F} \quad 9.282462$

Mulliken atomic charges:

$$
1
$$

$\begin{array}{lll}1 & \mathrm{H} & 0.213769\end{array}$

2 C -0.234491

3 C -0.242166

4 C -0.189756

5 C -0.302306

6 C -0.337551

7 C -0.350068

$8 \mathrm{H} \quad 0.178071$

$9 \mathrm{H} \quad 0.168847$

$10 \mathrm{H} \quad 0.170775$

$11 \mathrm{H} \quad 0.189736$

$12 \mathrm{H} \quad 0.180483$

$13 \mathrm{H} \quad 0.171815$

$14 \mathrm{H} \quad 0.185786$

$\begin{array}{lll}15 \mathrm{H} & 0.207842\end{array}$

$\begin{array}{lll}16 & \mathrm{C} & -0.350811\end{array}$

$\begin{array}{lll}17 & \mathrm{H} & 0.193807\end{array}$

$18 \mathrm{H} \quad 0.171751$

19 C 0.541318

$20 \mathrm{C} \quad 0.290575$

$21 \mathrm{H} \quad 0.161812$

22 C -0.284154

$23 \mathrm{H} \quad 0.225538$

$24 \mathrm{O}-0.555602$

$25 \mathrm{~F}-0.405020$

Sum of Mulliken charges $=0.00000$

Atomic charges with hydrogens summed into heavy atoms: 1

$1 \mathrm{H} \quad 0.000000$

2 C -0.020722

3 C -0.052430

4 C -0.009272

$\begin{array}{lll}5 \mathrm{C} & 0.047579\end{array}$

6 C 0.017082

$7 \mathrm{C} \quad 0.028548$

$8 \mathrm{H} \quad 0.000000$

$9 \mathrm{H} \quad 0.000000$

$10 \mathrm{H} \quad 0.000000$ 
$\begin{array}{rrr}11 & \mathrm{H} & 0.000000 \\ 12 & \mathrm{H} & 0.000000 \\ 13 & \mathrm{H} & 0.000000 \\ 14 & \mathrm{H} & 0.000000 \\ 15 & \mathrm{H} & 0.000000 \\ 16 & \mathrm{C} & 0.014748 \\ 17 & \mathrm{H} & 0.000000 \\ 18 & \mathrm{H} & 0.000000 \\ 19 & \mathrm{C} & 0.541318 \\ 20 & \mathrm{C} & 0.452387 \\ 21 & \mathrm{H} & 0.000000 \\ 22 & \mathrm{C} & -0.058616 \\ 23 & \mathrm{H} & 0.000000 \\ 24 & \mathrm{O} & -0.555602 \\ 25 & \mathrm{~F} & -0.405020\end{array}$

Sum of Mulliken charges $=0.00000$

Electronic spatial extent $(\mathrm{au}):<\mathrm{R} * * 2>=1533.8775$

Charge $=0.0000$ electrons

Dipole moment (field-independent basis, Debye):

$\mathrm{X}=4.5125 \mathrm{Y}=-1.7776 \mathrm{Z}=0.4576$ Tot $=4.8715$

Quadrupole moment (field-independent basis, Debye-Ang):

$\mathrm{XX}=-79.9740 \quad \mathrm{YY}=-75.1333 \quad \mathrm{ZZ}=-67.6301$

$\mathrm{XY}=3.6647 \quad \mathrm{XZ}=-0.7770 \quad \mathrm{YZ}=-0.8888$

Traceless Quadrupole moment (field-independent basis, Debye-Ang):

$\mathrm{XX}=-5.7282 \mathrm{YY}=-0.8875 \mathrm{ZZ}=6.6157$

$\mathrm{XY}=3.6647 \quad \mathrm{XZ}=-0.7770 \quad \mathrm{YZ}=-0.8888$

Octapole moment (field-independent basis, Debye-Ang**2):

$\mathrm{XXX}=2.6084 \mathrm{YYY}=-16.1337 \mathrm{ZZZ}=-0.1285 \mathrm{XYY}=10.8578$

$\mathrm{XXY}=-11.3136 \mathrm{XXZ}=-0.1699 \mathrm{XZZ}=-8.9348 \mathrm{YZZ}=-1.7016$

$\mathrm{YYZ}=1.5409 \mathrm{XYZ}=1.2159$

Hexadecapole moment (field-independent basis, Debye-Ang**3):

$X X X X=-800.6273 \mathrm{YYYY}=-665.6466 \mathrm{ZZZZ}=-511.5169 \mathrm{XXXY}=28.6944$

$\mathrm{XXXZ}=-0.1361 \mathrm{YYYX}=31.4961 \mathrm{YYYZ}=0.4460 \mathrm{ZZZX}=-2.5681$

$\mathrm{ZZZY}=-2.5367 \mathrm{XXYY}=-277.2938 \mathrm{XXZZ}=-205.9989 \mathrm{YYZZ}=-188.3219$

$\mathrm{XXYZ}=-0.8106 \mathrm{YYXZ}=-0.2198 \mathrm{ZZXY}=3.2244$

$\mathrm{N}-\mathrm{N}=7.895126009488 \mathrm{D}+02 \mathrm{E}-\mathrm{N}=-2.893322424689 \mathrm{D}+03 \quad \mathrm{KE}=5.598788621971 \mathrm{D}+02$

1|1|UNPC-UNKISPIRHF|6-31G(d)|C10H13F1O1|PCUSER|17-May-2006|0||\# RHF/6-

31G(D) GEOM=CONNECTIVITYI|Title Card Required $\| 0,1 \mid \mathrm{H}, 0,-2.14701318,0$, ,

$1.31693804|\mathrm{C}, 0,-1.27682272,0 ., 0.65389403| \mathrm{C}, 0,0 .,-1.22442246,-1.1651394$

2|C,0,0.,1.22442246,-1.16513942|C,0,0.,0.,-1.99432613|C,0,-1.2568334,1

$.2541007,-0.16825153|\mathrm{C}, 0,-1.2568334,-1.2541007,-0.16825153| \mathrm{H}, 0,-0.8838$

$5725,0 .,-2.64406371|\mathrm{H}, 0,-2.15871921,1.31718637,-0.78856706| \mathrm{H}, 0,-2.1587$

$1921,-1.31718637,-0.78856706|\mathrm{H}, 0,0 .,-2.10097106,-1.82305349| \mathrm{H}, 0,0 ., 2.1$

$0097106,-1.82305349|\mathrm{H}, 0,0.88385725,0 .,-2.64406371| \mathrm{H}, 0,-1.26263227,2.12$

$421982,0.50042273|\mathrm{H}, 0,-1.26263227,-2.12421982,0.50042273| \mathrm{C}, 0,1.2568334$

$, 1.2541007,-0.16825153 \mid \mathrm{H}, 0,1.26263227,2.12421982,0.50042273 \mathrm{lH}, 0,2.1587$

$1921,1.31718637,-0.78856706|\mathrm{C}, 0,0 ., 0 ., 1.48018292| \mathrm{C}, 0,1.2568334,-1.2541$

$007,-0.16825153|\mathrm{H}, 0,2.15871921,-1.31718637,-0.78856706| \mathrm{C}, 0,1.27682272$,

$0 ., 0.65389403|\mathrm{H}, 0,2.14701318,0 ., 1.31693804| \mathrm{O}, 0,0 ., 0 ., 2.70726517 \mathrm{~F}, 0,1$.

$26396712,-2.32451343,0.65434554||$ Version $=x 86-$ Win32-G03RevB.05IState $=1-$

$\mathrm{AlHF}=-560.550576|\mathrm{RMSD}=6.988 \mathrm{e}-006| \mathrm{Dipole}=-0.1086667,0.5714538,-1.826211$

$\mid \mathrm{PG}=\mathrm{C} 01[\mathrm{X}(\mathrm{C} 10 \mathrm{H} 13 \mathrm{~F} 1 \mathrm{O} 1)] \| @$

LIFE IS A CONTINUAL STRUGGLE AGAINST THE TENDENCY TO PRODUCE ENTROPY.

Job cpu time: 0 days 0 hours 2 minutes 59.0 seconds.

File lengths (MBytes): RWF $=32 \mathrm{Int}=0 \mathrm{D} 2 \mathrm{E}=0 \mathrm{Chk}=9 \mathrm{Scr}=1$

Normal termination of Gaussian 03 at Wed May 17 14:00:58 2006. 
Entering Link 1 = C: $\backslash$ G03W W11.exe PID= 2532.

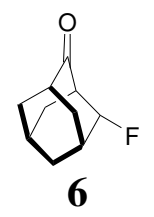

Copyright (c) 1988,1990,1992,1993,1995,1998,2003, Gaussian, Inc. All Rights Reserved.

This is the Gaussian(R) 03 program. It is based on the the Gaussian(R) 98 system (copyright 1998, Gaussian, Inc.), the Gaussian(R) 94 system (copyright 1995, Gaussian, Inc.), the Gaussian 92(TM) system (copyright 1992, Gaussian, Inc.), the Gaussian 90(TM) system (copyright 1990, Gaussian, Inc.), the Gaussian 88(TM) system (copyright 1988, Gaussian, Inc.), the Gaussian 86(TM) system (copyright 1986, Carnegie Mellon University), and the Gaussian 82(TM) system (copyright 1983, Carnegie Mellon University). Gaussian is a federally registered trademark of Gaussian, Inc.

This software contains proprietary and confidential information, including trade secrets, belonging to Gaussian, Inc.

This software is provided under written license and may be used, copied, transmitted, or stored only in accord with that written license.

The following legend is applicable only to US Government contracts under DFARS:

\section{RESTRICTED RIGHTS LEGEND}

Use, duplication or disclosure by the US Government is subject to restrictions as set forth in subparagraph (c)(1)(ii) of the Rights in Technical Data and Computer Software clause at DFARS 252.227-7013.

Gaussian, Inc.

Carnegie Office Park, Building 6, Pittsburgh, PA 15106 USA

The following legend is applicable only to US Government contracts under FAR:

\section{RESTRICTED RIGHTS LEGEND}

Use, reproduction and disclosure by the US Government is subject to restrictions as set forth in subparagraph (c) of the Commercial Computer Software - Restricted Rights clause at FAR 52.227-19.

Gaussian, Inc.

Carnegie Office Park, Building 6, Pittsburgh, PA 15106 USA

Warning -- This program may not be used in any manner that competes with the business of Gaussian, Inc. or will provide assistance to any competitor of Gaussian, Inc. The licensee of this program is prohibited from giving any competitor of Gaussian, Inc. access to this program. By using this program, the user acknowledges that Gaussian, Inc. is engaged in the business of creating and licensing software in the field of 
computational chemistry and represents and warrants to the licensee that it is not a competitor of Gaussian, Inc. and that it will not use this program in any manner prohibited above.

Cite this work as:

Gaussian 03, Revision B.05,

M. J. Frisch, G. W. Trucks, H. B. Schlegel, G. E. Scuseria,

M. A. Robb, J. R. Cheeseman, J. A. Montgomery, Jr., T. Vreven,

K. N. Kudin, J. C. Burant, J. M. Millam, S. S. Iyengar, J. Tomasi,

V. Barone, B. Mennucci, M. Cossi, G. Scalmani, N. Rega,

G. A. Petersson, H. Nakatsuji, M. Hada, M. Ehara, K. Toyota,

R. Fukuda, J. Hasegawa, M. Ishida, T. Nakajima, Y. Honda, O. Kitao,

H. Nakai, M. Klene, X. Li, J. E. Knox, H. P. Hratchian, J. B. Cross,

C. Adamo, J. Jaramillo, R. Gomperts, R. E. Stratmann, O. Yazyev,

A. J. Austin, R. Cammi, C. Pomelli, J. W. Ochterski, P. Y. Ayala,

K. Morokuma, G. A. Voth, P. Salvador, J. J. Dannenberg,

V. G. Zakrzewski, S. Dapprich, A. D. Daniels, M. C. Strain,

O. Farkas, D. K. Malick, A. D. Rabuck, K. Raghavachari,

J. B. Foresman, J. V. Ortiz, Q. Cui, A. G. Baboul, S. Clifford,

J. Cioslowski, B. B. Stefanov, G. Liu, A. Liashenko, P. Piskorz,

I. Komaromi, R. L. Martin, D. J. Fox, T. Keith, M. A. Al-Laham,

C. Y. Peng, A. Nanayakkara, M. Challacombe, P. M. W. Gill,

B. Johnson, W. Chen, M. W. Wong, C. Gonzalez, and J. A. Pople,

Gaussian, Inc., Pittsburgh PA, 2003.

$* * * * * * * * * * * * * * * * * * * * * * * * * * * * * * * * * * * * * * * * * * * * * * * *$

Gaussian 03: x86-Win32-G03RevB.05 8-Nov-2003 17-May-2006

$* * * * * * * * * * * * * * * * * * * * * * * * * * * * * * * * * * * * * * * * * * * * * * *$

$\%$ chk=C: $\backslash$ Documents and Settings $\backslash$ Nate Wilmot $\backslash$ Desktoplsterically unhinderedlfluoada $\mathrm{m} 2 . \mathrm{chk}$

$\%$ mem $=64 \mathrm{MB}$

$\%$ nproc $=1$

Will use up to 1 processors via shared memory.

\# rhf/6-31g(d) geom=connectivity

$1 / 38=1,57=2 / 1$;

$2 / 17=6,18=5,40=1 / 2$;

$3 / 5=1,6=6,7=1,11=1,16=1,25=1,30=1 / 1,2,3$;

$4 / 7=1 / 1$

$5 / 5=2,32=1,38=5 / 2$;

$6 / 7=2,8=2,9=2,10=2,28=1 / 1$;

$99 / 5=1,9=1 / 99$;

Title Card Required

Symbolic Z-matrix:

Charge $=0$ Multiplicity $=1$

$\begin{array}{lllll}\mathrm{H} & -2.47718 & 0.66866 & -0.98518\end{array}$

$\begin{array}{llll}\mathrm{C} & -1.46715 & 0.53304 & -0.58663\end{array}$

$\begin{array}{lllll}\mathrm{C} & 0.98885 & 0.39564 & -1.12304\end{array}$

$\begin{array}{llll}\text { C } & 0.23272 & 1.31948 & 1.10193\end{array}$

$\begin{array}{llll}\text { C } & 1.27499 & 1.43618 & -0.02475\end{array}$

$\begin{array}{llll}\text { C } & -1.17624 & 1.55316 & 0.52172\end{array}$

$\begin{array}{llll}\text { C } & -0.4176 & 0.64168 & -1.70182\end{array}$

$\begin{array}{llll}\mathrm{H} & 1.25169 & 2.4452 & -0.45154\end{array}$

$\begin{array}{llll}\mathrm{H} & -1.25245 & 2.5716 & 0.12284\end{array}$

$\mathrm{H} \quad \begin{array}{llll}-0.46536 & 1.63379 & -2.16633\end{array}$

$\begin{array}{llll}\mathrm{H} & 1.74575 & 0.4858 & -1.90982\end{array}$

$\begin{array}{llll}\mathrm{H} & 0.44053 & 2.0645 & 1.87892\end{array}$

$\begin{array}{llll}\mathrm{H} & 2.28242 & 1.28548 & 0.3814\end{array}$ 


$\begin{array}{lccc}\mathrm{H} & -1.92326 & 1.46261 & 1.32113 \\ \mathrm{H} & -0.62688 & -0.09426 & -2.4876 \\ \mathrm{C} & 0.29291 & -0.09528 & 1.70887 \\ \mathrm{H} & -0.45158 & -0.18415 & 2.50959 \\ \mathrm{H} & 1.27721 & -0.26561 & 2.16286 \\ \mathrm{C} & -1.33441 & -0.85675 & 0.02236 \\ \mathrm{C} & 1.0325 & -1.02 & -0.518 \\ \mathrm{C} & 0.02665 & -1.14761 & 0.62453 \\ \mathrm{H} & 0.04771 & -2.1524 & 1.05789 \\ \mathrm{O} & -2.24508 & -1.67833 & 0.00534 \\ \mathrm{H} & 0.83568 & -1.76846 & -1.29434 \\ \mathrm{~F} & 2.28412 & -1.25372 & -0.03706\end{array}$

Input orientation:

\begin{tabular}{|c|c|c|c|c|}
\hline \multirow{2}{*}{$\begin{array}{l}\text { Center } \\
\text { Number }\end{array}$} & \multirow{2}{*}{$\begin{array}{l}\text { Atomic } \\
\text { Number }\end{array}$} & \multirow{2}{*}{$\begin{array}{l}\text { Atomic } \\
\text { Type }\end{array}$} & \multicolumn{2}{|c|}{ Coordinates (Angstrom } \\
\hline & & & $\begin{array}{ll}X & Y\end{array}$ & Z \\
\hline 1 & 1 & -2.477180 & 0.668659 & -0.985179 \\
\hline 2 & 6 & -1.467147 & 0.533044 & -0.586633 \\
\hline 3 & 6 & 0.988854 & 0.395644 & -1.123036 \\
\hline 4 & 6 & 0.232719 & 1.319480 & 1.101931 \\
\hline 5 & 6 & 1.274991 & 1.436180 & -0.024747 \\
\hline 6 & 6 & -1.176239 & 1.553160 & 0.521719 \\
\hline 7 & 6 & -0.417602 & 0.641681 & -1.701818 \\
\hline 8 & 1 & 1.251692 & 2.445196 & -0.451544 \\
\hline 9 & 1 & -1.252452 & 2.571603 & 0.122837 \\
\hline 10 & 1 & -0.465357 & 1.633793 & -2.166326 \\
\hline 11 & 1 & 1.745754 & 0.485805 & -1.909825 \\
\hline 12 & 1 & 0.440528 & 2.064501 & 1.878917 \\
\hline 13 & 1 & 2.282416 & 1.285484 & 0.381401 \\
\hline 14 & 1 & -1.923262 & 1.462614 & 1.321129 \\
\hline 15 & 1 & -0.626883 & -0.094256 & -2.487597 \\
\hline 16 & 6 & 0.292906 & -0.095283 & 1.708865 \\
\hline 17 & 1 & -0.451577 & -0.184149 & 2.509587 \\
\hline 18 & 1 & 1.277210 & -0.265607 & 2.162858 \\
\hline 19 & 6 & -1.334412 & -0.856750 & 0.022360 \\
\hline 20 & 6 & 1.032499 & -1.020001 & -0.517999 \\
\hline 21 & 6 & 0.026654 & -1.147609 & 0.624525 \\
\hline 22 & 1 & 0.047705 & -2.152398 & 1.057885 \\
\hline 23 & 8 & -2.245083 & -1.678325 & 0.005338 \\
\hline 24 & 1 & 0.835680 & -1.768456 & -1.294342 \\
\hline 25 & 9 & 2.284118 & -1.253717 & -0.037057 \\
\hline
\end{tabular}

Distance matrix (angstroms):

$1 \mathrm{H} \quad 0.000000$

2 C $1.094256 \quad 0.000000$

$\begin{array}{lllll}3 & C & 3.479501 & 2.517647 & 0.000000\end{array}$

$\begin{array}{llllll}4 \mathrm{C} & 3.481831 & 2.521760 & 2.525013 & 0.000000\end{array}$

$\begin{array}{lllllll}5 & \mathrm{C} & 3.948456 & 2.941206 & 1.539749 & 1.539271 & 0.000000\end{array}$

$\begin{array}{lllllll}6 & \mathrm{C} & 2.178424 & 1.534180 & 2.955112 & 1.541562 & 2.514128\end{array}$

$\begin{array}{lllllll}7 & \mathrm{C} & 2.180862 & 1.535248 & 1.540663 & 2.956914 & 2.511706\end{array}$

$\begin{array}{lllllll}8 & \mathrm{H} & 4.164773 & 3.326659 & 2.172706 & 2.172287 & 1.095816\end{array}$

$\begin{array}{lllllll}9 \mathrm{H} & 2.519693 & 2.169139 & 3.363101 & 2.175356 & 2.774696\end{array}$

$\begin{array}{lllllll}10 \mathrm{H} & 2.524683 & 2.170406 & 2.176280 & 3.356726 & 2.766627\end{array}$

$\begin{array}{lllllll}11 & \mathrm{H} & 4.326844 & 3.475026 & 1.095475 & 3.472026 & 2.162949\end{array}$

$\begin{array}{llllllll}12 & \mathrm{H} & 4.320237 & 3.473259 & 3.478141 & 1.096334 & 2.171418\end{array}$

$\begin{array}{lllllll}13 & \mathrm{H} & 4.990167 & 3.944932 & 2.174500 & 2.172920 & 1.096618\end{array}$

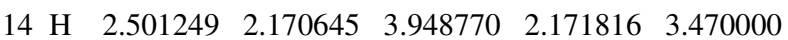

$\begin{array}{lllllll}15 \mathrm{H} & 2.502578 & 2.170994 & 2.170860 & 3.952503 & 3.467706\end{array}$

$\begin{array}{lllllll}16 & \mathrm{C} & 3.938890 & 2.960050 & 2.957197 & 1.540631 & 2.513023\end{array}$

$\begin{array}{lllllll}17 & \mathrm{H} & 4.128406 & 3.336514 & 3.950562 & 2.170403 & 3.468335\end{array}$

$\begin{array}{lllllll}18 \mathrm{H} & 4.987828 & 3.965986 & 3.364149 & 2.174633 & 2.771588\end{array}$ 
$\begin{array}{lllllll}19 & \mathrm{C} & 2.155905 & 1.523161 & 2.877148 & 2.890908 & 3.474007\end{array}$

$\begin{array}{lllllll}20 & \mathrm{C} & 3.922713 & 2.943619 & 1.540138 & 2.955841 & 2.516927\end{array}$

$\begin{array}{lllllll}21 & \mathrm{C} & 3.486999 & 2.554005 & 2.522187 & 2.521291 & 2.942085\end{array}$

$\begin{array}{lllllll}22 & \mathrm{H} & 4.302037 & 3.494398 & 3.483489 & 3.477083 & 3.944137\end{array}$

$\begin{array}{llllllll}23 & \mathrm{O} & 2.557993 & 2.417802 & 4.004113 & 4.040897 & 4.700209\end{array}$

$\begin{array}{lllllll}24 & \mathrm{H} & 4.124336 & 3.331781 & 2.176266 & 3.954875 & 3.474845\end{array}$

$\begin{array}{lllllll}25 & F & 5.221534 & 4.191246 & 2.361663 & 3.482366 & 2.872983\end{array}$

$\begin{array}{lll}6 \mathrm{C} & 0.000000\end{array}$

7 C $2.520008 \quad 0.000000$

$\begin{array}{lllll}8 \mathrm{H} & 2.763660 & 2.757244 & 0.000000\end{array}$

$\begin{array}{llllll}9 & \mathrm{H} & 1.096422 & 2.784051 & 2.572281 & 0.000000\end{array}$

$\begin{array}{lllllll}10 \mathrm{H} & 2.781626 & 1.096510 & 2.558732 & 2.596011 & 0.000000\end{array}$

$\begin{array}{lllllll}11 & \mathrm{H} & 3.948379 & 2.178916 & 2.491966 & 4.179894 & 2.504532\end{array}$

$\begin{array}{lllllll}12 & \mathrm{H} & 2.171956 & 3.947464 & 2.496791 & 2.491416 & 4.167749\end{array}$

$\begin{array}{lllllll}13 & \mathrm{H} & 3.471835 & 3.470502 & 1.761000 & 3.770445 & 3.763309\end{array}$

$\begin{array}{lllllll}14 & \mathrm{H} & 1.097861 & 3.475507 & 3.766718 & 1.765147 & 3.783799\end{array}$

$\begin{array}{lllllll}15 & \mathrm{H} & 3.474443 & 1.096746 & 3.758108 & 3.783188 & 1.765066\end{array}$

$\begin{array}{llllllll}16 & \mathrm{C} & 2.507004 & 3.560997 & 3.469968 & 3.466396 & 4.310659\end{array}$

$\begin{array}{llllllll}17 & \mathrm{H} & 2.737699 & 4.291746 & 4.310786 & 3.732579 & 5.016898\end{array}$

$\begin{array}{llllllll}18 & \mathrm{H} & 3.467083 & 4.316397 & 3.766193 & 4.314005 & 5.038462\end{array}$

$\begin{array}{lllllll}19 & \mathrm{C} & 2.466180 & 2.461428 & 4.220825 & 3.430804 & 3.427595\end{array}$

$\begin{array}{lllllll}20 \mathrm{C} & 3.546928 & 2.503080 & 3.472759 & 4.304799 & 3.464560\end{array}$

$\begin{array}{lllllll}21 & \mathrm{C} & 2.958323 & 2.968299 & 3.945491 & 3.964889 & 3.970784\end{array}$

$\begin{array}{llllllll}22 & \mathrm{H} & 3.939121 & 3.954663 & 4.986566 & 4.988077 & 4.999402\end{array}$

$\begin{array}{llllllll}23 & \mathrm{O} & 3.442611 & 3.411231 & 5.425827 & 4.365891 & 4.342082\end{array}$

$\begin{array}{lllllll}24 & \mathrm{H} & 4.287077 & 2.746910 & 4.317202 & 5.020438 & 3.745444\end{array}$

$\begin{array}{lllllll}25 & \mathrm{~F} & 4.490530 & 3.696384 & 3.862598 & 5.212098 & 4.520079\end{array}$ $\begin{array}{lllll}11 & 12 & 13 & 14 & 15\end{array}$

$\begin{array}{lll}11 \mathrm{H} & 0.000000\end{array}$

$\begin{array}{llll}12 & \mathrm{H} & 4.307025 & 0.000000\end{array}$

$\begin{array}{lllll}13 & \mathrm{H} & 2.485399 & 2.498394 & 0.000000\end{array}$

$\begin{array}{llllll}14 \mathrm{H} & 4.985469 & 2.502179 & 4.313026 & 0.000000\end{array}$

$\begin{array}{lllllll}15 \mathrm{H} & 2.509920 & 4.986587 & 4.312639 & 4.314028 & 0.000000\end{array}$

$\begin{array}{lllllll}16 & \mathrm{C} & 3.942506 & 2.171492 & 2.761672 & 2.736564 & 4.296081\end{array}$

$\begin{array}{lllllll}17 & \mathrm{H} & 4.980793 & 2.500004 & 3.763471 & 2.508011 & 5.001066\end{array}$

$\begin{array}{llllllll}18 \mathrm{H} & 4.167841 & 2.492000 & 2.567083 & 3.733400 & 5.028088\end{array}$

$\begin{array}{lllllll}19 & \mathrm{C} & 3.875979 & 3.889849 & 4.218948 & 2.722681 & 2.716962\end{array}$

$\begin{array}{lllllll}20 \mathrm{C} & 2.171028 & 3.950923 & 2.772450 & 4.275780 & 2.736763\end{array}$

$\begin{array}{llllllll}21 & \mathrm{C} & 3.470775 & 3.473103 & 3.326788 & 3.331770 & 3.349921\end{array}$

$\begin{array}{lllllll}22 & \mathrm{H} & 4.318656 & 4.314006 & 4.155792 & 4.125812 & 4.154692\end{array}$

$\begin{array}{lllllll}23 & \mathrm{O} & 4.927280 & 4.973083 & 5.424374 & 3.420581 & 3.367874\end{array}$

$\begin{array}{lllllll}24 & \mathrm{H} & 2.507737 & 4.991721 & 3.771963 & 4.989215 & 2.523072\end{array}$

$\begin{array}{lllllll}25 & \text { F } & 2.612093 & 4.252099 & 2.573451 & 5.188947 & 3.977867\end{array}$

$16 \mathrm{C} \quad 0.000000$

$\begin{array}{llll}17 & \mathrm{H} & 1.096953 & 0.000000\end{array}$

$\begin{array}{lllll}18 & \mathrm{H} & 1.097258 & 1.765094 & 0.000000\end{array}$

$\begin{array}{llllll}19 & \mathrm{C} & 2.464202 & 2.723616 & 3.428082 & 0.000000\end{array}$

$\begin{array}{lllllll}20 \mathrm{C} & 2.522107 & 3.473817 & 2.795709 & 2.433291 & 0.000000\end{array}$

$\begin{array}{lllllll}21 & \mathrm{C} & 1.534299 & 2.170349 & 2.169858 & 1.516477 & 1.527537\end{array}$

$\begin{array}{lllllll}22 & \mathrm{H} & 2.171548 & 2.496142 & 2.508511 & 2.158996 & 2.176133\end{array}$

$\begin{array}{llllllll}23 & \mathrm{O} & 3.442297 & 3.423520 & 4.365456 & 1.226620 & 3.383758\end{array}$

$\begin{array}{lllllll}24 & \mathrm{H} & 3.480426 & 4.317051 & 3.795489 & 2.697075 & 1.096189\end{array}$

$\begin{array}{llllllll}25 & \text { F } & 2.890526 & 3.887595 & 2.613398 & 3.640724 & 1.361058\end{array}$

$21 \mathrm{C} \quad 0.000000$ $\begin{array}{lllll}21 & 22 & 23 & 24 & 25\end{array}$

$\begin{array}{llll}22 & \mathrm{H} & 1.094461 & 0.000000\end{array}$

$\begin{array}{lllll}23 & \mathrm{O} & 2.413677 & 2.566998 & 0.000000\end{array}$

$\begin{array}{llllll}24 & \mathrm{H} & 2.173022 & 2.510237 & 3.344906 & 0.000000\end{array}$

$\begin{array}{lllllll}25 \mathrm{~F} & 2.354803 & 2.647276 & 4.549258 & 1.985874 & 0.000000\end{array}$

Stoichiometry C10H13FO

Framework group $\mathrm{C} 1[\mathrm{X}(\mathrm{C} 10 \mathrm{H} 13 \mathrm{FO})]$

Deg. of freedom 69 


\begin{tabular}{|c|c|c|c|c|c|}
\hline \multicolumn{6}{|c|}{$\begin{array}{l}\text { Full point group } \quad \mathrm{C} 1 \mathrm{NOp} \\
\text { Largest Abelian subgroup } \mathrm{C} 1 \\
\text { Largest concise Abelian subgroup C1 } \\
\text { Standard orientation: }\end{array}$} \\
\hline \multirow{2}{*}{$\begin{array}{l}\text { Center } \\
\text { Number }\end{array}$} & \multirow{2}{*}{\multicolumn{2}{|c|}{$\begin{array}{l}\text { Atomic } \\
\text { Number }\end{array}$}} & \multirow{2}{*}{$\begin{array}{l}\text { Atomic } \\
\text { Type }\end{array}$} & \multicolumn{2}{|c|}{ Coordinates (Angstroms) } \\
\hline & & & & X $\quad \mathrm{Y}$ & $\mathrm{Z}$ \\
\hline 1 & 1 & 0 & -2.477180 & 0.668659 & -0.985179 \\
\hline 2 & 6 & 0 & -1.467147 & 0.533044 & -0.586633 \\
\hline 3 & 6 & 0 & 0.988854 & 0.395644 & -1.123036 \\
\hline 4 & 6 & 0 & 0.232719 & 1.319480 & 1.101931 \\
\hline 5 & 6 & 0 & 1.274991 & 1.436180 & -0.024747 \\
\hline 6 & 6 & 0 & -1.176239 & 1.553160 & 0.521719 \\
\hline 7 & 6 & 0 & -0.417602 & 0.641681 & -1.701818 \\
\hline 8 & 1 & U & 1.251692 & 2.445196 & -0.451544 \\
\hline 9 & 1 & U & -1.252452 & 2.571603 & 0.122837 \\
\hline 10 & 1 & 0 & -0.465357 & 1.633793 & -2.166326 \\
\hline 11 & 1 & 0 & 1.745754 & 0.485805 & -1.909825 \\
\hline 12 & 1 & 0 & 0.440528 & 2.064501 & 1.878917 \\
\hline 13 & 1 & & 2.282416 & 1.285484 & 0.381401 \\
\hline 14 & 1 & & -1.923262 & 1.462614 & 1.321129 \\
\hline 15 & 1 & 0 & -0.626883 & -0.094256 & -2.487597 \\
\hline 16 & 6 & 0 & 0.292906 & -0.095283 & 1.708865 \\
\hline 17 & 1 & & -0.451577 & -0.184149 & 2.509587 \\
\hline 18 & 1 & & 1.277210 & -0.265607 & 2.162858 \\
\hline 19 & 6 & & -1.334412 & -0.856750 & 0.022360 \\
\hline 20 & 6 & 0 & 1.032499 & -1.020001 & -0.517999 \\
\hline 21 & 6 & U & 0.026654 & -1.147609 & 0.624525 \\
\hline 22 & 1 & U & 0.047705 & -2.152398 & 1.057885 \\
\hline 23 & 8 & & -2.245083 & -1.678325 & 0.005338 \\
\hline 24 & 1 & 0 & 0.835680 & -1.768456 & -1.294342 \\
\hline 25 & 9 & 0 & 2.284118 & -1.253717 & -0.037057 \\
\hline
\end{tabular}

Rotational constants (GHZ): $1.3349971 \quad 1.0842292 \quad 0.9278968$

Standard basis: 6-31G(d) $(6 \mathrm{D}, 7 \mathrm{~F})$

There are 206 symmetry adapted basis functions of A symmetry.

Integral buffers will be 262144 words long.

Raffenetti 1 integral format.

Two-electron integral symmetry is turned on.

206 basis functions, 388 primitive gaussians, 206 cartesian basis functions

45 alpha electrons 45 beta electrons nuclear repulsion energy $\quad 786.6968982059$ Hartrees.

NAtoms $=25$ NActive $=25$ NUniq $=25 \mathrm{SFac}=1.00 \mathrm{D}+00 \mathrm{NAtFMM}=60 \mathrm{Big}=\mathrm{F}$

One-electron integrals computed using PRISM.

NBasis $=206$ RedAO $=\mathrm{T}$ NBF $=206$

NBsUse $=206$ 1.00D-06 NBFU $=206$

Harris functional with IExCor $=205$ diagonalized for initial guess.

HarFok: IExCor $=205$ AccDes= 1.00D-06 IRadAn $=\quad 1 \mathrm{IDoV}=1$

ScaDFX $=1.0000001 .0000001 .0000001 .000000$

Initial guess orbital symmetries:

Occupied (A) (A) (A) (A) (A) (A) (A) (A) (A) (A) (A) (A)

(A) (A) (A) (A) (A) (A) (A) (A) (A) (A) (A) (A)

(A) $(\mathrm{A})(\mathrm{A})(\mathrm{A})(\mathrm{A})(\mathrm{A})(\mathrm{A})(\mathrm{A})(\mathrm{A})(\mathrm{A})(\mathrm{A})(\mathrm{A})$

(A) (A) (A) (A) (A) (A) (A) (A) (A)

Virtual (A) (A) (A) (A) (A) (A) (A) (A) (A) (A) (A) (A)

(A) $(\mathrm{A})(\mathrm{A})(\mathrm{A})(\mathrm{A})(\mathrm{A})(\mathrm{A})(\mathrm{A})(\mathrm{A})(\mathrm{A})(\mathrm{A})(\mathrm{A})$

(A) (A) (A) (A) (A) (A) (A) (A) (A) (A) (A) (A)

(A) $(\mathrm{A})(\mathrm{A})(\mathrm{A})(\mathrm{A})(\mathrm{A})(\mathrm{A})(\mathrm{A})(\mathrm{A})(\mathrm{A})(\mathrm{A})(\mathrm{A})$

(A) $(A)(A)(A)(A)(A)(A)(A)(A)(A)(A)(A)$

(A) (A) (A) (A) (A) (A) (A) (A) (A) (A) (A) (A)

(A) (A) (A) (A) (A) (A) (A) (A) (A) (A) (A) (A)

(A) $(\mathrm{A})(\mathrm{A})(\mathrm{A})(\mathrm{A})(\mathrm{A})(\mathrm{A})(\mathrm{A})(\mathrm{A})(\mathrm{A})(\mathrm{A})(\mathrm{A})$ 
(A) $(\mathrm{A})(\mathrm{A})(\mathrm{A})(\mathrm{A})(\mathrm{A})(\mathrm{A})(\mathrm{A})(\mathrm{A})(\mathrm{A})(\mathrm{A})(\mathrm{A})$

(A) (A) (A) (A) (A) (A) (A) (A) (A) (A) (A) (A)

(A) $(A)(A)(A)(A)(A)(A)(A)(A)(A)(A)(A)$

(A) (A) (A) (A) (A) (A) (A) (A) (A) (A) (A) (A)

(A) $(\mathrm{A})(\mathrm{A})(\mathrm{A})(\mathrm{A})(\mathrm{A})(\mathrm{A})(\mathrm{A})(\mathrm{A})(\mathrm{A})(\mathrm{A})(\mathrm{A})$

(A) (A) (A) (A) (A)

The electronic state of the initial guess is 1-A.

Warning! Cutoffs for single-point calculations used.

Requested convergence on RMS density matrix=1.00D-04 within 128 cycles.

Requested convergence on MAX density matrix $=1.00 \mathrm{D}-02$.

Requested convergence on energy $=5.00 \mathrm{D}-05$.

No special actions if energy rises.

SCF Done: $\mathrm{E}(\mathrm{RHF})=-560.574314233$ A.U. after 7 cycles

Convg $=0.6817 \mathrm{D}-05 \quad-\mathrm{V} / \mathrm{T}=2.0014$

$\mathrm{S} * * 2=0.0000$

$* * * * * * * * * * * * * * * * * * * * * * * * * * * * * * * * * * * * * * * * * * * * * * * * * * * * * * * * * * * * * * * * * * * * * * *$

Population analysis using the SCF density.

******************************************************************************

Orbital symmetries:

Occupied (A) (A) (A) (A) (A) (A) (A) (A) (A) (A) (A) (A)

(A) $(A)(A)(A)(A)(A)(A)(A)(A)(A)(A)(A)$

(A) $(\mathrm{A})(\mathrm{A})(\mathrm{A})(\mathrm{A})(\mathrm{A})(\mathrm{A})(\mathrm{A})(\mathrm{A})(\mathrm{A})(\mathrm{A})(\mathrm{A})$

$(\mathrm{A})(\mathrm{A})(\mathrm{A})(\mathrm{A})(\mathrm{A})(\mathrm{A})(\mathrm{A})(\mathrm{A})(\mathrm{A})$

Virtual (A) (A) (A) (A) (A) (A) (A) (A) (A) (A) (A) (A)

(A) $(\mathrm{A})(\mathrm{A})(\mathrm{A})(\mathrm{A})(\mathrm{A})(\mathrm{A})(\mathrm{A})(\mathrm{A})(\mathrm{A})(\mathrm{A})(\mathrm{A})$

(A) $(\mathrm{A})(\mathrm{A})(\mathrm{A})(\mathrm{A})(\mathrm{A})(\mathrm{A})(\mathrm{A})(\mathrm{A})(\mathrm{A})(\mathrm{A})(\mathrm{A})$

(A) $(A)(A)(A)(A)(A)(A)(A)(A)(A)(A)(A)$

(A) (A) (A) (A) (A) (A) (A) (A) (A) (A) (A) (A)

(A) $(\mathrm{A})(\mathrm{A})(\mathrm{A})(\mathrm{A})(\mathrm{A})(\mathrm{A})(\mathrm{A})(\mathrm{A})(\mathrm{A})(\mathrm{A})(\mathrm{A})$

(A) $(\mathrm{A})(\mathrm{A})(\mathrm{A})(\mathrm{A})(\mathrm{A})(\mathrm{A})(\mathrm{A})(\mathrm{A})(\mathrm{A})(\mathrm{A})(\mathrm{A})$

(A) $(\mathrm{A})(\mathrm{A})(\mathrm{A})(\mathrm{A})(\mathrm{A})(\mathrm{A})(\mathrm{A})(\mathrm{A})(\mathrm{A})(\mathrm{A})(\mathrm{A})$

(A) $(\mathrm{A})(\mathrm{A})(\mathrm{A})(\mathrm{A})(\mathrm{A})(\mathrm{A})(\mathrm{A})(\mathrm{A})(\mathrm{A})(\mathrm{A})(\mathrm{A})$

(A) $(A)(A)(A)(A)(A)(A)(A)(A)(A)(A)(A)$

(A) $(\mathrm{A})(\mathrm{A})(\mathrm{A})(\mathrm{A})(\mathrm{A})(\mathrm{A})(\mathrm{A})(\mathrm{A})(\mathrm{A})(\mathrm{A})(\mathrm{A})$

(A) $(\mathrm{A})(\mathrm{A})(\mathrm{A})(\mathrm{A})(\mathrm{A})(\mathrm{A})(\mathrm{A})(\mathrm{A})(\mathrm{A})(\mathrm{A})(\mathrm{A})$

(A) $(\mathrm{A})(\mathrm{A})(\mathrm{A})(\mathrm{A})(\mathrm{A})(\mathrm{A})(\mathrm{A})(\mathrm{A})(\mathrm{A})(\mathrm{A})(\mathrm{A})$

(A) $(\mathrm{A})(\mathrm{A})(\mathrm{A})(\mathrm{A})$

The electronic state is $1-\mathrm{A}$.

Alpha occ. eigenvalues -- -26.28510 -20.55411 -11.32923 -11.31887 -11.24368

Alpha occ. eigenvalues -- -11.24321 -11.24304 -11.24225 -11.23987 -11.23688

Alpha occ. eigenvalues -- -11.23629 -11.23011 -1.60163 -1.37515 -1.18276

Alpha occ. eigenvalues -- $-1.06736-1.05888-1.04006 \quad-0.92949-0.89202$

Alpha occ. eigenvalues -- $\quad-0.83408 \quad-0.82438 \quad-0.79476 \quad-0.72905 \quad-0.69493$

Alpha occ. eigenvalues -- $\quad-0.68058 \quad-0.67602 \quad-0.65568 \quad-0.63594 \quad-0.61332$

Alpha occ. eigenvalues -- $-0.60569-0.58505 \quad-0.57542 \quad-0.56928 \quad-0.56604$

Alpha occ. eigenvalues -- $-0.54719 \quad-0.53596 \quad-0.51332 \quad-0.49221 \quad-0.48417$

Alpha occ. eigenvalues -- $-0.47585 \quad-0.44084 \quad-0.43379 \quad-0.43029 \quad-0.39366$

$\begin{array}{lllllll}\text { Alpha virt. eigenvalues -- } & 0.14977 & 0.20205 & 0.25319 & 0.26270 & 0.27054\end{array}$

$\begin{array}{lllllll}\text { Alpha virt. eigenvalues -- } & 0.27485 & 0.28064 & 0.28977 & 0.29124 & 0.31012\end{array}$

$\begin{array}{lllllll}\text { Alpha virt. eigenvalues -- } & 0.31773 & 0.31988 & 0.34363 & 0.34754 & 0.35754\end{array}$

$\begin{array}{lllllll}\text { Alpha virt. eigenvalues -- } & 0.37799 & 0.38478 & 0.39656 & 0.40130 & 0.41102\end{array}$

$\begin{array}{lllllll}\text { Alpha virt. eigenvalues -- } & 0.42156 & 0.43586 & 0.44365 & 0.44995 & 0.48420\end{array}$

$\begin{array}{lllllll}\text { Alpha virt. eigenvalues -- } & 0.51769 & 0.54346 & 0.56198 & 0.70792 & 0.71784\end{array}$

$\begin{array}{lllllll}\text { Alpha virt. eigenvalues -- } & 0.72353 & 0.74171 & 0.75414 & 0.80166 & 0.81185\end{array}$

$\begin{array}{lllllll}\text { Alpha virt. eigenvalues -- } & 0.82885 & 0.85565 & 0.86647 & 0.88099 & 0.89537\end{array}$

$\begin{array}{lllllll}\text { Alpha virt. eigenvalues -- } & 0.89835 & 0.91026 & 0.93542 & 0.94777 & 0.97071\end{array}$

$\begin{array}{lllllll}\text { Alpha virt. eigenvalues -- } & 0.97204 & 0.99432 & 1.01195 & 1.01722 & 1.02906\end{array}$

$\begin{array}{lllllll}\text { Alpha virt. eigenvalues -- } & 1.04381 & 1.05467 & 1.07477 & 1.09846 & 1.12311\end{array}$

$\begin{array}{lllllll}\text { Alpha virt. eigenvalues -- } & 1.12578 & 1.12932 & 1.13354 & 1.13926 & 1.14190\end{array}$

$\begin{array}{lllllll}\text { Alpha virt. eigenvalues -- } & 1.17057 & 1.18748 & 1.19146 & 1.21115 & 1.21495\end{array}$ 
$\begin{array}{lllllll}\text { Alpha virt. eigenvalues -- } & 1.23160 & 1.24392 & 1.25841 & 1.32458 & 1.36285\end{array}$

$\begin{array}{lllllll}\text { Alpha virt. eigenvalues -- } & 1.38308 & 1.41125 & 1.42638 & 1.43517 & 1.44236\end{array}$

$\begin{array}{lllllll}\text { Alpha virt. eigenvalues -- } & 1.46722 & 1.48783 & 1.53188 & 1.56420 & 1.64735\end{array}$

$\begin{array}{lllllll}\text { Alpha virt. eigenvalues -- } & 1.65245 & 1.68025 & 1.70455 & 1.73100 & 1.77512\end{array}$

$\begin{array}{lllllll}\text { Alpha virt. eigenvalues -- } & 1.84091 & 1.85522 & 1.87985 & 1.89790 & 1.92077\end{array}$

$\begin{array}{lllllll}\text { Alpha virt. eigenvalues -- } & 1.93895 & 1.96494 & 1.97907 & 1.98967 & 2.04122\end{array}$

$\begin{array}{llllll}\text { Alpha virt. eigenvalues -- } & 2.06561 & 2.06794 & 2.10040 & 2.11940 & 2.12705\end{array}$

$\begin{array}{lllllll}\text { Alpha virt. eigenvalues -- } & 2.14088 & 2.17141 & 2.19691 & 2.21818 & 2.22637\end{array}$

$\begin{array}{llllll}\text { Alpha virt. eigenvalues -- } & 2.23782 & 2.28802 & 2.30589 & 2.35309 & 2.35613\end{array}$

$\begin{array}{llllll}\text { Alpha virt. eigenvalues -- } & 2.37228 & 2.38353 & 2.39533 & 2.41028 & 2.42165\end{array}$

$\begin{array}{lllllll}\text { Alpha virt. eigenvalues -- } & 2.47128 & 2.48317 & 2.49704 & 2.53062 & 2.55937\end{array}$

$\begin{array}{lllllll}\text { Alpha virt. eigenvalues -- } & 2.57986 & 2.60966 & 2.61507 & 2.62667 & 2.65659\end{array}$

$\begin{array}{llllll}\text { Alpha virt. eigenvalues -- } & 2.69767 & 2.72017 & 2.74236 & 2.74291 & 2.76126\end{array}$

$\begin{array}{lllllll}\text { Alpha virt. eigenvalues -- } & 2.78374 & 2.83224 & 2.86739 & 2.88527 & 2.92377\end{array}$

$\begin{array}{lllllll}\text { Alpha virt. eigenvalues -- } & 2.98516 & 2.98885 & 3.04090 & 3.05610 & 3.06704\end{array}$

$\begin{array}{llllll}\text { Alpha virt. eigenvalues -- } & 3.07594 & 3.08221 & 3.12398 & 3.14619 & 3.17020\end{array}$

$\begin{array}{lllllll}\text { Alpha virt. eigenvalues -- } & 3.20710 & 3.37827 & 3.38463 & 3.54186 & 4.49939\end{array}$

$\begin{array}{llllllll}\text { Alpha virt. eigenvalues -- } & 4.56171 & 4.69672 & 4.72152 & 4.74657 & 4.94599\end{array}$

$\begin{array}{llllll}\text { Alpha virt. eigenvalues -- } & 4.99555 & 5.12044 & 5.14493 & 5.22476 & 5.27197\end{array}$

Alpha virt. eigenvalues -- 5.39651

Condensed to atoms (all electrons):

$$
\begin{array}{llllll}
1 & 2 & 3 & 4 & 5 & 6
\end{array}
$$

$\begin{array}{llllllll}1 & \mathrm{H} & 0.474872 & 0.400859 & 0.003623 & 0.003686 & -0.000309 & -0.029010\end{array}$

$\begin{array}{llllllll}2 & \mathrm{C} & 0.400859 & 5.309577 & -0.055139 & -0.049463 & -0.000526 & 0.307663\end{array}$

$\begin{array}{llllllll}3 & C & 0.003623 & -0.055139 & 5.258138 & -0.041351 & 0.313744 & -0.002293\end{array}$

$\begin{array}{llllllll}4 & C & 0.003686 & -0.049463 & -0.041351 & 5.154879 & 0.329654 & 0.333680\end{array}$

$\begin{array}{llllllll}5 & \mathrm{C} & -0.000309 & -0.000526 & 0.313744 & 0.329654 & 5.192523 & -0.068451\end{array}$

$\begin{array}{llllllll}6 & C & -0.029010 & 0.307663 & -0.002293 & 0.333680 & -0.068451 & 5.205789\end{array}$

$\begin{array}{llllllll}7 & \mathrm{C} & -0.029136 & 0.312379 & 0.300283 & -0.005443 & -0.061411 & -0.060279\end{array}$

$\begin{array}{llllllll}8 \mathrm{H} & -0.000057 & -0.000116 & -0.040126 & -0.039532 & 0.401220 & -0.003147\end{array}$

$\begin{array}{llllllll}9 \mathrm{H} & -0.003173 & -0.037033 & 0.000389 & -0.037423 & -0.002801 & 0.396480\end{array}$

$\begin{array}{llllllll}10 \mathrm{H} & -0.002940 & -0.036673 & -0.032261 & 0.000541 & -0.003288 & -0.003905\end{array}$

$\begin{array}{llllllll}11 \mathrm{H} & -0.000056 & 0.004220 & 0.405776 & 0.004275 & -0.037945 & -0.000428\end{array}$

$\begin{array}{llllllll}12 \mathrm{H} & -0.000062 & 0.004440 & 0.004154 & 0.410771 & -0.036443 & -0.035676\end{array}$

$\begin{array}{llllllll}13 \mathrm{H} & 0.000010 & -0.000578 & -0.037512 & -0.037997 & 0.400464 & 0.004587\end{array}$

$\begin{array}{lllllll}14 \mathrm{H} & -0.002016 & -0.043053 & -0.000549 & -0.039843 & 0.004755 & 0.401587\end{array}$

$\begin{array}{llllllll}15 \mathrm{H} & -0.002015 & -0.043470 & -0.041806 & -0.000544 & 0.004806 & 0.004790\end{array}$

$\begin{array}{llllllll}16 & \mathrm{C} & -0.000426 & 0.004194 & -0.013510 & 0.329466 & -0.059979 & -0.072852\end{array}$

$\begin{array}{llllllll}17 & \mathrm{H} & -0.000054 & 0.001285 & -0.000583 & -0.039848 & 0.004778 & -0.004155\end{array}$

$\begin{array}{llllllll}18 & \mathrm{H} & 0.000012 & -0.000727 & 0.000976 & -0.036256 & -0.002356 & 0.004750\end{array}$

$\begin{array}{llllllll}19 & \mathrm{C} & -0.034648 & 0.331785 & 0.002242 & -0.006272 & -0.001345 & -0.055450\end{array}$

$\begin{array}{llllllll}20 & \mathrm{C} & -0.000246 & 0.000634 & 0.348133 & -0.004152 & -0.055000 & -0.000408\end{array}$

$\begin{array}{llllllll}21 & \mathrm{C} & 0.003751 & -0.103602 & -0.055617 & -0.045577 & -0.009318 & 0.005161\end{array}$

$\begin{array}{llllllll}22 & \mathrm{H} & -0.000076 & 0.003550 & 0.004097 & 0.003914 & -0.000477 & -0.000437\end{array}$

$\begin{array}{llllllll}23 & \mathrm{O} & 0.003715 & -0.063409 & 0.000193 & 0.000196 & -0.000050 & 0.001002\end{array}$

$\begin{array}{llllllll}24 & \mathrm{H} & -0.000039 & 0.001892 & -0.053378 & -0.000832 & 0.005357 & -0.000060\end{array}$

$\begin{array}{llllllll}25 & \mathrm{~F} & 0.000001 & -0.000024 & -0.035822 & -0.002524 & 0.004433 & 0.000001\end{array}$

$$
\begin{array}{llllll}
7 & 8 & 9 & 10 & 11 & 12
\end{array}
$$

$\begin{array}{llllllll}1 & \mathrm{H} & -0.029136 & -0.000057 & -0.003173 & -0.002940 & -0.000056 & -0.000062\end{array}$

$\begin{array}{llllllll}2 \mathrm{C} & 0.312379 & -0.000116 & -0.037033 & -0.036673 & 0.004220 & 0.004440\end{array}$

$\begin{array}{llllllll}3 & \mathrm{C} & 0.300283 & -0.040126 & 0.000389 & -0.032261 & 0.405776 & 0.004154\end{array}$

$\begin{array}{llllllll}4 & \mathrm{C} & -0.005443 & -0.039532 & -0.037423 & 0.000541 & 0.004275 & 0.410771\end{array}$

$\begin{array}{llllllll}5 & \mathrm{C} & -0.061411 & 0.401220 & -0.002801 & -0.003288 & -0.037945 & -0.036443\end{array}$

$\begin{array}{llllllll}6 & \mathrm{C} & -0.060279 & -0.003147 & 0.396480 & -0.003905 & -0.000428 & -0.035676\end{array}$

$\begin{array}{llllllll}7 & \mathrm{C} & 5.229452 & -0.003543 & -0.004189 & 0.395154 & -0.031350 & -0.000398\end{array}$

$\begin{array}{llllllll}8 & \mathrm{H} & -0.003543 & 0.533929 & 0.002508 & 0.002479 & -0.002718 & -0.002591\end{array}$

$\begin{array}{llllllll}9 \mathrm{H} & -0.004189 & 0.002508 & 0.526129 & 0.003109 & -0.000054 & -0.002541\end{array}$

$\begin{array}{lllllllll}10 \mathrm{H} & 0.395154 & 0.002479 & 0.003109 & 0.513155 & -0.002590 & -0.000060\end{array}$

$\begin{array}{llllllll}11 \mathrm{H} & -0.031350 & -0.002718 & -0.000054 & -0.002590 & 0.504690 & -0.000075\end{array}$

$\begin{array}{llllllll}12 \mathrm{H} & -0.000398 & -0.002591 & -0.002541 & -0.000060 & -0.000075 & 0.520249\end{array}$

$\begin{array}{llllllll}13 \mathrm{H} & 0.004879 & -0.024688 & -0.000086 & -0.000089 & -0.002149 & -0.002426\end{array}$

$\begin{array}{llllllll}14 \mathrm{H} & 0.004795 & -0.000062 & -0.023669 & -0.000072 & 0.000012 & -0.002809\end{array}$

$\begin{array}{llllllll}15 \mathrm{H} & 0.401288 & -0.000049 & -0.000079 & -0.022857 & -0.002943 & 0.000013\end{array}$

$\begin{array}{llllllll}16 & \mathrm{C} & 0.001425 & 0.004931 & 0.005002 & 0.000060 & -0.000554 & -0.035144\end{array}$ 
$\begin{array}{llllllll}17 & \mathrm{H} & 0.000016 & -0.000092 & -0.000023 & 0.000001 & 0.000012 & -0.002777\end{array}$

$\begin{array}{llllllll}18 & \mathrm{H} & 0.000003 & -0.000064 & -0.000107 & 0.000000 & -0.000061 & -0.002361\end{array}$

$\begin{array}{llllllll}19 & \mathrm{C} & -0.060713 & 0.000234 & 0.004653 & 0.004705 & -0.000549 & -0.000483\end{array}$

$\begin{array}{llllllll}20 & \mathrm{C} & -0.057613 & 0.004602 & 0.000119 & 0.003814 & -0.031412 & -0.000369\end{array}$

$\begin{array}{llllllll}21 & \mathrm{C} & 0.009427 & -0.000745 & -0.000844 & -0.000845 & 0.004871 & 0.004481\end{array}$

$\begin{array}{llllllll}22 & \mathrm{H} & -0.000460 & 0.000011 & 0.000013 & 0.000013 & -0.000063 & -0.000062\end{array}$

$\begin{array}{llllllll}23 & 0 & 0.000826 & 0.000000 & -0.000064 & -0.000061 & -0.000003 & -0.000002\end{array}$

$\begin{array}{llllllll}24 \mathrm{H} & -0.000751 & -0.000056 & 0.000003 & -0.000122 & -0.004143 & 0.000012\end{array}$

$\begin{array}{llllllll}25 & \mathrm{~F} & 0.001972 & 0.000040 & 0.000000 & -0.000026 & 0.000429 & -0.000014\end{array}$

$\begin{array}{llllll}13 & 14 & 15 & 16 & 17 & 18\end{array}$

$\begin{array}{llllllll}1 \mathrm{H} & 0.000010 & -0.002016 & -0.002015 & -0.000426 & -0.000054 & 0.000012\end{array}$

$\begin{array}{llllllll}2 & \mathrm{C} & -0.000578 & -0.043053 & -0.043470 & 0.004194 & 0.001285 & -0.000727\end{array}$

$\begin{array}{llllllll}3 & C & -0.037512 & -0.000549 & -0.041806 & -0.013510 & -0.000583 & 0.000976\end{array}$

$\begin{array}{llllllll}4 & C & -0.037997 & -0.039843 & -0.000544 & 0.329466 & -0.039848 & -0.036256\end{array}$

$\begin{array}{llllllll}5 & \mathrm{C} & 0.400464 & 0.004755 & 0.004806 & -0.059979 & 0.004778 & -0.002356\end{array}$

$\begin{array}{llllllll}6 & \mathrm{C} & 0.004587 & 0.401587 & 0.004790 & -0.072852 & -0.004155 & 0.004750\end{array}$

$\begin{array}{llllllll}7 & \mathrm{C} & 0.004879 & 0.004795 & 0.401288 & 0.001425 & 0.000016 & 0.000003\end{array}$

$\begin{array}{llllllll}8 \mathrm{H} & -0.024688 & -0.000062 & -0.000049 & 0.004931 & -0.000092 & -0.000064\end{array}$

$\begin{array}{llllllll}9 \mathrm{H} & -0.000086 & -0.023669 & -0.000079 & 0.005002 & -0.000023 & -0.000107\end{array}$

$\begin{array}{llllllll}10 \mathrm{H} & -0.000089 & -0.000072 & -0.022857 & 0.000060 & 0.000001 & 0.000000\end{array}$

$\begin{array}{llllllll}11 \mathrm{H} & -0.002149 & 0.000012 & -0.002943 & -0.000554 & 0.000012 & -0.000061\end{array}$

$\begin{array}{llllllll}12 \mathrm{H} & -0.002426 & -0.002809 & 0.000013 & -0.035144 & -0.002777 & -0.002361\end{array}$

$\begin{array}{llllllll}13 \mathrm{H} & 0.508404 & -0.000084 & -0.000086 & -0.002512 & -0.000037 & 0.001820\end{array}$

$\begin{array}{llllllll}14 \mathrm{H} & -0.000084 & 0.522614 & -0.000096 & -0.003835 & 0.002623 & -0.000024\end{array}$

$\begin{array}{llllllll}15 & \mathrm{H} & -0.000086 & -0.000096 & 0.520034 & 0.000009 & -0.000003 & 0.000002\end{array}$

$\begin{array}{llllllll}16 & \mathrm{C} & -0.002512 & -0.003835 & 0.000009 & 5.213221 & 0.402994 & 0.396908\end{array}$

$\begin{array}{llllllll}17 & \mathrm{H} & -0.000037 & 0.002623 & -0.000003 & 0.402994 & 0.519571 & -0.023301\end{array}$

$\begin{array}{llllllll}18 & \mathrm{H} & 0.001820 & -0.000024 & 0.000002 & 0.396908 & -0.023301 & 0.499570\end{array}$

$\begin{array}{llllllll}19 & \mathrm{C} & 0.000125 & -0.002004 & -0.001799 & -0.050110 & -0.002529 & 0.004704\end{array}$

$\begin{array}{llllllll}20 & \mathrm{C} & -0.004704 & 0.000034 & -0.003107 & -0.047742 & 0.004501 & -0.006062\end{array}$

$\begin{array}{llllllll}21 & \mathrm{C} & 0.000344 & 0.001206 & 0.001135 & 0.288023 & -0.043026 & -0.034340\end{array}$

$\begin{array}{lllllll}22 \mathrm{H} & -0.000063 & -0.000055 & -0.000051 & -0.030736 & -0.002117 & -0.002650\end{array}$

$\begin{array}{llllllll}23 & \mathrm{O} & 0.000001 & 0.000494 & 0.000649 & 0.000906 & 0.000479 & -0.000065\end{array}$

$\begin{array}{llllllll}24 & \mathrm{H} & 0.000049 & -0.000002 & 0.002843 & 0.005437 & -0.000066 & 0.000025\end{array}$

$\begin{array}{llllllll}25 \mathrm{~F} & 0.004465 & 0.000000 & 0.000000 & 0.003570 & 0.000024 & 0.004475\end{array}$

$\begin{array}{llllll}19 & 20 & 21 & 22 & 23 & 24\end{array}$

$\begin{array}{llllllll}1 & \mathrm{H} & -0.034648 & -0.000246 & 0.003751 & -0.000076 & 0.003715 & -0.000039\end{array}$

$\begin{array}{llllllll}2 \mathrm{C} & 0.331785 & 0.000634 & -0.103602 & 0.003550 & -0.063409 & 0.001892\end{array}$

$\begin{array}{llllllll}3 & \mathrm{C} & 0.002242 & 0.348133 & -0.055617 & 0.004097 & 0.000193 & -0.053378\end{array}$

$\begin{array}{llllllll}4 & C & -0.006272 & -0.004152 & -0.045577 & 0.003914 & 0.000196 & -0.000832\end{array}$

$\begin{array}{llllllll}5 & \mathrm{C} & -0.001345 & -0.055000 & -0.009318 & -0.000477 & -0.000050 & 0.005357\end{array}$

$\begin{array}{llllllll}6 & \mathrm{C} & -0.055450 & -0.000408 & 0.005161 & -0.000437 & 0.001002 & -0.000060\end{array}$

$\begin{array}{llllllll}7 & \mathrm{C} & -0.060713 & -0.057613 & 0.009427 & -0.000460 & 0.000826 & -0.000751\end{array}$

$\begin{array}{llllllll}8 \mathrm{H} & 0.000234 & 0.004602 & -0.000745 & 0.000011 & 0.000000 & -0.000056\end{array}$

$\begin{array}{llllllll}9 \mathrm{H} & 0.004653 & 0.000119 & -0.000844 & 0.000013 & -0.000064 & 0.000003\end{array}$

$\begin{array}{llllllll}10 \mathrm{H} & 0.004705 & 0.003814 & -0.000845 & 0.000013 & -0.000061 & -0.000122\end{array}$

$\begin{array}{llllllll}11 & \mathrm{H} & -0.000549 & -0.031412 & 0.004871 & -0.000063 & -0.000003 & -0.004143\end{array}$

$\begin{array}{llllllll}12 \mathrm{H} & -0.000483 & -0.000369 & 0.004481 & -0.000062 & -0.000002 & 0.000012\end{array}$

$\begin{array}{llllllll}13 \mathrm{H} & 0.000125 & -0.004704 & 0.000344 & -0.000063 & 0.000001 & 0.000049\end{array}$

$\begin{array}{llllllll}14 & \mathrm{H} & -0.002004 & 0.000034 & 0.001206 & -0.000055 & 0.000494 & -0.000002\end{array}$

$\begin{array}{llllllll}15 \mathrm{H} & -0.001799 & -0.003107 & 0.001135 & -0.000051 & 0.000649 & 0.002843\end{array}$

$\begin{array}{llllllll}16 & \mathrm{C} & -0.050110 & -0.047742 & 0.288023 & -0.030736 & 0.000906 & 0.005437\end{array}$

$\begin{array}{llllllll}17 & \mathrm{H} & -0.002529 & 0.004501 & -0.043026 & -0.002117 & 0.000479 & -0.000066\end{array}$

$\begin{array}{llllllll}18 & \mathrm{H} & 0.004704 & -0.006062 & -0.034340 & -0.002650 & -0.000065 & 0.000025\end{array}$

$\begin{array}{lllllllll}19 & \mathrm{C} & 4.559563 & -0.049367 & 0.291953 & -0.030712 & 0.553625 & 0.001479\end{array}$

$\begin{array}{llllllll}20 & \mathrm{C} & -0.049367 & 4.695851 & 0.324951 & -0.025440 & 0.000489 & 0.413442\end{array}$

$\begin{array}{llllllll}21 & \mathrm{C} & 0.291953 & 0.324951 & 5.419567 & 0.394650 & -0.061457 & -0.057610\end{array}$

$\begin{array}{llllllll}22 & \mathrm{H} & -0.030712 & -0.025440 & 0.394650 & 0.461798 & 0.003473 & -0.003321\end{array}$

$\begin{array}{llllllll}23 & \mathrm{O} & 0.553625 & 0.000489 & -0.061457 & 0.003473 & 8.114702 & 0.000683\end{array}$

$\begin{array}{llllllll}24 & \mathrm{H} & 0.001479 & 0.413442 & -0.057610 & -0.003321 & 0.000683 & 0.537142\end{array}$

$\begin{array}{llllllll}25 & \text { F } & 0.001708 & 0.190144 & -0.033305 & -0.000128 & -0.000004 & -0.027013\end{array}$ 25

$1 \mathrm{H} \quad 0.000001$

2 C -0.000024 

3 C -0.035822
4 C -0.002524
$5 \mathrm{C} \quad 0.004433$
$\begin{array}{ll}6 \mathrm{C} & 0.000001\end{array}$
7 C 0.001972
$8 \mathrm{H} \quad 0.000040$
$9 \mathrm{H} \quad 0.000000$
$10 \mathrm{H}-0.000026$
$11 \mathrm{H} \quad 0.000429$
$12 \mathrm{H} \quad-0.000014$
$13 \mathrm{H} \quad 0.004465$
$14 \mathrm{H} \quad 0.000000$
$15 \mathrm{H} \quad 0.000000$
$16 \mathrm{C} \quad 0.003570$
$17 \mathrm{H} \quad 0.000024$
$18 \mathrm{H} \quad 0.004475$
$19 \mathrm{C} \quad 0.001708$
$20 \mathrm{C} \quad 0.190144$
$21 \mathrm{C}-0.033305$
$22 \mathrm{H}-0.000128$
$\begin{array}{lll}23 & \mathrm{O} & -0.000004\end{array}$
$24 \mathrm{H}-0.027013$
$25 \mathrm{~F} \quad 9.309211$

Mulliken atomic charges: 1

$1 \mathrm{H} \quad 0.213736$

2 C -0.248664

3 C -0.231802

4 C -0.184003

5 C -0.322038

6 C -0.328938

7 C -0.346612

$8 \mathrm{H} \quad 0.167633$

$9 \mathrm{H} \quad 0.173679$

$10 \mathrm{H} \quad 0.182758$

$11 \mathrm{H} \quad 0.192807$

$12 \mathrm{H} \quad 0.180176$

$13 \mathrm{H} \quad 0.187863$

$14 \mathrm{H} \quad 0.180053$

$15 \mathrm{H} \quad 0.183336$

$16 \mathrm{C}-0.338745$

$\begin{array}{lll}17 \mathrm{H} & 0.182327\end{array}$

$18 \mathrm{H} \quad 0.195130$

19 C 0.539205

$20 \mathrm{C} \quad 0.298907$

$\begin{array}{lll}21 & \mathrm{C} & -0.303234\end{array}$

$22 \mathrm{H} \quad 0.225330$

$23 \mathrm{O}-0.556320$

$\begin{array}{lll}24 & \mathrm{H} & 0.179028\end{array}$

25 F -0.421612

Sum of Mulliken charges $=0.00000$

Atomic charges with hydrogens summed into heavy atoms:
$1 \mathrm{H} \quad 0.000000$
2 C -0.034928
3 C -0.038996
$\begin{array}{lll}4 & \mathrm{C} & -0.003827\end{array}$
$\begin{array}{lll}5 & \mathrm{C} & 0.033458\end{array}$
6 C 0.024794
7 C 0.019482
$8 \mathrm{H} \quad 0.000000$
$9 \mathrm{H} \quad 0.000000$
$10 \mathrm{H} \quad 0.000000$
$11 \mathrm{H} \quad 0.000000$ 
$12 \mathrm{H} \quad 0.000000$

$13 \mathrm{H} \quad 0.000000$

$14 \mathrm{H} \quad 0.000000$

$\begin{array}{lll}15 \mathrm{H} & 0.000000\end{array}$

$\begin{array}{ll}16 \mathrm{C} & 0.038711\end{array}$

$\begin{array}{lll}17 \mathrm{H} & 0.000000\end{array}$

$18 \mathrm{H} \quad 0.000000$

$19 \mathrm{C} \quad 0.539205$

$20 \mathrm{C} \quad 0.477935$

$21 \mathrm{C}-0.077903$

$22 \mathrm{H} \quad 0.000000$

$23 \mathrm{O}-0.556320$

$24 \mathrm{H} \quad 0.000000$

$25 \mathrm{~F}-0.421612$

Sum of Mulliken charges $=0.00000$

Electronic spatial extent $(\mathrm{au}):<\mathrm{R} * * 2>=1558.1456$

Charge $=0.0000$ electrons

Dipole moment (field-independent basis, Debye):

$\mathrm{X}=1.1385 \mathrm{Y}=2.9941 \mathrm{Z}=-0.4116$ Tot $=3.2296$

Quadrupole moment (field-independent basis, Debye-Ang):

$\mathrm{XX}=-82.9049 \quad \mathrm{YY}=-74.3329 \quad \mathrm{ZZ}=-67.4490$

$\mathrm{XY}=-4.8713 \quad \mathrm{XZ}=-0.4375 \quad \mathrm{YZ}=0.1935$

Traceless Quadrupole moment (field-independent basis, Debye-Ang):

$\mathrm{XX}=-8.0093 \mathrm{YY}=0.5627 \quad \mathrm{ZZ}=7.4466$

$\mathrm{XY}=-4.8713 \quad \mathrm{XZ}=-0.4375 \quad \mathrm{YZ}=0.1935$

Octapole moment (field-independent basis, Debye-Ang**2):

$\mathrm{XXX}=18.5137 \mathrm{YYY}=-5.6927 \mathrm{ZZZ}=-0.4736 \mathrm{XYY}=13.8566$

$\mathrm{XXY}=20.6063 \mathrm{XXZ}=-0.4725 \mathrm{XZZ}=3.9125 \mathrm{YZZ}=-6.4125$

$\mathrm{YYZ}=-1.6633 \mathrm{XYZ}=0.9059$

Hexadecapole moment (field-independent basis, Debye-Ang**3):

$\mathrm{XXXX}=-852.1314 \mathrm{YYYY}=-662.8638 \mathrm{ZZZZ}=-511.0297 \mathrm{XXXY}=-36.7497$

$\mathrm{XXXZ}=1.1066 \mathrm{YYYX}=-33.2683 \mathrm{YYYZ}=-2.8098 \mathrm{ZZZX}=-3.7526$

$Z Z Z Y=2.8063 X X Y Y=-282.3619 X X Z Z=-207.0457 \mathrm{YYZZ}=-188.7254$

$\mathrm{XXYZ}=-0.5701 \mathrm{YYXZ}=0.8011 \mathrm{ZZXY}=-3.8400$

$\mathrm{N}-\mathrm{N}=7.866968982059 \mathrm{D}+02 \mathrm{E}-\mathrm{N}=-2.887669059757 \mathrm{D}+03 \quad \mathrm{KE}=5.598014821746 \mathrm{D}+02$

1|1IUNPC-UNKISPIRHF|6-31G(d)|C10H13F1O1|PCUSER|17-May-2006|0||\# RHF/6-

31G(D) GEOM=CONNECTIVITYITitle Card Required॥0,1IH,0,-2.47717952,0.6

$6865877,-0.98517938|\mathrm{C}, 0,-1.46714744,0.53304372,-0.58663335| \mathrm{C}, 0,0.98885$

$356,0.39564375,-1.12303552|\mathrm{C}, 0,0.23271851,1.31947951,1.10193128| \mathrm{C}, 0,1$. $27499099,1.43617952,-0.02474728|\mathrm{C}, 0,-1.17623899,1.55316,0.52171877| \mathrm{C}, 0$ $,-0.41760202,0.64168128,-1.70181838 \mathrm{H}, 0,1.25169175,2.44519621,-0.45154$ $425|\mathrm{H}, 0,-1.25245242,2.5716028,0.12283663| \mathrm{H}, 0,-0.46535692,1.6337926,-2$. $16632648|\mathrm{H}, 0,1.7457543,0.48580476,-1.90982485| \mathrm{H}, 0,0.44052825,2.0645010$ $2,1.87891728|\mathrm{H}, 0,2.28241631,1.28548429,0.38140091| \mathrm{H}, 0,-1.92326193,1.46$ $261406,1.32112877|\mathrm{H}, 0,-0.62688263,-0.09425626,-2.48759744| \mathrm{C}, 0,0.292905$ $52,-0.09528336,1.7088653|\mathrm{H}, 0,-0.45157659,-0.18414861,2.50958656| \mathrm{H}, 0,1$. $27720952,-0.26560733,2.16285821|\mathrm{C}, 0,-1.33441182,-0.85675,0.0223601| \mathrm{C}, 0$ ,1.03249906,-1.02000137,-0.51799886|C,0,0.02665364,-1.14760942,0.62452 $533 \mid \mathrm{H}, 0,0.04770505,-2.15239849,1.05788541 \mathrm{OO}, 0,-2.24508297,-1.67832504$, $0.00533828 \mid \mathrm{H}, 0,0.83568039,-1.76845554,-1.29434218 \mathrm{IF}, 0,2.28411801,-1.25$ $371665,-0.03705663 \|$ Version $=x 86-$ Win32-G03RevB.05|State $=1-A \mid H F=-560.574$ $3142|\mathrm{RMSD}=6.817 \mathrm{e}-006| \mathrm{Dipole}=0.4479137,1.1779692,-0.1619406 \mid \mathrm{PG}=\mathrm{C} 01[\mathrm{X}(\mathrm{C}$ 10H13F1O1)]॥@

IT IS A SIMPLE TASK TO MAKE THINGS COMPLEX, BUT A COMPLEX TASK TO MAKE THEM SIMPLE.

Job cpu time: 0 days 0 hours 3 minutes 1.0 seconds.

File lengths (MBytes): $\mathrm{RWF}=32 \mathrm{Int}=0 \mathrm{D} 2 \mathrm{E}=0 \mathrm{Chk}=9 \mathrm{Scr}=1$ Normal termination of Gaussian 03 at Wed May 17 14:09:10 2006. 


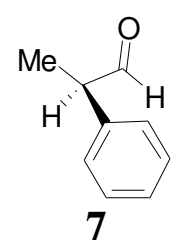

Entering Link 1 = C: $\backslash$ G03W W11 exe PID= 2188 .

Copyright (c) 1988,1990,1992,1993,1995,1998,2003, Gaussian, Inc. All Rights Reserved.

This is the Gaussian(R) 03 program. It is based on the the Gaussian(R) 98 system (copyright 1998, Gaussian, Inc.), the Gaussian(R) 94 system (copyright 1995, Gaussian, Inc.), the Gaussian 92(TM) system (copyright 1992, Gaussian, Inc.), the Gaussian 90(TM) system (copyright 1990, Gaussian, Inc.), the Gaussian 88(TM) system (copyright 1988, Gaussian, Inc.), the Gaussian 86(TM) system (copyright 1986, Carnegie Mellon University), and the Gaussian 82(TM) system (copyright 1983, Carnegie Mellon University). Gaussian is a federally registered trademark of Gaussian, Inc.

This software contains proprietary and confidential information, including trade secrets, belonging to Gaussian, Inc.

This software is provided under written license and may be used, copied, transmitted, or stored only in accord with that written license.

The following legend is applicable only to US Government contracts under DFARS:

\section{RESTRICTED RIGHTS LEGEND}

Use, duplication or disclosure by the US Government is subject to restrictions as set forth in subparagraph (c)(1)(ii) of the Rights in Technical Data and Computer Software clause at DFARS 252.227-7013.

Gaussian, Inc.

Carnegie Office Park, Building 6, Pittsburgh, PA 15106 USA

The following legend is applicable only to US Government contracts under FAR:

\section{RESTRICTED RIGHTS LEGEND}

Use, reproduction and disclosure by the US Government is subject to restrictions as set forth in subparagraph (c) of the Commercial Computer Software - Restricted Rights clause at FAR 52.227-19.

Gaussian, Inc.

Carnegie Office Park, Building 6, Pittsburgh, PA 15106 USA

Warning -- This program may not be used in any manner that competes with the business of Gaussian, Inc. or will provide assistance to any competitor of Gaussian, Inc. The licensee of this program is prohibited from giving any competitor of 
Gaussian, Inc. access to this program. By using this program, the user acknowledges that Gaussian, Inc. is engaged in the business of creating and licensing software in the field of computational chemistry and represents and warrants to the licensee that it is not a competitor of Gaussian, Inc. and that it will not use this program in any manner prohibited above.

Cite this work as:

Gaussian 03, Revision B.05,

M. J. Frisch, G. W. Trucks, H. B. Schlegel, G. E. Scuseria,

M. A. Robb, J. R. Cheeseman, J. A. Montgomery, Jr., T. Vreven,

K. N. Kudin, J. C. Burant, J. M. Millam, S. S. Iyengar, J. Tomasi,

V. Barone, B. Mennucci, M. Cossi, G. Scalmani, N. Rega,

G. A. Petersson, H. Nakatsuji, M. Hada, M. Ehara, K. Toyota,

R. Fukuda, J. Hasegawa, M. Ishida, T. Nakajima, Y. Honda, O. Kitao,

H. Nakai, M. Klene, X. Li, J. E. Knox, H. P. Hratchian, J. B. Cross,

C. Adamo, J. Jaramillo, R. Gomperts, R. E. Stratmann, O. Yazyev,

A. J. Austin, R. Cammi, C. Pomelli, J. W. Ochterski, P. Y. Ayala,

K. Morokuma, G. A. Voth, P. Salvador, J. J. Dannenberg,

V. G. Zakrzewski, S. Dapprich, A. D. Daniels, M. C. Strain,

O. Farkas, D. K. Malick, A. D. Rabuck, K. Raghavachari,

J. B. Foresman, J. V. Ortiz, Q. Cui, A. G. Baboul, S. Clifford,

J. Cioslowski, B. B. Stefanov, G. Liu, A. Liashenko, P. Piskorz,

I. Komaromi, R. L. Martin, D. J. Fox, T. Keith, M. A. Al-Laham,

C. Y. Peng, A. Nanayakkara, M. Challacombe, P. M. W. Gill,

B. Johnson, W. Chen, M. W. Wong, C. Gonzalez, and J. A. Pople, Gaussian, Inc., Pittsburgh PA, 2003.

*******************************************************

Gaussian 03: x86-Win32-G03RevB.05 8-Nov-2003

18-Apr-2006

$* * * * * * * * * * * * * * * * * * * * * * * * * * * * * * * * * * * * * * * * * * * * * *$

$\%$ chk=ph-me-h-h.chk

$\%$ mem $=64 \mathrm{MB}$

$\%$ nproc $=1$

Will use up to 1 processors via shared memory.

\# hf/6-31g(d) geom=connectivity

$1 / 38=1,57=2 / 1$;

$2 / 17=6,18=5,40=1 / 2$;

$3 / 5=1,6=6,7=1,11=9,16=1,25=1,30=1 / 1,2,3$;

$4 / / 1$

$5 / 5=2,32=1,38=5 / 2$;

$6 / 7=2,8=2,9=2,10=2,28=1 / 1$;

$99 / 5=1,9=1 / 99$;

Title Card Required

Symbolic Z-matrix:

Charge $=0$ Multiplicity $=1$

$\begin{array}{lrrr}\mathrm{C} & -0.13242 & -0.3165 & -0.06053 \\ \mathrm{C} & -2.89942 & 0.1545 & -0.24553 \\ \mathrm{C} & -0.62642 & 0.9845 & -0.23453 \\ \mathrm{C} & -1.04542 & -1.3745 & 0.02847 \\ \mathrm{C} & -2.41942 & -1.1435 & -0.06653 \\ \mathrm{C} & -1.99842 & 1.2185 & -0.32753 \\ \mathrm{H} & 0.06658 & 1.8195 & -0.29453 \\ \mathrm{H} & -0.67842 & -2.3885 & 0.16647 \\ \mathrm{H} & -3.11242 & -1.9775 & -0.00053 \\ \mathrm{H} & -2.36442 & 2.2335 & -0.46053 \\ \mathrm{H} & -3.96842 & 0.3365 & -0.31853\end{array}$




\begin{tabular}{lrrr}
$\mathrm{C}$ & 1.36958 & -0.5645 & 0.03247 \\
$\mathrm{H}$ & 1.50858 & -1.6225 & 0.30647 \\
$\mathrm{C}$ & 1.92858 & 0.2385 & 1.20147 \\
$\mathrm{O}$ & 2.79258 & 1.0825 & 1.11047 \\
$\mathrm{H}$ & 1.46158 & 0.0045 & 2.18447 \\
$\mathrm{C}$ & 2.12358 & -0.2745 & -1.27253 \\
$\mathrm{H}$ & 2.02158 & 0.7755 & -1.55953 \\
$\mathrm{H}$ & 1.73158 & -0.8945 & -2.08453 \\
$\mathrm{H}$ & 3.19158 & -0.4815 & -1.15453 \\
& \multicolumn{3}{l}{}
\end{tabular}

\begin{tabular}{|c|c|c|c|c|c|c|c|}
\hline \multirow{2}{*}{$\begin{array}{l}\text { Center } \\
\text { Number }\end{array}$} & \multirow{2}{*}{$\begin{array}{l}\text { Atomic } \\
\text { Number }\end{array}$} & \multirow{2}{*}{\multicolumn{2}{|c|}{$\begin{array}{r}\text { Atomic } \\
\text { Type }\end{array}$}} & \multicolumn{4}{|c|}{ Coordinates (Angstroms) } \\
\hline & & & & $\mathrm{X}$ & $\mathrm{Y}$ & & $\mathrm{Z}$ \\
\hline 1 & 6 & \multicolumn{2}{|c|}{-0.132417} & \multicolumn{4}{|c|}{$-0.316500 \quad-0.060528$} \\
\hline 2 & 6 & \multicolumn{2}{|c|}{-2.899417} & \multicolumn{2}{|c|}{0.154500} & \multicolumn{2}{|c|}{-0.245528} \\
\hline 3 & 6 & \multicolumn{2}{|c|}{-0.626417} & \multicolumn{2}{|c|}{0.984500} & \multicolumn{2}{|c|}{-0.234528} \\
\hline 4 & 6 & \multicolumn{2}{|c|}{-1.045417} & \multicolumn{2}{|c|}{-1.374500} & \multicolumn{2}{|c|}{0.028472} \\
\hline 5 & 6 & -2.419 & 417 & -1.143 & 3500 & -0.06 & 66528 \\
\hline 6 & 6 & -1.998 & 417 & 1.218 & 8500 & -0.32 & 27528 \\
\hline 7 & 1 & 0.066 & 583 & 1.819 & 500 & -0.29 & 4528 \\
\hline 8 & 1 & -0.678 & 417 & -2.388 & 8500 & 0.16 & 56472 \\
\hline 9 & 1 & -3.112 & 417 & -1.977 & 7500 & -0.00 & 00528 \\
\hline 10 & 1 & -2.36 & 4417 & 2.23 & 3500 & -0.4 & 60528 \\
\hline 11 & 1 & -3.968 & 8417 & 0.33 & 6500 & -0.3 & 18528 \\
\hline 12 & 6 & 1.369 & 9583 & -0.56 & 4500 & 0.03 & 32472 \\
\hline 13 & 1 & 1.508 & 3583 & -1.62 & 2500 & 0.30 & 06472 \\
\hline 14 & 6 & 1.928 & 3583 & 0.238 & 8500 & 1.20 & 01472 \\
\hline 15 & 8 & 2.792 & 2583 & 1.082 & 2500 & 1.11 & 10472 \\
\hline 16 & 1 & 1.461 & 1583 & 0.004 & 4500 & 2.18 & 84472 \\
\hline 17 & 6 & 2.123 & 3583 & -0.27 & 4500 & -1.2 & 72528 \\
\hline 18 & 1 & 2.021 & 1583 & 0.775 & 5500 & -1.5 & 59528 \\
\hline 19 & 1 & 1.731 & 1583 & -0.89 & 4500 & -2.08 & 84528 \\
\hline 20 & 1 & 3.191 & 1583 & -0.48 & 1500 & -1.1 & 54528 \\
\hline & Distanc & ce matrix (ar & & & & & \\
\hline & 1 & 2 & & 5 & & & \\
\hline $1 \mathrm{C}$ & 0.000000 & & & & & & \\
\hline $2 \mathrm{C}$ & 2.812891 & 0.000000 & & & & & \\
\hline $3 \mathrm{C}$ & 1.402467 & 2.419824 & 0.00 & 0000 & & & \\
\hline $4 \mathrm{C}$ & 1.400305 & 2.418725 & 2.41 & 0313 & 0.000 & 0000 & \\
\hline $5 \mathrm{C}$ & 2.431940 & 1.395437 & 2.78 & 7733 & 1.396 & 6518 & 0.000000 \\
\hline $6 \mathrm{C}$ & 2.430940 & 1.396646 & 1.39 & 4915 & 2.785 & 5425 & 2.413381 \\
\hline $7 \mathrm{H}$ & 2.157974 & 3.401732 & 1.08 & 36772 & 3.397 & 97427 & 3.874474 \\
\hline $8 \mathrm{H}$ & 2.154722 & 3.401387 & 3.39 & 7151 & 1.08 & 37166 & 2.152997 \\
\hline $9 \mathrm{H}$ & 3.412172 & 2.156576 & 3.87 & 4067 & 2.15 & 53355 & 1.086352 \\
\hline $10 \mathrm{H}$ & 3.412378 & 2.157473 & 2.1 & 52143 & 3.87 & 72537 & 3.400351 \\
\hline $11 \mathrm{H}$ & 3.899727 & 1.086837 & 3.4 & 05279 & 3.40 & 04682 & 2.157152 \\
\hline $12 \mathrm{C}$ & 1.525174 & 4.338042 & 2.5 & 40611 & 2.54 & 47222 & 3.834262 \\
\hline $13 \mathrm{H}$ & 2.129133 & 4.784652 & 3.4 & 12822 & 2.58 & 81028 & 3.974639 \\
\hline $14 \mathrm{C}$ & 2.479595 & 5.040878 & 3.0 & 24341 & 3.58 & 80834 & 4.735277 \\
\hline $15 \mathrm{O}$ & 3.447328 & 5.924423 & 3.6 & 75349 & 4.68 & 83782 & 5.788381 \\
\hline $16 \mathrm{H}$ & 2.771985 & 4.994569 & 3.3 & 42410 & 3.58 & 82600 & 4.631098 \\
\hline $17 \mathrm{C}$ & 2.561297 & 5.144832 & 3.1 & 97659 & 3.59 & 97939 & 4.780005 \\
\hline $18 \mathrm{H}$ & 2.842390 & 5.131128 & 2.9 & 68368 & 4.06 & 68259 & 5.063012 \\
\hline $19 \mathrm{H}$ & 2.811611 & 5.092002 & 3.5 & 37415 & 3.52 & 22343 & 4.622243 \\
\hline $20 \mathrm{H}$ & 3.503289 & 6.191208 & 4.1 & 91978 & 4.48 & 88776 & 5.753721 \\
\hline & 6 & $7 \quad 8$ & 9 & 1 & 0 & & \\
\hline $6 \mathrm{C}$ & 0.000000 & & & & & & \\
\hline $7 \mathrm{H}$ & 2.150934 & 0.000000 & & & & & \\
\hline $8 \mathrm{H}$ & 3.872581 & 4.298233 & 0.00 & 0000 & & & \\
\hline $9 \mathrm{H}$ & 3.400344 & 4.960815 & 2.47 & 4099 & 0.00 & 0000 & \\
\hline $10 \mathrm{H}$ & 1.087138 & 2.471581 & 4.9 & 59698 & 4.30 & 01584 & 0.000000 \\
\hline
\end{tabular}


$\begin{array}{lllllll}11 & \mathrm{H} & 2.158450 & 4.298964 & 4.299413 & 2.487661 & 2.488290\end{array}$

$\begin{array}{llllllll}12 & \mathrm{C} & 3.827808 & 2.736456 & 2.745767 & 4.699573 & 4.691973\end{array}$

$\begin{array}{lllllll}13 & \mathrm{H} & 4.557662 & 3.779938 & 2.321492 & 4.644773 & 5.518800\end{array}$

$\begin{array}{lllllll}14 & \mathrm{C} & 4.326612 & 2.864371 & 3.843020 & 5.636235 & 5.017182\end{array}$

$\begin{array}{llllllll}15 & \mathrm{O} & 5.004000 & 3.154088 & 4.998682 & 6.742918 & 5.512485\end{array}$

$\begin{array}{llllllll}16 & \mathrm{H} & 4.444720 & 3.374269 & 3.791883 & 5.442796 & 5.157785\end{array}$

$\begin{array}{llllllll}17 & \mathrm{C} & 4.484747 & 3.093957 & 3.793537 & 5.651008 & 5.204955\end{array}$

$\begin{array}{llllllll}18 & \mathrm{H} & 4.227821 & 2.551899 & 4.503329 & 6.030543 & 4.750848\end{array}$

$\begin{array}{lllllll}19 & \mathrm{H} & 4.633003 & 3.652687 & 3.620378 & 5.383334 & 5.403608\end{array}$

$\begin{array}{llllllll}20 & \mathrm{H} & 5.523588 & 3.974899 & 4.512049 & 6.581045 & 6.222700\end{array}$ $\begin{array}{lllll}11 & 12 & 13 & 14 & 15\end{array}$

$\begin{array}{lll}11 \mathrm{H} & 0.000000\end{array}$

$\begin{array}{llll}12 & \mathrm{C} & 5.424873 & 0.000000\end{array}$

$\begin{array}{lllll}13 \mathrm{H} & 5.850285 & 1.101708 & 0.000000\end{array}$

$\begin{array}{llllll}14 & \mathrm{C} & 6.090535 & 1.524418 & 2.107308 & 0.000000\end{array}$

$\begin{array}{llllllll}15 & \mathrm{O} & 6.950516 & 2.428914 & 3.100338 & 1.211244 & 0.000000\end{array}$

$\begin{array}{lllllll}16 \mathrm{H} & 5.988333 & 2.227853 & 2.485201 & 1.113164 & 2.021663\end{array}$

$\begin{array}{lllllll}17 & \mathrm{C} & 6.196443 & 1.534810 & 2.165311 & 2.534141 & 2.822711\end{array}$

$\begin{array}{lllllll}18 \mathrm{H} & 6.132936 & 2.180635 & 3.081482 & 2.814274 & 2.795995\end{array}$

$\begin{array}{lllllll}19 & \mathrm{H} & 6.092956 & 2.172932 & 2.509301 & 3.481421 & 3.904136\end{array}$

$\begin{array}{lllllll}20 \mathrm{H} & 7.254903 & 2.176130 & 2.503775 & 2.768448 & 2.781281\end{array}$

$\begin{array}{ll}16 \mathrm{H} & 0.000000\end{array}$

$\begin{array}{llll}17 & \mathrm{C} & 3.530855 & 0.000000\end{array}$

$\begin{array}{lllll}18 & \mathrm{H} & 3.863363 & 1.093285 & 0.000000\end{array}$

$\begin{array}{llllll}19 & \mathrm{H} & 4.370980 & 1.094261 & 1.774437 & 0.000000\end{array}$

$\begin{array}{lllllll}20 \mathrm{H} & 3.791836 & 1.094256 & 1.764362 & 1.779626 & 0.000000\end{array}$

Stoichiometry C9H10O

Framework group $\mathrm{C} 1[\mathrm{X}(\mathrm{C} 9 \mathrm{H} 10 \mathrm{O})]$

Deg. of freedom 54

Full point group $\quad \mathrm{C} 1 \quad \mathrm{NOp} 1$

Largest Abelian subgroup $\quad \mathrm{C} 1 \quad \mathrm{NOp} 1$

Largest concise Abelian subgroup C1 NOp 1 Standard orientation:

\begin{tabular}{|c|c|c|c|c|c|}
\hline \multirow{2}{*}{$\begin{array}{l}\text { Center } \\
\text { Number }\end{array}$} & \multirow{2}{*}{\multicolumn{2}{|c|}{$\begin{array}{l}\text { Atomic } \\
\text { Number }\end{array}$}} & \multirow{2}{*}{$\begin{array}{r}\text { Atomic } \\
\text { Type }\end{array}$} & \multicolumn{2}{|c|}{ Coordinates (Angstroms) } \\
\hline & & & & $\begin{array}{ll}X & Y\end{array}$ & $\mathrm{Z}$ \\
\hline 1 & 6 & 0 & 0.166986 & -0.305224 & -0.017991 \\
\hline 2 & 6 & 0 & 2.881998 & 0.429860 & 0.008716 \\
\hline 3 & 6 & 0 & 0.555032 & 0.984206 & 0.374060 \\
\hline 4 & 6 & 0 & 1.158173 & -1.215692 & -0.404583 \\
\hline 5 & 6 & 0 & 2.506972 & -0.854093 & -0.388858 \\
\hline 6 & 6 & 0 & 1.901353 & 1.348891 & 0.388633 \\
\hline 7 & 1 & 0 & -0.201105 & 1.707874 & 0.666695 \\
\hline 8 & 1 & 0 & 0.873299 & -2.218176 & -0.714102 \\
\hline 9 & 1 & 0 & 3.262348 & -1.575618 & -0.687135 \\
\hline 10 & 1 & 0 & 2.184588 & 2.353211 & 0.693575 \\
\hline 11 & 1 & 0 & 3.931080 & 0.713588 & 0.020529 \\
\hline 12 & 6 & 0 & -1.306676 & -0.698220 & -0.023679 \\
\hline 13 & 1 & 0 & -1.377022 & -1.691347 & -0.495390 \\
\hline 14 & 6 & 0 & -2.064065 & 0.248862 & -0.947394 \\
\hline 15 & 8 & 0 & -2.986784 & 0.955964 & -0.607233 \\
\hline 16 & 1 & 0 & -1.689785 & 0.265104 & -1.995623 \\
\hline 17 & 6 & 0 & -1.934268 & -0.755027 & 1.375800 \\
\hline 18 & 1 & 0 & -1.898347 & 0.223597 & 1.861883 \\
\hline 19 & 1 & 0 & -1.398758 & -1.473091 & 2.004307 \\
\hline 20 & 1 & 0 & -2.985061 & -1.054232 & 1.314910 \\
\hline
\end{tabular}

Rotational constants (GHZ): $\quad 2.8725897 \quad 0.8268204 \quad 0.7618703$

Standard basis: 6-31G(d) $(6 \mathrm{D}, 7 \mathrm{~F})$

There are 170 symmetry adapted basis functions of A symmetry.

Integral buffers will be 262144 words long.

Raffenetti 1 integral format. 
Two-electron integral symmetry is turned on.

170 basis functions, 320 primitive gaussians, 170 cartesian basis functions

36 alpha electrons 36 beta electrons nuclear repulsion energy $\quad 484.2885075609$ Hartrees.

NAtoms $=20$ NActive $=20 \mathrm{NUniq}=20 \mathrm{SFac}=1.00 \mathrm{D}+00 \mathrm{NAtFMM}=60 \mathrm{Big}=\mathrm{F}$

One-electron integrals computed using PRISM.

NBasis $=170$ RedAO $=\mathrm{T} \quad \mathrm{NBF}=170$

NBsUse $=170$ 1.00D-06 NBFU $=170$

Harris functional with IExCor= 205 diagonalized for initial guess.

ExpMin= 1.61D-01 ExpMax= 5.48D+03 ExpMxC= 8.25D+02 IAcc=1 IRadAn $=\quad 1$ AccDes $=1.00 \mathrm{D}-06$
HarFok: IExCor= 205 AccDes= $1.00 \mathrm{D}-06 \mathrm{IRadAn}=\quad 1 \mathrm{IDoV}=1$

ScaDFX $=1.0000001 .0000001 .0000001 .000000$

Initial guess orbital symmetries:

Occupied (A) (A) (A) (A) (A) (A) (A) (A) (A) (A) (A) (A)

(A) (A) (A) (A) (A) (A) (A) (A) (A) (A) (A) (A)

(A) $(\mathrm{A})(\mathrm{A})(\mathrm{A})(\mathrm{A})(\mathrm{A})(\mathrm{A})(\mathrm{A})(\mathrm{A})(\mathrm{A})(\mathrm{A})(\mathrm{A})$

Virtual (A) (A) (A) (A) (A) (A) (A) (A) (A) (A) (A) (A)

(A) (A) (A) (A) (A) (A) (A) (A) (A) (A) (A) (A)

(A) (A) (A) (A) (A) (A) (A) (A) (A) (A) (A) (A)

(A) $(\mathrm{A})(\mathrm{A})(\mathrm{A})(\mathrm{A})(\mathrm{A})(\mathrm{A})(\mathrm{A})(\mathrm{A})(\mathrm{A})(\mathrm{A})(\mathrm{A})$

(A) (A) (A) (A) (A) (A) (A) (A) (A) (A) (A) (A)

(A) (A) (A) (A) (A) (A) (A) (A) (A) (A) (A) (A)

(A) $(\mathrm{A})(\mathrm{A})(\mathrm{A})(\mathrm{A})(\mathrm{A})(\mathrm{A})(\mathrm{A})(\mathrm{A})(\mathrm{A})(\mathrm{A})(\mathrm{A})$

(A) (A) (A) (A) (A) (A) (A) (A) (A) (A) (A) (A)

(A) (A) (A) (A) (A) (A) (A) (A) (A) (A) (A) (A)

(A) $(\mathrm{A})(\mathrm{A})(\mathrm{A})(\mathrm{A})(\mathrm{A})(\mathrm{A})(\mathrm{A})(\mathrm{A})(\mathrm{A})(\mathrm{A})(\mathrm{A})$

(A) (A) (A) (A) (A) (A) (A) (A) (A) (A) (A) (A)

(A) (A)

The electronic state of the initial guess is 1-A.

Warning! Cutoffs for single-point calculations used.

Requested convergence on RMS density matrix=1.00D-04 within 128 cycles.

Requested convergence on MAX density matrix=1.00D-02.

Requested convergence on energy=5.00D-05.

No special actions if energy rises.

SCF Done: $\mathrm{E}(\mathrm{RHF})=-421.494370240 \quad$ A.U. after 6 cycles

Convg $=0.1934 \mathrm{D}-04 \quad-\mathrm{V} / \mathrm{T}=2.0022$

$\mathrm{S} * * 2=0.0000$

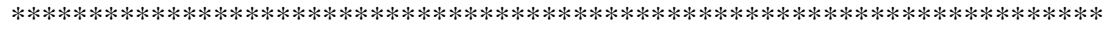

Population analysis using the SCF density.

*****************************************************************************

Orbital symmetries:

Occupied (A) (A) (A) (A) (A) (A) (A) (A) (A) (A) (A) (A)

(A) $(A)(A)(A)(A)(A)(A)(A)(A)(A)(A)(A)$

(A) $(\mathrm{A})(\mathrm{A})(\mathrm{A})(\mathrm{A})(\mathrm{A})(\mathrm{A})(\mathrm{A})(\mathrm{A})(\mathrm{A})(\mathrm{A})(\mathrm{A})$

Virtual (A) (A) (A) (A) (A) (A) (A) (A) (A) (A) (A) (A)

(A) (A) (A) (A) (A) (A) (A) (A) (A) (A) (A) (A)

(A) $(\mathrm{A})(\mathrm{A})(\mathrm{A})(\mathrm{A})(\mathrm{A})(\mathrm{A})(\mathrm{A})(\mathrm{A})(\mathrm{A})(\mathrm{A})(\mathrm{A})$

(A) $(A)(A)(A)(A)(A)(A)(A)(A)(A)(A)(A)$

(A) $(\mathrm{A})(\mathrm{A})(\mathrm{A})(\mathrm{A})(\mathrm{A})(\mathrm{A})(\mathrm{A})(\mathrm{A})(\mathrm{A})(\mathrm{A})(\mathrm{A})$

(A) $(\mathrm{A})(\mathrm{A})(\mathrm{A})(\mathrm{A})(\mathrm{A})(\mathrm{A})(\mathrm{A})(\mathrm{A})(\mathrm{A})(\mathrm{A})(\mathrm{A})$

(A) $(A)(A)(A)(A)(A)(A)(A)(A)(A)(A)(A)$

(A) $(\mathrm{A})(\mathrm{A})(\mathrm{A})(\mathrm{A})(\mathrm{A})(\mathrm{A})(\mathrm{A})(\mathrm{A})(\mathrm{A})(\mathrm{A})(\mathrm{A})$

(A) $(\mathrm{A})(\mathrm{A})(\mathrm{A})(\mathrm{A})(\mathrm{A})(\mathrm{A})(\mathrm{A})(\mathrm{A})(\mathrm{A})(\mathrm{A})(\mathrm{A})$

(A) (A) (A) (A) (A) (A) (A) (A) (A) (A) (A) (A)

(A) $(\mathrm{A})(\mathrm{A})(\mathrm{A})(\mathrm{A})(\mathrm{A})(\mathrm{A})(\mathrm{A})(\mathrm{A})(\mathrm{A})(\mathrm{A})(\mathrm{A})$

(A) $(\mathrm{A})$

The electronic state is 1-A.

Alpha occ. eigenvalues -- -20.56666 -11.33500 -11.25454 -11.25317 -11.24458

Alpha occ. eigenvalues -- -11.24398 -11.24335 -11.24210 -11.24156 -11.22277

Alpha occ. eigenvalues -- $-1.39027 \quad-1.16178$-1.08387 -1.01839 -0.99352 
Alpha occ. eigenvalues -- $\quad-0.90779-0.83431 \quad-0.82226 \quad-0.76099 \quad-0.70220$

Alpha occ. eigenvalues -- $\quad-0.67662 \quad-0.64950 \quad-0.63428 \quad-0.61549 \quad-0.60029$

Alpha occ. eigenvalues -- $\quad-0.58814 \quad-0.54723 \quad-0.53635 \quad-0.52374 \quad-0.51237$

$\begin{array}{llllll}\text { Alpha occ. eigenvalues -- } & -0.49992 & -0.48727 & -0.47811 & -0.41621 & -0.33995\end{array}$

Alpha occ. eigenvalues -- -0.33328

$\begin{array}{lllllll}\text { Alpha virt. eigenvalues -- } & 0.12379 & 0.13148 & 0.16995 & 0.23083 & 0.24928\end{array}$

$\begin{array}{llllllll}\text { Alpha virt. eigenvalues -- } & 0.26174 & 0.28414 & 0.30150 & 0.30567 & 0.31991\end{array}$

$\begin{array}{lllllll}\text { Alpha virt. eigenvalues -- } & 0.32223 & 0.33952 & 0.34460 & 0.36105 & 0.39529\end{array}$

$\begin{array}{llllllll}\text { Alpha virt. eigenvalues -- } & 0.41902 & 0.44104 & 0.46435 & 0.47741 & 0.50003\end{array}$

$\begin{array}{lllllll}\text { Alpha virt. eigenvalues -- } & 0.52375 & 0.53680 & 0.65189 & 0.71638 & 0.72796\end{array}$

$\begin{array}{llllllll}\text { Alpha virt. eigenvalues -- } & 0.73301 & 0.75730 & 0.76784 & 0.77385 & 0.77941\end{array}$

$\begin{array}{llllllll}\text { Alpha virt. eigenvalues -- } & 0.78860 & 0.80184 & 0.83009 & 0.83196 & 0.83355\end{array}$

$\begin{array}{lllllll}\text { Alpha virt. eigenvalues -- } & 0.85223 & 0.87772 & 0.88739 & 0.91508 & 0.95036\end{array}$

$\begin{array}{lllllll}\text { Alpha virt. eigenvalues -- } & 0.95723 & 0.98602 & 0.99688 & 1.02482 & 1.04578\end{array}$

$\begin{array}{lllllll}\text { Alpha virt. eigenvalues -- } & 1.08462 & 1.08650 & 1.09852 & 1.11099 & 1.11131\end{array}$

$\begin{array}{lllllll}\text { Alpha virt. eigenvalues -- } & 1.12330 & 1.14428 & 1.16168 & 1.18018 & 1.18486\end{array}$

$\begin{array}{lllllll}\text { Alpha virt. eigenvalues -- } & 1.20101 & 1.20724 & 1.23385 & 1.24218 & 1.27074\end{array}$

$\begin{array}{lllllll}\text { Alpha virt. eigenvalues -- } & 1.29173 & 1.31573 & 1.34358 & 1.36820 & 1.39568\end{array}$

$\begin{array}{lllllll}\text { Alpha virt. eigenvalues -- } & 1.41679 & 1.43462 & 1.49501 & 1.54790 & 1.57159\end{array}$

$\begin{array}{lllllll}\text { Alpha virt. eigenvalues -- } & 1.63446 & 1.69184 & 1.69837 & 1.70569 & 1.75756\end{array}$

$\begin{array}{lllllll}\text { Alpha virt. eigenvalues -- } & 1.76114 & 1.78632 & 1.82542 & 1.83764 & 1.90380\end{array}$

$\begin{array}{lllllll}\text { Alpha virt. eigenvalues -- } & 1.91256 & 2.00550 & 2.06808 & 2.08039 & 2.09621\end{array}$

$\begin{array}{lllllll}\text { Alpha virt. eigenvalues -- } & 2.12243 & 2.14069 & 2.15025 & 2.16884 & 2.22657\end{array}$

$\begin{array}{llllll}\text { Alpha virt. eigenvalues -- } & 2.27961 & 2.29566 & 2.30193 & 2.33228 & 2.36961\end{array}$

$\begin{array}{lllllll}\text { Alpha virt. eigenvalues -- } & 2.38591 & 2.42729 & 2.44248 & 2.45385 & 2.47119\end{array}$

$\begin{array}{llllll}\text { Alpha virt. eigenvalues -- } & 2.49471 & 2.52471 & 2.61361 & 2.62129 & 2.63100\end{array}$

$\begin{array}{lllllll}\text { Alpha virt. eigenvalues -- } & 2.63228 & 2.69787 & 2.74129 & 2.76939 & 2.80234\end{array}$

$\begin{array}{lllllll}\text { Alpha virt. eigenvalues -- } & 2.90199 & 2.91473 & 2.95917 & 2.99248 & 3.05862\end{array}$

$\begin{array}{lllllll}\text { Alpha virt. eigenvalues -- } & 3.08001 & 3.09521 & 3.10798 & 3.13799 & 3.17876\end{array}$

$\begin{array}{lllllll}\text { Alpha virt. eigenvalues -- } & 3.27713 & 3.35065 & 3.43283 & 3.77738 & 4.33243\end{array}$

$\begin{array}{lllllll}\text { Alpha virt. eigenvalues -- } & 4.50829 & 4.53428 & 4.56020 & 4.60731 & 4.67567\end{array}$

Alpha virt. eigenvalues -- $4.74694 \quad 4.81065 \quad 4.97346 \quad 5.12830$

Condensed to atoms (all electrons):

$$
\begin{array}{llllll}
1 & 2 & 3 & 4 & 5 & 6
\end{array}
$$

$\begin{array}{llllllll}1 & C & 4.880719 & -0.029938 & 0.542601 & 0.574784 & -0.042729 & -0.038511\end{array}$

$\begin{array}{llllllll}2 & \mathrm{C} & -0.029938 & 4.896383 & -0.045304 & -0.045245 & 0.551706 & 0.548061\end{array}$

$\begin{array}{llllllll}3 & C & 0.542601 & -0.045304 & 4.948996 & -0.041545 & -0.035373 & 0.544197\end{array}$

$\begin{array}{llllllll}4 & \mathrm{C} & 0.574784 & -0.045245 & -0.041545 & 4.913677 & 0.541610 & -0.035417\end{array}$

$\begin{array}{llllllll}5 & \mathrm{C} & -0.042729 & 0.551706 & -0.035373 & 0.541610 & 4.893526 & -0.042121\end{array}$

$\begin{array}{llllllll}6 & \mathrm{C} & -0.038511 & 0.548061 & 0.544197 & -0.035417 & -0.042121 & 4.887442\end{array}$

$\begin{array}{llllllll}7 & \mathrm{H} & -0.034050 & 0.002275 & 0.378615 & 0.002436 & 0.000137 & -0.025322\end{array}$

$\begin{array}{llllllll}8 & \mathrm{H} & -0.033289 & 0.002429 & 0.002776 & 0.380598 & -0.028704 & 0.000108\end{array}$

$\begin{array}{llllllll}9 \mathrm{H} & 0.002114 & -0.029415 & 0.000203 & -0.029264 & 0.381303 & 0.002428\end{array}$

$\begin{array}{llllllll}10 \mathrm{H} & 0.001840 & -0.029243 & -0.029372 & 0.000253 & 0.002407 & 0.381788\end{array}$

$\begin{array}{llllllll}11 \mathrm{H} & 0.000246 & 0.381897 & 0.002460 & 0.002386 & -0.029841 & -0.029624\end{array}$

$\begin{array}{llllllll}12 & \mathrm{C} & 0.315288 & 0.000179 & -0.057415 & -0.033291 & 0.003090 & 0.002042\end{array}$

$\begin{array}{llllllll}13 \mathrm{H} & -0.053432 & -0.000018 & 0.004323 & -0.003325 & 0.000335 & -0.000023\end{array}$

$\begin{array}{llllllll}14 & \mathrm{C} & -0.051564 & 0.000016 & -0.001721 & -0.000168 & -0.000108 & 0.000157\end{array}$

$\begin{array}{llllllll}15 & \mathrm{O} & -0.000713 & 0.000000 & 0.000340 & -0.000018 & 0.000000 & 0.000014\end{array}$

$\begin{array}{llllllll}16 \mathrm{H} & 0.004810 & -0.000004 & 0.001887 & 0.000315 & -0.000026 & -0.000066\end{array}$

$\begin{array}{llllllll}17 & \mathrm{C} & -0.055022 & 0.000008 & -0.001977 & -0.001177 & -0.000098 & 0.000113\end{array}$

$\begin{array}{llllllll}18 \mathrm{H} & -0.005459 & -0.000003 & 0.002613 & 0.000018 & 0.000004 & 0.000069\end{array}$

$\begin{array}{llllllll}19 \mathrm{H} & -0.001936 & -0.000004 & 0.000074 & 0.000682 & -0.000016 & -0.000034\end{array}$

$\begin{array}{llllllll}20 & \mathrm{H} & 0.005329 & 0.000000 & -0.000021 & -0.000022 & 0.000000 & 0.000000\end{array}$ $\begin{array}{llllll}7 & 8 & 9 & 10 & 11 & 12\end{array}$

$\begin{array}{llllllll}1 & \mathrm{C} & -0.034050 & -0.033289 & 0.002114 & 0.001840 & 0.000246 & 0.315288\end{array}$

$\begin{array}{llllllll}2 & \mathrm{C} & 0.002275 & 0.002429 & -0.029415 & -0.029243 & 0.381897 & 0.000179\end{array}$

$\begin{array}{llllllll}3 & \mathrm{C} & 0.378615 & 0.002776 & 0.000203 & -0.029372 & 0.002460 & -0.057415\end{array}$

$\begin{array}{llllllll}4 & \mathrm{C} & 0.002436 & 0.380598 & -0.029264 & 0.000253 & 0.002386 & -0.033291\end{array}$

$\begin{array}{llllllll}5 & \mathrm{C} & 0.000137 & -0.028704 & 0.381303 & 0.002407 & -0.029841 & 0.003090\end{array}$

$\begin{array}{llllllll}6 & \mathrm{C} & -0.025322 & 0.000108 & 0.002428 & 0.381788 & -0.029624 & 0.002042\end{array}$

$\begin{array}{llllllll}7 & \mathrm{H} & 0.454302 & -0.000107 & 0.000010 & -0.001194 & -0.000100 & -0.002686\end{array}$

$\begin{array}{llllllll}8 & \mathrm{H} & -0.000107 & 0.476473 & -0.001457 & 0.000010 & -0.000104 & -0.003144\end{array}$

$\begin{array}{llllllll}9 & \mathrm{H} & 0.000010 & -0.001457 & 0.469699 & -0.000103 & -0.001414 & -0.000100\end{array}$ 
$\begin{array}{lllllll}10 \mathrm{H} & -0.001194 & 0.000010 & -0.000103 & 0.466985 & -0.001380 & -0.000100\end{array}$

$\begin{array}{llllllll}11 \mathrm{H} & -0.000100 & -0.000104 & -0.001414 & -0.001380 & 0.469588 & 0.000005\end{array}$

$\begin{array}{llllllll}12 & \mathrm{C} & -0.002686 & -0.003144 & -0.000100 & -0.000100 & 0.000005 & 5.322189\end{array}$

$\begin{array}{llllllll}13 & \mathrm{H} & 0.000051 & 0.002550 & -0.000009 & 0.000002 & 0.000000 & 0.400346\end{array}$

$\begin{array}{llllllll}14 & \mathrm{C} & 0.002449 & 0.000087 & 0.000001 & -0.000006 & 0.000000 & 0.314029\end{array}$

$\begin{array}{llllllll}15 & \mathrm{O} & 0.001413 & 0.000000 & 0.000000 & 0.000000 & 0.000000 & -0.079320\end{array}$

$\begin{array}{llllllll}16 & \mathrm{H} & 0.000312 & 0.000027 & 0.000000 & -0.000001 & 0.000000 & -0.097685\end{array}$

$\begin{array}{llllllll}17 & \mathrm{C} & 0.000347 & 0.000081 & 0.000002 & 0.000001 & 0.000000 & 0.315117\end{array}$

$\begin{array}{llllllll}18 \mathrm{H} & 0.000712 & -0.000003 & 0.000000 & 0.000002 & 0.000000 & -0.032165\end{array}$

$\begin{array}{llllllll}19 \mathrm{H} & -0.000017 & 0.000030 & 0.000000 & 0.000000 & 0.000000 & -0.037006\end{array}$

$\begin{array}{llllllll}20 \mathrm{H} & -0.000013 & -0.000001 & 0.000000 & 0.000000 & 0.000000 & -0.035653\end{array}$ $\begin{array}{llllll}13 & 14 & 15 & 16 & 17 & 18\end{array}$

$\begin{array}{llllllll}1 & \mathrm{C} & -0.053432 & -0.051564 & -0.000713 & 0.004810 & -0.055022 & -0.005459\end{array}$

$\begin{array}{llllllll}2 & \mathrm{C} & -0.000018 & 0.000016 & 0.000000 & -0.000004 & 0.000008 & -0.000003\end{array}$

$\begin{array}{llllllll}3 \mathrm{C} & 0.004323 & -0.001721 & 0.000340 & 0.001887 & -0.001977 & 0.002613\end{array}$

$\begin{array}{llllllll}4 \mathrm{C} & -0.003325 & -0.000168 & -0.000018 & 0.000315 & -0.001177 & 0.000018\end{array}$

$\begin{array}{llllllll}5 & \mathrm{C} & 0.000335 & -0.000108 & 0.000000 & -0.000026 & -0.000098 & 0.000004\end{array}$

$\begin{array}{llllllll}6 \mathrm{C} & -0.000023 & 0.000157 & 0.000014 & -0.000066 & 0.000113 & 0.000069\end{array}$

$\begin{array}{llllllll}7 \mathrm{H} & 0.000051 & 0.002449 & 0.001413 & 0.000312 & 0.000347 & 0.000712\end{array}$

$\begin{array}{llllllll}8 \mathrm{H} & 0.002550 & 0.000087 & 0.000000 & 0.000027 & 0.000081 & -0.000003\end{array}$

$\begin{array}{llllllll}9 \mathrm{H} & -0.000009 & 0.000001 & 0.000000 & 0.000000 & 0.000002 & 0.000000\end{array}$

$\begin{array}{llllllll}10 \mathrm{H} & 0.000002 & -0.000006 & 0.000000 & -0.000001 & 0.000001 & 0.000002\end{array}$

$\begin{array}{llllllll}11 \mathrm{H} & 0.000000 & 0.000000 & 0.000000 & 0.000000 & 0.000000 & 0.000000\end{array}$

$\begin{array}{llllllll}12 & \mathrm{C} & 0.400346 & 0.314029 & -0.079320 & -0.097685 & 0.315117 & -0.032165\end{array}$

$\begin{array}{llllllll}13 & \mathrm{H} & 0.523921 & -0.037453 & 0.001585 & 0.002642 & -0.032128 & 0.003630\end{array}$

$\begin{array}{llllllll}14 & \mathrm{C} & -0.037453 & 4.554445 & 0.546807 & 0.389403 & -0.053791 & -0.004351\end{array}$

$\begin{array}{lllllllll}15 & \mathrm{O} & 0.001585 & 0.546807 & 8.056012 & -0.044845 & 0.009504 & 0.001766\end{array}$

$\begin{array}{llllllll}16 & \mathrm{H} & 0.002642 & 0.389403 & -0.044845 & 0.577149 & 0.004803 & -0.000002\end{array}$

$\begin{array}{lllllllll}17 & \mathrm{C} & -0.032128 & -0.053791 & 0.009504 & 0.004803 & 5.098423 & 0.392257\end{array}$

$\begin{array}{llllllll}18 & \mathrm{H} & 0.003630 & -0.004351 & 0.001766 & -0.000002 & 0.392257 & 0.511188\end{array}$

$\begin{array}{llllllll}19 \mathrm{H} & -0.002560 & 0.004273 & 0.000224 & -0.000035 & 0.389435 & -0.025930\end{array}$

$\begin{array}{llllllll}20 \mathrm{H} & -0.002580 & -0.002536 & 0.001489 & 0.000014 & 0.389890 & -0.024107\end{array}$ $19 \quad 20$

$\begin{array}{llll}1 & \mathrm{C} & -0.001936 & 0.005329\end{array}$

$\begin{array}{llll}2 & \mathrm{C} & -0.000004 & 0.000000\end{array}$

$\begin{array}{llll}3 \text { C } & 0.000074 & -0.000021\end{array}$

$\begin{array}{llll}4 \mathrm{C} & 0.000682 & -0.000022\end{array}$

$\begin{array}{llll}5 & \mathrm{C} & -0.000016 & 0.000000\end{array}$

$\begin{array}{llll}6 & \mathrm{C} & -0.000034 & 0.000000\end{array}$

$\begin{array}{llll}7 & \mathrm{H} & -0.000017 & -0.000013\end{array}$

$8 \mathrm{H} \quad 0.000030-0.000001$

$\begin{array}{llll}9 \mathrm{H} & 0.000000 & 0.000000\end{array}$

$\begin{array}{llll}10 \mathrm{H} & 0.000000 & 0.000000\end{array}$

$11 \mathrm{H} \quad 0.000000 \quad 0.000000$

12 C $-0.037006-0.035653$

$13 \mathrm{H}-0.002560-0.002580$

14 C $0.004273-0.002536$

$\begin{array}{llll}15 & \mathrm{O} & 0.000224 & 0.001489\end{array}$

$\begin{array}{llll}16 \mathrm{H} & -0.000035 & 0.000014\end{array}$

$\begin{array}{llll}17 & \mathrm{C} & 0.389435 & 0.389890\end{array}$

$\begin{array}{lllll}18 & \mathrm{H} & -0.025930 & -0.024107\end{array}$

$\begin{array}{llll}19 \mathrm{H} & 0.529951 & -0.025662\end{array}$

$\begin{array}{llll}20 \mathrm{H} & -0.025662 & 0.515024\end{array}$

Mulliken atomic charges:

1

1 C 0.018911

$\begin{array}{lll}2 & C & -0.203782\end{array}$

3 C -0.216355

4 C -0.227289

5 C -0.195100

6 C -0.195301

$\begin{array}{lll}7 & \mathrm{H} & 0.220431\end{array}$

$\begin{array}{lll}8 \mathrm{H} & 0.201640\end{array}$

$\begin{array}{lll}9 & \mathrm{H} & 0.206004\end{array}$ 
$\begin{array}{lll}10 \mathrm{H} & 0.208112\end{array}$

$11 \mathrm{H} \quad 0.205882$

12 C -0.293720

$13 \mathrm{H} \quad 0.192142$

$\begin{array}{lll}14 \text { C } & 0.340028\end{array}$

$\begin{array}{lll}15 & \mathrm{O} & -0.494259\end{array}$

$\begin{array}{ll}16 \mathrm{H} & 0.161302\end{array}$

$\begin{array}{lll}17 & \mathrm{C} & -0.455787\end{array}$

$\begin{array}{lll}18 \mathrm{H} & 0.179762\end{array}$

$19 \mathrm{H} \quad 0.168531$

$20 \mathrm{H} \quad 0.178847$

Sum of Mulliken charges $=0.00000$

Atomic charges with hydrogens summed into heavy atoms:

\begin{tabular}{ccc}
\multicolumn{4}{c}{1} \\
1 & $\mathrm{C}$ & 0.018911 \\
2 & $\mathrm{C}$ & 0.002101 \\
3 & $\mathrm{C}$ & 0.004076 \\
4 & $\mathrm{C}$ & -0.025649 \\
5 & $\mathrm{C}$ & 0.010904 \\
6 & $\mathrm{C}$ & 0.012811 \\
7 & $\mathrm{H}$ & 0.000000 \\
8 & $\mathrm{H}$ & 0.000000 \\
9 & $\mathrm{H}$ & 0.000000 \\
10 & $\mathrm{H}$ & 0.000000 \\
11 & $\mathrm{H}$ & 0.000000 \\
12 & $\mathrm{C}$ & -0.101578 \\
13 & $\mathrm{H}$ & 0.000000 \\
14 & $\mathrm{C}$ & 0.501330 \\
15 & $\mathrm{O}$ & -0.494259 \\
16 & $\mathrm{H}$ & 0.000000 \\
17 & $\mathrm{C}$ & 0.071354 \\
18 & $\mathrm{H}$ & 0.000000 \\
19 & $\mathrm{H}$ & 0.000000 \\
20 & $\mathrm{H}$ & 0.000000
\end{tabular}

Sum of Mulliken charges $=0.00000$

Electronic spatial extent $(\mathrm{au}):\langle\mathrm{R} * * 2\rangle=1588.3908$

Charge $=0.0000$ electrons

Dipole moment (field-independent basis, Debye):

$\mathrm{X}=2.5650 \mathrm{Y}=-1.7420 \quad \mathrm{Z}=-0.2857$ Tot $=3.1138$

Quadrupole moment (field-independent basis, Debye-Ang):

$\mathrm{XX}=-65.2678 \quad \mathrm{YY}=-56.6737 \quad \mathrm{ZZ}=-60.5767$

$\mathrm{XY}=6.3473 \mathrm{XZ}=-1.0761 \quad \mathrm{YZ}=3.4962$

Traceless Quadrupole moment (field-independent basis, Debye-Ang):

$\mathrm{XX}=-4.4284 \quad \mathrm{YY}=4.1657 \quad \mathrm{ZZ}=0.2627$

$\mathrm{XY}=6.3473 \mathrm{XZ}=-1.0761 \quad \mathrm{YZ}=3.4962$

Octapole moment (field-independent basis, Debye-Ang**2):

$\mathrm{XXX}=47.3377 \mathrm{YYY}=1.8864 \mathrm{ZZZ}=-5.1717 \mathrm{XYY}=6.1625$

$\mathrm{XXY}=-17.2062 \mathrm{XXZ}=2.2147 \mathrm{XZZ}=-12.2195 \mathrm{YZZ}=0.2972$

$\mathrm{YYZ}=1.0742 \mathrm{XYZ}=0.4295$

Hexadecapole moment (field-independent basis, Debye-Ang**3):

$\mathrm{XXXX}=-1521.8241 \mathrm{YYYY}=-361.6070 \mathrm{ZZZZ}=-248.2853 \mathrm{XXXY}=56.5224$

$\mathrm{XXXZ}=-23.4153 \mathrm{YYYX}=-0.4746 \mathrm{YYYZ}=14.6499 \mathrm{ZZZX}=12.6999$

$\mathrm{ZZZY}=-3.0570 \mathrm{XXYY}=-311.1753 \mathrm{XXZZ}=-300.8968 \mathrm{YYZZ}=-105.0787$

$\mathrm{XXYZ}=22.3503 \mathrm{YYXZ}=-1.6336 \mathrm{ZZXY}=-2.9406$

$\mathrm{N}-\mathrm{N}=4.842885075609 \mathrm{D}+02 \mathrm{E}-\mathrm{N}=-1.952204945113 \mathrm{D}+03 \mathrm{KE}=4.205681202161 \mathrm{D}+02$

1I1|UNPC-UNKISPIRHF|6-31G(d)|C9H10O1|PCUSERI18-Apr-2006|0||\# HF/6-31G(

D) GEOM=CONNECTIVITY॥Title Card Required $\| 0,1 \mid \mathrm{C}, 0,-0.13241667,-0.3165$

$,-0.06052778|\mathrm{C}, 0,-2.89941667,0.1545,-0.24552778| \mathrm{C}, 0,-0.62641667,0.9845$

$,-0.23452778|\mathrm{C}, 0,-1.04541667,-1.3745,0.02847222| \mathrm{C}, 0,-2.41941667,-1.143$

$5,-0.06652778|\mathrm{C}, 0,-1.99841667,1.2185,-0.32752778| \mathrm{H}, 0,0.06658333,1.8195$

$,-0.29452778|\mathrm{H}, 0,-0.67841667,-2.3885,0.16647222| \mathrm{H}, 0,-3.11241667,-1.977$

$5,-0.00052778 \mathrm{IH}, 0,-2.36441667,2.2335,-0.46052778 \mathrm{IH}, 0,-3.96841667,0.336$

$5,-0.31852778|\mathrm{C}, 0,1.36958333,-0.5645,0.03247222| \mathrm{H}, 0,1.50858333,-1.6225$

,0.30647222|C, $0,1.92858333,0.2385,1.20147222 \mid \mathrm{O}, 0,2.79258333,1.0825,1.1$ 
$1047222|\mathrm{H}, 0,1.46158333,0.0045,2.18447222| \mathrm{C}, 0,2.12358333,-0.2745,-1.272$

$52778|\mathrm{H}, 0,2.02158333,0.7755,-1.55952778| \mathrm{H}, 0,1.73158333,-0.8945,-2.0845$

2778|H,0,3.19158333,-0.4815,-1.15452778||Version=x86-Win32-G03RevB.05।

State $=1-A|H F=-421.4943702| R M S D=1.934 \mathrm{e}-005 \mid D i p o l e=-0.9313881,-0.7840152$

,-0.1363317|PG=C01 [X(C9H10O1)]||@

The impersonal hand of government can

never replace the helping hand of a neighbor.

-- Hubert H. Humphrey

Job cpu time: 0 days 0 hours 3 minutes 6.0 seconds.

File lengths (MBytes): $\mathrm{RWF}=26 \mathrm{Int}=0 \mathrm{D} 2 \mathrm{E}=0 \mathrm{Chk}=8 \mathrm{Scr}=1$

Normal termination of Gaussian 03 at Tue Apr 18 15:49:14 2006. 


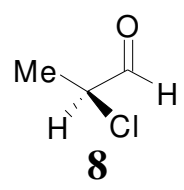

Entering Link 1 = C: $\mathrm{G} 03 \mathrm{~W} \backslash 11$.exe PID $=1820$.

Copyright (c) 1988,1990,1992,1993,1995,1998,2003, Gaussian, Inc. All Rights Reserved.

This is the Gaussian(R) 03 program. It is based on the the Gaussian(R) 98 system (copyright 1998, Gaussian, Inc.), the Gaussian(R) 94 system (copyright 1995, Gaussian, Inc.), the Gaussian 92(TM) system (copyright 1992, Gaussian, Inc.), the Gaussian 90(TM) system (copyright 1990, Gaussian, Inc.), the Gaussian 88(TM) system (copyright 1988, Gaussian, Inc.), the Gaussian 86(TM) system (copyright 1986, Carnegie Mellon University), and the Gaussian 82(TM) system (copyright 1983, Carnegie Mellon University). Gaussian is a federally registered trademark of Gaussian, Inc.

This software contains proprietary and confidential information, including trade secrets, belonging to Gaussian, Inc.

This software is provided under written license and may be used, copied, transmitted, or stored only in accord with that written license.

The following legend is applicable only to US Government contracts under DFARS:

\section{RESTRICTED RIGHTS LEGEND}

Use, duplication or disclosure by the US Government is subject to restrictions as set forth in subparagraph (c)(1)(ii) of the Rights in Technical Data and Computer Software clause at DFARS 252.227-7013.

Gaussian, Inc.

Carnegie Office Park, Building 6, Pittsburgh, PA 15106 USA

The following legend is applicable only to US Government contracts under FAR:

\section{RESTRICTED RIGHTS LEGEND}

Use, reproduction and disclosure by the US Government is subject to restrictions as set forth in subparagraph (c) of the Commercial Computer Software - Restricted Rights clause at FAR 52.227-19.

Gaussian, Inc.

Carnegie Office Park, Building 6, Pittsburgh, PA 15106 USA

Warning -- This program may not be used in any manner that competes with the business of Gaussian, Inc. or will provide assistance to any competitor of Gaussian, Inc. The licensee of this program is prohibited from giving any competitor of Gaussian, Inc. access to this program. By using this program, the user acknowledges that Gaussian, Inc. is engaged in the business of creating and licensing software in the field of 
computational chemistry and represents and warrants to the licensee that it is not a competitor of Gaussian, Inc. and that it will not use this program in any manner prohibited above.

Cite this work as:

Gaussian 03, Revision B.05,

M. J. Frisch, G. W. Trucks, H. B. Schlegel, G. E. Scuseria,

M. A. Robb, J. R. Cheeseman, J. A. Montgomery, Jr., T. Vreven,

K. N. Kudin, J. C. Burant, J. M. Millam, S. S. Iyengar, J. Tomasi,

V. Barone, B. Mennucci, M. Cossi, G. Scalmani, N. Rega,

G. A. Petersson, H. Nakatsuji, M. Hada, M. Ehara, K. Toyota,

R. Fukuda, J. Hasegawa, M. Ishida, T. Nakajima, Y. Honda, O. Kitao,

H. Nakai, M. Klene, X. Li, J. E. Knox, H. P. Hratchian, J. B. Cross,

C. Adamo, J. Jaramillo, R. Gomperts, R. E. Stratmann, O. Yazyev,

A. J. Austin, R. Cammi, C. Pomelli, J. W. Ochterski, P. Y. Ayala,

K. Morokuma, G. A. Voth, P. Salvador, J. J. Dannenberg,

V. G. Zakrzewski, S. Dapprich, A. D. Daniels, M. C. Strain,

O. Farkas, D. K. Malick, A. D. Rabuck, K. Raghavachari,

J. B. Foresman, J. V. Ortiz, Q. Cui, A. G. Baboul, S. Clifford,

J. Cioslowski, B. B. Stefanov, G. Liu, A. Liashenko, P. Piskorz,

I. Komaromi, R. L. Martin, D. J. Fox, T. Keith, M. A. Al-Laham,

C. Y. Peng, A. Nanayakkara, M. Challacombe, P. M. W. Gill,

B. Johnson, W. Chen, M. W. Wong, C. Gonzalez, and J. A. Pople, Gaussian, Inc., Pittsburgh PA, 2003.

*****************************************************

Gaussian 03: x86-Win32-G03RevB.05 8-Nov-2003

18-Apr-2006

$* * * * * * * * * * * * * * * * * * * * * * * * * * * * * * * * * * * * * * * * * * * * * *$

$\%$ chk $=$ cl-me-h-h.chk

$\%$ mem $=64 \mathrm{MB}$

$\%$ nproc $=1$

Will use up to 1 processors via shared memory.

\# hf/6-31g(d) geom=connectivity

$1 / 38=1,57=2 / 1$;

$2 / 17=6,18=5,40=1 / 2$;

$3 / 5=1,6=6,7=1,11=9,16=1,25=1,30=1 / 1,2,3$;

$4 / / 1$;

$5 / 5=2,32=1,38=5 / 2$;

$6 / 7=2,8=2,9=2,10=2,28=1 / 1$;

$99 / 5=1,9=1 / 99$;

Title Card Required

Symbolic Z-matrix:

Charge $=0$ Multiplicity $=1$

$\begin{array}{lrrr}\mathrm{C} & 1.02571 & -0.81704 & 0.24281 \\ \mathrm{H} & 1.99671 & -0.28104 & 0.26681 \\ \mathrm{O} & 0.95471 & -2.00504 & 0.03181 \\ \mathrm{C} & -0.19729 & 0.05596 & 0.51081 \\ \mathrm{H} & -0.14229 & 0.40596 & 1.54881 \\ \mathrm{C} & -1.51729 & -0.63504 & 0.22481 \\ \mathrm{H} & -1.57529 & -0.94504 & -0.82119 \\ \mathrm{H} & -2.35629 & 0.02796 & 0.44981 \\ \mathrm{H} & -1.59229 & -1.53104 & 0.84881 \\ \mathrm{Cl} & 0.00971 & 1.57296 & -0.49519\end{array}$

Input orientation:

Center Atomic Atomic Coordinates (Angstroms) 


\begin{tabular}{cccccc} 
Number & Number & Type & $X$ & $Y$ & $Z$ \\
\hline 1 & 6 & 0 & 1.025708 & -0.817042 & 0.242813 \\
2 & 1 & 0 & 1.996708 & -0.281042 & 0.266813 \\
3 & 8 & 0 & 0.954708 & -2.005042 & 0.031813 \\
4 & 6 & 0 & -0.197292 & 0.055958 & 0.510813 \\
5 & 1 & 0 & -0.142292 & 0.405958 & 1.548813 \\
6 & 6 & 0 & -1.517292 & -0.635042 & 0.224813 \\
7 & 1 & 0 & -1.575292 & -0.945042 & -0.821188 \\
8 & 1 & 0 & -2.356292 & 0.027958 & 0.449813 \\
9 & 1 & 0 & -1.592292 & -1.531042 & 0.848813 \\
10 & 17 & 0 & 0.009708 & 1.572958 & -0.495188
\end{tabular}

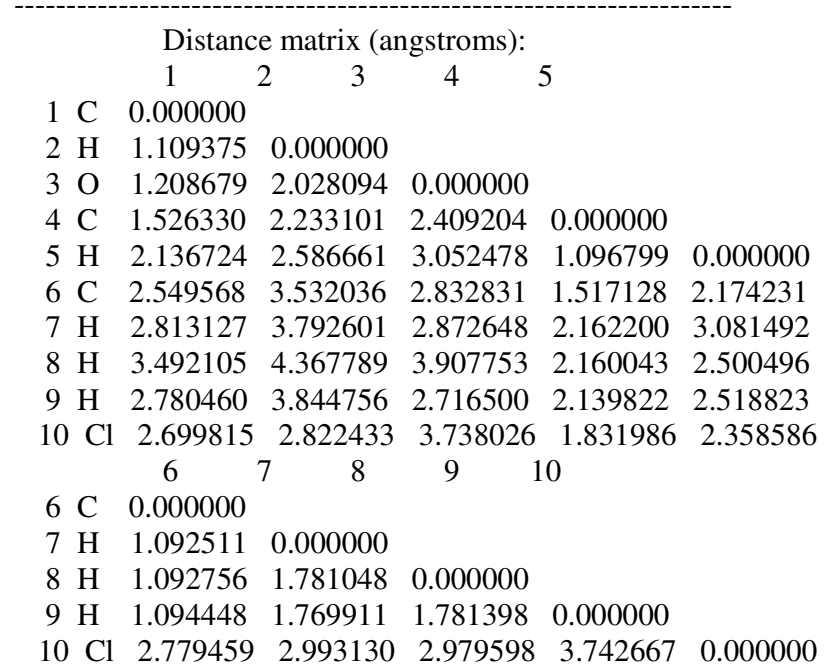

Stoichiometry $\mathrm{C} 3 \mathrm{H} 5 \mathrm{ClO}$

Framework group $\mathrm{C} 1[\mathrm{X}(\mathrm{C} 3 \mathrm{H} 5 \mathrm{ClO})]$

Deg. of freedom 24

Full point group $\quad \mathrm{C} 1 \quad \mathrm{NOp} 1$

Largest Abelian subgroup $\quad \mathrm{C} 1 \quad \mathrm{NOp} \quad 1$

Largest concise Abelian subgroup C1 NOp 1 Standard orientation:

\begin{tabular}{cccccc} 
Center & \multicolumn{2}{c}{ Atomic } & \multicolumn{2}{c}{ Atomic } & \multicolumn{2}{c}{ Coordinates (Angstroms) } \\
Number & Number & Type & X & Y & Z \\
- \hdashline 1 & 6 & 0 & -1.051219 & -0.800425 & 0.181250 \\
2 & 1 & 0 & -0.753380 & -1.839453 & 0.431102 \\
3 & 8 & 0 & -2.135748 & -0.539097 & -0.283948 \\
4 & 6 & 0 & 0.002799 & 0.261041 & 0.484597 \\
5 & 1 & 0 & 0.135661 & 0.304788 & 1.572441 \\
6 & 6 & 0 & -0.319463 & 1.626439 & -0.092906 \\
7 & 1 & 0 & -0.411860 & 1.576737 & -1.180368 \\
8 & 1 & 0 & 0.457503 & 2.348924 & 0.168730 \\
9 & 1 & 0 & -1.276589 & 1.967724 & 0.313606 \\
10 & 17 & 0 & 1.596585 & -0.386369 & -0.145387
\end{tabular}

Rotational constants (GHZ): $\quad 6.7488900 \quad 2.6798573 \quad 2.0674350$

Standard basis: 6-31G(d) $(6 \mathrm{D}, 7 \mathrm{~F})$

There are 89 symmetry adapted basis functions of A symmetry.

Integral buffers will be 262144 words long.

Raffenetti 1 integral format.

Two-electron integral symmetry is turned on.

89 basis functions, 184 primitive gaussians, 89 cartesian basis functions

24 alpha electrons 24 beta electrons

nuclear repulsion energy 213.6107127918 Hartrees.

NAtoms $=10$ NActive $=10 \mathrm{NUniq}=10 \mathrm{SFac}=1.00 \mathrm{D}+00 \mathrm{NAtFMM}=60 \mathrm{Big}=\mathrm{F}$

One-electron integrals computed using PRISM. 
NBasis $=89$ RedAO $=\mathrm{T}$ NBF $=89$

NBsUse $=891.00 \mathrm{D}-06 \mathrm{NBFU}=89$

Harris functional with IExCor= 205 diagonalized for initial guess.

ExpMin= 1.43D-01 ExpMax=2.52D+04 ExpMxC=3.78D+03 IAcc=1 IRadAn= $\quad 1$ AccDes= $1.00 \mathrm{D}-06$
HarFok: IExCor $=205$ AccDes= 1.00D-06 IRadAn $=\quad 1 \mathrm{IDoV}=1$

ScaDFX= 1.0000001 .0000001 .0000001 .000000

Initial guess orbital symmetries:

Occupied (A) (A) (A) (A) (A) (A) (A) (A) (A) (A) (A) (A)

$(\mathrm{A})(\mathrm{A})(\mathrm{A})(\mathrm{A})(\mathrm{A})(\mathrm{A})(\mathrm{A})(\mathrm{A})(\mathrm{A})(\mathrm{A})(\mathrm{A})(\mathrm{A})$

Virtual (A) (A) (A) (A) (A) (A) (A) (A) (A) (A) (A) (A)

(A) $(\mathrm{A})(\mathrm{A})(\mathrm{A})(\mathrm{A})(\mathrm{A})(\mathrm{A})(\mathrm{A})(\mathrm{A})(\mathrm{A})(\mathrm{A})(\mathrm{A})$

(A) $(A)(A)(A)(A)(A)(A)(A)(A)(A)(A)(A)$

(A) $(\mathrm{A})(\mathrm{A})(\mathrm{A})(\mathrm{A})(\mathrm{A})(\mathrm{A})(\mathrm{A})(\mathrm{A})(\mathrm{A})(\mathrm{A})(\mathrm{A})$

(A) $(A)(A)(A)(A)(A)(A)(A)(A)(A)(A)(A)$

(A) (A) (A) (A) (A)

The electronic state of the initial guess is 1-A.

Warning! Cutoffs for single-point calculations used.

Requested convergence on RMS density matrix=1.00D-04 within 128 cycles.

Requested convergence on MAX density matrix=1.00D-02.

Requested convergence on energy=5.00D-05.

No special actions if energy rises.

SCF Done: $\mathrm{E}(\mathrm{RHF})=-650.849127221 \quad$ A.U. after 6 cycles

Convg $=0.3487 \mathrm{D}-04 \quad-\mathrm{V} / \mathrm{T}=2.0011$

$\mathrm{S} * * 2=0.0000$

$* * * * * * * * * * * * * * * * * * * * * * * * * * * * * * * * * * * * * * * * * * * * * * * * * * * * * * * * * * * * * * * * * * * * * * *$

Population analysis using the SCF density.

$* * * * * * * * * * * * * * * * * * * * * * * * * * * * * * * * * * * * * * * * * * * * * * * * * * * * * * * * * * * * * * * * * * * * * *$

Orbital symmetries:

Occupied (A) (A) (A) (A) (A) (A) (A) (A) (A) (A) (A) (A)

(A) $(A)(A)(A)(A)(A)(A)(A)(A)(A)(A)(A)$

Virtual (A) (A) (A) (A) (A) (A) (A) (A) (A) (A) (A) (A)

(A) $(\mathrm{A})(\mathrm{A})(\mathrm{A})(\mathrm{A})(\mathrm{A})(\mathrm{A})(\mathrm{A})(\mathrm{A})(\mathrm{A})(\mathrm{A})(\mathrm{A})$

(A) $(\mathrm{A})(\mathrm{A})(\mathrm{A})(\mathrm{A})(\mathrm{A})(\mathrm{A})(\mathrm{A})(\mathrm{A})(\mathrm{A})(\mathrm{A})(\mathrm{A})$

(A) $(\mathrm{A})(\mathrm{A})(\mathrm{A})(\mathrm{A})(\mathrm{A})(\mathrm{A})(\mathrm{A})(\mathrm{A})(\mathrm{A})(\mathrm{A})(\mathrm{A})$

(A) $(\mathrm{A})(\mathrm{A})(\mathrm{A})(\mathrm{A})(\mathrm{A})(\mathrm{A})(\mathrm{A})(\mathrm{A})(\mathrm{A})(\mathrm{A})(\mathrm{A})$

(A) (A) (A) (A) (A)

The electronic state is $1-\mathrm{A}$.

Alpha occ. eigenvalues -- -104.82807 -20.59159 -11.35960 -11.32646 -11.24543

Alpha occ. eigenvalues -- $-10.56141 \quad-8.03062 \quad-8.02746 \quad-8.02737-1.41530$

Alpha occ. eigenvalues -- $\quad-1.15868 \quad-1.02254 \quad-0.92342 \quad-0.78837 \quad-0.70296$

Alpha occ. eigenvalues -- $\quad-0.65933-0.63677 \quad-0.57144 \quad-0.55237 \quad-0.54511$

Alpha occ. eigenvalues -- $-0.51064 \quad-0.47074-0.45121 \quad-0.43121$

$\begin{array}{lllllll}\text { Alpha virt. eigenvalues -- } & 0.11983 & 0.18313 & 0.24574 & 0.26356 & 0.27565\end{array}$

$\begin{array}{lllllll}\text { Alpha virt. eigenvalues -- } & 0.30923 & 0.32101 & 0.35324 & 0.41599 & 0.43650\end{array}$

$\begin{array}{lllllll}\text { Alpha virt. eigenvalues -- } & 0.56539 & 0.62527 & 0.64550 & 0.66319 & 0.71344\end{array}$

$\begin{array}{lllllll}\text { Alpha virt. eigenvalues -- } & 0.74480 & 0.76557 & 0.79931 & 0.80358 & 0.91305\end{array}$

$\begin{array}{lllllll}\text { Alpha virt. eigenvalues -- } & 0.93264 & 0.93431 & 1.01508 & 1.04138 & 1.08441\end{array}$

$\begin{array}{lllllll}\text { Alpha virt. eigenvalues -- } & 1.09544 & 1.10839 & 1.14524 & 1.15219 & 1.16896\end{array}$

$\begin{array}{lllllll}\text { Alpha virt. eigenvalues -- } & 1.19428 & 1.21459 & 1.24572 & 1.30302 & 1.32301\end{array}$

$\begin{array}{lllllll}\text { Alpha virt. eigenvalues -- } & 1.34904 & 1.38086 & 1.45238 & 1.64630 & 1.71862\end{array}$

$\begin{array}{lllllll}\text { Alpha virt. eigenvalues -- } & 1.79136 & 1.84063 & 1.88114 & 2.01787 & 2.05532\end{array}$

$\begin{array}{lllllll}\text { Alpha virt. eigenvalues -- } & 2.07842 & 2.18500 & 2.22865 & 2.27870 & 2.37690\end{array}$

$\begin{array}{lllllll}\text { Alpha virt. eigenvalues -- } & 2.42375 & 2.54888 & 2.59408 & 2.63865 & 2.70031\end{array}$

$\begin{array}{lllllll}\text { Alpha virt. eigenvalues -- } & 2.76004 & 2.90809 & 2.99955 & 3.20102 & 3.31153\end{array}$

Alpha virt. eigenvalues -- $4.24820 \quad 4.47583 \quad 4.62684 \quad 4.71196 \quad 4.85942$

Condensed to atoms (all electrons):

$\begin{array}{llllll}1 & 2 & 3 & 4 & 5 & 6\end{array}$

$\begin{array}{llllllll}1 & \mathrm{C} & 4.579787 & 0.396769 & 0.550755 & 0.264035 & -0.033240 & -0.041845\end{array}$

$\begin{array}{llllllll}2 & \mathrm{H} & 0.396769 & 0.542412 & -0.042853 & -0.093751 & 0.001538 & 0.004205\end{array}$

$\begin{array}{lllllllll}3 & \mathrm{O} & 0.550755 & -0.042853 & 8.022536 & -0.077648 & 0.000871 & 0.007908\end{array}$ 
$\begin{array}{llllllll}4 & \mathrm{C} & 0.264035 & -0.093751 & -0.077648 & 5.470940 & 0.379592 & 0.280557\end{array}$

$\begin{array}{llllllll}5 \mathrm{H} & -0.033240 & 0.001538 & 0.000871 & 0.379592 & 0.490129 & -0.032635\end{array}$

$\begin{array}{llllllll}6 \mathrm{C} & -0.041845 & 0.004205 & 0.007908 & 0.280557 & -0.032635 & 5.139922\end{array}$

$\begin{array}{llllllll}7 & \mathrm{H} & -0.003150 & 0.000007 & 0.001284 & -0.038133 & 0.003453 & 0.395294\end{array}$

$\begin{array}{llllllll}8 \mathrm{H} & 0.003922 & -0.000020 & 0.000223 & -0.037956 & -0.002720 & 0.393879\end{array}$

$\begin{array}{llllllll}9 \mathrm{H} & -0.003844 & 0.000039 & 0.002282 & -0.030892 & -0.001464 & 0.383179\end{array}$

$\begin{array}{llllllll}10 & C l & -0.059548 & 0.004781 & 0.002148 & 0.219015 & -0.046569 & -0.062094\end{array}$ $\begin{array}{llll}7 & 8 & 9 & 10\end{array}$

$\begin{array}{llllll}1 & \mathrm{C} & -0.003150 & 0.003922 & -0.003844 & -0.059548\end{array}$

$\begin{array}{llllll}2 & \mathrm{H} & 0.000007 & -0.000020 & 0.000039 & 0.004781\end{array}$

$\begin{array}{llllll}3 & \mathrm{O} & 0.001284 & 0.000223 & 0.002282 & 0.002148\end{array}$

$\begin{array}{llllll}4 & \mathrm{C} & -0.038133 & -0.037956 & -0.030892 & 0.219015\end{array}$

$\begin{array}{llllll}5 \mathrm{H} & 0.003453 & -0.002720 & -0.001464 & -0.046569\end{array}$

$\begin{array}{llllll}6 \mathrm{C} & 0.395294 & 0.393879 & 0.383179 & -0.062094\end{array}$

$\begin{array}{llllll}7 \mathrm{H} & 0.485619 & -0.023193 & -0.021671 & -0.000311\end{array}$

$\begin{array}{lllllll}8 \mathrm{H} & -0.023193 & 0.501427 & -0.024315 & -0.001814\end{array}$

$\begin{array}{llllll}9 \mathrm{H} & -0.021671 & -0.024315 & 0.494049 & 0.003949\end{array}$

$10 \mathrm{Cl}-0.000311 \quad-0.001814 \quad 0.003949 \quad 17.033139$

Mulliken atomic charges:
1
1 C 0.346359
$2 \mathrm{H} \quad 0.186873$
$\begin{array}{lll}3 & \mathrm{O} & -0.467507\end{array}$
$\begin{array}{lll}4 & \mathrm{C} & -0.335759\end{array}$
$\begin{array}{lll}5 \mathrm{H} & 0.241045\end{array}$
6 C -0.468371
$7 \mathrm{H} \quad 0.200802$
$8 \mathrm{H} \quad 0.190565$
$9 \mathrm{H} \quad 0.198689$
$10 \mathrm{Cl}-0.092696$

Sum of Mulliken charges $=0.00000$

Atomic charges with hydrogens summed into heavy atoms: 1

1 C 0.533232

$2 \mathrm{H} \quad 0.000000$

$3 \mathrm{O}-0.467507$

4 C -0.094713

$\begin{array}{lll}5 \mathrm{H} & 0.000000\end{array}$

$6 \mathrm{C} \quad 0.121685$

$7 \mathrm{H} \quad 0.000000$

$8 \mathrm{H} \quad 0.000000$

$9 \mathrm{H} \quad 0.000000$

$10 \mathrm{Cl}-0.092696$

Sum of Mulliken charges $=0.00000$

Electronic spatial extent $(\mathrm{au}):\langle\mathrm{R} * * 2\rangle=575.5880$

Charge $=0.0000$ electrons

Dipole moment (field-independent basis, Debye):

$\mathrm{X}=0.5638 \mathrm{Y}=0.6830 \mathrm{Z}=1.7611$ Tot $=1.9712$

Quadrupole moment (field-independent basis, Debye-Ang):

$\mathrm{XX}=-46.6518 \quad \mathrm{YY}=-34.6044 \quad \mathrm{ZZ}=-35.6424$

$\mathrm{XY}=-0.5077 \quad \mathrm{XZ}=-1.7983 \quad \mathrm{YZ}=-0.5406$

Traceless Quadrupole moment (field-independent basis, Debye-Ang):

$\mathrm{XX}=-7.6855 \mathrm{YY}=4.3618 \quad \mathrm{ZZ}=3.3238$

$\mathrm{XY}=-0.5077 \quad \mathrm{XZ}=-1.7983 \quad \mathrm{YZ}=-0.5406$

Octapole moment (field-independent basis, Debye-Ang**2):

$\mathrm{XXX}=14.1838 \mathrm{YYY}=-3.4220 \mathrm{ZZZ}=0.5850 \mathrm{XYY}=-1.7017$

$\mathrm{XXY}=3.1856 \mathrm{XXZ}=2.5537 \mathrm{XZZ}=-1.4119 \mathrm{YZZ}=-0.5806$

$\mathrm{YYZ}=0.6564 \mathrm{XYZ}=1.6871$

Hexadecapole moment (field-independent basis, Debye-Ang**3):

$\mathrm{XXXX}=-467.9364 \mathrm{YYYY}=-220.0885 \mathrm{ZZZZ}=-61.8883 \mathrm{XXXY}=-13.4198$

$\mathrm{XXXZ}=-11.1126 \mathrm{YYYX}=5.0493 \mathrm{YYYZ}=-0.1591 \mathrm{ZZZX}=2.1375$

$\mathrm{ZZZY}=-0.1850 \mathrm{XXYY}=-109.7545 \mathrm{XXZZ}=-83.0983 \mathrm{YYZZ}=-49.0389$

$\mathrm{XXYZ}=0.5751 \mathrm{YYXZ}=-1.1039 \mathrm{ZZXY}=0.6121$

$\mathrm{N}-\mathrm{N}=2.136107127918 \mathrm{D}+02 \mathrm{E}-\mathrm{N}=-1.969380969574 \mathrm{D}+03 \quad \mathrm{KE}=6.501385700465 \mathrm{D}+02$ 
1|1|UNPC-UNKISP|RHF|6-31G(d)|C3H5Cl1O1|PCUSER|18-Apr-2006|0||\# HF/6-31 G(D) GEOM=CONNECTIVITY॥Title Card Required $\| 0,1 \mid \mathrm{C}, 0,1.02570833,-0.817$

$04167,0.2428125|\mathrm{H}, 0,1.99670833,-0.28104167,0.2668125| \mathrm{O}, 0,0.95470833,-2$ $.00504167,0.0318125|\mathrm{C}, 0,-0.19729167,0.05595833,0.5108125| \mathrm{H}, 0,-0.142291$ $67,0.40595833,1.5488125|\mathrm{C}, 0,-1.51729167,-0.63504167,0.2248125| \mathrm{H}, 0,-1.5$ $7529167,-0.94504167,-0.8211875 \mathrm{lH}, 0,-2.35629167,0.02795833,0.4498125 \mathrm{lH}$, $0,-1.59229167,-1.53104167,0.8488125 \mid \mathrm{Cl}, 0,0.00970833,1.57295833,-0.4951$ $875 \|$ Version $=x 86-$ Win32-G03RevB $.05 \mid$ State $=1-A|H F=-650.8491272| \mathrm{RMSD}=3.487$ e-005|Dipole=-0.2282471,0.3086535,0.6738593|PG=C01 [X(C3H5Cl1O1)]||@

Once you get people laughing, they're

listening and you can tell them almost anything.

-- Herb Gardner

Job cpu time: 0 days 0 hours 0 minutes 32.0 seconds.

File lengths (MBytes): $\mathrm{RWF}=15 \mathrm{Int}=0 \mathrm{D} 2 \mathrm{E}=0 \mathrm{Chk}=7 \mathrm{Scr}=1$

Normal termination of Gaussian 03 at Tue Apr 18 16:01:53 2006. 


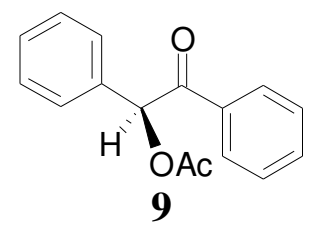

Entering Link 1 = C:\G03W11.exe PID= 3788.

Copyright (c) 1988,1990,1992,1993,1995,1998,2003, Gaussian, Inc. All Rights Reserved.

This is the Gaussian(R) 03 program. It is based on the the Gaussian(R) 98 system (copyright 1998, Gaussian, Inc.), the Gaussian(R) 94 system (copyright 1995, Gaussian, Inc.), the Gaussian 92(TM) system (copyright 1992, Gaussian, Inc.), the Gaussian 90(TM) system (copyright 1990, Gaussian, Inc.), the Gaussian 88(TM) system (copyright 1988, Gaussian, Inc.), the Gaussian 86(TM) system (copyright 1986, Carnegie Mellon University), and the Gaussian 82(TM) system (copyright 1983, Carnegie Mellon University). Gaussian is a federally registered trademark of Gaussian, Inc.

This software contains proprietary and confidential information, including trade secrets, belonging to Gaussian, Inc.

This software is provided under written license and may be used, copied, transmitted, or stored only in accord with that written license.

The following legend is applicable only to US Government contracts under DFARS:

\section{RESTRICTED RIGHTS LEGEND}

Use, duplication or disclosure by the US Government is subject to restrictions as set forth in subparagraph (c)(1)(ii) of the Rights in Technical Data and Computer Software clause at DFARS 252.227-7013.

Gaussian, Inc.

Carnegie Office Park, Building 6, Pittsburgh, PA 15106 USA

The following legend is applicable only to US Government contracts under FAR:

\section{RESTRICTED RIGHTS LEGEND}

Use, reproduction and disclosure by the US Government is subject to restrictions as set forth in subparagraph (c) of the Commercial Computer Software - Restricted Rights clause at FAR 52.227-19.

Gaussian, Inc.

Carnegie Office Park, Building 6, Pittsburgh, PA 15106 USA

Warning -- This program may not be used in any manner that competes with the business of Gaussian, Inc. or will provide assistance to any competitor of Gaussian, Inc. The licensee of this program is prohibited from giving any competitor of Gaussian, Inc. access to this program. By using this program, the user acknowledges that Gaussian, Inc. is engaged in the 
business of creating and licensing software in the field of computational chemistry and represents and warrants to the licensee that it is not a competitor of Gaussian, Inc. and that it will not use this program in any manner prohibited above.

Cite this work as:

Gaussian 03, Revision B.05,

M. J. Frisch, G. W. Trucks, H. B. Schlegel, G. E. Scuseria,

M. A. Robb, J. R. Cheeseman, J. A. Montgomery, Jr., T. Vreven,

K. N. Kudin, J. C. Burant, J. M. Millam, S. S. Iyengar, J. Tomasi,

V. Barone, B. Mennucci, M. Cossi, G. Scalmani, N. Rega,

G. A. Petersson, H. Nakatsuji, M. Hada, M. Ehara, K. Toyota,

R. Fukuda, J. Hasegawa, M. Ishida, T. Nakajima, Y. Honda, O. Kitao,

H. Nakai, M. Klene, X. Li, J. E. Knox, H. P. Hratchian, J. B. Cross,

C. Adamo, J. Jaramillo, R. Gomperts, R. E. Stratmann, O. Yazyev,

A. J. Austin, R. Cammi, C. Pomelli, J. W. Ochterski, P. Y. Ayala,

K. Morokuma, G. A. Voth, P. Salvador, J. J. Dannenberg,

V. G. Zakrzewski, S. Dapprich, A. D. Daniels, M. C. Strain,

O. Farkas, D. K. Malick, A. D. Rabuck, K. Raghavachari,

J. B. Foresman, J. V. Ortiz, Q. Cui, A. G. Baboul, S. Clifford,

J. Cioslowski, B. B. Stefanov, G. Liu, A. Liashenko, P. Piskorz,

I. Komaromi, R. L. Martin, D. J. Fox, T. Keith, M. A. Al-Laham,

C. Y. Peng, A. Nanayakkara, M. Challacombe, P. M. W. Gill,

B. Johnson, W. Chen, M. W. Wong, C. Gonzalez, and J. A. Pople, Gaussian, Inc., Pittsburgh PA, 2003.

$* * * * * * * * * * * * * * * * * * * * * * * * * * * * * * * * * * * * * * * * * * * * * *$

Gaussian 03: x86-Win32-G03RevB.05 8-Nov-2003

19-Apr-2006

***************************************************

$\%$ chk=oac-ph-h-ph.chk

$\%$ mem $=64 \mathrm{MB}$

$\%$ nproc $=1$

Will use up to 1 processors via shared memory.

\# hf/6-31g(d) geom=connectivity

$1 / 38=1,57=2 / 1$;

$2 / 17=6,18=5,40=1 / 2$;

$3 / 5=1,6=6,7=1,11=9,16=1,25=1,30=1 / 1,2,3$;

$4 / / 1$

$5 / 5=2,32=1,38=5 / 2$;

$6 / 7=2,8=2,9=2,10=2,28=1 / 1$;

$99 / 5=1,9=1 / 99$;

Title Card Required

Symbolic Z-matrix:

Charge $=0$ Multiplicity $=1$

\begin{tabular}{|c|c|c|c|}
\hline $\mathrm{H}$ & -2.37925 & -1.5921 & 1.95901 \\
\hline $\mathrm{C}$ & -2.70925 & -1.0191 & 1.09901 \\
\hline $\mathrm{C}$ & -3.49525 & 0.4549 & -1.13499 \\
\hline $\mathrm{C}$ & -1.73625 & -0.5421 & 0.20601 \\
\hline $\mathrm{C}$ & -4.05825 & -0.7631 & 0.87801 \\
\hline $\mathrm{C}$ & -4.45425 & -0.0251 & -0.24099 \\
\hline $\mathrm{C}$ & -2.14325 & 0.1979 & -0.91399 \\
\hline $\mathrm{H}$ & -4.80225 & -1.1381 & 1.57501 \\
\hline $\mathrm{H}$ & -5.50825 & 0.1759 & -0.41499 \\
\hline $\mathrm{H}$ & -1.41325 & 0.5849 & -1.61699 \\
\hline $\mathrm{H}$ & -3.80025 & 1.0289 & -2.00499 \\
\hline $\mathrm{C}$ & -0.30825 & -0.8641 & 0.50801 \\
\hline $\mathrm{O}$ & 0.00175 & -1.5181 & 1.48901 \\
\hline
\end{tabular}




$\begin{array}{lccc}\mathrm{C} & 0.80575 & -0.3151 & -0.41599 \\ \mathrm{H} & 0.51175 & -0.4381 & -1.46199 \\ \mathrm{C} & 1.11375 & 1.1369 & -0.10899 \\ \mathrm{C} & 1.72275 & 3.8119 & 0.46201 \\ \mathrm{C} & 1.41975 & 1.5279 & 1.20101 \\ \mathrm{C} & 1.12075 & 2.0949 & -1.12799 \\ \mathrm{C} & 1.42075 & 3.4279 & -0.84499 \\ \mathrm{C} & 1.72375 & 2.8589 & 1.48301 \\ \mathrm{H} & 1.41975 & 0.7849 & 1.99301 \\ \mathrm{H} & 0.89975 & 1.7959 & -2.14999 \\ \mathrm{H} & 1.42475 & 4.1629 & -1.64499 \\ \mathrm{H} & 1.96175 & 3.1519 & 2.50201 \\ \mathrm{H} & 1.95875 & 4.8489 & 0.68401 \\ \mathrm{C} & 2.00375 & -2.3411 & -0.64999 \\ \mathrm{O} & 1.09175 & -2.8101 & -1.29299 \\ \mathrm{O} & 2.01275 & -1.0641 & -0.18699 \\ \mathrm{C} & 3.26675 & -3.0621 & -0.24699 \\ \mathrm{H} & 3.31675 & -4.0231 & -0.75999 \\ \mathrm{H} & 3.25375 & -3.2241 & 0.83701 \\ \mathrm{H} & 4.14775 & -2.4571 & -0.48099\end{array}$

Input orientation:

\begin{tabular}{|c|c|c|c|c|c|}
\hline \multirow{2}{*}{$\begin{array}{l}\text { Center } \\
\text { Number }\end{array}$} & Atomic & \multicolumn{2}{|c|}{ Atomic } & \multicolumn{2}{|c|}{ Coordinates (Angstroms } \\
\hline & Numbe & & Type & X $\quad Y$ & $\mathrm{Z}$ \\
\hline 1 & 1 & 0 & -2.379246 & -1.592097 & 1.959007 \\
\hline 2 & 6 & 0 & -2.709246 & -1.019097 & 1.099007 \\
\hline 3 & 6 & 0 & -3.495246 & 0.454903 & -1.134993 \\
\hline 4 & 6 & 0 & -1.736246 & -0.542097 & 0.206007 \\
\hline 5 & 6 & 0 & -4.058246 & -0.763097 & 0.878007 \\
\hline 6 & 6 & 0 & -4.454246 & -0.025097 & -0.240993 \\
\hline 7 & 6 & 0 & -2.143246 & 0.197903 & -0.913993 \\
\hline 8 & 1 & 0 & -4.802246 & -1.138097 & 1.575007 \\
\hline 9 & 1 & 0 & -5.508246 & 0.175903 & -0.414993 \\
\hline 10 & 1 & 0 & -1.413246 & 0.584903 & -1.616993 \\
\hline 11 & 1 & 0 & -3.800246 & 1.028903 & -2.004993 \\
\hline 12 & 6 & 0 & -0.308246 & -0.864097 & 0.508007 \\
\hline 13 & 8 & 0 & 0.001754 & -1.518097 & 1.489007 \\
\hline 14 & 6 & 0 & 0.805754 & -0.315097 & -0.415993 \\
\hline 15 & 1 & 0 & 0.511754 & -0.438097 & -1.461993 \\
\hline 16 & 6 & 0 & 1.113754 & 1.136903 & -0.108993 \\
\hline 17 & 6 & 0 & 1.722754 & 3.811903 & 0.462007 \\
\hline 18 & 6 & 0 & 1.419754 & 1.527903 & 1.201007 \\
\hline 19 & 6 & 0 & 1.120754 & 2.094903 & -1.127993 \\
\hline 20 & 6 & 0 & 1.420754 & 3.427903 & -0.844993 \\
\hline 21 & 6 & 0 & 1.723754 & 2.858903 & 1.483007 \\
\hline 22 & 1 & 0 & 1.419754 & 0.784903 & 1.993007 \\
\hline 23 & 1 & 0 & 0.899754 & 1.795903 & -2.149993 \\
\hline 24 & 1 & 0 & 1.424754 & 4.162903 & -1.644993 \\
\hline 25 & 1 & 0 & 1.961754 & 3.151903 & 2.502007 \\
\hline 26 & 1 & 0 & 1.958754 & 4.848903 & 0.684007 \\
\hline 27 & 6 & 0 & 2.003754 & -2.341097 & -0.649993 \\
\hline 28 & 8 & 0 & 1.091754 & -2.810097 & -1.292993 \\
\hline 29 & 8 & 0 & 2.012754 & -1.064097 & -0.186993 \\
\hline 30 & 6 & 0 & 3.266754 & -3.062097 & -0.246993 \\
\hline 31 & 1 & 0 & 3.316754 & -4.023097 & -0.759993 \\
\hline 32 & 1 & 0 & 3.253754 & -3.224097 & 0.837007 \\
\hline 33 & 1 & 0 & 4.147754 & -2.457097 & -0.480993 \\
\hline
\end{tabular}

Distance matrix (angstroms):

$1 \mathrm{H} \quad 0.000000$

2 C $1.084817 \quad 0.000000$ 


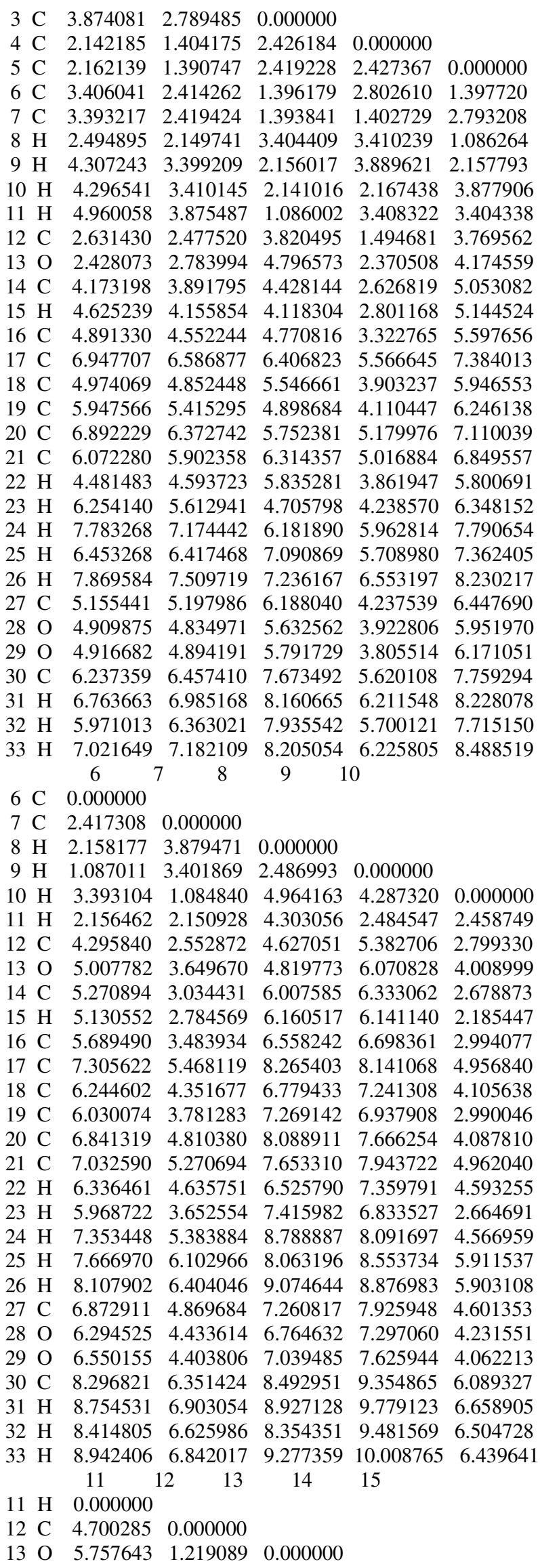


$\begin{array}{llllll}14 & \mathrm{C} & 5.054354 & 1.547958 & 2.392206 & 0.000000\end{array}$

$\begin{array}{lllllll}15 & \mathrm{H} & 4.586969 & 2.175954 & 3.183536 & 1.093472 & 0.000000\end{array}$

$\begin{array}{lllllll}16 \mathrm{C} & 5.268195 & 2.531161 & 3.292290 & 1.515723 & 2.161860\end{array}$

$\begin{array}{lllllll}17 & \mathrm{C} & 6.658431 & 5.098240 & 5.694337 & 4.317858 & 4.819834\end{array}$

$\begin{array}{lllllll}18 & \mathrm{C} & 6.146205 & 3.031154 & 3.372208 & 2.527515 & 3.432374\end{array}$

$\begin{array}{lllllll}19 & \mathrm{C} & 5.110942 & 3.670724 & 4.599415 & 2.532641 & 2.626505\end{array}$

$\begin{array}{lllllll}20 \mathrm{C} & 5.861710 & 4.820925 & 5.650136 & 3.817370 & 4.019070\end{array}$

$\begin{array}{llllllll}21 & \mathrm{C} & 6.784513 & 4.352054 & 4.703557 & 3.810932 & 4.583904\end{array}$

$\begin{array}{llllllll}22 & \mathrm{H} & 6.579661 & 2.812545 & 2.751100 & 2.718506 & 3.775873\end{array}$

$\begin{array}{lllllll}23 & \mathrm{H} & 4.764380 & 3.949662 & 5.003131 & 2.733480 & 2.369524\end{array}$

$\begin{array}{llllllll}24 \mathrm{H} & 6.103456 & 5.736674 & 6.642337 & 4.684665 & 4.694279\end{array}$

$\begin{array}{llllllll}25 & \mathrm{H} & 7.617140 & 5.025653 & 5.164946 & 4.676660 & 5.541110\end{array}$

$\begin{array}{lllllll}26 & \mathrm{H} & 7.415470 & 6.148873 & 6.709438 & 5.404286 & 5.886552\end{array}$

$\begin{array}{llllllll}27 & \mathrm{C} & 6.846849 & 2.977891 & 3.043132 & 2.365298 & 2.550846\end{array}$

$\begin{array}{llllllll}28 & \mathrm{O} & 6.259116 & 2.998419 & 3.255286 & 2.660066 & 2.447722\end{array}$

$\begin{array}{llllllll}29 & \mathrm{O} & 6.440244 & 2.431063 & 2.656918 & 1.438851 & 2.066519\end{array}$

$\begin{array}{lllllll}30 & \mathrm{C} & 8.352804 & 4.264019 & 4.007226 & 3.692031 & 3.993949\end{array}$

$\begin{array}{llllllll}31 & \mathrm{H} & 8.816145 & 4.972698 & 4.724643 & 4.491405 & 4.605763\end{array}$

$\begin{array}{llllllll}32 & \mathrm{H} & 8.713432 & 4.285520 & 3.729751 & 4.003123 & 4.534949\end{array}$

$\begin{array}{llllllll}33 & \mathrm{H} & 8.811667 & 4.834429 & 4.685289 & 3.970057 & 4.273081\end{array}$

$\begin{array}{ll}16 \mathrm{C} & 0.000000\end{array}$ $\begin{array}{lllll}16 & 17 & 18 & 19 & 20\end{array}$

$\begin{array}{llll}17 & \mathrm{C} & 2.802240 & 0.000000\end{array}$

$\begin{array}{lllll}18 & \mathrm{C} & 1.400934 & 2.419625 & 0.000000\end{array}$

$\begin{array}{llllll}19 & \mathrm{C} & 1.398633 & 2.416318 & 2.415602 & 0.000000\end{array}$

$\begin{array}{lllllll}20 & \mathrm{C} & 2.425825 & 1.395317 & 2.792153 & 1.395342 & 0.000000\end{array}$

$\begin{array}{lllllll}21 & \mathrm{C} & 2.423190 & 1.396657 & 1.394095 & 2.786508 & 2.415606\end{array}$

$\begin{array}{lllllll}22 & \mathrm{H} & 2.153124 & 3.405657 & 1.085962 & 3.397961 & 3.878104\end{array}$

$\begin{array}{lllllll}23 & \mathrm{H} & 2.155402 & 3.400607 & 3.401680 & 1.087532 & 2.153576\end{array}$

$\begin{array}{lllllll}24 & \mathrm{H} & 3.407740 & 2.156723 & 3.878526 & 2.153214 & 1.086389\end{array}$

$\begin{array}{lllllll}25 & \mathrm{H} & 3.405385 & 2.157388 & 2.150289 & 3.873168 & 3.401656\end{array}$

$\begin{array}{lllllll}26 \mathrm{H} & 3.888678 & 1.086439 & 3.403946 & 3.401486 & 2.155580\end{array}$

$\begin{array}{llllllll}27 & \mathrm{C} & 3.630601 & 6.258987 & 4.328558 & 4.548216 & 5.801661\end{array}$

$\begin{array}{llllllll}28 & \mathrm{O} & 4.120819 & 6.879613 & 5.014565 & 4.907860 & 6.262714\end{array}$

$\begin{array}{llllllll}29 & \mathrm{O} & 2.378799 & 4.927543 & 2.999443 & 3.414737 & 4.578372\end{array}$

$\begin{array}{lllllll}30 & \mathrm{C} & 4.720811 & 7.080854 & 5.155212 & 5.654744 & 6.773878\end{array}$

$\begin{array}{llllllll}31 & \mathrm{H} & 5.648240 & 8.088346 & 6.185283 & 6.510589 & 7.688917\end{array}$

$\begin{array}{llllllll}32 & \mathrm{H} & 4.949024 & 7.210401 & 5.106619 & 6.058273 & 7.101980\end{array}$

$\begin{array}{lllllll}33 & \mathrm{H} & 4.718090 & 6.787506 & 5.113837 & 5.504729 & 6.496326\end{array}$ $\begin{array}{lllll}21 & 22 & 23 & 24 & 25\end{array}$

$\begin{array}{lll}21 \mathrm{C} & 0.000000\end{array}$

$\begin{array}{llll}22 & \mathrm{H} & 2.157311 & 0.000000\end{array}$

$\begin{array}{lllll}23 & \mathrm{H} & 3.873969 & 4.296158 & 0.000000\end{array}$

$\begin{array}{llllll}24 & \mathrm{H} & 3.402088 & 4.964469 & 2.476558 & 0.000000\end{array}$

$\begin{array}{lllllll}25 & \mathrm{H} & 1.086671 & 2.481035 & 4.960613 & 4.302104 & 0.000000\end{array}$

$\begin{array}{llllllll}26 \mathrm{H} & 2.157250 & 4.303498 & 4.298121 & 2.485959 & 2.486954\end{array}$

$\begin{array}{llllllll}27 & \mathrm{C} & 5.627441 & 4.135019 & 4.536914 & 6.605095 & 6.333239\end{array}$

$\begin{array}{llllllll}28 & \mathrm{O} & 6.343750 & 4.881537 & 4.688982 & 6.989816 & 7.120700\end{array}$

$\begin{array}{llllllll}29 & \mathrm{O} & 4.273447 & 2.919392 & 3.643040 & 5.458300 & 5.000798\end{array}$

$\begin{array}{llllllll}30 & \mathrm{C} & 6.358615 & 4.819587 & 5.729246 & 7.586039 & 6.919091\end{array}$

$\begin{array}{llllllll}31 & \mathrm{H} & 7.411520 & 5.856149 & 6.452500 & 8.448283 & 7.997330\end{array}$

$\begin{array}{llllllll}32 & \mathrm{H} & 6.305641 & 4.557628 & 6.297927 & 8.004582 & 6.715271\end{array}$

$\begin{array}{lllllll}33 & \mathrm{H} & 6.163840 & 4.906447 & 5.605629 & 7.252174 & 6.718465\end{array}$ $\begin{array}{lllll}26 & 27 & 28 & 29 & 30\end{array}$

$\begin{array}{ll}26 \mathrm{H} & 0.000000\end{array}$

$\begin{array}{llll}27 & \mathrm{C} & 7.312844 & 0.000000\end{array}$

$\begin{array}{lllll}28 & \mathrm{O} & 7.957418 & 1.210435 & 0.000000\end{array}$

$\begin{array}{lllllll}29 & \mathrm{O} & 5.977050 & 1.358374 & 2.262740 & 0.000000\end{array}$

$\begin{array}{lllllll}30 \mathrm{C} & 8.072270 & 1.509112 & 2.426571 & 2.359686 & 0.000000\end{array}$

$\begin{array}{lllllll}31 & \mathrm{H} & 9.090747 & 2.136631 & 2.589611 & 3.283965 & 1.090500\end{array}$

$\begin{array}{lllllll}32 & \mathrm{H} & 8.177638 & 2.133860 & 3.063093 & 2.693373 & 1.096115\end{array}$

$\begin{array}{lllllll}33 & \mathrm{H} & 7.715347 & 2.153776 & 3.181680 & 2.566147 & 1.094048\end{array}$ $31 \quad 32 \quad 33$

$31 \mathrm{H} \quad 0.000000$ 
$\begin{array}{llll}32 & \mathrm{H} & 1.786835 & 0.000000\end{array}$

$\begin{array}{lllll}33 & \mathrm{H} & 1.794647 & 1.767668 & 0.000000\end{array}$

Stoichiometry $\mathrm{C} 16 \mathrm{H} 14 \mathrm{O} 3$

Framework group $\mathrm{C} 1[\mathrm{X}(\mathrm{C} 16 \mathrm{H} 14 \mathrm{O} 3)]$

Deg. of freedom 93

Full point group $\quad \mathrm{C} 1 \quad \mathrm{NOp} 1$

Largest Abelian subgroup $\quad \mathrm{C} 1 \quad$ NOp 1

Largest concise Abelian subgroup C1 NOp 1 Standard orientation:

\begin{tabular}{|c|c|c|c|c|}
\hline \multirow{2}{*}{$\begin{array}{l}\text { Center } \\
\text { Number }\end{array}$} & Atomic & Atomic & \multicolumn{2}{|c|}{ Coordinates (Angstroms } \\
\hline & Number & r Type & X $\quad Y$ & $\mathrm{Z}$ \\
\hline 1 & 1 & -2.615473 & -1.128607 & 1.979606 \\
\hline 2 & 6 & -2.845325 & -0.559485 & 1.085125 \\
\hline 3 & 6 & -3.374033 & 0.894347 & -1.236096 \\
\hline 4 & 6 & -1.799544 & -0.274747 & 0.192390 \\
\hline 5 & 6 & -4.139269 & -0.123554 & 0.820832 \\
\hline 6 & 6 & -4.406415 & 0.604679 & -0.341894 \\
\hline 7 & 6 & -2.077266 & 0.456996 & -0.971685 \\
\hline 8 & 1 & -4.940631 & -0.350684 & 1.518112 \\
\hline 9 & 1 & -5.417235 & 0.946182 & -0.549766 \\
\hline 10 & 1 & -1.287918 & 0.697691 & -1.675869 \\
\hline 11 & 1 & -3.578850 & 1.460336 & -2.140037 \\
\hline 12 & 6 & -0.438292 & -0.783077 & 0.542625 \\
\hline 13 & 8 & -0.241875 & -1.423075 & 1.561448 \\
\hline 14 & 6 & 0.757912 & -0.449122 & -0.381357 \\
\hline 15 & 1 & 0.465587 & -0.582634 & -1.426538 \\
\hline 16 & 6 & 1.268514 & 0.957291 & -0.139068 \\
\hline 17 & 6 & 2.250236 & 3.542596 & 0.313665 \\
\hline 18 & 6 & 1.607468 & 1.367624 & 1.156831 \\
\hline 19 & 6 & 1.430413 & 1.850036 & -1.203478 \\
\hline 20 & 6 & 1.916208 & 3.138761 & -0.979490 \\
\hline 21 & 6 & 2.096946 & 2.653743 & 1.380011 \\
\hline 22 & 1 & 1.487249 & 0.674539 & 1.984173 \\
\hline 23 & 1 & 1.184390 & 1.533328 & -2.214366 \\
\hline 24 & 1 & 2.039349 & 3.822948 & -1.814335 \\
\hline 25 & 1 & 2.358963 & 2.961916 & 2.388589 \\
\hline 26 & 1 & 2.630717 & 4.544871 & 0.489814 \\
\hline 27 & 6 & 1.652743 & -2.636029 & -0.487898 \\
\hline 28 & 8 & 0.692518 & -3.001447 & -1.127891 \\
\hline 29 & 8 & 1.839710 & -1.351350 & -0.088119 \\
\hline 30 & 6 & 2.791281 & -3.509620 & -0.021004 \\
\hline 31 & 1 & 2.709321 & -4.493131 & -0.484878 \\
\hline 32 & 1 & 2.737941 & -3.611521 & 1.069061 \\
\hline 33 & 1 & 3.754304 & -3.050899 & -0.264115 \\
\hline
\end{tabular}

Rotational constants (GHZ): $\quad 0.4152236 \quad 0.3269597 \quad 0.2187432$

Standard basis: 6-31G(d) (6D, 7F)

There are 313 symmetry adapted basis functions of A symmetry.

Integral buffers will be 262144 words long.

Raffenetti 1 integral format.

Two-electron integral symmetry is turned on.

313 basis functions, 588 primitive gaussians, 313 cartesian basis functions

67 alpha electrons 67 beta electrons

nuclear repulsion energy 1352.6540486791 Hartrees.

NAtoms $=33$ NActive $=33 \mathrm{NUniq}=33 \mathrm{SFac}=1.00 \mathrm{D}+00 \mathrm{NAtFMM}=60 \mathrm{Big}=\mathrm{F}$

One-electron integrals computed using PRISM.

NBasis $=313$ RedAO $=\mathrm{T}$ NBF $=313$

NBsUse $=3131.00 \mathrm{D}-06 \mathrm{NBFU}=313$

Harris functional with IExCor $=205$ diagonalized for initial guess.

ExpMin= 1.61D-01 ExpMax= 5.48D+03 ExpMxC= 8.25D+02 IAcc=1 IRadAn $=1$ AccDes= 1.00D-06

HarFok: IExCor $=205$ AccDes $=1.00 \mathrm{D}-06 \mathrm{IRadAn}=1 \mathrm{IDoV}=1$

ScaDFX $=1.0000001 .0000001 .0000001 .000000$ 
Initial guess orbital symmetries:

Occupied (A) (A) (A) (A) (A) (A) (A) (A) (A) (A) (A) (A)

(A) $(\mathrm{A})(\mathrm{A})(\mathrm{A})(\mathrm{A})(\mathrm{A})(\mathrm{A})(\mathrm{A})(\mathrm{A})(\mathrm{A})(\mathrm{A})(\mathrm{A})$

(A) (A) (A) (A) (A) (A) (A) (A) (A) (A) (A) (A)

(A) (A) (A) (A) (A) (A) (A) (A) (A) (A) (A) (A)

(A) (A) (A) (A) (A) (A) (A) (A) (A) (A) (A) (A)

(A) $(\mathrm{A})(\mathrm{A})(\mathrm{A})(\mathrm{A})(\mathrm{A})(\mathrm{A})$

Virtual (A) (A) (A) (A) (A) (A) (A) (A) (A) (A) (A) (A)

(A) $(A)(A)(A)(A)(A)(A)(A)(A)(A)(A)(A)$

(A) (A) (A) (A) (A) (A) (A) (A) (A) (A) (A) (A)

(A) $(\mathrm{A})(\mathrm{A})(\mathrm{A})(\mathrm{A})(\mathrm{A})(\mathrm{A})(\mathrm{A})(\mathrm{A})(\mathrm{A})(\mathrm{A})(\mathrm{A})$

(A) $(A)(A)(A)(A)(A)(A)(A)(A)(A)(A)(A)$

(A) (A) (A) (A) (A) (A) (A) (A) (A) (A) (A) (A)

(A) (A) (A) (A) (A) (A) (A) (A) (A) (A) (A) (A)

(A) (A) (A) (A) (A) (A) (A) (A) (A) (A) (A) (A)

(A) (A) (A) (A) (A) (A) (A) (A) (A) (A) (A) (A)

(A) (A) (A) (A) (A) (A) (A) (A) (A) (A) (A) (A)

(A) $(\mathrm{A})(\mathrm{A})(\mathrm{A})(\mathrm{A})(\mathrm{A})(\mathrm{A})(\mathrm{A})(\mathrm{A})(\mathrm{A})(\mathrm{A})(\mathrm{A})$

(A) (A) (A) (A) (A) (A) (A) (A) (A) (A) (A) (A)

(A) (A) (A) (A) (A) (A) (A) (A) (A) (A) (A) (A)

(A) $(\mathrm{A})(\mathrm{A})(\mathrm{A})(\mathrm{A})(\mathrm{A})(\mathrm{A})(\mathrm{A})(\mathrm{A})(\mathrm{A})(\mathrm{A})(\mathrm{A})$

(A) (A) (A) (A) (A) (A) (A) (A) (A) (A) (A) (A)

(A) (A) (A) (A) (A) (A) (A) (A) (A) (A) (A) (A)

(A) $(\mathrm{A})(\mathrm{A})(\mathrm{A})(\mathrm{A})(\mathrm{A})(\mathrm{A})(\mathrm{A})(\mathrm{A})(\mathrm{A})(\mathrm{A})(\mathrm{A})$

(A) $(\mathrm{A})(\mathrm{A})(\mathrm{A})(\mathrm{A})(\mathrm{A})(\mathrm{A})(\mathrm{A})(\mathrm{A})(\mathrm{A})(\mathrm{A})(\mathrm{A})$

(A) (A) (A) (A) (A) (A) (A) (A) (A) (A) (A) (A)

(A) (A) (A) (A) (A) (A) (A) (A) (A) (A) (A) (A)

(A) (A) (A) (A) (A) (A)

The electronic state of the initial guess is 1-A.

Warning! Cutoffs for single-point calculations used.

Requested convergence on RMS density matrix=1.00D-04 within 128 cycles.

Requested convergence on MAX density matrix $=1.00 \mathrm{D}-02$.

Requested convergence on energy $=5.00 \mathrm{D}-05$.

No special actions if energy rises.

SCF Done: $\mathrm{E}(\mathrm{RHF})=-838.657305375 \quad$ A.U. after 7 cycles

Convg $=0.6564 \mathrm{D}-05 \quad-\mathrm{V} / \mathrm{T}=2.0024$

$\mathrm{S} * * 2=0.0000$

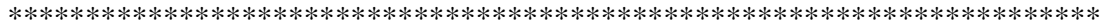

Population analysis using the SCF density.

******************************************************************************

Orbital symmetries:

Occupied (A) (A) (A) (A) (A) (A) (A) (A) (A) (A) (A) (A)

(A) $(A)(A)(A)(A)(A)(A)(A)(A)(A)(A)(A)$

(A) $(\mathrm{A})(\mathrm{A})(\mathrm{A})(\mathrm{A})(\mathrm{A})(\mathrm{A})(\mathrm{A})(\mathrm{A})(\mathrm{A})(\mathrm{A})(\mathrm{A})$

(A) $(\mathrm{A})(\mathrm{A})(\mathrm{A})(\mathrm{A})(\mathrm{A})(\mathrm{A})(\mathrm{A})(\mathrm{A})(\mathrm{A})(\mathrm{A})(\mathrm{A})$

(A) $(A)(A)(A)(A)(A)(A)(A)(A)(A)(A)(A)$

(A) $(\mathrm{A})(\mathrm{A})(\mathrm{A})(\mathrm{A})(\mathrm{A})(\mathrm{A})$

Virtual (A) (A) (A) (A) (A) (A) (A) (A) (A) (A) (A) (A)

(A) (A) (A) (A) (A) (A) (A) (A) (A) (A) (A) (A)

(A) $(\mathrm{A})(\mathrm{A})(\mathrm{A})(\mathrm{A})(\mathrm{A})(\mathrm{A})(\mathrm{A})(\mathrm{A})(\mathrm{A})(\mathrm{A})(\mathrm{A})$

(A) $(A)(A)(A)(A)(A)(A)(A)(A)(A)(A)(A)$

(A) (A) (A) (A) (A) (A) (A) (A) (A) (A) (A) (A)

(A) $(\mathrm{A})(\mathrm{A})(\mathrm{A})(\mathrm{A})(\mathrm{A})(\mathrm{A})(\mathrm{A})(\mathrm{A})(\mathrm{A})(\mathrm{A})(\mathrm{A})$

(A) (A) (A) (A) (A) (A) (A) (A) (A) (A) (A) (A)

(A) (A) (A) (A) (A) (A) (A) (A) (A) (A) (A) (A)

(A) (A) (A) (A) (A) (A) (A) (A) (A) (A) (A) (A)

(A) $(A)(A)(A)(A)(A)(A)(A)(A)(A)(A)(A)$

(A) (A) (A) (A) (A) (A) (A) (A) (A) (A) (A) (A)

(A) (A) (A) (A) (A) (A) (A) (A) (A) (A) (A) (A)

(A) (A) (A) (A) (A) (A) (A) (A) (A) (A) (A) (A) 
(A) $(\mathrm{A})(\mathrm{A})(\mathrm{A})(\mathrm{A})(\mathrm{A})(\mathrm{A})(\mathrm{A})(\mathrm{A})(\mathrm{A})(\mathrm{A})(\mathrm{A})$

(A) $(\mathrm{A})(\mathrm{A})(\mathrm{A})(\mathrm{A})(\mathrm{A})(\mathrm{A})(\mathrm{A})(\mathrm{A})(\mathrm{A})(\mathrm{A})(\mathrm{A})$

(A) $(\mathrm{A})(\mathrm{A})(\mathrm{A})(\mathrm{A})(\mathrm{A})(\mathrm{A})(\mathrm{A})(\mathrm{A})(\mathrm{A})(\mathrm{A})(\mathrm{A})$

(A) $(\mathrm{A})(\mathrm{A})(\mathrm{A})(\mathrm{A})(\mathrm{A})(\mathrm{A})(\mathrm{A})(\mathrm{A})(\mathrm{A})(\mathrm{A})(\mathrm{A})$

(A) $(\mathrm{A})(\mathrm{A})(\mathrm{A})(\mathrm{A})(\mathrm{A})(\mathrm{A})(\mathrm{A})(\mathrm{A})(\mathrm{A})(\mathrm{A})(\mathrm{A})$

(A) $(\mathrm{A})(\mathrm{A})(\mathrm{A})(\mathrm{A})(\mathrm{A})(\mathrm{A})(\mathrm{A})(\mathrm{A})(\mathrm{A})(\mathrm{A})(\mathrm{A})$

(A) $(\mathrm{A})(\mathrm{A})(\mathrm{A})(\mathrm{A})(\mathrm{A})(\mathrm{A})(\mathrm{A})(\mathrm{A})(\mathrm{A})(\mathrm{A})(\mathrm{A})$

(A) $(\mathrm{A})(\mathrm{A})(\mathrm{A})(\mathrm{A})(\mathrm{A})$

The electronic state is 1-A.

Alpha occ. eigenvalues -- -20.60871 -20.55742 -20.55174 -11.38517 -11.34332

Alpha occ. eigenvalues -- -11.31400 -11.25634 -11.25494 -11.25278 -11.25106

Alpha occ. eigenvalues -- -11.24857 -11.24705 -11.24549 -11.24537-11.24412

Alpha occ. eigenvalues -- -11.24310 -11.24296 -11.24195 -11.23817 -1.45865

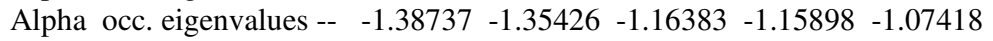

Alpha occ. eigenvalues -- $\quad-1.03609-1.02330 \quad-1.02033 \quad-1.01262 \quad-0.95572$

Alpha occ. eigenvalues -- $\quad-0.87051 \quad-0.83842 \quad-0.83489 \quad-0.81450 \quad-0.79507$

$\begin{array}{lllllll}\text { Alpha occ. eigenvalues -- } & -0.74506 & -0.72248 & -0.71152 & -0.68637 & -0.67883\end{array}$

Alpha occ. eigenvalues -- $-0.66824 \quad-0.64337 \quad-0.63739 \quad-0.62964 \quad-0.62261$

Alpha occ. eigenvalues -- $\quad-0.61104 \quad-0.59648 \quad-0.59458 \quad-0.59013 \quad-0.58793$

Alpha occ. eigenvalues -- $-0.56519 \quad-0.54521 \quad-0.53949-0.52300 \quad-0.52006$

Alpha occ. eigenvalues -- $-0.50604 \quad-0.50225 \quad-0.49992 \quad-0.49706 \quad-0.48528$

$\begin{array}{lllllll}\text { Alpha occ. eigenvalues -- } & -0.46776 & -0.44422 & -0.41529 & -0.34844 & -0.34119\end{array}$

Alpha occ. eigenvalues -- $-0.33968-0.33477$

$\begin{array}{llllll}\text { Alpha virt. eigenvalues -- } & 0.08001 & 0.12487 & 0.13535 & 0.13847 & 0.18732\end{array}$

$\begin{array}{lllllll}\text { Alpha virt. eigenvalues -- } & 0.20554 & 0.22931 & 0.23876 & 0.25636 & 0.25933\end{array}$

$\begin{array}{lllllll}\text { Alpha virt. eigenvalues -- } & 0.29198 & 0.29453 & 0.29764 & 0.30857 & 0.31166\end{array}$

$\begin{array}{llllll}\text { Alpha virt. eigenvalues -- } & 0.31819 & 0.32905 & 0.33421 & 0.33940 & 0.34586\end{array}$

$\begin{array}{lllllll}\text { Alpha virt. eigenvalues -- } & 0.35514 & 0.36833 & 0.40053 & 0.40177 & 0.42065\end{array}$

$\begin{array}{lllllll}\text { Alpha virt. eigenvalues -- } & 0.43328 & 0.44682 & 0.46635 & 0.46853 & 0.47265\end{array}$

$\begin{array}{lllllll}\text { Alpha virt. eigenvalues -- } & 0.48731 & 0.49196 & 0.50565 & 0.51835 & 0.53834\end{array}$

$\begin{array}{lllllll}\text { Alpha virt. eigenvalues -- } & 0.54205 & 0.56722 & 0.60128 & 0.65463 & 0.68708\end{array}$

$\begin{array}{lllllll}\text { Alpha virt. eigenvalues -- } & 0.70755 & 0.73060 & 0.73550 & 0.74098 & 0.74777\end{array}$

$\begin{array}{lllllll}\text { Alpha virt. eigenvalues -- } & 0.75781 & 0.76233 & 0.76674 & 0.77094 & 0.78256\end{array}$

$\begin{array}{lllllll}\text { Alpha virt. eigenvalues -- } & 0.78545 & 0.79132 & 0.80038 & 0.80668 & 0.81596\end{array}$

$\begin{array}{lllllll}\text { Alpha virt. eigenvalues -- } & 0.81900 & 0.82388 & 0.82869 & 0.83027 & 0.83115\end{array}$

$\begin{array}{lllllll}\text { Alpha virt. eigenvalues -- } & 0.83379 & 0.83446 & 0.84263 & 0.85554 & 0.86303\end{array}$

$\begin{array}{lllllll}\text { Alpha virt. eigenvalues -- } & 0.87670 & 0.88252 & 0.90868 & 0.92521 & 0.93102\end{array}$

$\begin{array}{lllllll}\text { Alpha virt. eigenvalues -- } & 0.93902 & 0.96424 & 0.98041 & 0.99290 & 1.01071\end{array}$

$\begin{array}{lllllll}\text { Alpha virt. eigenvalues -- } & 1.02889 & 1.04290 & 1.05896 & 1.07148 & 1.08483\end{array}$

$\begin{array}{llllll}\text { Alpha virt. eigenvalues -- } & 1.08819 & 1.09721 & 1.10081 & 1.10558 & 1.11046\end{array}$

$\begin{array}{lllllll}\text { Alpha virt. eigenvalues -- } & 1.11082 & 1.11740 & 1.12716 & 1.13262 & 1.13774\end{array}$

$\begin{array}{lllllll}\text { Alpha virt. eigenvalues -- } & 1.16113 & 1.16938 & 1.17617 & 1.18431 & 1.18736\end{array}$

$\begin{array}{lllllll}\text { Alpha virt. eigenvalues -- } & 1.19642 & 1.20400 & 1.21118 & 1.22030 & 1.23144\end{array}$

$\begin{array}{lllllll}\text { Alpha virt. eigenvalues -- } & 1.25861 & 1.26415 & 1.28772 & 1.29506 & 1.31586\end{array}$

$\begin{array}{lllllll}\text { Alpha virt. eigenvalues -- } & 1.32971 & 1.33731 & 1.35244 & 1.35796 & 1.38060\end{array}$

$\begin{array}{lllllll}\text { Alpha virt. eigenvalues -- } & 1.39276 & 1.41061 & 1.41928 & 1.42566 & 1.43655\end{array}$

$\begin{array}{lllllll}\text { Alpha virt. eigenvalues -- } & 1.45805 & 1.47913 & 1.49978 & 1.50741 & 1.51367\end{array}$

$\begin{array}{lllllll}\text { Alpha virt. eigenvalues -- } & 1.55314 & 1.57651 & 1.62049 & 1.64353 & 1.67049\end{array}$

$\begin{array}{lllllll}\text { Alpha virt. eigenvalues -- } & 1.67566 & 1.68373 & 1.69455 & 1.70061 & 1.70418\end{array}$

$\begin{array}{lllllll}\text { Alpha virt. eigenvalues -- } & 1.72203 & 1.74314 & 1.74657 & 1.75124 & 1.76209\end{array}$

$\begin{array}{lllllll}\text { Alpha virt. eigenvalues -- } & 1.76970 & 1.78049 & 1.78210 & 1.78875 & 1.80276\end{array}$

$\begin{array}{lllllll}\text { Alpha virt. eigenvalues -- } & 1.82710 & 1.92011 & 1.93844 & 1.95617 & 1.99204\end{array}$

$\begin{array}{lllllll}\text { Alpha virt. eigenvalues -- } & 2.01471 & 2.02908 & 2.04375 & 2.07063 & 2.09241\end{array}$

$\begin{array}{llllll}\text { Alpha virt. eigenvalues -- } & 2.10369 & 2.10903 & 2.12667 & 2.14112 & 2.14956\end{array}$

$\begin{array}{llllll}\text { Alpha virt. eigenvalues -- } & 2.15545 & 2.16997 & 2.17409 & 2.19564 & 2.22469\end{array}$

$\begin{array}{llllll}\text { Alpha virt. eigenvalues -- } & 2.24155 & 2.24770 & 2.27418 & 2.28920 & 2.29871\end{array}$

$\begin{array}{llllll}\text { Alpha virt. eigenvalues -- } & 2.30592 & 2.31160 & 2.35138 & 2.36268 & 2.36951\end{array}$

$\begin{array}{lllllll}\text { Alpha virt. eigenvalues -- } & 2.38085 & 2.38707 & 2.40136 & 2.43530 & 2.44121\end{array}$

$\begin{array}{llllll}\text { Alpha virt. eigenvalues -- } & 2.45013 & 2.45445 & 2.46177 & 2.47010 & 2.48235\end{array}$

$\begin{array}{lllllll}\text { Alpha virt. eigenvalues -- } & 2.49080 & 2.52495 & 2.53700 & 2.56705 & 2.58228\end{array}$

$\begin{array}{lllllll}\text { Alpha virt. eigenvalues -- } & 2.59316 & 2.60597 & 2.62641 & 2.63011 & 2.63404\end{array}$

$\begin{array}{llllll}\text { Alpha virt. eigenvalues -- } & 2.67975 & 2.70168 & 2.74156 & 2.74624 & 2.76370\end{array}$

$\begin{array}{lllllll}\text { Alpha virt. eigenvalues -- } & 2.79370 & 2.80778 & 2.86950 & 2.90072 & 2.91833\end{array}$

Alpha virt. eigenvalues -- $\begin{array}{llllll}2.92786 & 2.93553 & 2.95687 & 2.97801 & 2.99139\end{array}$ 
$\begin{array}{lllllll}\text { Alpha virt. eigenvalues -- } & 3.00530 & 3.03482 & 3.06378 & 3.06918 & 3.09605\end{array}$ $\begin{array}{lllllll}\text { Alpha virt. eigenvalues -- } & 3.10237 & 3.10440 & 3.10689 & 3.11908 & 3.14122\end{array}$ $\begin{array}{lllllll}\text { Alpha virt. eigenvalues -- } & 3.15587 & 3.21982 & 3.26033 & 3.33601 & 3.35763\end{array}$ $\begin{array}{lllllll}\text { Alpha virt. eigenvalues -- } & 3.37993 & 3.41962 & 3.46783 & 3.50930 & 3.55536\end{array}$ $\begin{array}{lllllll}\text { Alpha virt. eigenvalues -- } & 3.78177 & 3.79436 & 4.42206 & 4.47708 & 4.50362\end{array}$ $\begin{array}{lllllll}\text { Alpha virt. eigenvalues -- } & 4.51150 & 4.51963 & 4.53420 & 4.53731 & 4.56099\end{array}$ $\begin{array}{lllllll}\text { Alpha virt. eigenvalues -- } & 4.59419 & 4.65774 & 4.74059 & 4.75015 & 4.81496\end{array}$ $\begin{array}{llllll}\text { Alpha virt. eigenvalues -- } & 4.83002 & 4.87200 & 4.90284 & 5.05634 & 5.13124\end{array}$ Alpha virt. eigenvalues -- 5.15855

Condensed to atoms (all electrons):

$$
\begin{array}{cccccc}
1 & 2 & 3 & 4 & 5 & 6
\end{array}
$$

$\begin{array}{llllllll}1 & \mathrm{H} & 0.417145 & 0.375096 & 0.000047 & -0.026171 & -0.028187 & 0.002142\end{array}$

$\begin{array}{llllllll}2 & \mathrm{C} & 0.375096 & 4.908725 & -0.033318 & 0.528975 & 0.529963 & -0.042232\end{array}$

$\begin{array}{llllllll}3 & \mathrm{C} & 0.000047 & -0.033318 & 4.901207 & -0.033401 & -0.042677 & 0.549725\end{array}$

$\begin{array}{llllllll}4 & \mathrm{C} & -0.026171 & 0.528975 & -0.033401 & 5.009643 & -0.042343 & -0.029331\end{array}$

$\begin{array}{llllllll}5 & \mathrm{C} & -0.028187 & 0.529963 & -0.042677 & -0.042343 & 4.929832 & 0.539442\end{array}$

$\begin{array}{lllllll}6 \mathrm{C} & 0.002142 & -0.042232 & 0.549725 & -0.029331 & 0.539442 & 4.879490\end{array}$

$\begin{array}{llllllll}7 & \mathrm{C} & 0.002751 & -0.048783 & 0.541872 & 0.532021 & -0.033838 & -0.044179\end{array}$

$\begin{array}{llllllll}8 \mathrm{H} & -0.001077 & -0.028292 & 0.002381 & 0.001917 & 0.382798 & -0.029994\end{array}$

$\begin{array}{llllllll}9 \mathrm{H} & -0.000086 & 0.002201 & -0.029378 & 0.000226 & -0.029335 & 0.381048\end{array}$

$\begin{array}{llllllll}10 \mathrm{H} & -0.000094 & 0.002539 & -0.026075 & -0.031663 & 0.000113 & 0.002359\end{array}$

$\begin{array}{llllllll}11 & \mathrm{H} & 0.000009 & 0.000236 & 0.383000 & 0.001579 & 0.002315 & -0.028503\end{array}$

$\begin{array}{llllllll}12 & \mathrm{C} & -0.004517 & -0.032609 & 0.002253 & 0.343390 & 0.002776 & -0.000082\end{array}$

$\begin{array}{llllllll}13 & \mathrm{O} & 0.009490 & 0.001338 & -0.000045 & -0.076586 & 0.000737 & -0.000007\end{array}$

$\begin{array}{llllllll}14 \mathrm{C} & 0.000280 & 0.005104 & -0.000065 & -0.093800 & 0.000005 & -0.000010\end{array}$

$\begin{array}{llllllll}15 \mathrm{H} & -0.000007 & -0.000166 & 0.000061 & 0.006818 & -0.000012 & 0.000004\end{array}$

$\begin{array}{llllllll}16 & \mathrm{C} & -0.000012 & -0.000177 & -0.000040 & 0.001865 & -0.000005 & 0.000002\end{array}$

$\begin{array}{llllllll}17 & \mathrm{C} & 0.000000 & 0.000000 & 0.000000 & 0.000001 & 0.000000 & 0.000000\end{array}$

$\begin{array}{llllllll}18 & \mathrm{C} & -0.000004 & -0.000090 & 0.000005 & 0.001264 & 0.000000 & 0.000000\end{array}$

$\begin{array}{llllllll}19 & \mathrm{C} & 0.000000 & 0.000007 & -0.000011 & 0.000055 & -0.000001 & 0.000002\end{array}$

$\begin{array}{llllllll}20 \mathrm{C} & 0.000000 & 0.000000 & 0.000000 & -0.000008 & 0.000000 & 0.000000\end{array}$

$\begin{array}{llllllll}21 & \mathrm{C} & 0.000000 & -0.000003 & 0.000000 & -0.000012 & 0.000000 & 0.000000\end{array}$

$\begin{array}{llllllll}22 & \mathrm{H} & 0.000002 & -0.000012 & 0.000001 & 0.000241 & 0.000000 & 0.000000\end{array}$

$\begin{array}{llllllll}23 & \mathrm{H} & 0.000000 & 0.000001 & -0.000005 & 0.000015 & 0.000000 & 0.000000\end{array}$

$\begin{array}{llllllll}24 \mathrm{H} & 0.000000 & 0.000000 & 0.000000 & 0.000000 & 0.000000 & 0.000000\end{array}$

$\begin{array}{llllllll}25 & \mathrm{H} & 0.000000 & 0.000000 & 0.000000 & 0.000000 & 0.000000 & 0.000000\end{array}$

$\begin{array}{llllllll}26 \mathrm{H} & 0.000000 & 0.000000 & 0.000000 & 0.000000 & 0.000000 & 0.000000\end{array}$

$\begin{array}{llllllll}27 & \mathrm{C} & -0.000003 & -0.000034 & 0.000000 & 0.000555 & 0.000000 & 0.000000\end{array}$

$\begin{array}{llllllll}28 & \mathrm{O} & -0.000001 & 0.000021 & 0.000000 & -0.000676 & 0.000000 & 0.000000\end{array}$

$\begin{array}{llllllll}29 & \mathrm{O} & -0.000002 & -0.000040 & 0.000000 & 0.002174 & 0.000000 & 0.000000\end{array}$

$\begin{array}{llllllll}30 \mathrm{C} & 0.000000 & 0.000000 & 0.000000 & -0.000015 & 0.000000 & 0.000000\end{array}$

$\begin{array}{llllllll}31 \mathrm{H} & 0.000000 & 0.000000 & 0.000000 & 0.000000 & 0.000000 & 0.000000\end{array}$

$\begin{array}{llllllll}32 & \mathrm{H} & 0.000000 & 0.000000 & 0.000000 & 0.000002 & 0.000000 & 0.000000\end{array}$

$\begin{array}{llllllll}33 & \mathrm{H} & 0.000000 & 0.000000 & 0.000000 & 0.000000 & 0.000000 & 0.000000\end{array}$

$\begin{array}{llllll}7 & 8 & 9 & 10 & 11 & 12\end{array}$

$\begin{array}{llllllll}1 & \mathrm{H} & 0.002751 & -0.001077 & -0.000086 & -0.000094 & 0.000009 & -0.004517\end{array}$

$\begin{array}{llllllll}2 & \mathrm{C} & -0.048783 & -0.028292 & 0.002201 & 0.002539 & 0.000236 & -0.032609\end{array}$

$\begin{array}{llllllll}3 & \mathrm{C} & 0.541872 & 0.002381 & -0.029378 & -0.026075 & 0.383000 & 0.002253\end{array}$

$\begin{array}{llllllll}4 & C & 0.532021 & 0.001917 & 0.000226 & -0.031663 & 0.001579 & 0.343390\end{array}$

$\begin{array}{llllllll}5 & \mathrm{C} & -0.033838 & 0.382798 & -0.029335 & 0.000113 & 0.002315 & 0.002776\end{array}$

$\begin{array}{llllllll}6 & \mathrm{C} & -0.044179 & -0.029994 & 0.381048 & 0.002359 & -0.028503 & -0.000082\end{array}$

$\begin{array}{llllllll}7 & \mathrm{C} & 4.924144 & 0.000190 & 0.002543 & 0.373617 & -0.029167 & -0.030410\end{array}$

$\begin{array}{llllllll}8 \mathrm{H} & 0.000190 & 0.461989 & -0.001345 & 0.000009 & -0.000100 & -0.000079\end{array}$

$\begin{array}{llllllll}9 \mathrm{H} & 0.002543 & -0.001345 & 0.463365 & -0.000102 & -0.001335 & 0.000005\end{array}$

$\begin{array}{llllllll}10 \mathrm{H} & 0.373617 & 0.000009 & -0.000102 & 0.464028 & -0.001248 & -0.003203\end{array}$

$\begin{array}{llllllll}11 \mathrm{H} & -0.029167 & -0.000100 & -0.001335 & -0.001248 & 0.463802 & -0.000065\end{array}$

$\begin{array}{llllllll}12 & \mathrm{C} & -0.030410 & -0.000079 & 0.000005 & -0.003203 & -0.000065 & 4.436184\end{array}$

$\begin{array}{lllllllll}13 & \mathrm{O} & 0.002329 & 0.000001 & 0.000000 & 0.000011 & 0.000000 & 0.536131\end{array}$

$\begin{array}{llllllll}14 & \mathrm{C} & -0.000926 & 0.000001 & 0.000000 & 0.002670 & 0.000002 & 0.343613\end{array}$

$\begin{array}{llllllll}15 \mathrm{H} & -0.002214 & 0.000000 & 0.000000 & 0.000385 & -0.000004 & -0.046069\end{array}$

$\begin{array}{llllllll}16 & \mathrm{C} & -0.002052 & 0.000000 & 0.000000 & -0.000221 & -0.000002 & -0.048001\end{array}$

$\begin{array}{llllllll}17 & \mathrm{C} & 0.000005 & 0.000000 & 0.000000 & -0.000006 & 0.000000 & 0.000007\end{array}$

$\begin{array}{llllllll}18 & \mathrm{C} & -0.000275 & 0.000000 & 0.000000 & -0.000051 & 0.000000 & -0.004612\end{array}$

$\begin{array}{llllllll}19 & \mathrm{C} & -0.001546 & 0.000000 & 0.000000 & 0.002677 & -0.000004 & 0.000694\end{array}$ 
$\begin{array}{llllllll}20 & \mathrm{C} & -0.000030 & 0.000000 & 0.000000 & 0.000062 & 0.000000 & -0.000106\end{array}$

$\begin{array}{llllllll}21 & \mathrm{C} & -0.000008 & 0.000000 & 0.000000 & -0.000016 & 0.000000 & 0.000172\end{array}$

$\begin{array}{llllllll}22 & \mathrm{H} & -0.000050 & 0.000000 & 0.000000 & -0.000003 & 0.000000 & 0.001988\end{array}$

$\begin{array}{llllllll}23 \mathrm{H} & -0.000249 & 0.000000 & 0.000000 & 0.000589 & -0.000001 & 0.000044\end{array}$

$\begin{array}{llllllll}24 \mathrm{H} & 0.000000 & 0.000000 & 0.000000 & -0.000001 & 0.000000 & 0.000001\end{array}$

$\begin{array}{llllllll}25 & \mathrm{H} & 0.000000 & 0.000000 & 0.000000 & 0.000000 & 0.000000 & -0.000003\end{array}$

$\begin{array}{lllllll}26 \mathrm{H} & 0.000000 & 0.000000 & 0.000000 & 0.000000 & 0.000000 & 0.000000\end{array}$

$\begin{array}{llllllll}27 & \mathrm{C} & -0.000052 & 0.000000 & 0.000000 & -0.000003 & 0.000000 & 0.000275\end{array}$

$\begin{array}{llllllll}28 & \mathrm{O} & -0.000059 & 0.000000 & 0.000000 & -0.000048 & 0.000000 & 0.002562\end{array}$

$\begin{array}{llllllll}29 & \mathrm{O} & 0.000019 & 0.000000 & 0.000000 & 0.000019 & 0.000000 & -0.038561\end{array}$

$\begin{array}{llllllll}30 \mathrm{C} & 0.000000 & 0.000000 & 0.000000 & 0.000000 & 0.000000 & -0.000158\end{array}$

$\begin{array}{llllllll}31 \mathrm{H} & 0.000000 & 0.000000 & 0.000000 & 0.000000 & 0.000000 & 0.000001\end{array}$

$\begin{array}{llllllll}32 \mathrm{H} & 0.000000 & 0.000000 & 0.000000 & 0.000000 & 0.000000 & -0.000023\end{array}$

$\begin{array}{lllllllll}33 & \mathrm{H} & 0.000000 & 0.000000 & 0.000000 & 0.000000 & 0.000000 & 0.000025\end{array}$ $\begin{array}{llllll}13 & 14 & 15 & 16 & 17 & 18\end{array}$

$\begin{array}{llllllll}1 & \mathrm{H} & 0.009490 & 0.000280 & -0.000007 & -0.000012 & 0.000000 & -0.000004\end{array}$

$\begin{array}{llllllll}2 & \mathrm{C} & 0.001338 & 0.005104 & -0.000166 & -0.000177 & 0.000000 & -0.000090\end{array}$

$\begin{array}{llllllll}3 \mathrm{C} & -0.000045 & -0.000065 & 0.000061 & -0.000040 & 0.000000 & 0.000005\end{array}$

$\begin{array}{llllllll}4 \mathrm{C} & -0.076586 & -0.093800 & 0.006818 & 0.001865 & 0.000001 & 0.001264\end{array}$

$\begin{array}{llllllll}5 & \mathrm{C} & 0.000737 & 0.000005 & -0.000012 & -0.000005 & 0.000000 & 0.000000\end{array}$

$\begin{array}{llllllll}6 \mathrm{C} & -0.000007 & -0.000010 & 0.000004 & 0.000002 & 0.000000 & 0.000000\end{array}$

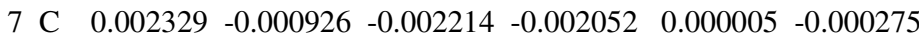

$\begin{array}{llllllll}8 \mathrm{H} & 0.000001 & 0.000001 & 0.000000 & 0.000000 & 0.000000 & 0.000000\end{array}$

$\begin{array}{llllllll}9 \mathrm{H} & 0.000000 & 0.000000 & 0.000000 & 0.000000 & 0.000000 & 0.000000\end{array}$

$\begin{array}{llllllll}10 \mathrm{H} & 0.000011 & 0.002670 & 0.000385 & -0.000221 & -0.000006 & -0.000051\end{array}$

$\begin{array}{llllllll}11 \mathrm{H} & 0.000000 & 0.000002 & -0.000004 & -0.000002 & 0.000000 & 0.000000\end{array}$

$\begin{array}{llllllll}12 & \mathrm{C} & 0.536131 & 0.343613 & -0.046069 & -0.048001 & 0.000007 & -0.004612\end{array}$

$\begin{array}{lllllllll}13 & \mathrm{O} & 8.168067 & -0.073329 & 0.001925 & -0.004800 & 0.000000 & 0.000722\end{array}$

$\begin{array}{llllllll}14 & \mathrm{C} & -0.073329 & 4.953619 & 0.406678 & 0.346019 & 0.000118 & -0.049830\end{array}$

$\begin{array}{llllllll}15 \mathrm{H} & 0.001925 & 0.406678 & 0.473526 & -0.045605 & -0.000020 & 0.004424\end{array}$

$\begin{array}{llllllll}16 & \mathrm{C} & -0.004800 & 0.346019 & -0.045605 & 4.851053 & -0.029382 & 0.541003\end{array}$

$\begin{array}{llllllll}17 & \mathrm{C} & 0.000000 & 0.000118 & -0.000020 & -0.029382 & 4.889390 & -0.044407\end{array}$

$\begin{array}{llllllll}18 & \mathrm{C} & 0.000722 & -0.049830 & 0.004424 & 0.541003 & -0.044407 & 4.934665\end{array}$

$\begin{array}{llllllll}19 & C & -0.000030 & -0.034243 & -0.002787 & 0.567013 & -0.045171 & -0.039564\end{array}$

$\begin{array}{llllllll}20 & \mathrm{C} & 0.000000 & 0.003609 & 0.000259 & -0.040661 & 0.552084 & -0.035528\end{array}$

$\begin{array}{llllllll}21 & \mathrm{C} & 0.000112 & 0.001675 & 0.000018 & -0.041043 & 0.545111 & 0.537708\end{array}$

$\begin{array}{llllllll}22 & \mathrm{H} & 0.005388 & -0.003635 & 0.000068 & -0.028651 & 0.002178 & 0.373047\end{array}$

$\begin{array}{llllllll}23 \mathrm{H} & -0.000001 & -0.002493 & 0.002285 & -0.034556 & 0.002350 & 0.002647\end{array}$

$\begin{array}{llllllll}24 \mathrm{H} & 0.000000 & -0.000108 & -0.000007 & 0.001948 & -0.028867 & 0.000222\end{array}$

$\begin{array}{llllllll}25 & \mathrm{H} & 0.000000 & -0.000098 & 0.000001 & 0.001902 & -0.029572 & -0.028752\end{array}$

$\begin{array}{llllllll}26 \mathrm{H} & 0.000000 & 0.000005 & 0.000000 & 0.000223 & 0.381876 & 0.002393\end{array}$

$\begin{array}{llllllll}27 & \mathrm{C} & 0.008486 & -0.029577 & -0.005333 & 0.004728 & 0.000000 & -0.000110\end{array}$

$\begin{array}{llllllll}28 & \mathrm{O} & -0.002410 & 0.002965 & 0.009648 & 0.000955 & 0.000000 & 0.000005\end{array}$

$\begin{array}{llllllll}29 & \mathrm{O} & -0.002551 & 0.151546 & -0.030519 & -0.049528 & 0.000013 & 0.002026\end{array}$

$\begin{array}{lllllll}30 \mathrm{C} & -0.000392 & 0.004680 & 0.000128 & -0.000010 & 0.000000 & -0.000018\end{array}$

$\begin{array}{llllllll}31 & \mathrm{H} & 0.000003 & -0.000125 & 0.000009 & 0.000001 & 0.000000 & 0.000000\end{array}$

$\begin{array}{llllllll}32 & \mathrm{H} & 0.000369 & -0.000251 & -0.000002 & -0.000010 & 0.000000 & 0.000003\end{array}$

$\begin{array}{llllllll}33 & \mathrm{H} & 0.000001 & -0.000188 & -0.000030 & -0.000020 & 0.000000 & 0.000002\end{array}$

$$
\begin{array}{llllll}
19 & 20 & 21 & 22 & 23 & 24
\end{array}
$$

$\begin{array}{llllllll}1 \mathrm{H} & 0.000000 & 0.000000 & 0.000000 & 0.000002 & 0.000000 & 0.000000\end{array}$

$\begin{array}{llllllll}2 \mathrm{C} & 0.000007 & 0.000000 & -0.000003 & -0.000012 & 0.000001 & 0.000000\end{array}$

$\begin{array}{llllllll}3 \mathrm{C} & -0.000011 & 0.000000 & 0.000000 & 0.000001 & -0.000005 & 0.000000\end{array}$

$\begin{array}{llllllll}4 \mathrm{C} & 0.000055 & -0.000008 & -0.000012 & 0.000241 & 0.000015 & 0.000000\end{array}$

$\begin{array}{llllllll}5 & \mathrm{C} & -0.000001 & 0.000000 & 0.000000 & 0.000000 & 0.000000 & 0.000000\end{array}$

$\begin{array}{llllllll}6 \mathrm{C} & 0.000002 & 0.000000 & 0.000000 & 0.000000 & 0.000000 & 0.000000\end{array}$

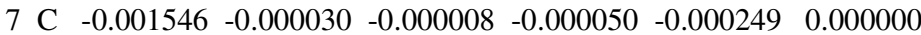

$\begin{array}{lllllll}8 \mathrm{H} & 0.000000 & 0.000000 & 0.000000 & 0.000000 & 0.000000 & 0.000000\end{array}$

$\begin{array}{llllllll}9 \mathrm{H} & 0.000000 & 0.000000 & 0.000000 & 0.000000 & 0.000000 & 0.000000\end{array}$

$\begin{array}{llllllll}10 \mathrm{H} & 0.002677 & 0.000062 & -0.000016 & -0.000003 & 0.000589 & -0.000001\end{array}$

$\begin{array}{llllllll}11 \mathrm{H} & -0.000004 & 0.000000 & 0.000000 & 0.000000 & -0.000001 & 0.000000\end{array}$

$\begin{array}{llllllll}12 & \mathrm{C} & 0.000694 & -0.000106 & 0.000172 & 0.001988 & 0.000044 & 0.000001\end{array}$

$\begin{array}{lllllllll}13 & \mathrm{O} & -0.000030 & 0.000000 & 0.000112 & 0.005388 & -0.000001 & 0.000000\end{array}$

$\begin{array}{llllllll}14 & \mathrm{C} & -0.034243 & 0.003609 & 0.001675 & -0.003635 & -0.002493 & -0.000108\end{array}$

$\begin{array}{llllllll}15 & \mathrm{H} & -0.002787 & 0.000259 & 0.000018 & 0.000068 & 0.002285 & -0.000007\end{array}$ 
$\begin{array}{llllllll}16 & \mathrm{C} & 0.567013 & -0.040661 & -0.041043 & -0.028651 & -0.034556 & 0.001948\end{array}$

$\begin{array}{llllllll}17 & \mathrm{C} & -0.045171 & 0.552084 & 0.545111 & 0.002178 & 0.002350 & -0.028867\end{array}$

$\begin{array}{llllllll}18 & \mathrm{C} & -0.039564 & -0.035528 & 0.537708 & 0.373047 & 0.002647 & 0.000222\end{array}$

$\begin{array}{llllllll}19 & \mathrm{C} & 4.910496 & 0.540064 & -0.035891 & 0.002579 & 0.379377 & -0.029069\end{array}$

$\begin{array}{llllllll}20 \mathrm{C} & 0.540064 & 4.897346 & -0.041364 & 0.000065 & -0.027038 & 0.381303\end{array}$

$\begin{array}{llllllll}21 & \mathrm{C} & -0.035891 & -0.041364 & 4.903460 & -0.024623 & 0.000118 & 0.002377\end{array}$

$\begin{array}{llllllll}22 & \mathrm{H} & 0.002579 & 0.000065 & -0.024623 & 0.427263 & -0.000099 & 0.000009\end{array}$

$\begin{array}{lllllllll}23 & \mathrm{H} & 0.379377 & -0.027038 & 0.000118 & -0.000099 & 0.474439 & -0.001368\end{array}$

$\begin{array}{llllllll}24 & \mathrm{H} & -0.029069 & 0.381303 & 0.002377 & 0.000009 & -0.001368 & 0.468189\end{array}$

$\begin{array}{llllllll}25 & \mathrm{H} & 0.000244 & 0.002397 & 0.382054 & -0.001093 & 0.000010 & -0.000102\end{array}$

$\begin{array}{llllllll}26 \mathrm{H} & 0.002495 & -0.029830 & -0.029650 & -0.000090 & -0.000104 & -0.001397\end{array}$

$\begin{array}{llllllll}27 & \mathrm{C} & -0.000105 & 0.000000 & 0.000002 & 0.000017 & -0.000009 & 0.000000\end{array}$

$\begin{array}{llllllll}28 & \mathrm{O} & -0.000030 & 0.000000 & 0.000000 & 0.000004 & -0.000002 & 0.000000\end{array}$

$\begin{array}{llllllll}29 & \mathrm{O} & 0.000108 & -0.000055 & 0.000098 & 0.001313 & 0.000052 & 0.000000\end{array}$

$\begin{array}{lllllll}30 \mathrm{C} & -0.000005 & 0.000000 & 0.000000 & 0.000004 & 0.000000 & 0.000000\end{array}$

$\begin{array}{llllllll}31 \mathrm{H} & 0.000000 & 0.000000 & 0.000000 & 0.000000 & 0.000000 & 0.000000\end{array}$

$\begin{array}{llllllll}32 \mathrm{H} & 0.000000 & 0.000000 & 0.000000 & 0.000001 & 0.000000 & 0.000000\end{array}$

$\begin{array}{llllllll}33 & \mathrm{H} & 0.000001 & 0.000000 & 0.000000 & 0.000000 & 0.000000 & 0.000000\end{array}$

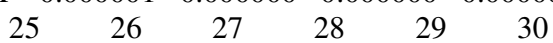

$\begin{array}{llllllll}1 \mathrm{H} & 0.000000 & 0.000000 & -0.000003 & -0.000001 & -0.000002 & 0.000000\end{array}$

$\begin{array}{llllllll}2 & \mathrm{C} & 0.000000 & 0.000000 & -0.000034 & 0.000021 & -0.000040 & 0.000000\end{array}$

$\begin{array}{llllllll}3 \mathrm{C} & 0.000000 & 0.000000 & 0.000000 & 0.000000 & 0.000000 & 0.000000\end{array}$

$\begin{array}{llllllll}4 \mathrm{C} & 0.000000 & 0.000000 & 0.000555 & -0.000676 & 0.002174 & -0.000015\end{array}$

$\begin{array}{llllllll}5 \mathrm{C} & 0.000000 & 0.000000 & 0.000000 & 0.000000 & 0.000000 & 0.000000\end{array}$

$\begin{array}{lllllll}6 \mathrm{C} & 0.000000 & 0.000000 & 0.000000 & 0.000000 & 0.000000 & 0.000000\end{array}$

$\begin{array}{llllllll}7 \mathrm{C} & 0.000000 & 0.000000 & -0.000052 & -0.000059 & 0.000019 & 0.000000\end{array}$

$\begin{array}{llllllll}8 \mathrm{H} & 0.000000 & 0.000000 & 0.000000 & 0.000000 & 0.000000 & 0.000000\end{array}$

$\begin{array}{llllllll}9 \mathrm{H} & 0.000000 & 0.000000 & 0.000000 & 0.000000 & 0.000000 & 0.000000\end{array}$

$\begin{array}{llllllll}10 \mathrm{H} & 0.000000 & 0.000000 & -0.000003 & -0.000048 & 0.000019 & 0.000000\end{array}$

$\begin{array}{llllllll}11 \mathrm{H} & 0.000000 & 0.000000 & 0.000000 & 0.000000 & 0.000000 & 0.000000\end{array}$

$\begin{array}{llllllll}12 & \mathrm{C} & -0.000003 & 0.000000 & 0.000275 & 0.002562 & -0.038561 & -0.000158\end{array}$

$\begin{array}{llllllll}13 & \mathrm{O} & 0.000000 & 0.000000 & 0.008486 & -0.002410 & -0.002551 & -0.000392\end{array}$

$\begin{array}{llllllll}14 \mathrm{C} & -0.000098 & 0.000005 & -0.029577 & 0.002965 & 0.151546 & 0.004680\end{array}$

$\begin{array}{llllllll}15 & \mathrm{H} & 0.000001 & 0.000000 & -0.005333 & 0.009648 & -0.030519 & 0.000128\end{array}$

$\begin{array}{llllllll}16 \mathrm{C} & 0.001902 & 0.000223 & 0.004728 & 0.000955 & -0.049528 & -0.000010\end{array}$

$\begin{array}{llllllll}17 & \mathrm{C} & -0.029572 & 0.381876 & 0.000000 & 0.000000 & 0.000013 & 0.000000\end{array}$

$\begin{array}{llllllll}18 & \mathrm{C} & -0.028752 & 0.002393 & -0.000110 & 0.000005 & 0.002026 & -0.000018\end{array}$

$\begin{array}{llllllll}19 & \mathrm{C} & 0.000244 & 0.002495 & -0.000105 & -0.000030 & 0.000108 & -0.000005\end{array}$

$\begin{array}{llllllll}20 & \mathrm{C} & 0.002397 & -0.029830 & 0.000000 & 0.000000 & -0.000055 & 0.000000\end{array}$

$\begin{array}{llllllll}21 & \mathrm{C} & 0.382054 & -0.029650 & 0.000002 & 0.000000 & 0.000098 & 0.000000\end{array}$

$\begin{array}{llllllll}22 & \mathrm{H} & -0.001093 & -0.000090 & 0.000017 & 0.000004 & 0.001313 & 0.000004\end{array}$

$\begin{array}{llllllll}23 & \mathrm{H} & 0.000010 & -0.000104 & -0.000009 & -0.000002 & 0.000052 & 0.000000\end{array}$

$\begin{array}{llllllll}24 \mathrm{H} & -0.000102 & -0.001397 & 0.000000 & 0.000000 & 0.000000 & 0.000000\end{array}$

$\begin{array}{llllllll}25 & \mathrm{H} & 0.464759 & -0.001402 & 0.000000 & 0.000000 & 0.000000 & 0.000000\end{array}$

$\begin{array}{llllllll}26 & \mathrm{H} & -0.001402 & 0.467987 & 0.000000 & 0.000000 & 0.000000 & 0.000000\end{array}$

$\begin{array}{lllllllll}27 & \mathrm{C} & 0.000000 & 0.000000 & 4.127954 & 0.566109 & 0.247739 & 0.375115\end{array}$

$\begin{array}{lllllllll}28 & \mathrm{O} & 0.000000 & 0.000000 & 0.566109 & 8.138215 & -0.077489 & -0.060217\end{array}$

$\begin{array}{lllllllll}29 & \mathrm{O} & 0.000000 & 0.000000 & 0.247739 & -0.077489 & 8.511190 & -0.089895\end{array}$

$\begin{array}{lllllllll}30 \mathrm{C} & 0.000000 & 0.000000 & 0.375115 & -0.060217 & -0.089895 & 5.203838\end{array}$

$\begin{array}{llllllll}31 & \mathrm{H} & 0.000000 & 0.000000 & -0.036446 & 0.002831 & 0.002988 & 0.382367\end{array}$

$\begin{array}{llllllll}32 & \mathrm{H} & 0.000000 & 0.000000 & -0.027988 & 0.000367 & 0.001498 & 0.377008\end{array}$

$\begin{array}{llllllll}33 & \mathrm{H} & 0.000000 & 0.000000 & -0.028949 & 0.001399 & 0.002633 & 0.375251\end{array}$

$\begin{array}{lll}31 & 32 & 33\end{array}$

$\begin{array}{lllll}1 \mathrm{H} & 0.000000 & 0.000000 & 0.000000\end{array}$

$\begin{array}{lllll}2 & \mathrm{C} & 0.000000 & 0.000000 & 0.000000\end{array}$

$\begin{array}{lllll}3 \mathrm{C} & 0.000000 & 0.000000 & 0.000000\end{array}$

$\begin{array}{lllll}4 \mathrm{C} & 0.000000 & 0.000002 & 0.000000\end{array}$

$\begin{array}{lllll}5 \mathrm{C} & 0.000000 & 0.000000 & 0.000000\end{array}$

$\begin{array}{lllll}6 \mathrm{C} & 0.000000 & 0.000000 & 0.000000\end{array}$

$\begin{array}{lllll}7 & \mathrm{C} & 0.000000 & 0.000000 & 0.000000\end{array}$

$\begin{array}{lllll}8 \mathrm{H} & 0.000000 & 0.000000 & 0.000000\end{array}$

$\begin{array}{lllll}9 \mathrm{H} & 0.000000 & 0.000000 & 0.000000\end{array}$

$\begin{array}{lllll}10 \mathrm{H} & 0.000000 & 0.000000 & 0.000000\end{array}$

$\begin{array}{lllll}11 \mathrm{H} & 0.000000 & 0.000000 & 0.000000\end{array}$ 
$\begin{array}{lllll}12 & \mathrm{C} & 0.000001 & -0.000023 & 0.000025\end{array}$

$\begin{array}{lllll}13 & \mathrm{O} & 0.000003 & 0.000369 & 0.000001\end{array}$

$\begin{array}{lllll}14 \mathrm{C} & -0.000125 & -0.000251 & -0.000188\end{array}$

$\begin{array}{lllll}15 \mathrm{H} & 0.000009 & -0.000002 & -0.000030\end{array}$

$\begin{array}{lllll}16 & \mathrm{C} & 0.000001 & -0.000010 & -0.000020\end{array}$

$\begin{array}{lllll}17 & \mathrm{C} & 0.000000 & 0.000000 & 0.000000\end{array}$

$\begin{array}{lllll}18 \mathrm{C} & 0.000000 & 0.000003 & 0.000002\end{array}$

$\begin{array}{lllll}19 \mathrm{C} & 0.000000 & 0.000000 & 0.000001\end{array}$

$\begin{array}{lllll}20 \mathrm{C} & 0.000000 & 0.000000 & 0.000000\end{array}$

$\begin{array}{lllll}21 \mathrm{C} & 0.000000 & 0.000000 & 0.000000\end{array}$

$\begin{array}{lllll}22 \mathrm{H} & 0.000000 & 0.000001 & 0.000000\end{array}$

$\begin{array}{lllll}23 & \mathrm{H} & 0.000000 & 0.000000 & 0.000000\end{array}$

$\begin{array}{lllll}24 \mathrm{H} & 0.000000 & 0.000000 & 0.000000\end{array}$

$\begin{array}{lllll}25 \mathrm{H} & 0.000000 & 0.000000 & 0.000000\end{array}$

$\begin{array}{lllll}26 \mathrm{H} & 0.000000 & 0.000000 & 0.000000\end{array}$

$\begin{array}{lllll}27 & \mathrm{C} & -0.036446 & -0.027988 & -0.028949\end{array}$

$\begin{array}{llllll}28 & \mathrm{O} & 0.002831 & 0.000367 & 0.001399\end{array}$

$\begin{array}{lllll}29 & \mathrm{O} & 0.002988 & 0.001498 & 0.002633\end{array}$

$\begin{array}{lllll}30 \mathrm{C} & 0.382367 & 0.377008 & 0.375251\end{array}$

$\begin{array}{llllll}31 & \mathrm{H} & 0.480818 & -0.018216 & -0.020199\end{array}$

$\begin{array}{llllll}32 & \mathrm{H} & -0.018216 & 0.472477 & -0.021275\end{array}$

$\begin{array}{lllll}33 & \mathrm{H} & -0.020199 & -0.021275 & 0.490067\end{array}$

Mulliken atomic charges:
$1 \mathrm{H} \quad 0.253199$
$\begin{array}{lll}2 & \mathrm{C} & -0.168449\end{array}$
3 C -0.215536
4 C -0.096735
5 C -0.211584
6 C -0.179877
7 C -0.185652
$8 \mathrm{H} \quad 0.211600$
$9 \mathrm{H} \quad 0.212194$
$10 \mathrm{H} \quad 0.213654$
$11 \mathrm{H} \quad 0.209485$
$\begin{array}{lll}12 & \mathrm{C} & 0.538377\end{array}$
13 O -0.574958
$\begin{array}{lll}14 & \mathrm{C} & 0.066088\end{array}$
$15 \mathrm{H} \quad 0.226537$
16 C 0.008063
$\begin{array}{lll}17 & \mathrm{C} & -0.195709\end{array}$
18 C -0.196895
19 C -0.217354
20 C -0.202569
21 C -0.200298
$22 \mathrm{H} \quad 0.244091$
$\begin{array}{lll}23 & \mathrm{H} & 0.203997\end{array}$
$\begin{array}{lll}24 \mathrm{H} & 0.206870\end{array}$
$\begin{array}{lll}25 \mathrm{H} & 0.209655\end{array}$
$\begin{array}{lll}26 \mathrm{H} & 0.207494\end{array}$
$\begin{array}{lll}27 & \mathrm{C} & 0.797629\end{array}$
$\begin{array}{lll}28 & \mathrm{O} & -0.584149\end{array}$
29 O -0.634775
$30 \mathrm{C}-0.567682$
$\begin{array}{lll}31 & \mathrm{H} & 0.205967\end{array}$
$\begin{array}{lll}32 & \mathrm{H} & 0.216039\end{array}$
$33 \mathrm{H} \quad 0.201282$

Sum of Mulliken charges $=0.00000$

Atomic charges with hydrogens summed into heavy atoms: 1

$1 \mathrm{H} \quad 0.000000$

2 C 0.084749

3 C -0.006051

4 C -0.096735 

5 C 0.000016
$\begin{array}{lll}6 & \mathrm{C} & 0.032317\end{array}$
$\begin{array}{lll}7 & \mathrm{C} & 0.028002\end{array}$
$8 \mathrm{H} \quad 0.000000$
$9 \mathrm{H} \quad 0.000000$
$10 \mathrm{H} \quad 0.000000$
$\begin{array}{lll}11 \mathrm{H} & 0.000000\end{array}$
12 C 0.538377
13 O -0.574958
$14 \mathrm{C} \quad 0.292625$
$\begin{array}{lll}15 \mathrm{H} & 0.000000\end{array}$
$16 \mathrm{C} \quad 0.008063$
$17 \mathrm{C} \quad 0.011786$
18 C 0.047196
19 C -0.013357
$\begin{array}{lll}20 \mathrm{C} & 0.004301\end{array}$
$21 \mathrm{C} \quad 0.009357$
$22 \mathrm{H} \quad 0.000000$
$23 \mathrm{H} \quad 0.000000$
$24 \mathrm{H} \quad 0.000000$
$25 \mathrm{H} \quad 0.000000$
$\begin{array}{lll}26 \mathrm{H} & 0.000000\end{array}$
$\begin{array}{lll}27 & \mathrm{C} & 0.797629\end{array}$
$\begin{array}{lll}28 & \mathrm{O} & -0.584149\end{array}$
29 O -0.634775
$30 \mathrm{C} \quad 0.055607$
$31 \mathrm{H} \quad 0.000000$
$\begin{array}{lll}32 & \mathrm{H} & 0.000000\end{array}$
$33 \mathrm{H} \quad 0.000000$

Sum of Mulliken charges $=0.00000$

Electronic spatial extent $(\mathrm{au}):<\mathrm{R} * * 2>=5211.4159$

Charge $=0.0000$ electrons

Dipole moment (field-independent basis, Debye):

$\mathrm{X}=0.0983 \mathrm{Y}=3.1260 \mathrm{Z}=-0.9537$ Tot $=3.2697$

Quadrupole moment (field-independent basis, Debye-Ang):

$\mathrm{XX}=-95.2777 \quad \mathrm{YY}=-108.8045 \quad \mathrm{ZZ}=-106.5345$

$\mathrm{XY}=-4.4496 \quad \mathrm{XZ}=0.3583 \quad \mathrm{YZ}=-4.8196$

Traceless Quadrupole moment (field-independent basis, Debye-Ang):

$\mathrm{XX}=8.2612 \mathrm{YY}=-5.2656 \mathrm{ZZ}=-2.9956$

$\mathrm{XY}=-4.4496 \quad \mathrm{XZ}=0.3583 \quad \mathrm{YZ}=-4.8196$

Octapole moment (field-independent basis, Debye-Ang**2):

$\mathrm{XXX}=-50.0708 \mathrm{YYY}=2.8951 \mathrm{ZZZ}=-2.4145 \mathrm{XYY}=56.7345$

$X X Y=-16.1635 X X Z=8.1875 X Z Z=5.3168 \quad Y Z Z=15.0927$

$\mathrm{YYZ}=10.3740 \mathrm{XYZ}=13.4106$

Hexadecapole moment (field-independent basis, Debye-Ang**3):

$X X X X=-3335.9070 \mathrm{YYYY}=-2558.1024 \mathrm{ZZZZ}=-681.5432 \mathrm{XXXY}=-153.0105$

$X X X Z=-29.2560 \mathrm{YYYX}=-12.8256 \mathrm{YYYZ}=-50.1185 \mathrm{ZZZX}=4.9959$

$\mathrm{ZZZY}=-10.5890 \mathrm{XXYY}=-952.2829 \mathrm{XXZZ}=-682.5482 \mathrm{YYZZ}=-561.7826$

$\mathrm{XXYZ}=-27.1322 \mathrm{YYXZ}=16.4270 \mathrm{ZZXY}=9.4028$

$\mathrm{N}-\mathrm{N}=1.352654048679 \mathrm{D}+03 \mathrm{E}-\mathrm{N}=-4.666525177883 \mathrm{D}+03 \mathrm{KE}=8.366380601557 \mathrm{D}+02$

1|1|UNPC-UNKISPIRHF|6-31G(d)|C16H14O3|PCUSERI19-Apr-2006|0||\# HF/6-31G

(D) GEOM=CONNECTIVITYIITitle Card Required $\| 0,1 \mid \mathrm{H}, 0,-2.37924627,-1.592$

09701,1.95900746|C,0,-2.70924627,-1.01909701,1.09900746|C,0,-3.4952462

7,0.45490299,-1.13499254|C,0,-1.73624627,-0.54209701,0.20600746|C,0,-4

$.05824627,-0.76309701,0.87800746 \mid \mathrm{C}, 0,-4.45424627,-0.02509701,-0.240992$

54|C,0,-2.14324627,0.19790299,-0.91399254|H,0,-4.80224627,-1.13809701,

$1.57500746|\mathrm{H}, 0,-5.50824627,0.17590299,-0.41499254| \mathrm{H}, 0,-1.41324627,0.58$

$490299,-1.61699254|\mathrm{H}, 0,-3.80024627,1.02890299,-2.00499254| \mathrm{C}, 0,-0.30824$

$627,-0.86409701,0.50800746|\mathrm{O}, 0,0.00175373,-1.51809701,1.48900746| \mathrm{C}, 0,0$

$.80575373,-0.31509701,-0.41599254 \mid \mathrm{H}, 0,0.51175373,-0.43809701,-1.461992$

54|C,0,1.11375373,1.13690299,-0.10899254|C,0,1.72275373,3.81190299,0.4

6200746|C,0,1.41975373,1.52790299,1.20100746|C,0,1.12075373,2.09490299

,-1.12799254|C,0,1.42075373,3.42790299,-0.84499254|C,0,1.72375373,2.85

$890299,1.48300746|\mathrm{H}, 0,1.41975373,0.78490299,1.99300746| \mathrm{H}, 0,0.89975373$, 
$1.79590299,-2.14999254|\mathrm{H}, 0,1.42475373,4.16290299,-1.64499254| \mathrm{H}, 0,1.961$

$75373,3.15190299,2.50200746|\mathrm{H}, 0,1.95875373,4.84890299,0.68400746| \mathrm{C}, 0,2$

$.00375373,-2.34109701,-0.64999254 \mid \mathrm{O}, 0,1.09175373,-2.81009701,-1.292992$

$54|\mathrm{O}, 0,2.01275373,-1.06409701,-0.18699254| \mathrm{C}, 0,3.26675373,-3.06209701,-$

$0.24699254|\mathrm{H}, 0,3.31675373,-4.02309701,-0.75999254| \mathrm{H}, 0,3.25375373,-3.22$

$409701,0.83700746 \mid \mathrm{H}, 0,4.14775373,-2.45709701,-0.48099254 \| V$ ersion=x86-

Win32-G03RevB.05|State $=1-\mathrm{AlHF}=-838.6573054|\mathrm{RMSD}=6.564 \mathrm{e}-006| \mathrm{Dipole}=-0.1$

476299,1.2393993,-0.3113866|PG=C01 [X(C16H14O3)]\|@

MANHOOD BEGINS BY TRYING TO SHAVE FACE,

AND ENDS BY TRYING TO SAVE FACE.

Job cpu time: 0 days 0 hours 5 minutes 17.0 seconds.

File lengths (MBytes): RWF $=60 \mathrm{Int}=0 \mathrm{D} 2 \mathrm{E}=0 \mathrm{Chk}=11 \mathrm{Scr}=1$

Normal termination of Gaussian 03 at Wed Apr 19 09:30:57 2006. 


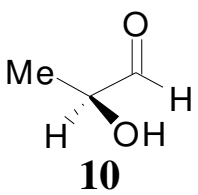

Entering Link 1 = C:\G03W11.exe PID $=3676$

Copyright (c) 1988,1990,1992,1993,1995,1998,2003, Gaussian, Inc. All Rights Reserved.

This is the Gaussian(R) 03 program. It is based on the the Gaussian(R) 98 system (copyright 1998, Gaussian, Inc.), the Gaussian(R) 94 system (copyright 1995, Gaussian, Inc.), the Gaussian 92(TM) system (copyright 1992, Gaussian, Inc.), the Gaussian 90(TM) system (copyright 1990, Gaussian, Inc.), the Gaussian 88(TM) system (copyright 1988, Gaussian, Inc.), the Gaussian 86(TM) system (copyright 1986, Carnegie Mellon University), and the Gaussian 82(TM) system (copyright 1983, Carnegie Mellon University). Gaussian is a federally registered trademark of Gaussian, Inc.

This software contains proprietary and confidential information, including trade secrets, belonging to Gaussian, Inc.

This software is provided under written license and may be used, copied, transmitted, or stored only in accord with that written license.

The following legend is applicable only to US Government contracts under DFARS:

\section{RESTRICTED RIGHTS LEGEND}

Use, duplication or disclosure by the US Government is subject to restrictions as set forth in subparagraph (c)(1)(ii) of the Rights in Technical Data and Computer Software clause at DFARS 252.227-7013.

Gaussian, Inc.

Carnegie Office Park, Building 6, Pittsburgh, PA 15106 USA

The following legend is applicable only to US Government contracts under FAR:

\section{RESTRICTED RIGHTS LEGEND}

Use, reproduction and disclosure by the US Government is subject to restrictions as set forth in subparagraph (c) of the Commercial Computer Software - Restricted Rights clause at FAR 52.227-19.

Gaussian, Inc.

Carnegie Office Park, Building 6, Pittsburgh, PA 15106 USA

Warning -- This program may not be used in any manner that competes with the business of Gaussian, Inc. or will provide assistance to any competitor of Gaussian, Inc. The licensee of this program is prohibited from giving any competitor of Gaussian, Inc. access to this program. By using this program, the user acknowledges that Gaussian, Inc. is engaged in the business of creating and licensing software in the field of computational chemistry and represents and warrants to the 
licensee that it is not a competitor of Gaussian, Inc. and that it will not use this program in any manner prohibited above.

Cite this work as:

Gaussian 03, Revision B.05,

M. J. Frisch, G. W. Trucks, H. B. Schlegel, G. E. Scuseria,

M. A. Robb, J. R. Cheeseman, J. A. Montgomery, Jr., T. Vreven,

K. N. Kudin, J. C. Burant, J. M. Millam, S. S. Iyengar, J. Tomasi,

V. Barone, B. Mennucci, M. Cossi, G. Scalmani, N. Rega,

G. A. Petersson, H. Nakatsuji, M. Hada, M. Ehara, K. Toyota,

R. Fukuda, J. Hasegawa, M. Ishida, T. Nakajima, Y. Honda, O. Kitao,

H. Nakai, M. Klene, X. Li, J. E. Knox, H. P. Hratchian, J. B. Cross,

C. Adamo, J. Jaramillo, R. Gomperts, R. E. Stratmann, O. Yazyev,

A. J. Austin, R. Cammi, C. Pomelli, J. W. Ochterski, P. Y. Ayala,

K. Morokuma, G. A. Voth, P. Salvador, J. J. Dannenberg,

V. G. Zakrzewski, S. Dapprich, A. D. Daniels, M. C. Strain,

O. Farkas, D. K. Malick, A. D. Rabuck, K. Raghavachari,

J. B. Foresman, J. V. Ortiz, Q. Cui, A. G. Baboul, S. Clifford,

J. Cioslowski, B. B. Stefanov, G. Liu, A. Liashenko, P. Piskorz,

I. Komaromi, R. L. Martin, D. J. Fox, T. Keith, M. A. Al-Laham,

C. Y. Peng, A. Nanayakkara, M. Challacombe, P. M. W. Gill,

B. Johnson, W. Chen, M. W. Wong, C. Gonzalez, and J. A. Pople, Gaussian, Inc., Pittsburgh PA, 2003.

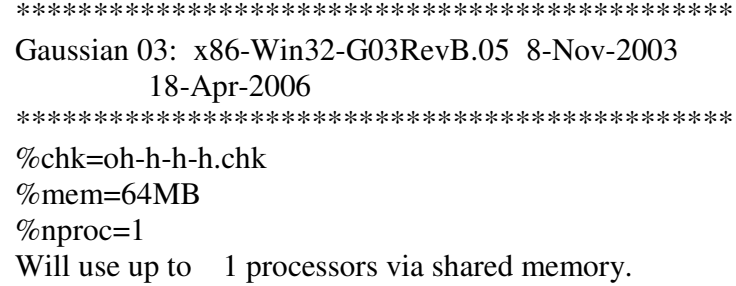

\# hf/6-31g(d) geom=connectivity

$1 / 38=1,57=2 / 1$;

$2 / 17=6,18=5,40=1 / 2$;

$3 / 5=1,6=6,7=1,11=9,16=1,25=1,30=1 / 1,2,3$;

$4 / / 1$

$5 / 5=2,32=1,38=5 / 2$;

$6 / 7=2,8=2,9=2,10=2,28=1 / 1$;

$99 / 5=1,9=1 / 99$;

Title Card Required

Symbolic Z-matrix:

Charge $=0$ Multiplicity $=1$

\begin{tabular}{|c|c|c|c|}
\hline $\mathrm{C}$ & 0.37118 & 0.12605 & -0.4767 \\
\hline $\mathrm{H}$ & 0.45018 & 0.02905 & -1.5767 \\
\hline $\mathrm{C}$ & 1.73618 & -0.21695 & 0.1353 \\
\hline $\mathrm{H}$ & 2.07518 & -1.21295 & -0.1707 \\
\hline $\mathrm{H}$ & 1.68518 & -0.17695 & 1.2283 \\
\hline $\mathrm{H}$ & 2.47418 & 0.51805 & -0.1987 \\
\hline $\mathrm{O}$ & -0.02183 & 1.43005 & -0.1287 \\
\hline $\mathrm{H}$ & -0.91483 & 1.34105 & 0.2583 \\
\hline $\mathrm{C}$ & -0.68183 & -0.87495 & -0.0407 \\
\hline $\mathrm{O}$ & -1.70983 & -0.52495 & 0.5033 \\
\hline $\mathrm{H}$ & -0.46983 & -1.94395 & -0.2447 \\
\hline
\end{tabular}

Input orientation:

Center Atomic Atomic Coordinates (Angstroms) 


$\begin{array}{cccccc}\text { Number } & \text { Number } & \text { Type } & X & Y & Z \\ -1 & 6 & 0 & 0.371175 & 0.126050 & -0.476700 \\ 2 & 1 & 0 & 0.450175 & 0.029050 & -1.576700 \\ 3 & 6 & 0 & 1.736175 & -0.216950 & 0.135300 \\ 4 & 1 & 0 & 2.075175 & -1.212950 & -0.170700 \\ 5 & 1 & 0 & 1.685175 & -0.176950 & 1.228300 \\ 6 & 1 & 0 & 2.474175 & 0.518050 & -0.198700 \\ 7 & 8 & 0 & -0.021825 & 1.430050 & -0.128700 \\ 8 & 1 & 0 & -0.914825 & 1.341050 & 0.258300 \\ 9 & 6 & 0 & -0.681825 & -0.874950 & -0.040700 \\ 10 & 8 & 0 & -1.709825 & -0.524950 & 0.503300 \\ 11 & 1 & 0 & -0.469825 & -1.943950 & -0.244700\end{array}$

Distance matrix (angstroms):

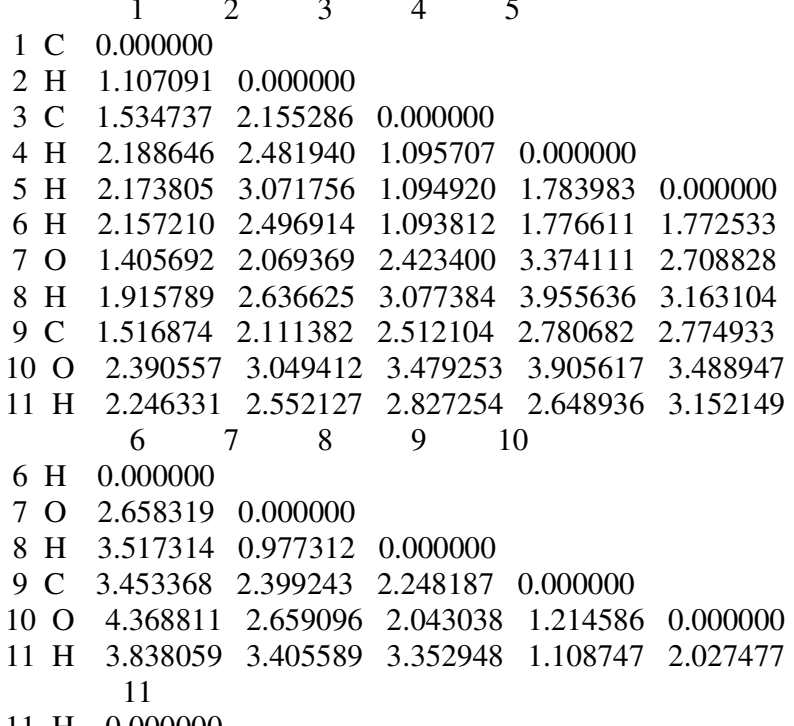

$11 \mathrm{H} \quad 0.000000$

Stoichiometry $\mathrm{C} 3 \mathrm{H} 6 \mathrm{O} 2$

Framework group $\mathrm{C} 1[\mathrm{X}(\mathrm{C} 3 \mathrm{H} 6 \mathrm{O} 2)]$

Deg. of freedom 27

Full point group $\quad \mathrm{C} 1 \quad \mathrm{NOp} 1$

Largest Abelian subgroup $\quad \mathrm{C} 1 \quad \mathrm{NOp} 1$

Largest concise Abelian subgroup C1 NOp 1

Standard orientation:

\begin{tabular}{|c|c|c|c|c|}
\hline \multirow{2}{*}{$\begin{array}{l}\text { Center } \\
\text { Number }\end{array}$} & Atomic & Atomic & \multicolumn{2}{|c|}{ Coordinates (Angstroms) } \\
\hline & Number & Type & $\begin{array}{ll}X & Y\end{array}$ & Z \\
\hline 1 & 6 & -0.444288 & 0.074805 & 0.421799 \\
\hline 2 & 1 & -0.631139 & 0.051829 & 1.512766 \\
\hline 3 & 6 & -1.615885 & -0.624067 & -0.281306 \\
\hline 4 & 1 & -1.759961 & -1.643290 & 0.094191 \\
\hline 5 & 1 & -1.444341 & -0.662269 & -1.362030 \\
\hline 6 & 1 & -2.532980 & -0.055691 & -0.101552 \\
\hline 7 & 8 & -0.311161 & 1.401694 & -0.022736 \\
\hline 8 & 1 & 0.619157 & 1.491150 & -0.308472 \\
\hline 9 & 6 & 0.848813 & -0.686620 & 0.200374 \\
\hline 10 & 8 & 1.831333 & -0.153070 & -0.274176 \\
\hline 11 & 1 & 0.856045 & -1.755428 & 0.495191 \\
\hline
\end{tabular}

Rotational constants (GHZ): $\quad 8.4627885 \quad 4.2130985 \quad 3.1372869$

Standard basis: 6-31G(d) $(6 \mathrm{D}, 7 \mathrm{~F})$

There are 87 symmetry adapted basis functions of A symmetry.

Integral buffers will be 262144 words long.

Raffenetti 1 integral format. 
Two-electron integral symmetry is turned on.

87 basis functions, 164 primitive gaussians, 87 cartesian basis functions

20 alpha electrons 20 beta electrons nuclear repulsion energy 178.7622270916 Hartrees.

NAtoms $=11$ NActive $=11 \mathrm{NUniq}=11 \mathrm{SFac}=1.00 \mathrm{D}+00 \mathrm{NAtFMM}=60 \mathrm{Big}=\mathrm{F}$

One-electron integrals computed using PRISM.

NBasis $=87$ RedAO $=\mathrm{T}$ NBF $=87$

NBsUse $=87$ 1.00D-06 NBFU $=87$

Harris functional with IExCor= 205 diagonalized for initial guess.

ExpMin= 1.61D-01 ExpMax= 5.48D+03 ExpMxC= 8.25D+02 IAcc $=1$ IRadAn $=\quad 1$ AccDes $=1.00 \mathrm{D}-06$
HarFok: IExCor= 205 AccDes= $1.00 \mathrm{D}-06 \mathrm{IRadAn}=\quad 1 \mathrm{IDoV}=1$

ScaDFX $=1.0000001 .0000001 .0000001 .000000$

Initial guess orbital symmetries:

Occupied (A) (A) (A) (A) (A) (A) (A) (A) (A) (A) (A) (A)

(A) (A) (A) (A) (A) (A) (A) (A)

Virtual (A) (A) (A) (A) (A) (A) (A) (A) (A) (A) (A) (A)

(A) $(\mathrm{A})(\mathrm{A})(\mathrm{A})(\mathrm{A})(\mathrm{A})(\mathrm{A})(\mathrm{A})(\mathrm{A})(\mathrm{A})(\mathrm{A})(\mathrm{A})$

(A) $(\mathrm{A})(\mathrm{A})(\mathrm{A})(\mathrm{A})(\mathrm{A})(\mathrm{A})(\mathrm{A})(\mathrm{A})(\mathrm{A})(\mathrm{A})(\mathrm{A})$

(A) (A) (A) (A) (A) (A) (A) (A) (A) (A) (A) (A)

(A) $(A)(A)(A)(A)(A)(A)(A)(A)(A)(A)(A)$

(A) (A) (A) (A) (A) (A) (A)

The electronic state of the initial guess is 1-A.

Warning! Cutoffs for single-point calculations used.

Requested convergence on RMS density matrix=1.00D-04 within 128 cycles.

Requested convergence on MAX density matrix=1.00D-02.

Requested convergence on energy $=5.00 \mathrm{D}-05$.

No special actions if energy rises.

Keep R1 integrals in memory in canonical form, NReq $=8138245$.

SCF Done: $\mathrm{E}(\mathrm{RHF})=-266.800440288$ A.U. after 6 cycles

Convg $=0.3128 \mathrm{D}-04 \quad-\mathrm{V} / \mathrm{T}=2.0027$

$\mathrm{S} * * 2=0.0000$

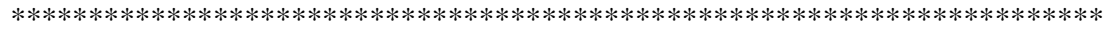

Population analysis using the SCF density.

$* * * * * * * * * * * * * * * * * * * * * * * * * * * * * * * * * * * * * * * * * * * * * * * * * * * * * * * * * * * * * * * * * * * * * *$

Orbital symmetries:

Occupied (A) (A) (A) (A) (A) (A) (A) (A) (A) (A) (A) (A)

(A) $(\mathrm{A})(\mathrm{A})(\mathrm{A})(\mathrm{A})(\mathrm{A})(\mathrm{A})(\mathrm{A})$

Virtual (A) (A) (A) (A) (A) (A) (A) (A) (A) (A) $(A)(A)$

(A) $(\mathrm{A})(\mathrm{A})(\mathrm{A})(\mathrm{A})(\mathrm{A})(\mathrm{A})(\mathrm{A})(\mathrm{A})(\mathrm{A})(\mathrm{A})(\mathrm{A})$

(A) $(\mathrm{A})(\mathrm{A})(\mathrm{A})(\mathrm{A})(\mathrm{A})(\mathrm{A})(\mathrm{A})(\mathrm{A})(\mathrm{A})(\mathrm{A})(\mathrm{A})$

(A) $(\mathrm{A})(\mathrm{A})(\mathrm{A})(\mathrm{A})(\mathrm{A})(\mathrm{A})(\mathrm{A})(\mathrm{A})(\mathrm{A})(\mathrm{A})(\mathrm{A})$

$(\mathrm{A})(\mathrm{A})(\mathrm{A})(\mathrm{A})(\mathrm{A})(\mathrm{A})(\mathrm{A})(\mathrm{A})(\mathrm{A})(\mathrm{A})(\mathrm{A})(\mathrm{A})$

(A) (A) (A) (A) (A) (A) (A)

The electronic state is $1-\mathrm{A}$.

Alpha occ. eigenvalues -- -20.59122 -20.56472 -11.35362 -11.30502 -11.23844

Alpha occ. eigenvalues -- $\quad-1.42049-1.36071 \quad-1.04997 \quad-0.91032 \quad-0.79731$

Alpha occ. eigenvalues -- $-0.72800 \quad-0.68403 \quad-0.65137 \quad-0.58747 \quad-0.56734$

$\begin{array}{lllllll}\text { Alpha occ. eigenvalues -- } & -0.54628 & -0.53923 & -0.52787 & -0.44901 & -0.43711\end{array}$

$\begin{array}{lllllll}\text { Alpha virt. eigenvalues -- } & 0.13132 & 0.23724 & 0.26571 & 0.27927 & 0.28138\end{array}$

$\begin{array}{lllllll}\text { Alpha virt. eigenvalues -- } & 0.31380 & 0.31764 & 0.37454 & 0.39962 & 0.45353\end{array}$

$\begin{array}{lllllll}\text { Alpha virt. eigenvalues -- } & 0.51765 & 0.70283 & 0.74832 & 0.78223 & 0.79546\end{array}$

$\begin{array}{lllllll}\text { Alpha virt. eigenvalues -- } & 0.84186 & 0.88356 & 0.92000 & 0.93670 & 0.96815\end{array}$

$\begin{array}{lllllll}\text { Alpha virt. eigenvalues -- } & 1.02263 & 1.07845 & 1.08957 & 1.11127 & 1.13727\end{array}$

$\begin{array}{lllllll}\text { Alpha virt. eigenvalues -- } & 1.15223 & 1.19961 & 1.21733 & 1.26469 & 1.28108\end{array}$

$\begin{array}{lllllll}\text { Alpha virt. eigenvalues -- } & 1.30305 & 1.35983 & 1.40286 & 1.52121 & 1.56557\end{array}$

$\begin{array}{lllllll}\text { Alpha virt. eigenvalues -- } & 1.60867 & 1.74529 & 1.76553 & 1.83014 & 1.91226\end{array}$

$\begin{array}{lllllll}\text { Alpha virt. eigenvalues -- } & 1.97958 & 2.04997 & 2.07649 & 2.12850 & 2.14880\end{array}$

$\begin{array}{llllll}\text { Alpha virt. eigenvalues -- } & 2.19521 & 2.26156 & 2.33680 & 2.35169 & 2.46775\end{array}$

$\begin{array}{lllllll}\text { Alpha virt. eigenvalues -- } & 2.50231 & 2.52636 & 2.63022 & 2.71603 & 2.76200\end{array}$

$\begin{array}{llllll}\text { Alpha virt. eigenvalues -- } & 2.81214 & 2.90384 & 2.92221 & 2.97916 & 3.20622\end{array}$ 


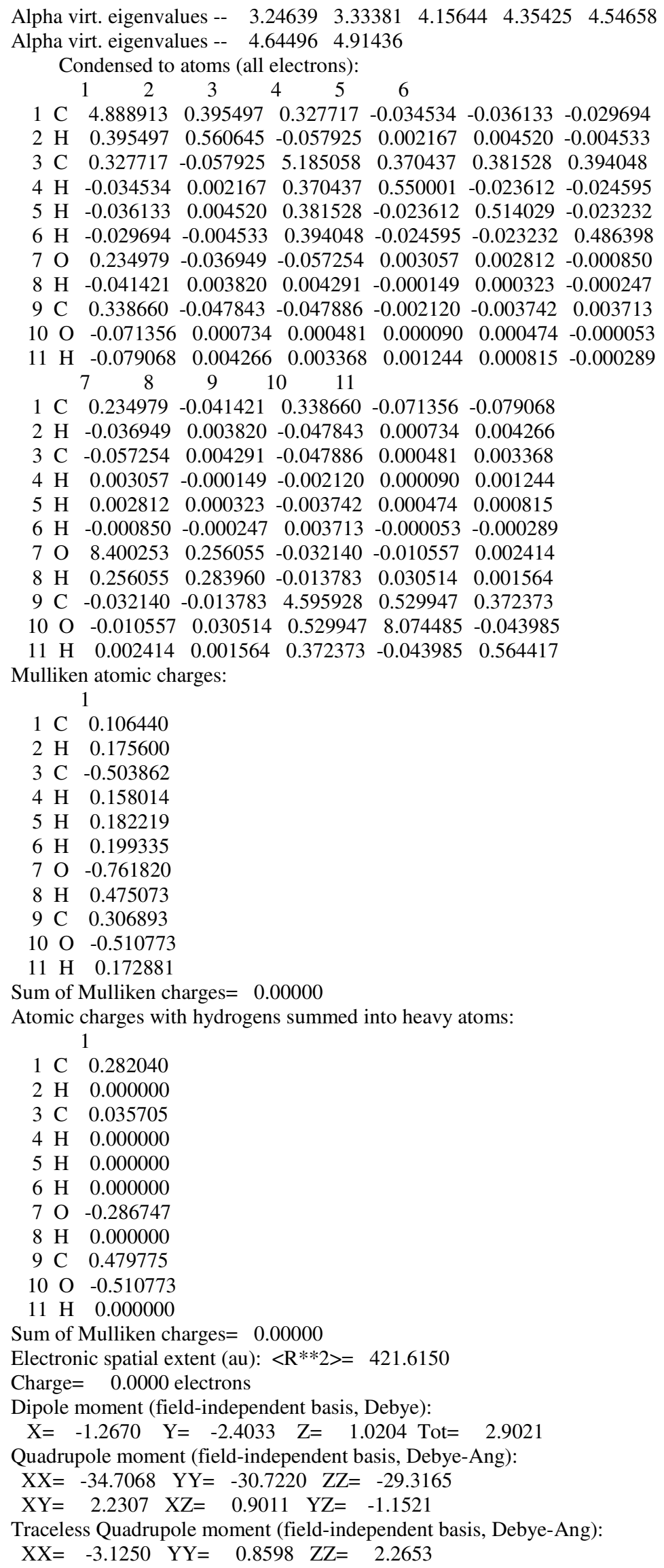


$\mathrm{XY}=2.2307 \mathrm{XZ}=0.9011 \mathrm{YZ}=-1.1521$

Octapole moment (field-independent basis, Debye-Ang**2):

$\mathrm{XXX}=-8.9975 \mathrm{YYY}=-1.2805 \mathrm{ZZZ}=-0.5707 \mathrm{XYY}=8.3487$

$\mathrm{XXY}=3.3677 \mathrm{XXZ}=2.6584 \mathrm{XZZ}=2.0328 \mathrm{YZZ}=1.2417$

$\mathrm{YYZ}=-0.7419 \mathrm{XYZ}=-1.4731$

Hexadecapole moment (field-independent basis, Debye-Ang**3):

$\mathrm{XXXX}=-327.4590 \mathrm{YYYY}=-153.2454 \mathrm{ZZZZ}=-54.9081 \mathrm{XXXY}=-1.5493$

$\mathrm{XXXZ}=1.8062 \mathrm{YYYX}=4.8676 \mathrm{YYYZ}=-5.6559 \mathrm{ZZZX}=-1.7286$

$\mathrm{ZZZY}=-0.1817 \mathrm{XXYY}=-75.2502 \mathrm{XXZZ}=-61.4489 \mathrm{YYZZ}=-35.6329$

$\mathrm{XXYZ}=-1.3375 \mathrm{YYXZ}=-1.7360 \mathrm{ZZXY}=-0.5047$

$\mathrm{N}-\mathrm{N}=1.787622270916 \mathrm{D}+02 \mathrm{E}-\mathrm{N}=-9.845884640686 \mathrm{D}+02 \quad \mathrm{KE}=2.660829791763 \mathrm{D}+02$

1I1|UNPC-UNKISP|RHF|6-31G(d)|C3H6O2|PCUSERI18-Apr-2006|0||\# HF/6-31G(D

) GEOM=CONNECTIVITY\|Title Card Required $\| 0,1 \mid \mathrm{C}, 0,0.371175,0.12605,-0$.

$4767|\mathrm{H}, 0,0.450175,0.02905,-1.5767| \mathrm{C}, 0,1.736175,-0.21695,0.1353 \mid \mathrm{H}, 0,2.0$

$75175,-1.21295,-0.1707|\mathrm{H}, 0,1.685175,-0.17695,1.2283| \mathrm{H}, 0,2.474175,0.518$

$05,-0.1987 \mathrm{IO}, 0,-0.021825,1.43005,-0.1287|\mathrm{H}, 0,-0.914825,1.34105,0.2583|$

$\mathrm{C}, 0,-0.681825,-0.87495,-0.0407 \mathrm{IO}, 0,-1.709825,-0.52495,0.5033 \mathrm{H}, 0,-0.46$

$9825,-1.94395,-0.2447 \|$ Version $=x 86-$ Win32-G03RevB $.05 \mid$ State $=1-A \mid H F=-266$.

$8004403|\mathrm{RMSD}=3.128 \mathrm{e}-005| \mathrm{Dipole}=0.6623354,-0.8488114,-0.3801536 \mid \mathrm{PG}=\mathrm{C} 01$

[X(C3H6O2)]\|@

Any fool can criticize, condemn, and complain -- and most do.

-- Dale Carnegie

Job cpu time: 0 days 0 hours 0 minutes 14.0 seconds.

File lengths (MBytes): $\mathrm{RWF}=11 \mathrm{Int}=0 \mathrm{D} 2 \mathrm{E}=0 \mathrm{Chk}=7 \mathrm{Scr}=1$

Normal termination of Gaussian 03 at Tue Apr 18 16:03:02 2006. 


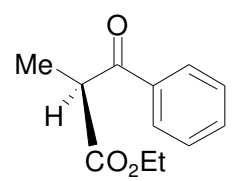

11

Entering Link 1 = C:\G03W11.exe PID= 2408.

Copyright (c) 1988,1990,1992,1993,1995,1998,2003, Gaussian, Inc. All Rights Reserved.

This is the Gaussian(R) 03 program. It is based on the the Gaussian(R) 98 system (copyright 1998, Gaussian, Inc.), the Gaussian(R) 94 system (copyright 1995, Gaussian, Inc.), the Gaussian 92(TM) system (copyright 1992, Gaussian, Inc.), the Gaussian 90(TM) system (copyright 1990, Gaussian, Inc.), the Gaussian 88(TM) system (copyright 1988, Gaussian, Inc.), the Gaussian 86(TM) system (copyright 1986, Carnegie Mellon University), and the Gaussian 82(TM) system (copyright 1983, Carnegie Mellon University). Gaussian is a federally registered trademark of Gaussian, Inc.

This software contains proprietary and confidential information, including trade secrets, belonging to Gaussian, Inc.

This software is provided under written license and may be used, copied, transmitted, or stored only in accord with that written license.

The following legend is applicable only to US Government contracts under DFARS:

\section{RESTRICTED RIGHTS LEGEND}

Use, duplication or disclosure by the US Government is subject to restrictions as set forth in subparagraph (c)(1)(ii) of the Rights in Technical Data and Computer Software clause at DFARS 252.227-7013.

Gaussian, Inc.

Carnegie Office Park, Building 6, Pittsburgh, PA 15106 USA

The following legend is applicable only to US Government contracts under FAR:

\section{RESTRICTED RIGHTS LEGEND}

Use, reproduction and disclosure by the US Government is subject to restrictions as set forth in subparagraph (c) of the

Commercial Computer Software - Restricted Rights clause at FAR 52.227-19.

Gaussian, Inc.

Carnegie Office Park, Building 6, Pittsburgh, PA 15106 USA

Warning -- This program may not be used in any manner that competes with the business of Gaussian, Inc. or will provide assistance to any competitor of Gaussian, Inc. The licensee of this program is prohibited from giving any competitor of Gaussian, Inc. access to this program. By using this program, the user acknowledges that Gaussian, Inc. is engaged in the 
business of creating and licensing software in the field of computational chemistry and represents and warrants to the licensee that it is not a competitor of Gaussian, Inc. and that it will not use this program in any manner prohibited above.

Cite this work as:

Gaussian 03, Revision B.05,

M. J. Frisch, G. W. Trucks, H. B. Schlegel, G. E. Scuseria,

M. A. Robb, J. R. Cheeseman, J. A. Montgomery, Jr., T. Vreven,

K. N. Kudin, J. C. Burant, J. M. Millam, S. S. Iyengar, J. Tomasi,

V. Barone, B. Mennucci, M. Cossi, G. Scalmani, N. Rega,

G. A. Petersson, H. Nakatsuji, M. Hada, M. Ehara, K. Toyota,

R. Fukuda, J. Hasegawa, M. Ishida, T. Nakajima, Y. Honda, O. Kitao,

H. Nakai, M. Klene, X. Li, J. E. Knox, H. P. Hratchian, J. B. Cross,

C. Adamo, J. Jaramillo, R. Gomperts, R. E. Stratmann, O. Yazyev,

A. J. Austin, R. Cammi, C. Pomelli, J. W. Ochterski, P. Y. Ayala,

K. Morokuma, G. A. Voth, P. Salvador, J. J. Dannenberg,

V. G. Zakrzewski, S. Dapprich, A. D. Daniels, M. C. Strain,

O. Farkas, D. K. Malick, A. D. Rabuck, K. Raghavachari,

J. B. Foresman, J. V. Ortiz, Q. Cui, A. G. Baboul, S. Clifford,

J. Cioslowski, B. B. Stefanov, G. Liu, A. Liashenko, P. Piskorz,

I. Komaromi, R. L. Martin, D. J. Fox, T. Keith, M. A. Al-Laham,

C. Y. Peng, A. Nanayakkara, M. Challacombe, P. M. W. Gill,

B. Johnson, W. Chen, M. W. Wong, C. Gonzalez, and J. A. Pople, Gaussian, Inc., Pittsburgh PA, 2003.

$* * * * * * * * * * * * * * * * * * * * * * * * * * * * * * * * * * * * * * * * * * * * * *$

Gaussian 03: x86-Win32-G03RevB.05 8-Nov-2003

19-Apr-2006

*****************************************************

$\%$ chk=co2et-me-h-ph.chk

$\%$ mem $=64 \mathrm{MB}$

$\%$ nproc $=1$

Will use up to 1 processors via shared memory.

\# hf/6-31g(d) geom=connectivity

$1 / 38=1,57=2 / 1$;

$2 / 17=6,18=5,40=1 / 2$;

$3 / 5=1,6=6,7=1,11=9,16=1,25=1,30=1 / 1,2,3$;

$4 / / 1$

$5 / 5=2,32=1,38=5 / 2$;

$6 / 7=2,8=2,9=2,10=2,28=1 / 1$;

$99 / 5=1,9=1 / 99$;

Title Card Required

Symbolic Z-matrix:

Charge $=0$ Multiplicity $=1$

$\begin{array}{lllr}\mathrm{C} & 0.70619 & 1.445 & 0.52489 \\ \mathrm{H} & 0.77219 & 0.992 & 1.51989 \\ \mathrm{C} & 1.52919 & 0.596 & -0.44211 \\ \mathrm{O} & 2.19119 & -0.396 & 0.19689 \\ \mathrm{C} & 3.01419 & -1.245 & -0.64111 \\ \mathrm{H} & 3.74319 & -0.619 & -1.16511 \\ \mathrm{H} & 2.37519 & -1.714 & -1.39611 \\ \mathrm{C} & 3.68219 & -2.265 & 0.26189 \\ \mathrm{H} & 4.31619 & -2.93 & -0.33511 \\ \mathrm{H} & 2.93819 & -2.876 & 0.78289 \\ \mathrm{H} & 4.30919 & -1.772 & 1.01189 \\ \mathrm{O} & 1.60319 & 0.797 & -1.63411 \\ \mathrm{C} & -0.77481 & 1.456 & 0.08189\end{array}$




$\begin{array}{lccc}\mathrm{O} & -1.27981 & 2.492 & -0.31511 \\ \mathrm{C} & -1.57681 & 0.194 & 0.16989 \\ \mathrm{C} & -3.19781 & -2.09 & 0.30189 \\ \mathrm{C} & -1.08081 & -0.998 & 0.72089 \\ \mathrm{C} & -2.89681 & 0.226 & -0.31211 \\ \mathrm{C} & -3.70081 & -0.907 & -0.24811 \\ \mathrm{C} & -1.88881 & -2.133 & 0.78489 \\ \mathrm{H} & -0.06381 & -1.053 & 1.09389 \\ \mathrm{H} & -3.26681 & 1.156 & -0.73111 \\ \mathrm{H} & -4.71881 & -0.872 & -0.62611 \\ \mathrm{H} & -1.49681 & -3.05 & 1.21489 \\ \mathrm{H} & -3.82481 & -2.976 & 0.35389 \\ \mathrm{C} & 1.27719 & 2.871 & 0.56089 \\ \mathrm{H} & 1.25719 & 3.312 & -0.43711 \\ \mathrm{H} & 2.31019 & 2.857 & 0.92489 \\ \mathrm{H} & 0.67919 & 3.501 & 1.22489\end{array}$

Input orientation:

\begin{tabular}{|c|c|c|c|c|c|}
\hline \multirow{2}{*}{$\begin{array}{l}\text { Center } \\
\text { Number }\end{array}$} & \multirow{2}{*}{$\begin{array}{l}\text { Atomic } \\
\text { Number }\end{array}$} & \multirow{2}{*}{\multicolumn{2}{|c|}{$\begin{array}{l}\text { Atomic } \\
\text { Type }\end{array}$}} & \multicolumn{2}{|c|}{ Coordinates (Angstroms) } \\
\hline & & & & $\begin{array}{ll}X & Y\end{array}$ & $\mathrm{Z}$ \\
\hline 1 & 6 & 0 & 0.706191 & 1.445000 & 0.524891 \\
\hline 2 & 1 & 0 & 0.772191 & 0.992000 & 1.519891 \\
\hline 3 & 6 & 0 & 1.529191 & 0.596000 & -0.442109 \\
\hline 4 & 8 & 0 & 2.191191 & -0.396000 & 0.196891 \\
\hline 5 & 6 & 0 & 3.014191 & -1.245000 & -0.641109 \\
\hline 6 & 1 & 0 & 3.743191 & -0.619000 & -1.165109 \\
\hline 7 & 1 & 0 & 2.375191 & -1.714000 & -1.396109 \\
\hline 8 & 6 & 0 & 3.682191 & -2.265000 & 0.261891 \\
\hline 9 & 1 & 0 & 4.316191 & -2.930000 & -0.335109 \\
\hline 10 & 1 & 0 & 2.938191 & -2.876000 & 0.782891 \\
\hline 11 & 1 & 0 & 4.309191 & -1.772000 & 1.011891 \\
\hline 12 & 8 & 0 & 1.603191 & 0.797000 & -1.634109 \\
\hline 13 & 6 & 0 & -0.774809 & 1.456000 & 0.081891 \\
\hline 14 & 8 & 0 & -1.279809 & 2.492000 & -0.315109 \\
\hline 15 & 6 & 0 & -1.576809 & 0.194000 & 0.169891 \\
\hline 16 & 6 & 0 & -3.197809 & -2.090000 & 0.301891 \\
\hline 17 & 6 & 0 & -1.080809 & -0.998000 & 0.720891 \\
\hline 18 & 6 & 0 & -2.896809 & 0.226000 & -0.312109 \\
\hline 19 & 6 & 0 & -3.700809 & -0.907000 & -0.248109 \\
\hline 20 & 6 & 0 & -1.888809 & -2.133000 & 0.784891 \\
\hline 21 & 1 & 0 & -0.063809 & -1.053000 & 1.093891 \\
\hline 22 & 1 & 0 & -3.266809 & 1.156000 & -0.731109 \\
\hline 23 & 1 & 0 & -4.718809 & -0.872000 & -0.626109 \\
\hline 24 & 1 & 0 & -1.496809 & -3.050000 & 1.214891 \\
\hline 25 & 1 & 0 & -3.824809 & -2.976000 & 0.353891 \\
\hline 26 & 6 & 0 & 1.277191 & 2.871000 & 0.560891 \\
\hline 27 & 1 & 0 & 1.257191 & 3.312000 & -0.437109 \\
\hline 28 & 1 & 0 & 2.310191 & 2.857000 & 0.924891 \\
\hline 29 & 1 & 0 & 0.679191 & 3.501000 & 1.224891 \\
\hline
\end{tabular}

Distance matrix (angstroms):

$1 \mathrm{C} \quad 0.000000$

$\begin{array}{llll}2 & \mathrm{H} & 1.095258 & 0.000000\end{array}$

$\begin{array}{lllll}3 \mathrm{C} & 1.527488 & 2.139932 & 0.000000\end{array}$

$\begin{array}{lllllll}4 & \mathrm{O} & 2.387905 & 2.385463 & 1.353007 & 0.000000\end{array}$

$\begin{array}{lllllll}5 & \mathrm{C} & 3.731289 & 3.834143 & 2.373627 & 1.449267 & 0.000000\end{array}$

$\begin{array}{lllllll}6 \mathrm{H} & 4.042223 & 4.316409 & 2.626928 & 2.076891 & 1.094483\end{array}$

$\begin{array}{lllllll}7 & \mathrm{H} & 4.056487 & 4.288951 & 2.638547 & 2.075724 & 1.094672\end{array}$

$\begin{array}{lllllll}8 & \mathrm{C} & 4.763386 & 4.545186 & 3.649157 & 2.391750 & 1.517245\end{array}$

$\begin{array}{lllllll}9 \mathrm{H} & 5.736926 & 5.602057 & 4.495720 & 3.349598 & 2.151294\end{array}$

$\begin{array}{lllllll}10 & \mathrm{H} & 4.870260 & 4.494013 & 3.942168 & 2.655523 & 2.166498\end{array}$ 
$\begin{array}{lllllll}11 & \mathrm{H} & 4.854675 & 4.517536 & 3.930641 & 2.653964 & 2.164986\end{array}$

$\begin{array}{lllllll}12 & \mathrm{O} & 2.426066 & 3.267461 & 1.211091 & 2.263085 & 2.673338\end{array}$

$\begin{array}{lllllll}13 & \mathrm{C} & 1.545875 & 2.162487 & 2.514476 & 3.498612 & 4.708997\end{array}$

$\begin{array}{llllllll}14 & \mathrm{O} & 2.397083 & 3.134953 & 3.391375 & 4.544285 & 5.701744\end{array}$

$\begin{array}{llllllll}15 & \mathrm{C} & 2.627378 & 2.824377 & 3.191141 & 3.814007 & 4.879111\end{array}$

$\begin{array}{llllllll}16 & \mathrm{C} & 5.271354 & 5.171378 & 5.487500 & 5.649954 & 6.339733\end{array}$

$\begin{array}{lllllll}17 & \mathrm{C} & 3.033156 & 2.834098 & 3.271927 & 3.367932 & 4.322624\end{array}$

$\begin{array}{llllllll}18 & \mathrm{C} & 3.894630 & 4.171875 & 4.443341 & 5.151088 & 6.100164\end{array}$

$\begin{array}{llllllll}19 & \mathrm{C} & 5.054808 & 5.171050 & 5.445140 & 5.930836 & 6.734977\end{array}$

$\begin{array}{llllllll}20 & \mathrm{C} & 4.427608 & 4.169745 & 4.542653 & 4.473177 & 5.182801\end{array}$

$\begin{array}{llllllll}21 & \mathrm{H} & 2.675194 & 2.249977 & 2.759737 & 2.514216 & 3.538527\end{array}$

$\begin{array}{lllllll}22 & \mathrm{H} & 4.176815 & 4.626815 & 4.837224 & 5.749752 & 6.724869\end{array}$

$\begin{array}{llllllll}23 \mathrm{H} & 6.010317 & 6.183114 & 6.420778 & 6.975099 & 7.742005\end{array}$

$\begin{array}{llllllll}24 & \mathrm{H} & 5.053151 & 4.645336 & 5.019526 & 4.656327 & 5.201142\end{array}$

$\begin{array}{lllllll}25 & \mathrm{H} & 6.332807 & 6.183606 & 6.485223 & 6.547771 & 7.124486\end{array}$

$\begin{array}{llllllll}26 \mathrm{C} & 1.536494 & 2.169181 & 2.499027 & 3.411917 & 4.626384\end{array}$

$\begin{array}{llllllll}27 & \mathrm{H} & 2.171344 & 3.073674 & 2.729591 & 3.876026 & 4.888242\end{array}$

$\begin{array}{lllllll}28 & \mathrm{H} & 2.174065 & 2.489517 & 2.755135 & 3.335589 & 4.446839\end{array}$

$\begin{array}{lllllll}29 & \mathrm{H} & 2.172065 & 2.527994 & 3.455490 & 4.304595 & 5.608805\end{array}$

$6 \mathrm{H} \quad 0.000000$

$\begin{array}{llll}7 & \mathrm{H} & 1.767430 & 0.000000\end{array}$

$\begin{array}{lllll}8 & \mathrm{C} & 2.179304 & 2.181929 & 0.000000\end{array}$

$\begin{array}{llllll}9 & \mathrm{H} & 2.521498 & 2.524254 & 1.095714 & 0.000000\end{array}$

$\begin{array}{lllllll}10 \mathrm{H} & 3.088167 & 2.532835 & 1.094668 & 1.775310 & 0.000000\end{array}$

$\begin{array}{lllllll}11 \mathrm{H} & 2.527666 & 3.089043 & 1.094842 & 1.776351 & 1.775077\end{array}$

$\begin{array}{lllllll}12 & \mathrm{O} & 2.608566 & 2.637755 & 4.158473 & 4.789394 & 4.595111\end{array}$

$\begin{array}{lllllll}13 & \mathrm{C} & 5.125715 & 4.707004 & 5.808880 & 6.732694 & 5.748391\end{array}$

$\begin{array}{llllllll}14 & \mathrm{O} & 5.969200 & 5.676092 & 6.898074 & 7.791900 & 6.914662\end{array}$

$\begin{array}{llllllll}15 & \mathrm{C} & 5.544871 & 4.659520 & 5.806223 & 6.688935 & 5.494169\end{array}$

$\begin{array}{lllllll}16 & \mathrm{C} & 7.245234 & 5.838057 & 6.882342 & 7.587593 & 6.204809\end{array}$

$\begin{array}{llllllll}17 & \mathrm{C} & 5.193420 & 4.115614 & 4.949964 & 5.828839 & 4.436563\end{array}$

$\begin{array}{lllllll}18 & \mathrm{C} & 6.747684 & 5.721245 & 7.058172 & 7.873261 & 6.698407\end{array}$

$\begin{array}{llllllll}19 & \mathrm{C} & 7.505796 & 6.235939 & 7.524158 & 8.268760 & 7.001160\end{array}$

$\begin{array}{llllllll}20 & \mathrm{C} & 6.149319 & 4.807704 & 5.597052 & 6.355441 & 4.883849\end{array}$

$\begin{array}{llllllll}21 & \mathrm{H} & 4.447998 & 3.547639 & 4.024138 & 4.974894 & 3.525912\end{array}$

$\begin{array}{llllllll}22 & \mathrm{H} & 7.244245 & 6.364848 & 7.808834 & 8.622882 & 7.553227\end{array}$

$\begin{array}{lllllll}23 & \mathrm{H} & 8.482922 & 7.185172 & 8.561880 & 9.270991 & 8.039337\end{array}$

$\begin{array}{lllllll}24 \mathrm{H} & 6.247540 & 4.857427 & 5.324141 & 6.017297 & 4.459386\end{array}$

$\begin{array}{llllllll}25 & \mathrm{H} & 8.070777 & 6.564689 & 7.541156 & 8.170234 & 6.777331\end{array}$

$\begin{array}{llllllll}26 & \mathrm{C} & 4.608723 & 5.104672 & 5.679078 & 6.609836 & 5.986336\end{array}$

$\begin{array}{llllllll}27 & \mathrm{H} & 4.707753 & 5.237393 & 6.121450 & 6.952010 & 6.527289\end{array}$

$\begin{array}{llllllll}28 & \mathrm{H} & 4.301647 & 5.126920 & 5.343860 & 6.253080 & 5.769041\end{array}$

$\begin{array}{lllllll}29 & \mathrm{H} & 5.663444 & 6.078016 & 6.572072 & 7.551101 & 6.779718\end{array}$ $\begin{array}{lllll}11 & 12 & 13 & 14 & 15\end{array}$

$\begin{array}{lll}11 \mathrm{H} & 0.000000\end{array}$

$\begin{array}{llll}12 & \mathrm{O} & 4.574223 & 0.000000\end{array}$

$\begin{array}{lllll}13 & \mathrm{C} & 6.093598 & 3.005632 & 0.000000\end{array}$

$\begin{array}{lllllll}14 & \mathrm{O} & 7.153988 & 3.595063 & 1.218987 & 0.000000\end{array}$

$\begin{array}{lllllll}15 & \mathrm{C} & 6.262517 & 3.705459 & 1.497862 & 2.367327 & 0.000000\end{array}$

$\begin{array}{lllllll}16 & \mathrm{C} & 7.547203 & 5.927265 & 4.300401 & 5.005411 & 2.803876\end{array}$

$\begin{array}{lllllll}17 & \mathrm{C} & 5.453059 & 3.996487 & 2.554226 & 3.645956 & 1.403738\end{array}$

$\begin{array}{lllllll}18 & \mathrm{C} & 7.594170 & 4.724799 & 2.484154 & 2.783784 & 1.405613\end{array}$

$\begin{array}{llllllll}19 & \mathrm{C} & 8.154503 & 5.740821 & 3.775466 & 4.173599 & 2.428642\end{array}$

$\begin{array}{llllllll}20 & \mathrm{C} & 6.212653 & 5.160477 & 3.823104 & 4.792860 & 2.427035\end{array}$

$\begin{array}{llllllll}21 & \mathrm{H} & 4.432473 & 3.693694 & 2.797275 & 4.003868 & 2.167476\end{array}$

$\begin{array}{lllllll}22 & \mathrm{H} & 8.307046 & 4.966003 & 2.638377 & 2.430251 & 2.143209\end{array}$

$\begin{array}{llllllll}23 & \mathrm{H} & 9.219427 & 6.615838 & 4.634219 & 4.820782 & 3.412057\end{array}$

$\begin{array}{lllllll}24 & \mathrm{H} & 5.948456 & 5.703175 & 4.702022 & 5.753412 & 3.409100\end{array}$

$\begin{array}{lllllll}25 & \mathrm{H} & 8.248911 & 6.902960 & 5.386939 & 6.068246 & 3.890535\end{array}$

$\begin{array}{lllllll}26 & \mathrm{C} & 5.563621 & 3.037396 & 2.538182 & 2.729334 & 3.932496\end{array}$

$\begin{array}{llllllll}27 & \mathrm{H} & 6.104208 & 2.806733 & 2.800557 & 2.669017 & 4.256986\end{array}$

$\begin{array}{lllllll}28 \mathrm{H} & 5.042937 & 3.360347 & 3.491515 & 3.815616 & 4.771830\end{array}$

$\begin{array}{lllllll}29 & \mathrm{H} & 6.405216 & 4.042187 & 2.757279 & 2.688375 & 4.139905\end{array}$ 


\begin{tabular}{|c|c|c|c|c|c|}
\hline & 16 & 18 & 19 & 20 & \\
\hline $16 \mathrm{C}$ & 0.000000 & & & & \\
\hline $17 \mathrm{C}$ & 2.418618 & 0.000000 & & & \\
\hline $18 \mathrm{C}$ & 2.414840 & 2.421388 & 0.000000 & & \\
\hline $19 \mathrm{C}$ & 1.398212 & 2.794931 & 1.390756 & 0.000000 & \\
\hline $20 \mathrm{C}$ & 1.395929 & 1.394699 & 2.790046 & 2.419403 & 0.000000 \\
\hline $21 \mathrm{H}$ & 3.394789 & 1.084640 & 3.411534 & 3.879439 & 2.143013 \\
\hline $22 \mathrm{H}$ & 3.407105 & 3.395087 & 1.085063 & 2.162779 & 3.874876 \\
\hline $23 \mathrm{H}$ & 2.158275 & 3.881408 & 2.150322 & 1.086477 & 3.404400 \\
\hline $24 \mathrm{H}$ & 2.156054 & 2.151231 & 3.876068 & 3.404473 & 1.086026 \\
\hline $25 \mathrm{H}$ & 1.086660 & 3.402456 & 3.399639 & 2.158365 & 2.155112 \\
\hline $26 \mathrm{C}$ & 6.686122 & 4.533754 & 5.018011 & 6.301448 & 5.925685 \\
\hline $27 \mathrm{H}$ & 7.040934 & 5.038185 & 5.176363 & 6.512868 & 6.406140 \\
\hline $28 \mathrm{H}$ & 7.429603 & 5.138241 & 5.963655 & 7.188584 & 6.523136 \\
\hline \multirow[t]{2}{*}{$29 \mathrm{H}$} & 6.866028 & 4.857223 & 5.086823 & 6.386282 & 6.207268 \\
\hline & 21 & $22 \quad 23$ & 24 & 25 & \\
\hline $21 \mathrm{H}$ & 0.000000 & & & & \\
\hline $22 \mathrm{H}$ & 4.297617 & 0.000000 & & & \\
\hline $23 \mathrm{H}$ & 4.965902 & 2.496420 & 0.000000 & & \\
\hline $24 \mathrm{H}$ & 2.460922 & 4.960872 & 4.302819 & 0.000000 & \\
\hline $25 \mathrm{H}$ & 4.288432 & 4.308365 & 2.487258 & 2.483220 & 0.000000 \\
\hline $26 \mathrm{C}$ & 4.180926 & 5.025776 & 7.167359 & 6.571228 & 7.762774 \\
\hline $27 \mathrm{H}$ & 4.810637 & 5.020094 & 7.297544 & 7.126617 & 8.123506 \\
\hline $28 \mathrm{H}$ & 4.577394 & 6.061243 & 8.106657 & 7.033491 & 8.484583 \\
\hline \multirow[t]{2}{*}{$29 \mathrm{H}$} & 4.616073 & 4.989577 & 7.189418 & 6.902947 & 7.937014 \\
\hline & 26 & $27 \quad 28$ & 29 & & \\
\hline $26 \mathrm{C}$ & 0.000000 & & & & \\
\hline $27 \mathrm{H}$ & 1.091277 & 0.000000 & & & \\
\hline $28 \mathrm{H}$ & 1.095345 & 1.780696 & 0.000000 & & \\
\hline $29 \mathrm{H}$ & 1.093343 & 1.769760 & 1.779016 & 0.000000 & \\
\hline \multicolumn{6}{|c|}{ Stoichiometry $\quad \mathrm{C} 12 \mathrm{H} 14 \mathrm{O} 3$} \\
\hline \multicolumn{6}{|c|}{ Framework group $\mathrm{C} 1[\mathrm{X}(\mathrm{C} 12 \mathrm{H} 14 \mathrm{O} 3)]$} \\
\hline \multicolumn{6}{|c|}{ Deg. of freedom 81} \\
\hline \multicolumn{6}{|c|}{ Full point group $\quad \mathrm{C} 1$} \\
\hline \multicolumn{6}{|c|}{$\begin{array}{llll}\text { Largest Abelian subgroup } & \mathrm{C} 1 & \text { NOp } & 1\end{array}$} \\
\hline Largest & $\begin{array}{r}\text { oncise Abe } \\
\text { Stanc }\end{array}$ & $\begin{array}{l}\text { elian subgro } \\
\text { dard orienta }\end{array}$ & $\operatorname{up~C1~} \mathrm{N}$ & Op 1 & \\
\hline
\end{tabular}

\begin{tabular}{|c|c|c|c|c|c|}
\hline \multirow{2}{*}{$\begin{array}{l}\text { Center } \\
\text { Number }\end{array}$} & Atomic & \multicolumn{2}{|c|}{ Atomic } & \multicolumn{2}{|c|}{ Coordinates (Angstroms } \\
\hline & Numbe & & Type & $\begin{array}{ll}X & Y\end{array}$ & $\mathrm{Z}$ \\
\hline 1 & 6 & 0 & -0.746680 & 1.370125 & -0.653809 \\
\hline 2 & 1 & 0 & -0.745175 & 0.848758 & -1.617014 \\
\hline 3 & 6 & 0 & -1.574004 & 0.548443 & 0.332899 \\
\hline 4 & 8 & 0 & -2.159032 & -0.515479 & -0.264129 \\
\hline 5 & 6 & 0 & -2.980327 & -1.345807 & 0.594011 \\
\hline 6 & 1 & 0 & -3.761073 & -0.722666 & 1.041250 \\
\hline 7 & 1 & 0 & -2.356392 & -1.731187 & 1.406719 \\
\hline 8 & 6 & 0 & -3.557713 & -2.455622 & -0.264458 \\
\hline 9 & 1 & 0 & -4.187625 & -3.109455 & 0.348977 \\
\hline 10 & 1 & 0 & -2.762830 & -3.062877 & -0.709093 \\
\hline 11 & 1 & 0 & -4.171012 & -2.045402 & -1.073322 \\
\hline 12 & 8 & 0 & -1.712811 & 0.825008 & 1.503790 \\
\hline 13 & 6 & 0 & 0.709918 & 1.483541 & -0.148645 \\
\hline 14 & 8 & 0 & 1.147567 & 2.567377 & 0.197320 \\
\hline 15 & 6 & 0 & 1.572418 & 0.259409 & -0.114171 \\
\hline 16 & 6 & 0 & 3.301273 & -1.945899 & -0.017232 \\
\hline 17 & 6 & 0 & 1.158130 & -0.989767 & -0.602438 \\
\hline 18 & 6 & 0 & 2.865573 & 0.388456 & 0.421408 \\
\hline 19 & 6 & 0 & 3.723007 & -0.705414 & 0.470965 \\
\hline 20 & 6 & 0 & 2.019647 & -2.085434 & -0.552569 \\
\hline 21 & 1 & 0 & 0.163287 & -1.119542 & -1.014613 \\
\hline 22 & 1 & 0 & 3.172366 & 1.361501 & 0.790762 \\
\hline 23 & 1 & 0 & 4.719535 & -0.595186 & 0.889551 \\
\hline
\end{tabular}




$\begin{array}{rrrrrr}24 & 1 & 0 & 1.690817 & -3.047342 & -0.934742 \\ 25 & 1 & 0 & 3.970153 & -2.801515 & 0.019500 \\ 26 & 6 & 0 & -1.380435 & 2.760727 & -0.813168 \\ 27 & 1 & 0 & -1.427429 & 3.268076 & 0.151857 \\ 28 & 1 & 0 & -2.393552 & 2.671627 & -1.219906 \\ 29 & 1 & 0 & -0.781702 & 3.373421 & -1.492524\end{array}$

Rotational constants (GHZ): $\quad 0.7905145 \quad 0.4338973 \quad 0.3093114$

Standard basis: 6-31G(d) $(6 \mathrm{D}, 7 \mathrm{~F})$

There are 253 symmetry adapted basis functions of A symmetry.

Integral buffers will be 262144 words long.

Raffenetti 1 integral format.

Two-electron integral symmetry is turned on.

253 basis functions, 476 primitive gaussians, 253 cartesian basis functions

55 alpha electrons 55 beta electrons

nuclear repulsion energy $\quad 972.7776633605$ Hartrees.

NAtoms $=29$ NActive $=29$ NUniq $=29 \mathrm{SFac}=1.00 \mathrm{D}+00 \mathrm{NAtFMM}=60 \mathrm{Big}=\mathrm{F}$

One-electron integrals computed using PRISM.

NBasis $=253$ RedAO $=\mathrm{T}$ NBF $=253$

NBsUse $=2531.00 \mathrm{D}-06 \mathrm{NBFU}=253$

Harris functional with IExCor= 205 diagonalized for initial guess.

ExpMin= 1.61D-01 ExpMax= 5.48D+03 ExpMxC= 8.25D+02 IAcc $=1$ IRadAn $=\quad 1$ AccDes=1.00D-06
HarFok: IExCor=205 AccDes= 1.00D-06 IRadAn $=\quad 1 \mathrm{IDoV}=1$

ScaDFX $=1.0000001 .0000001 .0000001 .000000$

Initial guess orbital symmetries:

Occupied (A) (A) (A) (A) (A) (A) (A) (A) (A) (A) (A) (A)

(A) (A) (A) (A) (A) (A) (A) (A) (A) (A) (A) (A)

(A) (A) (A) (A) (A) (A) (A) (A) (A) (A) (A) (A)

$(\mathrm{A})(\mathrm{A})(\mathrm{A})(\mathrm{A})(\mathrm{A})(\mathrm{A})(\mathrm{A})(\mathrm{A})(\mathrm{A})(\mathrm{A})(\mathrm{A})(\mathrm{A})$

(A) $(\mathrm{A})(\mathrm{A})(\mathrm{A})(\mathrm{A})(\mathrm{A})(\mathrm{A})$

Virtual (A) $(A)(A)(A)(A)(A)(A)(A)(A)(A)(A)(A)$

(A) $(A)(A)(A)(A)(A)(A)(A)(A)(A)(A)(A)$

(A) (A) (A) (A) (A) (A) (A) (A) (A) (A) (A) (A)

(A) $(\mathrm{A})(\mathrm{A})(\mathrm{A})(\mathrm{A})(\mathrm{A})(\mathrm{A})(\mathrm{A})(\mathrm{A})(\mathrm{A})(\mathrm{A})(\mathrm{A})$

$(\mathrm{A})(\mathrm{A})(\mathrm{A})(\mathrm{A})(\mathrm{A})(\mathrm{A})(\mathrm{A})(\mathrm{A})(\mathrm{A})(\mathrm{A})(\mathrm{A})(\mathrm{A})$

(A) (A) (A) (A) (A) (A) (A) (A) (A) (A) (A) (A)

(A) (A) (A) (A) (A) (A) (A) (A) (A) (A) (A) (A)

(A) (A) (A) (A) (A) (A) (A) (A) (A) (A) (A) (A)

(A) (A) (A) (A) (A) (A) (A) (A) (A) (A) (A) (A)

(A) $(\mathrm{A})(\mathrm{A})(\mathrm{A})(\mathrm{A})(\mathrm{A})(\mathrm{A})(\mathrm{A})(\mathrm{A})(\mathrm{A})(\mathrm{A})(\mathrm{A})$

(A) (A) (A) (A) (A) (A) (A) (A) (A) (A) (A) (A)

(A) (A) (A) (A) (A) (A) (A) (A) (A) (A) (A) (A)

(A) $(\mathrm{A})(\mathrm{A})(\mathrm{A})(\mathrm{A})(\mathrm{A})(\mathrm{A})(\mathrm{A})(\mathrm{A})(\mathrm{A})(\mathrm{A})(\mathrm{A})$

(A) (A) (A) (A) (A) (A) (A) (A) (A) (A) (A) (A)

(A) (A) (A) (A) (A) (A) (A) (A) (A) (A) (A) (A)

(A) $(\mathrm{A})(\mathrm{A})(\mathrm{A})(\mathrm{A})(\mathrm{A})(\mathrm{A})(\mathrm{A})(\mathrm{A})(\mathrm{A})(\mathrm{A})(\mathrm{A})$

(A) (A) (A) (A) (A) (A)

The electronic state of the initial guess is 1-A.

Warning! Cutoffs for single-point calculations used.

Requested convergence on RMS density matrix=1.00D-04 within 128 cycles.

Requested convergence on MAX density matrix $=1.00 \mathrm{D}-02$.

Requested convergence on energy $=5.00 \mathrm{D}-05$.

No special actions if energy rises.

SCF Done: $\mathrm{E}(\mathrm{RHF})=-687.181856994$ A.U. after 7 cycles

Convg $=0.8010 \mathrm{D}-05 \quad-\mathrm{V} / \mathrm{T}=2.0025$

$\mathrm{S} * * 2=0.0000$

$* * * * * * * * * * * * * * * * * * * * * * * * * * * * * * * * * * * * * * * * * * * * * * * * * * * * * * * * * * * * * * * * * * * * * * *$

Population analysis using the SCF density.

Orbital symmetries: 
Occupied (A) (A) (A) (A) (A) (A) (A) (A) (A) (A) (A) (A)

(A) (A) (A) (A) (A) (A) (A) (A) (A) (A) (A) (A)

(A) (A) (A) (A) (A) (A) (A) (A) (A) (A) (A) (A)

(A) $(\mathrm{A})(\mathrm{A})(\mathrm{A})(\mathrm{A})(\mathrm{A})(\mathrm{A})(\mathrm{A})(\mathrm{A})(\mathrm{A})(\mathrm{A})(\mathrm{A})$

(A) $(\mathrm{A})(\mathrm{A})(\mathrm{A})(\mathrm{A})(\mathrm{A})(\mathrm{A})$

Virtual (A) (A) (A) (A) (A) (A) (A) (A) (A) (A) (A) (A)

(A) (A) (A) (A) (A) (A) (A) (A) (A) (A) (A) (A)

(A) (A) (A) (A) (A) (A) (A) (A) (A) (A) (A) (A)

(A) $(\mathrm{A})(\mathrm{A})(\mathrm{A})(\mathrm{A})(\mathrm{A})(\mathrm{A})(\mathrm{A})(\mathrm{A})(\mathrm{A})(\mathrm{A})(\mathrm{A})$

(A) (A) (A) (A) (A) (A) (A) (A) (A) (A) (A) (A)

(A) $(\mathrm{A})(\mathrm{A})(\mathrm{A})(\mathrm{A})(\mathrm{A})(\mathrm{A})(\mathrm{A})(\mathrm{A})(\mathrm{A})(\mathrm{A})(\mathrm{A})$

(A) (A) (A) (A) (A) (A) (A) (A) (A) (A) (A) (A)

(A) (A) (A) (A) (A) (A) (A) (A) (A) (A) (A) (A)

(A) $(A)(A)(A)(A)(A)(A)(A)(A)(A)(A)(A)$

(A) (A) (A) (A) (A) (A) (A) (A) (A) (A) (A) (A)

(A) $(\mathrm{A})(\mathrm{A})(\mathrm{A})(\mathrm{A})(\mathrm{A})(\mathrm{A})(\mathrm{A})(\mathrm{A})(\mathrm{A})(\mathrm{A})(\mathrm{A})$

(A) (A) (A) (A) (A) (A) (A) (A) (A) (A) (A) (A)

(A) $(A)(A)(A)(A)(A)(A)(A)(A)(A)(A)(A)$

(A) $(\mathrm{A})(\mathrm{A})(\mathrm{A})(\mathrm{A})(\mathrm{A})(\mathrm{A})(\mathrm{A})(\mathrm{A})(\mathrm{A})(\mathrm{A})(\mathrm{A})$

(A) $(\mathrm{A})(\mathrm{A})(\mathrm{A})(\mathrm{A})(\mathrm{A})(\mathrm{A})(\mathrm{A})(\mathrm{A})(\mathrm{A})(\mathrm{A})(\mathrm{A})$

(A) $(\mathrm{A})(\mathrm{A})(\mathrm{A})(\mathrm{A})(\mathrm{A})(\mathrm{A})(\mathrm{A})(\mathrm{A})(\mathrm{A})(\mathrm{A})(\mathrm{A})$

(A) (A) (A) (A) (A) (A)

The electronic state is 1-A.

Alpha occ. eigenvalues -- -20.61826 -20.56043 -20.55895 -11.39360 -11.34502

Alpha occ. eigenvalues -- -11.29861 -11.26323 -11.25611 -11.25438 -11.25227

Alpha occ. eigenvalues -- -11.24838 -11.24740 -11.24481 -11.23529 -11.22719

Alpha occ. eigenvalues -- $\quad-1.46311 \quad-1.38868 \quad-1.36277 \quad-1.16231 \quad-1.09078$

Alpha occ. eigenvalues -- $-1.04347-1.03286 \quad-1.02417 \quad-0.91991 \quad-0.88622$

Alpha occ. eigenvalues -- $-0.84753 \quad-0.83198 \quad-0.79295 \quad-0.75145 \quad-0.71560$

Alpha occ. eigenvalues -- $-0.70566 \quad-0.68846 \quad-0.67196 \quad-0.65338 \quad-0.64572$

$\begin{array}{llllll}\text { Alpha occ. eigenvalues -- } & -0.62175 & -0.61168 & -0.60056 & -0.59128 & -0.58342\end{array}$

Alpha occ. eigenvalues -- $-0.56048 \quad-0.54587 \quad-0.53708 \quad-0.53351 \quad-0.52053$

Alpha occ. eigenvalues -- $\quad-0.51698 \quad-0.51613 \quad-0.50352 \quad-0.49910 \quad-0.49220$

$\begin{array}{llllll}\text { Alpha occ. eigenvalues -- } & -0.46116 & -0.44620 & -0.41608 & -0.34652 & -0.34199\end{array}$

$\begin{array}{lllllll}\text { Alpha virt. eigenvalues -- } & 0.08167 & 0.13120 & 0.17527 & 0.20939 & 0.22510\end{array}$

$\begin{array}{llllll}\text { Alpha virt. eigenvalues -- } & 0.23935 & 0.25828 & 0.27642 & 0.28723 & 0.29338\end{array}$

$\begin{array}{lllllll}\text { Alpha virt. eigenvalues -- } & 0.29512 & 0.30076 & 0.30930 & 0.31486 & 0.32534\end{array}$

$\begin{array}{lllllll}\text { Alpha virt. eigenvalues -- } & 0.33225 & 0.33630 & 0.34075 & 0.36389 & 0.36975\end{array}$

$\begin{array}{lllllll}\text { Alpha virt. eigenvalues -- } & 0.38321 & 0.40400 & 0.42259 & 0.43345 & 0.44635\end{array}$

$\begin{array}{lllllll}\text { Alpha virt. eigenvalues -- } & 0.46566 & 0.47820 & 0.49331 & 0.50765 & 0.53484\end{array}$

$\begin{array}{lllllll}\text { Alpha virt. eigenvalues -- } & 0.55812 & 0.60264 & 0.67584 & 0.70831 & 0.72490\end{array}$

$\begin{array}{lllllll}\text { Alpha virt. eigenvalues -- } & 0.72572 & 0.72788 & 0.73865 & 0.74364 & 0.75594\end{array}$

$\begin{array}{lllllll}\text { Alpha virt. eigenvalues -- } & 0.76445 & 0.76973 & 0.78258 & 0.80484 & 0.80802\end{array}$

$\begin{array}{lllllll}\text { Alpha virt. eigenvalues -- } & 0.82068 & 0.82242 & 0.82739 & 0.83095 & 0.85292\end{array}$

$\begin{array}{lllllll}\text { Alpha virt. eigenvalues -- } & 0.86099 & 0.87921 & 0.88871 & 0.90678 & 0.92131\end{array}$

$\begin{array}{lllllll}\text { Alpha virt. eigenvalues -- } & 0.93684 & 0.93961 & 0.96156 & 0.98339 & 1.00247\end{array}$

$\begin{array}{lllllll}\text { Alpha virt. eigenvalues -- } & 1.01039 & 1.01446 & 1.05694 & 1.05906 & 1.08222\end{array}$

$\begin{array}{lllllll}\text { Alpha virt. eigenvalues -- } & 1.08753 & 1.09519 & 1.10502 & 1.10971 & 1.11235\end{array}$

$\begin{array}{lllllll}\text { Alpha virt. eigenvalues -- } & 1.12787 & 1.14416 & 1.15270 & 1.15919 & 1.16744\end{array}$

$\begin{array}{lllllll}\text { Alpha virt. eigenvalues -- } & 1.17208 & 1.17623 & 1.18503 & 1.18964 & 1.19854\end{array}$

$\begin{array}{lllllll}\text { Alpha virt. eigenvalues -- } & 1.22263 & 1.22511 & 1.23452 & 1.24202 & 1.26142\end{array}$

$\begin{array}{lllllll}\text { Alpha virt. eigenvalues -- } & 1.30896 & 1.31575 & 1.32400 & 1.35444 & 1.36135\end{array}$

$\begin{array}{lllllll}\text { Alpha virt. eigenvalues -- } & 1.40034 & 1.41548 & 1.42812 & 1.43580 & 1.45739\end{array}$

$\begin{array}{lllllll}\text { Alpha virt. eigenvalues -- } & 1.49522 & 1.50603 & 1.53142 & 1.55021 & 1.57214\end{array}$

$\begin{array}{lllllll}\text { Alpha virt. eigenvalues -- } & 1.58677 & 1.64798 & 1.66463 & 1.67717 & 1.68743\end{array}$

$\begin{array}{lllllll}\text { Alpha virt. eigenvalues -- } & 1.69622 & 1.71097 & 1.73477 & 1.74909 & 1.75921\end{array}$

$\begin{array}{lllllll}\text { Alpha virt. eigenvalues -- } & 1.77846 & 1.78780 & 1.84200 & 1.88522 & 1.90233\end{array}$

$\begin{array}{lllllll}\text { Alpha virt. eigenvalues -- } & 1.94053 & 1.95965 & 2.02550 & 2.03683 & 2.05480\end{array}$

$\begin{array}{llllll}\text { Alpha virt. eigenvalues -- } & 2.06511 & 2.08163 & 2.09392 & 2.10843 & 2.12151\end{array}$

$\begin{array}{llllll}\text { Alpha virt. eigenvalues -- } & 2.14148 & 2.15801 & 2.16715 & 2.19058 & 2.22458\end{array}$

$\begin{array}{lllllll}\text { Alpha virt. eigenvalues -- } & 2.23396 & 2.24189 & 2.26467 & 2.27529 & 2.30341\end{array}$

$\begin{array}{llllll}\text { Alpha virt. eigenvalues -- } & 2.30534 & 2.33426 & 2.35553 & 2.36702 & 2.38528\end{array}$

$\begin{array}{lllllll}\text { Alpha virt. eigenvalues -- } & 2.39067 & 2.40082 & 2.44336 & 2.45054 & 2.45618\end{array}$

$\begin{array}{llllll}\text { Alpha virt. eigenvalues -- } & 2.46867 & 2.47087 & 2.52193 & 2.53310 & 2.58842\end{array}$ 
$\begin{array}{llllll}\text { Alpha virt. eigenvalues -- } & 2.60133 & 2.61842 & 2.62792 & 2.64679 & 2.65561\end{array}$ $\begin{array}{llllll}\text { Alpha virt. eigenvalues -- } & 2.66048 & 2.68343 & 2.70128 & 2.71836 & 2.75596\end{array}$ $\begin{array}{lllllll}\text { Alpha virt. eigenvalues -- } & 2.84430 & 2.84930 & 2.91577 & 2.92303 & 2.93600\end{array}$ $\begin{array}{lllllll}\text { Alpha virt. eigenvalues -- } & 2.95338 & 2.95842 & 2.99796 & 3.00972 & 3.03191\end{array}$ $\begin{array}{lllllll}\text { Alpha virt. eigenvalues -- } & 3.07659 & 3.09485 & 3.10316 & 3.11166 & 3.18310\end{array}$ $\begin{array}{lllllll}\text { Alpha virt. eigenvalues -- } & 3.23471 & 3.32090 & 3.34289 & 3.35437 & 3.41979\end{array}$ $\begin{array}{lllllll}\text { Alpha virt. eigenvalues -- } & 3.51073 & 3.53223 & 3.78992 & 4.38449 & 4.45578\end{array}$ $\begin{array}{lllllll}\text { Alpha virt. eigenvalues -- } & 4.50539 & 4.53445 & 4.56381 & 4.61207 & 4.63299\end{array}$ $\begin{array}{lllllll}\text { Alpha virt. eigenvalues -- } & 4.72826 & 4.74738 & 4.81251 & 4.83563 & 4.87881\end{array}$ Alpha virt. eigenvalues -- $4.90430 \quad 5.07961 \quad 5.16769$

Condensed to atoms (all electrons):

$$
\begin{array}{llllll}
1 & 2 & 3 & 4 & 5 & 6
\end{array}
$$

$\begin{array}{llllllll}1 & C & 5.440615 & 0.397629 & 0.335634 & -0.079584 & 0.004916 & -0.000120\end{array}$

$\begin{array}{llllllll}2 & \mathrm{H} & 0.397629 & 0.480830 & -0.045417 & 0.003222 & -0.000027 & -0.000010\end{array}$

$\begin{array}{llllllll}3 & \mathrm{C} & 0.335634 & -0.045417 & 4.182966 & 0.254291 & -0.025468 & -0.003676\end{array}$

$\begin{array}{llllllll}4 & \mathrm{O} & -0.079584 & 0.003222 & 0.254291 & 8.497845 & 0.151901 & -0.033123\end{array}$

$\begin{array}{llllllll}5 \mathrm{C} & 0.004916 & -0.000027 & -0.025468 & 0.151901 & 4.837481 & 0.402191\end{array}$

$\begin{array}{llllllll}6 \mathrm{H} & -0.000120 & -0.000010 & -0.003676 & -0.033123 & 0.402191 & 0.519688\end{array}$

$\begin{array}{llllllll}7 & \mathrm{H} & -0.000148 & 0.000014 & -0.002851 & -0.034080 & 0.402155 & -0.036060\end{array}$

$\begin{array}{llllllll}8 & \mathrm{C} & -0.000018 & -0.000058 & 0.003330 & -0.045654 & 0.339777 & -0.039666\end{array}$

$\begin{array}{llllllll}9 & \mathrm{H} & 0.000002 & 0.000000 & -0.000031 & 0.002556 & -0.037415 & -0.000510\end{array}$

$\begin{array}{llllllll}10 \mathrm{H} & -0.000015 & 0.000001 & -0.000154 & 0.000363 & -0.033834 & 0.003766\end{array}$

$\begin{array}{lllllllll}11 & \mathrm{H} & -0.000013 & 0.000007 & -0.000098 & 0.000649 & -0.033780 & -0.003759\end{array}$

$\begin{array}{lllllllll}12 & \mathrm{O} & -0.074931 & 0.002335 & 0.563420 & -0.077315 & 0.001116 & 0.003148\end{array}$

$\begin{array}{llllllll}13 & \mathrm{C} & 0.317808 & -0.034903 & -0.038860 & 0.000528 & -0.000144 & -0.000010\end{array}$

$\begin{array}{llllllll}14 & \mathrm{O} & -0.078773 & 0.000861 & -0.000210 & -0.000008 & 0.000001 & 0.000000\end{array}$

$\begin{array}{llllllll}15 & \mathrm{C} & -0.112315 & 0.003604 & 0.001443 & 0.000065 & 0.000011 & 0.000001\end{array}$

$\begin{array}{llllllll}16 & \mathrm{C} & -0.000016 & 0.000000 & -0.000005 & 0.000000 & 0.000000 & 0.000000\end{array}$

$\begin{array}{llllllll}17 & \mathrm{C} & -0.001586 & -0.001283 & 0.000506 & -0.004554 & 0.000235 & -0.000011\end{array}$

$\begin{array}{llllllll}18 & \mathrm{C} & 0.005544 & -0.000131 & -0.000162 & 0.000005 & -0.000001 & 0.000000\end{array}$

$\begin{array}{llllllll}19 & C & -0.000001 & -0.000007 & -0.000003 & 0.000000 & 0.000000 & 0.000000\end{array}$

$\begin{array}{llllllll}20 \mathrm{C} & -0.000130 & 0.000067 & -0.000067 & 0.000073 & -0.000006 & 0.000000\end{array}$

$\begin{array}{llllllll}21 & \mathrm{H} & -0.000267 & 0.000004 & -0.002813 & 0.009566 & -0.000603 & 0.000054\end{array}$

$\begin{array}{llllllll}22 & \mathrm{H} & 0.000339 & -0.000008 & -0.000015 & 0.000000 & 0.000000 & 0.000000\end{array}$

$\begin{array}{llllllll}23 \mathrm{H} & 0.000001 & 0.000000 & 0.000000 & 0.000000 & 0.000000 & 0.000000\end{array}$

$\begin{array}{llllllll}24 & \mathrm{H} & 0.000005 & -0.000002 & 0.000000 & -0.000002 & 0.000001 & 0.000000\end{array}$

$\begin{array}{llllllll}25 & \mathrm{H} & 0.000000 & 0.000000 & 0.000000 & 0.000000 & 0.000000 & 0.000000\end{array}$

$\begin{array}{llllllll}26 & \mathrm{C} & 0.316422 & -0.029634 & -0.041777 & 0.000958 & -0.000191 & 0.000047\end{array}$

$\begin{array}{llllllll}27 & \mathrm{H} & -0.034269 & 0.003320 & -0.004437 & 0.000168 & -0.000006 & -0.000002\end{array}$

$\begin{array}{llllllll}28 \mathrm{H} & -0.040481 & -0.002247 & -0.003732 & 0.000620 & -0.000045 & -0.000004\end{array}$

$\begin{array}{llllllll}29 & \mathrm{H} & -0.035849 & -0.002260 & 0.004684 & -0.000066 & 0.000003 & 0.000000\end{array}$

$$
\begin{array}{llllll}
7 & 8 & 9 & 10 & 11 & 12
\end{array}
$$

$\begin{array}{llllllll}1 \mathrm{C} & -0.000148 & -0.000018 & 0.000002 & -0.000015 & -0.000013 & -0.074931\end{array}$

$\begin{array}{llllllll}2 \mathrm{H} & 0.000014 & -0.000058 & 0.000000 & 0.000001 & 0.000007 & 0.002335\end{array}$

$\begin{array}{llllllll}3 \mathrm{C} & -0.002851 & 0.003330 & -0.000031 & -0.000154 & -0.000098 & 0.563420\end{array}$

$\begin{array}{llllllll}4 & \mathrm{O} & -0.034080 & -0.045654 & 0.002556 & 0.000363 & 0.000649 & -0.077315\end{array}$

$\begin{array}{llllllll}5 & \mathrm{C} & 0.402155 & 0.339777 & -0.037415 & -0.033834 & -0.033780 & 0.001116\end{array}$

$\begin{array}{llllllll}6 \mathrm{H} & -0.036060 & -0.039666 & -0.000510 & 0.003766 & -0.003759 & 0.003148\end{array}$

$\begin{array}{llllllll}7 & \mathrm{H} & 0.520266 & -0.039737 & -0.000459 & -0.003703 & 0.003759 & 0.002895\end{array}$

$\begin{array}{llllllll}8 & \mathrm{C} & -0.039737 & 5.115735 & 0.387870 & 0.387234 & 0.387604 & 0.000560\end{array}$

$\begin{array}{lllllllll}9 \mathrm{H} & -0.000459 & 0.387870 & 0.524124 & -0.024290 & -0.023897 & 0.000009\end{array}$

$\begin{array}{lllllllll}10 \mathrm{H} & -0.003703 & 0.387234 & -0.024290 & 0.523343 & -0.026966 & -0.000007\end{array}$

$\begin{array}{llllllll}11 & \mathrm{H} & 0.003759 & 0.387604 & -0.023897 & -0.026966 & 0.517603 & -0.000006\end{array}$

$\begin{array}{llllllll}12 & \mathrm{O} & 0.002895 & 0.000560 & 0.000009 & -0.000007 & -0.000006 & 8.141117\end{array}$

$\begin{array}{llllllll}13 & \mathrm{C} & 0.000063 & 0.000001 & 0.000000 & 0.000000 & 0.000000 & 0.006102\end{array}$

$\begin{array}{llllllll}14 & \mathrm{O} & 0.000000 & 0.000000 & 0.000000 & 0.000000 & 0.000000 & -0.000360\end{array}$

$\begin{array}{llllllll}15 \mathrm{C} & 0.000002 & 0.000000 & 0.000000 & 0.000000 & 0.000000 & 0.000028\end{array}$

$\begin{array}{llllllll}16 \mathrm{C} & 0.000000 & 0.000000 & 0.000000 & 0.000000 & 0.000000 & 0.000000\end{array}$

$\begin{array}{llllllll}17 & \mathrm{C} & 0.000123 & -0.000023 & 0.000000 & -0.000046 & 0.000000 & -0.000343\end{array}$

$\begin{array}{lllllll}18 \mathrm{C} & 0.000000 & 0.000000 & 0.000000 & 0.000000 & 0.000000 & -0.000007\end{array}$

$\begin{array}{llllllll}19 \mathrm{C} & 0.000000 & 0.000000 & 0.000000 & 0.000000 & 0.000000 & 0.000001\end{array}$

$\begin{array}{llllllll}20 \mathrm{C} & 0.000006 & -0.000002 & 0.000000 & 0.000006 & 0.000000 & -0.000003\end{array}$

$\begin{array}{llllllll}21 & \mathrm{H} & -0.000042 & -0.000021 & 0.000000 & 0.000282 & 0.000001 & -0.000070\end{array}$

$\begin{array}{llllllll}22 & \mathrm{H} & 0.000000 & 0.000000 & 0.000000 & 0.000000 & 0.000000 & -0.000001\end{array}$ 


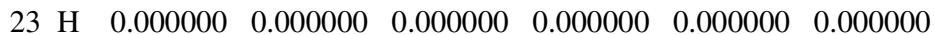
$\begin{array}{llllllll}24 \mathrm{H} & -0.000001 & 0.000000 & 0.000000 & 0.000002 & 0.000000 & 0.000000\end{array}$ $\begin{array}{llllllll}25 \mathrm{H} & 0.000000 & 0.000000 & 0.000000 & 0.000000 & 0.000000 & 0.000000\end{array}$ $\begin{array}{llllllll}26 & \mathrm{C} & -0.000010 & 0.000001 & 0.000000 & 0.000000 & -0.000001 & 0.000661\end{array}$ $\begin{array}{llllllll}27 & \mathrm{H} & 0.000000 & 0.000000 & 0.000000 & 0.000000 & 0.000000 & 0.003483\end{array}$ $\begin{array}{llllllll}28 \mathrm{H} & 0.000002 & -0.000001 & 0.000000 & 0.000000 & 0.000001 & -0.000009\end{array}$ $\begin{array}{llllllll}29 & \mathrm{H} & 0.000000 & 0.000000 & 0.000000 & 0.000000 & 0.000000 & 0.000018\end{array}$
$\begin{array}{llllll}13 & 14 & 15 & 16 & 17 & 18\end{array}$

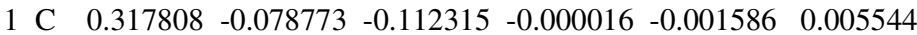

$\begin{array}{llllllll}2 \mathrm{H} & -0.034903 & 0.000861 & 0.003604 & 0.000000 & -0.001283 & -0.000131\end{array}$

$\begin{array}{llllllll}3 \mathrm{C} & -0.038860 & -0.000210 & 0.001443 & -0.000005 & 0.000506 & -0.000162\end{array}$

$\begin{array}{llllllll}4 & \mathrm{O} & 0.000528 & -0.000008 & 0.000065 & 0.000000 & -0.004554 & 0.000005\end{array}$

$\begin{array}{llllllll}5 & \mathrm{C} & -0.000144 & 0.000001 & 0.000011 & 0.000000 & 0.000235 & -0.000001\end{array}$

$\begin{array}{llllllll}6 \mathrm{H} & -0.000010 & 0.000000 & 0.000001 & 0.000000 & -0.000011 & 0.000000\end{array}$

$\begin{array}{llllllll}7 & \mathrm{H} & 0.000063 & 0.000000 & 0.000002 & 0.000000 & 0.000123 & 0.000000\end{array}$

$\begin{array}{llllllll}8 \mathrm{C} & 0.000001 & 0.000000 & 0.000000 & 0.000000 & -0.000023 & 0.000000\end{array}$

$\begin{array}{llllllll}9 \mathrm{H} & 0.000000 & 0.000000 & 0.000000 & 0.000000 & 0.000000 & 0.000000\end{array}$

$\begin{array}{llllllll}10 \mathrm{H} & 0.000000 & 0.000000 & 0.000000 & 0.000000 & -0.000046 & 0.000000\end{array}$

$\begin{array}{llllllll}11 \mathrm{H} & 0.000000 & 0.000000 & 0.000000 & 0.000000 & 0.000000 & 0.000000\end{array}$

$\begin{array}{lllllllll}12 & \mathrm{O} & 0.006102 & -0.000360 & 0.000028 & 0.000000 & -0.000343 & -0.000007\end{array}$

$\begin{array}{lllllllll}13 & \mathrm{C} & 4.364937 & 0.568804 & 0.368927 & -0.000030 & -0.031945 & -0.034189\end{array}$

$\begin{array}{llllllll}14 & \mathrm{O} & 0.568804 & 8.122242 & -0.082161 & -0.000006 & 0.002385 & 0.001358\end{array}$

$\begin{array}{lllllllll}15 & \mathrm{C} & 0.368927 & -0.082161 & 5.020051 & -0.028561 & 0.529690 & 0.532075\end{array}$

$\begin{array}{llllllll}16 & \mathrm{C} & -0.000030 & -0.000006 & -0.028561 & 4.880310 & -0.044438 & -0.042988\end{array}$

$\begin{array}{llllllll}17 & \mathrm{C} & -0.031945 & 0.002385 & 0.529690 & -0.044438 & 4.969905 & -0.048463\end{array}$

$\begin{array}{llllllll}18 & \mathrm{C} & -0.034189 & 0.001358 & 0.532075 & -0.042988 & -0.048463 & 4.904668\end{array}$

$\begin{array}{llllllll}19 & \mathrm{C} & 0.002717 & 0.000713 & -0.043108 & 0.538533 & -0.034029 & 0.532152\end{array}$

$\begin{array}{llllllll}20 & \mathrm{C} & 0.001852 & -0.000049 & -0.034993 & 0.550968 & 0.537521 & -0.033003\end{array}$

$\begin{array}{llllllll}21 & \mathrm{H} & -0.001975 & 0.000002 & -0.029602 & 0.002132 & 0.368397 & 0.002399\end{array}$

$\begin{array}{llllllll}22 & \mathrm{H} & -0.004533 & 0.009579 & -0.026564 & 0.002161 & 0.002864 & 0.375272\end{array}$

$\begin{array}{llllllll}23 & \mathrm{H} & -0.000076 & 0.000000 & 0.001923 & -0.030065 & 0.000173 & -0.027979\end{array}$

$\begin{array}{llllllll}24 \mathrm{H} & -0.000070 & 0.000000 & 0.001579 & -0.028572 & -0.029515 & 0.000249\end{array}$

$\begin{array}{llllllll}25 & \mathrm{H} & 0.000005 & 0.000000 & 0.000214 & 0.381282 & 0.002617 & 0.002182\end{array}$

$\begin{array}{llllllll}26 & \mathrm{C} & -0.043845 & 0.002248 & 0.003594 & 0.000000 & 0.000129 & -0.000081\end{array}$

$\begin{array}{llllllll}27 & \mathrm{H} & -0.003393 & 0.001750 & 0.000041 & 0.000000 & -0.000009 & -0.000003\end{array}$

$\begin{array}{llllllll}28 & \mathrm{H} & 0.004828 & 0.000392 & -0.000013 & 0.000000 & -0.000002 & 0.000001\end{array}$

$\begin{array}{llllllll}29 & \mathrm{H} & -0.004393 & 0.001928 & 0.000086 & 0.000000 & -0.000015 & -0.000006\end{array}$

$$
\begin{array}{llllll}
19 & 20 & 21 & 22 & 23 & 24
\end{array}
$$

$\begin{array}{llllllll}1 & \mathrm{C} & -0.000001 & -0.000130 & -0.000267 & 0.000339 & 0.000001 & 0.000005\end{array}$

$\begin{array}{llllllll}2 \mathrm{H} & -0.000007 & 0.000067 & 0.000004 & -0.000008 & 0.000000 & -0.000002\end{array}$

$\begin{array}{llllllll}3 \mathrm{C} & -0.000003 & -0.000067 & -0.002813 & -0.000015 & 0.000000 & 0.000000\end{array}$

$\begin{array}{llllllll}4 & \mathrm{O} & 0.000000 & 0.000073 & 0.009566 & 0.000000 & 0.000000 & -0.000002\end{array}$

$\begin{array}{llllllll}5 \mathrm{C} & 0.000000 & -0.000006 & -0.000603 & 0.000000 & 0.000000 & 0.000001\end{array}$

$\begin{array}{llllllll}6 \mathrm{H} & 0.000000 & 0.000000 & 0.000054 & 0.000000 & 0.000000 & 0.000000\end{array}$

$\begin{array}{llllllll}7 & \mathrm{H} & 0.000000 & 0.000006 & -0.000042 & 0.000000 & 0.000000 & -0.000001\end{array}$

$\begin{array}{llllllll}8 \mathrm{C} & 0.000000 & -0.000002 & -0.000021 & 0.000000 & 0.000000 & 0.000000\end{array}$

$\begin{array}{llllllll}9 \mathrm{H} & 0.000000 & 0.000000 & 0.000000 & 0.000000 & 0.000000 & 0.000000\end{array}$

$\begin{array}{lllllll}10 \mathrm{H} & 0.000000 & 0.000006 & 0.000282 & 0.000000 & 0.000000 & 0.000002\end{array}$

$\begin{array}{llllllll}11 \mathrm{H} & 0.000000 & 0.000000 & 0.000001 & 0.000000 & 0.000000 & 0.000000\end{array}$

$\begin{array}{llllllll}12 & \mathrm{O} & 0.000001 & -0.000003 & -0.000070 & -0.000001 & 0.000000 & 0.000000\end{array}$

$\begin{array}{llllllll}13 & \mathrm{C} & 0.002717 & 0.001852 & -0.001975 & -0.004533 & -0.000076 & -0.000070\end{array}$

$\begin{array}{llllllll}14 & \mathrm{O} & 0.000713 & -0.000049 & 0.000002 & 0.009579 & 0.000000 & 0.000000\end{array}$

$\begin{array}{llllllll}15 & \mathrm{C} & -0.043108 & -0.034993 & -0.029602 & -0.026564 & 0.001923 & 0.001579\end{array}$

$\begin{array}{llllllll}16 & \mathrm{C} & 0.538533 & 0.550968 & 0.002132 & 0.002161 & -0.030065 & -0.028572\end{array}$

$\begin{array}{llllllll}17 & \mathrm{C} & -0.034029 & 0.537521 & 0.368397 & 0.002864 & 0.000173 & -0.029515\end{array}$

$\begin{array}{llllllll}18 & \mathrm{C} & 0.532152 & -0.033003 & 0.002399 & 0.375272 & -0.027979 & 0.000249\end{array}$

$\begin{array}{llllllll}19 & \mathrm{C} & 4.929504 & -0.042593 & 0.000106 & -0.028259 & 0.382581 & 0.002316\end{array}$

$\begin{array}{lllllllll}20 & \mathrm{C} & -0.042593 & 4.903240 & -0.023736 & 0.000017 & 0.002396 & 0.383061\end{array}$

$\begin{array}{llllllll}21 & \mathrm{H} & 0.000106 & -0.023736 & 0.434184 & -0.000086 & 0.000009 & -0.000968\end{array}$

$\begin{array}{llllllll}22 & \mathrm{H} & -0.028259 & 0.000017 & -0.000086 & 0.416028 & -0.001073 & 0.000009\end{array}$

$\begin{array}{llllllll}23 & \mathrm{H} & 0.382581 & 0.002396 & 0.000009 & -0.001073 & 0.461076 & -0.000100\end{array}$

$\begin{array}{llllllll}24 \mathrm{H} & 0.002316 & 0.383061 & -0.000968 & 0.000009 & -0.000100 & 0.465077\end{array}$

$\begin{array}{llllllll}25 & \mathrm{H} & -0.029270 & -0.029661 & -0.000096 & -0.000085 & -0.001328 & -0.001363\end{array}$

$\begin{array}{llllllll}26 & \mathrm{C} & 0.000000 & 0.000002 & 0.000156 & -0.000008 & 0.000000 & 0.000000\end{array}$ 
$\begin{array}{llllllll}27 & \mathrm{H} & 0.000000 & 0.000000 & -0.000003 & -0.000001 & 0.000000 & 0.000000\end{array}$

$\begin{array}{llllllll}28 \mathrm{H} & 0.000000 & 0.000000 & 0.000003 & 0.000000 & 0.000000 & 0.000000\end{array}$

$\begin{array}{llllllll}29 & \mathrm{H} & 0.000000 & 0.000000 & -0.000005 & -0.000001 & 0.000000 & 0.000000\end{array}$

$$
\begin{array}{lllll}
25 & 26 & 27 & 28 & 29
\end{array}
$$

$\begin{array}{lllllll}1 & \mathrm{C} & 0.000000 & 0.316422 & -0.034269 & -0.040481 & -0.035849\end{array}$

$\begin{array}{llllllll}2 & \mathrm{H} & 0.000000 & -0.029634 & 0.003320 & -0.002247 & -0.002260\end{array}$

$\begin{array}{lllllll}3 \mathrm{C} & 0.000000 & -0.041777 & -0.004437 & -0.003732 & 0.004684\end{array}$

$\begin{array}{lllllll}4 & \mathrm{O} & 0.000000 & 0.000958 & 0.000168 & 0.000620 & -0.000066\end{array}$

$\begin{array}{lllllll}5 \mathrm{C} & 0.000000 & -0.000191 & -0.000006 & -0.000045 & 0.000003\end{array}$

$\begin{array}{lllllll}6 & \mathrm{H} & 0.000000 & 0.000047 & -0.000002 & -0.000004 & 0.000000\end{array}$

$\begin{array}{lllllll}7 \mathrm{H} & 0.000000 & -0.000010 & 0.000000 & 0.000002 & 0.000000\end{array}$

$\begin{array}{lllllll}8 & \mathrm{C} & 0.000000 & 0.000001 & 0.000000 & -0.000001 & 0.000000\end{array}$

$\begin{array}{lllllll}9 \mathrm{H} & 0.000000 & 0.000000 & 0.000000 & 0.000000 & 0.000000\end{array}$

$\begin{array}{lllllll}10 \mathrm{H} & 0.000000 & 0.000000 & 0.000000 & 0.000000 & 0.000000\end{array}$

$\begin{array}{lllllll}11 \mathrm{H} & 0.000000 & -0.000001 & 0.000000 & 0.000001 & 0.000000\end{array}$

$\begin{array}{lllllll}12 & \mathrm{O} & 0.000000 & 0.000661 & 0.003483 & -0.000009 & 0.000018\end{array}$

$\begin{array}{lllllll}13 & \mathrm{C} & 0.000005 & -0.043845 & -0.003393 & 0.004828 & -0.004393\end{array}$

$\begin{array}{lllllll}14 & \mathrm{O} & 0.000000 & 0.002248 & 0.001750 & 0.000392 & 0.001928\end{array}$

$\begin{array}{lllllll}15 & \mathrm{C} & 0.000214 & 0.003594 & 0.000041 & -0.000013 & 0.000086\end{array}$

$\begin{array}{lllllll}16 \mathrm{C} & 0.381282 & 0.000000 & 0.000000 & 0.000000 & 0.000000\end{array}$

$\begin{array}{lllllll}17 & \mathrm{C} & 0.002617 & 0.000129 & -0.000009 & -0.000002 & -0.000015\end{array}$

$\begin{array}{lllllll}18 \mathrm{C} & 0.002182 & -0.000081 & -0.000003 & 0.000001 & -0.000006\end{array}$

$\begin{array}{lllllll}19 \mathrm{C} & -0.029270 & 0.000000 & 0.000000 & 0.000000 & 0.000000\end{array}$

$\begin{array}{lllllll}20 \mathrm{C} & -0.029661 & 0.000002 & 0.000000 & 0.000000 & 0.000000\end{array}$

$\begin{array}{lllllll}21 \mathrm{H} & -0.000096 & 0.000156 & -0.000003 & 0.000003 & -0.000005\end{array}$

$\begin{array}{llllllll}22 & \mathrm{H} & -0.000085 & -0.000008 & -0.000001 & 0.000000 & -0.000001\end{array}$

$\begin{array}{lllllll}23 & \mathrm{H} & -0.001328 & 0.000000 & 0.000000 & 0.000000 & 0.000000\end{array}$

$\begin{array}{lllllll}24 & \mathrm{H} & -0.001363 & 0.000000 & 0.000000 & 0.000000 & 0.000000\end{array}$

$\begin{array}{lllllll}25 \mathrm{H} & 0.463358 & 0.000000 & 0.000000 & 0.000000 & 0.000000\end{array}$

$\begin{array}{lllllll}26 & \mathrm{C} & 0.000000 & 5.086354 & 0.393580 & 0.388470 & 0.395666\end{array}$

$\begin{array}{llllllll}27 & \mathrm{H} & 0.000000 & 0.393580 & 0.472241 & -0.024605 & -0.020883\end{array}$

$\begin{array}{llllllll}28 & \mathrm{H} & 0.000000 & 0.388470 & -0.024605 & 0.535806 & -0.025653\end{array}$

$\begin{array}{lllllll}29 & \mathrm{H} & 0.000000 & 0.395666 & -0.020883 & -0.025653 & 0.497386\end{array}$

Mulliken atomic charges:

$$
1
$$

1 C -0.360400

$2 \mathrm{H} \quad 0.224094$

3 C 0.823499

$4 \mathrm{O}-0.648423$

5 C -0.008269

$6 \mathrm{H} \quad 0.188057$

$\begin{array}{lll}7 \mathrm{H} & 0.187808\end{array}$

8 C -0.496934

$9 \mathrm{H} \quad 0.172040$

$10 \mathrm{H} \quad 0.174020$

$11 \mathrm{H} \quad 0.178896$

12 O -0.571842

13 C 0.561795

$\begin{array}{lll}14 & \mathrm{O} & -0.550697\end{array}$

$15 \mathrm{C}-0.106014$

16 C -0.180705

$\begin{array}{lll}17 & \mathrm{C} & -0.218283\end{array}$

18 C -0.168892

19 C -0.211354

$20 \mathrm{C}-0.214965$

$21 \mathrm{H} \quad 0.242990$

$22 \mathrm{H} \quad 0.254365$

$23 \mathrm{H} \quad 0.212462$

$24 \mathrm{H} \quad 0.208293$

$25 \mathrm{H} \quad 0.212145$

$26 \mathrm{C}-0.472744$

$\begin{array}{lll}27 \mathrm{H} & 0.213027\end{array}$

$28 \mathrm{H} \quad 0.166668$

$29 \mathrm{H} \quad 0.189360$ 
Sum of Mulliken charges $=0.00000$

Atomic charges with hydrogens summed into heavy atoms: 1

1 C -0.136306

$\begin{array}{lll}2 \mathrm{H} & 0.000000\end{array}$

$\begin{array}{lll}3 & \mathrm{C} & 0.823499\end{array}$

$4 \mathrm{O}-0.648423$

5 C 0.367596

$6 \mathrm{H} \quad 0.000000$

$7 \mathrm{H} \quad 0.000000$

$8 \mathrm{C} \quad 0.028022$

$9 \mathrm{H} \quad 0.000000$

$10 \mathrm{H} \quad 0.000000$

$11 \mathrm{H} \quad 0.000000$

12 O -0.571842

$13 \mathrm{C} \quad 0.561795$

$14 \mathrm{O} \quad-0.550697$

$15 \mathrm{C}-0.106014$

$\begin{array}{lll}16 & \mathrm{C} & 0.031441\end{array}$

$\begin{array}{lll}17 \mathrm{C} & 0.024708\end{array}$

$18 \mathrm{C} \quad 0.085473$

19 C 0.001109

$20 \mathrm{C}-0.006672$

$\begin{array}{lll}21 \mathrm{H} & 0.000000\end{array}$

$22 \mathrm{H} \quad 0.000000$

$23 \mathrm{H} \quad 0.000000$

$24 \mathrm{H} \quad 0.000000$

$25 \mathrm{H} \quad 0.000000$

$\begin{array}{lll}26 \mathrm{C} & 0.096312\end{array}$

$27 \mathrm{H} \quad 0.000000$

$28 \mathrm{H} \quad 0.000000$

$29 \mathrm{H} \quad 0.000000$

Sum of Mulliken charges $=0.00000$

Electronic spatial extent $(\mathrm{au}):<\mathrm{R} * * 2>=3642.2594$

Charge $=0.0000$ electrons

Dipole moment (field-independent basis, Debye):

$\mathrm{X}=-0.7321 \mathrm{Y}=-4.1032 \mathrm{Z}=-2.2318 \mathrm{Tot}=4.7279$

Quadrupole moment (field-independent basis, Debye-Ang):

$\mathrm{XX}=-76.5328 \quad \mathrm{YY}=-89.0626 \quad \mathrm{ZZ}=-93.4773$

$\mathrm{XY}=-2.1898 \mathrm{XZ}=3.7956 \quad \mathrm{YZ}=-4.3032$

Traceless Quadrupole moment (field-independent basis, Debye-Ang):

$\mathrm{XX}=9.8247 \quad \mathrm{YY}=-2.7050 \quad \mathrm{ZZ}=-7.1197$

$\mathrm{XY}=-2.1898 \quad \mathrm{XZ}=3.7956 \quad \mathrm{YZ}=-4.3032$

Octapole moment (field-independent basis, Debye-Ang**2):

$\mathrm{XXX}=-7.0330 \mathrm{YYY}=-30.4140 \mathrm{ZZZ}=-2.9594 \mathrm{XYY}=-15.8456$

$\mathrm{XXY}=-25.0308 \mathrm{XXZ}=16.8820 \mathrm{XZZ}=-4.8698 \mathrm{YZZ}=2.3177$

$\mathrm{YYZ}=-7.1835 \mathrm{XYZ}=9.3312$

Hexadecapole moment (field-independent basis, Debye-Ang**3):

$X X X X=-2699.4080 \mathrm{YYYY}=-1596.0929 \mathrm{ZZZZ}=-327.2342 \mathrm{XXXY}=11.4078$

$\mathrm{XXXZ}=47.1124 \mathrm{YYYX}=-48.8738 \mathrm{YYYZ}=1.8552 \mathrm{ZZZX}=8.2807$

$\mathrm{ZZZY}=-5.3243 \mathrm{XXYY}=-701.8686 \mathrm{XXZZ}=-572.5916 \mathrm{YYZZ}=-314.2578$

$\mathrm{XXYZ}=-17.9749 \mathrm{YYXZ}=-12.4463 \mathrm{ZZXY}=24.7252$

$\mathrm{N}-\mathrm{N}=9.727776633605 \mathrm{D}+02 \mathrm{E}-\mathrm{N}=-3.553562803323 \mathrm{D}+03 \mathrm{KE}=6.854954808669 \mathrm{D}+02$

1/1|UNPC-UNKISP|RHF|6-31G(d)|C12H14O3|PCUSER|19-Apr-2006|0||\# HF/6-31G

(D) GEOM=CONNECTIVITYIITitle Card Required\|0,1।C,0,0.70619091,1.445,0

$.52489091 \mathrm{IH}, 0,0.77219091,0.992,1.51989091 \mid \mathrm{C}, 0,1.52919091,0.596,-0.4421$

0909|O,0,2.19119091,-0.396,0.19689091|C,0,3.01419091,-1.245,-0.6411090

9|H,0,3.74319091,-0.619,-1.16510909|H,0,2.37519091,-1.714,-1.39610909|

C, $0,3.68219091,-2.265,0.26189091|\mathrm{H}, 0,4.31619091,-2.93,-0.33510909| \mathrm{H}, 0$,

$2.93819091,-2.876,0.78289091 \mathrm{IH}, 0,4.30919091,-1.772,1.01189091 \mathrm{IO}, 0,1.60$

$319091,0.797,-1.63410909 \mid \mathrm{C}, 0,-0.77480909,1.456,0.08189091 \mathrm{IO}, 0,-1.27980$

909,2.492,-0.31510909|C,0,-1.57680909,0.194,0.16989091|C,0,-3.19780909

$,-2.09,0.30189091 \mathrm{IC}, 0,-1.08080909,-0.998,0.72089091 \mathrm{IC}, 0,-2.89680909,0$.

226,-0.31210909|C, $0,-3.70080909,-0.907,-0.24810909 \mid \mathrm{C}, 0,-1.88880909,-2$. 
$133,0.78489091|\mathrm{H}, 0,-0.06380909,-1.053,1.09389091| \mathrm{H}, 0,-3.26680909,1.156$ ,-0.73110909|H,0,-4.71880909,-0.872,-0.62610909|H,0,-1.49680909,-3.05, $1.21489091 \mathrm{IH}, 0,-3.82480909,-2.976,0.35389091 \mathrm{IC}, 0,1.27719091,2.871,0.56$ $089091|\mathrm{H}, 0,1.25719091,3.312,-0.43710909| \mathrm{H}, 0,2.31019091,2.857,0.9248909$ $1 \mid \mathrm{H}, 0,0.67919091,3.501,1.22489091 \|$ Version $=x 86-\mathrm{Win} 32-\mathrm{G} 03$ RevB.05IState $=$ $1-\mathrm{AlHF}=-687.181857|\mathrm{RMSD}=8.010 \mathrm{e}-006| \mathrm{Dipole}=0.404905,-1.5347409,0.969854$

7|PG=C01 [X(C12H14O3)]\|@

EDUCATION WITHOUT COMMON SENSE IS A LOAD OF BOOKS ON THE BACK OF AN ASS.

Job cpu time: 0 days 0 hours 3 minutes 17.0 seconds.

File lengths (MBytes): $\mathrm{RWF}=43 \mathrm{Int}=0 \mathrm{D} 2 \mathrm{E}=0 \mathrm{Chk}=9 \mathrm{Scr}=1$

Normal termination of Gaussian 03 at Wed Apr 19 09:05:58 2006. 


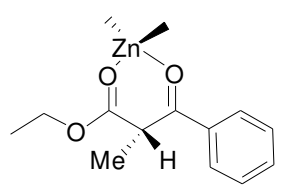

12

Entering Link 1 = C:\G03WV11.exe PID= 1244

Copyright (c) 1988,1990,1992,1993,1995,1998,2003, Gaussian, Inc. All Rights Reserved.

This is the Gaussian(R) 03 program. It is based on the the Gaussian(R) 98 system (copyright 1998, Gaussian, Inc.), the Gaussian(R) 94 system (copyright 1995, Gaussian, Inc.), the Gaussian 92(TM) system (copyright 1992, Gaussian, Inc.), the Gaussian 90(TM) system (copyright 1990, Gaussian, Inc.), the Gaussian 88(TM) system (copyright 1988, Gaussian, Inc.), the Gaussian 86(TM) system (copyright 1986, Carnegie Mellon University), and the Gaussian 82(TM) system (copyright 1983, Carnegie Mellon University). Gaussian is a federally registered trademark of Gaussian, Inc.

This software contains proprietary and confidential information, including trade secrets, belonging to Gaussian, Inc.

This software is provided under written license and may be used, copied, transmitted, or stored only in accord with that written license.

The following legend is applicable only to US Government contracts under DFARS:

\section{RESTRICTED RIGHTS LEGEND}

Use, duplication or disclosure by the US Government is subject to restrictions as set forth in subparagraph (c)(1)(ii) of the Rights in Technical Data and Computer Software clause at DFARS 252.227-7013.

Gaussian, Inc.

Carnegie Office Park, Building 6, Pittsburgh, PA 15106 USA

The following legend is applicable only to US Government contracts under FAR:

\section{RESTRICTED RIGHTS LEGEND}

Use, reproduction and disclosure by the US Government is subject to restrictions as set forth in subparagraph (c) of the Commercial Computer Software - Restricted Rights clause at FAR 52.227-19.

Gaussian, Inc.

Carnegie Office Park, Building 6, Pittsburgh, PA 15106 USA

Warning -- This program may not be used in any manner that competes with the business of Gaussian, Inc. or will provide assistance to any competitor of Gaussian, Inc. The licensee of this program is prohibited from giving any competitor of Gaussian, Inc. access to this program. By using this program, 
the user acknowledges that Gaussian, Inc. is engaged in the business of creating and licensing software in the field of computational chemistry and represents and warrants to the licensee that it is not a competitor of Gaussian, Inc. and that it will not use this program in any manner prohibited above.

Cite this work as:

Gaussian 03, Revision B.05,

M. J. Frisch, G. W. Trucks, H. B. Schlegel, G. E. Scuseria,

M. A. Robb, J. R. Cheeseman, J. A. Montgomery, Jr., T. Vreven,

K. N. Kudin, J. C. Burant, J. M. Millam, S. S. Iyengar, J. Tomasi,

V. Barone, B. Mennucci, M. Cossi, G. Scalmani, N. Rega,

G. A. Petersson, H. Nakatsuji, M. Hada, M. Ehara, K. Toyota,

R. Fukuda, J. Hasegawa, M. Ishida, T. Nakajima, Y. Honda, O. Kitao,

H. Nakai, M. Klene, X. Li, J. E. Knox, H. P. Hratchian, J. B. Cross,

C. Adamo, J. Jaramillo, R. Gomperts, R. E. Stratmann, O. Yazyev,

A. J. Austin, R. Cammi, C. Pomelli, J. W. Ochterski, P. Y. Ayala,

K. Morokuma, G. A. Voth, P. Salvador, J. J. Dannenberg,

V. G. Zakrzewski, S. Dapprich, A. D. Daniels, M. C. Strain,

O. Farkas, D. K. Malick, A. D. Rabuck, K. Raghavachari,

J. B. Foresman, J. V. Ortiz, Q. Cui, A. G. Baboul, S. Clifford,

J. Cioslowski, B. B. Stefanov, G. Liu, A. Liashenko, P. Piskorz,

I. Komaromi, R. L. Martin, D. J. Fox, T. Keith, M. A. Al-Laham,

C. Y. Peng, A. Nanayakkara, M. Challacombe, P. M. W. Gill,

B. Johnson, W. Chen, M. W. Wong, C. Gonzalez, and J. A. Pople, Gaussian, Inc., Pittsburgh PA, 2003.

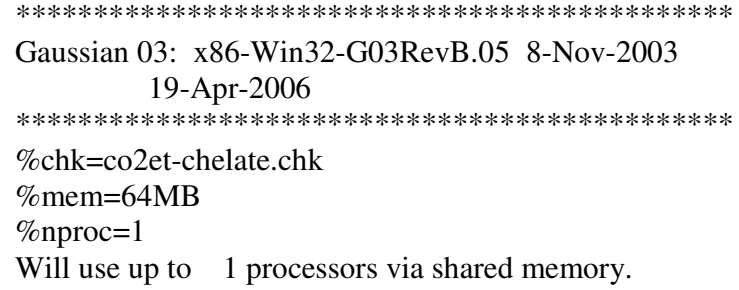

\# hf/6-31g(d) geom=connectivity

$1 / 38=1,57=2 / 1$

$2 / 17=6,18=5,40=1 / 2$;

$3 / 5=1,6=6,7=1,11=9,16=1,25=1,30=1 / 1,2,3$;

$4 / / 1$

$5 / 5=2,32=1,38=5 / 2$;

$6 / 7=2,8=2,9=2,10=2,28=1 / 1$;

$99 / 5=1,9=1 / 99$;

Title Card Required

Symbolic Z-matrix:

$\begin{array}{lrrr}\text { Charge }=0 & \text { Multiplicity }=1 & \\ \mathrm{C} & 0.65541 & -0.56871 & 1.2366 \\ \mathrm{H} & 0.80941 & -1.59771 & 1.6536 \\ \mathrm{O} & 2.78041 & -1.10271 & 0.2516 \\ \mathrm{C} & 3.88941 & -0.95471 & -0.6484 \\ \mathrm{H} & 4.66241 & -1.47771 & -0.0564 \\ \mathrm{H} & 4.18241 & 0.10729 & -0.7494 \\ \mathrm{C} & 3.64441 & -1.61371 & -1.9894 \\ \mathrm{H} & 4.57641 & -1.62271 & -2.5704 \\ \mathrm{H} & 2.89341 & -1.07871 & -2.5864 \\ \mathrm{H} & 3.30941 & -2.65371 & -1.8874 \\ \mathrm{C} & -1.73759 & -1.51871 & 1.1226 \\ \mathrm{C} & -3.59059 & -3.45871 & 1.8886\end{array}$




$\begin{array}{lccc}\mathrm{C} & -1.73059 & -2.02171 & 2.4286 \\ \mathrm{C} & -2.68559 & -1.98771 & 0.2056 \\ \mathrm{C} & -3.60259 & -2.95971 & 0.5906 \\ \mathrm{C} & -2.65559 & -2.98571 & 2.8066 \\ \mathrm{H} & -1.01259 & -1.65171 & 3.1706 \\ \mathrm{H} & -2.71059 & -1.58971 & -0.8174 \\ \mathrm{H} & -4.34059 & -3.32571 & -0.1324 \\ \mathrm{H} & -2.65259 & -3.37171 & 3.8326 \\ \mathrm{H} & -4.31759 & -4.22071 & 2.1906 \\ \mathrm{C} & 0.84841 & 0.46529 & 2.3426 \\ \mathrm{H} & 1.84641 & 0.37829 & 2.7906 \\ \mathrm{H} & 0.11441 & 0.33329 & 3.1476 \\ \mathrm{H} & 0.73741 & 1.50029 & 1.9596 \\ \mathrm{C} & 1.69941 & -0.31571 & 0.1596 \\ \mathrm{C} & -0.76659 & -0.48671 & 0.6916 \\ \mathrm{O} & -1.16659 & 0.38129 & -0.1044 \\ \mathrm{O} & 1.69441 & 0.52029 & -0.7654 \\ \mathrm{Zn} & 0.10541 & 1.59229 & -0.8894 \\ \mathrm{Cl} & 0.33541 & 3.32029 & 0.4756 \\ \mathrm{Cl} & -0.42559 & 1.65329 & -3.0244\end{array}$

Input orientation:

\begin{tabular}{|c|c|c|c|c|c|}
\hline \multirow{2}{*}{$\begin{array}{l}\text { Center } \\
\text { Number }\end{array}$} & \multirow{2}{*}{\multicolumn{2}{|c|}{$\begin{array}{l}\text { Atomic } \\
\text { Number }\end{array}$}} & \multirow{2}{*}{$\begin{array}{l}\text { Atomic } \\
\text { Type }\end{array}$} & \multicolumn{2}{|c|}{ Coordinates (Angstroms } \\
\hline & & & & $\begin{array}{ll}X & Y\end{array}$ & $\mathrm{Z}$ \\
\hline 1 & 6 & 0 & 0.655408 & -0.568713 & 1.236603 \\
\hline 2 & 1 & 0 & 0.809408 & -1.597713 & 1.653603 \\
\hline 3 & 8 & 0 & 2.780408 & -1.102713 & 0.251603 \\
\hline 4 & 6 & 0 & 3.889408 & -0.954713 & -0.648397 \\
\hline 5 & 1 & 0 & 4.662408 & -1.477713 & -0.056397 \\
\hline 6 & 1 & 0 & 4.182408 & 0.107287 & -0.749397 \\
\hline 7 & 6 & 0 & 3.644408 & -1.613713 & -1.989397 \\
\hline 8 & 1 & 0 & 4.576408 & -1.622713 & -2.570397 \\
\hline 9 & 1 & 0 & 2.893408 & -1.078713 & -2.586397 \\
\hline 10 & 1 & 0 & 3.309408 & -2.653713 & -1.887397 \\
\hline 11 & 6 & 0 & -1.737592 & -1.518713 & 1.122603 \\
\hline 12 & 6 & 0 & -3.590592 & -3.458713 & 1.888603 \\
\hline 13 & 6 & 0 & -1.730592 & -2.021713 & 2.428603 \\
\hline 14 & 6 & 0 & -2.685592 & -1.987713 & 0.205603 \\
\hline 15 & 6 & 0 & -3.602592 & -2.959713 & 0.590603 \\
\hline 16 & 6 & 0 & -2.655592 & -2.985713 & 2.806603 \\
\hline 17 & 1 & 0 & -1.012592 & -1.651713 & 3.170603 \\
\hline 18 & 1 & 0 & -2.710592 & -1.589713 & -0.817397 \\
\hline 19 & 1 & 0 & -4.340592 & -3.325713 & -0.132397 \\
\hline 20 & 1 & 0 & -2.652592 & -3.371713 & 3.832603 \\
\hline 21 & 1 & 0 & -4.317592 & -4.220713 & 2.190603 \\
\hline 22 & 6 & 0 & 0.848408 & 0.465287 & 2.342603 \\
\hline 23 & 1 & 0 & 1.846408 & 0.378287 & 2.790603 \\
\hline 24 & 1 & 0 & 0.114408 & 0.333287 & 3.147603 \\
\hline 25 & 1 & 0 & 0.737408 & 1.500287 & 1.959603 \\
\hline 26 & 6 & 0 & 1.699408 & -0.315713 & 0.159603 \\
\hline 27 & 6 & 0 & -0.766592 & -0.486713 & 0.691603 \\
\hline 28 & 8 & 0 & -1.166592 & 0.381287 & -0.104397 \\
\hline 29 & 8 & 0 & 1.694408 & 0.520287 & -0.765397 \\
\hline 30 & 30 & 0 & 0.105408 & 1.592287 & -0.889397 \\
\hline 31 & 17 & 0 & 0.335408 & 3.320287 & 0.475603 \\
\hline 32 & 17 & 0 & -0.425592 & 1.653287 & -3.024397 \\
\hline
\end{tabular}

Distance matrix (angstroms):

$1 \mathrm{C} \quad 0.000000$

$\begin{array}{llll}2 \mathrm{H} & 1.120913 & 0.000000\end{array}$

$\begin{array}{lllll}3 & \mathrm{O} & 2.402292 & 2.468901 & 0.000000\end{array}$ 


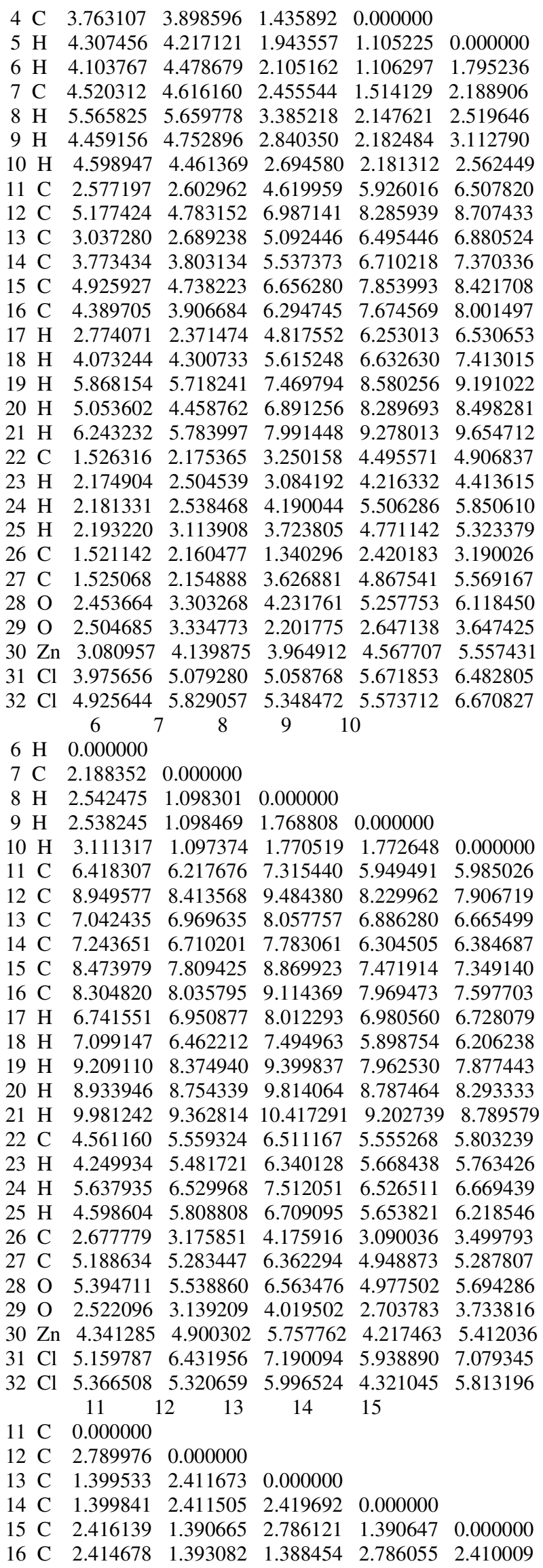


$\begin{array}{lllllll}17 & \mathrm{H} & 2.176607 & 3.399244 & 1.096808 & 3.420972 & 3.882701\end{array}$

$\begin{array}{lllllll}18 \mathrm{H} & 2.171490 & 3.404408 & 3.418119 & 1.097979 & 2.157551\end{array}$

$\begin{array}{lllllll}19 & \mathrm{H} & 3.408208 & 2.159775 & 3.882169 & 2.154881 & 1.096052\end{array}$

$\begin{array}{lllllll}20 \mathrm{H} & 3.408069 & 2.160220 & 2.154948 & 3.882225 & 3.403352\end{array}$

$\begin{array}{lllllll}21 & \mathrm{H} & 3.885592 & 1.095617 & 3.403647 & 3.404400 & 2.159015\end{array}$

$\begin{array}{llllllll}22 & \mathrm{C} & 3.480237 & 5.942105 & 3.583826 & 4.803450 & 5.883156\end{array}$

$\begin{array}{llllllll}23 & \mathrm{H} & 4.384734 & 6.715441 & 4.322727 & 5.728805 & 6.758243\end{array}$

$\begin{array}{llllllll}24 & \mathrm{H} & 3.310654 & 5.448979 & 3.076851 & 4.677863 & 5.585534\end{array}$

$\begin{array}{lllllll}25 & \mathrm{H} & 3.992562 & 6.582424 & 4.326138 & 5.192262 & 6.371920\end{array}$

$\begin{array}{llllllll}26 & \mathrm{C} & 3.766636 & 6.391556 & 4.452381 & 4.693179 & 5.940345\end{array}$

$\begin{array}{llllllll}27 & \mathrm{C} & 1.481089 & 4.270898 & 2.510516 & 2.484302 & 3.764150\end{array}$

$\begin{array}{llllllll}28 & \mathrm{O} & 2.332717 & 4.959176 & 3.536749 & 2.831187 & 4.192780\end{array}$

$\begin{array}{llllllll}29 & \mathrm{O} & 4.415958 & 7.127930 & 5.328604 & 5.139777 & 6.481307\end{array}$

$\begin{array}{llllllll}30 & \mathrm{Zn} & 4.138008 & 6.847649 & 5.238417 & 4.669594 & 6.054781\end{array}$

$\begin{array}{llllllll}31 & C l & 5.303947 & 7.960206 & 6.051407 & 6.113445 & 7.413465\end{array}$

$\begin{array}{lllllll}32 & \mathrm{Cl} & 5.383357 & 7.764492 & 6.704018 & 5.366319 & 6.666433\end{array}$

$16 \mathrm{C} \quad 0.000000$

$\begin{array}{lllll}16 & 17 & 18 & 19 & 20\end{array}$

$\begin{array}{llll}17 & \mathrm{H} & 2.147441 & 0.000000\end{array}$

$\begin{array}{lllll}18 \mathrm{H} & 3.883969 & 4.334881 & 0.000000\end{array}$

$\begin{array}{llllll}19 & \mathrm{H} & 3.404783 & 4.978722 & 2.477866 & 0.000000\end{array}$

$\begin{array}{lllllll}20 & \mathrm{H} & 1.096212 & 2.467031 & 4.980099 & 4.309604 & 0.000000\end{array}$

$\begin{array}{llllllll}21 & \mathrm{H} & 2.160307 & 4.299208 & 4.307282 & 2.489555 & 2.487808\end{array}$

$\begin{array}{llllllll}22 & \mathrm{C} & 4.939910 & 2.937787 & 5.184121 & 6.886438 & 5.403672\end{array}$

$\begin{array}{llllllll}23 & \mathrm{H} & 5.620032 & 3.526922 & 6.136525 & 7.780907 & 5.948888\end{array}$

$\begin{array}{lllllll}24 & \mathrm{H} & 4.336467 & 2.282736 & 5.234480 & 6.632775 & 4.674670\end{array}$

$\begin{array}{lllllll}25 & \mathrm{H} & 5.688062 & 3.803173 & 5.398938 & 7.311144 & 6.223874\end{array}$

$\begin{array}{lllllll}26 \mathrm{C} & 5.753393 & 4.266844 & 4.693155 & 6.754773 & 6.462969\end{array}$

$\begin{array}{llllllll}27 & \mathrm{C} & 3.779755 & 2.750124 & 2.696818 & 4.638143 & 4.663272\end{array}$

$\begin{array}{llllllll}28 & \mathrm{O} & 4.693371 & 3.857775 & 2.603295 & 4.880257 & 5.638544\end{array}$

$\begin{array}{llllllll}29 & \mathrm{O} & 6.631268 & 5.247621 & 4.884550 & 7.184263 & 7.428706\end{array}$

$\begin{array}{llllllll}30 & \mathrm{Zn} & 6.499355 & 5.315737 & 4.249725 & 6.672832 & 7.385468\end{array}$

$\begin{array}{lllllll}31 & \mathrm{Cl} & 7.358348 & 5.813855 & 5.920985 & 8.148862 & 8.061046\end{array}$

$\begin{array}{lllllll}32 & \mathrm{Cl} & 7.777775 & 7.045965 & 4.539727 & 6.962854 & 8.787981\end{array}$ $\begin{array}{lllll}21 & 22 & 23 & 24 & 25\end{array}$

$\begin{array}{lll}21 \mathrm{H} & 0.000000\end{array}$

$\begin{array}{llll}22 & \mathrm{C} & 6.976335 & 0.000000\end{array}$

$\begin{array}{lllll}23 & \mathrm{H} & 7.713994 & 1.097396 & 0.000000\end{array}$

$\begin{array}{llllll}24 \mathrm{H} & 6.426304 & 1.097363 & 1.768982 & 0.000000\end{array}$

$\begin{array}{lllllll}25 \mathrm{H} & 7.637816 & 1.109160 & 1.783066 & 1.778022 & 0.000000\end{array}$

$\begin{array}{lllllll}26 & \mathrm{C} & 7.455084 & 2.469747 & 2.724960 & 3.444063 & 2.731904\end{array}$

$\begin{array}{llllllll}27 & \mathrm{C} & 5.366503 & 2.498065 & 3.461473 & 2.735050 & 2.796070\end{array}$

$\begin{array}{llllllll}28 & \mathrm{O} & 6.031105 & 3.170976 & 4.178421 & 3.495536 & 3.022825\end{array}$

$\begin{array}{llllllll}29 & \text { O } & 8.207263 & 3.221553 & 3.562079 & 4.224090 & 3.049897\end{array}$

$\begin{array}{lllllll}30 & \mathrm{Zn} & 7.927187 & 3.502571 & 4.248209 & 4.228774 & 2.919707\end{array}$

$\begin{array}{lllllll}31 & \mathrm{Cl} & 9.025426 & 3.449621 & 4.037042 & 4.013800 & 2.382490\end{array}$

$\begin{array}{llllllll}32 & \mathrm{Cl} & 8.766286 & 5.642615 & 6.371957 & 6.334634 & 5.120179\end{array}$

$26 \mathrm{C} \quad 0.000000$

$\begin{array}{llll}27 & \mathrm{C} & 2.528522 & 0.000000\end{array}$

$\begin{array}{lllll}28 & \mathrm{O} & 2.961328 & 1.243801 & 0.000000\end{array}$

$\begin{array}{lllllll}29 & \mathrm{O} & 1.246814 & 3.032065 & 2.939654 & 0.000000\end{array}$

$\begin{array}{lllllll}30 & \mathrm{Zn} & 2.698463 & 2.753577 & 1.923728 & 1.920802 & 0.000000\end{array}$

$\begin{array}{llllllll}31 & \mathrm{Cl} & 3.896261 & 3.969170 & 3.351138 & 3.350666 & 2.214071\end{array}$

$\begin{array}{llllllll}32 & \mathrm{Cl} & 4.304700 & 4.301690 & 3.270086 & 3.298662 & 2.200888\end{array}$ $31 \quad 32$

$\begin{array}{lll}31 \mathrm{Cl} & 0.000000\end{array}$

$\begin{array}{llll}32 & \mathrm{Cl} & 3.950697 & 0.000000\end{array}$

Stoichiometry $\mathrm{C} 12 \mathrm{H} 14 \mathrm{Cl} 2 \mathrm{O} 3 \mathrm{Zn}$

Framework group C1[X(C12H14Cl2O3Zn)]

Deg. of freedom 90

Full point group $\quad \mathrm{C} 1 \quad \mathrm{NOp} 1$

Largest Abelian subgroup $\quad \mathrm{C} 1 \quad$ NOp 1

Largest concise Abelian subgroup C1 NOp 1 
Standard orientation:

\begin{tabular}{|c|c|c|c|c|c|}
\hline \multirow{2}{*}{$\begin{array}{l}\text { Center } \\
\text { Number }\end{array}$} & \multirow{2}{*}{$\begin{array}{l}\text { Atomic } \\
\text { Number }\end{array}$} & \multirow{2}{*}{\multicolumn{2}{|c|}{$\begin{array}{r}\text { Atomic } \\
\text { Type }\end{array}$}} & \multicolumn{2}{|c|}{ Coordinates (Angstroms) } \\
\hline & & & & $\begin{array}{ll}X & Y\end{array}$ & $\mathrm{Z}$ \\
\hline 1 & 6 & 0 & 0.484354 & 1.062297 & 0.958702 \\
\hline 2 & 1 & 0 & 1.201170 & 1.921886 & 0.897651 \\
\hline 3 & 8 & 0 & -1.018554 & 2.809551 & 0.280928 \\
\hline 4 & 6 & 0 & -2.238638 & 3.376240 & -0.221110 \\
\hline 5 & 1 & 0 & -2.152138 & 4.387010 & 0.217506 \\
\hline 6 & 1 & 0 & -3.121757 & 2.876146 & 0.219231 \\
\hline 7 & 6 & 0 & -2.289121 & 3.405325 & -1.734118 \\
\hline 8 & 1 & 0 & -3.153339 & 3.998620 & -2.061828 \\
\hline 9 & 1 & 0 & -2.399490 & 2.401986 & -2.167438 \\
\hline 10 & 1 & 0 & -1.391684 & 3.860269 & -2.172138 \\
\hline 11 & 6 & 0 & 2.564501 & -0.089729 & -0.035164 \\
\hline 12 & 6 & 0 & 5.298076 & -0.046831 & -0.591666 \\
\hline 13 & 6 & 0 & 3.461354 & 0.464692 & 0.885143 \\
\hline 14 & 6 & 0 & 3.047800 & -0.631033 & -1.232230 \\
\hline 15 & 6 & 0 & 4.410550 & -0.600956 & -1.507740 \\
\hline 16 & 6 & 0 & 4.821234 & 0.482460 & 0.605477 \\
\hline 17 & 1 & 0 & 3.107934 & 0.872885 & 1.839848 \\
\hline 18 & 1 & 0 & 2.355523 & -1.082279 & -1.955202 \\
\hline 19 & 1 & 0 & 4.783172 & -1.023292 & -2.448014 \\
\hline 20 & 1 & 0 & 5.521849 & 0.910157 & 1.332041 \\
\hline 21 & 1 & 0 & 6.371755 & -0.029185 & -0.809100 \\
\hline 22 & 6 & 0 & 0.233102 & 0.709088 & 2.422176 \\
\hline 23 & 1 & 0 & -0.157645 & 1.574304 & 2.972626 \\
\hline 24 & 1 & 0 & 1.156730 & 0.391430 & 2.922385 \\
\hline 25 & 1 & 0 & -0.499792 & -0.117174 & 2.524115 \\
\hline 26 & 6 & 0 & -0.838135 & 1.482579 & 0.335596 \\
\hline 27 & 6 & 0 & 1.109192 & -0.127296 & 0.237397 \\
\hline 28 & 8 & 0 & 0.477468 & -1.125102 & -0.152920 \\
\hline 29 & 8 & 0 & -1.765549 & 0.774372 & -0.103598 \\
\hline 30 & 30 & 0 & -1.443548 & -1.118517 & -0.051021 \\
\hline 31 & 17 & 0 & -2.023563 & -1.857621 & 1.953828 \\
\hline 32 & 17 & 0 & -2.108694 & -1.905839 & -1.995658 \\
\hline
\end{tabular}

Rotational constants (GHZ): $\quad 0.3601549 \quad 0.2026250 \quad 0.1759578$

Standard basis: $6-31 \mathrm{G}(\mathrm{d})(6 \mathrm{D}, 7 \mathrm{~F})$

There are 327 symmetry adapted basis functions of A symmetry.

Integral buffers will be 262144 words long.

Raffenetti 1 integral format.

Two-electron integral symmetry is turned on.

327 basis functions, 684 primitive gaussians, 330 cartesian basis functions

87 alpha electrons 87 beta electrons

nuclear repulsion energy 2120.6133978253 Hartrees.

NAtoms $=32$ NActive $=32 \mathrm{NUniq}=32 \mathrm{SFac}=1.00 \mathrm{D}+00 \mathrm{NAtFMM}=60 \mathrm{Big}=\mathrm{F}$

One-electron integrals computed using PRISM.

NBasis $=327$ RedAO $=\mathrm{T}$ NBF $=327$

$\mathrm{NBsUse}=327$ 1.00D-06 NBFU $=327$

Harris functional with IExCor= 205 diagonalized for initial guess.

ExpMin= 4.93D-02 ExpMax= 8.24D+04 ExpMxC=1.24D+04 IAcc=2 IRadAn $=4$ AccDes $=0.00 D+00$

HarFok: IExCor $=205$ AccDes $=0.00 D+00 \mathrm{IRadAn}=\quad 4 \mathrm{IDoV}=1$

ScaDFX= 1.0000001 .0000001 .0000001 .000000

Initial guess orbital symmetries:

Occupied (A) (A) (A) (A) (A) (A) (A) (A) (A) (A) (A) (A)

(A) (A) (A) (A) (A) (A) (A) (A) (A) (A) (A) (A)

(A) (A) (A) (A) (A) (A) (A) (A) (A) (A) (A) $(\mathrm{A})$

(A) $(\mathrm{A})(\mathrm{A})(\mathrm{A})(\mathrm{A})(\mathrm{A})(\mathrm{A})(\mathrm{A})(\mathrm{A})(\mathrm{A})(\mathrm{A})(\mathrm{A})$

$(\mathrm{A})(\mathrm{A})(\mathrm{A})(\mathrm{A})(\mathrm{A})(\mathrm{A})(\mathrm{A})(\mathrm{A})(\mathrm{A})(\mathrm{A})(\mathrm{A})(\mathrm{A})$

(A) (A) (A) (A) (A) (A) (A) (A) (A) (A) (A) (A)

(A) $(\mathrm{A})(\mathrm{A})(\mathrm{A})(\mathrm{A})(\mathrm{A})(\mathrm{A})(\mathrm{A})(\mathrm{A})(\mathrm{A})(\mathrm{A})(\mathrm{A})$

(A) $(\mathrm{A})(\mathrm{A})$ 
Virtual (A) (A) (A) (A) (A) (A) (A) (A) (A) (A) (A) (A)

(A) $(A)(A)(A)(A)(A)(A)(A)(A)(A)(A)(A)$

(A) $(\mathrm{A})(\mathrm{A})(\mathrm{A})(\mathrm{A})(\mathrm{A})(\mathrm{A})(\mathrm{A})(\mathrm{A})(\mathrm{A})(\mathrm{A})(\mathrm{A})$

(A) (A) (A) (A) (A) (A) (A) (A) (A) (A) (A) (A)

(A) $(\mathrm{A})(\mathrm{A})(\mathrm{A})(\mathrm{A})(\mathrm{A})(\mathrm{A})(\mathrm{A})(\mathrm{A})(\mathrm{A})(\mathrm{A})(\mathrm{A})$

(A) (A) (A) (A) (A) (A) (A) (A) (A) (A) (A) (A)

(A) (A) (A) (A) (A) (A) (A) (A) (A) (A) (A) (A)

(A) $(\mathrm{A})(\mathrm{A})(\mathrm{A})(\mathrm{A})(\mathrm{A})(\mathrm{A})(\mathrm{A})(\mathrm{A})(\mathrm{A})(\mathrm{A})(\mathrm{A})$

(A) (A) (A) (A) (A) (A) (A) (A) (A) (A) (A) (A)

(A) $(\mathrm{A})(\mathrm{A})(\mathrm{A})(\mathrm{A})(\mathrm{A})(\mathrm{A})(\mathrm{A})(\mathrm{A})(\mathrm{A})(\mathrm{A})(\mathrm{A})$

(A) $(\mathrm{A})(\mathrm{A})(\mathrm{A})(\mathrm{A})(\mathrm{A})(\mathrm{A})(\mathrm{A})(\mathrm{A})(\mathrm{A})(\mathrm{A})(\mathrm{A})$

(A) $(\mathrm{A})(\mathrm{A})(\mathrm{A})(\mathrm{A})(\mathrm{A})(\mathrm{A})(\mathrm{A})(\mathrm{A})(\mathrm{A})(\mathrm{A})(\mathrm{A})$

(A) $(\mathrm{A})(\mathrm{A})(\mathrm{A})(\mathrm{A})(\mathrm{A})(\mathrm{A})(\mathrm{A})(\mathrm{A})(\mathrm{A})(\mathrm{A})(\mathrm{A})$

(A) $(\mathrm{A})(\mathrm{A})(\mathrm{A})(\mathrm{A})(\mathrm{A})(\mathrm{A})(\mathrm{A})(\mathrm{A})(\mathrm{A})(\mathrm{A})(\mathrm{A})$

(A) (A) (A) (A) (A) (A) (A) (A) (A) (A) (A) (A)

(A) $(\mathrm{A})(\mathrm{A})(\mathrm{A})(\mathrm{A})(\mathrm{A})(\mathrm{A})(\mathrm{A})(\mathrm{A})(\mathrm{A})(\mathrm{A})(\mathrm{A})$

(A) $(\mathrm{A})(\mathrm{A})(\mathrm{A})(\mathrm{A})(\mathrm{A})(\mathrm{A})(\mathrm{A})(\mathrm{A})(\mathrm{A})(\mathrm{A})(\mathrm{A})$

(A) (A) (A) (A) (A) (A) (A) (A) (A) (A) (A) (A)

(A) $(A)(A)(A)(A)(A)(A)(A)(A)(A)(A)(A)$

(A) (A) (A) (A) (A) (A) (A) (A) (A) (A) (A) (A)

The electronic state of the initial guess is 1-A.

Requested convergence on RMS density matrix=1.00D-04 within 128 cycles.

Requested convergence on MAX density matrix $=1.00 \mathrm{D}-02$.

Requested convergence on energy=1.00D-02.

No special actions if energy rises.

SCF Done: $\mathrm{E}(\mathrm{RHF})=-3383.77681030 \quad$ A.U. after 5 cycles

Convg $=0.3506 \mathrm{D}-04 \quad-\mathrm{V} / \mathrm{T}=2.0005$

$\mathrm{S} * * 2=0.0000$

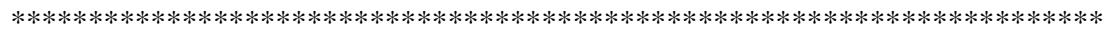

Population analysis using the SCF density.

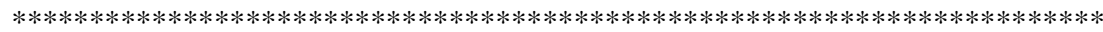

Orbital symmetries:

Occupied (A) (A) (A) (A) (A) (A) (A) (A) (A) (A) (A) (A)

(A) (A) (A) (A) (A) (A) (A) (A) (A) (A) (A) (A)

(A) (A) (A) (A) (A) (A) (A) (A) (A) (A) (A) (A)

(A) $(\mathrm{A})(\mathrm{A})(\mathrm{A})(\mathrm{A})(\mathrm{A})(\mathrm{A})(\mathrm{A})(\mathrm{A})(\mathrm{A})(\mathrm{A})(\mathrm{A})$

(A) (A) (A) (A) (A) (A) (A) (A) (A) (A) (A) (A)

(A) (A) (A) (A) (A) (A) (A) (A) (A) (A) (A) (A)

(A) $(\mathrm{A})(\mathrm{A})(\mathrm{A})(\mathrm{A})(\mathrm{A})(\mathrm{A})(\mathrm{A})(\mathrm{A})(\mathrm{A})(\mathrm{A})(\mathrm{A})$

(A) $(\mathrm{A})(\mathrm{A})$

Virtual (A) (A) (A) (A) (A) (A) (A) (A) (A) (A) (A) (A)

(A) $(A)(A)(A)(A)(A)(A)(A)(A)(A)(A)(A)$

(A) (A) (A) (A) (A) (A) (A) (A) (A) (A) (A) (A)

(A) $(\mathrm{A})(\mathrm{A})(\mathrm{A})(\mathrm{A})(\mathrm{A})(\mathrm{A})(\mathrm{A})(\mathrm{A})(\mathrm{A})(\mathrm{A})(\mathrm{A})$

(A) (A) (A) (A) (A) (A) (A) (A) (A) (A) (A) (A)

(A) (A) (A) (A) (A) (A) (A) (A) (A) (A) (A) (A)

(A) $(\mathrm{A})(\mathrm{A})(\mathrm{A})(\mathrm{A})(\mathrm{A})(\mathrm{A})(\mathrm{A})(\mathrm{A})(\mathrm{A})(\mathrm{A})(\mathrm{A})$

(A) (A) (A) (A) (A) (A) (A) (A) (A) (A) (A) (A)

(A) (A) (A) (A) (A) (A) (A) (A) (A) (A) (A) (A)

(A) $(\mathrm{A})(\mathrm{A})(\mathrm{A})(\mathrm{A})(\mathrm{A})(\mathrm{A})(\mathrm{A})(\mathrm{A})(\mathrm{A})(\mathrm{A})(\mathrm{A})$

(A) (A) (A) (A) (A) (A) (A) (A) (A) (A) (A) (A)

(A) (A) (A) (A) (A) (A) (A) (A) (A) (A) (A) (A)

(A) (A) (A) (A) (A) (A) (A) (A) (A) (A) (A) (A)

(A) (A) (A) (A) (A) (A) (A) (A) (A) (A) (A) (A)

(A) (A) (A) (A) (A) (A) (A) (A) (A) (A) (A) (A)

(A) (A) (A) (A) (A) (A) (A) (A) (A) (A) (A) (A)

(A) (A) (A) (A) (A) (A) (A) (A) (A) (A) (A) (A)

(A) (A) (A) (A) (A) (A) (A) (A) (A) (A) (A) (A)

(A) (A) (A) (A) (A) (A) (A) (A) (A) (A) (A) (A)

(A) $(\mathrm{A})(\mathrm{A})(\mathrm{A})(\mathrm{A})(\mathrm{A})(\mathrm{A})(\mathrm{A})(\mathrm{A})(\mathrm{A})(\mathrm{A})(\mathrm{A})$ 
The electronic state is 1-A.

Alpha occ. eigenvalues -- -353.28641-104.72071-104.70585 -44.36479 -38.93283

Alpha occ. eigenvalues -- -38.93271 -38.93196 -20.67034 -20.64432 -20.63854

Alpha occ. eigenvalues -- -11.47853 -11.42876 -11.33990 -11.33192 -11.29833

Alpha occ. eigenvalues -- -11.29718 -11.29025 -11.28709 -11.28200 -11.27875

Alpha occ. eigenvalues -- -11.27833 -11.24920 -10.45603 -10.44058 -7.92455

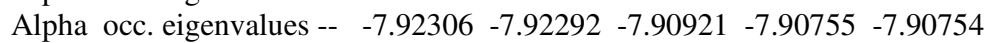

Alpha occ. eigenvalues -- $\quad-5.63458-3.84302 \quad-3.84191 \quad-3.84173 \quad-1.53037$

Alpha occ. eigenvalues -- $\quad-1.47369-1.42562-1.20732-1.16506-1.08626$

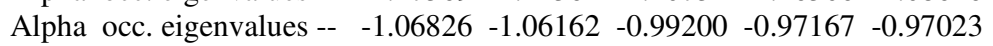

$\begin{array}{lllllll}\text { Alpha occ. eigenvalues -- } & -0.95009 & -0.90269 & -0.87399 & -0.84226 & -0.83090\end{array}$

Alpha occ. eigenvalues -- $-0.80692 \quad-0.78837 \quad-0.77059-0.76997 \quad-0.76597$

$\begin{array}{llllll}\text { Alpha occ. eigenvalues -- } & -0.75260 & -0.74646 & -0.72821 & -0.71034 & -0.70355\end{array}$

Alpha occ. eigenvalues -- $-0.69426-0.67389-0.66364-0.64676-0.64435$

Alpha occ. eigenvalues -- $-0.63123 \quad-0.61514 \quad-0.59125 \quad-0.58911 \quad-0.58223$

$\begin{array}{llllll}\text { Alpha occ. eigenvalues -- } & -0.57345 & -0.56252 & -0.55945 & -0.54519 & -0.53949\end{array}$

$\begin{array}{lllllll}\text { Alpha occ. eigenvalues -- } & -0.53687 & -0.52184 & -0.50980 & -0.49431 & -0.42609\end{array}$

$\begin{array}{lllllll}\text { Alpha occ. eigenvalues -- } & -0.39820 & -0.38691 & -0.38341 & -0.37978 & -0.37554\end{array}$

Alpha occ. eigenvalues -- $-0.36536-0.36208$

$\begin{array}{lllllll}\text { Alpha virt. eigenvalues -- } & 0.02585 & 0.08797 & 0.09703 & 0.14028 & 0.15887\end{array}$

$\begin{array}{lllllll}\text { Alpha virt. eigenvalues -- } & 0.18234 & 0.19074 & 0.19359 & 0.20194 & 0.22055\end{array}$

$\begin{array}{lllllll}\text { Alpha virt. eigenvalues -- } & 0.23789 & 0.24463 & 0.26377 & 0.26708 & 0.27360\end{array}$

$\begin{array}{llllllll}\text { Alpha virt. eigenvalues -- } & 0.28392 & 0.28553 & 0.28784 & 0.30129 & 0.30895\end{array}$

$\begin{array}{lllllll}\text { Alpha virt. eigenvalues -- } & 0.31456 & 0.32372 & 0.32638 & 0.33519 & 0.34322\end{array}$

$\begin{array}{lllllll}\text { Alpha virt. eigenvalues -- } & 0.35126 & 0.37729 & 0.37922 & 0.39131 & 0.41187\end{array}$

$\begin{array}{lllllll}\text { Alpha virt. eigenvalues -- } & 0.43589 & 0.44349 & 0.44488 & 0.46050 & 0.46521\end{array}$

$\begin{array}{lllllll}\text { Alpha virt. eigenvalues -- } & 0.49062 & 0.51487 & 0.52958 & 0.55513 & 0.58319\end{array}$

$\begin{array}{lllllll}\text { Alpha virt. eigenvalues -- } & 0.63195 & 0.65233 & 0.67689 & 0.68388 & 0.68596\end{array}$

$\begin{array}{llllllll}\text { Alpha virt. eigenvalues -- } & 0.69489 & 0.70534 & 0.70763 & 0.71455 & 0.72218\end{array}$

$\begin{array}{llllllll}\text { Alpha virt. eigenvalues -- } & 0.72677 & 0.74874 & 0.75337 & 0.76170 & 0.76192\end{array}$

$\begin{array}{lllllll}\text { Alpha virt. eigenvalues -- } & 0.77757 & 0.78322 & 0.79022 & 0.79505 & 0.79680\end{array}$

$\begin{array}{lllllll}\text { Alpha virt. eigenvalues -- } & 0.80309 & 0.80561 & 0.81016 & 0.81529 & 0.82331\end{array}$

$\begin{array}{lllllll}\text { Alpha virt. eigenvalues -- } & 0.83933 & 0.86161 & 0.86508 & 0.87423 & 0.88415\end{array}$

$\begin{array}{lllllll}\text { Alpha virt. eigenvalues -- } & 0.89160 & 0.90967 & 0.92277 & 0.94093 & 0.95126\end{array}$

$\begin{array}{lllllll}\text { Alpha virt. eigenvalues -- } & 0.96849 & 0.97632 & 0.99245 & 1.01178 & 1.03538\end{array}$

$\begin{array}{lllllll}\text { Alpha virt. eigenvalues -- } & 1.05374 & 1.06347 & 1.06451 & 1.07477 & 1.08284\end{array}$

$\begin{array}{lllllll}\text { Alpha virt. eigenvalues -- } & 1.09438 & 1.10696 & 1.11672 & 1.12366 & 1.13553\end{array}$

$\begin{array}{lllllll}\text { Alpha virt. eigenvalues -- } & 1.14659 & 1.15337 & 1.15518 & 1.16757 & 1.17060\end{array}$

$\begin{array}{lllllll}\text { Alpha virt. eigenvalues -- } & 1.18927 & 1.19213 & 1.20213 & 1.21173 & 1.22334\end{array}$

$\begin{array}{lllllll}\text { Alpha virt. eigenvalues -- } & 1.22464 & 1.23634 & 1.23856 & 1.24308 & 1.24889\end{array}$

$\begin{array}{lllllll}\text { Alpha virt. eigenvalues -- } & 1.25486 & 1.25613 & 1.26161 & 1.27792 & 1.29689\end{array}$

$\begin{array}{lllllll}\text { Alpha virt. eigenvalues -- } & 1.30686 & 1.32280 & 1.32943 & 1.33270 & 1.36168\end{array}$

$\begin{array}{lllllll}\text { Alpha virt. eigenvalues -- } & 1.37858 & 1.38845 & 1.39955 & 1.41099 & 1.41736\end{array}$

$\begin{array}{lllllll}\text { Alpha virt. eigenvalues -- } & 1.45426 & 1.46558 & 1.49381 & 1.51929 & 1.53923\end{array}$

$\begin{array}{lllllll}\text { Alpha virt. eigenvalues -- } & 1.57754 & 1.59284 & 1.60305 & 1.64633 & 1.66232\end{array}$

$\begin{array}{lllllll}\text { Alpha virt. eigenvalues -- } & 1.67374 & 1.68521 & 1.69379 & 1.70789 & 1.71857\end{array}$

$\begin{array}{lllllll}\text { Alpha virt. eigenvalues -- } & 1.72527 & 1.73372 & 1.74500 & 1.79325 & 1.80021\end{array}$

$\begin{array}{lllllll}\text { Alpha virt. eigenvalues -- } & 1.83861 & 1.87380 & 1.87987 & 1.90997 & 1.94704\end{array}$

$\begin{array}{lllllll}\text { Alpha virt. eigenvalues -- } & 1.97048 & 1.99325 & 2.00185 & 2.01314 & 2.02388\end{array}$

$\begin{array}{llllll}\text { Alpha virt. eigenvalues -- } & 2.05037 & 2.06964 & 2.09024 & 2.10007 & 2.11336\end{array}$

$\begin{array}{llllll}\text { Alpha virt. eigenvalues -- } & 2.12805 & 2.14751 & 2.16391 & 2.18057 & 2.18889\end{array}$

$\begin{array}{lllllll}\text { Alpha virt. eigenvalues -- } & 2.20996 & 2.21733 & 2.24687 & 2.25871 & 2.30012\end{array}$

$\begin{array}{llllll}\text { Alpha virt. eigenvalues -- } & 2.31227 & 2.32132 & 2.33387 & 2.35346 & 2.36596\end{array}$

$\begin{array}{lllllll}\text { Alpha virt. eigenvalues -- } & 2.41409 & 2.41864 & 2.43541 & 2.44433 & 2.48262\end{array}$

$\begin{array}{lllllll}\text { Alpha virt. eigenvalues -- } & 2.50013 & 2.52195 & 2.53684 & 2.55900 & 2.56852\end{array}$

$\begin{array}{lllllll}\text { Alpha virt. eigenvalues -- } & 2.57450 & 2.58897 & 2.59686 & 2.60646 & 2.61082\end{array}$

$\begin{array}{llllll}\text { Alpha virt. eigenvalues -- } & 2.61640 & 2.62856 & 2.64382 & 2.64959 & 2.67441\end{array}$

$\begin{array}{lllllll}\text { Alpha virt. eigenvalues -- } & 2.69123 & 2.69748 & 2.70711 & 2.74739 & 2.77231\end{array}$

$\begin{array}{llllll}\text { Alpha virt. eigenvalues -- } & 2.79926 & 2.81139 & 2.83261 & 2.85210 & 2.85630\end{array}$

$\begin{array}{lllllll}\text { Alpha virt. eigenvalues -- } & 2.87048 & 2.88043 & 2.90986 & 2.94110 & 2.95083\end{array}$

$\begin{array}{lllllll}\text { Alpha virt. eigenvalues -- } & 2.96054 & 2.99480 & 3.02387 & 3.04883 & 3.06057\end{array}$

$\begin{array}{lllllll}\text { Alpha virt. eigenvalues -- } & 3.07145 & 3.09587 & 3.16130 & 3.25230 & 3.30027\end{array}$

$\begin{array}{lllllll}\text { Alpha virt. eigenvalues -- } & 3.31426 & 3.36836 & 3.38669 & 3.42840 & 3.53327\end{array}$

$\begin{array}{lllllll}\text { Alpha virt. eigenvalues -- } & 3.54369 & 3.75259 & 4.42331 & 4.46436 & 4.47908\end{array}$ 
$\begin{array}{lllllll}\text { Alpha virt. eigenvalues -- } & 4.49306 & 4.54031 & 4.59400 & 4.61090 & 4.69605\end{array}$ Alpha virt. eigenvalues -- $4.71176 \quad 4.72607 \quad 4.74666 \quad 4.82166 \quad 4.83005$ $\begin{array}{llllll}\text { Alpha virt. eigenvalues -- } & 4.83699 & 4.84882 & 5.05803 & 5.09710 & 48.59123\end{array}$ Condensed to atoms (all electrons):

$$
\begin{array}{llllll}
1 & 2 & 3 & 4 & 5 & 6
\end{array}
$$

$\begin{array}{llllllll}1 & \mathrm{C} & 5.515403 & 0.394709 & -0.065471 & 0.002948 & -0.000028 & -0.000120\end{array}$

$\begin{array}{llllllll}2 & \mathrm{H} & 0.394709 & 0.423023 & 0.003509 & -0.000065 & -0.000036 & 0.000016\end{array}$

$\begin{array}{llllllll}3 & \mathrm{O} & -0.065471 & 0.003509 & 8.412303 & 0.130063 & -0.041826 & -0.027651\end{array}$

$\begin{array}{lllllllll}4 \mathrm{C} & 0.002948 & -0.000065 & 0.130063 & 4.885594 & 0.410785 & 0.395568\end{array}$

$\begin{array}{llllllll}5 & \mathrm{H} & -0.000028 & -0.000036 & -0.041826 & 0.410785 & 0.499085 & -0.027787\end{array}$

$\begin{array}{llllllll}6 \mathrm{H} & -0.000120 & 0.000016 & -0.027651 & 0.395568 & -0.027787 & 0.481212\end{array}$

$\begin{array}{llllllll}7 & \mathrm{C} & -0.000358 & 0.000005 & -0.038608 & 0.329757 & -0.040954 & -0.037833\end{array}$

$\begin{array}{llllllll}8 & \mathrm{H} & 0.000005 & 0.000000 & 0.002481 & -0.033964 & -0.001327 & -0.000130\end{array}$

$\begin{array}{llllllll}9 \mathrm{H} & 0.000054 & -0.000003 & -0.000816 & -0.030338 & 0.002857 & -0.003198\end{array}$

$\begin{array}{llllllll}10 \mathrm{H} & -0.000050 & 0.000007 & 0.000169 & -0.036549 & -0.002802 & 0.003360\end{array}$

$\begin{array}{llllllll}11 & \mathrm{C} & -0.080308 & 0.006945 & -0.000084 & 0.000003 & 0.000000 & 0.000000\end{array}$

$\begin{array}{llllllll}12 & \mathrm{C} & 0.000006 & 0.000007 & 0.000000 & 0.000000 & 0.000000 & 0.000000\end{array}$

$\begin{array}{llllllll}13 & \mathrm{C} & -0.005406 & 0.000551 & -0.000004 & 0.000000 & 0.000000 & 0.000000\end{array}$

$\begin{array}{llllllll}14 \mathrm{C} & 0.004090 & 0.000076 & 0.000000 & 0.000000 & 0.000000 & 0.000000\end{array}$

$\begin{array}{llllllll}15 \mathrm{C} & -0.000059 & -0.000055 & 0.000000 & 0.000000 & 0.000000 & 0.000000\end{array}$

$\begin{array}{llllllll}16 & \mathrm{C} & -0.000289 & -0.000143 & 0.000000 & 0.000000 & 0.000000 & 0.000000\end{array}$

$\begin{array}{llllllll}17 & \mathrm{H} & 0.000128 & -0.000152 & -0.000001 & 0.000000 & 0.000000 & 0.000000\end{array}$

$\begin{array}{llllllll}18 \mathrm{H} & 0.000154 & 0.000007 & 0.000000 & 0.000000 & 0.000000 & 0.000000\end{array}$

$\begin{array}{llllllll}19 & \mathrm{H} & 0.000001 & 0.000000 & 0.000000 & 0.000000 & 0.000000 & 0.000000\end{array}$

$\begin{array}{llllllll}20 \mathrm{H} & 0.000005 & -0.000002 & 0.000000 & 0.000000 & 0.000000 & 0.000000\end{array}$

$\begin{array}{llllllll}21 \mathrm{H} & 0.000000 & 0.000000 & 0.000000 & 0.000000 & 0.000000 & 0.000000\end{array}$

$\begin{array}{llllllll}22 & \mathrm{C} & 0.265612 & -0.023055 & 0.000017 & -0.000210 & 0.000010 & 0.000021\end{array}$

$\begin{array}{llllllll}23 & \mathrm{H} & -0.037653 & -0.003057 & 0.001631 & -0.000062 & -0.000004 & -0.000001\end{array}$

$\begin{array}{llllllll}24 \mathrm{H} & -0.037830 & -0.002879 & -0.000034 & 0.000003 & 0.000000 & 0.000000\end{array}$

$\begin{array}{llllllll}25 & \mathrm{H} & -0.030292 & 0.002353 & 0.000119 & 0.000001 & 0.000000 & -0.000001\end{array}$

$\begin{array}{llllllll}26 & \mathrm{C} & 0.336837 & -0.033965 & 0.328772 & -0.028273 & 0.003227 & -0.004761\end{array}$

$\begin{array}{llllllll}27 & \mathrm{C} & 0.271450 & -0.043050 & 0.001513 & -0.000027 & -0.000001 & -0.000004\end{array}$

$\begin{array}{llllllll}28 & \mathrm{O} & -0.058563 & 0.001652 & 0.000028 & 0.000002 & 0.000000 & 0.000000\end{array}$

$\begin{array}{lllllllll}29 & \mathrm{O} & -0.055086 & 0.001392 & -0.083977 & -0.001799 & 0.000174 & 0.002687\end{array}$

$\begin{array}{lllllll}30 \mathrm{Zn} & -0.006556 & -0.000291 & -0.001453 & 0.000157 & 0.000009 & 0.000647\end{array}$

$\begin{array}{llllllll}31 & \mathrm{Cl} & 0.000003 & -0.000055 & -0.000015 & -0.000006 & 0.000000 & -0.000006\end{array}$

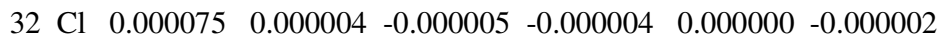

$$
\begin{array}{llllll}
7 & 8 & 9 & 10 & 11 & 12
\end{array}
$$

$\begin{array}{llllllll}1 & \mathrm{C} & -0.000358 & 0.000005 & 0.000054 & -0.000050 & -0.080308 & 0.000006\end{array}$

$\begin{array}{llllllll}2 \mathrm{H} & 0.000005 & 0.000000 & -0.000003 & 0.000007 & 0.006945 & 0.000007\end{array}$

$\begin{array}{llllllll}3 & \mathrm{O} & -0.038608 & 0.002481 & -0.000816 & 0.000169 & -0.000084 & 0.000000\end{array}$

$\begin{array}{llllllll}4 \mathrm{C} & 0.329757 & -0.033964 & -0.030338 & -0.036549 & 0.000003 & 0.000000\end{array}$

$\begin{array}{llllllll}5 \mathrm{H} & -0.040954 & -0.001327 & 0.002857 & -0.002802 & 0.000000 & 0.000000\end{array}$

$\begin{array}{llllllll}6 & \mathrm{H} & -0.037833 & -0.000130 & -0.003198 & 0.003360 & 0.000000 & 0.000000\end{array}$

$\begin{array}{llllllll}7 \mathrm{C} & 5.160815 & 0.384071 & 0.390020 & 0.387036 & 0.000000 & 0.000000\end{array}$

$\begin{array}{llllllll}8 \mathrm{H} & 0.384071 & 0.507292 & -0.021129 & -0.023381 & 0.000000 & 0.000000\end{array}$

$\begin{array}{llllllll}9 \mathrm{H} & 0.390020 & -0.021129 & 0.460152 & -0.025830 & 0.000000 & 0.000000\end{array}$

$\begin{array}{llllllll}10 \mathrm{H} & 0.387036 & -0.023381 & -0.025830 & 0.519635 & 0.000000 & 0.000000\end{array}$

$\begin{array}{llllllll}11 & \mathrm{C} & 0.000000 & 0.000000 & 0.000000 & 0.000000 & 4.968218 & -0.029454\end{array}$

$\begin{array}{llllllll}12 & \mathrm{C} & 0.000000 & 0.000000 & 0.000000 & 0.000000 & -0.029454 & 4.873190\end{array}$

$\begin{array}{llllllll}13 & \mathrm{C} & 0.000000 & 0.000000 & 0.000000 & 0.000000 & 0.525273 & -0.043193\end{array}$

$\begin{array}{llllllll}14 \mathrm{C} & 0.000000 & 0.000000 & 0.000000 & 0.000000 & 0.535993 & -0.041543\end{array}$

$\begin{array}{llllllll}15 & \mathrm{C} & 0.000000 & 0.000000 & 0.000000 & 0.000000 & -0.041063 & 0.540926\end{array}$

$\begin{array}{llllllll}16 \mathrm{C} & 0.000000 & 0.000000 & 0.000000 & 0.000000 & -0.036854 & 0.544335\end{array}$

$\begin{array}{llllllll}17 \mathrm{H} & 0.000000 & 0.000000 & 0.000000 & 0.000000 & -0.036420 & 0.002170\end{array}$

$\begin{array}{llllllll}18 \mathrm{H} & 0.000000 & 0.000000 & 0.000000 & 0.000000 & -0.027452 & 0.002099\end{array}$

$\begin{array}{llllllll}19 \mathrm{H} & 0.000000 & 0.000000 & 0.000000 & 0.000000 & 0.001926 & -0.027712\end{array}$

$\begin{array}{llllllll}20 \mathrm{H} & 0.000000 & 0.000000 & 0.000000 & 0.000000 & 0.001799 & -0.026502\end{array}$

$\begin{array}{llllllll}21 \mathrm{H} & 0.000000 & 0.000000 & 0.000000 & 0.000000 & 0.000190 & 0.379542\end{array}$

$\begin{array}{llllllll}22 & \mathrm{C} & -0.000002 & 0.000000 & 0.000000 & 0.000001 & -0.000326 & 0.000000\end{array}$

$\begin{array}{llllllll}23 & \mathrm{H} & 0.000003 & 0.000000 & 0.000000 & 0.000000 & -0.000108 & 0.000000\end{array}$

$\begin{array}{llllllll}24 \mathrm{H} & 0.000000 & 0.000000 & 0.000000 & 0.000000 & -0.000327 & 0.000000\end{array}$

$\begin{array}{llllllll}25 & \mathrm{H} & 0.000000 & 0.000000 & 0.000000 & 0.000000 & 0.000416 & 0.000000\end{array}$

$\begin{array}{llllllll}26 & \mathrm{C} & -0.002643 & 0.000122 & 0.001145 & 0.000018 & 0.002723 & 0.000000\end{array}$ 
$\begin{array}{llllllll}27 & \mathrm{C} & 0.000023 & 0.000000 & -0.000010 & -0.000001 & 0.382127 & -0.000165\end{array}$

$\begin{array}{llllllll}28 & \mathrm{O} & 0.000000 & 0.000000 & -0.000001 & 0.000000 & -0.076823 & -0.000005\end{array}$ $\begin{array}{lllllllll}29 & \mathrm{O} & -0.001673 & 0.000045 & 0.000339 & 0.000068 & 0.000239 & 0.000000\end{array}$

$\begin{array}{llllllll}30 & \mathrm{Zn} & 0.000371 & -0.000038 & 0.000675 & 0.000103 & -0.003873 & -0.000003\end{array}$ $\begin{array}{llllllll}31 & \mathrm{Cl} & 0.000001 & 0.000000 & -0.000002 & 0.000000 & -0.000112 & 0.000000\end{array}$ $\begin{array}{llllllll}32 & \mathrm{Cl} & -0.000056 & 0.000000 & 0.000185 & 0.000000 & 0.000004 & 0.000000\end{array}$
$\begin{array}{llllll}13 & 14 & 15 & 16 & 17 & 18\end{array}$

$\begin{array}{llllllll}1 & \mathrm{C} & -0.005406 & 0.004090 & -0.000059 & -0.000289 & 0.000128 & 0.000154\end{array}$

$\begin{array}{llllllll}2 \mathrm{H} & 0.000551 & 0.000076 & -0.000055 & -0.000143 & -0.000152 & 0.000007\end{array}$

$\begin{array}{llllllll}3 & \mathrm{O} & -0.000004 & 0.000000 & 0.000000 & 0.000000 & -0.000001 & 0.000000\end{array}$

$\begin{array}{llllllll}4 \mathrm{C} & 0.000000 & 0.000000 & 0.000000 & 0.000000 & 0.000000 & 0.000000\end{array}$

$\begin{array}{llllllll}5 \mathrm{H} & 0.000000 & 0.000000 & 0.000000 & 0.000000 & 0.000000 & 0.000000\end{array}$

$\begin{array}{llllllll}6 \mathrm{H} & 0.000000 & 0.000000 & 0.000000 & 0.000000 & 0.000000 & 0.000000\end{array}$

$\begin{array}{llllllll}7 \mathrm{C} & 0.000000 & 0.000000 & 0.000000 & 0.000000 & 0.000000 & 0.000000\end{array}$

$\begin{array}{lllllll}8 \mathrm{H} & 0.000000 & 0.000000 & 0.000000 & 0.000000 & 0.000000 & 0.000000\end{array}$

$\begin{array}{llllllll}9 \mathrm{H} & 0.000000 & 0.000000 & 0.000000 & 0.000000 & 0.000000 & 0.000000\end{array}$

$\begin{array}{llllllll}10 \mathrm{H} & 0.000000 & 0.000000 & 0.000000 & 0.000000 & 0.000000 & 0.000000\end{array}$

$\begin{array}{llllllll}11 & \mathrm{C} & 0.525273 & 0.535993 & -0.041063 & -0.036854 & -0.036420 & -0.027452\end{array}$

$\begin{array}{llllllll}12 & \mathrm{C} & -0.043193 & -0.041543 & 0.540926 & 0.544335 & 0.002170 & 0.002099\end{array}$

$\begin{array}{llllllll}13 & C & 4.934632 & -0.031993 & -0.037874 & 0.545186 & 0.376792 & 0.002091\end{array}$

$\begin{array}{lllllllll}14 & \mathrm{C} & -0.031993 & 4.886518 & 0.535096 & -0.036892 & 0.001944 & 0.374704\end{array}$

$\begin{array}{llllllll}15 & \mathrm{C} & -0.037874 & 0.535096 & 4.918135 & -0.039117 & 0.000115 & -0.023978\end{array}$

$\begin{array}{llllllll}16 & \mathrm{C} & 0.545186 & -0.036892 & -0.039117 & 4.896506 & -0.020928 & 0.000048\end{array}$

$\begin{array}{llllllll}17 & \mathrm{H} & 0.376792 & 0.001944 & 0.000115 & -0.020928 & 0.451068 & -0.000082\end{array}$

$\begin{array}{llllllll}18 & \mathrm{H} & 0.002091 & 0.374704 & -0.023978 & 0.000048 & -0.000082 & 0.398660\end{array}$

$\begin{array}{llllllll}19 \mathrm{H} & 0.000246 & -0.026032 & 0.380028 & 0.002254 & 0.000009 & -0.001068\end{array}$

$\begin{array}{llllllll}20 & \mathrm{H} & -0.028504 & 0.000253 & 0.002227 & 0.380765 & -0.001182 & 0.000008\end{array}$

$\begin{array}{llllllll}21 & \mathrm{H} & 0.002619 & 0.002306 & -0.027623 & -0.028279 & -0.000095 & -0.000080\end{array}$

$\begin{array}{llllllll}22 & \mathrm{C} & -0.001993 & -0.000068 & 0.000000 & 0.000060 & -0.000415 & -0.000005\end{array}$

$\begin{array}{llllllll}23 & \mathrm{H} & 0.000061 & 0.000001 & 0.000000 & -0.000001 & 0.000064 & 0.000000\end{array}$

$\begin{array}{llllllll}24 \mathrm{H} & -0.000263 & -0.000024 & 0.000000 & 0.000074 & 0.001783 & 0.000000\end{array}$

$\begin{array}{lllllllll}25 & \mathrm{H} & -0.000005 & 0.000004 & 0.000000 & -0.000001 & 0.000020 & 0.000000\end{array}$

$\begin{array}{llllllll}26 & \mathrm{C} & 0.000371 & -0.000126 & 0.000001 & 0.000003 & 0.000119 & -0.000002\end{array}$

$\begin{array}{llllllll}27 & \mathrm{C} & -0.038975 & -0.034636 & 0.003470 & 0.003277 & -0.002006 & -0.002695\end{array}$

$\begin{array}{llllllll}28 & \mathrm{O} & 0.000923 & 0.001392 & 0.000377 & -0.000049 & 0.000037 & 0.001114\end{array}$

$\begin{array}{llllllll}29 & \mathrm{O} & -0.000005 & 0.000005 & 0.000000 & 0.000000 & 0.000000 & 0.000000\end{array}$

$\begin{array}{llllllll}30 & \mathrm{Zn} & 0.000150 & 0.000121 & 0.000079 & -0.000017 & -0.000002 & 0.000459\end{array}$

$\begin{array}{llllllll}31 & \mathrm{Cl} & 0.000003 & -0.000001 & 0.000000 & 0.000000 & 0.000000 & -0.000002\end{array}$

$\begin{array}{llllllll}32 & \mathrm{Cl} & 0.000000 & -0.000055 & 0.000000 & 0.000000 & 0.000000 & 0.000096\end{array}$

$$
\begin{array}{llllll}
19 & 20 & 21 & 22 & 23 & 24
\end{array}
$$

$\begin{array}{llllllll}1 & \mathrm{C} & 0.000001 & 0.000005 & 0.000000 & 0.265612 & -0.037653 & -0.037830\end{array}$

$\begin{array}{llllllll}2 \mathrm{H} & 0.000000 & -0.000002 & 0.000000 & -0.023055 & -0.003057 & -0.002879\end{array}$

$\begin{array}{llllllll}3 & \mathrm{O} & 0.000000 & 0.000000 & 0.000000 & 0.000017 & 0.001631 & -0.000034\end{array}$

$\begin{array}{llllllll}4 \mathrm{C} & 0.000000 & 0.000000 & 0.000000 & -0.000210 & -0.000062 & 0.000003\end{array}$

$\begin{array}{llllllll}5 \mathrm{H} & 0.000000 & 0.000000 & 0.000000 & 0.000010 & -0.000004 & 0.000000\end{array}$

$\begin{array}{llllllll}6 \mathrm{H} & 0.000000 & 0.000000 & 0.000000 & 0.000021 & -0.000001 & 0.000000\end{array}$

$\begin{array}{llllllll}7 \mathrm{C} & 0.000000 & 0.000000 & 0.000000 & -0.000002 & 0.000003 & 0.000000\end{array}$

$\begin{array}{llllllll}8 \mathrm{H} & 0.000000 & 0.000000 & 0.000000 & 0.000000 & 0.000000 & 0.000000\end{array}$

$\begin{array}{llllllll}9 \mathrm{H} & 0.000000 & 0.000000 & 0.000000 & 0.000000 & 0.000000 & 0.000000\end{array}$

$\begin{array}{llllllll}10 \mathrm{H} & 0.000000 & 0.000000 & 0.000000 & 0.000001 & 0.000000 & 0.000000\end{array}$

$\begin{array}{llllllll}11 & \mathrm{C} & 0.001926 & 0.001799 & 0.000190 & -0.000326 & -0.000108 & -0.000327\end{array}$

$\begin{array}{llllllll}12 & \mathrm{C} & -0.027712 & -0.026502 & 0.379542 & 0.000000 & 0.000000 & 0.000000\end{array}$

$\begin{array}{llllllll}13 & C & 0.000246 & -0.028504 & 0.002619 & -0.001993 & 0.000061 & -0.000263\end{array}$

$\begin{array}{llllllll}14 & \mathrm{C} & -0.026032 & 0.000253 & 0.002306 & -0.000068 & 0.000001 & -0.000024\end{array}$

$\begin{array}{llllllll}15 & C & 0.380028 & 0.002227 & -0.027623 & 0.000000 & 0.000000 & 0.000000\end{array}$

$\begin{array}{llllllll}16 & \mathrm{C} & 0.002254 & 0.380765 & -0.028279 & 0.000060 & -0.000001 & 0.000074\end{array}$

$\begin{array}{llllllll}17 & \mathrm{H} & 0.000009 & -0.001182 & -0.000095 & -0.000415 & 0.000064 & 0.001783\end{array}$

$\begin{array}{llllllll}18 \mathrm{H} & -0.001068 & 0.000008 & -0.000080 & -0.000005 & 0.000000 & 0.000000\end{array}$

$\begin{array}{llllllll}19 & \mathrm{H} & 0.439407 & -0.000092 & -0.001307 & 0.000000 & 0.000000 & 0.000000\end{array}$

$\begin{array}{llllllll}20 \mathrm{H} & -0.000092 & 0.447032 & -0.001329 & -0.000001 & 0.000000 & -0.000003\end{array}$

$\begin{array}{llllllll}21 & \mathrm{H} & -0.001307 & -0.001329 & 0.444067 & 0.000000 & 0.000000 & 0.000000\end{array}$

$\begin{array}{llllllll}22 & \mathrm{C} & 0.000000 & -0.000001 & 0.000000 & 5.281275 & 0.388875 & 0.390652\end{array}$

$\begin{array}{llllllll}23 & \mathrm{H} & 0.000000 & 0.000000 & 0.000000 & 0.388875 & 0.476861 & -0.021903\end{array}$

$\begin{array}{llllllll}24 & \mathrm{H} & 0.000000 & -0.000003 & 0.000000 & 0.390652 & -0.021903 & 0.483156\end{array}$ 
$\begin{array}{llllllll}25 & \mathrm{H} & 0.000000 & 0.000000 & 0.000000 & 0.365285 & -0.017619 & -0.017753\end{array}$

$\begin{array}{llllllll}26 & \mathrm{C} & 0.000000 & 0.000000 & 0.000000 & -0.044123 & -0.004683 & 0.004731\end{array}$

$\begin{array}{llllllll}27 & \mathrm{C} & -0.000093 & -0.000089 & 0.000004 & -0.049153 & 0.005184 & -0.003530\end{array}$

$\begin{array}{llllllll}28 & \mathrm{O} & 0.000000 & 0.000000 & 0.000000 & 0.001720 & -0.000036 & -0.000027\end{array}$

$\begin{array}{llllllll}29 & \mathrm{O} & 0.000000 & 0.000000 & 0.000000 & 0.000797 & -0.000035 & -0.000024\end{array}$

$\begin{array}{llllllll}30 & \mathrm{Zn} & -0.000004 & 0.000001 & 0.000000 & -0.001676 & 0.000382 & 0.000273\end{array}$

$\begin{array}{llllllll}31 & \mathrm{Cl} & 0.000000 & 0.000000 & 0.000000 & -0.031946 & 0.000517 & 0.000526\end{array}$

$\begin{array}{llllllll}32 & \mathrm{Cl} & 0.000000 & 0.000000 & 0.000000 & -0.000029 & 0.000000 & 0.000000\end{array}$

$$
\begin{array}{llllll}
25 & 26 & 27 & 28 & 29 & 30
\end{array}
$$

$\begin{array}{llllllll}1 & \mathrm{C} & -0.030292 & 0.336837 & 0.271450 & -0.058563 & -0.055086 & -0.006556\end{array}$

$\begin{array}{llllllll}2 & \mathrm{H} & 0.002353 & -0.033965 & -0.043050 & 0.001652 & 0.001392 & -0.000291\end{array}$

$\begin{array}{lllllllll}3 & \mathrm{O} & 0.000119 & 0.328772 & 0.001513 & 0.000028 & -0.083977 & -0.001453\end{array}$

$\begin{array}{llllllll}4 \mathrm{C} & 0.000001 & -0.028273 & -0.000027 & 0.000002 & -0.001799 & 0.000157\end{array}$

$\begin{array}{llllllll}5 \mathrm{H} & 0.000000 & 0.003227 & -0.000001 & 0.000000 & 0.000174 & 0.000009\end{array}$

$\begin{array}{llllllll}6 & \mathrm{H} & -0.000001 & -0.004761 & -0.000004 & 0.000000 & 0.002687 & 0.000647\end{array}$

$\begin{array}{llllllll}7 \mathrm{C} & 0.000000 & -0.002643 & 0.000023 & 0.000000 & -0.001673 & 0.000371\end{array}$

$\begin{array}{llllllll}8 \mathrm{H} & 0.000000 & 0.000122 & 0.000000 & 0.000000 & 0.000045 & -0.000038\end{array}$

$\begin{array}{llllllll}9 \mathrm{H} & 0.000000 & 0.001145 & -0.000010 & -0.000001 & 0.000339 & 0.000675\end{array}$

$\begin{array}{llllllll}10 \mathrm{H} & 0.000000 & 0.000018 & -0.000001 & 0.000000 & 0.000068 & 0.000103\end{array}$

$\begin{array}{llllllll}11 & \mathrm{C} & 0.000416 & 0.002723 & 0.382127 & -0.076823 & 0.000239 & -0.003873\end{array}$

$\begin{array}{llllllll}12 & \mathrm{C} & 0.000000 & 0.000000 & -0.000165 & -0.000005 & 0.000000 & -0.000003\end{array}$

$\begin{array}{llllllll}13 & \mathrm{C} & -0.000005 & 0.000371 & -0.038975 & 0.000923 & -0.000005 & 0.000150\end{array}$

$\begin{array}{llllllll}14 \mathrm{C} & 0.000004 & -0.000126 & -0.034636 & 0.001392 & 0.000005 & 0.000121\end{array}$

$\begin{array}{llllllll}15 & \mathrm{C} & 0.000000 & 0.000001 & 0.003470 & 0.000377 & 0.000000 & 0.000079\end{array}$

$\begin{array}{llllllll}16 & \mathrm{C} & -0.000001 & 0.000003 & 0.003277 & -0.000049 & 0.000000 & -0.000017\end{array}$

$\begin{array}{llllllll}17 \mathrm{H} & 0.000020 & 0.000119 & -0.002006 & 0.000037 & 0.000000 & -0.000002\end{array}$

$\begin{array}{llllllll}18 \mathrm{H} & 0.000000 & -0.000002 & -0.002695 & 0.001114 & 0.000000 & 0.000459\end{array}$

$\begin{array}{llllllll}19 \mathrm{H} & 0.000000 & 0.000000 & -0.000093 & 0.000000 & 0.000000 & -0.000004\end{array}$

$\begin{array}{llllllll}20 \mathrm{H} & 0.000000 & 0.000000 & -0.000089 & 0.000000 & 0.000000 & 0.000001\end{array}$

$\begin{array}{llllllll}21 & \mathrm{H} & 0.000000 & 0.000000 & 0.000004 & 0.000000 & 0.000000 & 0.000000\end{array}$

$\begin{array}{llllllll}22 & \mathrm{C} & 0.365285 & -0.044123 & -0.049153 & 0.001720 & 0.000797 & -0.001676\end{array}$

$\begin{array}{llllllll}23 & \mathrm{H} & -0.017619 & -0.004683 & 0.005184 & -0.000036 & -0.000035 & 0.000382\end{array}$

$\begin{array}{llllllll}24 & \mathrm{H} & -0.017753 & 0.004731 & -0.003530 & -0.000027 & -0.000024 & 0.000273\end{array}$

$\begin{array}{llllllll}25 & \mathrm{H} & 0.394185 & -0.004493 & -0.004545 & 0.000515 & 0.000476 & 0.005107\end{array}$

$\begin{array}{llllllll}26 & \mathrm{C} & -0.004493 & 4.156326 & -0.034827 & 0.003966 & 0.426660 & -0.013456\end{array}$

$\begin{array}{lllllllll}27 & \mathrm{C} & -0.004545 & -0.034827 & 4.539949 & 0.415499 & 0.004981 & -0.014328\end{array}$

$\begin{array}{lllllllll}28 & \mathrm{O} & 0.000515 & 0.003966 & 0.415499 & 8.281619 & -0.006961 & 0.086227\end{array}$

$\begin{array}{llllllll}29 & \mathrm{O} & 0.000476 & 0.426660 & 0.004981 & -0.006961 & 8.319353 & 0.082774\end{array}$

$\begin{array}{llllllll}30 & \mathrm{Zn} & 0.005107 & -0.013456 & -0.014328 & 0.086227 & 0.082774 & 28.318100\end{array}$

$\begin{array}{llllllll}31 & \mathrm{Cl} & 0.023257 & -0.000074 & 0.000481 & -0.012010 & -0.012288 & 0.233372\end{array}$

$\begin{array}{llllllll}32 & \mathrm{Cl} & 0.000018 & -0.000854 & -0.000988 & -0.012882 & -0.012231 & 0.265415\end{array}$

$31 \quad 32$

$\begin{array}{llll}1 & \mathrm{C} & 0.000003 & 0.000075\end{array}$

$\begin{array}{llll}2 \mathrm{H} & -0.000055 & 0.000004\end{array}$

3 O $-0.000015-0.000005$

4 C $-0.000006-0.000004$

$\begin{array}{llll}5 \mathrm{H} & 0.000000 & 0.000000\end{array}$

$6 \mathrm{H}-0.000006-0.000002$

$\begin{array}{llll}7 & \mathrm{C} & 0.000001 & -0.000056\end{array}$

$8 \mathrm{H} \quad 0.000000 \quad 0.000000$

$9 \mathrm{H}-0.000002 \quad 0.000185$

$\begin{array}{lll}10 \mathrm{H} & 0.000000 & 0.000000\end{array}$

$\begin{array}{llll}11 & \mathrm{C} & -0.000112 & 0.000004\end{array}$

$\begin{array}{llll}12 \mathrm{C} & 0.000000 & 0.000000\end{array}$

$\begin{array}{llll}13 \mathrm{C} & 0.000003 & 0.000000\end{array}$

$14 \mathrm{C}-0.000001-0.000055$

$\begin{array}{llll}15 \mathrm{C} & 0.000000 & 0.000000\end{array}$

$\begin{array}{lll}16 \mathrm{C} & 0.000000 & 0.000000\end{array}$

$\begin{array}{llll}17 \mathrm{H} & 0.000000 & 0.000000\end{array}$

$\begin{array}{llll}18 \mathrm{H} & -0.000002 & 0.000096\end{array}$

$\begin{array}{llll}19 \mathrm{H} & 0.000000 & 0.000000\end{array}$

$\begin{array}{llll}20 \mathrm{H} & 0.000000 & 0.000000\end{array}$

$\begin{array}{llll}21 & \mathrm{H} & 0.000000 & 0.000000\end{array}$

22 C $-0.031946-0.000029$ 
$\begin{array}{lll}23 \mathrm{H} & 0.000517 & 0.000000\end{array}$

$\begin{array}{llll}24 & \mathrm{H} & 0.000526 & 0.000000\end{array}$

$\begin{array}{llll}25 \mathrm{H} & 0.023257 & 0.000018\end{array}$

$\begin{array}{llll}26 & \mathrm{C} & -0.000074 & -0.000854\end{array}$

$\begin{array}{llll}27 & \mathrm{C} & 0.000481 & -0.000988\end{array}$

$\begin{array}{lllll}28 & \mathrm{O} & -0.012010 & -0.012882\end{array}$

$\begin{array}{llll}29 & \mathrm{O} & -0.012288 & -0.012231\end{array}$

$\begin{array}{llll}30 & \mathrm{Zn} & 0.233372 & 0.265415\end{array}$

$31 \mathrm{Cl} 17.430572-0.009414$

$32 \mathrm{Cl}-0.009414 \quad 17.390392$

Mulliken atomic charges:

$$
1
$$

1 C -0.413411

$2 \mathrm{H} \quad 0.272552$

$3 \mathrm{O}-0.620661$

4 C -0.023588

$\begin{array}{lll}5 \mathrm{H} & 0.198618\end{array}$

$6 \mathrm{H} \quad 0.217983$

7 C -0.529976

$8 \mathrm{H} \quad 0.185955$

$9 \mathrm{H} \quad 0.225900$

$10 \mathrm{H} \quad 0.178215$

11 C -0.092651

$\begin{array}{lll}12 & \mathrm{C} & -0.173699\end{array}$

13 C -0.200685

14 C -0.171133

$15 \mathrm{C}-0.210683$

16 C -0.209940

$\begin{array}{lll}17 & \mathrm{H} & 0.227036\end{array}$

$18 \mathrm{H} \quad 0.275926$

$19 \mathrm{H} \quad 0.232434$

$20 \mathrm{H} \quad 0.225611$

$21 \mathrm{H} \quad 0.229985$

22 C -0.541324

$23 \mathrm{H} \quad 0.211582$

$\begin{array}{lll}24 & \mathrm{H} & 0.203399\end{array}$

$\begin{array}{lll}25 \mathrm{H} & 0.282954\end{array}$

$26 \mathrm{C} \quad 0.907260$

$\begin{array}{lll}27 \mathrm{C} & 0.601167\end{array}$

28 O -0.627716

29 O -0.665912

$30 \mathrm{Zn} 1.047272$

$31 \mathrm{Cl}-0.622801$

$32 \mathrm{Cl}-0.619670$

Sum of Mulliken charges $=0.00000$

Atomic charges with hydrogens summed into heavy atoms:

$$
1
$$

1 C -0.140859

$2 \mathrm{H} \quad 0.000000$

300.620661

4 C 0.393013

$5 \mathrm{H} \quad 0.000000$

$6 \mathrm{H} \quad 0.000000$

7 C 0.060094

$8 \mathrm{H} \quad 0.000000$

$9 \mathrm{H} \quad 0.000000$

$10 \mathrm{H} \quad 0.000000$

$11 \mathrm{C}-0.092651$

12 C 0.056286

13 C 0.026351

$14 \mathrm{C} \quad 0.104793$

15 C 0.021752

$16 \mathrm{C} \quad 0.015671$

$17 \mathrm{H} \quad 0.000000$ 
$\begin{array}{llr}18 & \mathrm{H} & 0.000000 \\ 19 & \mathrm{H} & 0.000000 \\ 20 & \mathrm{H} & 0.000000 \\ 21 & \mathrm{H} & 0.000000 \\ 22 & \mathrm{C} & 0.156612 \\ 23 & \mathrm{H} & 0.000000 \\ 24 & \mathrm{H} & 0.000000 \\ 25 & \mathrm{H} & 0.000000 \\ 26 & \mathrm{C} & 0.907260 \\ 27 & \mathrm{C} & 0.601167 \\ 28 & \mathrm{O} & -0.627716 \\ 29 & \mathrm{O} & -0.665912 \\ 30 & \mathrm{Zn} & 1.047272 \\ 31 & \mathrm{Cl} & -0.622801 \\ 32 & \mathrm{Cl} & -0.619670\end{array}$

Sum of Mulliken charges $=0.00000$

Electronic spatial extent $(\mathrm{au}):<\mathrm{R} * * 2>=6757.1636$

Charge $=0.0000$ electrons

Dipole moment (field-independent basis, Debye):

$\mathrm{X}=10.4215 \mathrm{Y}=11.4937 \mathrm{Z}=1.5920$ Tot $=15.5964$

Quadrupole moment (field-independent basis, Debye-Ang):

$\mathrm{XX}=-115.1890 \quad \mathrm{YY}=-134.9493 \quad \mathrm{ZZ}=-136.9963$

$\mathrm{XY}=-23.4720 \quad \mathrm{XZ}=-1.6717 \quad \mathrm{YZ}=2.1534$

Traceless Quadrupole moment (field-independent basis, Debye-Ang):

$\mathrm{XX}=13.8559 \mathrm{YY}=-5.9044 \mathrm{ZZ}=-7.9514$

$\mathrm{XY}=-23.4720 \quad \mathrm{XZ}=-1.6717 \quad \mathrm{YZ}=2.1534$

Octapole moment (field-independent basis, Debye-Ang**2):

$\mathrm{XXX}=92.6957 \mathrm{YYY}=41.8164 \mathrm{ZZZ}=6.1513 \mathrm{XYY}=-30.9122$

$\mathrm{XXY}=53.9395 \mathrm{XXZ}=-13.8834 \mathrm{XZZ}=34.8471 \mathrm{YZZ}=31.9310$

$\mathrm{YYZ}=-6.0143 \mathrm{XYZ}=22.8826$

Hexadecapole moment (field-independent basis, Debye-Ang**3):

$X X X X=-4321.5074$ YYYY $=-2231.1446 Z Z Z Z=-1506.4256 X X X Y=-90.0281$

$X X X Z=-74.5971 \mathrm{YYYX}=-148.9587 \mathrm{YYYZ}=-23.4033 \mathrm{ZZZX}=-15.2949$

$Z Z Z Y=23.9216 X X Y Y=-1253.7937 X X Z Z=-1102.1057 \mathrm{YYZZ}=-678.8059$

$\mathrm{XXYZ}=51.5755 \mathrm{YYXZ}=16.4397 \mathrm{ZZXY}=-87.3855$

$\mathrm{N}-\mathrm{N}=2.120613397825 \mathrm{D}+03 \mathrm{E}-\mathrm{N}=-1.229793289063 \mathrm{D}+04 \mathrm{KE}=3.381960823296 \mathrm{D}+03$

1/1|UNPC-UNKISPIRHF|6-31G(d)|C12H14Cl2O3Zn1|PCUSER|19-Apr-2006|0||\# HF

/6-31G(D) GEOM=CONNECTIVITY\|Title Card Required\|I0,1|C,0,0.65540805,-

$0.56871264,1.23660345|\mathrm{H}, 0,0.80940805,-1.59771264,1.65360345| \mathrm{O}, 0,2.7804$

$0805,-1.10271264,0.25160345 \mid \mathrm{C}, 0,3.88940805,-0.95471264,-0.64839655 \mathrm{lH}, 0$

,4.66240805,-1.47771264,-0.05639655|H,0,4.18240805,0.10728736,-0.74939

655|C,0,3.64440805,-1.61371264,-1.98939655|H,0,4.57640805,-1.62271264,

$-2.57039655|\mathrm{H}, 0,2.89340805,-1.07871264,-2.58639655| \mathrm{H}, 0,3.30940805,-2.6$

$5371264,-1.88739655|\mathrm{C}, 0,-1.73759195,-1.51871264,1.12260345| \mathrm{C}, 0,-3.5905$

$9195,-3.45871264,1.88860345|\mathrm{C}, 0,-1.73059195,-2.02171264,2.42860345| \mathrm{C}, 0$ ,-2.68559195,-1.98771264,0.20560345|C,0,-3.60259195,-2.95971264,0.5906 $0345 \mid \mathrm{C}, 0,-2.65559195,-2.98571264,2.80660345 \mathrm{H}, 0,-1.01259195,-1.6517126$ 4,3.17060345|H,0,-2.71059195,-1.58971264,-0.81739655|H,0,-4.34059195,-$3.32571264,-0.13239655 \mathrm{H}, 0,-2.65259195,-3.37171264,3.83260345 \mathrm{IH}, 0,-4.3$ $1759195,-4.22071264,2.19060345|\mathrm{C}, 0,0.84840805,0.46528736,2.34260345| \mathrm{H}$, $0,1.84640805,0.37828736,2.79060345 \mid \mathrm{H}, 0,0.11440805,0.33328736,3.1476034$ $5|\mathrm{H}, 0,0.73740805,1.50028736,1.95960345| \mathrm{C}, 0,1.69940805,-0.31571264,0.15$ $960345|\mathrm{C}, 0,-0.76659195,-0.48671264,0.69160345| \mathrm{O}, 0,-1.16659195,0.381287$ $36,-0.10439655 \mid \mathrm{O}, 0,1.69440805,0.52028736,-0.76539655 \mathrm{IZn}, 0,0.10540805,1$ .59228736,-0.88939655|Cl,0,0.33540805,3.32028736,0.47560345|Cl,0,-0.42 $559195,1.65328736,-3.02439655||$ Version $=x 86-W i n 32-G 03 R e v B .05 \mid$ State $=1-A \mid$ $\mathrm{HF}=-3383.7768103 \mid \mathrm{RMSD}=3.506 \mathrm{e}-005$ IDipole $=0.7837273,-5.193753,3.1721306 \mathrm{I}$ $\mathrm{PG}=\mathrm{C} 01[\mathrm{X}(\mathrm{C} 12 \mathrm{H} 14 \mathrm{Cl} 2 \mathrm{O} 3 \mathrm{Zn} 1)] \| @$

IF YOU GIVE A BOY AND A PIG EVERYTHING THEY WANT, YOU'LL GET A BAD BOY AND A GOOD PIG.

Job cpu time: 0 days 0 hours 10 minutes 18.0 seconds.

File lengths (MBytes): RWF $=65 \mathrm{Int}=\quad 0 \mathrm{D} 2 \mathrm{E}=0 \mathrm{Chk}=11 \mathrm{Scr}=1$ 
Normal termination of Gaussian 03 at Wed Apr 19 09:24:59 2006. 
Entering Link 1 = C: $\backslash$ G03W W11.exe PID= 4072.

Copyright (c) 1988,1990,1992,1993,1995,1998,2003, Gaussian, Inc. All Rights Reserved.

This is the Gaussian(R) 03 program. It is based on the the Gaussian(R) 98 system (copyright 1998, Gaussian, Inc.), the Gaussian(R) 94 system (copyright 1995, Gaussian, Inc.), the Gaussian 92(TM) system (copyright 1992, Gaussian, Inc.), the Gaussian 90(TM) system (copyright 1990, Gaussian, Inc.), the Gaussian 88(TM) system (copyright 1988, Gaussian, Inc.), the Gaussian 86(TM) system (copyright 1986, Carnegie Mellon University), and the Gaussian 82(TM) system (copyright 1983, Carnegie Mellon University). Gaussian is a federally registered trademark of Gaussian, Inc.

This software contains proprietary and confidential information, including trade secrets, belonging to Gaussian, Inc.

This software is provided under written license and may be used, copied, transmitted, or stored only in accord with that written license.

The following legend is applicable only to US Government contracts under DFARS:

\section{RESTRICTED RIGHTS LEGEND}

Use, duplication or disclosure by the US Government is subject to restrictions as set forth in subparagraph (c)(1)(ii) of the Rights in Technical Data and Computer Software clause at DFARS 252.227-7013.

Gaussian, Inc.

Carnegie Office Park, Building 6, Pittsburgh, PA 15106 USA

The following legend is applicable only to US Government contracts under FAR:

\section{RESTRICTED RIGHTS LEGEND}

Use, reproduction and disclosure by the US Government is subject to restrictions as set forth in subparagraph (c) of the Commercial Computer Software - Restricted Rights clause at FAR 52.227-19.

Gaussian, Inc.

Carnegie Office Park, Building 6, Pittsburgh, PA 15106 USA

Warning -- This program may not be used in any manner that competes with the business of Gaussian, Inc. or will provide assistance to any competitor of Gaussian, Inc. The licensee of this program is prohibited from giving any competitor of Gaussian, Inc. access to this program. By using this program, the user acknowledges that Gaussian, Inc. is engaged in the business of creating and licensing software in the field of 
computational chemistry and represents and warrants to the licensee that it is not a competitor of Gaussian, Inc. and that it will not use this program in any manner prohibited above.

Cite this work as:

Gaussian 03, Revision B.05,

M. J. Frisch, G. W. Trucks, H. B. Schlegel, G. E. Scuseria,

M. A. Robb, J. R. Cheeseman, J. A. Montgomery, Jr., T. Vreven,

K. N. Kudin, J. C. Burant, J. M. Millam, S. S. Iyengar, J. Tomasi,

V. Barone, B. Mennucci, M. Cossi, G. Scalmani, N. Rega,

G. A. Petersson, H. Nakatsuji, M. Hada, M. Ehara, K. Toyota,

R. Fukuda, J. Hasegawa, M. Ishida, T. Nakajima, Y. Honda, O. Kitao,

H. Nakai, M. Klene, X. Li, J. E. Knox, H. P. Hratchian, J. B. Cross,

C. Adamo, J. Jaramillo, R. Gomperts, R. E. Stratmann, O. Yazyev,

A. J. Austin, R. Cammi, C. Pomelli, J. W. Ochterski, P. Y. Ayala,

K. Morokuma, G. A. Voth, P. Salvador, J. J. Dannenberg,

V. G. Zakrzewski, S. Dapprich, A. D. Daniels, M. C. Strain,

O. Farkas, D. K. Malick, A. D. Rabuck, K. Raghavachari,

J. B. Foresman, J. V. Ortiz, Q. Cui, A. G. Baboul, S. Clifford,

J. Cioslowski, B. B. Stefanov, G. Liu, A. Liashenko, P. Piskorz,

I. Komaromi, R. L. Martin, D. J. Fox, T. Keith, M. A. Al-Laham,

C. Y. Peng, A. Nanayakkara, M. Challacombe, P. M. W. Gill,

B. Johnson, W. Chen, M. W. Wong, C. Gonzalez, and J. A. Pople, Gaussian, Inc., Pittsburgh PA, 2003.

******************************************************

Gaussian 03: x86-Win32-G03RevB.05 8-Nov-2003

$$
\text { 21-Apr-2006 }
$$

*****************************************************

$\%$ chk=e-ph-me.chk

$\%$ mem $=64 \mathrm{MB}$

$\%$ nproc $=1$

Will use up to 1 processors via shared memory.

\# hf/6-31g(d) geom=connectivity

$1 / 38=1,57=2 / 1$

$2 / 17=6,18=5,40=1 / 2$;

$3 / 5=1,6=6,7=1,11=9,16=1,25=1,30=1 / 1,2,3$;

$4 / / 1$

$5 / 5=2,32=1,38=5 / 2$;

$6 / 7=2,8=2,9=2,10=2,28=1 / 1$;

$99 / 5=1,9=1 / 99$;

Title Card Required

Symbolic Z-matrix:

Charge $=0$ Multiplicity $=1$

$\begin{array}{lrrr}\mathrm{C} & 1.93795 & -0.30145 & -0.25116 \\ \mathrm{H} & 2.02695 & -0.52245 & -1.31216 \\ \mathrm{C} & 0.87995 & 0.38455 & 0.21484 \\ \mathrm{H} & 0.82295 & 0.58655 & 1.28484 \\ \mathrm{C} & -0.28305 & 0.94455 & -0.58316 \\ \mathrm{H} & -0.28005 & 2.02555 & -0.38416 \\ \mathrm{C} & -0.17805 & 0.79655 & -2.10716 \\ \mathrm{H} & -0.15505 & -0.25245 & -2.42216 \\ \mathrm{H} & 0.72895 & 1.28255 & -2.48516 \\ \mathrm{H} & -1.03505 & 1.26855 & -2.60216 \\ \mathrm{C} & -1.60605 & 0.37855 & -0.08316 \\ \mathrm{C} & -4.05405 & -0.64045 & 0.84784 \\ \mathrm{C} & -2.56305 & 1.22855 & 0.49284 \\ \mathrm{C} & -1.89705 & -0.99245 & -0.18216\end{array}$




\begin{tabular}{|c|c|c|}
\hline $\mathrm{C}$ & $-3.11405-1.49745$ & 0.27984 \\
\hline $\mathrm{C}$ & $-3.77905 \quad 0.72055$ & 0.95484 \\
\hline $\mathrm{H}$ & $-2.36905 \quad 2.29455$ & 0.58584 \\
\hline $\mathrm{H}$ & $-1.17405-1.67645$ & -0.61916 \\
\hline $\mathrm{H}$ & $\begin{array}{ll}-3.32605 & -2.55945\end{array}$ & 0.19784 \\
\hline $\mathrm{H}$ & $-4.51305 \quad 1.38955$ & 1.39884 \\
\hline $\mathrm{H}$ & $-5.00105-1.03445$ & 1.20784 \\
\hline $\mathrm{C}$ & $3.04595-0.82145$ & 0.61084 \\
\hline $\mathrm{H}$ & $3.13395-1.90645$ & 0.49884 \\
\hline $\mathrm{H}$ & $2.89395-0.59245$ & 1.66884 \\
\hline $\mathrm{Cl}$ & $4.58295-0.08845$ & 0.10684 \\
\hline
\end{tabular}

Input orientation:

\begin{tabular}{|c|c|c|c|c|}
\hline \multirow{2}{*}{$\begin{array}{l}\text { Center } \\
\text { Number }\end{array}$} & Atomic & Atomic & \multicolumn{2}{|c|}{ Coordinates (Angstrom } \\
\hline & Number & r Type & $\begin{array}{ll}X & Y\end{array}$ & $\mathrm{Z}$ \\
\hline 1 & 6 & 1.937948 & -0.301448 & -0.251156 \\
\hline 2 & 1 & 2.026948 & -0.522448 & -1.312156 \\
\hline 3 & 6 & 0.879948 & 0.384552 & 0.214844 \\
\hline 4 & 1 & 0.822948 & 0.586552 & 1.284844 \\
\hline 5 & 6 & -0.283052 & 0.944552 & -0.583156 \\
\hline 6 & 1 & -0.280052 & 2.025552 & -0.384156 \\
\hline 7 & 6 & -0.178052 & 0.796552 & -2.107156 \\
\hline 8 & 1 & -0.155052 & -0.252448 & -2.422156 \\
\hline 9 & 1 & 0.728948 & 1.282552 & -2.485156 \\
\hline 10 & 1 & -1.035052 & 1.268552 & -2.602156 \\
\hline 11 & 6 & -1.606052 & 0.378552 & -0.083156 \\
\hline 12 & 6 & -4.054052 & -0.640448 & 0.847844 \\
\hline 13 & 6 & -2.563052 & 1.228552 & 0.492844 \\
\hline 14 & 6 & -1.897052 & -0.992448 & -0.182156 \\
\hline 15 & 6 & -3.114052 & -1.497448 & 0.279844 \\
\hline 16 & 6 & -3.779052 & 0.720552 & 0.954844 \\
\hline 17 & 1 & -2.369052 & 2.294552 & 0.585844 \\
\hline 18 & 1 & -1.174052 & -1.676448 & -0.619156 \\
\hline 19 & 1 & -3.326052 & -2.559448 & 0.197844 \\
\hline 20 & 1 & -4.513052 & 1.389552 & 1.398844 \\
\hline 21 & 1 & -5.001052 & -1.034448 & 1.207844 \\
\hline 22 & 6 & 3.045948 & -0.821448 & 0.610844 \\
\hline 23 & 1 & 3.133948 & -1.906448 & 0.498844 \\
\hline 24 & 1 & 2.893948 & -0.592448 & 1.668844 \\
\hline 25 & 17 & 4.582948 & -0.088448 & 0.106844 \\
\hline
\end{tabular}

Distance matrix (angstroms):
$1 \mathrm{C} \quad 0.000000$
$\begin{array}{llll}2 & \mathrm{H} & 1.087420 & 0.000000\end{array}$
$\begin{array}{lllll}3 & \mathrm{C} & 1.344290 & 2.114234 & 0.000000\end{array}$
$\begin{array}{llllll}4 \mathrm{H} & 2.095487 & 3.069838 & 1.090391 & 0.000000\end{array}$
$\begin{array}{lllllll}5 & \mathrm{C} & 2.568186 & 2.831895 & 1.517555 & 2.200187 & 0.000000\end{array}$
$\begin{array}{lllllll}6 \mathrm{H} & 3.217474 & 3.560300 & 2.096970 & 2.464324 & 1.099168\end{array}$
$\begin{array}{lllllll}7 & \mathrm{C} & 3.021224 & 2.689575 & 2.584723 & 3.542847 & 1.534765\end{array}$
$\begin{array}{lllllll}8 \mathrm{H} & 3.016006 & 2.462950 & 2.903578 & 3.924571 & 2.197980\end{array}$
$\begin{array}{lllllll}9 \mathrm{H} & 2.993575 & 2.513714 & 2.849422 & 3.834860 & 2.180824\end{array}$
$\begin{array}{llllllll}10 & \mathrm{H} & 4.102539 & 3.774603 & 3.519115 & 4.361887 & 2.178725\end{array}$
$\begin{array}{lllllll}11 & \mathrm{C} & 3.612556 & 3.939661 & 2.503804 & 2.795484 & 1.523379\end{array}$
$\begin{array}{lllllll}12 & \mathrm{C} & 6.101376 & 6.454307 & 5.078944 & 5.047933 & 4.333639\end{array}$
$\begin{array}{llllllll}13 & \mathrm{C} & 4.811802 & 5.233749 & 3.555822 & 3.536159 & 2.537091\end{array}$
$\begin{array}{lllllll}14 & \mathrm{C} & 3.897367 & 4.110423 & 3.124975 & 3.470408 & 2.552992\end{array}$
$\begin{array}{lllllll}15 & \mathrm{C} & 5.218724 & 5.469458 & 4.415675 & 4.566514 & 3.837016\end{array}$
$\begin{array}{lllllll}16 & \mathrm{C} & 5.931527 & 6.355625 & 4.729353 & 4.615762 & 3.825916\end{array}$
$\begin{array}{lllllll}17 & \mathrm{H} & 5.098042 & 5.555422 & 3.787049 & 3.687103 & 2.745989\end{array}$
$\begin{array}{lllllll}18 \mathrm{H} & 3.422074 & 3.472516 & 3.026911 & 3.568528 & 2.768541\end{array}$
$\begin{array}{lllllll}19 \mathrm{H} & 5.745421 & 5.923181 & 5.133991 & 5.319125 & 4.706148\end{array}$ 
$\begin{array}{lllllll}20 & \mathrm{H} & 6.870035 & 7.333271 & 5.612159 & 5.397286 & 4.692467\end{array}$

$\begin{array}{llllllll}21 & \mathrm{H} & 7.128513 & 7.483671 & 6.130724 & 6.045870 & 5.420668\end{array}$

$\begin{array}{lllllll}22 & \mathrm{C} & 1.497033 & 2.196746 & 2.510539 & 2.716334 & 3.953054\end{array}$

$\begin{array}{lllllll}23 & \mathrm{H} & 2.137508 & 2.533895 & 3.226430 & 3.489064 & 4.579827\end{array}$

$\begin{array}{lllllll}24 \mathrm{H} & 2.164490 & 3.105310 & 2.669240 & 2.413822 & 4.186550\end{array}$

$\begin{array}{lllllll}25 & \mathrm{Cl} & 2.677603 & 2.955512 & 3.734649 & 3.997613 & 5.022066\end{array}$

$$
\begin{array}{lllll}
6 & 7 & 8 & 9 & 10
\end{array}
$$

$6 \mathrm{H} \quad 0.000000$

$\begin{array}{llll}7 & \mathrm{C} & 2.118861 & 0.000000\end{array}$

$\begin{array}{lllll}8 & \mathrm{H} & 3.059143 & 1.095516 & 0.000000\end{array}$

$\begin{array}{llllll}9 \mathrm{H} & 2.446289 & 1.096234 & 1.772470 & 0.000000\end{array}$

$\begin{array}{lllllll}10 & \mathrm{H} & 2.462234 & 1.096475 & 1.766420 & 1.767931 & 0.000000\end{array}$

$\begin{array}{lllllll}11 & \mathrm{C} & 2.135764 & 2.512068 & 2.823913 & 3.469733 & 2.731941\end{array}$

$\begin{array}{llllllll}12 & \mathrm{C} & 4.782097 & 5.081375 & 5.103493 & 6.138722 & 4.965999\end{array}$

$\begin{array}{lllllll}13 & \mathrm{C} & 2.572242 & 3.554553 & 4.060671 & 4.439444 & 3.451870\end{array}$

$\begin{array}{lllllll}14 & \mathrm{C} & 3.429842 & 3.140240 & 2.932535 & 4.168370 & 3.422216\end{array}$

$\begin{array}{lllllll}15 & \mathrm{C} & 4.569899 & 4.424963 & 4.196011 & 5.490198 & 4.503212\end{array}$

$\begin{array}{lllllll}16 \mathrm{C} & 3.967234 & 4.727454 & 5.048191 & 5.698378 & 4.525714\end{array}$

$\begin{array}{lllllll}17 & \mathrm{H} & 2.318875 & 3.781102 & 4.520738 & 4.478034 & 3.604938\end{array}$

$\begin{array}{lllllll}18 \mathrm{H} & 3.815660 & 3.053177 & 2.513354 & 3.982342 & 3.553116\end{array}$

$\begin{array}{llllllll}19 & \mathrm{H} & 5.535257 & 5.146423 & 4.716131 & 6.196973 & 5.267093\end{array}$

$\begin{array}{lllllll}20 \mathrm{H} & 4.637011 & 5.606774 & 6.023983 & 6.524988 & 5.302747\end{array}$

$\begin{array}{llllllll}21 & \mathrm{H} & 5.846871 & 6.132138 & 6.105091 & 7.199975 & 5.962304\end{array}$

$\begin{array}{llllllll}22 & \mathrm{C} & 4.489734 & 4.516594 & 4.446263 & 4.402331 & 5.598753\end{array}$

$\begin{array}{llllllll}23 & \mathrm{H} & 5.281639 & 5.006674 & 4.699519 & 4.985780 & 6.089120\end{array}$

$\begin{array}{llllllll}24 & \mathrm{H} & 4.598153 & 5.062083 & 5.113539 & 5.045648 & 6.094408\end{array}$

$\begin{array}{lllllll}25 & \mathrm{Cl} & 5.325302 & 5.324673 & 5.373210 & 4.842667 & 6.382950\end{array}$

$$
\begin{array}{lllll}
11 & 12 & 13 & 14 & 15
\end{array}
$$

$\begin{array}{lll}11 \mathrm{C} & 0.000000\end{array}$

$\begin{array}{llll}12 & \mathrm{C} & 2.810307 & 0.000000\end{array}$

$\begin{array}{lllll}13 & \mathrm{C} & 1.403611 & 2.417078 & 0.000000\end{array}$

$\begin{array}{llllll}14 \mathrm{C} & 1.405035 & 2.416082 & 2.414958 & 0.000000\end{array}$

$\begin{array}{lllllll}15 & \mathrm{C} & 2.434175 & 1.393080 & 2.789273 & 1.396266 & 0.000000\end{array}$

$\begin{array}{lllllll}16 & \mathrm{C} & 2.432352 & 1.392622 & 1.396483 & 2.787304 & 2.411923\end{array}$

$\begin{array}{llllllll}17 & \mathrm{H} & 2.168130 & 3.394421 & 1.087493 & 3.408369 & 3.876587\end{array}$

$\begin{array}{lllllll}18 & \mathrm{H} & 2.167244 & 3.394081 & 3.406595 & 1.086993 & 2.145657\end{array}$

$\begin{array}{lllllll}19 & \mathrm{H} & 3.416022 & 2.152915 & 3.875324 & 2.154514 & 1.086053\end{array}$

$\begin{array}{lllllll}20 \mathrm{H} & 3.416006 & 2.152947 & 2.156214 & 3.875170 & 3.397666\end{array}$

$\begin{array}{lllllll}21 & \mathrm{H} & 3.897342 & 1.087035 & 3.402387 & 3.401276 & 2.153212\end{array}$

$\begin{array}{llllllll}22 & \mathrm{C} & 4.854147 & 7.106260 & 5.973048 & 5.009126 & 6.205815\end{array}$

$\begin{array}{llllllll}23 & \mathrm{H} & 5.294105 & 7.306976 & 6.502620 & 5.158500 & 6.265201\end{array}$

$\begin{array}{llllllll}24 & \mathrm{H} & 4.925682 & 6.996503 & 5.871786 & 5.151687 & 6.232528\end{array}$

$\begin{array}{lllllll}25 & \mathrm{Cl} & 6.209502 & 8.686285 & 7.276593 & 6.549133 & 7.826814\end{array}$

$16 \mathrm{C} \quad 0.000000$

$\begin{array}{lllll}16 & 17 & 18 & 19 & 20\end{array}$

$\begin{array}{llll}17 & \mathrm{H} & 2.145166 & 0.000000\end{array}$

$\begin{array}{lllll}18 \mathrm{H} & 3.874159 & 4.318436 & 0.000000\end{array}$

$\begin{array}{llllll}19 & \mathrm{H} & 3.396566 & 4.962631 & 2.465417 & 0.000000\end{array}$

$\begin{array}{lllllll}20 \mathrm{H} & 1.087866 & 2.465102 & 4.962016 & 4.294877 & 0.000000\end{array}$

$\begin{array}{llllllll}21 & \mathrm{H} & 2.153443 & 4.289120 & 4.289058 & 2.480192 & 2.480000\end{array}$

$\begin{array}{lllllll}22 & \mathrm{C} & 7.005478 & 6.247584 & 4.477982 & 6.617673 & 7.915046\end{array}$

$\begin{array}{llllllll}23 & \mathrm{H} & 7.409361 & 6.923798 & 4.456645 & 6.499893 & 8.375573\end{array}$

$\begin{array}{llllllll}24 & \mathrm{H} & 6.838325 & 6.099740 & 4.791516 & 6.687401 & 7.672345\end{array}$

$\begin{array}{lllllll}25 & \mathrm{Cl} & 8.443733 & 7.364675 & 6.015968 & 8.286519 & 9.305427\end{array}$ $\begin{array}{lllll}21 & 22 & 23 & 24 & 25\end{array}$

$\begin{array}{lll}21 \mathrm{H} & 0.000000\end{array}$

$\begin{array}{llll}22 & \mathrm{C} & 8.071926 & 0.000000\end{array}$

$\begin{array}{lllll}23 & \mathrm{H} & 8.212265 & 1.094309 & 0.000000\end{array}$

$\begin{array}{llllll}24 & \mathrm{H} & 7.920790 & 1.093119 & 1.775696 & 0.000000\end{array}$

$\begin{array}{lllllll}25 & \mathrm{Cl} & 9.693306 & 1.775859 & 2.357624 & 2.355118 & 0.000000\end{array}$

Stoichiometry $\mathrm{C} 11 \mathrm{H} 13 \mathrm{Cl}$

Framework group $\mathrm{C} 1[\mathrm{X}(\mathrm{C} 11 \mathrm{H} 13 \mathrm{Cl})]$

Deg. of freedom 69

Full point group $\quad$ C1 NOp 1 


\begin{tabular}{|c|c|c|c|c|c|}
\hline \multirow{2}{*}{$\begin{array}{l}\text { Center } \\
\text { Number }\end{array}$} & & \multicolumn{2}{|c|}{ Atomic } & \multicolumn{2}{|c|}{ Coordinates (Angstroms) } \\
\hline & Number & & Type & $\begin{array}{ll}\mathrm{X} & \mathrm{Y}\end{array}$ & $\mathrm{Z}$ \\
\hline 1 & 6 & 0 & -1.953226 & -0.108868 & 0.287490 \\
\hline 2 & 1 & 0 & -2.098649 & 0.385272 & 1.245175 \\
\hline 3 & 6 & 0 & -0.861006 & 0.148945 & -0.452581 \\
\hline 4 & 1 & 0 & -0.747443 & -0.366160 & -1.406899 \\
\hline 5 & 6 & 0 & 0.271231 & 1.105685 & -0.127525 \\
\hline 6 & 1 & 0 & 0.298006 & 1.820614 & -0.961989 \\
\hline 7 & 6 & 0 & 0.088277 & 1.947531 & 1.142644 \\
\hline 8 & 1 & 0 & 0.030390 & 1.330003 & 2.045674 \\
\hline 9 & 1 & 0 & -0.827194 & 2.547782 & 1.084834 \\
\hline 10 & 1 & 0 & 0.928303 & 2.640140 & 1.272670 \\
\hline 11 & 6 & 0 & 1.606680 & 0.373508 & -0.093230 \\
\hline 12 & 6 & 0 & 4.078525 & -0.962948 & -0.052093 \\
\hline 13 & 6 & 0 & 2.606504 & 0.687892 & -1.026847 \\
\hline 14 & 6 & 0 & 1.867102 & -0.624873 & 0.860467 \\
\hline 15 & 6 & 0 & 3.095944 & -1.287520 & 0.880571 \\
\hline 16 & 6 & 0 & 3.834292 & 0.022897 & -1.004906 \\
\hline 17 & 1 & 0 & 2.437027 & 1.454630 & -1.779197 \\
\hline 18 & 1 & 0 & 1.110887 & -0.893744 & 1.593544 \\
\hline 19 & 1 & 0 & 3.284014 & -2.057766 & 1.622772 \\
\hline 20 & 1 & 0 & 4.601561 & 0.275892 & -1.733428 \\
\hline 21 & 1 & 0 & 5.034746 & -1.479702 & -0.036183 \\
\hline 22 & 6 & 0 & -3.026636 & -1.073853 & -0.109626 \\
\hline 23 & 1 & 0 & -3.140056 & -1.848145 & 0.655305 \\
\hline 24 & 1 & 0 & -2.818301 & -1.558829 & -1.066864 \\
\hline 25 & 17 & 0 & -4.572906 & $5-0.212963$ & $3-0.256564$ \\
\hline
\end{tabular}

Rotational constants (GHZ): $\quad 2.0821031 \quad 0.2937774 \quad 0.2832245$

Standard basis: 6-31G(d) $(6 \mathrm{D}, 7 \mathrm{~F})$

There are 210 symmetry adapted basis functions of A symmetry.

Integral buffers will be 262144 words long.

Raffenetti 1 integral format.

Two-electron integral symmetry is turned on.

210 basis functions, 412 primitive gaussians, 210 cartesian basis functions

48 alpha electrons 48 beta electrons nuclear repulsion energy 705.2067331835 Hartrees.

NAtoms $=25$ NActive $=25$ NUniq $=25 \mathrm{SFac}=1.00 \mathrm{D}+00 \mathrm{NAtFMM}=60 \mathrm{Big}=\mathrm{F}$

One-electron integrals computed using PRISM.

NBasis $=210$ RedAO $=\mathrm{T} \quad \mathrm{NBF}=210$

NBsUse $=2101.00 \mathrm{D}-06 \mathrm{NBFU}=210$

Harris functional with IExCor= 205 diagonalized for initial guess.

ExpMin= 1.43D-01 ExpMax= 2.52D+04 ExpMxC=3.78D+03 IAcc=1 IRadAn $=\quad 1$ AccDes $=1.00 \mathrm{D}-06$
HarFok: IExCor= 205 AccDes= 1.00D-06 IRadAn $=\quad 1 \mathrm{IDoV}=1$

ScaDFX $=1.0000001 .0000001 .0000001 .000000$

Initial guess orbital symmetries:

Occupied (A) (A) (A) (A) (A) (A) (A) (A) (A) (A) (A) (A)

(A) (A) (A) (A) (A) (A) (A) (A) (A) (A) (A) (A)

(A) $(\mathrm{A})(\mathrm{A})(\mathrm{A})(\mathrm{A})(\mathrm{A})(\mathrm{A})(\mathrm{A})(\mathrm{A})(\mathrm{A})(\mathrm{A})(\mathrm{A})$

$(\mathrm{A})(\mathrm{A})(\mathrm{A})(\mathrm{A})(\mathrm{A})(\mathrm{A})(\mathrm{A})(\mathrm{A})(\mathrm{A})(\mathrm{A})(\mathrm{A})(\mathrm{A})$

Virtual (A) (A) (A) (A) (A) (A) (A) (A) (A) (A) (A) (A)

(A) (A) (A) (A) (A) (A) (A) (A) (A) (A) (A) (A)

(A) (A) (A) (A) (A) (A) (A) (A) (A) (A) (A) (A)

(A) (A) (A) (A) (A) (A) (A) (A) (A) (A) (A) (A)

(A) $(\mathrm{A})(\mathrm{A})(\mathrm{A})(\mathrm{A})(\mathrm{A})(\mathrm{A})(\mathrm{A})(\mathrm{A})(\mathrm{A})(\mathrm{A})(\mathrm{A})$

$(\mathrm{A})(\mathrm{A})(\mathrm{A})(\mathrm{A})(\mathrm{A})(\mathrm{A})(\mathrm{A})(\mathrm{A})(\mathrm{A})(\mathrm{A})(\mathrm{A})(\mathrm{A})$

(A) (A) (A) (A) (A) (A) (A) (A) (A) (A) (A) (A)

(A) $(\mathrm{A})(\mathrm{A})(\mathrm{A})(\mathrm{A})(\mathrm{A})(\mathrm{A})(\mathrm{A})(\mathrm{A})(\mathrm{A})(\mathrm{A})(\mathrm{A})$

(A) $(\mathrm{A})(\mathrm{A})(\mathrm{A})(\mathrm{A})(\mathrm{A})(\mathrm{A})(\mathrm{A})(\mathrm{A})(\mathrm{A})(\mathrm{A})(\mathrm{A})$ 
(A) $(\mathrm{A})(\mathrm{A})(\mathrm{A})(\mathrm{A})(\mathrm{A})(\mathrm{A})(\mathrm{A})(\mathrm{A})(\mathrm{A})(\mathrm{A})(\mathrm{A})$

(A) (A) (A) (A) (A) (A) (A) (A) (A) (A) (A) (A)

(A) $(A)(A)(A)(A)(A)(A)(A)(A)(A)(A)(A)$

(A) (A) (A) (A) (A) (A) (A) (A) (A) (A) (A) (A)

(A) (A) (A) (A) (A) (A)

The electronic state of the initial guess is 1-A.

Warning! Cutoffs for single-point calculations used.

Requested convergence on RMS density matrix=1.00D-04 within 128 cycles.

Requested convergence on MAX density matrix=1.00D-02.

Requested convergence on energy $=5.00 \mathrm{D}-05$.

No special actions if energy rises.

SCF Done: $\mathrm{E}(\mathrm{RHF})=-883.585416480 \quad$ A.U. after 6 cycles

Convg $=0.1066 \mathrm{D}-04 \quad-\mathrm{V} / \mathrm{T}=2.0010$

$\mathrm{S} * * 2=0.0000$

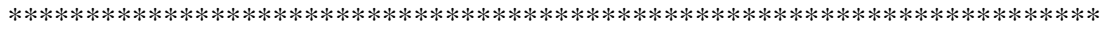

Population analysis using the SCF density.

$* * * * * * * * * * * * * * * * * * * * * * * * * * * * * * * * * * * * * * * * * * * * * * * * * * * * * * * * * * * * * * * * * * * * * * *$

Orbital symmetries:

Occupied (A) (A) (A) (A) (A) (A) (A) (A) (A) (A) (A) (A)

(A) $(\mathrm{A})(\mathrm{A})(\mathrm{A})(\mathrm{A})(\mathrm{A})(\mathrm{A})(\mathrm{A})(\mathrm{A})(\mathrm{A})(\mathrm{A})(\mathrm{A})$

(A) $(A)(A)(A)(A)(A)(A)(A)(A)(A)(A)(A)$

(A) $(A)(A)(A)(A)(A)(A)(A)(A)(A)(A)(A)$

Virtual (A) (A) (A) (A) (A) (A) (A) (A) (A) (A) (A) (A)

(A) $(\mathrm{A})(\mathrm{A})(\mathrm{A})(\mathrm{A})(\mathrm{A})(\mathrm{A})(\mathrm{A})(\mathrm{A})(\mathrm{A})(\mathrm{A})(\mathrm{A})$

(A) $(\mathrm{A})(\mathrm{A})(\mathrm{A})(\mathrm{A})(\mathrm{A})(\mathrm{A})(\mathrm{A})(\mathrm{A})(\mathrm{A})(\mathrm{A})(\mathrm{A})$

(A) $(\mathrm{A})(\mathrm{A})(\mathrm{A})(\mathrm{A})(\mathrm{A})(\mathrm{A})(\mathrm{A})(\mathrm{A})(\mathrm{A})(\mathrm{A})(\mathrm{A})$

(A) $(A)(A)(A)(A)(A)(A)(A)(A)(A)(A)(A)$

(A) $(\mathrm{A})(\mathrm{A})(\mathrm{A})(\mathrm{A})(\mathrm{A})(\mathrm{A})(\mathrm{A})(\mathrm{A})(\mathrm{A})(\mathrm{A})(\mathrm{A})$

(A) $(\mathrm{A})(\mathrm{A})(\mathrm{A})(\mathrm{A})(\mathrm{A})(\mathrm{A})(\mathrm{A})(\mathrm{A})(\mathrm{A})(\mathrm{A})(\mathrm{A})$

(A) $(A)(A)(A)(A)(A)(A)(A)(A)(A)(A)(A)$

(A) $(\mathrm{A})(\mathrm{A})(\mathrm{A})(\mathrm{A})(\mathrm{A})(\mathrm{A})(\mathrm{A})(\mathrm{A})(\mathrm{A})(\mathrm{A})(\mathrm{A})$

(A) $(\mathrm{A})(\mathrm{A})(\mathrm{A})(\mathrm{A})(\mathrm{A})(\mathrm{A})(\mathrm{A})(\mathrm{A})(\mathrm{A})(\mathrm{A})(\mathrm{A})$

(A) $(A)(A)(A)(A)(A)(A)(A)(A)(A)(A)(A)$

(A) $(\mathrm{A})(\mathrm{A})(\mathrm{A})(\mathrm{A})(\mathrm{A})(\mathrm{A})(\mathrm{A})(\mathrm{A})(\mathrm{A})(\mathrm{A})(\mathrm{A})$

$(\mathrm{A})(\mathrm{A})(\mathrm{A})(\mathrm{A})(\mathrm{A})(\mathrm{A})(\mathrm{A})(\mathrm{A})(\mathrm{A})(\mathrm{A})(\mathrm{A})(\mathrm{A})$

(A) $(\mathrm{A})(\mathrm{A})(\mathrm{A})(\mathrm{A})(\mathrm{A})$

The electronic state is $1-\mathrm{A}$.

Alpha occ. eigenvalues -- -104.80642 -11.29575 -11.24891 -11.24674 -11.24477

Alpha occ. eigenvalues -- -11.24211 -11.23726 -11.23677 -11.23532 -11.23504

Alpha occ. eigenvalues -- $-11.23344-11.22298-10.54077$-8.01012 -8.00681

Alpha occ. eigenvalues -- $-8.00675 \quad-1.15673-1.14470-1.09386-1.02839$

$\begin{array}{lllllll}\text { Alpha occ. eigenvalues -- } & -1.01121 & -0.98187 & -0.91418 & -0.86146 & -0.82409\end{array}$

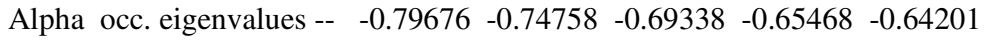

Alpha occ. eigenvalues -- $-0.63159-0.61427 \quad-0.59610 \quad-0.59006 \quad-0.58085$

Alpha occ. eigenvalues -- $-0.55143 \quad-0.54719-0.53169-0.50885 \quad-0.50329$

$\begin{array}{lllllll}\text { Alpha occ. eigenvalues -- } & -0.48531 & -0.48185 & -0.47108 & -0.43387 & -0.42812\end{array}$

Alpha occ. eigenvalues -- $-0.36218-0.33330-0.32253$

$\begin{array}{lllllll}\text { Alpha virt. eigenvalues -- } & 0.13446 & 0.13961 & 0.16928 & 0.21756 & 0.23309\end{array}$

$\begin{array}{lllllll}\text { Alpha virt. eigenvalues -- } & 0.24778 & 0.25007 & 0.26467 & 0.28450 & 0.29797\end{array}$

$\begin{array}{llllll}\text { Alpha virt. eigenvalues -- } & 0.30021 & 0.31310 & 0.31936 & 0.32689 & 0.34377\end{array}$

$\begin{array}{lllllll}\text { Alpha virt. eigenvalues -- } & 0.34912 & 0.35725 & 0.36388 & 0.39031 & 0.42043\end{array}$

$\begin{array}{lllllll}\text { Alpha virt. eigenvalues -- } & 0.42643 & 0.47075 & 0.48697 & 0.50133 & 0.50340\end{array}$

$\begin{array}{lllllll}\text { Alpha virt. eigenvalues -- } & 0.53347 & 0.57745 & 0.59012 & 0.64631 & 0.65024\end{array}$

$\begin{array}{llllllll}\text { Alpha virt. eigenvalues -- } & 0.65707 & 0.67850 & 0.68603 & 0.72717 & 0.74088\end{array}$

$\begin{array}{lllllll}\text { Alpha virt. eigenvalues -- } & 0.74217 & 0.75443 & 0.77199 & 0.77966 & 0.78926\end{array}$

$\begin{array}{lllllll}\text { Alpha virt. eigenvalues -- } & 0.80151 & 0.81802 & 0.81924 & 0.83398 & 0.83870\end{array}$

$\begin{array}{llllllll}\text { Alpha virt. eigenvalues -- } & 0.84053 & 0.84571 & 0.86761 & 0.88241 & 0.89897\end{array}$

$\begin{array}{llllll}\text { Alpha virt. eigenvalues -- } & 0.90561 & 0.92362 & 0.94166 & 0.97991 & 1.00281\end{array}$

$\begin{array}{lllllll}\text { Alpha virt. eigenvalues -- } & 1.01260 & 1.03214 & 1.06319 & 1.08881 & 1.09168\end{array}$

$\begin{array}{lllllll}\text { Alpha virt. eigenvalues -- } & 1.10246 & 1.10676 & 1.11561 & 1.11820 & 1.12701\end{array}$ 
$\begin{array}{lllllll}\text { Alpha virt. eigenvalues -- } & 1.14190 & 1.14413 & 1.15975 & 1.16838 & 1.17541\end{array}$ $\begin{array}{lllllll}\text { Alpha virt. eigenvalues -- } & 1.19323 & 1.19705 & 1.20683 & 1.21372 & 1.21924\end{array}$ $\begin{array}{lllllll}\text { Alpha virt. eigenvalues -- } & 1.22842 & 1.24855 & 1.26641 & 1.27761 & 1.32392\end{array}$ $\begin{array}{lllllll}\text { Alpha virt. eigenvalues -- } & 1.34224 & 1.35614 & 1.38854 & 1.41924 & 1.42724\end{array}$ $\begin{array}{lllllll}\text { Alpha virt. eigenvalues -- } & 1.44053 & 1.46326 & 1.50885 & 1.54572 & 1.55513\end{array}$ $\begin{array}{lllllll}\text { Alpha virt. eigenvalues -- } & 1.59612 & 1.65252 & 1.68610 & 1.70068 & 1.70778\end{array}$ $\begin{array}{lllllll}\text { Alpha virt. eigenvalues -- } & 1.71760 & 1.76387 & 1.77258 & 1.78334 & 1.79391\end{array}$ $\begin{array}{lllllll}\text { Alpha virt. eigenvalues -- } & 1.88101 & 1.92057 & 1.96605 & 2.02411 & 2.06161\end{array}$ $\begin{array}{lllllll}\text { Alpha virt. eigenvalues -- } & 2.09547 & 2.11283 & 2.12961 & 2.15390 & 2.16983\end{array}$ $\begin{array}{llllll}\text { Alpha virt. eigenvalues -- } & 2.20221 & 2.23253 & 2.24237 & 2.29605 & 2.30840\end{array}$ $\begin{array}{lllllll}\text { Alpha virt. eigenvalues -- } & 2.33141 & 2.35435 & 2.38637 & 2.39796 & 2.41483\end{array}$ $\begin{array}{lllllll}\text { Alpha virt. eigenvalues -- } & 2.44166 & 2.46043 & 2.46204 & 2.50131 & 2.52302\end{array}$ $\begin{array}{lllllll}\text { Alpha virt. eigenvalues -- } & 2.58048 & 2.60357 & 2.61758 & 2.62655 & 2.63661\end{array}$ $\begin{array}{lllllll}\text { Alpha virt. eigenvalues -- } & 2.64575 & 2.66404 & 2.69785 & 2.73177 & 2.74897\end{array}$ $\begin{array}{llllll}\text { Alpha virt. eigenvalues -- } & 2.87831 & 2.89927 & 2.91809 & 2.95506 & 2.99273\end{array}$ $\begin{array}{lllllll}\text { Alpha virt. eigenvalues -- } & 3.04560 & 3.07608 & 3.10142 & 3.11177 & 3.14719\end{array}$ $\begin{array}{lllllll}\text { Alpha virt. eigenvalues -- } & 3.20525 & 3.24096 & 3.34762 & 3.43805 & 3.78444\end{array}$ $\begin{array}{lllllll}\text { Alpha virt. eigenvalues -- } & 4.51472 & 4.52254 & 4.54113 & 4.58055 & 4.64449\end{array}$ $\begin{array}{lllllll}\text { Alpha virt. eigenvalues -- } & 4.66320 & 4.75316 & 4.76264 & 4.80459 & 4.84101\end{array}$ Alpha virt. eigenvalues -- 5.000385 .13965

Condensed to atoms (all electrons):

$$
\begin{array}{llllll}
1 & 2 & 3 & 4 & 5 & 6
\end{array}
$$

$\begin{array}{llllllll}1 & C & 5.051322 & 0.390874 & 0.666638 & -0.041249 & -0.041395 & 0.000203\end{array}$

$\begin{array}{llllllll}2 & \mathrm{H} & 0.390874 & 0.475235 & -0.030473 & 0.003083 & -0.004304 & 0.000050\end{array}$

$\begin{array}{llllllll}3 & \mathrm{C} & 0.666638 & -0.030473 & 4.960452 & 0.388595 & 0.361506 & -0.048073\end{array}$

$\begin{array}{llllllll}4 \mathrm{H} & -0.041249 & 0.003083 & 0.388595 & 0.493095 & -0.036562 & -0.001370\end{array}$

$\begin{array}{llllllll}5 & \mathrm{C} & -0.041395 & -0.004304 & 0.361506 & -0.036562 & 5.037860 & 0.424614\end{array}$

$\begin{array}{llllllll}6 & \mathrm{H} & 0.000203 & 0.000050 & -0.048073 & -0.001370 & 0.424614 & 0.535172\end{array}$

$\begin{array}{llllllll}7 & \mathrm{C} & -0.004956 & 0.001296 & -0.053624 & 0.003000 & 0.346097 & -0.039528\end{array}$

$\begin{array}{llllllll}8 \mathrm{H} & 0.000438 & -0.000030 & -0.001989 & -0.000054 & -0.034620 & 0.004214\end{array}$

$\begin{array}{llllllll}9 \mathrm{H} & -0.000135 & -0.000215 & -0.000391 & -0.000044 & -0.038591 & -0.003951\end{array}$

$\begin{array}{llllllll}10 \mathrm{H} & 0.000091 & 0.000041 & 0.003867 & -0.000071 & -0.034508 & -0.002636\end{array}$

$\begin{array}{llllllll}11 & \mathrm{C} & -0.002215 & 0.000102 & -0.065615 & 0.000503 & 0.317867 & -0.062707\end{array}$

$\begin{array}{llllllll}12 & \mathrm{C} & 0.000000 & 0.000000 & 0.000010 & -0.000006 & 0.000186 & -0.000021\end{array}$

$\begin{array}{llllllll}13 & C & -0.000013 & -0.000003 & -0.000521 & 0.000683 & -0.035022 & -0.001446\end{array}$

$\begin{array}{llllllll}14 \mathrm{C} & 0.000878 & -0.000014 & -0.001295 & 0.000641 & -0.048095 & 0.003725\end{array}$

$\begin{array}{llllllll}15 & \mathrm{C} & 0.000016 & 0.000000 & 0.000208 & -0.000029 & 0.001914 & -0.000018\end{array}$

$\begin{array}{llllllll}16 & \mathrm{C} & 0.000002 & 0.000000 & -0.000121 & -0.000016 & 0.003284 & 0.000394\end{array}$

$\begin{array}{llllllll}17 & \mathrm{H} & -0.000001 & 0.000000 & 0.000115 & 0.000037 & -0.003228 & 0.002591\end{array}$

$\begin{array}{llllllll}18 \mathrm{H} & 0.001465 & 0.000004 & 0.002698 & 0.000061 & -0.001396 & 0.000038\end{array}$

$\begin{array}{llllllll}19 \mathrm{H} & 0.000000 & 0.000000 & -0.000003 & 0.000000 & -0.000097 & 0.000002\end{array}$

$\begin{array}{llllllll}20 \mathrm{H} & 0.000000 & 0.000000 & 0.000002 & 0.000000 & -0.000103 & -0.000009\end{array}$ $\begin{array}{llllllll}21 \mathrm{H} & 0.000000 & 0.000000 & 0.000000 & 0.000000 & 0.000005 & 0.000000\end{array}$ $\begin{array}{llllllll}22 & \mathrm{C} & 0.321383 & -0.040555 & -0.039652 & -0.003541 & 0.002476 & -0.000070\end{array}$

$\begin{array}{llllllll}23 & \mathrm{H} & -0.040749 & -0.001025 & -0.000451 & 0.000104 & -0.000089 & -0.000002\end{array}$ $\begin{array}{llllllll}24 \mathrm{H} & -0.043702 & 0.002679 & 0.002876 & 0.003174 & 0.000106 & -0.000005\end{array}$ $\begin{array}{llllllll}25 \mathrm{Cl} & -0.081694 & 0.000930 & 0.000050 & 0.000179 & -0.000212 & 0.000030\end{array}$

$$
\begin{array}{llllll}
7 & 8 & 9 & 10 & 11 & 12
\end{array}
$$

$\begin{array}{llllllll}1 & \mathrm{C} & -0.004956 & 0.000438 & -0.000135 & 0.000091 & -0.002215 & 0.000000\end{array}$

$\begin{array}{llllllll}2 \mathrm{H} & 0.001296 & -0.000030 & -0.000215 & 0.000041 & 0.000102 & 0.000000\end{array}$

$\begin{array}{llllllll}3 \mathrm{C} & -0.053624 & -0.001989 & -0.000391 & 0.003867 & -0.065615 & 0.000010\end{array}$

$\begin{array}{llllllll}4 \mathrm{H} & 0.003000 & -0.000054 & -0.000044 & -0.000071 & 0.000503 & -0.000006\end{array}$

$\begin{array}{llllllll}5 \mathrm{C} & 0.346097 & -0.034620 & -0.038591 & -0.034508 & 0.317867 & 0.000186\end{array}$

$\begin{array}{llllllll}6 \mathrm{H} & -0.039528 & 0.004214 & -0.003951 & -0.002636 & -0.062707 & -0.000021\end{array}$

$\begin{array}{llllllll}7 & \mathrm{C} & 5.127663 & 0.388161 & 0.388333 & 0.392280 & -0.060422 & 0.000012\end{array}$

$\begin{array}{llllllll}8 & \mathrm{H} & 0.388161 & 0.538851 & -0.027719 & -0.026368 & -0.005858 & -0.000004\end{array}$

$\begin{array}{llllllll}9 \mathrm{H} & 0.388333 & -0.027719 & 0.531060 & -0.025296 & 0.006193 & 0.000000\end{array}$

$\begin{array}{llllllll}10 \mathrm{H} & 0.392280 & -0.026368 & -0.025296 & 0.516929 & -0.001920 & -0.000006\end{array}$ $\begin{array}{llllllll}11 & \mathrm{C} & -0.060422 & -0.005858 & 0.006193 & -0.001920 & 4.888778 & -0.029822\end{array}$

$\begin{array}{llllllll}12 & \mathrm{C} & 0.000012 & -0.000004 & 0.000000 & -0.000006 & -0.029822 & 4.897110\end{array}$

$\begin{array}{llllllll}13 & C & -0.001290 & 0.000110 & -0.000013 & 0.000642 & 0.574108 & -0.046128\end{array}$

$\begin{array}{llllllll}14 \mathrm{C} & -0.003547 & 0.002061 & -0.000027 & 0.000385 & 0.545893 & -0.046655\end{array}$

$\begin{array}{llllllll}15 & C & 0.000101 & 0.000062 & 0.000000 & -0.000054 & -0.037691 & 0.549336\end{array}$

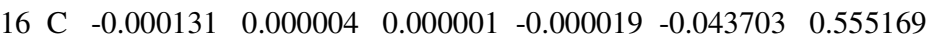


$\begin{array}{llllllll}17 & \mathrm{H} & 0.000115 & -0.000003 & -0.000003 & 0.000039 & -0.031304 & 0.002463\end{array}$

$\begin{array}{llllllll}18 \mathrm{H} & -0.000117 & 0.000693 & -0.000030 & 0.000019 & -0.033434 & 0.002436\end{array}$

$\begin{array}{llllllll}19 & \mathrm{H} & 0.000001 & 0.000002 & 0.000000 & 0.000000 & 0.001822 & -0.029891\end{array}$

$\begin{array}{llllllll}20 & \mathrm{H} & 0.000002 & 0.000000 & 0.000000 & 0.000001 & 0.002085 & -0.030103\end{array}$

$\begin{array}{llllllll}21 & \mathrm{H} & 0.000000 & 0.000000 & 0.000000 & 0.000000 & 0.000252 & 0.382192\end{array}$

$\begin{array}{llllllll}22 & \mathrm{C} & -0.000005 & -0.000021 & -0.000039 & 0.000001 & -0.000129 & 0.000000\end{array}$

$\begin{array}{llllllll}23 & \mathrm{H} & 0.000003 & -0.000004 & 0.000003 & 0.000000 & 0.000015 & 0.000000\end{array}$

$\begin{array}{llllllll}24 \mathrm{H} & 0.000002 & -0.000001 & -0.000001 & 0.000000 & -0.000009 & 0.000000\end{array}$

$\begin{array}{lllllllll}25 & \mathrm{Cl} & -0.000007 & 0.000008 & 0.000005 & 0.000000 & -0.000005 & 0.000000\end{array}$

$$
\begin{array}{llllll}
13 & 14 & 15 & 16 & 17 & 18
\end{array}
$$

$\begin{array}{llllllll}1 & \mathrm{C} & -0.000013 & 0.000878 & 0.000016 & 0.000002 & -0.000001 & 0.001465\end{array}$

$\begin{array}{llllllll}2 \mathrm{H} & -0.000003 & -0.000014 & 0.000000 & 0.000000 & 0.000000 & 0.000004\end{array}$

$\begin{array}{llllllll}3 & \mathrm{C} & -0.000521 & -0.001295 & 0.000208 & -0.000121 & 0.000115 & 0.002698\end{array}$

$\begin{array}{llllllll}4 \mathrm{H} & 0.000683 & 0.000641 & -0.000029 & -0.000016 & 0.000037 & 0.000061\end{array}$

$\begin{array}{llllllll}5 & \mathrm{C} & -0.035022 & -0.048095 & 0.001914 & 0.003284 & -0.003228 & -0.001396\end{array}$

$\begin{array}{llllllll}6 \mathrm{H} & -0.001446 & 0.003725 & -0.000018 & 0.000394 & 0.002591 & 0.000038\end{array}$

$\begin{array}{llllllll}7 & \mathrm{C} & -0.001290 & -0.003547 & 0.000101 & -0.000131 & 0.000115 & -0.000117\end{array}$

$\begin{array}{llllllll}8 \mathrm{H} & 0.000110 & 0.002061 & 0.000062 & 0.000004 & -0.000003 & 0.000693\end{array}$

$\begin{array}{llllllll}9 \mathrm{H} & -0.000013 & -0.000027 & 0.000000 & 0.000001 & -0.000003 & -0.000030\end{array}$

$\begin{array}{llllllll}10 \mathrm{H} & 0.000642 & 0.000385 & -0.000054 & -0.000019 & 0.000039 & 0.000019\end{array}$

$\begin{array}{lllllllll}11 & \mathrm{C} & 0.574108 & 0.545893 & -0.037691 & -0.043703 & -0.031304 & -0.033434\end{array}$

$\begin{array}{llllllll}12 & \mathrm{C} & -0.046128 & -0.046655 & 0.549336 & 0.555169 & 0.002463 & 0.002436\end{array}$

$\begin{array}{llllllll}13 & C & 4.922027 & -0.040495 & -0.034901 & 0.539385 & 0.380476 & 0.002532\end{array}$

$\begin{array}{llllllll}14 & \mathrm{C} & -0.040495 & 4.931994 & 0.544900 & -0.034861 & 0.002676 & 0.379422\end{array}$

$\begin{array}{llllllll}15 & \mathrm{C} & -0.034901 & 0.544900 & 4.889076 & -0.042841 & 0.000138 & -0.029133\end{array}$

$\begin{array}{llllllll}16 & \mathrm{C} & 0.539385 & -0.034861 & -0.042841 & 4.893982 & -0.030454 & 0.000146\end{array}$

$\begin{array}{llllllll}17 & \mathrm{H} & 0.380476 & 0.002676 & 0.000138 & -0.030454 & 0.478044 & -0.000103\end{array}$

$\begin{array}{llllllll}18 \mathrm{H} & 0.002532 & 0.379422 & -0.029133 & 0.000146 & -0.000103 & 0.472916\end{array}$

$\begin{array}{llllllll}19 & \mathrm{H} & 0.000253 & -0.029952 & 0.382243 & 0.002451 & 0.000010 & -0.001340\end{array}$

$\begin{array}{llllllll}20 \mathrm{H} & -0.029350 & 0.000230 & 0.002441 & 0.381200 & -0.001437 & 0.000010\end{array}$

$\begin{array}{llllllll}21 & \mathrm{H} & 0.002445 & 0.002484 & -0.030510 & -0.030358 & -0.000109 & -0.000109\end{array}$

$\begin{array}{llllllll}22 & \mathrm{C} & 0.000000 & -0.000028 & 0.000000 & 0.000000 & 0.000000 & 0.000011\end{array}$

$\begin{array}{llllllll}23 & \mathrm{H} & 0.000000 & 0.000004 & 0.000000 & 0.000000 & 0.000000 & -0.000013\end{array}$

$\begin{array}{llllllll}24 \mathrm{H} & 0.000000 & -0.000001 & 0.000000 & 0.000000 & 0.000000 & 0.000001\end{array}$

$\begin{array}{llllllll}25 \mathrm{Cl} & 0.000000 & 0.000000 & 0.000000 & 0.000000 & 0.000000 & 0.000001\end{array}$

$\begin{array}{llllll}19 & 20 & 21 & 22 & 23 & 24\end{array}$

$\begin{array}{llllllll}1 & \mathrm{C} & 0.000000 & 0.000000 & 0.000000 & 0.321383 & -0.040749 & -0.043702\end{array}$

$\begin{array}{llllllll}2 \mathrm{H} & 0.000000 & 0.000000 & 0.000000 & -0.040555 & -0.001025 & 0.002679\end{array}$

$\begin{array}{llllllll}3 \mathrm{C} & -0.000003 & 0.000002 & 0.000000 & -0.039652 & -0.000451 & 0.002876\end{array}$

$\begin{array}{llllllll}4 \mathrm{H} & 0.000000 & 0.000000 & 0.000000 & -0.003541 & 0.000104 & 0.003174\end{array}$

$\begin{array}{llllllll}5 & \mathrm{C} & -0.000097 & -0.000103 & 0.000005 & 0.002476 & -0.000089 & 0.000106\end{array}$

$\begin{array}{llllllll}6 \mathrm{H} & 0.000002 & -0.000009 & 0.000000 & -0.000070 & -0.000002 & -0.000005\end{array}$

$\begin{array}{llllllll}7 & \mathrm{C} & 0.000001 & 0.000002 & 0.000000 & -0.000005 & 0.000003 & 0.000002\end{array}$

$\begin{array}{llllllll}8 & \mathrm{H} & 0.000002 & 0.000000 & 0.000000 & -0.000021 & -0.000004 & -0.000001\end{array}$

$\begin{array}{llllllll}9 \mathrm{H} & 0.000000 & 0.000000 & 0.000000 & -0.000039 & 0.000003 & -0.000001\end{array}$

$\begin{array}{llllllll}10 \mathrm{H} & 0.000000 & 0.000001 & 0.000000 & 0.000001 & 0.000000 & 0.000000\end{array}$

$\begin{array}{llllllll}11 & \mathrm{C} & 0.001822 & 0.002085 & 0.000252 & -0.000129 & 0.000015 & -0.000009\end{array}$

$\begin{array}{llllllll}12 & \mathrm{C} & -0.029891 & -0.030103 & 0.382192 & 0.000000 & 0.000000 & 0.000000\end{array}$

$\begin{array}{llllllll}13 \mathrm{C} & 0.000253 & -0.029350 & 0.002445 & 0.000000 & 0.000000 & 0.000000\end{array}$

$\begin{array}{llllllll}14 & C & -0.029952 & 0.000230 & 0.002484 & -0.000028 & 0.000004 & -0.000001\end{array}$

$\begin{array}{llllllll}15 & \mathrm{C} & 0.382243 & 0.002441 & -0.030510 & 0.000000 & 0.000000 & 0.000000\end{array}$

$\begin{array}{llllllll}16 \mathrm{C} & 0.002451 & 0.381200 & -0.030358 & 0.000000 & 0.000000 & 0.000000\end{array}$

$\begin{array}{llllllll}17 \mathrm{H} & 0.000010 & -0.001437 & -0.000109 & 0.000000 & 0.000000 & 0.000000\end{array}$

$\begin{array}{llllllll}18 \mathrm{H} & -0.001340 & 0.000010 & -0.000109 & 0.000011 & -0.000013 & 0.000001\end{array}$

$\begin{array}{llllllll}19 & \mathrm{H} & 0.473878 & -0.000108 & -0.001480 & 0.000000 & 0.000000 & 0.000000\end{array}$

$\begin{array}{llllllll}20 \mathrm{H} & -0.000108 & 0.474965 & -0.001541 & 0.000000 & 0.000000 & 0.000000\end{array}$

$\begin{array}{llllllll}21 \mathrm{H} & -0.001480 & -0.001541 & 0.476733 & 0.000000 & 0.000000 & 0.000000\end{array}$

$\begin{array}{lllllllll}22 & \mathrm{C} & 0.000000 & 0.000000 & 0.000000 & 5.194383 & 0.386900 & 0.390830\end{array}$

$\begin{array}{llllllll}23 & \mathrm{H} & 0.000000 & 0.000000 & 0.000000 & 0.386900 & 0.516032 & -0.025862\end{array}$

$\begin{array}{llllllll}24 & \mathrm{H} & 0.000000 & 0.000000 & 0.000000 & 0.390830 & -0.025862 & 0.493882\end{array}$

$\begin{array}{llllllll}25 \mathrm{Cl} & 0.000000 & 0.000000 & 0.000000 & 0.202834 & -0.051550 & -0.044518\end{array}$ 25

1 C -0.081694

$2 \mathrm{H} \quad 0.000930$ 


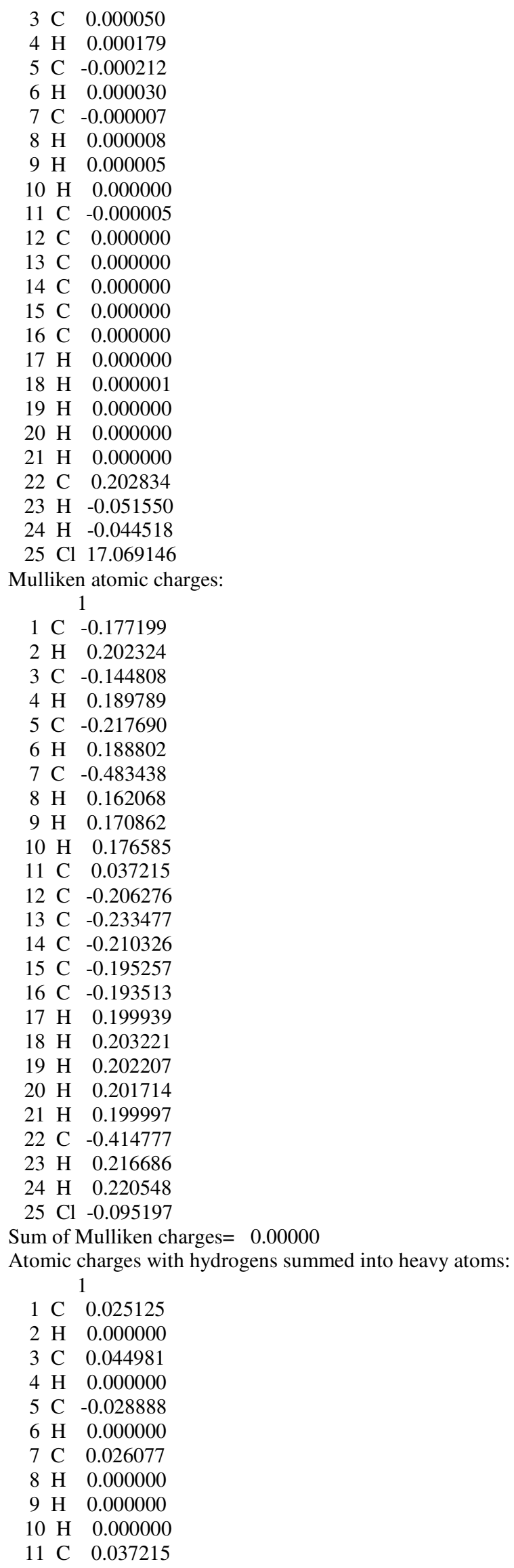

Atomic charges with hydrogens summed into heavy atoms: 1

1 C 0.025125

$2 \mathrm{H} \quad 0.000000$

3 C 0.044981

$4 \mathrm{H} \quad 0.000000$

$\begin{array}{lll}5 & \mathrm{C} & -0.028888\end{array}$

$6 \mathrm{H} \quad 0.000000$

$\begin{array}{lll}7 & \mathrm{C} & 0.026077\end{array}$

$8 \mathrm{H} \quad 0.000000$

$9 \mathrm{H} \quad 0.000000$

$10 \mathrm{H} \quad 0.000000$

$11 \mathrm{C} \quad 0.037215$ 
12 C -0.006279

13 C -0.033538

14 C -0.007104

15 C 0.006950

$16 \mathrm{C} \quad 0.008201$

$\begin{array}{lll}17 \mathrm{H} & 0.000000\end{array}$

$18 \mathrm{H} \quad 0.000000$

$19 \mathrm{H} \quad 0.000000$

$20 \mathrm{H} \quad 0.000000$

$\begin{array}{lll}21 \mathrm{H} & 0.000000\end{array}$

$22 \mathrm{C} \quad 0.022457$

$23 \mathrm{H} \quad 0.000000$

$24 \mathrm{H} \quad 0.000000$

$25 \mathrm{Cl}-0.095197$

Sum of Mulliken charges $=0.00000$

Electronic spatial extent $(\mathrm{au}):<\mathrm{R} * * 2>=3754.2750$

Charge $=0.0000$ electrons

Dipole moment (field-independent basis, Debye):

$\mathrm{X}=2.3635 \mathrm{Y}=-0.6748 \quad \mathrm{Z}=0.1488$ Tot $=2.4624$

Quadrupole moment (field-independent basis, Debye-Ang):

$\mathrm{XX}=-91.1466 \mathrm{YY}=-77.1025 \quad \mathrm{ZZ}=-77.1513$

$\mathrm{XY}=1.0212 \mathrm{XZ}=-3.4543 \quad \mathrm{YZ}=-2.8330$

Traceless Quadrupole moment (field-independent basis, Debye-Ang):

$\mathrm{XX}=-9.3465 \mathrm{YY}=4.6976 \quad \mathrm{ZZ}=4.6489$

$\mathrm{XY}=1.0212 \mathrm{XZ}=-3.4543 \mathrm{YZ}=-2.8330$

Octapole moment (field-independent basis, Debye-Ang $* * 2$ ):

$\mathrm{XXX}=80.9663 \mathrm{YYY}=-2.2171 \mathrm{ZZZ}=-1.6354 \mathrm{XYY}=-9.6828$

$\mathrm{XXY}=-16.3435 \mathrm{XXZ}=-4.2057 \mathrm{XZZ}=-5.4720 \mathrm{YZZ}=-1.5913$

$\mathrm{YYZ}=1.9475 \mathrm{XYZ}=-10.3588$

Hexadecapole moment (field-independent basis, Debye-Ang**3):

$X X X X=-4398.4332$ YYYY $=-536.0219 \mathrm{ZZZZ}=-352.7151 \mathrm{XXXY}=-82.8939$

$\mathrm{XXXZ}=-79.5737 \mathrm{YYYX}=12.1276 \mathrm{YYYZ}=-11.5994 \mathrm{ZZZX}=-4.2765$

$Z Z Z Y=-11.8661 X X Y Y=-760.0731 X X Z Z=-747.3368 Y Y Z Z=-140.4689$

$\mathrm{XXYZ}=-30.8364 \mathrm{YYXZ}=10.8971 \mathrm{ZZXY}=8.6407$

$\mathrm{N}-\mathrm{N}=7.052067331835 \mathrm{D}+02 \mathrm{E}-\mathrm{N}=-3.491098092327 \mathrm{D}+03 \mathrm{KE}=8.826593846306 \mathrm{D}+02$

1|1|UNPC-UNKISP|RHF|6-31G(d)|C11H13Cl1|PCUSER|21-Apr-2006|0||\# HF/6-31

G(D) GEOM=CONNECTIVITY॥Title Card Required॥0,1IC,0,1.93794792,-0.301

$44792,-0.25115625|\mathrm{H}, 0,2.02694792,-0.52244792,-1.31215625| \mathrm{C}, 0,0.8799479$

$2,0.38455208,0.21484375|\mathrm{H}, 0,0.82294792,0.58655208,1.28484375| \mathrm{C}, 0,-0.28$

$305208,0.94455208,-0.58315625 \mid \mathrm{H}, 0,-0.28005208,2.02555208,-0.38415625 \mathrm{IC}$

$, 0,-0.17805208,0.79655208,-2.10715625 \mathrm{H}, 0,-0.15505208,-0.25244792,-2.4$

$2215625|\mathrm{H}, 0,0.72894792,1.28255208,-2.48515625| \mathrm{H}, 0,-1.03505208,1.268552$

$08,-2.60215625|\mathrm{C}, 0,-1.60605208,0.37855208,-0.08315625| \mathrm{C}, 0,-4.05405208$,

$-0.64044792,0.84784375|\mathrm{C}, 0,-2.56305208,1.22855208,0.49284375| \mathrm{C}, 0,-1.89$

$705208,-0.99244792,-0.18215625|\mathrm{C}, 0,-3.11405208,-1.49744792,0.27984375|$

$\mathrm{C}, 0,-3.77905208,0.72055208,0.95484375 \mid \mathrm{H}, 0,-2.36905208,2.29455208,0.585$

$84375 \mathrm{lH}, 0,-1.17405208,-1.67644792,-0.61915625 \mathrm{lH}, 0,-3.32605208,-2.55944$

$792,0.19784375|\mathrm{H}, 0,-4.51305208,1.38955208,1.39884375| \mathrm{H}, 0,-5.00105208$,$1.03444792,1.20784375|\mathrm{C}, 0,3.04594792,-0.82144792,0.61084375| \mathrm{H}, 0,3.1339$ $4792,-1.90644792,0.49884375 \mathrm{lH}, 0,2.89394792,-0.59244792,1.66884375 \mathrm{ICl}, 0$ ,4.58294792,-0.08844792,0.10684375॥Version=x86-Win32-G03RevB.05IState $=1-\mathrm{AlHF}=-883.5854165|\mathrm{RMSD}=1.066 \mathrm{e}-005| \mathrm{Dipole}=-0.927038,-0.2259977,0.167$ 5772|PG=C01 [X(C11H13Cl1)]\|@

In the beginning the Universe was created.

This has made a lot of people very angry

and been widely regarded as a bad move.

-D.Adams

Job cpu time: 0 days 0 hours 2 minutes 20.0 seconds.

File lengths (MBytes): RWF $=33 \mathrm{Int}=0 \mathrm{D} 2 \mathrm{E}=0 \mathrm{Chk}=10 \mathrm{Scr}=1$ Normal termination of Gaussian 03 at Fri Apr 21 16:51:40 2006. 


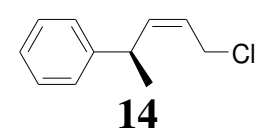

Entering Link 1 = C: $\mathrm{G}$ 03W11.exe PID= 2856.

Copyright (c) 1988,1990,1992,1993,1995,1998,2003, Gaussian, Inc. All Rights Reserved.

This is the Gaussian(R) 03 program. It is based on the the Gaussian(R) 98 system (copyright 1998, Gaussian, Inc.), the Gaussian(R) 94 system (copyright 1995, Gaussian, Inc.), the Gaussian 92(TM) system (copyright 1992, Gaussian, Inc.), the Gaussian 90(TM) system (copyright 1990, Gaussian, Inc.), the Gaussian 88(TM) system (copyright 1988, Gaussian, Inc.), the Gaussian 86(TM) system (copyright 1986, Carnegie Mellon University), and the Gaussian 82(TM) system (copyright 1983, Carnegie Mellon University). Gaussian is a federally registered trademark of Gaussian, Inc.

This software contains proprietary and confidential information, including trade secrets, belonging to Gaussian, Inc.

This software is provided under written license and may be used, copied, transmitted, or stored only in accord with that written license.

The following legend is applicable only to US Government contracts under DFARS:

\section{RESTRICTED RIGHTS LEGEND}

Use, duplication or disclosure by the US Government is subject to restrictions as set forth in subparagraph (c)(1)(ii) of the Rights in Technical Data and Computer Software clause at DFARS 252.227-7013.

Gaussian, Inc.

Carnegie Office Park, Building 6, Pittsburgh, PA 15106 USA

The following legend is applicable only to US Government contracts under FAR:

\section{RESTRICTED RIGHTS LEGEND}

Use, reproduction and disclosure by the US Government is subject to restrictions as set forth in subparagraph (c) of the

Commercial Computer Software - Restricted Rights clause at FAR 52.227-19.

Gaussian, Inc.

Carnegie Office Park, Building 6, Pittsburgh, PA 15106 USA

Warning -- This program may not be used in any manner that competes with the business of Gaussian, Inc. or will provide assistance to any competitor of Gaussian, Inc. The licensee of this program is prohibited from giving any competitor of Gaussian, Inc. access to this program. By using this program, the user acknowledges that Gaussian, Inc. is engaged in the business of creating and licensing software in the field of computational chemistry and represents and warrants to the licensee that it is not a competitor of Gaussian, Inc. and that 
it will not use this program in any manner prohibited above.

Cite this work as:

Gaussian 03, Revision B.05,

M. J. Frisch, G. W. Trucks, H. B. Schlegel, G. E. Scuseria,

M. A. Robb, J. R. Cheeseman, J. A. Montgomery, Jr., T. Vreven,

K. N. Kudin, J. C. Burant, J. M. Millam, S. S. Iyengar, J. Tomasi,

V. Barone, B. Mennucci, M. Cossi, G. Scalmani, N. Rega,

G. A. Petersson, H. Nakatsuji, M. Hada, M. Ehara, K. Toyota,

R. Fukuda, J. Hasegawa, M. Ishida, T. Nakajima, Y. Honda, O. Kitao,

H. Nakai, M. Klene, X. Li, J. E. Knox, H. P. Hratchian, J. B. Cross,

C. Adamo, J. Jaramillo, R. Gomperts, R. E. Stratmann, O. Yazyev,

A. J. Austin, R. Cammi, C. Pomelli, J. W. Ochterski, P. Y. Ayala,

K. Morokuma, G. A. Voth, P. Salvador, J. J. Dannenberg,

V. G. Zakrzewski, S. Dapprich, A. D. Daniels, M. C. Strain,

O. Farkas, D. K. Malick, A. D. Rabuck, K. Raghavachari,

J. B. Foresman, J. V. Ortiz, Q. Cui, A. G. Baboul, S. Clifford,

J. Cioslowski, B. B. Stefanov, G. Liu, A. Liashenko, P. Piskorz,

I. Komaromi, R. L. Martin, D. J. Fox, T. Keith, M. A. Al-Laham,

C. Y. Peng, A. Nanayakkara, M. Challacombe, P. M. W. Gill,

B. Johnson, W. Chen, M. W. Wong, C. Gonzalez, and J. A. Pople, Gaussian, Inc., Pittsburgh PA, 2003.

$* * * * * * * * * * * * * * * * * * * * * * * * * * * * * * * * * * * * * * * * * * * * * *$

Gaussian 03: x86-Win32-G03RevB.05 8-Nov-2003

$$
\text { 23-Apr-2006 }
$$

****************************************************

\%chk=9-10_alkene.chk

$\%$ mem $=64 \mathrm{MB}$

$\%$ nproc $=1$

Will use up to 1 processors via shared memory.

\# hf/6-31g(d) geom=connectivity

$1 / 38=1,57=2 / 1$;

$2 / 17=6,18=5,40=1 / 2$;

$3 / 5=1,6=6,7=1,11=9,16=1,25=1,30=1 / 1,2,3$;

$4 / / 1$

$5 / 5=2,32=1,38=5 / 2$;

$6 / 7=2,8=2,9=2,10=2,28=1 / 1$;

$99 / 5=1,9=1 / 99$;

Title Card Required

Symbolic Z-matrix:

Charge $=0$ Multiplicity $=1$

$\begin{array}{llll}\text { C } & 2.16831 & -0.12075 & 0.49001\end{array}$

$\begin{array}{llll}\text { C } & 1.33631 & -0.64375 & -0.42799\end{array}$

$\begin{array}{llll}\mathrm{H} & 1.57431 & -1.63775 & -0.80699\end{array}$

$\begin{array}{lllll}\text { C } & 0.10431 & 0.00425 & -1.02499\end{array}$

$\begin{array}{llll}\mathrm{H} & 0.15231 & 1.08725 & -0.85599\end{array}$

$\begin{array}{llll}\text { C } & 0.08931 & -0.16975 & -2.54899\end{array}$

$\mathrm{H} \quad 0.07731 \quad-1.22475-2.84699$

$\begin{array}{lllll}\mathrm{H} & 0.97531 & 0.29025 & -2.99999\end{array}$

$\begin{array}{lllll}\mathrm{H} & -0.79469 & 0.30725 & -2.98699\end{array}$

$\begin{array}{lllll}\text { C } & -1.17069 & -0.52275 & -0.38599\end{array}$

$\begin{array}{llll}\text { C } & -3.53269 & -1.45775 & 0.82201\end{array}$

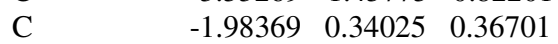

$\begin{array}{llll}\text { C } & -1.56269 & -1.86575 & -0.51699\end{array}$

$\begin{array}{llll}\text { C } & -2.73569 & -2.32775 & 0.08201\end{array}$

$\begin{array}{llll}\text { C } & -3.15669 & -0.12475 & 0.96501\end{array}$

$\begin{array}{llll}\mathrm{H} & -1.71069 & 1.38525 & 0.49101\end{array}$ 


$\begin{array}{lrrc}\mathrm{H} & -0.95469 & -2.56475 & -1.08599 \\ \mathrm{H} & -3.02669 & -3.36975 & -0.02799 \\ \mathrm{H} & -3.77769 & 0.55525 & 1.54301 \\ \mathrm{H} & -4.44569 & -1.81875 & 1.28801 \\ \mathrm{H} & 3.02431 & -0.72175 & 0.79601 \\ \mathrm{C} & 2.04431 & 1.19525 & 1.19001 \\ \mathrm{H} & 2.19331 & 1.06025 & 2.26501 \\ \mathrm{H} & 1.07331 & 1.67325 & 1.04601 \\ \mathrm{Cl} & 3.29631 & 2.30225 & 0.59501\end{array}$

Input orientation:

\begin{tabular}{|c|c|c|c|c|}
\hline \multirow{2}{*}{$\begin{array}{l}\text { Center } \\
\text { Number }\end{array}$} & \multirow{2}{*}{$\begin{array}{l}\text { Atomic } \\
\text { Number }\end{array}$} & \multirow{2}{*}{$\begin{array}{l}\text { Atomic } \\
\text { Type }\end{array}$} & \multicolumn{2}{|c|}{ Coordinates (Angstroms) } \\
\hline & & & $\begin{array}{ll}X & Y\end{array}$ & $\mathrm{Z}$ \\
\hline 1 & 6 & 2.168313 & -0.120750 & 0.490010 \\
\hline 2 & 6 & 1.336313 & -0.643750 & -0.427990 \\
\hline 3 & 1 & 1.574313 & -1.637750 & -0.806990 \\
\hline 4 & 6 & 0.104313 & 0.004250 & -1.024990 \\
\hline 5 & 1 & 0.152313 & 1.087250 & -0.855990 \\
\hline 6 & 6 & 0.089313 & -0.169750 & -2.548990 \\
\hline 7 & 1 & 0.077313 & -1.224750 & -2.846990 \\
\hline 8 & 1 & 0.975313 & 0.290250 & -2.999990 \\
\hline 9 & 1 & -0.794688 & 0.307250 & -2.986990 \\
\hline 10 & 6 & -1.170688 & -0.522750 & -0.385990 \\
\hline 11 & 6 & -3.532688 & -1.457750 & 0.822010 \\
\hline 12 & 6 & -1.983688 & 0.340250 & 0.367010 \\
\hline 13 & 6 & -1.562688 & -1.865750 & -0.516990 \\
\hline 14 & 6 & -2.735688 & -2.327750 & 0.082010 \\
\hline 15 & 6 & -3.156688 & -0.124750 & 0.965010 \\
\hline 16 & 1 & -1.710688 & 1.385250 & 0.491010 \\
\hline 17 & 1 & -0.954688 & -2.564750 & -1.085990 \\
\hline 18 & 1 & -3.026688 & -3.369750 & -0.027990 \\
\hline 19 & 1 & -3.777688 & 0.555250 & 1.543010 \\
\hline 20 & 1 & -4.445688 & -1.818750 & 1.288010 \\
\hline 21 & 1 & 3.024313 & -0.721750 & 0.796010 \\
\hline 22 & 6 & 2.044313 & 1.195250 & 1.190010 \\
\hline 23 & 1 & 2.193313 & 1.060250 & 2.265010 \\
\hline 24 & 1 & 1.073313 & 1.673250 & 1.046010 \\
\hline 25 & 17 & 3.296313 & 2.302250 & 0.595010 \\
\hline
\end{tabular}

Distance matrix (angstroms):

\begin{tabular}{|c|c|c|c|c|c|}
\hline & 1 & 3 & 4 & 5 & \\
\hline $1 \mathrm{C}$ & 0.000000 & & & & \\
\hline $2 \mathrm{C}$ & 1.344796 & 0.000000 & & & \\
\hline $3 \mathrm{H}$ & 2.082387 & 1.090101 & 0.000000 & & \\
\hline $4 \mathrm{C}$ & 2.563386 & 1.514641 & 2.214630 & 0.000000 & \\
\hline $5 \mathrm{H}$ & 2.708364 & 2.140 & & 157 & 0.000000 \\
\hline $6 \mathrm{C}$ & 3.682413 & 2.505659 & 2.719341 & & 9566 \\
\hline $7 \mathrm{H}$ & 4.089825 & 2.788226 & 3821 & 2.197 & 057 \\
\hline $8 \mathrm{H}$ & 3.71 & 2.760047 & & & \\
\hline $9 \mathrm{H}$ & 4.58 & 3.4 & & & \\
\hline $10 \mathrm{C}$ & 3.4 & 270 & & & \\
\hline $11 \mathrm{C}$ & & 2372 & & & 461 \\
\hline $12 \mathrm{C}$ & 4.179325 & 2841 & 4.2 & & 204 \\
\hline $13 \mathrm{C}$ & 4.24 & & & & \\
\hline $14 \mathrm{C}$ & 5.393193 & 892 & 494 & & 750 \\
\hline $15 \mathrm{C}$ & & 532 & & & 6669 \\
\hline $16 \mathrm{H}$ & 4.161091 & 4336 & 4.6 & & 8185 \\
\hline 17 & 4.267322 & & & & \\
\hline $18 \mathrm{H}$ & & & 4.97 & & \\
\hline $19 \mathrm{H}$ & & 5.610297 & 6.243048 & & 4.6 \\
\hline & 6.874955 & 6.144657 & 6.376691 & 5.419944 & 5.84663 \\
\hline $21 \mathrm{H}$ & 1.089758 & 2.086529 & 2.347587 & 3.517032 & 3.77491 \\
\hline
\end{tabular}




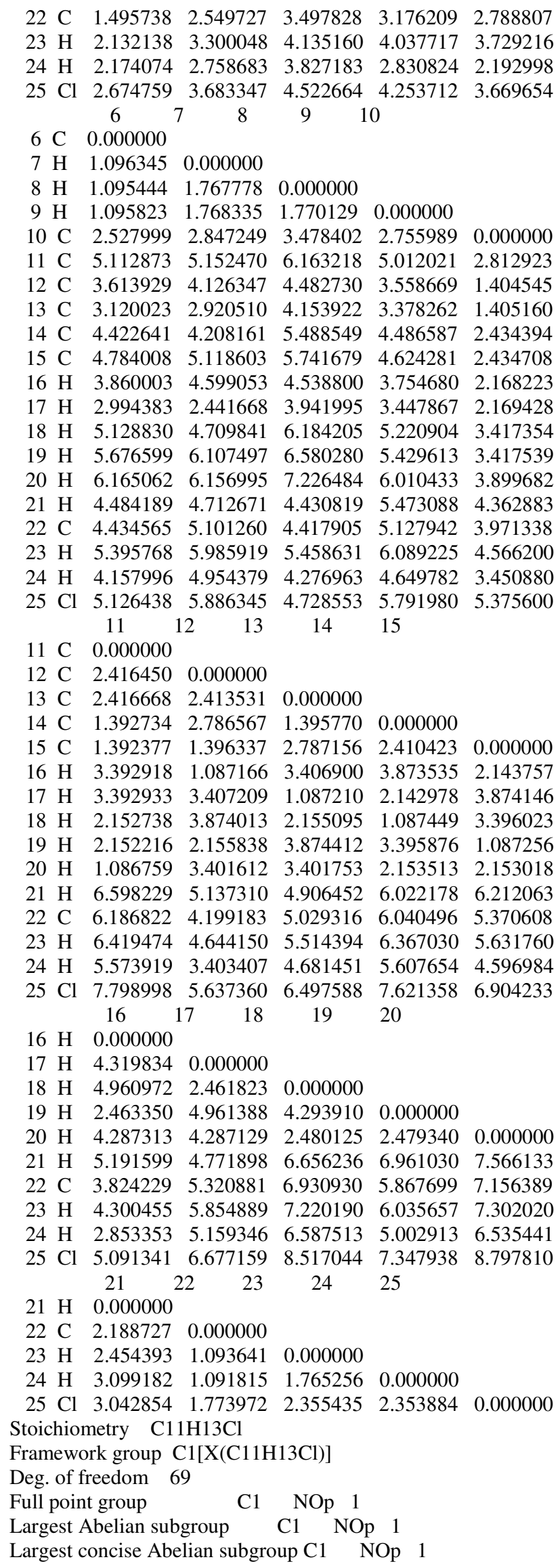


Standard orientation:

\begin{tabular}{|c|c|c|c|c|c|}
\hline \multirow{2}{*}{$\begin{array}{l}\text { Center } \\
\text { Number }\end{array}$} & \multirow{2}{*}{$\begin{array}{l}\text { Atomic } \\
\text { Number }\end{array}$} & \multirow{2}{*}{\multicolumn{2}{|c|}{$\begin{array}{l}\text { Atomic } \\
\text { Type }\end{array}$}} & \multicolumn{2}{|c|}{ Coordinates (Angstroms) } \\
\hline & & & & $\begin{array}{ll}X & Y\end{array}$ & $\mathrm{Z}$ \\
\hline 1 & 6 & 0 & -1.887059 & 0.229772 & 1.158657 \\
\hline 2 & 6 & 0 & -0.861437 & 1.014168 & 0.782757 \\
\hline 3 & 1 & 0 & -0.591529 & 1.834129 & 1.448441 \\
\hline 4 & 6 & 0 & -0.041531 & 0.907358 & -0.486291 \\
\hline 5 & 1 & 0 & -0.594877 & 0.308338 & -1.220278 \\
\hline 6 & 6 & 0 & 0.130783 & 2.287277 & -1.133755 \\
\hline 7 & 1 & 0 & 0.645936 & 2.994960 & -0.473623 \\
\hline 8 & 1 & 0 & -0.842995 & 2.720837 & -1.386299 \\
\hline 9 & 1 & 0 & 0.714005 & 2.213719 & -2.058563 \\
\hline 10 & 6 & 0 & 1.297717 & 0.233832 & -0.232419 \\
\hline 11 & 6 & 0 & 3.758346 & -1.052524 & 0.218275 \\
\hline 12 & 6 & 0 & 1.577908 & -1.009600 & -0.822434 \\
\hline 13 & 6 & 0 & 2.273939 & 0.817390 & 0.592762 \\
\hline 14 & 6 & 0 & 3.494885 & 0.178343 & 0.814314 \\
\hline 15 & 6 & 0 & 2.800296 & -1.646494 & -0.599070 \\
\hline 16 & 1 & 0 & 0.845486 & -1.491465 & -1.465314 \\
\hline 17 & 1 & 0 & 2.089560 & 1.775452 & 1.072500 \\
\hline 18 & 1 & 0 & 4.241116 & 0.641921 & 1.455234 \\
\hline 19 & 1 & 0 & 3.004522 & -2.607679 & -1.064408 \\
\hline 20 & 1 & 0 & 4.709327 & -1.549121 & 0.391689 \\
\hline 21 & 1 & 0 & -2.381227 & 0.462462 & 2.101644 \\
\hline 22 & 6 & 0 & -2.423619 & -0.973318 & 0.450201 \\
\hline 23 & 1 & 0 & -2.548082 & -1.797310 & 1.158437 \\
\hline 24 & 1 & 0 & -1.779027 & -1.323850 & -0.358309 \\
\hline 25 & 17 & 0 & -4.013740 & -0.594095 & -0.238773 \\
\hline
\end{tabular}

Rotational constants (GHZ): $1.5979749 \quad 0.3676734 \quad 0.3366005$

Standard basis: 6-31G(d) $(6 \mathrm{D}, 7 \mathrm{~F})$

There are 210 symmetry adapted basis functions of A symmetry.

Integral buffers will be 262144 words long.

Raffenetti 1 integral format.

Two-electron integral symmetry is turned on.

210 basis functions, 412 primitive gaussians, 210 cartesian basis functions

48 alpha electrons 48 beta electrons nuclear repulsion energy 720.6321232396 Hartrees.

NAtoms $=25$ NActive $=25 \mathrm{NUniq}=25 \mathrm{SFac}=1.00 \mathrm{D}+00 \mathrm{NAtFMM}=60 \mathrm{Big}=\mathrm{F}$

One-electron integrals computed using PRISM.

$\mathrm{NBasis}=210 \mathrm{RedAO}=\mathrm{T} \mathrm{NBF}=210$

NBsUse $=2101.00 \mathrm{D}-06 \mathrm{NBFU}=210$

Harris functional with IExCor $=205$ diagonalized for initial guess.

ExpMin= 1.43D-01 ExpMax=2.52D+04 ExpMxC=3.78D+03 IAcc=1 IRadAn= 1 AccDes=1.00D-06

HarFok: IExCor $=205$ AccDes $=1.00 D-06$ IRadAn $=\quad 1 \mathrm{IDoV}=1$

ScaDFX $=1.0000001 .0000001 .0000001 .000000$

Initial guess orbital symmetries:

Occupied (A) (A) (A) (A) (A) (A) (A) (A) (A) (A) (A) (A)

(A) (A) (A) (A) (A) (A) (A) (A) (A) (A) (A) (A)

(A) $(\mathrm{A})(\mathrm{A})(\mathrm{A})(\mathrm{A})(\mathrm{A})(\mathrm{A})(\mathrm{A})(\mathrm{A})(\mathrm{A})(\mathrm{A})(\mathrm{A})$

(A) (A) (A) (A) (A) (A) (A) (A) (A) (A) (A) (A)

Virtual (A) (A) (A) (A) (A) (A) (A) (A) (A) (A) (A) (A)

(A) $(\mathrm{A})(\mathrm{A})(\mathrm{A})(\mathrm{A})(\mathrm{A})(\mathrm{A})(\mathrm{A})(\mathrm{A})(\mathrm{A})(\mathrm{A})(\mathrm{A})$

(A) (A) (A) (A) (A) (A) (A) (A) (A) (A) (A) (A)

(A) $(\mathrm{A})(\mathrm{A})(\mathrm{A})(\mathrm{A})(\mathrm{A})(\mathrm{A})(\mathrm{A})(\mathrm{A})(\mathrm{A})(\mathrm{A})(\mathrm{A})$

(A) (A) (A) (A) (A) (A) (A) (A) (A) (A) (A) (A)

(A) $(\mathrm{A})(\mathrm{A})(\mathrm{A})(\mathrm{A})(\mathrm{A})(\mathrm{A})(\mathrm{A})(\mathrm{A})(\mathrm{A})(\mathrm{A})(\mathrm{A})$

(A) $(\mathrm{A})(\mathrm{A})(\mathrm{A})(\mathrm{A})(\mathrm{A})(\mathrm{A})(\mathrm{A})(\mathrm{A})(\mathrm{A})(\mathrm{A})(\mathrm{A})$

(A) (A) (A) (A) (A) (A) (A) (A) (A) (A) (A) (A)

(A) $(\mathrm{A})(\mathrm{A})(\mathrm{A})(\mathrm{A})(\mathrm{A})(\mathrm{A})(\mathrm{A})(\mathrm{A})(\mathrm{A})(\mathrm{A})(\mathrm{A})$

(A) (A) (A) (A) (A) (A) (A) (A) (A) (A) (A) (A)

(A) $(\mathrm{A})(\mathrm{A})(\mathrm{A})(\mathrm{A})(\mathrm{A})(\mathrm{A})(\mathrm{A})(\mathrm{A})(\mathrm{A})(\mathrm{A})(\mathrm{A})$ 
(A) $(\mathrm{A})(\mathrm{A})(\mathrm{A})(\mathrm{A})(\mathrm{A})(\mathrm{A})(\mathrm{A})(\mathrm{A})(\mathrm{A})(\mathrm{A})(\mathrm{A})$

(A) $(\mathrm{A})(\mathrm{A})(\mathrm{A})(\mathrm{A})(\mathrm{A})(\mathrm{A})(\mathrm{A})(\mathrm{A})(\mathrm{A})(\mathrm{A})(\mathrm{A})$

(A) (A) (A) (A) (A) (A)

The electronic state of the initial guess is 1-A.

Warning! Cutoffs for single-point calculations used.

Requested convergence on RMS density matrix=1.00D-04 within 128 cycles.

Requested convergence on MAX density matrix=1.00D-02.

Requested convergence on energy $=5.00 \mathrm{D}-05$.

No special actions if energy rises.

SCF Done: $\mathrm{E}(\mathrm{RHF})=-883.581993752 \quad$ A.U. after 6 cycles

Convg $=0.1169 \mathrm{D}-04 \quad-\mathrm{V} / \mathrm{T}=2.0010$

$\mathrm{S}^{* * 2}=0.0000$

$* * * * * * * * * * * * * * * * * * * * * * * * * * * * * * * * * * * * * * * * * * * * * * * * * * * * * * * * * * * * * * * * * * * * * * *$

Population analysis using the SCF density.

******************************************************************************

Orbital symmetries:

Occupied (A) (A) (A) (A) (A) (A) (A) (A) (A) (A) (A) (A)

(A) $(\mathrm{A})(\mathrm{A})(\mathrm{A})(\mathrm{A})(\mathrm{A})(\mathrm{A})(\mathrm{A})(\mathrm{A})(\mathrm{A})(\mathrm{A})(\mathrm{A})$

$(\mathrm{A})(\mathrm{A})(\mathrm{A})(\mathrm{A})(\mathrm{A})(\mathrm{A})(\mathrm{A})(\mathrm{A})(\mathrm{A})(\mathrm{A})(\mathrm{A})(\mathrm{A})$

(A) $(\mathrm{A})(\mathrm{A})(\mathrm{A})(\mathrm{A})(\mathrm{A})(\mathrm{A})(\mathrm{A})(\mathrm{A})(\mathrm{A})(\mathrm{A})(\mathrm{A})$

Virtual (A) (A) (A) (A) (A) (A) (A) (A) (A) (A) (A) (A)

(A) $(\mathrm{A})(\mathrm{A})(\mathrm{A})(\mathrm{A})(\mathrm{A})(\mathrm{A})(\mathrm{A})(\mathrm{A})(\mathrm{A})(\mathrm{A})(\mathrm{A})$

(A) $(\mathrm{A})(\mathrm{A})(\mathrm{A})(\mathrm{A})(\mathrm{A})(\mathrm{A})(\mathrm{A})(\mathrm{A})(\mathrm{A})(\mathrm{A})(\mathrm{A})$

(A) $(\mathrm{A})(\mathrm{A})(\mathrm{A})(\mathrm{A})(\mathrm{A})(\mathrm{A})(\mathrm{A})(\mathrm{A})(\mathrm{A})(\mathrm{A})(\mathrm{A})$

(A) $(\mathrm{A})(\mathrm{A})(\mathrm{A})(\mathrm{A})(\mathrm{A})(\mathrm{A})(\mathrm{A})(\mathrm{A})(\mathrm{A})(\mathrm{A})(\mathrm{A})$

(A) $(\mathrm{A})(\mathrm{A})(\mathrm{A})(\mathrm{A})(\mathrm{A})(\mathrm{A})(\mathrm{A})(\mathrm{A})(\mathrm{A})(\mathrm{A})(\mathrm{A})$

(A) $(\mathrm{A})(\mathrm{A})(\mathrm{A})(\mathrm{A})(\mathrm{A})(\mathrm{A})(\mathrm{A})(\mathrm{A})(\mathrm{A})(\mathrm{A})(\mathrm{A})$

(A) $(\mathrm{A})(\mathrm{A})(\mathrm{A})(\mathrm{A})(\mathrm{A})(\mathrm{A})(\mathrm{A})(\mathrm{A})(\mathrm{A})(\mathrm{A})(\mathrm{A})$

(A) $(\mathrm{A})(\mathrm{A})(\mathrm{A})(\mathrm{A})(\mathrm{A})(\mathrm{A})(\mathrm{A})(\mathrm{A})(\mathrm{A})(\mathrm{A})(\mathrm{A})$

(A) $(\mathrm{A})(\mathrm{A})(\mathrm{A})(\mathrm{A})(\mathrm{A})(\mathrm{A})(\mathrm{A})(\mathrm{A})(\mathrm{A})(\mathrm{A})(\mathrm{A})$

(A) $(\mathrm{A})(\mathrm{A})(\mathrm{A})(\mathrm{A})(\mathrm{A})(\mathrm{A})(\mathrm{A})(\mathrm{A})(\mathrm{A})(\mathrm{A})(\mathrm{A})$

(A) $(\mathrm{A})(\mathrm{A})(\mathrm{A})(\mathrm{A})(\mathrm{A})(\mathrm{A})(\mathrm{A})(\mathrm{A})(\mathrm{A})(\mathrm{A})(\mathrm{A})$

(A) $(A)(A)(A)(A)(A)(A)(A)(A)(A)(A)(A)$

(A) (A) (A) (A) (A) (A)

The electronic state is $1-\mathrm{A}$.

Alpha occ. eigenvalues -- -104.80702 -11.29498 -11.24801 -11.24672 -11.24565

Alpha occ. eigenvalues -- -11.24188 -11.23855 -11.23801 -11.23649 -11.23635

Alpha occ. eigenvalues -- $-11.23384-11.22351-10.54135$-8.01071 -8.00738

Alpha occ. eigenvalues -- $\quad-8.00733-1.15839-1.14509-1.09433-1.02776$

Alpha occ. eigenvalues -- $\quad-1.01262 \quad-0.98511 \quad-0.91253 \quad-0.86431 \quad-0.82573$

Alpha occ. eigenvalues -- $-0.79860 \quad-0.73993 \quad-0.69691 \quad-0.67219-0.63646$

$\begin{array}{llllll}\text { Alpha occ. eigenvalues -- } & -0.63261 & -0.60714 & -0.59451 & -0.58513 & -0.58246\end{array}$

Alpha occ. eigenvalues -- $-0.55774 \quad-0.54324 \quad-0.53812-0.51730 \quad-0.50421$

$\begin{array}{llllll}\text { Alpha occ. eigenvalues -- } & -0.48410 & -0.48173 & -0.47039 & -0.43236 & -0.42936\end{array}$

Alpha occ. eigenvalues -- $-0.36068-0.33465-0.32226$

$\begin{array}{llllll}\text { Alpha virt. eigenvalues -- } & 0.13182 & 0.13806 & 0.16603 & 0.20438 & 0.22942\end{array}$

$\begin{array}{lllllll}\text { Alpha virt. eigenvalues -- } & 0.23600 & 0.25472 & 0.27179 & 0.28657 & 0.29856\end{array}$

$\begin{array}{lllllll}\text { Alpha virt. eigenvalues -- } & 0.31046 & 0.31854 & 0.32434 & 0.33845 & 0.34389\end{array}$

$\begin{array}{lllllll}\text { Alpha virt. eigenvalues -- } & 0.35113 & 0.35659 & 0.37680 & 0.38883 & 0.41290\end{array}$

$\begin{array}{lllllll}\text { Alpha virt. eigenvalues -- } & 0.42268 & 0.47429 & 0.49180 & 0.50301 & 0.51524\end{array}$

$\begin{array}{lllllll}\text { Alpha virt. eigenvalues -- } & 0.52957 & 0.58054 & 0.59424 & 0.63557 & 0.65282\end{array}$

$\begin{array}{lllllll}\text { Alpha virt. eigenvalues -- } & 0.66337 & 0.67382 & 0.70074 & 0.70949 & 0.73213\end{array}$

$\begin{array}{llllllll}\text { Alpha virt. eigenvalues -- } & 0.74834 & 0.75754 & 0.76996 & 0.77520 & 0.78010\end{array}$

$\begin{array}{llllllll}\text { Alpha virt. eigenvalues -- } & 0.80116 & 0.80954 & 0.82781 & 0.83252 & 0.83995\end{array}$

$\begin{array}{lllllll}\text { Alpha virt. eigenvalues -- } & 0.84003 & 0.84776 & 0.85793 & 0.87645 & 0.89557\end{array}$

$\begin{array}{llllllll}\text { Alpha virt. eigenvalues -- } & 0.89848 & 0.93005 & 0.94579 & 0.98664 & 1.00256\end{array}$

$\begin{array}{lllllll}\text { Alpha virt. eigenvalues -- } & 1.02714 & 1.04257 & 1.06365 & 1.07117 & 1.08292\end{array}$

$\begin{array}{lllllll}\text { Alpha virt. eigenvalues -- } & 1.09286 & 1.10867 & 1.11710 & 1.11951 & 1.13576\end{array}$

$\begin{array}{lllllll}\text { Alpha virt. eigenvalues -- } & 1.14715 & 1.14847 & 1.16517 & 1.17243 & 1.17769\end{array}$

$\begin{array}{lllllll}\text { Alpha virt. eigenvalues -- } & 1.18653 & 1.19437 & 1.21312 & 1.21937 & 1.22814\end{array}$ 
$\begin{array}{llllll}\text { Alpha virt. eigenvalues -- } & 1.23982 & 1.25010 & 1.25802 & 1.27024 & 1.30758\end{array}$

$\begin{array}{lllllll}\text { Alpha virt. eigenvalues -- } & 1.33699 & 1.36559 & 1.38998 & 1.41009 & 1.42081\end{array}$

$\begin{array}{lllllll}\text { Alpha virt. eigenvalues -- } & 1.43360 & 1.44491 & 1.46422 & 1.51988 & 1.55911\end{array}$

$\begin{array}{lllllll}\text { Alpha virt. eigenvalues -- } & 1.60700 & 1.67640 & 1.69817 & 1.70501 & 1.73233\end{array}$

$\begin{array}{lllllll}\text { Alpha virt. eigenvalues -- } & 1.74300 & 1.76911 & 1.77147 & 1.79235 & 1.81694\end{array}$

$\begin{array}{lllllll}\text { Alpha virt. eigenvalues -- } & 1.82669 & 1.90503 & 1.98659 & 2.02278 & 2.05621\end{array}$

$\begin{array}{llllll}\text { Alpha virt. eigenvalues -- } & 2.09832 & 2.12342 & 2.13646 & 2.14839 & 2.16803\end{array}$

$\begin{array}{lllllll}\text { Alpha virt. eigenvalues -- } & 2.18974 & 2.23809 & 2.28100 & 2.28445 & 2.30609\end{array}$

$\begin{array}{llllll}\text { Alpha virt. eigenvalues -- } & 2.33245 & 2.35491 & 2.37469 & 2.38663 & 2.40573\end{array}$

$\begin{array}{lllllll}\text { Alpha virt. eigenvalues -- } & 2.44546 & 2.46084 & 2.46724 & 2.47201 & 2.51686\end{array}$

$\begin{array}{lllllll}\text { Alpha virt. eigenvalues -- } & 2.53941 & 2.56858 & 2.61574 & 2.62170 & 2.63270\end{array}$

$\begin{array}{lllllll}\text { Alpha virt. eigenvalues -- } & 2.63719 & 2.68323 & 2.72739 & 2.75148 & 2.82776\end{array}$

$\begin{array}{lllllll}\text { Alpha virt. eigenvalues -- } & 2.85178 & 2.88505 & 2.92641 & 2.94223 & 2.98337\end{array}$

$\begin{array}{lllllll}\text { Alpha virt. eigenvalues -- } & 3.01821 & 3.07321 & 3.09891 & 3.10912 & 3.15480\end{array}$

$\begin{array}{lllllll}\text { Alpha virt. eigenvalues -- } & 3.19347 & 3.24088 & 3.37740 & 3.44005 & 3.78446\end{array}$

$\begin{array}{lllllll}\text { Alpha virt. eigenvalues -- } & 4.51379 & 4.52326 & 4.53958 & 4.57306 & 4.65512\end{array}$

$\begin{array}{lllllll}\text { Alpha virt. eigenvalues -- } & 4.65880 & 4.75238 & 4.77862 & 4.80708 & 4.88310\end{array}$

Alpha virt. eigenvalues -- $5.00432 \quad 5.14067$

Condensed to atoms (all electrons):

$$
\begin{array}{llllll}
1 & 2 & 3 & 4 & 5 & 6
\end{array}
$$

$\begin{array}{llllllll}1 & \mathrm{C} & 5.025350 & 0.667791 & -0.033220 & -0.040429 & -0.000369 & 0.000547\end{array}$

$\begin{array}{llllllll}2 & \mathrm{C} & 0.667791 & 4.971134 & 0.387506 & 0.350923 & -0.044561 & -0.066134\end{array}$

$\begin{array}{llllllll}3 \mathrm{H} & -0.033220 & 0.387506 & 0.495155 & -0.038917 & 0.003010 & -0.002569\end{array}$

$\begin{array}{llllllll}4 & \mathrm{C} & -0.040429 & 0.350923 & -0.038917 & 5.078713 & 0.423353 & 0.350568\end{array}$

$\begin{array}{lllllllll}5 & \mathrm{H} & -0.000369 & -0.044561 & 0.003010 & 0.423353 & 0.522321 & -0.041231\end{array}$

$\begin{array}{llllllll}6 & \mathrm{C} & 0.000547 & -0.066134 & -0.002569 & 0.350568 & -0.041231 & 5.134167\end{array}$

$\begin{array}{llllllll}7 & \mathrm{H} & 0.000103 & -0.003483 & 0.002288 & -0.035307 & 0.004123 & 0.387838\end{array}$

$\begin{array}{lllllllll}8 \mathrm{H} & 0.000102 & -0.000366 & 0.000206 & -0.037862 & -0.003290 & 0.387987\end{array}$

$\begin{array}{llllllll}9 \mathrm{H} & -0.000052 & 0.004964 & -0.000049 & -0.037228 & -0.002403 & 0.391037\end{array}$

$\begin{array}{llllllll}10 & C & -0.001856 & -0.063939 & -0.000456 & 0.320786 & -0.054815 & -0.066066\end{array}$

$\begin{array}{lllllllll}11 & \mathrm{C} & 0.000000 & 0.000005 & 0.000000 & 0.000198 & -0.000018 & 0.000002\end{array}$

$\begin{array}{llllllll}12 & C & 0.000317 & -0.001621 & -0.000044 & -0.034379 & -0.001719 & -0.000642\end{array}$

$\begin{array}{llllllll}13 & \mathrm{C} & 0.000187 & -0.002635 & 0.001048 & -0.045843 & 0.003061 & -0.002965\end{array}$

$\begin{array}{llllllll}14 & C & -0.000009 & 0.000035 & 0.000014 & 0.002079 & -0.000033 & 0.000143\end{array}$

$\begin{array}{llllllll}15 \mathrm{C} & 0.000001 & -0.000113 & 0.000003 & 0.003300 & 0.000393 & -0.000101\end{array}$

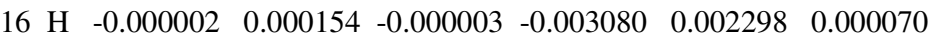

$\begin{array}{llllllll}17 & \mathrm{H} & 0.000036 & 0.000294 & 0.000288 & -0.001410 & -0.000015 & 0.000023\end{array}$

$\begin{array}{llllllll}18 \mathrm{H} & 0.000000 & 0.000001 & 0.000000 & -0.000099 & 0.000002 & 0.000001\end{array}$

$\begin{array}{llllllll}19 & \mathrm{H} & 0.000000 & 0.000003 & 0.000000 & -0.000106 & -0.000009 & 0.000002\end{array}$

$\begin{array}{llllllll}20 \mathrm{H} & 0.000000 & 0.000000 & 0.000000 & 0.000006 & 0.000000 & 0.000000\end{array}$

$\begin{array}{llllllll}21 & \mathrm{H} & 0.392991 & -0.030871 & -0.003654 & 0.002752 & 0.000068 & -0.000107\end{array}$

$\begin{array}{llllllll}22 & \mathrm{C} & 0.321282 & -0.042860 & 0.002955 & -0.007499 & -0.001868 & 0.000230\end{array}$

$\begin{array}{llllllll}23 & \mathrm{H} & -0.038793 & 0.001195 & -0.000094 & 0.000095 & 0.000072 & -0.000008\end{array}$

$\begin{array}{llllllll}24 & \mathrm{H} & -0.039097 & 0.000618 & 0.000047 & 0.000256 & 0.000391 & 0.000061\end{array}$

$\begin{array}{lllllll}25 \mathrm{Cl} & -0.083861 & -0.000933 & -0.000223 & 0.000216 & 0.000410 & 0.000010\end{array}$

$$
\begin{array}{llllll}
7 & 8 & 9 & 10 & 11 & 12
\end{array}
$$

$\begin{array}{llllllll}1 & \mathrm{C} & 0.000103 & 0.000102 & -0.000052 & -0.001856 & 0.000000 & 0.000317\end{array}$

$\begin{array}{llllllll}2 \mathrm{C} & -0.003483 & -0.000366 & 0.004964 & -0.063939 & 0.000005 & -0.001621\end{array}$

$\begin{array}{llllllll}3 \mathrm{H} & 0.002288 & 0.000206 & -0.000049 & -0.000456 & 0.000000 & -0.000044\end{array}$

$\begin{array}{llllllll}4 & \mathrm{C} & -0.035307 & -0.037862 & -0.037228 & 0.320786 & 0.000198 & -0.034379\end{array}$

$\begin{array}{llllllll}5 \mathrm{H} & 0.004123 & -0.003290 & -0.002403 & -0.054815 & -0.000018 & -0.001719\end{array}$

$\begin{array}{llllllll}6 & \mathrm{C} & 0.387838 & 0.387987 & 0.391037 & -0.066066 & 0.000002 & -0.000642\end{array}$

$\begin{array}{llllllll}7 & \mathrm{H} & 0.543803 & -0.027183 & -0.026257 & -0.005016 & -0.000003 & 0.000034\end{array}$

$\begin{array}{llllllll}8 & \mathrm{H} & -0.027183 & 0.524488 & -0.024276 & 0.005768 & 0.000000 & -0.000019\end{array}$

$\begin{array}{llllllll}9 \mathrm{H} & -0.026257 & -0.024276 & 0.519374 & -0.000820 & -0.000006 & 0.000552\end{array}$

$\begin{array}{llllllll}10 \mathrm{C} & -0.005016 & 0.005768 & -0.000820 & 4.846346 & -0.030071 & 0.575036\end{array}$

$\begin{array}{llllllll}11 & C & -0.000003 & 0.000000 & -0.000006 & -0.030071 & 4.897577 & -0.046164\end{array}$

$\begin{array}{llllllll}12 & \mathrm{C} & 0.000034 & -0.000019 & 0.000552 & 0.575036 & -0.046164 & 4.921908\end{array}$

$\begin{array}{llllllll}13 & \mathrm{C} & 0.001576 & -0.000007 & 0.000381 & 0.549893 & -0.046531 & -0.038914\end{array}$

$\begin{array}{llllllll}14 & \mathrm{C} & 0.000058 & 0.000001 & -0.000064 & -0.036852 & 0.550540 & -0.035549\end{array}$

$\begin{array}{llllllll}15 & \mathrm{C} & 0.000004 & 0.000001 & -0.000023 & -0.042725 & 0.554792 & 0.541542\end{array}$

$\begin{array}{lllllll}16 \mathrm{H} & -0.000004 & -0.000002 & 0.000029 & -0.032028 & 0.002455 & 0.380223\end{array}$

$\begin{array}{llllllll}17 & \mathrm{H} & 0.000494 & -0.000020 & 0.000025 & -0.034088 & 0.002442 & 0.002399\end{array}$

$\begin{array}{llllllll}18 \mathrm{H} & 0.000002 & 0.000000 & 0.000000 & 0.001822 & -0.029781 & 0.000257\end{array}$ 


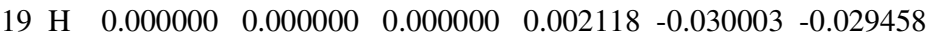

$\begin{array}{llllllll}20 \mathrm{H} & 0.000000 & 0.000000 & 0.000000 & 0.000258 & 0.382212 & 0.002474\end{array}$

$\begin{array}{llllllll}21 \mathrm{H} & 0.000000 & -0.000012 & 0.000002 & -0.000152 & 0.000000 & -0.000001\end{array}$

$\begin{array}{llllllll}22 & \mathrm{C} & -0.000006 & 0.000000 & 0.000002 & -0.000037 & 0.000000 & -0.000355\end{array}$

$\begin{array}{llllllll}23 & \mathrm{H} & 0.000000 & 0.000000 & 0.000000 & 0.000059 & 0.000000 & -0.000002\end{array}$

$\begin{array}{llllllll}24 \mathrm{H} & -0.000001 & -0.000017 & 0.000006 & -0.000268 & -0.000001 & 0.000899\end{array}$

$\begin{array}{llllllll}25 & \mathrm{Cl} & 0.000000 & 0.000014 & -0.000002 & -0.000015 & 0.000000 & 0.000002\end{array}$ $\begin{array}{llllll}13 & 14 & 15 & 16 & 17 & 18\end{array}$

$\begin{array}{llllllll}1 & \mathrm{C} & 0.000187 & -0.000009 & 0.000001 & -0.000002 & 0.000036 & 0.000000\end{array}$

$\begin{array}{llllllll}2 & \mathrm{C} & -0.002635 & 0.000035 & -0.000113 & 0.000154 & 0.000294 & 0.000001\end{array}$

$\begin{array}{llllllll}3 \mathrm{H} & 0.001048 & 0.000014 & 0.000003 & -0.000003 & 0.000288 & 0.000000\end{array}$

$\begin{array}{llllllll}4 \mathrm{C} & -0.045843 & 0.002079 & 0.003300 & -0.003080 & -0.001410 & -0.000099\end{array}$

$\begin{array}{llllllll}5 & \mathrm{H} & 0.003061 & -0.000033 & 0.000393 & 0.002298 & -0.000015 & 0.000002\end{array}$

$\begin{array}{lllllll}6 \mathrm{C} & -0.002965 & 0.000143 & -0.000101 & 0.000070 & 0.000023 & 0.000001\end{array}$

$\begin{array}{llllllll}7 & \mathrm{H} & 0.001576 & 0.000058 & 0.000004 & -0.000004 & 0.000494 & 0.000002\end{array}$

$\begin{array}{llllllll}8 & \mathrm{H} & -0.000007 & 0.000001 & 0.000001 & -0.000002 & -0.000020 & 0.000000\end{array}$

$\begin{array}{llllllll}9 \mathrm{H} & 0.000381 & -0.000064 & -0.000023 & 0.000029 & 0.000025 & 0.000000\end{array}$

$\begin{array}{llllllll}10 & \mathrm{C} & 0.549893 & -0.036852 & -0.042725 & -0.032028 & -0.034088 & 0.001822\end{array}$

$\begin{array}{llllllll}11 & \mathrm{C} & -0.046531 & 0.550540 & 0.554792 & 0.002455 & 0.002442 & -0.029781\end{array}$

$\begin{array}{llllllll}12 & \mathrm{C} & -0.038914 & -0.035549 & 0.541542 & 0.380223 & 0.002399 & 0.000257\end{array}$

$\begin{array}{llllllll}13 & \mathrm{C} & 4.932612 & 0.547579 & -0.035365 & 0.002595 & 0.381305 & -0.029913\end{array}$

$\begin{array}{llllllll}14 & \mathrm{C} & 0.547579 & 4.882098 & -0.042419 & 0.000160 & -0.028486 & 0.381910\end{array}$

$\begin{array}{llllllll}15 & \mathrm{C} & -0.035365 & -0.042419 & 4.889604 & -0.029990 & 0.000185 & 0.002461\end{array}$

$\begin{array}{llllllll}16 \mathrm{H} & 0.002595 & 0.000160 & -0.029990 & 0.481969 & -0.000106 & 0.000010\end{array}$

$\begin{array}{llllllll}17 & \mathrm{H} & 0.381305 & -0.028486 & 0.000185 & -0.000106 & 0.479256 & -0.001389\end{array}$

$\begin{array}{lllllllll}18 \mathrm{H} & -0.029913 & 0.381910 & 0.002461 & 0.000010 & -0.001389 & 0.473576\end{array}$

$\begin{array}{llllllll}19 & \mathrm{H} & 0.000232 & 0.002460 & 0.381086 & -0.001413 & 0.000010 & -0.000109\end{array}$

$\begin{array}{llllllll}20 \mathrm{H} & 0.002513 & -0.030363 & -0.030473 & -0.000111 & -0.000112 & -0.001483\end{array}$

$\begin{array}{llllllll}21 \mathrm{H} & -0.000008 & 0.000000 & 0.000000 & 0.000000 & 0.000000 & 0.000000\end{array}$

$\begin{array}{llllllll}22 & \mathrm{C} & 0.000005 & 0.000001 & -0.000003 & -0.000092 & 0.000000 & 0.000000\end{array}$

$\begin{array}{llllllll}23 & \mathrm{H} & 0.000000 & 0.000000 & 0.000000 & -0.000004 & 0.000000 & 0.000000\end{array}$

$\begin{array}{llllllll}24 & \mathrm{H} & 0.000001 & -0.000001 & 0.000006 & 0.000590 & -0.000001 & 0.000000\end{array}$

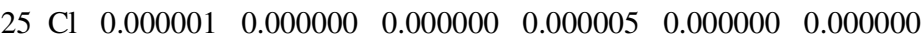

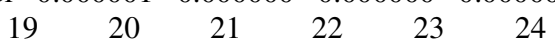

$\begin{array}{llllllll}1 & \mathrm{C} & 0.000000 & 0.000000 & 0.392991 & 0.321282 & -0.038793 & -0.039097\end{array}$

$\begin{array}{llllllll}2 & \mathrm{C} & 0.000003 & 0.000000 & -0.030871 & -0.042860 & 0.001195 & 0.000618\end{array}$

$\begin{array}{llllllll}3 \mathrm{H} & 0.000000 & 0.000000 & -0.003654 & 0.002955 & -0.000094 & 0.000047\end{array}$

$\begin{array}{llllllll}4 \mathrm{C} & -0.000106 & 0.000006 & 0.002752 & -0.007499 & 0.000095 & 0.000256\end{array}$

$\begin{array}{llllllll}5 & \mathrm{H} & -0.000009 & 0.000000 & 0.000068 & -0.001868 & 0.000072 & 0.000391\end{array}$

$\begin{array}{llllllll}6 \mathrm{C} & 0.000002 & 0.000000 & -0.000107 & 0.000230 & -0.000008 & 0.000061\end{array}$

$\begin{array}{llllllll}7 \mathrm{H} & 0.000000 & 0.000000 & 0.000000 & -0.000006 & 0.000000 & -0.000001\end{array}$

$\begin{array}{llllllll}8 \mathrm{H} & 0.000000 & 0.000000 & -0.000012 & 0.000000 & 0.000000 & -0.000017\end{array}$

$\begin{array}{llllllll}9 \mathrm{H} & 0.000000 & 0.000000 & 0.000002 & 0.000002 & 0.000000 & 0.000006\end{array}$

$\begin{array}{llllllll}10 & \mathrm{C} & 0.002118 & 0.000258 & -0.000152 & -0.000037 & 0.000059 & -0.000268\end{array}$

$\begin{array}{llllllll}11 & \mathrm{C} & -0.030003 & 0.382212 & 0.000000 & 0.000000 & 0.000000 & -0.000001\end{array}$

$\begin{array}{llllllll}12 & \mathrm{C} & -0.029458 & 0.002474 & -0.000001 & -0.000355 & -0.000002 & 0.000899\end{array}$

$\begin{array}{llllllll}13 & \mathrm{C} & 0.000232 & 0.002513 & -0.000008 & 0.000005 & 0.000000 & 0.000001\end{array}$

$\begin{array}{llllllll}14 & \mathrm{C} & 0.002460 & -0.030363 & 0.000000 & 0.000001 & 0.000000 & -0.000001\end{array}$

$\begin{array}{llllllll}15 \mathrm{C} & 0.381086 & -0.030473 & 0.000000 & -0.000003 & 0.000000 & 0.000006\end{array}$

$\begin{array}{llllllll}16 & \mathrm{H} & -0.001413 & -0.000111 & 0.000000 & -0.000092 & -0.000004 & 0.000590\end{array}$

$\begin{array}{llllllll}17 & \mathrm{H} & 0.000010 & -0.000112 & 0.000000 & 0.000000 & 0.000000 & -0.000001\end{array}$

$\begin{array}{llllllll}18 & \mathrm{H} & -0.000109 & -0.001483 & 0.000000 & 0.000000 & 0.000000 & 0.000000\end{array}$

$\begin{array}{llllllll}19 & \mathrm{H} & 0.474324 & -0.001546 & 0.000000 & 0.000000 & 0.000000 & 0.000000\end{array}$

$\begin{array}{llllllll}20 \mathrm{H} & -0.001546 & 0.476105 & 0.000000 & 0.000000 & 0.000000 & 0.000000\end{array}$

$\begin{array}{llllllll}21 \mathrm{H} & 0.000000 & 0.000000 & 0.483127 & -0.042609 & -0.002415 & 0.002746\end{array}$

$\begin{array}{llllllll}22 & \mathrm{C} & 0.000000 & 0.000000 & -0.042609 & 5.210987 & 0.390643 & 0.389079\end{array}$

$\begin{array}{llllllll}23 & \mathrm{H} & 0.000000 & 0.000000 & -0.002415 & 0.390643 & 0.505117 & -0.027646\end{array}$

$\begin{array}{lllllllll}24 & \mathrm{H} & 0.000000 & 0.000000 & 0.002746 & 0.389079 & -0.027646 & 0.499708\end{array}$

$\begin{array}{lllllllll}25 & \mathrm{Cl} & 0.000000 & 0.000000 & 0.001877 & 0.203125 & -0.049740 & -0.046010\end{array}$ 25

1 C -0.083861

2 C -0.000933

$3 \mathrm{H}-0.000223$

4 C 0.000216 


\begin{abstract}
$\begin{array}{lll}5 \mathrm{H} & 0.000410\end{array}$
$6 \mathrm{C} \quad 0.000010$

$7 \mathrm{H} \quad 0.000000$

$8 \mathrm{H} \quad 0.000014$

$9 \mathrm{H}-0.000002$

$10 \mathrm{C}-0.000015$

$11 \mathrm{C} \quad 0.000000$

$12 \mathrm{C} \quad 0.000002$

$13 \mathrm{C} \quad 0.000001$

$14 \mathrm{C} \quad 0.000000$

$15 \mathrm{C} \quad 0.000000$

$16 \mathrm{H} \quad 0.000005$

$17 \mathrm{H} \quad 0.000000$

$18 \mathrm{H} \quad 0.000000$

$19 \mathrm{H} \quad 0.000000$

$20 \mathrm{H} \quad 0.000000$

$21 \mathrm{H} \quad 0.001877$

$22 \mathrm{C} \quad 0.203125$

$23 \mathrm{H}-0.049740$

$24 \mathrm{H}-0.046010$

$25 \mathrm{Cl} 17.067477$
\end{abstract}

Mulliken atomic charges:

$$
1
$$

$1 \mathrm{C}-0.171018$

2 C -0.127105

$3 \mathrm{H} \quad 0.186710$

$\begin{array}{lll}4 \mathrm{C} & -0.251087\end{array}$

$5 \mathrm{H} \quad 0.190829$

6 C -0.472864

$\begin{array}{lll}7 \mathrm{H} & 0.156934\end{array}$

$8 \mathrm{H} \quad 0.174486$

$9 \mathrm{H} \quad 0.174809$

$10 \mathrm{C} \quad 0.067115$

11 C -0.207645

$\begin{array}{lll}12 & \mathrm{C} & -0.236777\end{array}$

13 C -0.220809

14 C -0.193299

15 C -0.192167

$16 \mathrm{H} \quad 0.196276$

$\begin{array}{lll}17 \mathrm{H} & 0.198869\end{array}$

$18 \mathrm{H} \quad 0.202731$

$19 \mathrm{H} \quad 0.202407$

$20 \mathrm{H} \quad 0.200521$

$21 \mathrm{H} \quad 0.196265$

22 C -0.422981

$23 \mathrm{H} \quad 0.221521$

$24 \mathrm{H} \quad 0.218633$

$25 \mathrm{Cl}-0.092352$

Sum of Mulliken charges $=0.00000$

Atomic charges with hydrogens summed into heavy atoms: 1

1 C 0.025247

2 C 0.059605

$3 \mathrm{H} \quad 0.000000$

4 C -0.060258

$\begin{array}{lll}5 \mathrm{H} & 0.000000\end{array}$

$6 \mathrm{C} \quad 0.033365$

$7 \mathrm{H} \quad 0.000000$

$8 \mathrm{H} \quad 0.000000$

$9 \mathrm{H} \quad 0.000000$

$10 \mathrm{C} \quad 0.067115$

$11 \mathrm{C}-0.007124$

12 C -0.040501

13 C -0.021940 
14 C 0.009431

$15 \mathrm{C} \quad 0.010240$

$16 \mathrm{H} \quad 0.000000$

$17 \mathrm{H} \quad 0.000000$

$18 \mathrm{H} \quad 0.000000$

$19 \mathrm{H} \quad 0.000000$

$20 \mathrm{H} \quad 0.000000$

$\begin{array}{lll}21 \mathrm{H} & 0.000000\end{array}$

$22 \mathrm{C} \quad 0.017173$

$23 \mathrm{H} \quad 0.000000$

$24 \mathrm{H} \quad 0.000000$

$25 \mathrm{Cl}-0.092352$

Sum of Mulliken charges $=0.00000$

Electronic spatial extent $(\mathrm{au}):<\mathrm{R} * * 2>=3250.5998$

Charge $=0.0000$ electrons

Dipole moment (field-independent basis, Debye):

$\mathrm{X}=2.2951 \mathrm{Y}=-0.0990 \mathrm{Z}=0.6956 \mathrm{Tot}=2.4003$

Quadrupole moment (field-independent basis, Debye-Ang):

$\mathrm{XX}=-88.8681 \quad \mathrm{YY}=-75.4444 \quad \mathrm{ZZ}=-78.8540$

$\mathrm{XY}=-0.9004 \quad \mathrm{XZ}=-0.4383 \quad \mathrm{YZ}=2.7937$

Traceless Quadrupole moment (field-independent basis, Debye-Ang):

$\mathrm{XX}=-7.8126 \mathrm{YY}=5.6111 \mathrm{ZZ}=2.2015$

$\mathrm{XY}=-0.9004 \quad \mathrm{XZ}=-0.4383 \quad \mathrm{YZ}=2.7937$

Octapole moment (field-independent basis, Debye-Ang**2):

$\mathrm{XXX}=58.1492 \mathrm{YYY}=-6.7075 \mathrm{ZZZ}=0.9083 \mathrm{XYY}=1.1395$

$\mathrm{XXY}=-5.2537 \mathrm{XXZ}=20.6638 \mathrm{XZZ}=-12.9151 \mathrm{YZZ}=1.2809$

$\mathrm{YYZ}=-0.0430 \mathrm{XYZ}=9.7560$

Hexadecapole moment (field-independent basis, Debye-Ang**3):

$X X X X=-3400.9195 \mathrm{YYYY}=-718.9240 \mathrm{ZZZZ}=-367.9901 \mathrm{XXXY}=-70.0854$

$\mathrm{XXXZ}=58.2254 \mathrm{YYYX}=1.2312 \mathrm{YYYZ}=18.3163 \mathrm{ZZZX}=-4.4361$

$\mathrm{ZZZY}=6.8069 \mathrm{XXYY}=-654.2214 \mathrm{XXZZ}=-606.6464 \mathrm{YYZZ}=-174.0231$

$\mathrm{XXYZ}=8.1975 \mathrm{YYXZ}=-8.6092 \mathrm{ZZXY}=10.0371$

$\mathrm{N}-\mathrm{N}=7.206321232396 \mathrm{D}+02 \mathrm{E}-\mathrm{N}=-3.521905322942 \mathrm{D}+03 \quad \mathrm{KE}=8.826874325322 \mathrm{D}+02$

1|1|UNPC-UNKISPIRHF|6-31G(d)|C11H13Cl1|PCUSER|23-Apr-2006|0||\# HF/6-31

G(D) GEOM=CONNECTIVITYIITitle Card Required\|0,1|C,0,2.1683125,-0.1207

$5,0.49001042|\mathrm{C}, 0,1.3363125,-0.64375,-0.42798958| \mathrm{H}, 0,1.5743125,-1.63775$

,-0.80698958IC $, 0,0.1043125,0.00425,-1.02498958 \mathrm{IH}, 0,0.1523125,1.08725,-$ $0.85598958 \mid \mathrm{C}, 0,0.0893125,-0.16975,-2.54898958 \mathrm{IH}, 0,0.0773125,-1.22475,-$ $2.84698958 \mathrm{lH}, 0,0.9753125,0.29025,-2.99998958 \mid \mathrm{H}, 0,-0.7946875,0.30725,-2$ $.98698958|\mathrm{C}, 0,-1.1706875,-0.52275,-0.38598958| \mathrm{C}, 0,-3.5326875,-1.45775$, $0.82201042|\mathrm{C}, 0,-1.9836875,0.34025,0.36701042| \mathrm{C}, 0,-1.5626875,-1.86575,-$ $0.51698958 \mid \mathrm{C}, 0,-2.7356875,-2.32775,0.08201042 \mathrm{IC}, 0,-3.1566875,-0.12475$, $0.96501042 \mathrm{lH}, 0,-1.7106875,1.38525,0.49101042 \mid \mathrm{H}, 0,-0.9546875,-2.56475,-$ $1.08598958 \mathrm{lH}, 0,-3.0266875,-3.36975,-0.02798958 \mathrm{lH}, 0,-3.7776875,0.55525$, $1.54301042|\mathrm{H}, 0,-4.4456875,-1.81875,1.28801042| \mathrm{H}, 0,3.0243125,-0.72175,0$ $.79601042 \mathrm{lC}, 0,2.0443125,1.19525,1.19001042 \mid \mathrm{H}, 0,2.1933125,1.06025,2.265$ $01042|\mathrm{H}, 0,1.0733125,1.67325,1.04601042| \mathrm{Cl}, 0,3.2963125,2.30225,0.595010$ $42 \|$ Version $=x 86-$ Win32-G03RevB.05|State $=1-A|H F=-883.5819938| \mathrm{RMSD}=1.169 \mathrm{e}$ -005|Dipole=-0.7064939,-0.6137301,0.1264684|PG=C01 [X(C11H13Cl1)]||@

CHEMISTRY BEGAN BY SAYING IT WOULD CHANGE THE BASER METALS INTO GOLD. BY NOT DOING THAT IT HAS DONE MUCH GREATER THINGS. -- RALPH WALDO EMERSON

Job cpu time: 0 days 0 hours 2 minutes 6.0 seconds.

File lengths (MBytes): RWF $=33 \mathrm{Int}=0 \mathrm{D} 2 \mathrm{E}=0 \mathrm{Chk}=9 \mathrm{Scr}=1$ Normal termination of Gaussian 03 at Sun Apr 23 12:47:23 2006. 
Entering Link $1=\mathrm{C}: \mathrm{G} 03 \mathrm{~W} \backslash 11$.exe PID $=600$.

Copyright (c) 1988,1990,1992,1993,1995,1998,2003, Gaussian, Inc. All Rights Reserved.

This is the Gaussian(R) 03 program. It is based on the the Gaussian(R) 98 system (copyright 1998, Gaussian, Inc.), the Gaussian(R) 94 system (copyright 1995, Gaussian, Inc.), the Gaussian 92(TM) system (copyright 1992, Gaussian, Inc.), the Gaussian 90(TM) system (copyright 1990, Gaussian, Inc.), the Gaussian 88(TM) system (copyright 1988, Gaussian, Inc.), the Gaussian 86(TM) system (copyright 1986, Carnegie Mellon University), and the Gaussian 82(TM) system (copyright 1983, Carnegie Mellon University). Gaussian is a federally registered trademark of Gaussian, Inc.

This software contains proprietary and confidential information, including trade secrets, belonging to Gaussian, Inc.

This software is provided under written license and may be used, copied, transmitted, or stored only in accord with that written license.

The following legend is applicable only to US Government contracts under DFARS:

\section{RESTRICTED RIGHTS LEGEND}

Use, duplication or disclosure by the US Government is subject to restrictions as set forth in subparagraph (c)(1)(ii) of the Rights in Technical Data and Computer Software clause at DFARS 252.227-7013.

Gaussian, Inc.

Carnegie Office Park, Building 6, Pittsburgh, PA 15106 USA

The following legend is applicable only to US Government contracts under FAR:

\section{RESTRICTED RIGHTS LEGEND}

Use, reproduction and disclosure by the US Government is subject to restrictions as set forth in subparagraph (c) of the Commercial Computer Software - Restricted Rights clause at FAR 52.227-19.

Gaussian, Inc.

Carnegie Office Park, Building 6, Pittsburgh, PA 15106 USA

Warning -- This program may not be used in any manner that competes with the business of Gaussian, Inc. or will provide assistance to any competitor of Gaussian, Inc. The licensee of this program is prohibited from giving any competitor of Gaussian, Inc. access to this program. By using this program, the user acknowledges that Gaussian, Inc. is engaged in the business of creating and licensing software in the field of computational chemistry and represents and warrants to the licensee that it is not a competitor of Gaussian, Inc. and that 
it will not use this program in any manner prohibited above.

Cite this work as:

Gaussian 03, Revision B.05,

M. J. Frisch, G. W. Trucks, H. B. Schlegel, G. E. Scuseria,

M. A. Robb, J. R. Cheeseman, J. A. Montgomery, Jr., T. Vreven,

K. N. Kudin, J. C. Burant, J. M. Millam, S. S. Iyengar, J. Tomasi,

V. Barone, B. Mennucci, M. Cossi, G. Scalmani, N. Rega,

G. A. Petersson, H. Nakatsuji, M. Hada, M. Ehara, K. Toyota,

R. Fukuda, J. Hasegawa, M. Ishida, T. Nakajima, Y. Honda, O. Kitao,

H. Nakai, M. Klene, X. Li, J. E. Knox, H. P. Hratchian, J. B. Cross,

C. Adamo, J. Jaramillo, R. Gomperts, R. E. Stratmann, O. Yazyev,

A. J. Austin, R. Cammi, C. Pomelli, J. W. Ochterski, P. Y. Ayala,

K. Morokuma, G. A. Voth, P. Salvador, J. J. Dannenberg,

V. G. Zakrzewski, S. Dapprich, A. D. Daniels, M. C. Strain,

O. Farkas, D. K. Malick, A. D. Rabuck, K. Raghavachari,

J. B. Foresman, J. V. Ortiz, Q. Cui, A. G. Baboul, S. Clifford,

J. Cioslowski, B. B. Stefanov, G. Liu, A. Liashenko, P. Piskorz,

I. Komaromi, R. L. Martin, D. J. Fox, T. Keith, M. A. Al-Laham,

C. Y. Peng, A. Nanayakkara, M. Challacombe, P. M. W. Gill,

B. Johnson, W. Chen, M. W. Wong, C. Gonzalez, and J. A. Pople, Gaussian, Inc., Pittsburgh PA, 2003.

$* * * * * * * * * * * * * * * * * * * * * * * * * * * * * * * * * * * * * * * * * * * * * *$

Gaussian 03: x86-Win32-G03RevB.05 8-Nov-2003

$$
\text { 23-Apr-2006 }
$$

****************************************************

$\%$ chk=17_alkene.chk

$\%$ mem $=64 \mathrm{MB}$

$\%$ nproc $=1$

Will use up to 1 processors via shared memory.

\# hf/6-31g(d) geom=connectivity

$1 / 38=1,57=2 / 1$;

$2 / 17=6,18=5,40=1 / 2$;

$3 / 5=1,6=6,7=1,11=9,16=1,25=1,30=1 / 1,2,3$;

$4 / / 1$

$5 / 5=2,32=1,38=5 / 2$;

$6 / 7=2,8=2,9=2,10=2,28=1 / 1$;

$99 / 5=1,9=1 / 99$;

Title Card Required

Symbolic Z-matrix:

Charge $=0$ Multiplicity $=1$

$\begin{array}{llcc}\mathrm{C} & -1.93929 & 0.36879 & -0.23416 \\ \mathrm{C} & -0.74529 & 0.06579 & -0.77316 \\ \mathrm{H} & -0.70129 & -0.42721 & -1.74216 \\ \mathrm{C} & 0.58371 & 0.32579 & -0.09916 \\ \mathrm{H} & 0.42171 & 0.69879 & 0.92184 \\ \mathrm{C} & 1.46571 & 1.37479 & -0.85416 \\ \mathrm{O} & 1.28371 & -0.92521 & -0.04616 \\ \mathrm{C} & 0.90571 & -1.72021 & 1.07484 \\ \mathrm{H} & -0.18429 & -1.77321 & 1.16584 \\ \mathrm{H} & 1.26171 & -2.74321 & 0.90684 \\ \mathrm{O} & 1.43571 & -1.21221 & 2.29484 \\ \mathrm{C} & 2.82471 & -1.48221 & 2.41884 \\ \mathrm{H} & 3.17971 & -1.03121 & 3.34884 \\ \mathrm{H} & 3.38571 & -1.05021 & 1.58684 \\ \mathrm{H} & 3.00071 & -2.56121 & 2.46884 \\ \mathrm{C} & 1.81771 & 0.92479 & -2.28716\end{array}$




$\begin{array}{lrrr}\mathrm{H} & 2.32271 & -0.04721 & -2.29116 \\ \mathrm{H} & 2.48771 & 1.64479 & -2.77016 \\ \mathrm{H} & 0.92571 & 0.84379 & -2.91716 \\ \mathrm{C} & 2.79071 & 1.56979 & -0.08516 \\ \mathrm{H} & 3.39971 & 2.35979 & -0.53916 \\ \mathrm{H} & 3.39271 & 0.65479 & -0.08616 \\ \mathrm{H} & 2.60471 & 1.85179 & 0.95684 \\ \mathrm{C} & 0.74771 & 2.73779 & -0.92116 \\ \mathrm{H} & 1.39171 & 3.50179 & -1.37116 \\ \mathrm{H} & 0.47071 & 3.08579 & 0.07984 \\ \mathrm{H} & -0.16429 & 2.68679 & -1.52616 \\ \mathrm{H} & -1.98129 & 0.84879 & 0.74184 \\ \mathrm{C} & -3.25429 & 0.09379 & -0.89016 \\ \mathrm{H} & -3.13929 & -0.34921 & -1.88416 \\ \mathrm{H} & -3.82929 & 1.01879 & -0.98616 \\ \mathrm{Cl} & -4.18729 & -1.03921 & 0.10884\end{array}$

Input orientation:

\begin{tabular}{|c|c|c|c|c|c|}
\hline \multirow{2}{*}{$\begin{array}{l}\text { Center } \\
\text { Number }\end{array}$} & \multirow{2}{*}{\multicolumn{2}{|c|}{$\begin{array}{l}\text { Atomic } \\
\text { Number }\end{array}$}} & \multirow{2}{*}{$\begin{array}{l}\text { Atomic } \\
\text { Type }\end{array}$} & \multicolumn{2}{|c|}{ Coordinates (Angstroms) } \\
\hline & & & & X $\quad \mathrm{Y}$ & $\mathrm{Z}$ \\
\hline 1 & 6 & 0 & -1.939286 & 0.368786 & -0.234161 \\
\hline 2 & 6 & 0 & -0.745286 & 0.065786 & -0.773161 \\
\hline 3 & 1 & 0 & -0.701286 & -0.427214 & -1.742161 \\
\hline 4 & 6 & 0 & 0.583714 & 0.325786 & -0.099161 \\
\hline 5 & 1 & 0 & 0.421714 & 0.698786 & 0.921839 \\
\hline 6 & 6 & 0 & 1.465714 & 1.374786 & -0.854161 \\
\hline 7 & 8 & 0 & 1.283714 & -0.925214 & -0.046161 \\
\hline 8 & 6 & 0 & 0.905714 & -1.720214 & 1.074839 \\
\hline 9 & 1 & 0 & -0.184286 & -1.773214 & 1.165839 \\
\hline 10 & 1 & 0 & 1.261714 & -2.743214 & 0.906839 \\
\hline 11 & 8 & 0 & 1.435714 & -1.212214 & 2.294839 \\
\hline 12 & 6 & 0 & 2.824714 & -1.482214 & 2.418839 \\
\hline 13 & 1 & 0 & 3.179714 & -1.031214 & 3.348839 \\
\hline 14 & 1 & 0 & 3.385714 & -1.050214 & 1.586839 \\
\hline 15 & 1 & 0 & 3.000714 & -2.561214 & 2.468839 \\
\hline 16 & 6 & 0 & 1.817714 & 0.924786 & -2.287161 \\
\hline 17 & 1 & 0 & 2.322714 & -0.047214 & -2.291161 \\
\hline 18 & 1 & 0 & 2.487714 & 1.644786 & -2.770161 \\
\hline 19 & 1 & 0 & 0.925714 & 0.843786 & -2.917161 \\
\hline 20 & 6 & 0 & 2.790714 & 1.569786 & -0.085161 \\
\hline 21 & 1 & 0 & 3.399714 & 2.359786 & -0.539161 \\
\hline 22 & 1 & 0 & 3.392714 & 0.654786 & -0.086161 \\
\hline 23 & 1 & 0 & 2.604714 & 1.851786 & 0.956839 \\
\hline 24 & 6 & 0 & 0.747714 & 2.737786 & -0.921161 \\
\hline 25 & 1 & 0 & 1.391714 & 3.501786 & -1.371161 \\
\hline 26 & 1 & 0 & 0.470714 & 3.085786 & 0.079839 \\
\hline 27 & 1 & 0 & -0.164286 & 2.686786 & -1.526161 \\
\hline 28 & 1 & 0 & -1.981286 & 0.848786 & 0.741839 \\
\hline 29 & 6 & 0 & -3.254286 & 0.093786 & -0.890161 \\
\hline 30 & 1 & 0 & -3.139286 & -0.349214 & -1.884161 \\
\hline 31 & 1 & 0 & -3.829286 & 1.018786 & -0.986161 \\
\hline 32 & 17 & 0 & -4.187286 & -1.039214 & $\begin{array}{ll}4 & 0.108839\end{array}$ \\
\hline
\end{tabular}

Distance matrix (angstroms):
$1 \mathrm{C} \quad 0.000000$
$\begin{array}{llll}2 & \mathrm{C} & 1.344606 & 0.000000\end{array}$
$\begin{array}{lllll}3 \mathrm{H} & 2.107208 & 1.088093 & 0.000000\end{array}$
$\begin{array}{llllll}4 & \mathrm{C} & 2.526975 & 1.512652 & 2.217585 & 0.000000\end{array}$
$\begin{array}{lllllll}5 & \mathrm{H} & 2.649445 & 2.153045 & 3.102564 & 1.099006 & 0.000000\end{array}$
$\begin{array}{lllllll}6 & \mathrm{C} & 3.604228 & 2.570713 & 2.954934 & 1.564720 & 2.168199\end{array}$
$\begin{array}{lllllll}7 & \mathrm{O} & 3.478147 & 2.372225 & 2.657940 & 1.434507 & 2.077846\end{array}$ 
$\begin{array}{lllllll}8 \mathrm{C} & 3.764496 & 3.054620 & 3.491388 & 2.380772 & 2.471685\end{array}$

$\begin{array}{lllllll}9 \mathrm{H} & 3.102932 & 2.730634 & 3.245839 & 2.568239 & 2.556864\end{array}$

$\begin{array}{lllllll}10 \mathrm{H} & 4.607909 & 3.839392 & 4.029197 & 3.300073 & 3.543048\end{array}$

$\begin{array}{lllllll}11 & \mathrm{O} & 4.504001 & 3.975257 & 4.634691 & 2.970283 & 2.562274\end{array}$

$\begin{array}{lllllll}12 & \mathrm{C} & 5.758499 & 5.032899 & 5.555144 & 3.825084 & 3.573819\end{array}$

$\begin{array}{lllllll}13 & \mathrm{H} & 6.403284 & 5.796544 & 6.430028 & 4.524309 & 4.060763\end{array}$

$\begin{array}{lllllll}14 & \mathrm{H} & 5.803898 & 4.886739 & 5.307913 & 3.547841 & 3.505214\end{array}$

$\begin{array}{lllllll}15 & \mathrm{H} & 6.347811 & 5.607514 & 5.999273 & 4.557552 & 4.435318\end{array}$

$\begin{array}{lllllll}16 & \mathrm{C} & 4.317290 & 3.098233 & 2.910376 & 2.582422 & 3.506790\end{array}$

$\begin{array}{lllllll}17 & \mathrm{H} & 4.750679 & 3.424867 & 3.096833 & 2.822785 & 3.807057\end{array}$

$\begin{array}{llllllll}18 & \mathrm{H} & 5.259068 & 4.115038 & 3.939504 & 3.535423 & 4.335220\end{array}$

$\begin{array}{lllllll}19 & \mathrm{H} & 3.953775 & 2.827412 & 2.375541 & 2.885552 & 3.874656\end{array}$

$\begin{array}{lllllll}20 \mathrm{C} & 4.882366 & 3.903672 & 4.350600 & 2.533492 & 2.717508\end{array}$

$\begin{array}{llllllll}21 & \mathrm{H} & 5.706315 & 4.743229 & 5.102233 & 3.501516 & 3.709707\end{array}$

$\begin{array}{lllllll}22 & \mathrm{H} & 5.341715 & 4.235792 & 4.546856 & 2.828231 & 3.137649\end{array}$

$\begin{array}{llllllll}23 & \mathrm{H} & 4.926023 & 4.171953 & 4.838190 & 2.743766 & 2.469033\end{array}$

$\begin{array}{llllllll}24 & \mathrm{C} & 3.647478 & 3.064398 & 3.576432 & 2.553493 & 2.767751\end{array}$

$\begin{array}{llllllll}25 & \mathrm{H} & 4.712114 & 4.090290 & 4.467139 & 3.515370 & 3.749074\end{array}$

$\begin{array}{lllllll}26 & \mathrm{H} & 3.645379 & 3.365511 & 4.127280 & 2.768106 & 2.531627\end{array}$

$\begin{array}{llllllll}27 & \mathrm{H} & 3.192650 & 2.788227 & 3.167337 & 2.858348 & 3.207529\end{array}$

$\begin{array}{lllllll}28 & \mathrm{H} & 1.088458 & 2.106184 & 3.071943 & 2.749552 & 2.414396\end{array}$

$\begin{array}{lllllll}29 & \mathrm{C} & 1.495054 & 2.511883 & 2.741378 & 3.925525 & 4.142746\end{array}$

$\begin{array}{lllllll}30 & \mathrm{H} & 2.162874 & 2.671663 & 2.443377 & 4.183608 & 4.653242\end{array}$

$\begin{array}{lllllll}31 & \mathrm{H} & 2.135440 & 3.234909 & 3.528007 & 4.554293 & 4.670532\end{array}$

$\begin{array}{lllllll}32 & \mathrm{Cl} & 2.674625 & 3.721063 & 3.994113 & 4.966783 & 4.992444\end{array}$ $\begin{array}{lllll}6 & 7 & 8 & 9 & 10\end{array}$

$\begin{array}{lll}6 \mathrm{C} & 0.000000\end{array}$

$\begin{array}{llll}7 & \mathrm{O} & 2.444583 & 0.000000\end{array}$

$\begin{array}{lllll}8 \mathrm{C} & 3.689670 & 1.425325 & 0.000000\end{array}$

$\begin{array}{llllll}9 & \mathrm{H} & 4.088130 & 2.084004 & 1.095075 & 0.000000\end{array}$

$\begin{array}{lllllll}10 \mathrm{H} & 4.483376 & 2.052758 & 1.096125 & 1.760368 & 0.000000\end{array}$

$\begin{array}{lllllll}11 & \mathrm{O} & 4.075496 & 2.363420 & 1.423855 & 2.052745 & 2.073832\end{array}$

$\begin{array}{lllllll}12 & \mathrm{C} & 4.552127 & 2.959925 & 2.354897 & 3.272426 & 2.513809\end{array}$

$\begin{array}{llllllll}13 & \mathrm{H} & 5.137299 & 3.889997 & 3.288901 & 4.078302 & 3.545847\end{array}$

$\begin{array}{lllllll}14 & \mathrm{H} & 3.940242 & 2.664717 & 2.619436 & 3.666725 & 2.800004\end{array}$

$\begin{array}{lllllll}15 & \mathrm{H} & 5.375002 & 3.456850 & 2.653214 & 3.530294 & 2.344587\end{array}$

$\begin{array}{lllllll}16 & \mathrm{C} & 1.542690 & 2.954613 & 4.373878 & 4.817719 & 4.895406\end{array}$

$\begin{array}{lllllll}17 & \mathrm{H} & 2.195792 & 2.624963 & 4.017060 & 4.605972 & 4.315245\end{array}$

$\begin{array}{lllllll}18 \mathrm{H} & 2.188250 & 3.933789 & 5.348829 & 5.857850 & 5.854737\end{array}$

$\begin{array}{lllllll}19 & \mathrm{H} & 2.197619 & 3.391189 & 4.744529 & 4.975106 & 5.253803\end{array}$

$\begin{array}{lllllll}20 & \mathrm{C} & 1.544348 & 2.915063 & 3.965214 & 4.646641 & 4.682294\end{array}$

$\begin{array}{lllllll}21 & \mathrm{H} & 2.193127 & 3.938493 & 5.046923 & 5.730076 & 5.718616\end{array}$

$\begin{array}{llllllll}22 & \mathrm{H} & 2.195803 & 2.635504 & 3.629561 & 4.500846 & 4.132023\end{array}$

$\begin{array}{lllllll}23 & \mathrm{H} & 2.191933 & 3.234622 & 3.957235 & 4.578518 & 4.787502\end{array}$

$\begin{array}{lllllll}24 & \mathrm{C} & 1.542006 & 3.804010 & 4.886997 & 5.057006 & 5.800616\end{array}$

$\begin{array}{llllllll}25 & \mathrm{H} & 2.190181 & 4.622296 & 5.786916 & 6.061829 & 6.648775\end{array}$

$\begin{array}{lllllll}26 & \mathrm{H} & 2.188584 & 4.094504 & 4.927158 & 5.021783 & 5.940274\end{array}$

$\begin{array}{llllllll}27 & \mathrm{H} & 2.197687 & 4.163370 & 5.227978 & 5.209497 & 6.118649\end{array}$

$\begin{array}{llllllll}28 & \mathrm{H} & 3.834801 & 3.798453 & 3.878842 & 3.206847 & 4.842183\end{array}$

$\begin{array}{llllllll}29 & \mathrm{C} & 4.890875 & 4.726959 & 4.945444 & 4.139774 & 5.627791\end{array}$

$\begin{array}{lllllll}30 & \mathrm{H} & 5.023853 & 4.824204 & 5.195897 & 4.479096 & 5.734956\end{array}$

$\begin{array}{llllllll}31 & \mathrm{H} & 5.308595 & 5.550271 & 5.845517 & 5.070739 & 6.607146\end{array}$

$\begin{array}{lllllll}32 & \mathrm{Cl} & 6.221830 & 5.474382 & 5.228343 & 4.204761 & 5.764722\end{array}$ $\begin{array}{lllll}11 & 12 & 13 & 14 & 15\end{array}$

$\begin{array}{lll}11 & \mathrm{O} & 0.000000\end{array}$

$\begin{array}{llll}12 & \mathrm{C} & 1.420421 & 0.000000\end{array}$

$\begin{array}{llll}13 \mathrm{H} & 2.045779 & 1.092852 & 0.000000\end{array}$

$\begin{array}{llllll}14 \mathrm{H} & 2.080867 & 1.092506 & 1.774103 & 0.000000\end{array}$

$\begin{array}{lllllll}15 & \mathrm{H} & 2.073476 & 1.094403 & 1.774075 & 1.791444 & 0.000000\end{array}$

$\begin{array}{lllllll}16 & \mathrm{C} & 5.070248 & 5.380905 & 6.119271 & 4.622459 & 6.014251\end{array}$

$\begin{array}{llllllll}17 & \mathrm{H} & 4.814082 & 4.949276 & 5.788981 & 4.144257 & 5.425632\end{array}$

$\begin{array}{lllllll}18 \mathrm{H} & 5.909600 & 6.067736 & 6.714313 & 5.201238 & 6.738006\end{array}$

$\begin{array}{llllllll}19 & \mathrm{H} & 5.626027 & 6.122857 & 6.918013 & 5.470361 & 6.701391\end{array}$

$\begin{array}{lllllll}20 & \mathrm{C} & 3.903838 & 3.947895 & 4.325376 & 3.164492 & 4.861294\end{array}$ 
Center Atomic Atomic Coordinates (Angstroms) Number Number Type X $\quad$ Y $\quad$ Z 


\begin{tabular}{cccccc}
\hline 1 & 6 & 0 & -1.887573 & -0.474383 & -0.404588 \\
2 & 6 & 0 & -0.807098 & -0.597476 & 0.386227 \\
3 & 1 & 0 & -0.930345 & -0.905956 & 1.422372 \\
4 & 6 & 0 & 0.607889 & -0.290667 & -0.051718 \\
5 & 1 & 0 & 0.604469 & 0.137821 & -1.063746 \\
6 & 6 & 0 & 1.536262 & -1.550060 & -0.071824 \\
7 & 8 & 0 & 1.138652 & 0.669379 & 0.872627 \\
8 & 6 & 0 & 0.778944 & 2.008439 & 0.542359 \\
9 & 1 & 0 & -0.292921 & 2.083745 & 0.331112 \\
10 & 1 & 0 & 0.977467 & 2.642933 & 1.413849 \\
11 & 8 & 0 & 1.496304 & 2.492371 & -0.588379 \\
12 & 6 & 0 & 2.842906 & 2.806303 & -0.263256 \\
13 & 1 & 0 & 3.349828 & 3.127819 & -1.176483 \\
14 & 1 & 0 & 3.365504 & 1.933675 & 0.135469 \\
15 & 1 & 0 & 2.879497 & 3.627195 & 0.459596 \\
16 & 6 & 0 & 1.673595 & -2.201810 & 1.319670 \\
17 & 1 & 0 & 2.042917 & -1.487892 & 2.063812 \\
18 & 1 & 0 & 2.378195 & -3.040456 & 1.291374 \\
19 & 1 & 0 & 0.717563 & -2.597790 & 1.677898 \\
20 & 6 & 0 & 2.948712 & -1.130829 & -0.534686 \\
21 & 1 & 0 & 3.605447 & -2.001709 & -0.641289 \\
22 & 1 & 0 & 3.421471 & -0.453881 & 0.184946 \\
23 & 1 & 0 & 2.911850 & -0.622475 & -1.504274 \\
24 & 6 & 0 & 1.001042 & -2.601372 & -1.064832 \\
25 & 1 & 0 & 1.689272 & -3.449820 & -1.150894 \\
26 & 1 & 0 & 0.878872 & -2.171241 & -2.064780 \\
27 & 1 & 0 & 0.031870 & -3.002367 & -0.748163 \\
28 & 1 & 0 & -1.763334 & -0.151612 & -1.436636 \\
29 & 6 & 0 & -3.288848 & -0.757596 & 0.032910 \\
30 & 1 & 0 & -3.338145 & -1.117857 & 1.065040 \\
31 & 1 & 0 & -3.743854 & -1.507922 & -0.619353 \\
32 & 17 & 0 & -4.252956 & 0.729441 & -0.073847 \\
----------------------------------------------------------- \\
\hline
\end{tabular}

Rotational constants (GHZ): $\quad 0.7778263 \quad 0.3727537 \quad 0.2775442$

Standard basis: 6-31G(d) $(6 \mathrm{D}, 7 \mathrm{~F})$

There are 237 symmetry adapted basis functions of A symmetry.

Integral buffers will be 262144 words long.

Raffenetti 1 integral format.

Two-electron integral symmetry is turned on.

237 basis functions, 464 primitive gaussians, 237 cartesian basis functions

56 alpha electrons 56 beta electrons

nuclear repulsion energy $\quad 950.4203400965$ Hartrees.

NAtoms $=32$ NActive $=32 \mathrm{NUniq}=32 \mathrm{SFac}=1.00 \mathrm{D}+00 \mathrm{NAtFMM}=60 \mathrm{Big}=\mathrm{F}$

One-electron integrals computed using PRISM.

NBasis $=237$ RedAO $=\mathrm{T}$ NBF $=237$

NBsUse $=2371.00 \mathrm{D}-06 \mathrm{NBFU}=237$

Harris functional with IExCor $=205$ diagonalized for initial guess.

ExpMin= 1.43D-01 ExpMax= 2.52D+04 ExpMxC=3.78D+03 IAcc=1 IRadAn $=1$ AccDes= 1.00D-06

HarFok: IExCor $=205$ AccDes $=1.00 \mathrm{D}-06 \mathrm{IRadAn}=1 \mathrm{IDoV}=1$

ScaDFX $=1.0000001 .0000001 .0000001 .000000$

Initial guess orbital symmetries:

Occupied (A) (A) (A) (A) (A) (A) (A) (A) (A) (A) (A) (A)

(A) (A) (A) (A) (A) (A) (A) (A) (A) (A) (A) (A)

(A) (A) (A) (A) (A) (A) (A) (A) (A) (A) (A) (A)

(A) (A) (A) (A) (A) (A) (A) (A) (A) (A) (A) (A)

(A) $(\mathrm{A})(\mathrm{A})(\mathrm{A})(\mathrm{A})(\mathrm{A})(\mathrm{A})(\mathrm{A})$

Virtual (A) (A) (A) (A) (A) (A) (A) (A) (A) (A) (A) (A)

(A) (A) (A) (A) (A) (A) (A) (A) (A) (A) (A) (A)

(A) $(\mathrm{A})(\mathrm{A})(\mathrm{A})(\mathrm{A})(\mathrm{A})(\mathrm{A})(\mathrm{A})(\mathrm{A})(\mathrm{A})(\mathrm{A})(\mathrm{A})$

(A) $(A)(A)(A)(A)(A)(A)(A)(A)(A)(A)(A)$

(A) $(\mathrm{A})(\mathrm{A})(\mathrm{A})(\mathrm{A})(\mathrm{A})(\mathrm{A})(\mathrm{A})(\mathrm{A})(\mathrm{A})(\mathrm{A})(\mathrm{A})$

(A) $(A)(A)(A)(A)(A)(A)(A)(A)(A)(A)(A)$

(A) $(\mathrm{A})(\mathrm{A})(\mathrm{A})(\mathrm{A})(\mathrm{A})(\mathrm{A})(\mathrm{A})(\mathrm{A})(\mathrm{A})(\mathrm{A})(\mathrm{A})$ 
(A) $(\mathrm{A})(\mathrm{A})(\mathrm{A})(\mathrm{A})(\mathrm{A})(\mathrm{A})(\mathrm{A})(\mathrm{A})(\mathrm{A})(\mathrm{A})(\mathrm{A})$

(A) (A) (A) (A) (A) (A) (A) (A) (A) (A) (A) (A)

(A) $(A)(A)(A)(A)(A)(A)(A)(A)(A)(A)(A)$

(A) (A) (A) (A) (A) (A) (A) (A) (A) (A) (A) (A)

(A) $(\mathrm{A})(\mathrm{A})(\mathrm{A})(\mathrm{A})(\mathrm{A})(\mathrm{A})(\mathrm{A})(\mathrm{A})(\mathrm{A})(\mathrm{A})(\mathrm{A})$

(A) (A) (A) (A) (A) (A) (A) (A) (A) (A) (A) (A)

(A) $(\mathrm{A})(\mathrm{A})(\mathrm{A})(\mathrm{A})(\mathrm{A})(\mathrm{A})(\mathrm{A})(\mathrm{A})(\mathrm{A})(\mathrm{A})(\mathrm{A})$

(A) $(\mathrm{A})(\mathrm{A})(\mathrm{A})(\mathrm{A})(\mathrm{A})(\mathrm{A})(\mathrm{A})(\mathrm{A})(\mathrm{A})(\mathrm{A})(\mathrm{A})$

(A)

The electronic state of the initial guess is 1-A.

Warning! Cutoffs for single-point calculations used.

Requested convergence on RMS density matrix=1.00D-04 within 128 cycles.

Requested convergence on MAX density matrix $=1.00 \mathrm{D}-02$.

Requested convergence on energy=5.00D-05.

No special actions if energy rises.

SCF Done: $\mathrm{E}(\mathrm{RHF})=-998.905272020$ A.U. after 6 cycles

Convg $=0.7633 \mathrm{D}-05 \quad-\mathrm{V} / \mathrm{T}=2.0013$

$\mathrm{S}^{* * 2}=0.0000$

******************************************************************************

Population analysis using the SCF density.

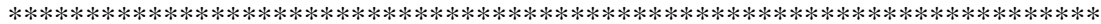

Orbital symmetries:

Occupied (A) (A) (A) (A) (A) (A) (A) (A) (A) (A) (A) (A)

(A) $(A)(A)(A)(A)(A)(A)(A)(A)(A)(A)(A)$

(A) $(\mathrm{A})(\mathrm{A})(\mathrm{A})(\mathrm{A})(\mathrm{A})(\mathrm{A})(\mathrm{A})(\mathrm{A})(\mathrm{A})(\mathrm{A})(\mathrm{A})$

$(\mathrm{A})(\mathrm{A})(\mathrm{A})(\mathrm{A})(\mathrm{A})(\mathrm{A})(\mathrm{A})(\mathrm{A})(\mathrm{A})(\mathrm{A})(\mathrm{A})(\mathrm{A})$

(A) $(A)(A)(A)(A)(A)(A)(A)$

Virtual (A) (A) (A) (A) (A) (A) (A) (A) (A) (A) (A) (A)

(A) (A) (A) (A) (A) (A) (A) (A) (A) (A) (A) (A)

(A) (A) (A) (A) (A) (A) (A) (A) (A) (A) (A) (A)

(A) (A) (A) (A) (A) (A) (A) (A) (A) (A) (A) (A)

(A) (A) (A) (A) (A) (A) (A) (A) (A) (A) (A) (A)

(A) (A) (A) (A) (A) (A) (A) (A) (A) (A) (A) (A)

(A) (A) (A) (A) (A) (A) (A) (A) (A) (A) (A) (A)

(A) (A) (A) (A) (A) (A) (A) (A) (A) (A) (A) (A)

(A) (A) (A) (A) (A) (A) (A) (A) (A) (A) (A) (A)

(A) (A) (A) (A) (A) (A) (A) (A) (A) (A) (A) (A)

(A) (A) (A) (A) (A) (A) (A) (A) (A) (A) (A) (A)

(A) (A) (A) (A) (A) (A) (A) (A) (A) (A) (A) (A)

(A) (A) (A) (A) (A) (A) (A) (A) (A) (A) (A) (A)

(A) $(\mathrm{A})(\mathrm{A})(\mathrm{A})(\mathrm{A})(\mathrm{A})(\mathrm{A})(\mathrm{A})(\mathrm{A})(\mathrm{A})(\mathrm{A})(\mathrm{A})$

(A) $(\mathrm{A})(\mathrm{A})(\mathrm{A})(\mathrm{A})(\mathrm{A})(\mathrm{A})(\mathrm{A})(\mathrm{A})(\mathrm{A})(\mathrm{A})(\mathrm{A})$

(A)

The electronic state is $1-\mathrm{A}$.

Alpha occ. eigenvalues -- -104.81025 -20.57019 -20.56869 -11.33092 -11.29963

Alpha occ. eigenvalues -- -11.29000 -11.27107 -11.24947 -11.24374 -11.24237

Alpha occ. eigenvalues -- -11.21621 -11.21194 -11.21057 -10.54459 -8.01394

Alpha occ. eigenvalues -- $\quad-8.01062 \quad-8.01055-1.40576 \quad-1.34174 \quad-1.15044$

Alpha occ. eigenvalues -- $\quad-1.10707 \quad-1.05614 \quad-0.97142 \quad-0.94524 \quad-0.92953$

Alpha occ. eigenvalues -- $-0.92634 \quad-0.88824 \quad-0.84023 \quad-0.77851 \quad-0.74286$

Alpha occ. eigenvalues -- $\quad-0.69195 \quad-0.67599 \quad-0.67276 \quad-0.64734 \quad-0.63041$

Alpha occ. eigenvalues -- $\quad-0.61432 \quad-0.60387 \quad-0.59637 \quad-0.58159 \quad-0.57409$

Alpha occ. eigenvalues -- $\quad-0.56111 \quad-0.55088 \quad-0.54007 \quad-0.53005 \quad-0.52635$

Alpha occ. eigenvalues -- $-0.51386 \quad-0.50797 \quad-0.47912-0.46865 \quad-0.45778$

Alpha occ. eigenvalues -- $-0.45207 \quad-0.44324 \quad-0.43857 \quad-0.43447 \quad-0.42240$

Alpha occ. eigenvalues -- -0.36441

$\begin{array}{lllllll}\text { Alpha virt. eigenvalues -- } & 0.14929 & 0.21022 & 0.22266 & 0.23912 & 0.25187\end{array}$

$\begin{array}{llllll}\text { Alpha virt. eigenvalues -- } & 0.26831 & 0.26923 & 0.27882 & 0.28974 & 0.29330\end{array}$

$\begin{array}{lllllll}\text { Alpha virt. eigenvalues -- } & 0.29618 & 0.30165 & 0.30552 & 0.31056 & 0.32384\end{array}$

$\begin{array}{lllllll}\text { Alpha virt. eigenvalues -- } & 0.33129 & 0.33624 & 0.34193 & 0.34952 & 0.36904\end{array}$ 
$\begin{array}{lllllll}\text { Alpha virt. eigenvalues -- } & 0.37457 & 0.38744 & 0.39802 & 0.40684 & 0.41983\end{array}$ $\begin{array}{lllllll}\text { Alpha virt. eigenvalues -- } & 0.42294 & 0.43336 & 0.43734 & 0.46487 & 0.47490\end{array}$ $\begin{array}{llllllll}\text { Alpha virt. eigenvalues -- } & 0.51416 & 0.57449 & 0.58917 & 0.64852 & 0.65375\end{array}$ $\begin{array}{lllllll}\text { Alpha virt. eigenvalues -- } & 0.67912 & 0.69488 & 0.69920 & 0.71344 & 0.71641\end{array}$ $\begin{array}{lllllll}\text { Alpha virt. eigenvalues -- } & 0.73358 & 0.75036 & 0.76164 & 0.77126 & 0.77465\end{array}$ $\begin{array}{lllllll}\text { Alpha virt. eigenvalues -- } & 0.79349 & 0.80746 & 0.81554 & 0.83778 & 0.85311\end{array}$ $\begin{array}{llllll}\text { Alpha virt. eigenvalues -- } & 0.87343 & 0.90395 & 0.90864 & 0.92469 & 0.93683\end{array}$ $\begin{array}{lllllll}\text { Alpha virt. eigenvalues -- } & 0.96487 & 0.96796 & 1.00159 & 1.02842 & 1.03169\end{array}$ $\begin{array}{lllllll}\text { Alpha virt. eigenvalues -- } & 1.04393 & 1.06225 & 1.08555 & 1.09206 & 1.09672\end{array}$ $\begin{array}{llllll}\text { Alpha virt. eigenvalues -- } & 1.11360 & 1.12273 & 1.13018 & 1.13262 & 1.14157\end{array}$ $\begin{array}{lllllll}\text { Alpha virt. eigenvalues -- } & 1.14486 & 1.14789 & 1.15902 & 1.16483 & 1.16931\end{array}$ $\begin{array}{lllllll}\text { Alpha virt. eigenvalues -- } & 1.17791 & 1.18378 & 1.18790 & 1.19797 & 1.20531\end{array}$ $\begin{array}{lllllll}\text { Alpha virt. eigenvalues -- } & 1.20842 & 1.21738 & 1.21797 & 1.23623 & 1.24523\end{array}$ $\begin{array}{lllllll}\text { Alpha virt. eigenvalues -- } & 1.26003 & 1.26513 & 1.28810 & 1.30889 & 1.34691\end{array}$ $\begin{array}{lllllll}\text { Alpha virt. eigenvalues -- } & 1.34919 & 1.38377 & 1.40553 & 1.43300 & 1.44730\end{array}$ $\begin{array}{llllll}\text { Alpha virt. eigenvalues -- } & 1.47495 & 1.50330 & 1.51970 & 1.52902 & 1.57469\end{array}$ $\begin{array}{lllllll}\text { Alpha virt. eigenvalues -- } & 1.59619 & 1.61834 & 1.66463 & 1.70985 & 1.74635\end{array}$ $\begin{array}{lllllll}\text { Alpha virt. eigenvalues -- } & 1.76066 & 1.77004 & 1.79144 & 1.83009 & 1.84152\end{array}$ $\begin{array}{lllllll}\text { Alpha virt. eigenvalues -- } & 1.85997 & 1.87669 & 1.92641 & 1.93532 & 1.99680\end{array}$ $\begin{array}{lllllll}\text { Alpha virt. eigenvalues -- } & 2.01768 & 2.03468 & 2.07010 & 2.08017 & 2.11099\end{array}$ $\begin{array}{lllllll}\text { Alpha virt. eigenvalues -- } & 2.16381 & 2.17171 & 2.19472 & 2.19991 & 2.23263\end{array}$ $\begin{array}{llllll}\text { Alpha virt. eigenvalues -- } & 2.27077 & 2.28998 & 2.30014 & 2.33110 & 2.34610\end{array}$ $\begin{array}{lllllll}\text { Alpha virt. eigenvalues -- } & 2.36150 & 2.37941 & 2.40440 & 2.42056 & 2.42563\end{array}$ $\begin{array}{lllllll}\text { Alpha virt. eigenvalues -- } & 2.44585 & 2.45158 & 2.47746 & 2.48821 & 2.50311\end{array}$ $\begin{array}{lllllll}\text { Alpha virt. eigenvalues -- } & 2.53267 & 2.53555 & 2.57915 & 2.59026 & 2.60701\end{array}$ $\begin{array}{lllllll}\text { Alpha virt. eigenvalues -- } & 2.61159 & 2.61823 & 2.62119 & 2.64168 & 2.67787\end{array}$ $\begin{array}{lllllll}\text { Alpha virt. eigenvalues -- } & 2.69252 & 2.77588 & 2.80294 & 2.82875 & 2.88220\end{array}$ $\begin{array}{lllllll}\text { Alpha virt. eigenvalues -- } & 2.92023 & 2.93162 & 2.94086 & 3.03628 & 3.06524\end{array}$ $\begin{array}{lllllll}\text { Alpha virt. eigenvalues -- } & 3.08928 & 3.10675 & 3.13417 & 3.22151 & 3.24150\end{array}$ $\begin{array}{lllllll}\text { Alpha virt. eigenvalues -- } & 3.28627 & 3.33255 & 3.41152 & 4.32483 & 4.43038\end{array}$ $\begin{array}{lllllll}\text { Alpha virt. eigenvalues -- } & 4.52314 & 4.60243 & 4.67903 & 4.68601 & 4.75230\end{array}$ $\begin{array}{lllllll}\text { Alpha virt. eigenvalues -- } & 4.75503 & 4.78153 & 4.79455 & 4.85220 & 4.95969\end{array}$ Alpha virt. eigenvalues -- 5.13525

Condensed to atoms (all electrons):

$$
\begin{array}{llllll}
1 & 2 & 3 & 4 & 5 & 6
\end{array}
$$

$\begin{array}{llllllll}1 & C & 5.060112 & 0.633258 & -0.038762 & -0.037411 & 0.005430 & -0.002301\end{array}$

$\begin{array}{llllllll}2 & \mathrm{C} & 0.633258 & 5.029455 & 0.385060 & 0.363681 & -0.060488 & -0.059947\end{array}$

$\begin{array}{lllllllll}3 \mathrm{H} & -0.038762 & 0.385060 & 0.472696 & -0.024846 & 0.002557 & -0.002771\end{array}$

$\begin{array}{llllllll}4 & \mathrm{C} & -0.037411 & 0.363681 & -0.024846 & 4.829588 & 0.411077 & 0.382213\end{array}$

$\begin{array}{llllllll}5 \mathrm{H} & 0.005430 & -0.060488 & 0.002557 & 0.411077 & 0.509712 & -0.039190\end{array}$

$\begin{array}{llllllll}6 \mathrm{C} & -0.002301 & -0.059947 & -0.002771 & 0.382213 & -0.039190 & 5.050017\end{array}$

$\begin{array}{llllllll}7 & \mathrm{O} & 0.000436 & -0.048336 & 0.001064 & 0.163998 & -0.030487 & -0.050567\end{array}$

$\begin{array}{lllllllll}8 & \mathrm{C} & -0.001601 & -0.009597 & 0.000172 & -0.029251 & -0.007694 & 0.003856\end{array}$

$\begin{array}{llllllll}9 \mathrm{H} & 0.003026 & 0.006725 & 0.000083 & -0.006547 & -0.000717 & -0.000167\end{array}$

$\begin{array}{llllllll}10 \mathrm{H} & -0.000009 & 0.000055 & -0.000007 & 0.004574 & 0.000281 & -0.000152\end{array}$

$\begin{array}{llllllll}11 & \mathrm{O} & 0.000014 & 0.000209 & 0.000003 & -0.002628 & 0.008222 & -0.000565\end{array}$

$\begin{array}{llllllll}12 & \mathrm{C} & -0.000001 & -0.000010 & 0.000000 & -0.000317 & -0.000315 & 0.000047\end{array}$

$\begin{array}{llllllll}13 \mathrm{H} & 0.000000 & 0.000000 & 0.000000 & -0.000021 & -0.000022 & 0.000003\end{array}$

$\begin{array}{llllllll}14 \mathrm{H} & 0.000000 & 0.000007 & 0.000000 & -0.000284 & -0.000145 & 0.000136\end{array}$

$\begin{array}{llllllll}15 \mathrm{H} & 0.000000 & 0.000001 & 0.000000 & 0.000052 & 0.000043 & -0.000010\end{array}$

$\begin{array}{llllllll}16 & \mathrm{C} & 0.000600 & -0.006088 & 0.000466 & -0.058625 & 0.004136 & 0.355008\end{array}$

$\begin{array}{llllllll}17 & \mathrm{H} & -0.000009 & 0.000411 & 0.000259 & -0.005358 & 0.000043 & -0.032239\end{array}$

$\begin{array}{llllllll}18 & \mathrm{H} & -0.000005 & -0.000148 & 0.000027 & 0.004663 & -0.000045 & -0.035182\end{array}$

$\begin{array}{llllllll}19 \mathrm{H} & 0.000155 & 0.002240 & -0.000475 & -0.005274 & -0.000131 & -0.034248\end{array}$

$\begin{array}{llllllll}20 & \mathrm{C} & -0.000099 & 0.005056 & -0.000092 & -0.065230 & -0.005995 & 0.360195\end{array}$

$\begin{array}{llllllll}21 \mathrm{H} & -0.000001 & -0.000067 & 0.000002 & 0.004743 & -0.000062 & -0.035118\end{array}$

$\begin{array}{llllllll}22 & \mathrm{H} & 0.000000 & -0.000036 & 0.000001 & -0.004826 & 0.000254 & -0.032034\end{array}$

$\begin{array}{llllllll}23 & \mathrm{H} & 0.000004 & -0.000072 & -0.000002 & -0.004373 & 0.002731 & -0.036126\end{array}$

$\begin{array}{llllllll}24 & \mathrm{C} & -0.001034 & -0.003462 & 0.000447 & -0.061658 & -0.000458 & 0.357108\end{array}$

$\begin{array}{llllllll}25 & \mathrm{H} & 0.000054 & 0.000191 & -0.000003 & 0.004319 & -0.000076 & -0.032109\end{array}$

$\begin{array}{llllllll}26 \mathrm{H} & 0.000518 & -0.000444 & 0.000004 & -0.002898 & 0.002451 & -0.037625\end{array}$

$\begin{array}{llllllll}27 & \mathrm{H} & 0.001282 & 0.004021 & -0.000027 & -0.004321 & 0.000139 & -0.034171\end{array}$

$\begin{array}{llllllll}28 & \mathrm{H} & 0.391469 & -0.034876 & 0.002881 & -0.002308 & 0.003065 & 0.000002\end{array}$

$\begin{array}{llllllll}29 & \mathrm{C} & 0.319135 & -0.042420 & -0.002539 & 0.002451 & 0.000137 & -0.000141\end{array}$ 
$\begin{array}{lllllll}30 \mathrm{H} & -0.044212 & 0.003048 & 0.002857 & 0.000134 & 0.000008 & -0.000007\end{array}$ $\begin{array}{llllllll}31 & \mathrm{H} & -0.039940 & 0.000037 & 0.000067 & -0.000104 & -0.000007 & 0.000012\end{array}$

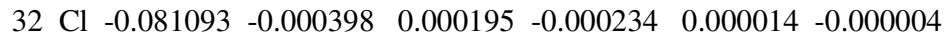
$\begin{array}{cccccccc} & 7 & 8 & 9 & 10 & 11 & 12 & \\ \text { C } & 0.000436 & -0.001601 & 0.003026 & -0.000009 & 0.000014 & -0.000001\end{array}$

$\begin{array}{llllllll}2 & \mathrm{C} & -0.048336 & -0.009597 & 0.006725 & 0.000055 & 0.000209 & -0.000010\end{array}$

$\begin{array}{llllllll}3 \mathrm{H} & 0.001064 & 0.000172 & 0.000083 & -0.000007 & 0.000003 & 0.000000\end{array}$

$\begin{array}{llllllll}4 & \mathrm{C} & 0.163998 & -0.029251 & -0.006547 & 0.004574 & -0.002628 & -0.000317\end{array}$

$\begin{array}{llllllll}5 & \mathrm{H} & -0.030487 & -0.007694 & -0.000717 & 0.000281 & 0.008222 & -0.000315\end{array}$

$\begin{array}{llllllll}6 & \mathrm{C} & -0.050567 & 0.003856 & -0.000167 & -0.000152 & -0.000565 & 0.000047\end{array}$

$\begin{array}{lllllllll}7 & \mathrm{O} & 8.529697 & 0.214109 & -0.038629 & -0.039124 & -0.053087 & 0.000981\end{array}$

$\begin{array}{llllllll}8 & \mathrm{C} & 0.214109 & 4.566387 & 0.406990 & 0.406781 & 0.225522 & -0.047023\end{array}$

$\begin{array}{lllllllll}9 \mathrm{H} & -0.038629 & 0.406990 & 0.517131 & -0.037454 & -0.038031 & 0.004628\end{array}$

$\begin{array}{llllllll}10 & \mathrm{H} & -0.039124 & 0.406781 & -0.037454 & 0.540827 & -0.039115 & -0.007926\end{array}$

$\begin{array}{llllllll}11 & \mathrm{O} & -0.053087 & 0.225522 & -0.038031 & -0.039115 & 8.426713 & 0.203154\end{array}$

$\begin{array}{llllllll}12 & \mathrm{C} & 0.000981 & -0.047023 & 0.004628 & -0.007926 & 0.203154 & 4.848000\end{array}$

$\begin{array}{llllllll}13 & \mathrm{H} & -0.000067 & 0.004745 & -0.000074 & -0.000194 & -0.030907 & 0.399819\end{array}$

$\begin{array}{llllllll}14 & \mathrm{H} & 0.005263 & -0.006778 & -0.000062 & -0.000448 & -0.036631 & 0.390115\end{array}$

$\begin{array}{llllllll}15 \mathrm{H} & -0.000337 & -0.007294 & -0.000071 & 0.006800 & -0.039129 & 0.390091\end{array}$

$\begin{array}{llllllll}16 & \mathrm{C} & -0.000880 & -0.000094 & -0.000009 & 0.000004 & -0.000001 & 0.000000\end{array}$

$\begin{array}{llllllll}17 & \mathrm{H} & 0.003806 & -0.000072 & 0.000010 & -0.000010 & 0.000002 & 0.000000\end{array}$

$\begin{array}{llllllll}18 \mathrm{H} & -0.000013 & 0.000004 & 0.000000 & 0.000000 & 0.000000 & 0.000000\end{array}$

$\begin{array}{llllllll}19 & \mathrm{H} & 0.000101 & -0.000010 & 0.000001 & 0.000000 & 0.000000 & 0.000000\end{array}$

$\begin{array}{llllllll}20 & \mathrm{C} & 0.002283 & -0.000192 & 0.000027 & 0.000013 & 0.000380 & -0.000788\end{array}$

$\begin{array}{llllllll}21 \mathrm{H} & 0.000090 & 0.000007 & 0.000000 & 0.000000 & 0.000002 & 0.000010\end{array}$

$\begin{array}{llllllll}22 & \mathrm{H} & 0.002302 & -0.000101 & 0.000010 & -0.000004 & 0.000043 & -0.000842\end{array}$

$\begin{array}{llllllll}23 & \mathrm{H} & 0.000057 & -0.000055 & -0.000002 & 0.000004 & 0.000249 & 0.000023\end{array}$

$\begin{array}{llllllll}24 & \mathrm{C} & 0.001948 & -0.000113 & 0.000006 & 0.000001 & 0.000005 & -0.000001\end{array}$

$\begin{array}{lllllllll}25 & \mathrm{H} & -0.000032 & 0.000002 & 0.000000 & 0.000000 & 0.000000 & 0.000000\end{array}$

$\begin{array}{llllllll}26 \mathrm{H} & -0.000002 & -0.000005 & 0.000000 & 0.000000 & -0.000004 & 0.000000\end{array}$

$\begin{array}{llllllll}27 & \mathrm{H} & 0.000005 & -0.000003 & 0.000000 & 0.000000 & 0.000000 & 0.000000\end{array}$

$\begin{array}{llllllll}28 \mathrm{H} & 0.000043 & 0.000046 & -0.000005 & -0.000001 & -0.000006 & 0.000000\end{array}$

$\begin{array}{llllllll}29 & \mathrm{C} & -0.000061 & -0.000004 & 0.000003 & -0.000001 & 0.000000 & 0.000000\end{array}$

$\begin{array}{llllllll}30 \mathrm{H} & -0.000001 & -0.000001 & 0.000002 & 0.000000 & 0.000000 & 0.000000\end{array}$

$\begin{array}{llllllll}31 \mathrm{H} & 0.000000 & -0.000001 & 0.000004 & 0.000000 & 0.000000 & 0.000000\end{array}$

$\begin{array}{llllllll}32 & \mathrm{Cl} & 0.000014 & -0.000016 & 0.000157 & 0.000000 & 0.000000 & 0.000000\end{array}$ $\begin{array}{llllll}13 & 14 & 15 & 16 & 17 & 18\end{array}$

$\begin{array}{llllllll}1 & \mathrm{C} & 0.000000 & 0.000000 & 0.000000 & 0.000600 & -0.000009 & -0.000005\end{array}$

$\begin{array}{llllllll}2 & \mathrm{C} & 0.000000 & 0.000007 & 0.000001 & -0.006088 & 0.000411 & -0.000148\end{array}$

$\begin{array}{llllllll}3 \mathrm{H} & 0.000000 & 0.000000 & 0.000000 & 0.000466 & 0.000259 & 0.000027\end{array}$

$\begin{array}{llllllll}4 \mathrm{C} & -0.000021 & -0.000284 & 0.000052 & -0.058625 & -0.005358 & 0.004663\end{array}$

$\begin{array}{llllllll}5 \mathrm{H} & -0.000022 & -0.000145 & 0.000043 & 0.004136 & 0.000043 & -0.000045\end{array}$

$\begin{array}{llllllll}6 & \mathrm{C} & 0.000003 & 0.000136 & -0.000010 & 0.355008 & -0.032239 & -0.035182\end{array}$

$\begin{array}{llllllll}7 & \mathrm{O} & -0.000067 & 0.005263 & -0.000337 & -0.000880 & 0.003806 & -0.000013\end{array}$

$\begin{array}{llllllll}8 \mathrm{C} & 0.004745 & -0.006778 & -0.007294 & -0.000094 & -0.000072 & 0.000004\end{array}$

$\begin{array}{llllllll}9 \mathrm{H} & -0.000074 & -0.000062 & -0.000071 & -0.000009 & 0.000010 & 0.000000\end{array}$

$\begin{array}{llllllll}10 \mathrm{H} & -0.000194 & -0.000448 & 0.006800 & 0.000004 & -0.000010 & 0.000000\end{array}$

$\begin{array}{llllllll}11 & \mathrm{O} & -0.030907 & -0.036631 & -0.039129 & -0.000001 & 0.000002 & 0.000000\end{array}$

$\begin{array}{llllllll}12 & \mathrm{C} & 0.399819 & 0.390115 & 0.390091 & 0.000000 & 0.000000 & 0.000000\end{array}$

$\begin{array}{llllllll}13 & \mathrm{H} & 0.511563 & -0.030845 & -0.030739 & 0.000000 & 0.000000 & 0.000000\end{array}$

$\begin{array}{llllllll}14 \mathrm{H} & -0.030845 & 0.549700 & -0.039951 & -0.000007 & 0.000001 & -0.000001\end{array}$

$\begin{array}{llllllll}15 \mathrm{H} & -0.030739 & -0.039951 & 0.573522 & 0.000000 & 0.000000 & 0.000000\end{array}$

$\begin{array}{llllllll}16 & \mathrm{C} & 0.000000 & -0.000007 & 0.000000 & 5.168389 & 0.390760 & 0.391722\end{array}$

$\begin{array}{llllllll}17 & \mathrm{H} & 0.000000 & 0.000001 & 0.000000 & 0.390760 & 0.506382 & -0.026322\end{array}$

$\begin{array}{lllllllll}18 \mathrm{H} & 0.000000 & -0.000001 & 0.000000 & 0.391722 & -0.026322 & 0.538174\end{array}$

$\begin{array}{llllllll}19 & \mathrm{H} & 0.000000 & 0.000000 & 0.000000 & 0.386579 & -0.025556 & -0.027948\end{array}$

$\begin{array}{llllllll}20 \mathrm{C} & 0.000018 & -0.000330 & 0.000023 & -0.076535 & -0.003115 & -0.004906\end{array}$

$\begin{array}{lllllllll}21 & \mathrm{H} & -0.000001 & -0.000005 & 0.000000 & -0.003980 & 0.000066 & 0.002034\end{array}$

$\begin{array}{llllllll}22 & \mathrm{H} & 0.000022 & 0.000500 & 0.000026 & -0.003610 & 0.002090 & 0.000042\end{array}$

$\begin{array}{llllllll}23 & \mathrm{H} & 0.000006 & -0.000131 & 0.000007 & 0.005123 & -0.000069 & -0.000012\end{array}$

$\begin{array}{llllllll}24 \mathrm{C} & 0.000000 & 0.000002 & 0.000000 & -0.071497 & 0.004607 & -0.003976\end{array}$

$\begin{array}{llllllll}25 \mathrm{H} & 0.000000 & 0.000000 & 0.000000 & -0.003512 & -0.000060 & 0.002080\end{array}$

$\begin{array}{lllllll}26 \mathrm{H} & 0.000000 & 0.000000 & 0.000000 & 0.005154 & -0.000100 & -0.000059\end{array}$

$\begin{array}{lllllllll}27 & \mathrm{H} & 0.000000 & 0.000000 & 0.000000 & -0.003732 & -0.000048 & 0.000029\end{array}$ 
$\begin{array}{lllllll}28 \mathrm{H} & 0.000000 & 0.000000 & 0.000000 & 0.000005 & 0.000000 & 0.000000\end{array}$ $\begin{array}{llllllll}29 & \mathrm{C} & 0.000000 & 0.000000 & 0.000000 & 0.000000 & 0.000000 & 0.000000\end{array}$ $\begin{array}{lllllll}30 \mathrm{H} & 0.000000 & 0.000000 & 0.000000 & 0.000000 & 0.000000 & 0.000000\end{array}$ $\begin{array}{llllllll}31 \mathrm{H} & 0.000000 & 0.000000 & 0.000000 & 0.000000 & 0.000000 & 0.000000\end{array}$ $\begin{array}{llllllll}32 & \mathrm{Cl} & 0.000000 & 0.000000 & 0.000000 & 0.000000 & 0.000000 & 0.000000\end{array}$ $\begin{array}{llllll}19 & 20 & 21 & 22 & 23 & 24\end{array}$

$\begin{array}{llllllll}1 & \mathrm{C} & 0.000155 & -0.000099 & -0.000001 & 0.000000 & 0.000004 & -0.001034\end{array}$

$\begin{array}{llllllll}2 & \mathrm{C} & 0.002240 & 0.005056 & -0.000067 & -0.000036 & -0.000072 & -0.003462\end{array}$

$\begin{array}{llllllll}3 \mathrm{H} & -0.000475 & -0.000092 & 0.000002 & 0.000001 & -0.000002 & 0.000447\end{array}$

$\begin{array}{llllllll}4 & \mathrm{C} & -0.005274 & -0.065230 & 0.004743 & -0.004826 & -0.004373 & -0.061658\end{array}$

$\begin{array}{llllllll}5 \mathrm{H} & -0.000131 & -0.005995 & -0.000062 & 0.000254 & 0.002731 & -0.000458\end{array}$

$\begin{array}{llllllll}6 \mathrm{C} & -0.034248 & 0.360195 & -0.035118 & -0.032034 & -0.036126 & 0.357108\end{array}$

$\begin{array}{llllllll}7 & \mathrm{O} & 0.000101 & 0.002283 & 0.000090 & 0.002302 & 0.000057 & 0.001948\end{array}$

$\begin{array}{llllllll}8 \mathrm{C} & -0.000010 & -0.000192 & 0.000007 & -0.000101 & -0.000055 & -0.000113\end{array}$

$\begin{array}{llllllll}9 & \mathrm{H} & 0.000001 & 0.000027 & 0.000000 & 0.000010 & -0.000002 & 0.000006\end{array}$

$\begin{array}{llllllll}10 \mathrm{H} & 0.000000 & 0.000013 & 0.000000 & -0.000004 & 0.000004 & 0.000001\end{array}$

$\begin{array}{llllllll}11 & \mathrm{O} & 0.000000 & 0.000380 & 0.000002 & 0.000043 & 0.000249 & 0.000005\end{array}$

$\begin{array}{llllllll}12 & \mathrm{C} & 0.000000 & -0.000788 & 0.000010 & -0.000842 & 0.000023 & -0.000001\end{array}$

$\begin{array}{llllllll}13 \mathrm{H} & 0.000000 & 0.000018 & -0.000001 & 0.000022 & 0.000006 & 0.000000\end{array}$

$\begin{array}{llllllll}14 \mathrm{H} & 0.000000 & -0.000330 & -0.000005 & 0.000500 & -0.000131 & 0.000002\end{array}$

$\begin{array}{llllllll}15 \mathrm{H} & 0.000000 & 0.000023 & 0.000000 & 0.000026 & 0.000007 & 0.000000\end{array}$

$\begin{array}{llllllll}16 & \mathrm{C} & 0.386579 & -0.076535 & -0.003980 & -0.003610 & 0.005123 & -0.071497\end{array}$

$\begin{array}{lllllllll}17 & \mathrm{H} & -0.025556 & -0.003115 & 0.000066 & 0.002090 & -0.000069 & 0.004607\end{array}$

$\begin{array}{llllllll}18 & \mathrm{H} & -0.027948 & -0.004906 & 0.002034 & 0.000042 & -0.000012 & -0.003976\end{array}$

$\begin{array}{llllllll}19 & \mathrm{H} & 0.549312 & 0.005127 & -0.000075 & -0.000034 & -0.000112 & -0.003730\end{array}$

$\begin{array}{llllllll}20 \mathrm{C} & 0.005127 & 5.164651 & 0.389259 & 0.390740 & 0.388420 & -0.072450\end{array}$

$\begin{array}{llllllll}21 & \mathrm{H} & -0.000075 & 0.389259 & 0.543064 & -0.027576 & -0.026768 & -0.004903\end{array}$

$\begin{array}{llllllll}22 & \mathrm{H} & -0.000034 & 0.390740 & -0.027576 & 0.515858 & -0.025282 & 0.004793\end{array}$

$\begin{array}{llllllll}23 \mathrm{H} & -0.000112 & 0.388420 & -0.026768 & -0.025282 & 0.539350 & -0.003074\end{array}$

$\begin{array}{llllllll}24 & \mathrm{C} & -0.003730 & -0.072450 & -0.004903 & 0.004793 & -0.003074 & 5.166439\end{array}$

$\begin{array}{llllllll}25 & \mathrm{H} & 0.000048 & -0.005205 & 0.002088 & -0.000025 & 0.000108 & 0.388960\end{array}$

$\begin{array}{llllllll}26 & \mathrm{H} & -0.000068 & -0.003431 & 0.000088 & -0.000079 & 0.002113 & 0.387856\end{array}$

$\begin{array}{llllllll}27 & \mathrm{H} & 0.002356 & 0.005004 & -0.000037 & -0.000101 & -0.000088 & 0.387281\end{array}$

$\begin{array}{llllllll}28 & \mathrm{H} & 0.000000 & -0.000013 & 0.000000 & 0.000000 & -0.000002 & 0.000050\end{array}$

$\begin{array}{llllllll}29 & \mathrm{C} & 0.000003 & 0.000001 & 0.000000 & 0.000000 & 0.000000 & -0.000009\end{array}$

$\begin{array}{llllllll}30 \mathrm{H} & 0.000004 & 0.000000 & 0.000000 & 0.000000 & 0.000000 & 0.000000\end{array}$

$\begin{array}{llllllll}31 \mathrm{H} & -0.000001 & 0.000000 & 0.000000 & 0.000000 & 0.000000 & 0.000010\end{array}$

$\begin{array}{llllllll}32 & \mathrm{Cl} & 0.000000 & 0.000000 & 0.000000 & 0.000000 & 0.000000 & 0.000000\end{array}$

$$
\begin{array}{llllll}
25 & 26 & 27 & 28 & 29 & 30
\end{array}
$$

$\begin{array}{llllllll}1 & \mathrm{C} & 0.000054 & 0.000518 & 0.001282 & 0.391469 & 0.319135 & -0.044212\end{array}$

$\begin{array}{llllllll}2 \mathrm{C} & 0.000191 & -0.000444 & 0.004021 & -0.034876 & -0.042420 & 0.003048\end{array}$

$\begin{array}{llllllll}3 \mathrm{H} & -0.000003 & 0.000004 & -0.000027 & 0.002881 & -0.002539 & 0.002857\end{array}$

$\begin{array}{llllllll}4 & \mathrm{C} & 0.004319 & -0.002898 & -0.004321 & -0.002308 & 0.002451 & 0.000134\end{array}$

$\begin{array}{llllllll}5 & \mathrm{H} & -0.000076 & 0.002451 & 0.000139 & 0.003065 & 0.000137 & 0.000008\end{array}$

$\begin{array}{llllllll}6 & \mathrm{C} & -0.032109 & -0.037625 & -0.034171 & 0.000002 & -0.000141 & -0.000007\end{array}$

$\begin{array}{llllllll}7 & \mathrm{O} & -0.000032 & -0.000002 & 0.000005 & 0.000043 & -0.000061 & -0.000001\end{array}$

$\begin{array}{llllllll}8 & \mathrm{C} & 0.000002 & -0.000005 & -0.000003 & 0.000046 & -0.000004 & -0.000001\end{array}$

$\begin{array}{llllllll}9 \mathrm{H} & 0.000000 & 0.000000 & 0.000000 & -0.000005 & 0.000003 & 0.000002\end{array}$

$\begin{array}{llllllll}10 \mathrm{H} & 0.000000 & 0.000000 & 0.000000 & -0.000001 & -0.000001 & 0.000000\end{array}$

$\begin{array}{llllllll}11 & \mathrm{O} & 0.000000 & -0.000004 & 0.000000 & -0.000006 & 0.000000 & 0.000000\end{array}$

$\begin{array}{llllllll}12 & \mathrm{C} & 0.000000 & 0.000000 & 0.000000 & 0.000000 & 0.000000 & 0.000000\end{array}$

$\begin{array}{llllllll}13 \mathrm{H} & 0.000000 & 0.000000 & 0.000000 & 0.000000 & 0.000000 & 0.000000\end{array}$

$\begin{array}{lllllll}14 \mathrm{H} & 0.000000 & 0.000000 & 0.000000 & 0.000000 & 0.000000 & 0.000000\end{array}$

$\begin{array}{llllllll}15 \mathrm{H} & 0.000000 & 0.000000 & 0.000000 & 0.000000 & 0.000000 & 0.000000\end{array}$

$\begin{array}{llllllll}16 & \mathrm{C} & -0.003512 & 0.005154 & -0.003732 & 0.000005 & 0.000000 & 0.000000\end{array}$

$\begin{array}{llllllll}17 & \mathrm{H} & -0.000060 & -0.000100 & -0.000048 & 0.000000 & 0.000000 & 0.000000\end{array}$

$\begin{array}{llllllll}18 \mathrm{H} & 0.002080 & -0.000059 & 0.000029 & 0.000000 & 0.000000 & 0.000000\end{array}$

$\begin{array}{llllllll}19 & \mathrm{H} & 0.000048 & -0.000068 & 0.002356 & 0.000000 & 0.000003 & 0.000004\end{array}$

$\begin{array}{llllllll}20 & \mathrm{C} & -0.005205 & -0.003431 & 0.005004 & -0.000013 & 0.000001 & 0.000000\end{array}$

$\begin{array}{llllllll}21 \mathrm{H} & 0.002088 & 0.000088 & -0.000037 & 0.000000 & 0.000000 & 0.000000\end{array}$

$\begin{array}{llllllll}22 & \mathrm{H} & -0.000025 & -0.000079 & -0.000101 & 0.000000 & 0.000000 & 0.000000\end{array}$

$\begin{array}{llllllll}23 & \mathrm{H} & 0.000108 & 0.002113 & -0.000088 & -0.000002 & 0.000000 & 0.000000\end{array}$

$\begin{array}{llllllll}24 & \mathrm{C} & 0.388960 & 0.387856 & 0.387281 & 0.000050 & -0.000009 & 0.000000\end{array}$

$\begin{array}{llllllll}25 & \mathrm{H} & 0.532070 & -0.026224 & -0.026889 & 0.000000 & 0.000000 & 0.000000\end{array}$ 


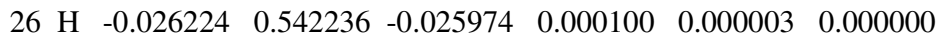

$\begin{array}{llllllll}27 & \mathrm{H} & -0.026889 & -0.025974 & 0.531228 & -0.000014 & 0.000048 & 0.000002\end{array}$

$\begin{array}{llllllll}28 & \mathrm{H} & 0.000000 & 0.000100 & -0.000014 & 0.470818 & -0.038163 & 0.002616\end{array}$

$\begin{array}{llllllll}29 & \mathrm{C} & 0.000000 & 0.000003 & 0.000048 & -0.038163 & 5.200171 & 0.391377\end{array}$

$\begin{array}{llllllll}30 \mathrm{H} & 0.000000 & 0.000000 & 0.000002 & 0.002616 & 0.391377 & 0.492825\end{array}$

$\begin{array}{llllllll}31 & \mathrm{H} & 0.000000 & -0.000002 & -0.000013 & -0.001255 & 0.385831 & -0.025899\end{array}$

$\begin{array}{llllllll}32 & \mathrm{Cl} & 0.000000 & 0.000000 & 0.000002 & 0.001004 & 0.203166 & -0.044515\end{array}$ $31 \quad 32$

1 C $-0.039940-0.081093$

$\begin{array}{llll}2 & \mathrm{C} & 0.000037 & -0.000398\end{array}$

$\begin{array}{llll}3 \mathrm{H} & 0.000067 & 0.000195\end{array}$

4 C $-0.000104-0.000234$

$\begin{array}{llll}5 & \mathrm{H} & -0.000007 & 0.000014\end{array}$

$6 \mathrm{C} \quad 0.000012-0.000004$

$\begin{array}{llll}7 & \mathrm{O} & 0.000000 & 0.000014\end{array}$

8 C $\quad-0.000001-0.000016$

$9 \mathrm{H} \quad 0.000004 \quad 0.000157$

$10 \mathrm{H} \quad 0.000000 \quad 0.000000$

$\begin{array}{llll}11 & \mathrm{O} & 0.000000 & 0.000000\end{array}$

$\begin{array}{llll}12 \mathrm{C} & 0.000000 & 0.000000\end{array}$

$\begin{array}{llll}13 \mathrm{H} & 0.000000 & 0.000000\end{array}$

$\begin{array}{llll}14 \mathrm{H} & 0.000000 & 0.000000\end{array}$

$15 \mathrm{H} \quad 0.000000 \quad 0.000000$

$\begin{array}{lll}16 \mathrm{C} & 0.000000 & 0.000000\end{array}$

$\begin{array}{llll}17 & \mathrm{H} & 0.000000 & 0.000000\end{array}$

$\begin{array}{llll}18 \mathrm{H} & 0.000000 & 0.000000\end{array}$

$\begin{array}{llll}19 \mathrm{H} & -0.000001 & 0.000000\end{array}$

$\begin{array}{llll}20 \mathrm{C} & 0.000000 & 0.000000\end{array}$

$\begin{array}{llll}21 \mathrm{H} & 0.000000 & 0.000000\end{array}$

$\begin{array}{llll}22 \mathrm{H} & 0.000000 & 0.000000\end{array}$

$\begin{array}{llll}23 \mathrm{H} & 0.000000 & 0.000000\end{array}$

$\begin{array}{llll}24 \mathrm{C} & 0.000010 & 0.000000\end{array}$

$\begin{array}{llll}25 \mathrm{H} & 0.000000 & 0.000000\end{array}$

$\begin{array}{llll}26 & \mathrm{H} & -0.000002 & 0.000000\end{array}$

$\begin{array}{llll}27 & \mathrm{H} & -0.000013 & 0.000002\end{array}$

$\begin{array}{llll}28 \mathrm{H} & -0.001255 & 0.001004\end{array}$

$\begin{array}{llll}29 & \mathrm{C} & 0.385831 & 0.203166\end{array}$

$\begin{array}{llll}30 \mathrm{H} & -0.025899 & -0.044515\end{array}$

$\begin{array}{llll}31 & \mathrm{H} & 0.512356 & -0.050538\end{array}$

$32 \mathrm{Cl}-0.050538 \quad 17.062876$

Mulliken atomic charges:

1 C -0.169011

$\begin{array}{lll}2 & \mathrm{C} & -0.167068\end{array}$

$3 \mathrm{H} \quad 0.200682$

4 C 0.145019

$5 \mathrm{H} \quad 0.195533$

6 C -0.043927

$\begin{array}{lll}7 & \mathrm{O} & -0.664574\end{array}$

8 C 0.281286

$9 \mathrm{H} \quad 0.182965$

$10 \mathrm{H} \quad 0.165103$

$\begin{array}{lll}11 & \mathrm{O} & -0.624414\end{array}$

$\begin{array}{lll}12 & \mathrm{C} & -0.179647\end{array}$

$13 \mathrm{H} \quad 0.176691$

$14 \mathrm{H} \quad 0.169895$

$15 \mathrm{H} \quad 0.146966$

16 C -0.479375

$17 \mathrm{H} \quad 0.184523$

$18 \mathrm{H} \quad 0.159843$

$19 \mathrm{H} \quad 0.151736$

$20 \mathrm{C}-0.472814$

$21 \mathrm{H} \quad 0.157139$

$\begin{array}{lll}22 & \mathrm{H} & 0.177867\end{array}$ 

$23 \mathrm{H} \quad 0.157973$
24 C -0.473150
$\begin{array}{lll}25 \mathrm{H} & 0.164217\end{array}$
$26 \mathrm{H} \quad 0.156391$
$\begin{array}{lll}27 \mathrm{H} & 0.164021\end{array}$
$\begin{array}{lll}28 \mathrm{H} & 0.204544\end{array}$
29 C -0.418987
$\begin{array}{lll}30 \mathrm{H} & 0.221764\end{array}$
$\begin{array}{lll}31 & \mathrm{H} & 0.219442\end{array}$
$32 \mathrm{Cl}-0.090632$

Sum of Mulliken charges $=0.00000$

Atomic charges with hydrogens summed into heavy atoms: 1

1 C 0.035533

$2 \mathrm{C} \quad 0.033614$

$3 \mathrm{H} \quad 0.000000$

4 C 0.340552

$\begin{array}{lll}5 \mathrm{H} & 0.000000\end{array}$

$\begin{array}{lll}6 & \mathrm{C} & -0.043927\end{array}$

$\begin{array}{lll}7 & \mathrm{O} & -0.664574\end{array}$

8 C 0.629353

$9 \mathrm{H} \quad 0.000000$

$10 \mathrm{H} \quad 0.000000$

$110 \quad-0.624414$

12 C 0.313905

$13 \mathrm{H} \quad 0.000000$

$14 \mathrm{H} \quad 0.000000$

$\begin{array}{ll}15 \mathrm{H} & 0.000000\end{array}$

$16 \mathrm{C} \quad 0.016727$

$17 \mathrm{H} \quad 0.000000$

$18 \mathrm{H} \quad 0.000000$

$19 \mathrm{H} \quad 0.000000$

$20 \mathrm{C} \quad 0.020164$

$21 \mathrm{H} \quad 0.000000$

$22 \mathrm{H} \quad 0.000000$

$23 \mathrm{H} \quad 0.000000$

$24 \mathrm{C} \quad 0.011479$

$25 \mathrm{H} \quad 0.000000$

$26 \mathrm{H} \quad 0.000000$

$27 \mathrm{H} \quad 0.000000$

$28 \mathrm{H} \quad 0.000000$

$29 \mathrm{C} \quad 0.022220$

$30 \mathrm{H} \quad 0.000000$

$\begin{array}{lll}31 \mathrm{H} & 0.000000\end{array}$

$32 \mathrm{Cl}-0.090632$

Sum of Mulliken charges $=0.00000$

Electronic spatial extent $(\mathrm{au}):<\mathrm{R} * * 2>=3992.6095$

Charge $=0.0000$ electrons

Dipole moment (field-independent basis, Debye):

$\mathrm{X}=1.5084 \mathrm{Y}=-1.5239 \mathrm{Z}=0.3956 \mathrm{Tot}=2.1804$

Quadrupole moment (field-independent basis, Debye-Ang):

$\mathrm{XX}=-93.6295 \quad \mathrm{YY}=-86.4996 \quad \mathrm{ZZ}=-89.4722$

$\mathrm{XY}=8.5635 \mathrm{XZ}=-0.7393 \quad \mathrm{YZ}=2.6686$

Traceless Quadrupole moment (field-independent basis, Debye-Ang):

$\mathrm{XX}=-3.7624 \mathrm{YY}=3.3675 \mathrm{ZZ}=0.3949$

$\mathrm{XY}=8.5635 \mathrm{XZ}=-0.7393 \mathrm{YZ}=2.6686$

Octapole moment (field-independent basis, Debye-Ang $* * 2$ ):

$\mathrm{XXX}=58.3970 \mathrm{YYY}=26.7627 \mathrm{ZZZ}=-0.0552 \mathrm{XYY}=8.2968$

$\mathrm{XXY}=-4.9043 \mathrm{XXZ}=-0.5201 \mathrm{XZZ}=-6.2278 \mathrm{YZZ}=5.4641$

$\mathrm{YYZ}=11.9964 \mathrm{XYZ}=2.8409$

Hexadecapole moment (field-independent basis, Debye-Ang**3):

$X X X X=-3487.1563 \mathrm{YYYY}=-1615.9813 \mathrm{ZZZZ}=-369.0447 \mathrm{XXXY}=147.1962$

$X X X Z=-6.9593 \mathrm{YYYX}=37.4627 \mathrm{YYYZ}=25.0600 \mathrm{ZZZX}=-3.6959$

$Z Z Z Y=-3.9784 X X Y Y=-743.1640 X X Z Z=-608.5251 \mathrm{YYZZ}=-338.2881$ 
$\mathrm{XXYZ}=-6.8292 \mathrm{YYXZ}=4.4069 \mathrm{ZZXY}=15.9135$

$\mathrm{N}-\mathrm{N}=9.504203400965 \mathrm{D}+02 \mathrm{E}-\mathrm{N}=-4.255903724983 \mathrm{D}+03 \quad \mathrm{KE}=9.975666436207 \mathrm{D}+02$

1I1|UNPC-UNKISP|RHF|6-31G(d)|C10H19C11O2|PCUSER|23-Apr-2006|0||\# HF/6-

31G(D) GEOM=CONNECTIVITY॥Title Card Required $\| 0,1 \mid \mathrm{C}, 0,-1.93928571,0.3$

$6878571,-0.23416071 \mathrm{IC}, 0,-0.74528571,0.06578571,-0.77316071 \mid \mathrm{H}, 0,-0.7012$

$8571,-0.42721429,-1.74216071 \mathrm{IC}, 0,0.58371429,0.32578571,-0.09916071 \mathrm{lH}, 0$

$, 0.42171429,0.69878571,0.92183929 \mid \mathrm{C}, 0,1.46571429,1.37478571,-0.8541607$ $1|\mathrm{O}, 0,1.28371429,-0.92521429,-0.04616071| \mathrm{C}, 0,0.90571429,-1.72021429,1$.

07483929|H,0,-0.18428571,-1.77321429,1.16583929|H,0,1.26171429,-2.7432 $1429,0.90683929|\mathrm{O}, 0,1.43571429,-1.21221429,2.29483929| \mathrm{C}, 0,2.82471429,-$ $1.48221429,2.41883929|\mathrm{H}, 0,3.17971429,-1.03121429,3.34883929| \mathrm{H}, 0,3.3857$ $1429,-1.05021429,1.58683929|\mathrm{H}, 0,3.00071429,-2.56121429,2.46883929| \mathrm{C}, 0$, $1.81771429,0.92478571,-2.28716071 \mid \mathrm{H}, 0,2.32271429,-0.04721429,-2.291160$ $71|\mathrm{H}, 0,2.48771429,1.64478571,-2.77016071| \mathrm{H}, 0,0.92571429,0.84378571,-2$. $91716071 \mathrm{IC}, 0,2.79071429,1.56978571,-0.08516071 \mid \mathrm{H}, 0,3.39971429,2.359785$ $71,-0.53916071|\mathrm{H}, 0,3.39271429,0.65478571,-0.08616071| \mathrm{H}, 0,2.60471429,1$. $85178571,0.95683929|\mathrm{C}, 0,0.74771429,2.73778571,-0.92116071| \mathrm{H}, 0,1.391714$ 29,3.50178571,-1.37116071|H,0,0.47071429,3.08578571,0.07983929|H,0,-0. $16428571,2.68678571,-1.52616071|\mathrm{H}, 0,-1.98128571,0.84878571,0.74183929|$ C,0,-3.25428571,0.09378571,-0.89016071|H,0,-3.13928571,-0.34921429,-1. $88416071 \mid \mathrm{H}, 0,-3.82928571,1.01878571,-0.98616071 \mathrm{ICl}, 0,-4.18728571,-1.03$ $921429,0.10883929||$ Version $=x 86-$ Win32-G03RevB.05|State $=1-A \mid H F=-998.9052$ 72|RMSD $=7.633 \mathrm{e}-006 \mid$ Dipole $=0.5967799,0.407652,-0.462122 \mid \mathrm{PG}=\mathrm{C} 01[\mathrm{X}(\mathrm{C} 10 \mathrm{H} 1$ 9Cl1O2)]\|@

OLD HORSE! OLD HORSE! WHAT BROUGHT YOU HERE?

FROM SACARAP' TO PORTLAND PIER

I'VE CARTED STONE THIS MANY A YEAR;

TILL, KILLED BY BLOWS AND SORE ABUSE,

THEY SALTED ME DOWN FOR SAILORS' USE.

THE SAILORS THEY DO ME DESPISE;

THEY TURN ME OVER AND DAMN MY EYES;

CUT OFF MY MEAT, AND SCRAPE MY BONES, AND PITCH ME OVER TO DAVY JONES.

SAILORS' COMPLAINT ABOUT THE BEEF SERVED ON SHIPBOARD, CIRCA 1835.

Job cpu time: 0 days 0 hours 5 minutes 39.0 seconds.

File lengths (MBytes): RWF $=39 \mathrm{Int}=0 \mathrm{D} 2 \mathrm{E}=0 \mathrm{Chk}=9 \mathrm{Scr}=1$

Normal termination of Gaussian 03 at Sun Apr 23 12:07:19 2006. 


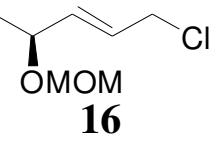

Entering Link 1 = C:\G03WV11.exe PID= 892.

Copyright (c) 1988,1990,1992,1993,1995,1998,2003, Gaussian, Inc. All Rights Reserved.

This is the Gaussian(R) 03 program. It is based on the the Gaussian(R) 98 system (copyright 1998, Gaussian, Inc.), the Gaussian(R) 94 system (copyright 1995, Gaussian, Inc.), the Gaussian 92(TM) system (copyright 1992, Gaussian, Inc.), the Gaussian 90(TM) system (copyright 1990, Gaussian, Inc.), the Gaussian 88(TM) system (copyright 1988, Gaussian, Inc.), the Gaussian 86(TM) system (copyright 1986, Carnegie Mellon University), and the Gaussian 82(TM) system (copyright 1983, Carnegie Mellon University). Gaussian is a federally registered trademark of Gaussian, Inc.

This software contains proprietary and confidential information, including trade secrets, belonging to Gaussian, Inc.

This software is provided under written license and may be used, copied, transmitted, or stored only in accord with that written license.

The following legend is applicable only to US Government contracts under DFARS:

\section{RESTRICTED RIGHTS LEGEND}

Use, duplication or disclosure by the US Government is subject to restrictions as set forth in subparagraph (c)(1)(ii) of the Rights in Technical Data and Computer Software clause at DFARS 252.227-7013.

Gaussian, Inc.

Carnegie Office Park, Building 6, Pittsburgh, PA 15106 USA

The following legend is applicable only to US Government contracts under FAR:

RESTRICTED RIGHTS LEGEND

Use, reproduction and disclosure by the US Government is subject to restrictions as set forth in subparagraph (c) of the Commercial Computer Software - Restricted Rights clause at FAR 52.227-19.

Gaussian, Inc.

Carnegie Office Park, Building 6, Pittsburgh, PA 15106 USA

Warning -- This program may not be used in any manner that competes with the business of Gaussian, Inc. or will provide assistance to any competitor of Gaussian, Inc. The licensee of this program is prohibited from giving any competitor of Gaussian, Inc. access to this program. By using this program, the user acknowledges that Gaussian, Inc. is engaged in the business of creating and licensing software in the field of computational chemistry and represents and warrants to the licensee that it is not a competitor of Gaussian, Inc. and that 
it will not use this program in any manner prohibited above.

Cite this work as:

Gaussian 03, Revision B.05,

M. J. Frisch, G. W. Trucks, H. B. Schlegel, G. E. Scuseria,

M. A. Robb, J. R. Cheeseman, J. A. Montgomery, Jr., T. Vreven,

K. N. Kudin, J. C. Burant, J. M. Millam, S. S. Iyengar, J. Tomasi,

V. Barone, B. Mennucci, M. Cossi, G. Scalmani, N. Rega,

G. A. Petersson, H. Nakatsuji, M. Hada, M. Ehara, K. Toyota,

R. Fukuda, J. Hasegawa, M. Ishida, T. Nakajima, Y. Honda, O. Kitao,

H. Nakai, M. Klene, X. Li, J. E. Knox, H. P. Hratchian, J. B. Cross,

C. Adamo, J. Jaramillo, R. Gomperts, R. E. Stratmann, O. Yazyev,

A. J. Austin, R. Cammi, C. Pomelli, J. W. Ochterski, P. Y. Ayala,

K. Morokuma, G. A. Voth, P. Salvador, J. J. Dannenberg,

V. G. Zakrzewski, S. Dapprich, A. D. Daniels, M. C. Strain,

O. Farkas, D. K. Malick, A. D. Rabuck, K. Raghavachari,

J. B. Foresman, J. V. Ortiz, Q. Cui, A. G. Baboul, S. Clifford,

J. Cioslowski, B. B. Stefanov, G. Liu, A. Liashenko, P. Piskorz,

I. Komaromi, R. L. Martin, D. J. Fox, T. Keith, M. A. Al-Laham,

C. Y. Peng, A. Nanayakkara, M. Challacombe, P. M. W. Gill,

B. Johnson, W. Chen, M. W. Wong, C. Gonzalez, and J. A. Pople, Gaussian, Inc., Pittsburgh PA, 2003.

$* * * * * * * * * * * * * * * * * * * * * * * * * * * * * * * * * * * * * * * * * * * * * *$

Gaussian 03: x86-Win32-G03RevB.05 8-Nov-2003

$$
\text { 23-Apr-2006 }
$$

*****************************************************

$\%$ chk=11_alkene.chk

$\%$ mem $=64 \mathrm{MB}$

$\%$ nproc $=1$

Will use up to 1 processors via shared memory.

\# hf/6-31g(d) geom=connectivity

$1 / 38=1,57=2 / 1$;

$2 / 17=6,18=5,40=1 / 2$;

$3 / 5=1,6=6,7=1,11=9,16=1,25=1,30=1 / 1,2,3$;

$4 / / 1$

$5 / 5=2,32=1,38=5 / 2$;

$6 / 7=2,8=2,9=2,10=2,28=1 / 1$;

$99 / 5=1,9=1 / 99$;

Title Card Required

Symbolic Z-matrix:

Charge $=0$ Multiplicity $=1$

$\begin{array}{llll}\text { C } & -1.49208 & -0.26503 & 0.89251\end{array}$

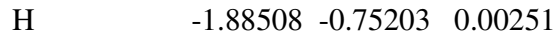

$\begin{array}{llll}\text { C } & -0.17508 & -0.32703 & 1.15551\end{array}$

$\begin{array}{llll}\mathrm{H} & 0.22592 & 0.16597 & 2.03951\end{array}$

$\begin{array}{llll}\mathrm{C} & 0.83092 & -1.02403 & 0.28051\end{array}$

$\begin{array}{lllll}\mathrm{H} & 0.36292 & -1.45703 & -0.61249\end{array}$

$\begin{array}{lllll}\text { C } & -2.49108 & 0.43997 & 1.75351\end{array}$

$\begin{array}{llll}\mathrm{H} & -2.03508 & 0.87897 & 2.64651\end{array}$

$\begin{array}{llll}\mathrm{H} & -3.27608 & -0.25303 & 2.06851\end{array}$

$\begin{array}{lllll}\mathrm{Cl} & -3.24908 & 1.75097 & 0.82651\end{array}$

$\begin{array}{llll}\mathrm{C} & 1.51192 & -2.14603 & 1.05651\end{array}$

$\begin{array}{llll}\mathrm{H} & 0.79192 & -2.90403 & 1.38251\end{array}$

$\mathrm{H} \quad 2.03892-1.76103 \quad 1.93751$

$\begin{array}{llll}\mathrm{H} & 2.26792 & -2.63303 & 0.43051\end{array}$

$\begin{array}{lllll}\mathrm{O} & 1.83992 & -0.08003 & -0.07849\end{array}$

C $\quad 1.52392 \quad 0.62797 \quad-1.27149$ 


$\begin{array}{lrrr}\mathrm{H} & 0.50492 & 1.02697 & -1.22749 \\ \mathrm{H} & 2.20192 & 1.48497 & -1.35549 \\ \mathrm{O} & 1.63892 & -0.19103 & -2.43149 \\ \mathrm{C} & 2.98992 & -0.54703 & -2.69249 \\ \mathrm{H} & 3.01292 & -1.15303 & -3.60249 \\ \mathrm{H} & 3.40192 & -1.14103 & -1.87149 \\ \mathrm{H} & 3.59992 & 0.34697 & -2.85649 \\ & & & \end{array}$

\begin{tabular}{|c|c|c|c|c|c|}
\hline \multirow{2}{*}{$\begin{array}{l}\text { Center } \\
\text { Number }\end{array}$} & Atomic & \multicolumn{2}{|c|}{ Atomic } & \multicolumn{2}{|c|}{ Coordinates (Angstroms) } \\
\hline & Number & & Type & $\begin{array}{ll}X & Y\end{array}$ & $\mathrm{Z}$ \\
\hline 1 & 6 & 0 & -1.492080 & -0.265034 & 0.892511 \\
\hline 2 & 1 & 0 & -1.885080 & -0.752034 & 0.002511 \\
\hline 3 & 6 & 0 & -0.175080 & -0.327034 & 1.155511 \\
\hline 4 & 1 & 0 & 0.225920 & 0.165966 & 2.039511 \\
\hline 5 & 6 & 0 & 0.830920 & -1.024034 & 0.280511 \\
\hline 6 & 1 & 0 & 0.362920 & -1.457034 & -0.612489 \\
\hline 7 & 6 & 0 & -2.491080 & 0.439966 & 1.753511 \\
\hline 8 & 1 & 0 & -2.035080 & 0.878966 & 2.646511 \\
\hline 9 & 1 & 0 & -3.276080 & -0.253034 & 2.068511 \\
\hline 10 & 17 & 0 & -3.249080 & 1.750966 & 0.826511 \\
\hline 11 & 6 & 0 & 1.511920 & -2.146034 & 1.056511 \\
\hline 12 & 1 & 0 & 0.791920 & -2.904034 & 1.382511 \\
\hline 13 & 1 & 0 & 2.038920 & -1.761034 & 1.937511 \\
\hline 14 & 1 & 0 & 2.267920 & -2.633034 & 0.430511 \\
\hline 15 & 8 & 0 & 1.839920 & -0.080034 & -0.078489 \\
\hline 16 & 6 & 0 & 1.523920 & 0.627966 & -1.271489 \\
\hline 17 & 1 & 0 & 0.504920 & 1.026966 & -1.227489 \\
\hline 18 & 1 & 0 & 2.201920 & 1.484966 & -1.355489 \\
\hline 19 & 8 & 0 & 1.638920 & -0.191034 & -2.431489 \\
\hline 20 & 6 & 0 & 2.989920 & -0.547034 & -2.692489 \\
\hline 21 & 1 & 0 & 3.012920 & -1.153034 & -3.602489 \\
\hline 22 & 1 & 0 & 3.401920 & -1.141034 & -1.871489 \\
\hline 23 & 1 & 0 & 3.599920 & 0.346966 & -2.856489 \\
\hline
\end{tabular}

Distance matrix (angstroms):
$1 \mathrm{C} \quad 0.000000$
$\begin{array}{llll}2 & \mathrm{H} & 1.087988 & 0.000000\end{array}$
$\begin{array}{lllll}3 & \mathrm{C} & 1.344434 & 2.105738 & 0.000000\end{array}$
$\begin{array}{llllll}4 \mathrm{H} & 2.110188 & 3.073827 & 1.088718 & 0.000000\end{array}$
$\begin{array}{lllllll}5 \mathrm{C} & 2.519316 & 2.743706 & 1.504483 & 2.208213 & 0.000000\end{array}$
$\begin{array}{lllllll}6 & \mathrm{H} & 2.669628 & 2.434903 & 2.166141 & 3.112234 & 1.097252\end{array}$
$\begin{array}{lllllll}7 & \mathrm{C} & 1.495442 & 2.203202 & 2.511921 & 2.745717 & 3.917743\end{array}$
$\begin{array}{lllllll}8 & \mathrm{H} & 2.163354 & 3.110209 & 2.671538 & 2.447231 & 4.175323\end{array}$
$\begin{array}{lllllll}9 \mathrm{H} & 2.136768 & 2.540126 & 3.233457 & 3.527096 & 4.545199\end{array}$
$\begin{array}{lllllll}10 \mathrm{Cl} & 2.675007 & 2.967235 & 3.725024 & 4.007396 & 4.964387\end{array}$
$\begin{array}{llllllll}11 & \mathrm{C} & 3.548108 & 3.820178 & 2.482847 & 2.822309 & 1.524736\end{array}$
$\begin{array}{lllllll}12 & \mathrm{H} & 3.524355 & 3.701599 & 2.761801 & 3.190126 & 2.179524\end{array}$
$\begin{array}{llllllll}13 & \mathrm{H} & 3.974670 & 4.489998 & 2.751304 & 2.647773 & 2.179009\end{array}$
$\begin{array}{lllllll}14 & \mathrm{H} & 4.467490 & 4.579165 & 3.436788 & 3.820085 & 2.162487\end{array}$
$\begin{array}{llllllll}15 & \mathrm{O} & 3.475527 & 3.785997 & 2.375708 & 2.674217 & 1.427620\end{array}$
$\begin{array}{lllllll}16 & \mathrm{C} & 3.817932 & 3.892140 & 3.112709 & 3.586219 & 2.370244\end{array}$
$\begin{array}{llllllll}17 & \mathrm{H} & 3.186169 & 3.223328 & 2.823899 & 3.390052 & 2.566504\end{array}$
$\begin{array}{llllllll}18 & \mathrm{H} & 4.664937 & 4.853030 & 3.903664 & 4.143714 & 3.294119\end{array}$
$\begin{array}{llllllll}19 & \mathrm{O} & 4.567014 & 4.319451 & 4.021898 & 4.702538 & 2.949864\end{array}$
$\begin{array}{lllllll}20 & \mathrm{C} & 5.746310 & 5.574108 & 4.987257 & 5.526291 & 3.705069\end{array}$
$\begin{array}{llllllll}21 & \mathrm{H} & 6.425620 & 6.094853 & 5.786552 & 6.429564 & 4.455946\end{array}$
$\begin{array}{lllllll}22 & \mathrm{H} & 5.688436 & 5.622772 & 4.756075 & 5.204916 & 3.354822\end{array}$
$\begin{array}{llllllll}23 & \mathrm{H} & 6.352795 & 6.282269 & 5.549869 & 5.948735 & 4.403155\end{array}$
$6 \mathrm{H} \quad 0.000000$

$\begin{array}{ccccc}6 & 7 & 8 & 9 & 10\end{array}$




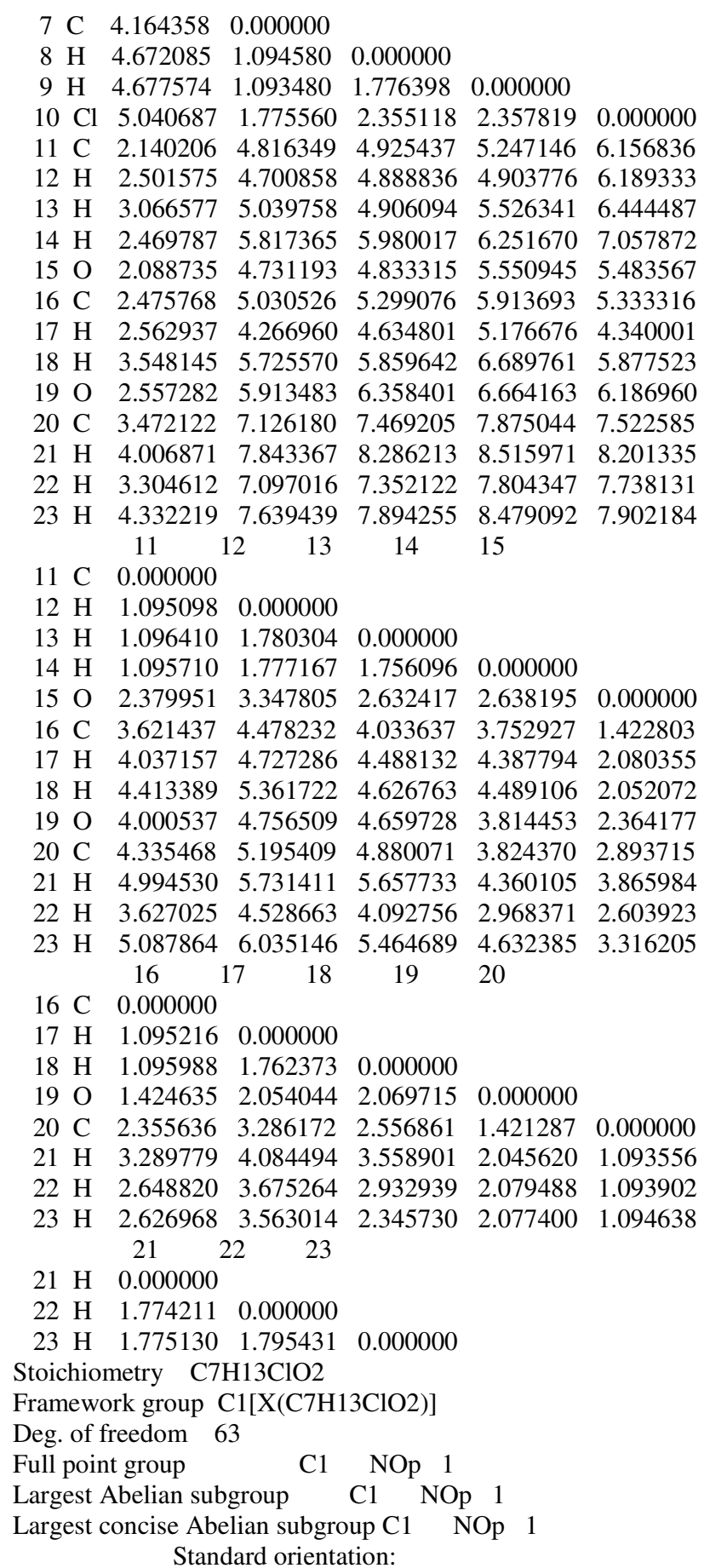

\begin{tabular}{|c|c|c|c|c|}
\hline Center & Atomic & Atomic & Coordinate & es (Angstrom \\
\hline Number & Number & Type & $\begin{array}{ll}X & Y\end{array}$ & $\mathrm{Z}$ \\
\hline 1 & 6 & 1.591302 & 0.579479 & -0.474431 \\
\hline 2 & 1 & 1.387059 & 0.189613 & -1.469422 \\
\hline 3 & 6 & 0.575747 & 1.030308 & 0.282474 \\
\hline 4 & 1 & 0.772202 & 1.413138 & 1.282550 \\
\hline 5 & 6 & -0.866756 & 1.022506 & -0.144835 \\
\hline 6 & 1 & -0.993559 & 0.582151 & -1.141815 \\
\hline 7 & 6 & 3.023832 & 0.572922 & -0.045291 \\
\hline 8 & 1 & 3.162497 & 1.007303 & 0.949792 \\
\hline 9 & 1 & 3.635559 & 1.133077 & -0.757832 \\
\hline
\end{tabular}




$\begin{array}{rrrrrr}10 & 17 & 0 & 3.618991 & -1.099284 & 0.000852 \\ 11 & 6 & 0 & -1.411277 & 2.446332 & -0.177024 \\ 12 & 1 & 0 & -0.868017 & 3.068461 & -0.896095 \\ 13 & 1 & 0 & -1.353405 & 2.919610 & 0.810282 \\ 14 & 1 & 0 & -2.470753 & 2.439010 & -0.456379 \\ 15 & 8 & 0 & -1.617405 & 0.300552 & 0.831591 \\ 16 & 6 & 0 & -1.686403 & -1.091549 & 0.545823 \\ 17 & 1 & 0 & -0.690457 & -1.493336 & 0.330981 \\ 18 & 1 & 0 & -2.057368 & -1.610098 & 1.437272 \\ 19 & 8 & 0 & -2.536181 & -1.373409 & -0.562337 \\ 20 & 6 & 0 & -3.898982 & -1.103359 & -0.262496 \\ 21 & 1 & 0 & -4.498362 & -1.359852 & -1.140459 \\ 22 & 1 & 0 & -4.046477 & -0.042183 & -0.041653 \\ 23 & 1 & 0 & -4.237848 & -1.716044 & 0.578943\end{array}$

Rotational constants (GHZ): $1.7880229 \quad 0.4482390 \quad 0.3782159$

Standard basis: 6-31G(d) $(6 \mathrm{D}, 7 \mathrm{~F})$

There are 180 symmetry adapted basis functions of A symmetry.

Integral buffers will be 262144 words long.

Raffenetti 1 integral format.

Two-electron integral symmetry is turned on.

180 basis functions, 356 primitive gaussians, 180 cartesian basis functions

44 alpha electrons 44 beta electrons

nuclear repulsion energy $\quad 612.4168047588$ Hartrees.

NAtoms $=23$ NActive $=23 \mathrm{NUniq}=23 \mathrm{SFac}=1.00 \mathrm{D}+00 \mathrm{NAtFMM}=60 \mathrm{Big}=\mathrm{F}$

One-electron integrals computed using PRISM.

NBasis $=180$ RedAO $=\mathrm{T}$ NBF $=180$

$\mathrm{NBsUse}=180$ 1.00D-06 NBFU $=180$

Harris functional with IExCor= 205 diagonalized for initial guess.

ExpMin= 1.43D-01 ExpMax=2.52D+04 ExpMxC=3.78D+03 IAcc=1 IRadAn= 1 AccDes=1.00D-06
HarFok: IExCor=205 AccDes= 1.00D-06 IRadAn= 1 IDoV=1

ScaDFX $=1.0000001 .0000001 .0000001 .000000$

Initial guess orbital symmetries:

Occupied (A) (A) (A) (A) (A) (A) (A) (A) (A) (A) (A) $(A)$

(A) $(\mathrm{A})(\mathrm{A})(\mathrm{A})(\mathrm{A})(\mathrm{A})(\mathrm{A})(\mathrm{A})(\mathrm{A})(\mathrm{A})(\mathrm{A})(\mathrm{A})$

(A) $(\mathrm{A})(\mathrm{A})(\mathrm{A})(\mathrm{A})(\mathrm{A})(\mathrm{A})(\mathrm{A})(\mathrm{A})(\mathrm{A})(\mathrm{A})(\mathrm{A})$

(A) $(A)(A)(A)(A)(A)(A)(A)$

Virtual (A) (A) (A) (A) (A) (A) (A) (A) (A) (A) (A) (A)

(A) (A) (A) (A) (A) (A) (A) (A) (A) (A) (A) (A)

(A) $(\mathrm{A})(\mathrm{A})(\mathrm{A})(\mathrm{A})(\mathrm{A})(\mathrm{A})(\mathrm{A})(\mathrm{A})(\mathrm{A})(\mathrm{A})(\mathrm{A})$

(A) $(A)(A)(A)(A)(A)(A)(A)(A)(A)(A)(A)$

(A) (A) (A) (A) (A) (A) (A) (A) (A) (A) (A) (A)

(A) (A) (A) (A) (A) (A) (A) (A) (A) (A) (A) (A)

(A) (A) (A) (A) (A) (A) (A) (A) (A) (A) (A) (A)

(A) (A) (A) (A) (A) (A) (A) (A) (A) (A) (A) (A)

(A) $(\mathrm{A})(\mathrm{A})(\mathrm{A})(\mathrm{A})(\mathrm{A})(\mathrm{A})(\mathrm{A})(\mathrm{A})(\mathrm{A})(\mathrm{A})(\mathrm{A})$

(A) (A) (A) (A) (A) (A) (A) (A) (A) (A) (A) (A)

(A) $(\mathrm{A})(\mathrm{A})(\mathrm{A})(\mathrm{A})(\mathrm{A})(\mathrm{A})(\mathrm{A})(\mathrm{A})(\mathrm{A})(\mathrm{A})(\mathrm{A})$

(A) $(\mathrm{A})(\mathrm{A})(\mathrm{A})$

The electronic state of the initial guess is 1-A.

Warning! Cutoffs for single-point calculations used.

Requested convergence on RMS density matrix=1.00D-04 within 128 cycles.

Requested convergence on MAX density matrix=1.00D-02.

Requested convergence on energy $=5.00 \mathrm{D}-05$.

No special actions if energy rises.

SCF Done: $\mathrm{E}(\mathrm{RHF})=-881.806303427$ A.U. after 6 cycles

Convg $=0.8022 \mathrm{D}-05 \quad-\mathrm{V} / \mathrm{T}=2.0013$

$\mathrm{S} * * 2=0.0000$

$* * * * * * * * * * * * * * * * * * * * * * * * * * * * * * * * * * * * * * * * * * * * * * * * * * * * * * * * * * * * * * * * * * * * * * *$

Population analysis using the SCF density. 
Orbital symmetries:

Occupied (A) (A) (A) (A) (A) (A) (A) (A) (A) (A) (A) (A)

(A) (A) (A) (A) (A) (A) (A) (A) (A) (A) (A) (A)

(A) (A) (A) (A) (A) (A) (A) (A) (A) (A) (A) (A)

(A) $(\mathrm{A})(\mathrm{A})(\mathrm{A})(\mathrm{A})(\mathrm{A})(\mathrm{A})(\mathrm{A})$

Virtual (A) (A) (A) (A) (A) (A) (A) (A) (A) (A) (A) (A)

(A) $(A)(A)(A)(A)(A)(A)(A)(A)(A)(A)(A)$

(A) (A) (A) (A) (A) (A) (A) (A) (A) (A) (A) (A)

(A) (A) (A) (A) (A) (A) (A) (A) (A) (A) (A) (A)

(A) $(\mathrm{A})(\mathrm{A})(\mathrm{A})(\mathrm{A})(\mathrm{A})(\mathrm{A})(\mathrm{A})(\mathrm{A})(\mathrm{A})(\mathrm{A})(\mathrm{A})$

(A) (A) (A) (A) (A) (A) (A) (A) (A) (A) (A) (A)

(A) (A) (A) (A) (A) (A) (A) (A) (A) (A) (A) (A)

(A) $(A)(A)(A)(A)(A)(A)(A)(A)(A)(A)(A)$

(A) (A) (A) (A) (A) (A) (A) (A) (A) (A) (A) (A)

(A) (A) (A) (A) (A) (A) (A) (A) (A) (A) (A) (A)

(A) $(\mathrm{A})(\mathrm{A})(\mathrm{A})(\mathrm{A})(\mathrm{A})(\mathrm{A})(\mathrm{A})(\mathrm{A})(\mathrm{A})(\mathrm{A})(\mathrm{A})$

(A) (A) (A) (A)

The electronic state is $1-\mathrm{A}$.

Alpha occ. eigenvalues -- -104.81021 -20.57100 -20.56898 -11.33114 -11.29959

Alpha occ. eigenvalues -- -11.29089 -11.27135 -11.24967 -11.24396 -11.22160

Alpha occ. eigenvalues -- $-10.54453 \quad-8.01389-8.01056-8.01049-1.40812$

Alpha occ. eigenvalues -- $\quad-1.34294 \quad-1.15019 \quad-1.07998 \quad-1.01417 \quad-0.95452$

Alpha occ. eigenvalues -- $\quad-0.91711 \quad-0.87452 \quad-0.81801 \quad-0.77589 \quad-0.69649$

Alpha occ. eigenvalues -- $-0.67854 \quad-0.67690 \quad-0.64835 \quad-0.61842 \quad-0.60158$

Alpha occ. eigenvalues -- $-0.59797 \quad-0.58734 \quad-0.55959-0.54911 \quad-0.53660$

Alpha occ. eigenvalues -- $-0.52296 \quad-0.51911 \quad-0.49811 \quad-0.46215 \quad-0.44674$

Alpha occ. eigenvalues -- $-0.44021 \quad-0.43530 \quad-0.42690 \quad-0.36627$

$\begin{array}{lllllll}\text { Alpha virt. eigenvalues -- } & 0.15036 & 0.21316 & 0.23380 & 0.24447 & 0.26201\end{array}$

$\begin{array}{lllllll}\text { Alpha virt. eigenvalues -- } & 0.27280 & 0.28286 & 0.29287 & 0.29705 & 0.30948\end{array}$

$\begin{array}{lllllll}\text { Alpha virt. eigenvalues -- } & 0.31672 & 0.31834 & 0.32147 & 0.34033 & 0.35057\end{array}$

$\begin{array}{lllllll}\text { Alpha virt. eigenvalues -- } & 0.36541 & 0.38429 & 0.40644 & 0.41463 & 0.43464\end{array}$

$\begin{array}{lllllll}\text { Alpha virt. eigenvalues -- } & 0.44740 & 0.50786 & 0.56827 & 0.58785 & 0.64637\end{array}$

$\begin{array}{lllllll}\text { Alpha virt. eigenvalues -- } & 0.65372 & 0.67722 & 0.70005 & 0.71896 & 0.72793\end{array}$

$\begin{array}{llllllll}\text { Alpha virt. eigenvalues -- } & 0.73546 & 0.75810 & 0.77052 & 0.77950 & 0.79283\end{array}$

$\begin{array}{lllllll}\text { Alpha virt. eigenvalues -- } & 0.81192 & 0.81679 & 0.83051 & 0.88561 & 0.89194\end{array}$

$\begin{array}{lllllll}\text { Alpha virt. eigenvalues -- } & 0.91363 & 0.92587 & 0.94953 & 0.98134 & 1.02501\end{array}$

$\begin{array}{lllllll}\text { Alpha virt. eigenvalues -- } & 1.04870 & 1.06150 & 1.07090 & 1.09005 & 1.09773\end{array}$

$\begin{array}{lllllll}\text { Alpha virt. eigenvalues -- } & 1.11283 & 1.12612 & 1.13207 & 1.14465 & 1.14602\end{array}$

$\begin{array}{lllllll}\text { Alpha virt. eigenvalues -- } & 1.15179 & 1.16322 & 1.18024 & 1.18337 & 1.19388\end{array}$

$\begin{array}{lllllll}\text { Alpha virt. eigenvalues -- } & 1.20110 & 1.21164 & 1.23847 & 1.24205 & 1.26662\end{array}$

$\begin{array}{llllll}\text { Alpha virt. eigenvalues -- } & 1.28583 & 1.32095 & 1.33838 & 1.36527 & 1.42411\end{array}$

$\begin{array}{lllllll}\text { Alpha virt. eigenvalues -- } & 1.42863 & 1.45712 & 1.48359 & 1.52214 & 1.54841\end{array}$

$\begin{array}{lllllll}\text { Alpha virt. eigenvalues -- } & 1.57325 & 1.59747 & 1.61345 & 1.70623 & 1.73089\end{array}$

$\begin{array}{llllll}\text { Alpha virt. eigenvalues -- } & 1.75819 & 1.80729 & 1.84626 & 1.85764 & 1.89487\end{array}$

$\begin{array}{lllllll}\text { Alpha virt. eigenvalues -- } & 1.91641 & 1.97617 & 1.98786 & 2.06365 & 2.08090\end{array}$

$\begin{array}{llllll}\text { Alpha virt. eigenvalues -- } & 2.10771 & 2.17227 & 2.20552 & 2.26247 & 2.26695\end{array}$

$\begin{array}{llllll}\text { Alpha virt. eigenvalues -- } & 2.29621 & 2.32690 & 2.34208 & 2.34883 & 2.37565\end{array}$

$\begin{array}{lllllll}\text { Alpha virt. eigenvalues -- } & 2.38589 & 2.43645 & 2.46336 & 2.47321 & 2.50989\end{array}$

$\begin{array}{llllll}\text { Alpha virt. eigenvalues -- } & 2.51697 & 2.55405 & 2.55686 & 2.58508 & 2.60563\end{array}$

$\begin{array}{llllll}\text { Alpha virt. eigenvalues -- } & 2.61924 & 2.68564 & 2.70920 & 2.78402 & 2.84117\end{array}$

$\begin{array}{lllllll}\text { Alpha virt. eigenvalues -- } & 2.86759 & 2.89022 & 2.93265 & 2.97282 & 3.06703\end{array}$

$\begin{array}{lllllll}\text { Alpha virt. eigenvalues -- } & 3.08462 & 3.18655 & 3.22779 & 3.27920 & 3.31497\end{array}$

$\begin{array}{lllllll}\text { Alpha virt. eigenvalues -- } & 3.34740 & 4.30892 & 4.38787 & 4.51794 & 4.63611\end{array}$

$\begin{array}{lllllll}\text { Alpha virt. eigenvalues -- } & 4.67458 & 4.68315 & 4.77551 & 4.78069 & 4.84816\end{array}$

Alpha virt. eigenvalues -- 4.97174

Condensed to atoms (all electrons):

$\begin{array}{llllll}1 & 2 & 3 & 4 & 5 & 6\end{array}$

$\begin{array}{llllllll}1 & \mathrm{C} & 5.061125 & 0.391950 & 0.634612 & -0.037306 & -0.037908 & 0.005991\end{array}$

$\begin{array}{llllllll}2 & \mathrm{H} & 0.391950 & 0.471709 & -0.034952 & 0.002911 & -0.002750 & 0.002914\end{array}$

$\begin{array}{llllllll}3 \mathrm{C} & 0.634612 & -0.034952 & 5.040610 & 0.383932 & 0.360717 & -0.060045\end{array}$

$\begin{array}{llllllll}4 \mathrm{H} & -0.037306 & 0.002911 & 0.383932 & 0.476643 & -0.027846 & 0.002638\end{array}$

$\begin{array}{llllllll}5 & \mathrm{C} & -0.037908 & -0.002750 & 0.360717 & -0.027846 & 4.786130 & 0.411435\end{array}$

$\begin{array}{llllllll}6 & \mathrm{H} & 0.005991 & 0.002914 & -0.060045 & 0.002638 & 0.411435 & 0.509403\end{array}$ 
$\begin{array}{llllllll}7 & \mathrm{C} & 0.318056 & -0.038324 & -0.042019 & -0.002643 & 0.002566 & 0.000107\end{array}$

$\begin{array}{llllllll}8 \mathrm{H} & -0.043958 & 0.002618 & 0.002861 & 0.002873 & 0.000124 & 0.000008\end{array}$

$\begin{array}{llllllll}9 \mathrm{H} & -0.040142 & -0.001170 & 0.000013 & 0.000062 & -0.000105 & -0.000006\end{array}$

$\begin{array}{llllllll}10 \mathrm{Cl} & -0.080211 & 0.000863 & -0.000307 & 0.000190 & -0.000234 & 0.000011\end{array}$

$\begin{array}{llllllll}11 & \mathrm{C} & -0.001284 & 0.000121 & -0.077327 & -0.001883 & 0.370842 & -0.039684\end{array}$

$\begin{array}{llllllll}12 & \mathrm{H} & 0.000233 & 0.000041 & 0.001518 & 0.000363 & -0.035773 & 0.000156\end{array}$

$\begin{array}{llllllll}13 \mathrm{H} & 0.000535 & 0.000006 & -0.004699 & 0.001859 & -0.034056 & 0.003837\end{array}$

$\begin{array}{llllllll}14 & \mathrm{H} & -0.000065 & -0.000004 & 0.005545 & -0.000091 & -0.034586 & -0.003605\end{array}$

$\begin{array}{llllllll}15 & \mathrm{O} & 0.000289 & 0.000033 & -0.051328 & 0.001139 & 0.165029 & -0.030475\end{array}$

$\begin{array}{llllllll}16 & \mathrm{C} & -0.000949 & 0.000035 & -0.007218 & 0.000104 & -0.029562 & -0.007573\end{array}$

$\begin{array}{llllllll}17 & \mathrm{H} & 0.002701 & 0.000053 & 0.005823 & 0.000085 & -0.006460 & -0.000800\end{array}$

$\begin{array}{llllllll}18 & \mathrm{H} & -0.000018 & -0.000001 & 0.000025 & -0.000006 & 0.004635 & 0.000285\end{array}$

$\begin{array}{lllllllll}19 & \mathrm{O} & 0.000002 & -0.000006 & 0.000235 & 0.000001 & -0.003361 & 0.008528\end{array}$

$\begin{array}{llllllll}20 & \mathrm{C} & -0.000001 & 0.000000 & -0.000004 & 0.000000 & -0.000431 & -0.000386\end{array}$

$\begin{array}{llllllll}21 & \mathrm{H} & 0.000000 & 0.000000 & 0.000001 & 0.000000 & -0.000033 & -0.000019\end{array}$

$\begin{array}{llllllll}22 & \mathrm{H} & -0.000001 & 0.000000 & 0.000017 & 0.000000 & -0.000649 & -0.000285\end{array}$

$\begin{array}{llllllll}23 & \mathrm{H} & 0.000000 & 0.000000 & 0.000002 & 0.000000 & 0.000092 & 0.000053\end{array}$

$$
\begin{array}{llllll}
7 & 8 & 9 & 10 & 11 & 12
\end{array}
$$

$\begin{array}{llllllll}1 & \mathrm{C} & 0.318056 & -0.043958 & -0.040142 & -0.080211 & -0.001284 & 0.000233\end{array}$

$\begin{array}{llllllll}2 \mathrm{H} & -0.038324 & 0.002618 & -0.001170 & 0.000863 & 0.000121 & 0.000041\end{array}$

$\begin{array}{llllllll}3 & \mathrm{C} & -0.042019 & 0.002861 & 0.000013 & -0.000307 & -0.077327 & 0.001518\end{array}$

$\begin{array}{llllllll}4 \mathrm{H} & -0.002643 & 0.002873 & 0.000062 & 0.000190 & -0.001883 & 0.000363\end{array}$

$\begin{array}{llllllll}5 \mathrm{C} & 0.002566 & 0.000124 & -0.000105 & -0.000234 & 0.370842 & -0.035773\end{array}$

$\begin{array}{llllllll}6 & \mathrm{H} & 0.000107 & 0.000008 & -0.000006 & 0.000011 & -0.039684 & 0.000156\end{array}$

$\begin{array}{llllllll}7 & \mathrm{C} & 5.200151 & 0.391343 & 0.386003 & 0.203056 & -0.000186 & -0.000004\end{array}$

$\begin{array}{lllllllll}8 & \mathrm{H} & 0.391343 & 0.492560 & -0.025854 & -0.044492 & -0.000008 & 0.000000\end{array}$

$\begin{array}{llllllll}9 \mathrm{H} & 0.386003 & -0.025854 & 0.512928 & -0.050615 & 0.000015 & -0.000001\end{array}$

$\begin{array}{llllllll}10 \mathrm{Cl} & 0.203056 & -0.044492 & -0.050615 & 17.062335 & -0.000003 & 0.000000\end{array}$

$\begin{array}{llllllll}11 & \mathrm{C} & -0.000186 & -0.000008 & 0.000015 & -0.000003 & 5.131920 & 0.379586\end{array}$

$\begin{array}{llllllll}12 \mathrm{H} & -0.000004 & 0.000000 & -0.000001 & 0.000000 & 0.379586 & 0.534531\end{array}$

$\begin{array}{llllllll}13 \mathrm{H} & 0.000001 & 0.000001 & 0.000000 & 0.000000 & 0.387481 & -0.024648\end{array}$

$\begin{array}{llllllll}14 \mathrm{H} & 0.000002 & 0.000000 & 0.000000 & 0.000000 & 0.386616 & -0.023163\end{array}$

$\begin{array}{llllllll}15 & \mathrm{O} & -0.000059 & -0.000001 & 0.000000 & 0.000014 & -0.054306 & 0.002886\end{array}$

$\begin{array}{llllllll}16 & \mathrm{C} & -0.000005 & -0.000001 & 0.000000 & -0.000011 & 0.004185 & -0.000117\end{array}$

$\begin{array}{llllllll}17 & \mathrm{H} & 0.000003 & 0.000001 & 0.000003 & 0.000091 & -0.000043 & 0.000011\end{array}$

$\begin{array}{llllllll}18 & \mathrm{H} & -0.000001 & 0.000000 & 0.000000 & 0.000000 & -0.000182 & 0.000002\end{array}$

$\begin{array}{llllllll}19 & \mathrm{O} & 0.000000 & 0.000000 & 0.000000 & 0.000000 & -0.000250 & 0.000002\end{array}$

$\begin{array}{llllllll}20 \mathrm{C} & 0.000000 & 0.000000 & 0.000000 & 0.000000 & -0.000069 & 0.000003\end{array}$

$\begin{array}{llllllll}21 \mathrm{H} & 0.000000 & 0.000000 & 0.000000 & 0.000000 & 0.000003 & 0.000000\end{array}$

$\begin{array}{llllllll}22 \mathrm{H} & 0.000000 & 0.000000 & 0.000000 & 0.000000 & 0.000116 & 0.000001\end{array}$

$\begin{array}{llllllll}23 & \mathrm{H} & 0.000000 & 0.000000 & 0.000000 & 0.000000 & -0.000009 & 0.000000\end{array}$

$$
\begin{array}{llllll}
13 & 14 & 15 & 16 & 17 & 18
\end{array}
$$

$\begin{array}{llllllll}1 \mathrm{C} & 0.000535 & -0.000065 & 0.000289 & -0.000949 & 0.002701 & -0.000018\end{array}$

$\begin{array}{llllllll}2 \mathrm{H} & 0.000006 & -0.000004 & 0.000033 & 0.000035 & 0.000053 & -0.000001\end{array}$

$\begin{array}{llllllll}3 & \mathrm{C} & -0.004699 & 0.005545 & -0.051328 & -0.007218 & 0.005823 & 0.000025\end{array}$

$\begin{array}{llllllll}4 \mathrm{H} & 0.001859 & -0.000091 & 0.001139 & 0.000104 & 0.000085 & -0.000006\end{array}$

$\begin{array}{llllllll}5 \mathrm{C} & -0.034056 & -0.034586 & 0.165029 & -0.029562 & -0.006460 & 0.004635\end{array}$

$\begin{array}{llllllll}6 \mathrm{H} & 0.003837 & -0.003605 & -0.030475 & -0.007573 & -0.000800 & 0.000285\end{array}$

$\begin{array}{llllllll}7 & \mathrm{C} & 0.000001 & 0.000002 & -0.000059 & -0.000005 & 0.000003 & -0.000001\end{array}$

$\begin{array}{llllllll}8 & \mathrm{H} & 0.000001 & 0.000000 & -0.000001 & -0.000001 & 0.000001 & 0.000000\end{array}$

$\begin{array}{llllllll}9 \mathrm{H} & 0.000000 & 0.000000 & 0.000000 & 0.000000 & 0.000003 & 0.000000\end{array}$

$\begin{array}{llllllll}10 \mathrm{Cl} & 0.000000 & 0.000000 & 0.000014 & -0.000011 & 0.000091 & 0.000000\end{array}$

$\begin{array}{llllllll}11 & \mathrm{C} & 0.387481 & 0.386616 & -0.054306 & 0.004185 & -0.000043 & -0.000182\end{array}$

$\begin{array}{llllllll}12 \mathrm{H} & -0.024648 & -0.023163 & 0.002886 & -0.000117 & 0.000011 & 0.000002\end{array}$

$\begin{array}{llllllll}13 & \mathrm{H} & 0.527242 & -0.027621 & 0.000389 & -0.000091 & -0.000001 & -0.000002\end{array}$

$\begin{array}{llllllll}14 \mathrm{H} & -0.027621 & 0.522402 & 0.001236 & -0.000194 & 0.000003 & 0.000000\end{array}$

$\begin{array}{llllllll}15 & \mathrm{O} & 0.000389 & 0.001236 & 8.524851 & 0.212840 & -0.037880 & -0.039476\end{array}$

$\begin{array}{llllllll}16 & \mathrm{C} & -0.000091 & -0.000194 & 0.212840 & 4.564513 & 0.407567 & 0.407853\end{array}$

$\begin{array}{llllllll}17 & \mathrm{H} & -0.000001 & 0.000003 & -0.037880 & 0.407567 & 0.517945 & -0.037505\end{array}$

$\begin{array}{llllllll}18 \mathrm{H} & -0.000002 & 0.000000 & -0.039476 & 0.407853 & -0.037505 & 0.538894\end{array}$

$\begin{array}{llllllll}19 & \mathrm{O} & -0.000001 & 0.000066 & -0.052447 & 0.226501 & -0.038053 & -0.039946\end{array}$

$\begin{array}{llllllll}20 \mathrm{C} & 0.000004 & -0.000078 & -0.000094 & -0.048360 & 0.004721 & -0.007789\end{array}$

$\begin{array}{llllllll}21 & \mathrm{H} & 0.000000 & 0.000002 & 0.000000 & 0.004651 & -0.000051 & -0.000226\end{array}$

$\begin{array}{llllllll}22 & \mathrm{H} & -0.000001 & 0.000483 & 0.006997 & -0.006882 & -0.000014 & -0.000507\end{array}$ 


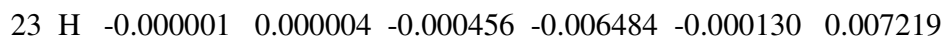

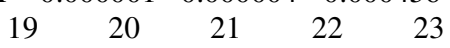

$\begin{array}{lllllll}1 \mathrm{C} & 0.000002 & -0.000001 & 0.000000 & -0.000001 & 0.000000\end{array}$

$\begin{array}{lllllll}2 \mathrm{H} & -0.000006 & 0.000000 & 0.000000 & 0.000000 & 0.000000\end{array}$

$\begin{array}{lllllll}3 \mathrm{C} & 0.000235 & -0.000004 & 0.000001 & 0.000017 & 0.000002\end{array}$

$\begin{array}{lllllll}4 \mathrm{H} & 0.000001 & 0.000000 & 0.000000 & 0.000000 & 0.000000\end{array}$

$\begin{array}{lllllll}5 & \mathrm{C} & -0.003361 & -0.000431 & -0.000033 & -0.000649 & 0.000092\end{array}$

$\begin{array}{lllllll}6 \mathrm{H} & 0.008528 & -0.000386 & -0.000019 & -0.000285 & 0.000053\end{array}$

$\begin{array}{lllllll}7 & \mathrm{C} & 0.000000 & 0.000000 & 0.000000 & 0.000000 & 0.000000\end{array}$

$\begin{array}{lllllll}8 \mathrm{H} & 0.000000 & 0.000000 & 0.000000 & 0.000000 & 0.000000\end{array}$

$\begin{array}{lllllll}9 \mathrm{H} & 0.000000 & 0.000000 & 0.000000 & 0.000000 & 0.000000\end{array}$

$\begin{array}{lllllll}10 \mathrm{Cl} & 0.000000 & 0.000000 & 0.000000 & 0.000000 & 0.000000\end{array}$

$\begin{array}{lllllll}11 & \mathrm{C} & -0.000250 & -0.000069 & 0.000003 & 0.000116 & -0.000009\end{array}$

$\begin{array}{lllllll}12 & \mathrm{H} & 0.000002 & 0.000003 & 0.000000 & 0.000001 & 0.000000\end{array}$

$\begin{array}{lllllll}13 \mathrm{H} & -0.000001 & 0.000004 & 0.000000 & -0.000001 & -0.000001\end{array}$

$\begin{array}{lllllll}14 \mathrm{H} & 0.000066 & -0.000078 & 0.000002 & 0.000483 & 0.000004\end{array}$

$\begin{array}{llllllll}15 & \mathrm{O} & -0.052447 & -0.000094 & 0.000000 & 0.006997 & -0.000456\end{array}$

$\begin{array}{lllllll}16 & \mathrm{C} & 0.226501 & -0.048360 & 0.004651 & -0.006882 & -0.006484\end{array}$

$\begin{array}{lllllll}17 & \mathrm{H} & -0.038053 & 0.004721 & -0.000051 & -0.000014 & -0.000130\end{array}$

$\begin{array}{lllllll}18 & \mathrm{H} & -0.039946 & -0.007789 & -0.000226 & -0.000507 & 0.007219\end{array}$

$\begin{array}{llllllll}19 & \mathrm{O} & 8.427639 & 0.202861 & -0.030840 & -0.036804 & -0.038723\end{array}$

$\begin{array}{llllllll}20 & \mathrm{C} & 0.202861 & 4.847412 & 0.398498 & 0.391955 & 0.389141\end{array}$

$\begin{array}{llllllll}21 & \mathrm{H} & -0.030840 & 0.398498 & 0.514005 & -0.031521 & -0.030475\end{array}$

$\begin{array}{lllllll}22 & \mathrm{H} & -0.036804 & 0.391955 & -0.031521 & 0.547116 & -0.038558\end{array}$

$\begin{array}{lllllll}23 & \mathrm{H} & -0.038723 & 0.389141 & -0.030475 & -0.038558 & 0.571336\end{array}$

Mulliken atomic charges:
1
1 C -0.173652
$2 \mathrm{H} \quad 0.203953$
3 C -0.158010
$\begin{array}{lll}4 \mathrm{H} & 0.196975\end{array}$
5 C 0.112184
$6 \mathrm{H} \quad 0.197515$
$\begin{array}{lll}7 & \mathrm{C} & -0.418047\end{array}$
$8 \mathrm{H} \quad 0.221926$
$9 \mathrm{H} \quad 0.218871$
$10 \mathrm{Cl}-0.090688$
$11 \mathrm{C}-0.485652$
$12 \mathrm{H} \quad 0.164372$
$13 \mathrm{H} \quad 0.169766$
$14 \mathrm{H} \quad 0.173049$
$\begin{array}{lll}15 & \mathrm{O} & -0.649181\end{array}$
$16 \mathrm{C} \quad 0.279200$
$\begin{array}{lll}17 & \mathrm{H} & 0.181929\end{array}$
$18 \mathrm{H} \quad 0.166746$
19 O -0.625401
$20 \mathrm{C}-0.177386$
$21 \mathrm{H} \quad 0.176006$
$\begin{array}{lll}22 & \mathrm{H} & 0.168538\end{array}$
$23 \mathrm{H} \quad 0.146988$

Sum of Mulliken charges $=0.00000$

Atomic charges with hydrogens summed into heavy atoms:$$
1
$$
1 C 0.030301
$2 \mathrm{H} \quad 0.000000$
$3 \mathrm{C} \quad 0.038965$
$4 \mathrm{H} \quad 0.000000$
$\begin{array}{lll}5 & \mathrm{C} & 0.309699\end{array}$
$6 \mathrm{H} \quad 0.000000$
$7 \mathrm{C} \quad 0.022750$
$8 \mathrm{H} \quad 0.000000$
$9 \mathrm{H} \quad 0.000000$
$10 \mathrm{Cl}-0.090688$
$11 \mathrm{C} \quad 0.021535$ 
$12 \mathrm{H} \quad 0.000000$

$13 \mathrm{H} \quad 0.000000$

$\begin{array}{lll}14 \mathrm{H} & 0.000000\end{array}$

$\begin{array}{lll}15 & \mathrm{O} & -0.649181\end{array}$

$16 \mathrm{C} \quad 0.627874$

$17 \mathrm{H} \quad 0.000000$

$\begin{array}{lll}18 \mathrm{H} & 0.000000\end{array}$

$\begin{array}{lll}19 & \mathrm{O} & -0.625401\end{array}$

$20 \mathrm{C} \quad 0.314147$

$21 \mathrm{H} \quad 0.000000$

$22 \mathrm{H} \quad 0.000000$

$23 \mathrm{H} \quad 0.000000$

Sum of Mulliken charges $=0.00000$

Electronic spatial extent $(\mathrm{au}):<\mathrm{R} * * 2>=2840.5995$

Charge $=0.0000$ electrons

Dipole moment (field-independent basis, Debye):

$\mathrm{X}=-1.0693 \mathrm{Y}=2.0849 \mathrm{Z}=0.0577$ Tot $=2.3438$

Quadrupole moment (field-independent basis, Debye-Ang):

$\mathrm{XX}=-68.3454 \mathrm{YY}=-69.0065 \quad \mathrm{ZZ}=-68.8861$

$\mathrm{XY}=7.2753 \mathrm{XZ}=-0.5100 \mathrm{YZ}=-2.6136$

Traceless Quadrupole moment (field-independent basis, Debye-Ang):

$\mathrm{XX}=0.4006 \mathrm{YY}=-0.2605 \mathrm{ZZ}=-0.1401$

$\mathrm{XY}=7.2753 \quad \mathrm{XZ}=-0.5100 \quad \mathrm{YZ}=-2.6136$

Octapole moment (field-independent basis, Debye-Ang $* * 2$ ):

$\mathrm{XXX}=-63.0773 \mathrm{YYY}=-7.9068 \mathrm{ZZZ}=0.2662 \mathrm{XYY}=-3.0945$

$\mathrm{XXY}=12.8266 \mathrm{XXZ}=-1.8114 \mathrm{XZZ}=2.6661 \mathrm{YZZ}=-1.4017$

$\mathrm{YYZ}=6.9345 \mathrm{XYZ}=7.4566$

Hexadecapole moment (field-independent basis, Debye-Ang**3):

$X X X X=-2725.0477 \mathrm{YYYY}=-745.5269 \mathrm{ZZZZ}=-171.3538 \mathrm{XXXY}=123.8332$

$\mathrm{XXXZ}=10.7057 \mathrm{YYYX}=-13.9060 \mathrm{YYYZ}=-1.4807 \mathrm{ZZZX}=2.4679$

$Z Z Z Y=3.3845 X X Y Y=-582.1293 X X Z Z=-491.0649 \mathrm{YYZZ}=-148.8259$

$\mathrm{XXYZ}=-10.9552 \mathrm{YYXZ}=-8.2708 \mathrm{ZZXY}=10.2365$

$\mathrm{N}-\mathrm{N}=6.124168047588 \mathrm{D}+02 \mathrm{E}-\mathrm{N}=-3.307848684648 \mathrm{D}+03 \quad \mathrm{KE}=8.806829991341 \mathrm{D}+02$

1I1IUNPC-UNKISPIRHF|6-31G(d)|C7H13Cl1O2IPCUSERI23-Apr-2006|0||\# HF/6-3

1G(D) GEOM=CONNECTIVITY॥Title Card Required $\| 0,1 \mathrm{IC}, 0,-1.49207955,-0.2$

$6503409,0.89251136|\mathrm{H}, 0,-1.88507955,-0.75203409,0.00251136| \mathrm{C}, 0,-0.17507$

$955,-0.32703409,1.15551136|\mathrm{H}, 0,0.22592045,0.16596591,2.03951136| \mathrm{C}, 0,0$.

$83092045,-1.02403409,0.28051136 \mid \mathrm{H}, 0,0.36292045,-1.45703409,-0.61248864$

IC, $0,-2.49107955,0.43996591,1.75351136 \mid \mathrm{H}, 0,-2.03507955,0.87896591,2.64$

$651136|\mathrm{H}, 0,-3.27607955,-0.25303409,2.06851136| \mathrm{Cl}, 0,-3.24907955,1.75096$

$591,0.82651136 \mid \mathrm{C}, 0,1.51192045,-2.14603409,1.05651136 \mathrm{lH}, 0,0.79192045,-2$ $.90403409,1.38251136 \mathrm{H}, 0,2.03892045,-1.76103409,1.93751136 \mid \mathrm{H}, 0,2.26792$ $045,-2.63303409,0.43051136|\mathrm{O}, 0,1.83992045,-0.08003409,-0.07848864| \mathrm{C}, 0$, $1.52392045,0.62796591,-1.27148864 \mid \mathrm{H}, 0,0.50492045,1.02696591,-1.2274886$ $4|\mathrm{H}, 0,2.20192045,1.48496591,-1.35548864| \mathrm{O}, 0,1.63892045,-0.19103409,-2$. $43148864|\mathrm{C}, 0,2.98992045,-0.54703409,-2.69248864| \mathrm{H}, 0,3.01292045,-1.1530$ $3409,-3.60248864|\mathrm{H}, 0,3.40192045,-1.14103409,-1.87148864| \mathrm{H}, 0,3.59992045$ $, 0.34696591,-2.85648864 \|$ Version $=x 86-$ Win32-G03RevB $.05 \mid$ State $=1-A \mid H F=-88$ $1.8063034|\mathrm{RMSD}=8.022 \mathrm{e}-006| \mathrm{Dipole}=0.5038121,-0.6479744,0.4202514 \mid \mathrm{PG}=\mathrm{C} 01$ [X(C7H13Cl1O2)]॥@

DESK: A WASTEBASKET WITH DRAWERS.

Job cpu time: 0 days 0 hours 2 minutes 9.0 seconds.

File lengths (MBytes): RWF $=28 \mathrm{Int}=0 \mathrm{D} 2 \mathrm{E}=0 \mathrm{Chk}=8 \mathrm{Scr}=1$

Normal termination of Gaussian 03 at Sun Apr 23 11:17:12 2006. 